\title{
A Phase I Cultural Resources Survey of the Orbit Pipeline Project JefFerson, LiberTy, AND Chambers Counties, TeXas
}

Prepared For:

\author{
Energy Transfer Company \\ Prepared by: \\ Jennifer Cochran, MA, RPA \\ Abby Peyton, MA, RPA \\ Karissa Basse, Ph.D.

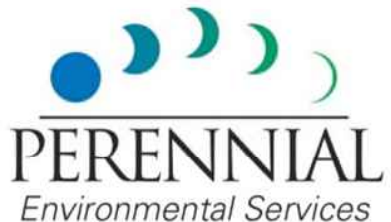

Principal Investigator - Jennifer Cochran, MA, RPA

Perennial Environmental Services, LLC

5424 W. US Hwy 290, Suite 208

Austin, TX 78735

jcochran@perennialenv.com

512-358-0345

Lead Agency:

United States Army Corps of Engineers Galveston District

SWG-2019-00074

Texas Antiquities Permit \#8690

Perennial Report No. 19-008

March 2019

(Revised January 2020) 


\section{MANAGEMENT SUMMARY}

Energy Transfer Company (ETC) is proposing to construct the Orbit Pipeline Project (Project) located in Jefferson, Liberty, and Chambers counties, Texas. The Project consists of approximately 68.7 miles (mi) (110.6 kilometer [km]) of new 20.0-inch (in) (50.8-centimeter [cm]) diameter pipeline that will be used to transmit ethane and propane. The Project is located within the jurisdictional boundary of the United States Army Corps of Engineers (USACE) - Galveston District.

At the request of ETC, Perennial Environmental Services, LLC (Perennial) conducted an intensive Phase I cultural resources investigation for the proposed Project to comply with anticipated USACE permitting requirements. Archaeological investigations for the Project were conducted in accordance with Section 106 of the National Historic Preservation Act (NHPA) and Texas State Historical Preservation Office (SHPO) standards. Additionally, the Project traverses several discontinuous publicly-owned tracts that fall under the jurisdiction of the Antiquities Code of Texas (Code). The results of survey investigations conducted under Texas Antiquities Permit (TAP) \#8690 (issued December 17, 2018, and amended on August 30, 2019) across six discontinuous publicly -owned tracts are also presented herein.

Consistent with USACE application requirements, and in accordance with Section 106 of the NHPA of 1966, as amended (36 CFR 800) and the Code, the proposed Project must make a reasonable and good faith effort to identify historic properties within the Project Area of Potential Effect (APE) and to take into account any potential effects, direct or indirect, the proposed undertaking could have on properties listed or considered eligible for listing in the National Register of Historic Places (NRHP) or for designation as a State Antiquities Landmark (SAL), as warranted.

As the Project footprint was not finalized at the time of field investigations, survey efforts were concentrated within the vicinity of delineated wetland and waterbody features along the length of the route within a 300.0-foot- (ft-) (91.4-meter- [m-]) wide Environmental Survey Area (ESA). The anticipated depths of impact for the Project will range from 4.0 to $7.0 \mathrm{ft}$. (1.2 to $2.1 \mathrm{~m}$ ) along the pipeline centerline, with limited deeper impacts at horizontal directional drill (HDD) and bore locations, including Cow Island Bayou, Hillebrandt Bayou, Lower Neches Valley River Authority canals, Nolte Canal, the Trinity River, Turtle Bayou, Whites Bayou, Willow Marsh Bayou, and public road crossings. The anticipated depths of impact for temporary workspace areas within the Project APE corridor would not exceed 0.6 to $1.0 \mathrm{ft}$. $(0.1$ to $0.3 \mathrm{~m})$. The overall APE for direct effects for the Project measured 2307.19 acres (ac) (933.68 ha), while APE for Code-permitted tracts totaled 210.0 ac (85.06 ha). As presented herein, the Project ESA is coterminous with the Project APE, which is also referred to as the USACE permit areas. Only the areas adjacent to the USACE permit areas and the entirety of Code-permitted areas were surveyed for cultural resources. 
Jennifer Cochran served as the Principal Investigator, and field efforts were conducted by Sarah Boudreaux, Rafael Cortez, Wyatt Ellison, Rachel Kelley, William Kinkner, Colene Knaub, Jonathan Laird, Alejandro Martinez, and Thomas Ross across multiple field mobilizations between October 1, 2018, and November 12, 2019.

Investigations included an archival background review and intensive pedestrian surveys augmented by shovel testing in the vicinity of delineated wetland and waterbody features. Archival research determined that there are no previously recorded sites within, or directly adjacent to the Project APE, and approximately 32.7\% of the Project APE has been previously surveyed for cultural resources. However, many of these surveys are outdated and do not meet modern survey standards. Additionally, many of these previously surveys were conducted for USACE-permitted projects, and the entire Project was not surveyed for the presence of cultural resources. As such, all areas surrounding identified wetland and waterbody for this Project were surveyed.

Perennial biologists delineated a total of 490 wetland areas. Of the 490 wetlands identified within the Project APE, 290 were characterized as palustrine emergent (PEM) wetlands, 106 were characterized as palustrine forested (PFO) wetlands, 4 were classified as PFO-Cypress wetlands, and 90 were characterized as palustrine scrub-shrub (PSS) wetlands. Perennial biologists also identified 270 waterbodies that ranged from perennial streams to ephemeral streams. Of the 270 waterbodies delineated, 36 were classified as having perennial flow, 66 were classified as having intermittent flow, and 148 were classified as ephemeral flow. Additionally, 20 open water features were delineated and classified as manmade ponds.

Survey efforts were concentrated in the vicinity of these features where land access was voluntarily granted in accordance with a scope of work for the Project approved by the USACE on August 28, 2018, and the Texas Historical Commission (THC) on October 1, 2018. Following the approval of the scope of work, ETC extended the eastern terminus of the Project to the western bank of the Neches River, and other minor reroutes were also implemented. Perennial applied the approved survey methodology to all new Project components.

For Code-permitted tracts, surveys were conducted across the entire length of the Project APE corridor in accordance with a stand-alone scope submitted to the THC on December 14, 2018, and amended August 30, 2019. Additionally, following the receipt of TAP\#8690, the proposed Project was routed onto two additional publicly-owned tracts belonging to the Texas Department of Corrections (DOC) - Stiles Unit and Jefferson County. The TAP \#8690 was revised and amended on August 30, 2019. Following this August 30, 2019 amendment, ETC added additional route options across the DOC - Stiles Unit tract. This additional mileage was surveyed using the same methods as stated in the previously presented revised TAP scope of work for this property. 
In all, the survey investigations included the excavation of a total of 1,250 shovel tests of which 1,171 shovel tests were excavated within the Project APE. The remaining 79 shovel tests were excavated outside of USACE permit areas but within the Project workspace. For the purposes of this report, only investigations within the Project APE will be discussed.

Survey investigations within the Project APE resulted in entirely negative findings. No archeological sites were encountered within the survey areas reported herein. Additionally, no historic standing structures or landscape features such as historic-age canals were observed with any USACE permit areas. Overall, the surveys documented predominately inundated landscapes with a low probability for intact cultural resources. Numerous existing pipeline corridors and modern canal features are traversed by the Project. While some of the modern canals could be historic in nature or connected to a greater network of irrigation features used historically to supply agricultural crops with water, it is important to note that the majority of the waterways associated with these features will be bypassed via bore/HDD. As such, any impacts to these waterbodies as well as the associated canal structure will be entirely avoided. Additionally, all visual impacts from the proposed pipeline corridor will be temporary in nature.

To date, field surveys have been completed for all accessible wetland and waterbody features along the pipeline route, as well as the total length of the Project survey corridor across all Codepermitted tracts. Prior to the beginning of November 2019, field surveys had not been conducted along the eastern banks and associated bottomlands of the Trinity River due to multiple flooding events that resulted in heavy inundation beginning in September 2018 which prevented access or survey investigations of any kind. Additionally, field surveys did not occur along portions of the Project containing denied landowner permissions.

On November 6, 2019, a survey crew was able to access previously inaccessible areas associated with the Trinity River due to several months of normalized weather conditions throughout portions of Eastern Texas. Even under normal conditions, large portions of this area remain constantly inundated due to strong hydrological influences and the geomorphic position of the landscape. However, crews were able to traverse inundated areas by foot to access portions of the Project located immediately adjacent to the Trinity River. While the area located immediately adjacent to the Trinity River was not inundated at the time of survey, wetlands with strong hydrological indicators still dominate the landscape.

Of the 760 delineated wetland and waterbody features, 74 features were originally not surveyed for cultural resources due to restricted land access including denied landowner permissions and significant inundation. Of these 74 features, 38 feature locations ( 27 streams and 42 wetlands) will be bypassed via horizontal directional drill (HDD) or bore trenchless construction methods resulting in no impacts to these features. The remaining five features (including multiple crossing locations of the same feature) are located along the eastern banks of the Trinity River. While these features were surveyed for cultural resources with negative findings, the presence of buried deposits exists within the vicinity of these features. However, access to these features with heavy 
machinery is not feasible due to the remote location and constant hydrological influences (e.g. inundation and saturation) associated with the floodplain setting of the Trinity River. As such, these five features are proposed to be monitored by a qualified Archeological Monitor during construction efforts. Appendix $\mathbf{C}$ provides each wetland and waterbody feature crossed by the Project with management recommendations and associated comments, while Appendix F provides a Cultural Monitoring Plan to evaluate the five features that will not be avoided during construction efforts. Appendix $\mathbf{F}$ also includes a table in response to a letter issued by the USACE Staff Archeologist, Mr. Jerry Androy, on May 17, 2019, indicating that the 74 aforementioned permit areas associated with the Project would require cultural resources investigations. The table lists each permit area, the reason surveys were not originally conducted, and justification for/against the need for monitoring.

Based on the results of the survey effort reported here, no cultural resources will be affected by any construction activities within the Project APE. Aside from Cultural Monitoring at five features (including multiple crossing locations of the same feature) within the Trinity River floodplain, it is Perennial's opinion that no further cultural resources investigations are warranted for the Project. Should archaeological remains be encountered during construction, work in the immediate area will cease, and a qualified archaeologist will be called upon to evaluate the remains and provide recommendations for how to manage the resources under the State's Historic Preservation Plan. 


\section{TABLE OF CONTENTS}

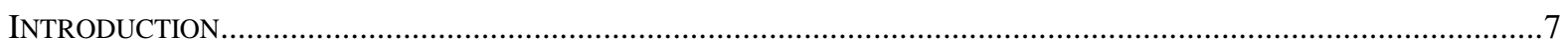

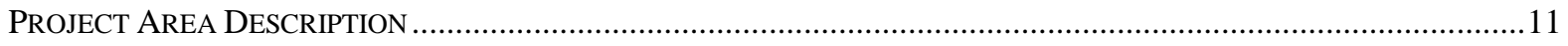

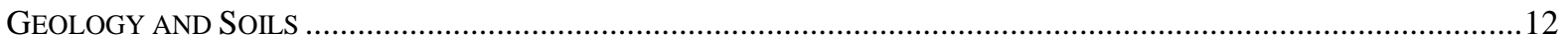

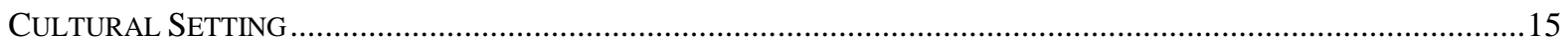

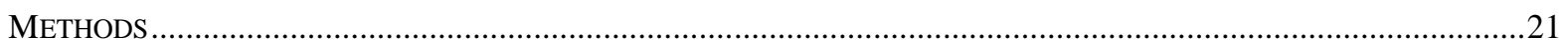

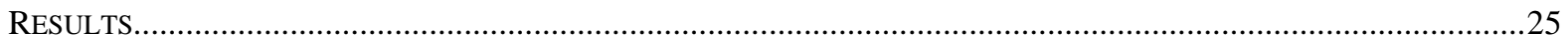

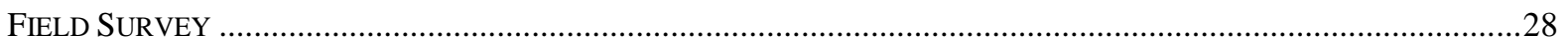

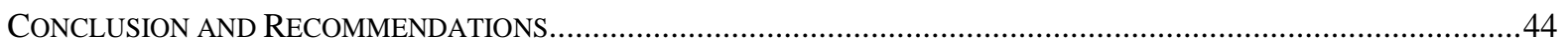

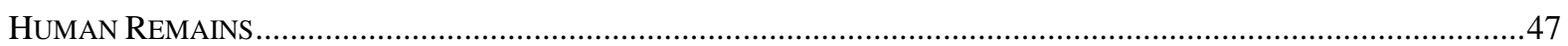

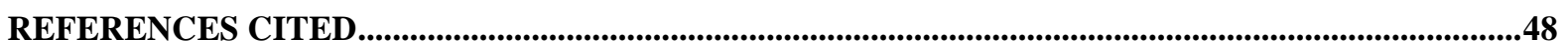

APPENDIX A: AGENCY CORRESPONDENCE

APPENDIX B: PROJECT MAPPING EXHIBITS

APPENDIX C: WETLAND AND WATERBODY DATA TABLES

APPENDIX D: SHOVEL TEST DATA FOR USACE PERMIT AREAS

APPENDIX E: SHOVEl TeST DATA FOR CODE-PERMitTED TRACTS

APPENDIX F: ARCHEOLOGICAL MONITORING PLAN

APPENDIX G: UNANTICIPATED DISCOVERY PLAN

\section{FIGURES}

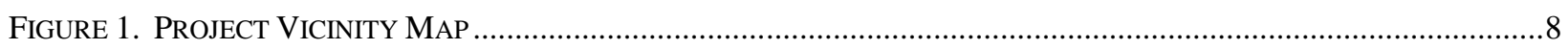

FIGURE 2. TYPICAL VIEW OF PROJECT APE WITHIN EXISTING CORRIDORS ..................................................................11

FIGURE 3. VIEW OF EXPANSIVE INUNDATED AREAS CHARACTERISTIC OF THE PROJECT APE .....................................12

FIGURE 4. VIEW OF FLOODED ACCESS POINT ON THE EASTERN SIDE OF THE TRINITY RIVER.......................................24

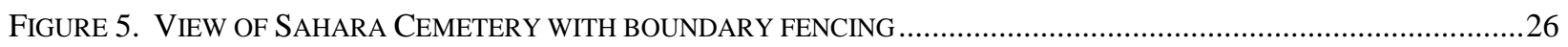

FiguRE 6. VIEW OF NON-INUNDATED AREAS IN TRINITY RIVER BOTTOMLANDS WITHIN PROJECT APE, FACING

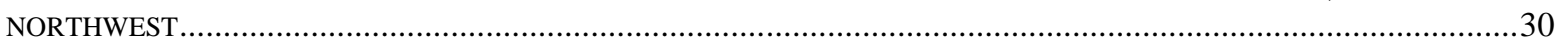

FIGURE 7. VIEW OF INUNDATED AREAS IN TRINITY RIVER BOTTOMLANDS WITHIN PROJECT APE, FACING SOUTHEAST

FIGURE 8. VIEW OF NON-INUNDATED AREAS IN TRINITY RIVER BOTTOMLANDS WITHIN PROJECT APE, WEST .............31

FIGURE 9. VIEW OF NON-INUNDATED AREAS IN TRINITY RIVER BOTTOMLANDS WITHIN PROJECT APE, FACING SOUTH

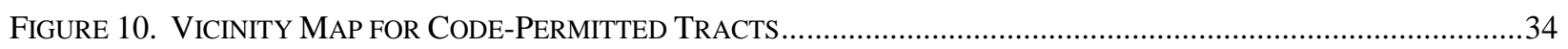

FIGURE 11. OVERVIEW OF JEFFERSON COUNTY TRACTS, FACING NORTHWEST …….....................................................

Figure 12. OVERVIEW OF JEFFERSON COUNTY TRACT (ADJACENT TO DOC - STILES UNIT TRACT), FACING WEST ...35

FIGURE 13. OVERVIEW OF CITY OF BEAUMONT TRACTS, FACING NORTHEAST........................................................36 


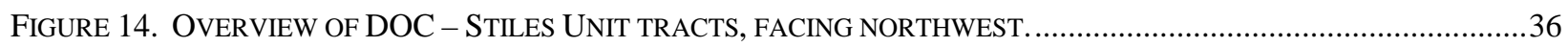

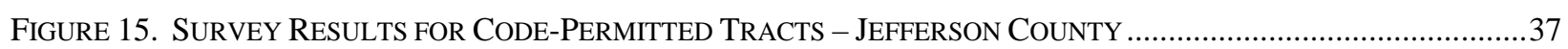

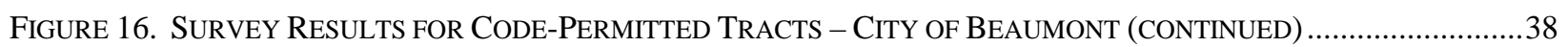

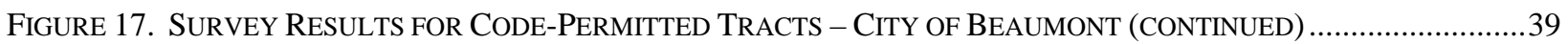

Figure 18. Survey Results For Code-PERmitTed Tracts, DOC - Stiles Unit AND JeFFERSON COUNTY..........40

Figure 19. Survey Results FOr CODE-PERmitTed TraCts, DOC - Stiles Unit AND JEFFERSON COUNTY..........41

FIGURE 20. SurVEy RESUlts FOR CODE-PERMitTED TRACTS, DOC - STILES UNIT AND JEFFERSON COUNTY..........42

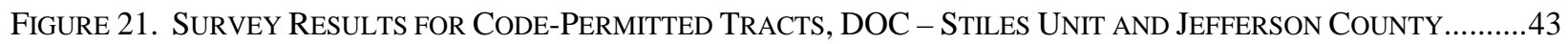

\section{TABLES}

TABLE 1 SOIL MAPPING UNITS CORRESPONDING TO ABBOTT'S GEOARCHEOLOGICAL MODEL (2001) .......................13

TABLE 2. SITES PREVIOUSLY RECORDED WITHIN THE 1.0 MI BACKGROUND REVIEW RADIUS .................................25

TABLE 3. CEMETERIES RECORDED WITHIN THE 1.0 MI BACKGROUND REVIEW RADIUS ........................................26

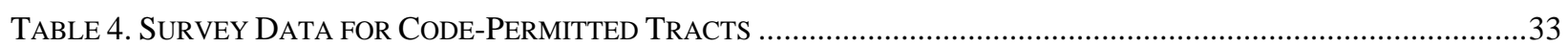




\section{INTRODUCTION}

Energy Transfer Company (ETC) is proposing to construct the Orbit Pipeline Project (Project) located in Jefferson, Liberty, and Chambers counties, Texas. The Project consists of approximately 68.7 miles (mi) (110.6 kilometer [km]) of new 20.0-inch (in) (50.8-centimeter [cm]) diameter pipeline that will be used to transmit ethane and propane. The Project is located within the jurisdictional boundary of the United States Army Corps of Engineers (USACE) - Galveston District.

At the request of ETC, Perennial Environmental Services, LLC (Perennial) conducted an intensive Phase I cultural resources investigation for the proposed Project to comply with anticipated USACE permitting requirements. Archaeological investigations for the Project were conducted in accordance with Section 106 of the National Historic Preservation Act (NHPA) and Texas State Historical Preservation Office (SHPO) standards. Additionally, the Project traverses several discontinuous publicly-owned tracts that fall under the jurisdiction of the Antiquities Code of Texas (Code). The results of survey investigations conducted under Texas Antiquities Permit (TAP) \#8690 (issued December 17, 2018, and amended on August 30, 2019) across six discontinuous publicly-owned tracts are also presented herein.

Consistent with USACE application requirements, and in accordance with Section 106 of the NHPA of 1966, as amended (36 CFR 800) and the Code, the proposed Project must make a reasonable and good faith effort to identify historic properties within the Project Area of Potential Effect (APE) and to take into account any potential effects, direct or indirect, the proposed undertaking could have on properties listed or considered eligible for listing in the National Register of Historic Places (NRHP) or for designation as a State Antiquities Landmark (SAL), as warranted.

As the Project footprint was not finalized at the time of field investigations, survey efforts were concentrated within the vicinity of delineated wetland and waterbody features along the length of the route within a 300.0-foot- (ft-) (91.4-meter- [m-]) wide Environmental Survey Area (ESA). The anticipated depths of impact for the Project will range from 4.0 to $7.0 \mathrm{ft}$. (1.2 to $2.1 \mathrm{~m}$ ) along the pipeline centerline, with limited deeper impacts at horizontal directional drill (HDD) and bore locations, including Cow Island Bayou, Hillebrandt Bayou, Lower Neches Valley River Authority canals, Nolte Canal, the Trinity River, Turtle Bayou, Whites Bayou, Willow Marsh Bayou, and public road crossings. The anticipated depths of impact for temporary workspace areas within the Project APE corridor would not exceed 0.6 to $1.0 \mathrm{ft}$. $(0.1$ to $0.3 \mathrm{~m})$. The overall APE for direct effects for the Project measured 2307.19 acres (ac) (933.68 ha), while APE for Code-permitted tracts totaled 210.0 ac (85.06 ha). As presented herein, the Project ESA is coterminous with the Project APE, which is also referred to as the USACE permit areas. Only the areas adjacent to the USACE permit areas and the entirety of Code-permitted areas were surveyed for cultural resources. 


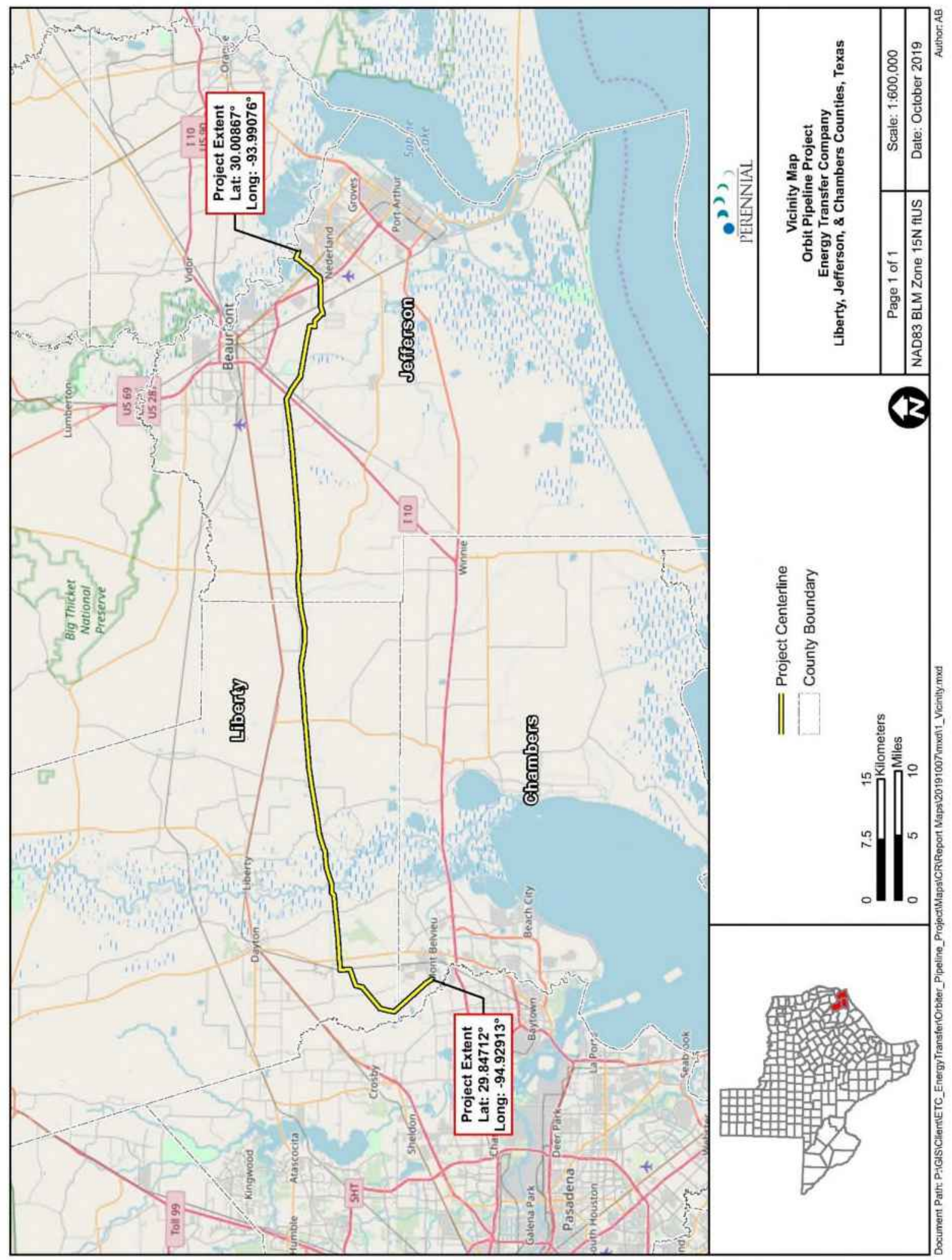

Figure 1. Project Vicinity Map 
Jennifer Cochran served as the Principal Investigator, and field efforts were conducted by Sarah Boudreaux, Rafael Cortez, Wyatt Ellison, Rachel Kelley, William Kinkner, Colene Knaub, Jonathan Laird, Alejandro Martinez, and Thomas Ross across multiple field mobilizations between October 1, 2018, and November 12, 2019.

Investigations included an archival background review and intensive pedestrian surveys augmented by shovel testing in the vicinity of delineated wetland and waterbody features. Archival research determined that there are no previously recorded sites within, or directly adjacent to the Project APE, and approximately 32.7\% of the Project APE has been previously surveyed for cultural resources. However, many of these surveys are outdated and do not meet modern survey standards. Additionally, many of these previously surveys were conducted for USACE-permitted projects, and the entire Project was not surveyed for the presence of cultural resources. As such, all areas surrounding identified wetland and waterbody for this Project were surveyed.

Perennial biologists delineated a total of 490 wetland areas. Of the 490 wetlands identified within the Project APE, 290 were characterized as palustrine emergent (PEM) wetlands, 106 were characterized as palustrine forested (PFO) wetlands, 4 were classified as PFO-Cypress wetlands, and 90 were characterized as palustrine scrub-shrub (PSS) wetlands. Perennial biologists also identified 270 waterbodies that ranged from perennial streams to ephemeral streams. Of the 270 waterbodies delineated, 36 were classified as having perennial flow, 66 were classified as having intermittent flow, and 148 were classified as ephemeral flow. Additionally, 20 open water features were delineated and classified as manmade ponds.

Survey efforts were concentrated in the vicinity of these features where land access was voluntarily granted in accordance with a scope of work for the Project approved by the USACE on August 28, 2018, and the Texas Historical Commission (THC) on October 1, 2018. Following the approval of the scope of work, ETC extended the eastern terminus of the Project to the western bank of the Neches River, and other minor reroutes were also implemented. Perennial applied the approved survey methodology to all new Project components.

For Code-permitted tracts, surveys were conducted across the entire length of the Project APE corridor in accordance with a stand-alone scope submitted to the THC on December 14, 2018, and amended August 30, 2019. Additionally, following the receipt of TAP\#8690, the proposed Project was routed onto two additional publicly-owned tracts belonging to the Texas Department of Corrections (DOC) - Stiles Unit and Jefferson County. The TAP \#8690 was revised and amended on August 30, 2019. Following this August 30, 2019, amendment, ETC added additional route options across the DOC - Stiles Unit tract. This additional mileage was surveyed using the same methods as stated in the previously presented revised TAP scope of work for this property. 
In all, the survey investigations included the excavation of a total of 1,250 shovel tests of which 1,171 shovel tests were excavated within the Project APE. The remaining 79 shovel tests were excavated outside USACE permit areas but within the Project workspace. For the purposes of this report, only investigations within the Project APE will be discussed.

Survey investigations within the Project APE resulted in entirely negative findings. No archeological sites were encountered within the survey areas reported herein. Additionally, no historic standing structures or landscape features such as historic-age canals were observed with any USACE permit areas. Overall, the surveys documented predominately inundated landscapes with a low probability for intact cultural resources. Numerous existing pipeline corridors and modern canal features are traversed by the Project. While some of the modern canals could be historic in nature or connected to a greater network of irrigation features used historically to supply agricultural crops with water, it is important to note that the majority of the waterways associated with these features will be bypassed via bore/HDD. As such, any impacts to these waterbodies as well as the associated canal structure will be entirely avoided. Additionally, all visual impacts from the proposed pipeline corridor will be temporary in nature.

Should cultural materials be encountered during this phase of the Project, then the Archeological Monitor would follow the procedures outlined in the Cultural Monitoring Plan, which would require construction to stop in the immediate area of discovery, and initiation of notification protocols needed to ensure the find is adequately evaluated in accordance with Section 106 of the NHPA. Details of the Archeological Monitoring Plan are presented in Appendix F.

Project records associated with Code-permitted tracts will be curated at the Texas Archeological Research Laboratory (TARL), while all original records for non-Code-permitted portions of the Project will be permanently housed at Perennial's laboratory in Austin. Copies of the records will be provided electronically to ETC.

Agency correspondence for the Project is presented in Appendix A. Mapping exhibits for the Project APE are provided in Appendix B, and corresponding data tables detailing the level of effort for each delineated wetland or waterbody feature is provided in Appendix C. Shovel test data for the USACE permit areas are provided in Appendix D, while shovel test data for the Codepermitted tracts are provided in Appendix E. The Archeological Monitoring Plan is presented in Appendix F and the Unanticipated Discovery Plan is provided in Appendix G. 


\section{Project Area Description}

The Project traverses a combination of highly developed oil and gas landscapes, expansive lowland agricultural fields that have been heavily modified to facilitate rice production, and pockets of dense bottomland forests (Figures 2 and 3). Many of the natural waterways have been channelized for flood control and irrigation purposes resulting in the alteration of natural levee and terrace formations.

ETC has attempted to minimize impacts to wetland and waterbody features to the greatest extent practicable by co-locating a majority of the overall route with the existing Energy Transfer Mariner Pipeline constructed in 2014. Additionally, the HDD or bore installation technique will be implemented to avoid impacts to larger waterbody features, namely Cow Island Bayou, Hillebrandt Bayou, Lower Neches Valley River Authority canals, Nolte Canal, the Trinity River, Turtle Bayou, Whites Bayou, and Willow Marsh Bayou.

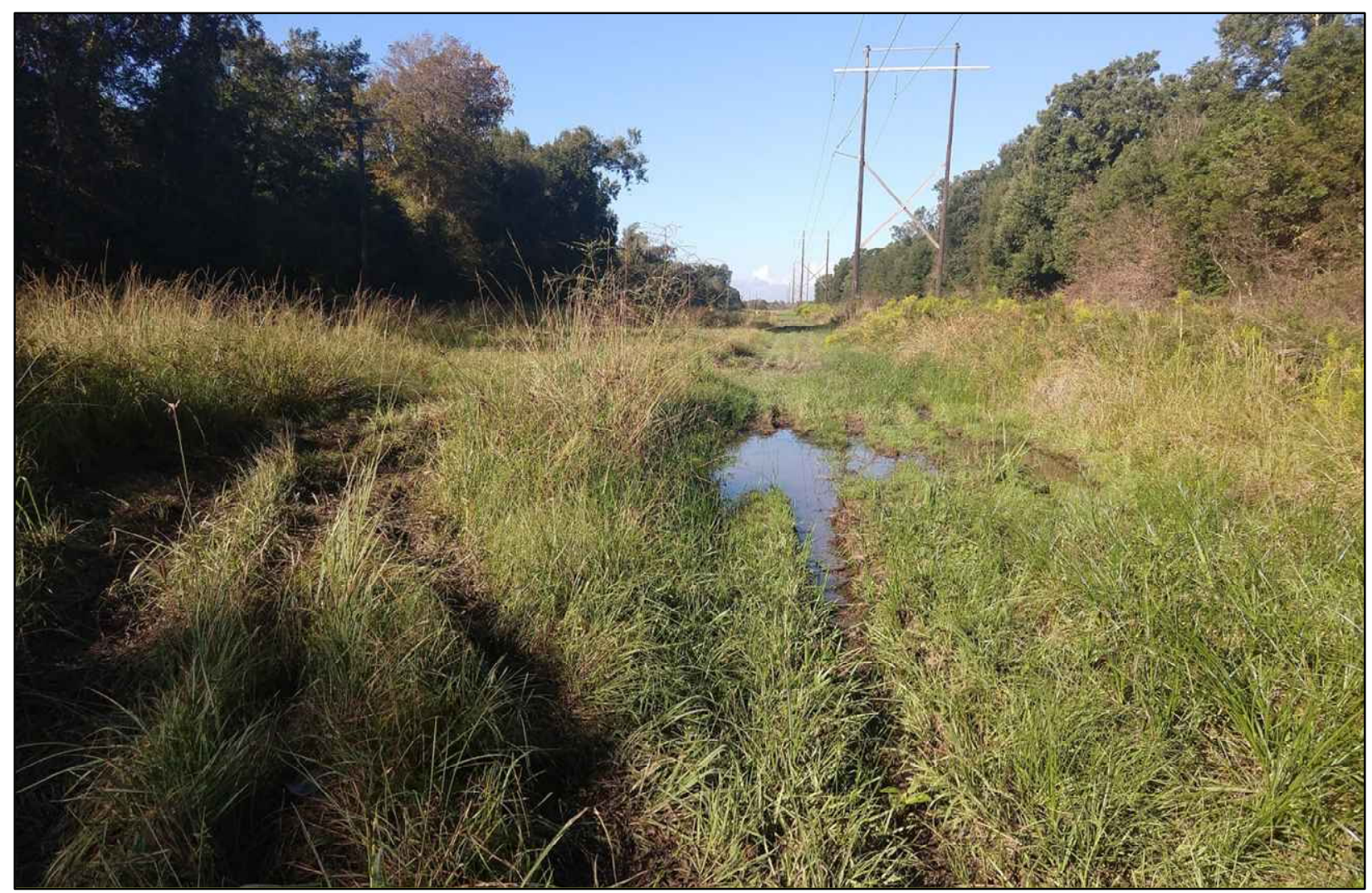

Figure 2. Typical view of Project APE within existing corridors 


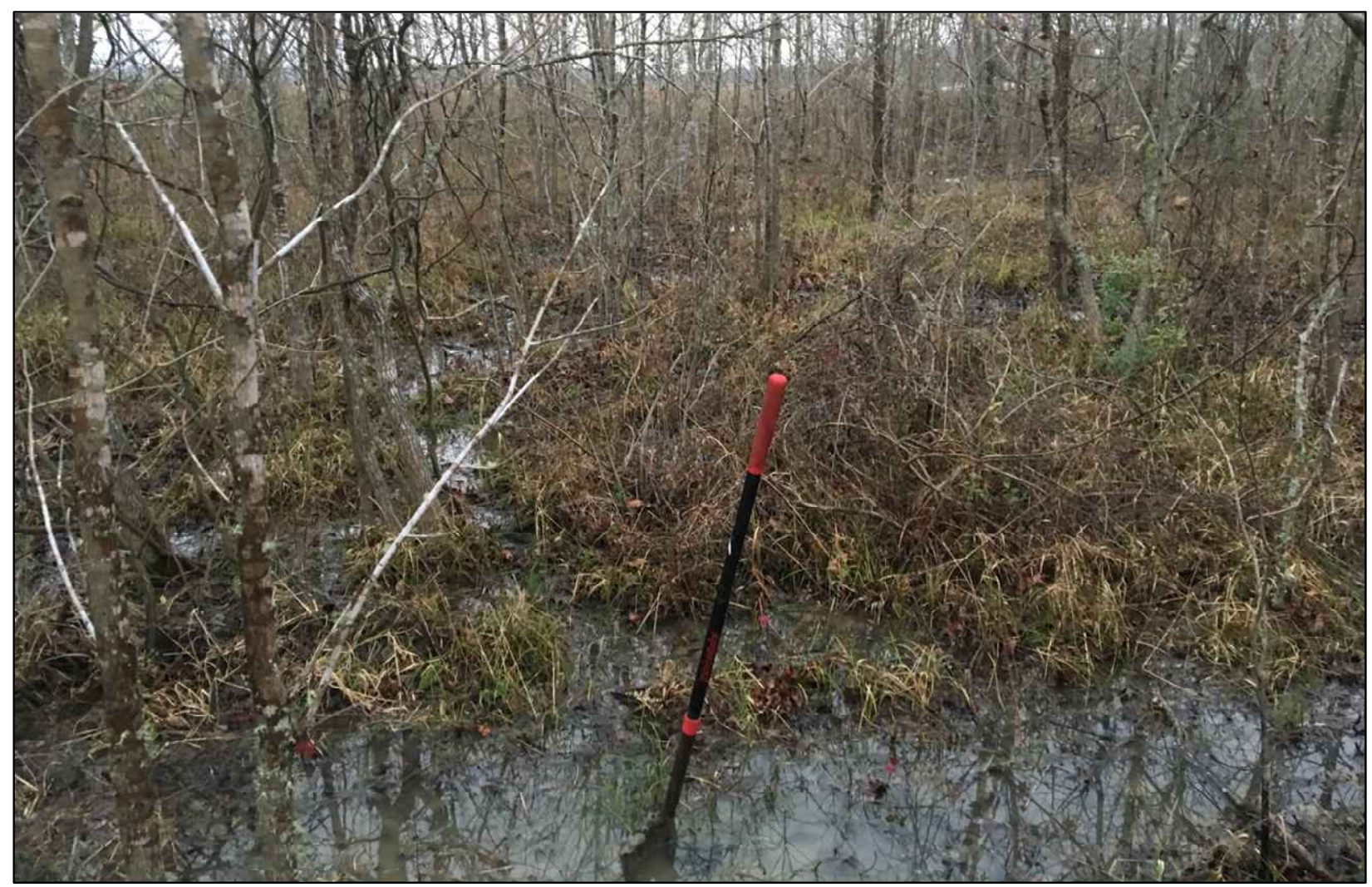

Figure 3. View of expansive inundated areas characteristic of the Project APE

\section{ENVIRONMENTAL SETTING}

The Project APE is located within the Northern Humid Gulf Coastal Prairies of the Western Gulf Coastal Plain ecoregion. The Western Gulf Coastal Plains ecoregion is a relatively flat expanse of grassland heavily utilized to grow rice, grain, cotton and soybeans, and for oil and gas production in recent decades (Griffith et al. 2007, 73).

Native vegetation includes clusters of hardwoods including Sweetgum, Sugarberry, and Loblolly pine interspersed among grasslands of bluestem, switchgrass, and yellow Indiangrass (Griffith et al. 2007, 74). The invasive species Chinese tallow accounts for a large percentage of the vegetation in this area (Griffith et al. 2007, 74-75). This region has an extended history of modification and the majority of the area has been converted to cropland, rangeland, and urban and industrial uses. As the soils are poorly draining, and the region remains inundated for extended periods, expansive networks of drainage channels and canals crisscross the landscape (Griffith 2007, 74).

\section{GEOLOGY AND SOILS}

Geologically, the Project APE is intermittently underlain by the Beaumont Formation (Qbs and Qbc) (USGS 2019). The Beaumont Formation (Qbs) consists of sandy deposits concentrated on point-bars, natural levee ridges, and poorly defined meander-belt ridge and pimple mounds in the 
southwestern extent of the Project. The Beaumont Formation (Qbc) consists of predominantly clay deposits in flood basins, coastal lakes, and former stream channels.

Holocene-age alluvial deposits (Qal) are located in only two portions of the Project area: in the vicinity of the Trinity River, and at the eastern terminus adjacent to the Neches River. These deposits are comprised of sand, silt, and clay, and were found to consist of expansive inundated lowland areas.

According to the United States Department of Agriculture (USDA) National Resources Conservation Service (NRCS) Web Soil Survey for Chambers, Liberty, and Jefferson counties, the Project lies within 39 soil mapping units. Of these, seven soils units are included in James Abbott's analysis of the geoarcheological potential of soil units within the greater Houston area (2001) (Table 1). Two soils units (Voss and Kaman) are referenced as having a high, or moderate to high geoarcheological potential, while the remaining soil units have a low geoarcheological potential. Both the Voss and Kaman soils units are situated in the vicinity of the Trinity River.

\begin{tabular}{|c|c|c|c|}
\hline \multicolumn{4}{|c|}{$\begin{array}{l}\text { Table } 1 \\
\text { Soil Mapping Units corresponding to Abbott's geoarcheological model (2001) }\end{array}$} \\
\hline $\begin{array}{l}\text { Mapping Unit } \\
\text { (Map Symbol) }\end{array}$ & Texture and Drainage & $\begin{array}{c}\text { General } \\
\text { Location }\end{array}$ & $\begin{array}{l}\text { Geoarcheological } \\
\text { Potential }\end{array}$ \\
\hline $\begin{array}{l}\text { Beaumont clay, } 0 \text { to } 1 \\
\text { percent slopes (BeaA) }\end{array}$ & $\begin{array}{l}\text { The Beaumont series consists of very deep, } \\
\text { poorly drained, very slowly permeable soils on } \\
\text { coastal plains. These nearly level soils formed } \\
\text { in clayey sediments on the Beaumont } \\
\text { Formation of the Pleistocene Age. Slopes } \\
\text { range from } 0 \text { to } 1 \text { percent. }\end{array}$ & $\begin{array}{l}\text { Flats, Coastal } \\
\text { Plains }\end{array}$ & Low \\
\hline $\begin{array}{c}\text { Mocarey-Yeaton } \\
\text { complex, } 0 \text { to } 1 \\
\text { percent slopes (MofA) }\end{array}$ & $\begin{array}{l}\text { The Mocarey series consists of very deep, } \\
\text { moderately well drained, moderately slow } \\
\text { permeable soils. These nearly level soils } \\
\text { formed in loamy fluviomarine deposits of the } \\
\text { Beaumont Formation. Slope ranges from } 0 \text { to } 1 \\
\text { percent. } \\
\text { The Yeaton series consists of very deep, } \\
\text { moderately well drained soils. These nearly } \\
\text { level soils formed in loamy fluviomarine } \\
\text { deposits of the Beaumont Formation. These } \\
\text { soils have been leveled for rice irrigation. } \\
\text { Slope ranges from } 0 \text { to } 1 \text { percent. }\end{array}$ & $\begin{array}{l}\text { Flats, Coastal } \\
\text { Plains }\end{array}$ & Low \\
\hline $\begin{array}{c}\text { Voss sand, } 0 \text { to } 1 \\
\text { percent slopes, } \\
\text { occasionally flooded } \\
\text { (VorA) }\end{array}$ & $\begin{array}{l}\text { The Voss series consists of very deep, } \\
\text { moderately well drained, rapidly permeable } \\
\text { soils that formed from deep sandy sediments. } \\
\text { These nearly level to very gently sloping soils } \\
\text { are on flood plains. Slopes range from } 0 \text { to } 3 \\
\text { percent. }\end{array}$ & $\begin{array}{l}\text { River Valleys, } \\
\text { Coastal Plains }\end{array}$ & High \\
\hline
\end{tabular}


Table 1

Soil Mapping Units corresponding to Abbott's geoarcheological model (2001)

\begin{tabular}{|c|c|c|c|}
\hline $\begin{array}{l}\text { Mapping Unit } \\
\text { (Map Symbol) }\end{array}$ & Texture and Drainage & $\begin{array}{c}\text { General } \\
\text { Location }\end{array}$ & $\begin{array}{c}\text { Geoarcheological } \\
\text { Potential }\end{array}$ \\
\hline $\begin{array}{l}\text { Kaman clay, } 0 \text { to } 1 \\
\text { percent slopes, } \\
\text { frequently flooded } \\
\qquad(\text { KanA })\end{array}$ & $\begin{array}{l}\text { The Kaman series consists of very deep, } \\
\text { somewhat poorly drained, very slowly } \\
\text { permeable soils that formed in clayey } \\
\text { alluvium. These nearly level soils occur on } \\
\text { floodplains. Slope ranges from } 0 \text { to } 1 \text { percent. }\end{array}$ & $\begin{array}{l}\text { Flood Plains, } \\
\text { River Valleys }\end{array}$ & Moderate to High \\
\hline $\begin{array}{l}\text { Morey-Levac complex, } \\
0 \text { to } 1 \text { percent slopes } \\
\text { (MorA) }\end{array}$ & $\begin{array}{l}\text { The Morey series consists of very deep, } \\
\text { somewhat poorly drained very slowly } \\
\text { permeable soils. These nearly level soils } \\
\text { formed in loamy fluviomarine deposits of the } \\
\text { Beaumont Formation. Slope ranges from } 0 \text { to } 1 \\
\text { percent. } \\
\text { The Levac series consists of very deep, } \\
\text { somewhat poorly drained soils. These nearly } \\
\text { level soils formed in loamy fluviomarine } \\
\text { deposits of the Beaumont Formation. These } \\
\text { soils have been leveled for rice irrigation. } \\
\text { Slope ranges from } 0 \text { to } 1 \text { percent. }\end{array}$ & $\begin{array}{c}\text { Flats, Coastal } \\
\text { Plains }\end{array}$ & Low \\
\hline $\begin{array}{l}\text { Beaumont silty clay, } 0 \\
\text { to } 1 \text { percent slopes, } \\
\text { rarely flooded (BebA) }\end{array}$ & Beaumont- see above & $\begin{array}{c}\text { Flats, Coastal } \\
\text { Plains }\end{array}$ & Low \\
\hline $\begin{array}{l}\text { Beaumont-Urban land } \\
\text { complex, } 0 \text { to } 1 \\
\text { percent slopes (BecA) }\end{array}$ & $\begin{array}{l}\text { Beaumont- see above } \\
\text { Urban Land consists of very high runoff. }\end{array}$ & $\begin{array}{c}\text { Flats, Coastal } \\
\text { Plains }\end{array}$ & Low \\
\hline
\end{tabular}




\section{Cultural Setting}

\section{INTRODUCTION}

Based on technological and stylistic changes evident in the archeological record, the sequence of recognized cultural manifestations in the central and upper Texas coastal region has been traditionally divided into four periods: the Paleoindian period (ca. 11,500 to 8,000 B.P.), the Archaic period (8,000 to 2,000 B.P.), the Ceramic period (2,000 to 300 B.P.), and the Historic period (after 300 B.P.). These periods are further divided into finer-grained temporal divisions depending on often subtle changes in cultural practices as observed in the archaeological record.

\section{Paleoindian Period (CA. 11,500 to 8,000 B.P.)}

The character of the first human occupation of the coastal regions of Texas during the Paleoindian period is considered to be relatively sparse (Ricklis 2004, 184). While a number of Paleoindian artifacts - notably projectile points - have been encountered in the coastal region of Texas, none of these were identified in discrete Paleoindian contexts - the Paleoindian material was either found on the surface, or was mixed with later cultural material (Hester 1980, 4; Patterson 1980, 6; Ricklis 2004, 184). The seeming lack of contextualized Paleoindian cultural material identified to date in the Texas coast may be due to a combination of various site formation processes, such as the rise of sea levels in the Late Pleistocene, which inundated approximately 30 to $40 \mathrm{~km}$ of exposed coastline in the Texas coast (land that may have been utilized by Paleoindian peoples) (Aten 1983, 178-210). Holocene erosion and site burial by alluvium deposition may also have obscured or obliterated some Paleoindian sites (Abbott 2001; Hester 1980). Due to the lack of contextualized cultural material, it is difficult to provide an assessment that goes beyond a generalized overview of Texas coast inhabitant lifeways during the Paleoindian period. It is doubtless that the Paleoindian peoples of the Texas coast were, like their counterparts elsewhere in Texas and beyond, hunters and gatherers. Moreover, based on the observation that the highquality stone material used to make the projectile points encountered in the Texas coast was procured from elsewhere, it is likely that Paleoindian people of the Texas coast engaged in longdistance trade networks and/or large-scale migratory rounds (Ricklis 2004, 184). Early Paleoindian points are represented by Clovis and Folsom points, and Late Paleoindian projectile points identified in the Texas coastal regions include San Patrice and Scottsbluff (Ricklis 2004, 184). One notable Paleoindian site in the upper Texas coast is the McFaddin Beach Site (41JF50) in Jefferson County, a secondary context site located on the east side of Galveston Bay (Bousman et al 2004). This site is characterized by a high concentration of cultural material deposited on the beach by tidal action, which includes over one hundred Paleoindian projectile points, more than any other site or county in Texas. It is unknown, however, whether this high density of artifact distribution is due to nearby ancient human occupation, dynamic coastal processes, or overdiligence by the beachcombers and archaeologists working in the area (Brown 2004). 


\section{ArChaic Period (CA. 8,000 To 2,000 B.P.)}

In the upper coastal region of Texas, the Archaic period is generally divided into Early Archaic (8,000 to 6,000 B.P.), Middle Archaic (6,000 to 3,000 B.P.), and Late Archaic (3,000 to 2,000 B.P.). The Archaic period on the upper Texas coast is marked by sea-level rise and climatic fluctuation during the middle to late Holocene, from 9,000 to 2,000 B.P. (Aten 1983, 152-157). Archaeologically, this period is generally marked by an increase in the proliferation of sites in the region, mainly along stream courses and shoreline, reflecting larger occupational patterns observed elsewhere in Texas. The Archaic period is further characterized by reduced group mobility and well-defined social territories, as exemplified by a significant increase in the representation of local chert in tool manufacture (Ricklis 2004, 185; Story 1990). Specialized hunting and gathering represented the main subsistence strategy for inhabitants of the central Texas coast in the Archaic period. Generally, Archaic sites near the coastline demonstrate a dietary focus on marine resources, while the remains of terrestrial mammals are better represented in Archaic sites further inland.

\section{EARLY ARCHAIC (CA. 8,000 TO 6,000 B.P.)}

While it was previously thought that habitation and exploitation of the upper Texas coastline was relatively constricted in the Early and Middle Archaic periods (Aten 1983), it has been suggested that many of these early sites may be in fact buried or obscured in submerged upland stream margins; transgressive marine waters; and later Holocene bay, estuarine, and alluvial sediments, as the sea level changed and finally stabilized at the start of the Late Archaic period (Ricklis 2004, 186). Nevertheless, Early Archaic occupation at the shoreline, where identified, was small in scale in comparison with later habitation of the region. Moreover, the period between 6,800 and 5,900 B.P. is considered to have represented a time of occupational hiatus in the region, marked by a break in shell midden deposition. This identified lack of cultural material is associated with fluctuations in sea level that may have destabilized the biotic balance of the coastal region, leading to the depletion of exploitable resources for human populations (Ricklis 2004, 187).

Inland from the coast, a number of sites with components dating to the Early Archaic have been recorded. Notable sites include 41GV53 and the Eagle's Ridge site (41CH252) in Galveston and Chambers counties, respectively (Ricklis 2004). Both of these sites are characterized by dense stratified shellfish (rangia) middens, hearths, and artifact scatters associated with long occupational sequences starting in the Early Archaic. Technologically, this period is associated with early side-notched and corner-notched projectile points that include the Keithville, Neches River, and Trinity types (Ricklis 2004, 185).

\section{MidDLE ARCHAIC (CA. 6,000 TO 3,500 B.P.)}

Evidence for human occupation in the upper Texas coast initially shows a marked increase from previous periods after ca. 6,000 B.P., as demonstrated by a significant rise in the number of 
archaeological sites associated with this period. Sites with an Early Archaic component, such as 41GV53 and Eagle's Ridge Site (41CH252), also show continuous occupation through the first half of the Middle Archaic period. Exploitation of local resources, particularly estuarine and marine fish and shellfish, continued. The artifact assemblages most commonly associated with this period in the upper Texas coast demonstrate a marked degree of tool diversification: different types of projectile points, such as the Carrollton, Morhiss, Palmillas, and Travis appear in the archaeological record (Ensor and Ricklis 1998). Scrapers, bifacial drills, awls, utilized flakes, and beads are also documented in this period (Ricklis 2004). Obsidian and non-local high-grade chert were also recovered from Middle Archaic sites such as the McKinzie Site (41NU221), which may hint at the existence of an extensive trade network in this period (Ricklis 2004). The Middle Archaic is also the period when cemeteries are first identified in the archaeological record, located in the inland prairies of the upper Texas coast. Ernest Witte site, (41AU36) located in the lower Brazos River drainage system, is the largest cemetery in the upper Texas coast and was first established in the Middle Archaic. Like many cemeteries in this period, Ernest Witte site was also continuously used through the Late Archaic (Ricklis 2004).

Similar to the occupational hiatus observed in the latter part of the Early Archaic, the period between 4,000 to 3,000 B.P. is also marked with distinguishable break in the deposition of shell middens in certain portions of the Texas coastal region. While inland sites such as Eagle's Ridge (41CH252) are continuously occupied or utilized through the Middle Archaic and beyond, sites close to the shoreline, the portion of the coast between Galveston Bay and Baffin Bay in particular, may have experienced fluctuating sea levels. These sea level variations likely disrupted the coastal biome and caused the depletion of food resources commonly exploited by Middle Archaic peoples. Sea levels ultimately stabilized in 3,000 B.P.

\section{LATE ARCHAIC (CA. 3,500 TO 2,000 B.P.)}

The Late Archaic occupation of the central and upper Texas coastal areas is characterized by a significant increase in the population, based on the proliferation of shell midden sites along the shores of bays and in estuarine zones (Ricklis 2004, 187). This population increase was likely facilitated by the stabilization of the sea level around 3,000 B.P. and subsequent strengthening of the regional biome, which provided a plentiful and reliable source of food for the inhabitants of the area. Moreover, although the main focus of the subsistence strategy of these Late Archaic communities seems to have been estuarine and marine resources, as shown by the abundance of fish and shellfish remains in Late Archaic sites, reptiles and mammals were also similarly targeted, pointing to a diversified diet (Ricklis 2004). Sites in this period show variability among each other in terms of occupational intensity and size, which has led to the suggestion that not all communities in the area were alike (Dillehay 1975; Ricklis 2004). Hall (1981) argues that the highly productive environments such as river valley bottoms, estuaries, and bays that formed during the late Holocene allowed Late Archaic groups to operate within smaller, more exclusive territories. The territorialization of the landscape is further supported by the establishment and continued use of earlier cemeteries, such as Ernest Witte site, which has been interpreted to indicate the expression 
of distinct social identities and territorial ties between discrete social units in the Texas coast (Ricklis 2004, 186; Story 1985, 40).

Technologically, the Late Archaic is characterized by the adoption of dart points such as Yarbrough, Kent, and Gary types, which are found in both shoreline and inland sites (Gadus and Howard 1990). It has been suggested that the development and application of technologies such as fish weirs may have also allowed for higher levels of efficiency in the exploitation of coastal and riverine food resources, although empirical evidence for such device lacks from the region (Aten 1983; Ricklis 2004).

Significant Late Archaic sites in this region include sites 41HR80 and 41HR85, known collectively as Harris County Boys' School. These sites are defined by an extensive midden and a cemetery, established and occupied from approximately 3,500 to 1,500 B.P. The midden is comprised mainly of Rangia shell, with a significant quantity of lithic debitage and broken or reworked bifacial stone tools, bone tools, and beads (Aten 1983). Other significant Late Archaic sites include the Ernest Witte site (41AU36) and the Eagle's Ridge site (41CH252), which, although established in earlier periods, grew in size in the Late Archaic (Ricklis 2004).

\section{Ceramic Period (2,000 To 300 B.P.)}

The Ceramic Period, sometimes referred to as the Late Prehistoric Period, is distinguishable from earlier periods in particular due to the development and adoption of two new technologies: ceramics and the bow and arrow. Ceramics first appear in the archaeological record in the upper and central Texas coastal regions in 2,000 B.P., potentially via diffusion from the east, most likely the Mississippi River area (Ricklis 2004). The adoption of ceramic technology is largely interpreted to have represented a development in cooking and storage efficiency in comparison to earlier periods. However, the extent to which ceramics influenced other aspects of life and community in the Ceramic Period is still contested, as the majority of material culture found in Ceramic Period sites does not seem to differ greatly from that of the Archaic Period (Ricklis 2004, 189; Shafer et al 1975; Takac et al. 2000, 17). The bow and arrow are first identified in the archaeological record in the second half of the Ceramic Period around 1,200 B.P., replacing the atlatl and spear as the dominant projectile technology and thus, mirroring technological developments elsewhere in inland Texas and beyond (Ricklis 2004, 194). The Ceramic Period is generally divided into Early Ceramic and Late Prehistoric Periods, after Ensor et al. (1990) and Story et al. (1990).

Although the Ceramic Period differs from earlier periods in technological terms, the high degree of occupational permanence observed in many sites established in Archaic Period has led to the suggestion that Ceramic Period communities largely followed subsistence and settlement practices established in previous periods (Ricklis 2004, 189). Nevertheless, the nature of the larger regional cultural affiliations of central and upper Texas coast communities is still under debate. Two perspectives have been proposed. One, advocated by Shafer (1975) and Aten (1983), proposes 
that coastal Texas communities in the Ceramic Period were largely affiliated with the preMississippian Woodland cultures of eastern United States, an association that is supported by perceived ceramic stylistic and technological similarities between the two groups (Ricklis 2004, 189). The second perspective positions upper and central Texas groups in the Ceramic Period as part of the more circumscribed, archaeologically distinct Mossy Grove cultural tradition (Story 1990), with closer ties to coastal Louisiana groups than to eastern Woodland cultural traditions. This second perspective is generally favored in contemporary literature (Ricklis 2004; Ricklis and Weinstein 2005).

\section{EARLY CERAMiC PERIOD(2,000 TO 1,200 B.P.)}

As there is a high degree of occupational continuity between the Late Archaic and Early Ceramic period, Early Ceramic sites in the central and upper Texas coast are generally characterized by rangia shell middens along coastal bays or river margins, with a noticeable regional population increase (Ricklis 2004, 192). Early Ceramic sites are identified in the archaeological record only by the presence of certain material culture, namely the co-occurrence of sandy or clay paste Tchefuncte and Mandeville ceramics and dart points, primarily Gary and Kent types (Aten 1983, 303; Ricklis 2004). As sandy-pasted ceramics are associated with cultural traditions prior to the development and adoption of horticultural practices at the larger regional scale in places such as East Texas, it is thought that the subsistence strategies of the Early Ceramic period in the Texas coast was largely dependent on hunting and gathering, similarly to earlier periods (Ricklis 2004, 193). Significant Early Ceramic sites in the area include Eagle's Ridge site (41CH252), inhabited since the Late Paleoindian (Ensor and Ricklis 1998; Ricklis 2004). The site of Mitchell Ridge (41GV66) on Galveston Island is significant in the Late Prehistoric Period, although initial occupation likely dates to the end of the Early Ceramic Period and extends into the Historic Period (Ricklis 1994).

\section{Historic Period (Post 300 B.P.)}

The Trinity River region was dominated by the Atakapan Indians when the first Europeans arrived in the area in the 1700's (Kliener 2019c). The Louisiana Purchase in 1803 encouraged additional people to move to the region from the southern states, and the population of the region increased steadily until the mid- $19^{\text {th }}$ century due to the abundant land available.

Economically, Antebellum Trinity River Region was dominated by plantations along the Trinity River that raised cotton, sugarcane, tobacco, indigo, grains, and vegetables. A large number of lumber mills were also in operation during this time and remained a mainstay in economic stability. The economy received a further boost in 1840 when an extensive ranch was built and cattle drives began to move through the area. 


\section{LIBERTY COUNTY}

River travel via steamboat to and from the Gulf of Mexico was the dominant mode of transportation for people and products in the region from 1838 to 1878 . The United States governmental assistance for making the Trinity River more navigable came after 1880, and by 1940, a total of 236 miles of waterway had been constructed. Attempts to build an extensive railroad system were interrupted by the Civil War, but construction continued after Reconstruction (Kliener 2019c). This rail system caused the Port of Liberty to see significantly less traffic.

The population within Liberty County remained stable from 1870 to 1900 when it doubled, signaling an important growth in the county's agriculture and industry (Kliener 2019c). During the same timeframe, Liberty County became the center for Texas cattle production and rice industry when irrigation plants were developed. Oil prospecting and production began about 1901. The population steadily grew, as did the agricultural and cattle industry. As with the rest of the Trinity River region, lumber milling remained an important county industry.

\section{JEFFERSON COUNTY}

Jefferson County was formed in 1836 as one of the original counties in the Republic of Texas (Kliener 2016b). By the 1840s, shingle manufacture and timber exports helped to supplement an existing domestic economy. After 1880, rail transportation increased significantly. The Port Arthur Ship Canal was dug in 1897 and 1898 from Sabine Pass to Port Arthur (Kliener 2016b).

In the early 1900s, population in Jefferson County grew from a major influx followed by the Spindletop oilfield opening in 1901. Additionally, there was significant migrations of the Cajun French from Louisiana to Jefferson County in 1910, as a result of damaged cotton crops by insect infestation (Kliener 2016b).

Spindletop transformed the Beaumont area into a major industrial center. By the 1950s, the Spindletop field was still active, however sulfur mining was also gaining popularity in the region. By the 1970s, rice and cattle were the dominant agricultural products, and many of the residents were employed in the petrochemical, shipbuilding, and rubber industries (Kliener 2016b).

\section{CHAMBERS COUNTY}

In 1858, Chambers County was formed from Liberty and Jefferson counties. Industry was small scale at this time with only a steam sawmill and a shipyard (Kliener 2016a). Railroad routes did not reach into Chambers County so the county was dependent on steam traffic and other water transportation from Galveston. Between 1910 and 1930, tenant farming increased in the area. Additionally, rice farming increased greatly on the area in the 1900s due to the development of a canal system (Kliener 2016a). Lumber was also an important industry during the early twentieth century as well. 


\section{METHODS}

\section{BACKGROUND REVIEW}

Prior to initiating fieldwork, Perennial conducted a records and literature review of the THC's Texas Archeological Sites Atlas (Atlas) online database and the NRHP database to identify previously recorded cultural resource sites, historic structures, properties listed in the NRHP, designated historic districts, or SAL which could potentially be affected by the proposed undertaking. Previously recorded cultural resource site forms, reports of archaeological investigations, general historical documents, and secondary sources concerning the background of the area were reviewed. The records search included a review of all site records and previous surveys on file within $1.00 \mathrm{mi}(1.61 \mathrm{~km})$ of the Project.

In addition to a records and literature search, archaeologists gathered information from secondary sources concerning the prehistoric and historic background of the area. Documents associated with the history of the area were used to model prehistoric and historic settlement patterns in relation to the landscape and terrain characteristics as well as cultural patterns and regional trends. NRCS soil data, USGS 7.5 minute topographic quadrangles, aerial photographs, and contemporary geologic and physiographic features were also examined.

\section{Field Survey Methods}

The cultural resources surveys were conducted by one to two crews comprised of up to three archeologists each under the supervision of the Principal Investigator who provided logistical and technical support, as well as oversight of all staff. Phase I survey investigations were concentrated in the vicinity of delineated wetland or waterbody features, and survey efforts were conducted in accordance with a scope of work for the Project approved by the USACE on August 28, 2018, and the THC on October 1, 2018. Following the approval of the scope of work, ETC extended the eastern terminus of the Project to the western bank of the Neches River, and other minor reroutes were also implemented. Perennial applied the approved survey methodology to all new Project components. Additionally, several route options were presented across the DOC - Stiles Unit. These tracts were added to the Project in late August 2019, and the additional route options were added to the Project in late September and October 2019. Any reroutes across public tracts were surveyed in accordance with the previously approved scope of work associated with TAP \#8690 Project.

In practice, the archeological field crew utilized data collected by Perennial field biologists to identify feature locations within the Project APE. In general, the Texas State Minimum Archeological Survey Standards (TSMASS) require that a minimum of 16 shovel tests be excavated per $1.0 \mathrm{mi}(1.6 \mathrm{~km})$ for rights-of-way (ROWs) measuring up to $100.0 \mathrm{ft}$. $(30.0 \mathrm{~m})$ in width (one survey transect). For the majority of the Project length, intensive survey investigations took place within an approximately $300.0 \mathrm{ft}$. $(91.5 \mathrm{~m})$ wide corridor. Within the vicinity of 
delineated wetland and waterbody features, shovel tests were excavated along three evenly spaced transects at intervals ranging from 98.4 to $328.0 \mathrm{ft}$. $(30.0$ to $100.0 \mathrm{~m})$, depending on the archaeological sensitivity of the landscape.

Perennial conducted a preliminary probability assessment for the Project APE utilizing multiple data sources, including location of previously recorded sites, soils and geology, aerial imagery, as well as topographic and historic maps. Overall, the probability classifications are meant to be a guidance tool that serves to inform survey strategy and level of effort while ensuring adherence to state and federal standards and guidelines. The three probability classifications and the characteristics for each probability area are defined as follows:

High Probability Areas (HPAs) were defined as areas that are likely to contain prehistoric and/or historic-age archaeological sites. HPAs generally include settings along major waterbodies, such as the Trinity River and bayous, and incorporate other high probability settings such as alluvial terraces, Holocene-aged soil deposits, remnant natural levees, benches, and topographic rises in floodplain settings (relatively unaffected by natural or human-induced processes), or proximity to previously recorded sites or historic structures. In HPAs, survey efforts extended beyond the water feature by a variable distance of 164.0 to $492.0 \mathrm{ft}$. (50.0 to $150.0 \mathrm{~m}$ ) to include any adjacent prominent landforms, terrace projections, remnant natural levees, benches, topographic rises in floodplain settings, (relatively unaffected by natural or human-induced processes), as well as any previously recorded sites or historic structures. Surveys across HPA settings included shovel testing intervals ranging from 82.0 to $164.0 \mathrm{ft}$. $(25.0-50.0 \mathrm{~m})$ along parallel transects. In cases where the pipeline will be installed via HDD under major waterway crossing resulting in no impacts to high probability alluvial settings, surveys were concentrated in the drill entry and exit workspaces.

Medium Probability Areas (MPAs) were defined as areas that exhibit landscape characteristics of an HPA, but that show evidence of human-induced or natural disturbances that would impact the integrity of any previously unrecorded archaeological deposits. MPAs are also defined as areas in which resources, such as lithic raw materials, may be located. Generally, the remnants of historic structures and artifact scatters are often found in MPAs. In MPAs, survey efforts extended beyond the water feature by a variable distance of 164.0 to $492.0 \mathrm{ft}$. (50.0 to $150.0 \mathrm{~m}$ ) to include any adjacent landscape features and/or potential raw material resources. Surveys across these settings included shovel testing intervals ranging from 165.0 to $330.0 \mathrm{ft}$. (50.0 to $75.0 \mathrm{~m}$ ) along parallel transects.

Low Probability Areas (LPAs) were defined as areas consisting of inundated lowlands, swamps and sloughs, as well as expansive wetlands within agricultural settings. LPAs consisted of areas where soils are of Pleistocene-age or older. LPAs also included locations with significant disturbance from oil and gas, and/or modern development that have created varying levels of landscape modification. Survey efforts in LPAs were conducted within roughly 164.0 to $492.0 \mathrm{ft}$. 
(50.0 to $150.0 \mathrm{~m}$ ) of either side of the delineated feature boundaries. Surveys across these settings included shovel testing intervals ranging from 246.0 to $328.0 \mathrm{ft}$. (75.0 to $100.0 \mathrm{~m}$ ) along parallel transects. Much of the Project traverses irrigations ditches across low-lying agricultural fields. Much is these areas were considered low probability for cultural resources due to the extensive ground-disturbing activities needed construct water control features.

In areas where wetlands were delineated across large areas with a discontinuous or irregular boundary, survey investigations consisted primarily of pedestrian surveys across feature clusters with shovel testing efforts conducted at the discretion of the Principal Investigator or crew chief. In lieu of shovel testing in areas with standing water, field archeologists recorded inundation points with GPS units to demonstrate the extent of waterlogged settings. These inundation points are demonstrated on the survey results maps provided in Appendix B.

In general, shovel tests measured approximately $30.0 \mathrm{~cm}$ (12.0 in) in diameter and were excavated by natural strata. Where possible, shovel tests were excavated to a depth commensurable with the Project impacts per the THC/CTA survey standards, or to where pre-Holocene sterile substrates are encountered, if possible, unless manual shovel testing is unable to penetrate hard clay soils. All soil matrices were sifted through 6.3-millimeter (1/4-inch) mesh hardware cloth unless dominated by clay. Clayey matrix was finely divided by trowel and visually inspected.

For each of the shovel tests, the following information was recorded on shovel test logs: location, maximum depth, and the number of soil strata. For each soil stratum, thickness, texture, color, and the presence or absence and nature of cultural materials was recorded. Perennial maintained a selective artifact collection survey strategy whereby only representative samples of artifacts by category and diagnostic artifacts would have been collected from each newly recorded or revisited site and housed temporarily at Perennial's laboratory for analysis. Any collected artifacts would have been be catalogued, analyzed, and prepared for submittal to an approved permanent curatorial facility or returned to the landowner upon request at the completion of the Section 106 consultation process. In practice, the survey investigations as detailed herein resulted in negative findings, as such the artifact collection strategy was not implemented.

The field crew recorded all shovel test locations, isolated finds, archaeological sites and associated features using a handheld GPS device (as encountered). Also, each archaeologist was equipped with a compass, shovel test and photographic logs, daily journal forms, and appropriate state site forms. Each crew also utilized topographic maps and aerial photographs of the APE, a digital camera, and a cellular telephone to maintain contact with the Principal Investigator (terrain permitting).

In addition to shovel testing, the THC/Council of Texas Archeologists (CTA) survey standards require that mechanical trenching be conducted in areas of the Project APE with deep alluvial sediments, namely the Trinity River crossing, and the western banks of the Neches River. Overall, 
both of these settings were noted on multiple field visits to consist of heavily inundated settings with up to $2.0 \mathrm{ft}$. $(0.6 \mathrm{~m})$ of standing water in areas. Access these areas with heavy machinery is not a feasible option due to the limited access points associated with these areas (Figure 4).

Field surveys across Code-permitted tracts were conducted along 100\% of the length of the Project APE for each tract. Shovel tests were conducted in non-inundated areas along three parallel transects (when capable) at intervals ranging from 98.4 to $328.0 \mathrm{ft}$. (30.0 to $100.0 \mathrm{~m}$ ) apart. Overall, surveys across these tracts noted extensive disturbance from modern development, as well as widespread inundation that prevented traditional shovel testing in some areas. In such cases, inundation points were taken with hand-held GPS units during pedestrian surveys. Based on the underlying geology and the low potential for buried cultural deposits for soils found within the Code-permitted tracts, trenching was not warranted across these areas.

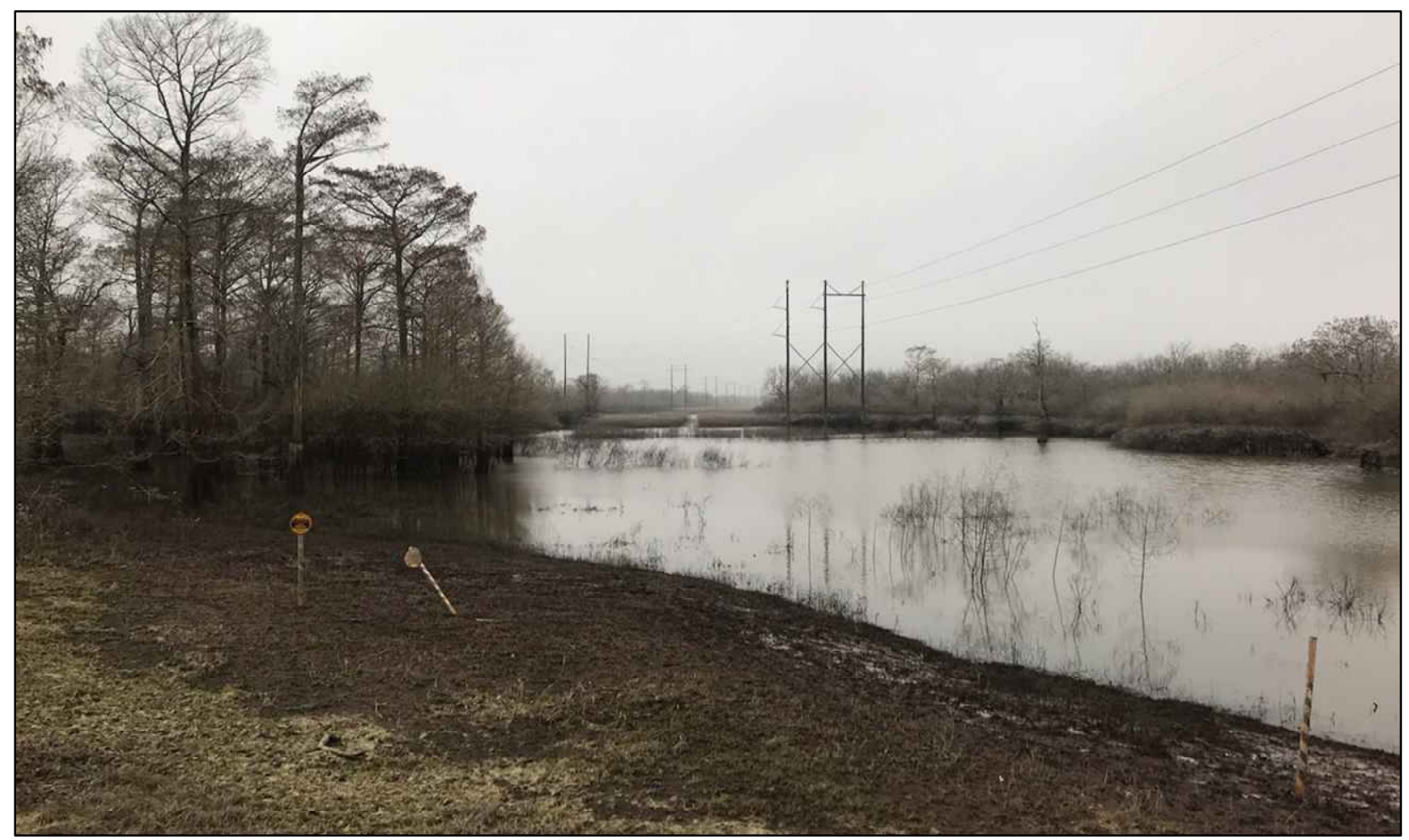

Figure 4. View of flooded access point on the eastern side of the Trinity River 


\section{RESULTS}

\section{BACKGROUND REVIEW}

Based upon a background literature review on the THC's Atlas, ten archaeological sites (Table 2) and eight cemeteries (Table 3) are mapped within a $1.0 \mathrm{mi}(1.6 \mathrm{~km})$ of the Project APE. None of the 10 archaeological sites identified are located within or adjacent to the Project APE. These sites consist either of historic artifact scatters or farmsteads located in agricultural settings, or prehistoric shell middens documented on the banks of the Neches River. Site 41JF66 is mapped within $400.0 \mathrm{ft}$. $(121.95 \mathrm{~m})$ of the Project APE and is recorded as a single point on the Atlas, suggesting that the boundaries of the site were not well defined. For this reason, additional archival research and a more intensive shovel testing regime were implemented in this area to better define the limits and characteristics of the site within the APE.

Of the eight cemeteries identified within the $1.0 \mathrm{mi}(1.6 \mathrm{~km})$ background review radius, one cemetery (Sahara Cemetery [LC-C070]) is located approximately $130.0 \mathrm{ft}$. (40.0 m) north of Project APE. The cemetery is delineated by a barbed-wire boundary fence, and is located within an isolated stand of trees situated within an expansive open pasture (Figure 5). Construction activities associated with the Project are not anticipated to encroach on the 25-foot cemetery buffer.

\begin{tabular}{|c|c|c|c|c|}
\hline \multicolumn{5}{|c|}{ Table 2. Sites previously recorded within the 1.0 mi background review radius } \\
\hline Site Trinomial & Site Description & $\begin{array}{c}\text { Year } \\
\text { Recorded }\end{array}$ & $\begin{array}{c}\text { NRHP } \\
\text { Eligibility }\end{array}$ & $\begin{array}{c}\text { Distance from Project } \\
\text { APE }\end{array}$ \\
\hline 41LB121 & Prehistoric Lithic Scatter & 2013 & Not Eligible & $0.58 \mathrm{mi}(0.93 \mathrm{~km})$ \\
\hline 41JF66 & $\begin{array}{c}\text { Tyrrell Tenant Farmstead - } \\
\text { Historic, Early 20th Century }\end{array}$ & 1990 & Undetermined & $360.0 \mathrm{ft.}(109.7 \mathrm{~m})$ \\
\hline 41JF97 & Historic Scatter & 2011 & Not Eligible & $0.37 \mathrm{mi}(0.59 \mathrm{~km})$ \\
\hline 41JF99 & Historic Isolated Find & 2013 & Not Eligible & $0.13 \mathrm{mi}(0.20 \mathrm{~km})$ \\
\hline 41JF101 & Historic Scatter & 2016 & Not Eligible & $0.4 \mathrm{mi}(0.64 \mathrm{~km})$ \\
\hline 41JF5 & Shell Midden/Campsite & 1940 & Undetermined & $0.63 \mathrm{mi}(1.01 \mathrm{~km})$ \\
\hline 41OR110 & Shell Midden & 2013 & Not Eligible & $0.29 \mathrm{mi}(0.46 \mathrm{~km})$ \\
\hline 41OR8 & Shell Midden & 1940 & Undetermined & $0.88 \mathrm{mi}(1.41 \mathrm{~km})$ \\
\hline 41OR9 & Shell Midden/Human Burial & 1940 & Undetermined & $0.8 \mathrm{mi}(1.28 \mathrm{~km})$ \\
\hline 41OR10 & Shell Midden & 1940 & Undetermined & $0.68 \mathrm{mi}(1.09 \mathrm{~km})$ \\
\hline
\end{tabular}




\begin{tabular}{|c|c|c|}
\hline \multicolumn{3}{|c|}{ Table 3. Cemeteries recorded within the 1.0 mi background review radius } \\
\hline Cemetery Name & Cemetery Number & Distance from Project APE \\
\hline Freeman Cemetery & N/A & $0.78 \mathrm{mi}(1.25 \mathrm{~km})$ \\
\hline Sahara Cemetery & LB-C070 & $130.0 \mathrm{ft} .(39.6 \mathrm{~m})$ \\
\hline Quarters Cemetery & LB-C075 & $0.43 \mathrm{mi}(0.69 \mathrm{~km})$ \\
\hline Unknown Cemetery & LB-C067 & $0.93 \mathrm{mi}(1.49 \mathrm{~km})$ \\
\hline Redmond Field & LB-C132 & $0.76 \mathrm{mi} 1.22 \mathrm{~km})$ \\
\hline Miller Cemetery & LB-C068 & $0.6 \mathrm{mi}(0.96 \mathrm{~km})$ \\
\hline Memorial Gardens & JF-C026 & $0.19 \mathrm{mi}(0.30 \mathrm{~km})$ \\
\hline Unknown Cemetery & JF-C007 & $0.74 \mathrm{mi}(1.19 \mathrm{~km})$ \\
\hline
\end{tabular}

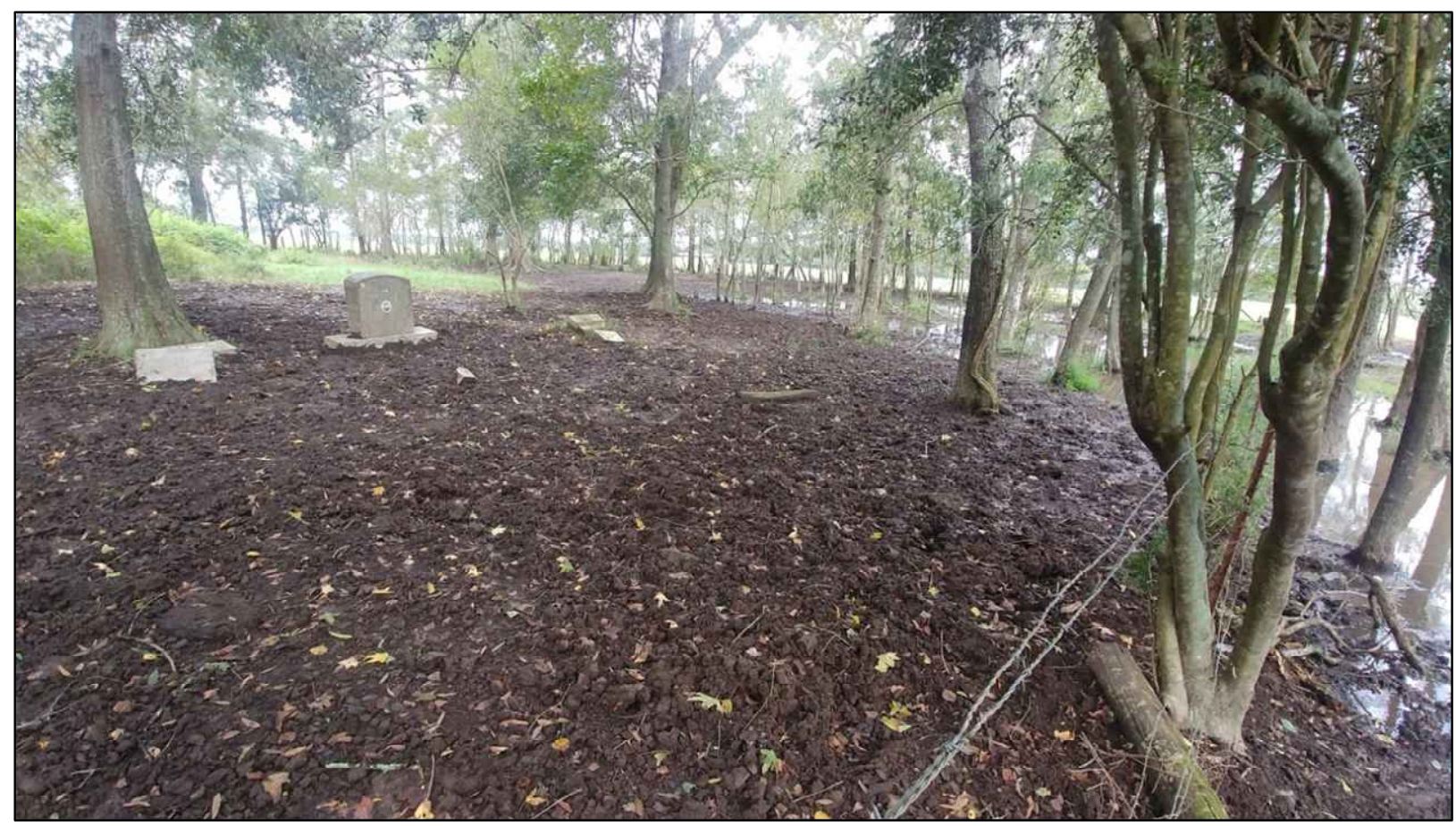

Figure 5. View of Sahara Cemetery with boundary fencing

The background review also determined that a total of 60 previous archaeological projects have been conducted within $1.0 \mathrm{mi}(1.6 \mathrm{~km})$ of the Project. The vast majority of these archaeological investigations consist of Phase I surveys for large-scale energy infrastructure projects. The lead agency on these projects is typically either the USACE Galveston District or the Federal Energy Regulatory Commission (FERC). Many of the remaining surveys were conducted in support of municipal transportation projects. 
Twenty-seven of the 60 surveys overlap or intersect portions of the Project route. These previous survey investigations constitute approximately $32.7 \%$ overlap with the current Project APE (Appendix B). However, many of these surveys are outdated and do not meet modern survey standards. Additionally, many of these previously surveys were conducted for USACE-permitted projects, and the entire Project was not surveyed for the presence of cultural resources. As such, all areas surrounding identified wetland and waterbody for this Project were surveyed. 


\section{FieLd SURVEY}

Perennial archaeologists Colene Knaub, William Kinkner, Jonathan Laird, Rachel Kelley, Rafael Cortez, Thomas Ross, and Wyatt Ellison conducted an intensive cultural resources survey of the Project APE between October 1, 2018, and November 12, 2019. The objectives of the survey were to (1) locate cultural resource sites within the APE in the vicinity of delineated wetland and waterbody features; (2) delineate the vertical and horizontal extent of any newly identified sites within the APE; (3) provide a preliminary evaluation of each site's eligibility for listing in the NRHP; and (4) assess any potential for the Project to directly or indirectly affect historic properties, or other sensitive cultural resources.

In addition to pedestrian surveys, investigations included the excavation of a total of 1,250 shovel tests of which 1,171 shovel tests were excavated adjacent to delineated wetland and waterbody features, also referred to as USACE permit areas (Appendix B). The remaining 79 shovel tests were excavated outside of USACE permit areas but within the Project workspace. For the purposes of this report, only investigations within the Project APE will be discussed.

In practice, the survey crews were strategically staggered to follow Perennial biological surveys to ensure adequate survey coverage within the USACE permit areas, which include expansive wetlands in forested areas, inundated agricultural fields that have existed in a fallow state for several years, drainage canals and ditches, and natural waterway crossings. Appendix $\mathbf{C}$ details the level of effort for each delineated feature.

Survey efforts across the USACE permit areas resulted in entirely negative findings with no cultural materials noted on the surface or within any shovel tests. Additionally, no historic standing structures or features were observed with any USACE permit areas. Large portions of the Project APE consist of heavily modified agricultural landscapes, or inundated lowlands underlain by clayey, saturated soils. Overall, the majority of the Project was determined to have a low probability for cultural resources based on the prevalence of previous disturbance and land modification from oil and gas development and agricultural practices. Greenfield settings with intact forested areas were also noted to have a low probability for cultural resources based on the presence of expansive inundated or wetland areas. These probability determinations are also reflected in the broader archeological dataset, which shows extensive survey efforts conducted on behalf of prolific oil and gas development, with sparse archeological findings.

The exception to this patterning would be the Trinity River basin. The initial assessment of this area determined that the Trinity River crossing, and the associated bottomlands that extend the east of the River, are underlain by Holocene-age alluvial deposits with soils characterized as having a high geoarcheological potential (NRCS 2019; Abbott 2001). The Project APE crosses the Trinity River in an area where numerous previous survey investigations converge, and survey and trenching efforts in support of these projects were negative for cultural materials and documented a disturbed soil profile (Wilcox et al. 2007; Brownlow and Clark 2006). 
Prior to the beginning of November 2019, field surveys had not been conducted along the eastern banks and associated bottomlands of the Trinity River due to multiple flooding events that resulted in heavy inundation beginning in September 2018 which prevented access or survey investigations of any kind. For more than one year, field crews have attempted to survey the Trinity River bottomlands on multiple occasions. Access to this area was determined to be not feasible due to the presence of up to $2.0 \mathrm{ft}$. $(6.0 \mathrm{~m})$ of standing water for the entire $2.6 \mathrm{mi}(4.1 \mathrm{~km}) \mathrm{stretch}$ extending from the eastern banks of the Trinity River to Self Bayou. However, on November 6, 2019, a survey crew was able to access previously inaccessible areas associated with the Trinity River due to several months of normalized weather conditions throughout portions of Eastern Texas. This landscape, which is characterized by swampy marshes and ridge and swale topography. Even under normal conditions, large portions of this area remain constantly inundated due to strong hydrological influences and the geomorphic position of the landscape. While the area located immediately adjacent to the Trinity River was not inundated at the time of survey, wetlands with strong hydrological indicators still dominate the landscape. In non-inundated areas, shovel tests were excavated at 82.0-to $-164-\mathrm{ft}(25.0$ - to 50-m) intervals along three transects. Figure 6 present a representative photo of the non-inundated areas. A total of 96 shovel tests were excavated within this location, all of which were negative for cultural resources. Inundated locations within this area consisted of low-lying mapped wetland features that likely hold water much of the year (Figures 7-9). These areas are located between the only access/entry path to the eastern side of the Trinity River, and they had to be traversed on foot. It is not feasible to gain access to the drier areas near the river with heavy equipment.

Overall, deep impacts associated with the HDD exit workspace will be confined to the existing, permanent easement with temporary workspace extending across the broader APE corridor at select locales. As such, Perennial recommends that Construction Monitoring be conducted by a qualified archaeologist during construction efforts in the vicinity of the HDD crossing the Trinity River near MP 17.0-17.2.

Of the 760 delineated wetland and waterbody features, 74 features were originally not surveyed for cultural resources due to restricted land access including denied landowner permissions and significant inundation. Of these 74 features, 38 feature locations (27 streams and 42 wetlands) will be bypassed via horizontal directional drill (HDD) or bore trenchless construction methods resulting in no impacts to these features. The remaining five features (including multiple crossing locations of the same feature) are located along the eastern banks of the Trinity River. While these features were surveyed for cultural resources with negative findings, the presence of buried deposits exists within the vicinity of these features. However, access to these features with heavy machinery is not feasible due to the remote location and constant hydrological influences (e.g. inundation and saturation) associated with the floodplain setting of the Trinity River. As such, these five features are proposed to be monitored by a qualified Archeological Monitor during construction efforts. Appendix $\mathbf{C}$ provides each wetland and waterbody feature crossed by the Project with management recommendations and associated comments, while Appendix F 
provides a Cultural Monitoring Plan to evaluate the five features that will not be avoided during construction efforts. Appendix F also includes a table in response to a letter issued by the USACE Staff Archeologist, Mr. Jerry Androy, on May 17, 2019, indicating that the 74 aforementioned permit areas associated with the Project would require cultural resources investigations. The table lists each permit area, the reason surveys were not originally conducted, and justification for/against the need for monitoring.

Should cultural materials be encountered during this phase of the Project, then the Archeological Monitor would follow the procedures outlined in the Cultural Monitoring Plan, which would require construction to stop in the immediate area of discovery, and initiation of notification protocols needed to ensure the find is adequately evaluated in accordance with Section 106 of the NHPA. Details of the Archeological Monitoring Plan are presented in Appendix F

\section{CODE-PermitTed Tracts}

The Project APE crosses six tracts owned by Jefferson County, the City of Beaumont, and the DOC - Stiles Unit (Figure 6). These properties fall under the jurisdiction of the Texas Antiquities Code (TAC [13 TAC $\$ 22.1-22.6]$ ). As such, survey investigations were conducted for $100 \%$ of the Project APE across these properties by Perennial archaeologists Sarah Boudreaux, Wyatt Ellison, Colene Knaub, William Kinkner, Jonathan Laird, and Alejandro Martinez on December 20, 2018, September 25-27, 2019, and October 10, 2019.

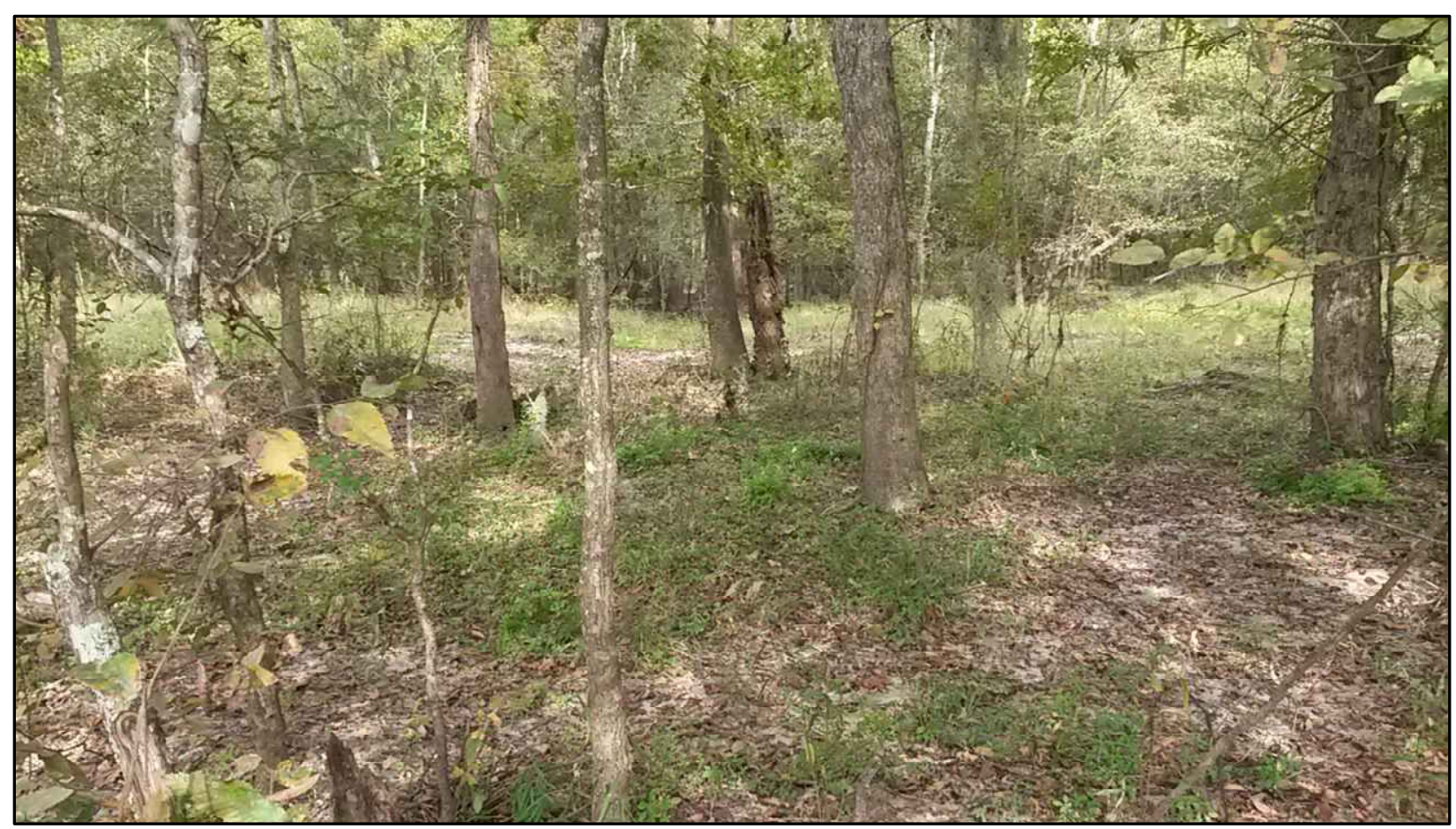

Figure 6. View of non-inundated areas in Trinity River Bottomlands within Project APE, facing northwest 


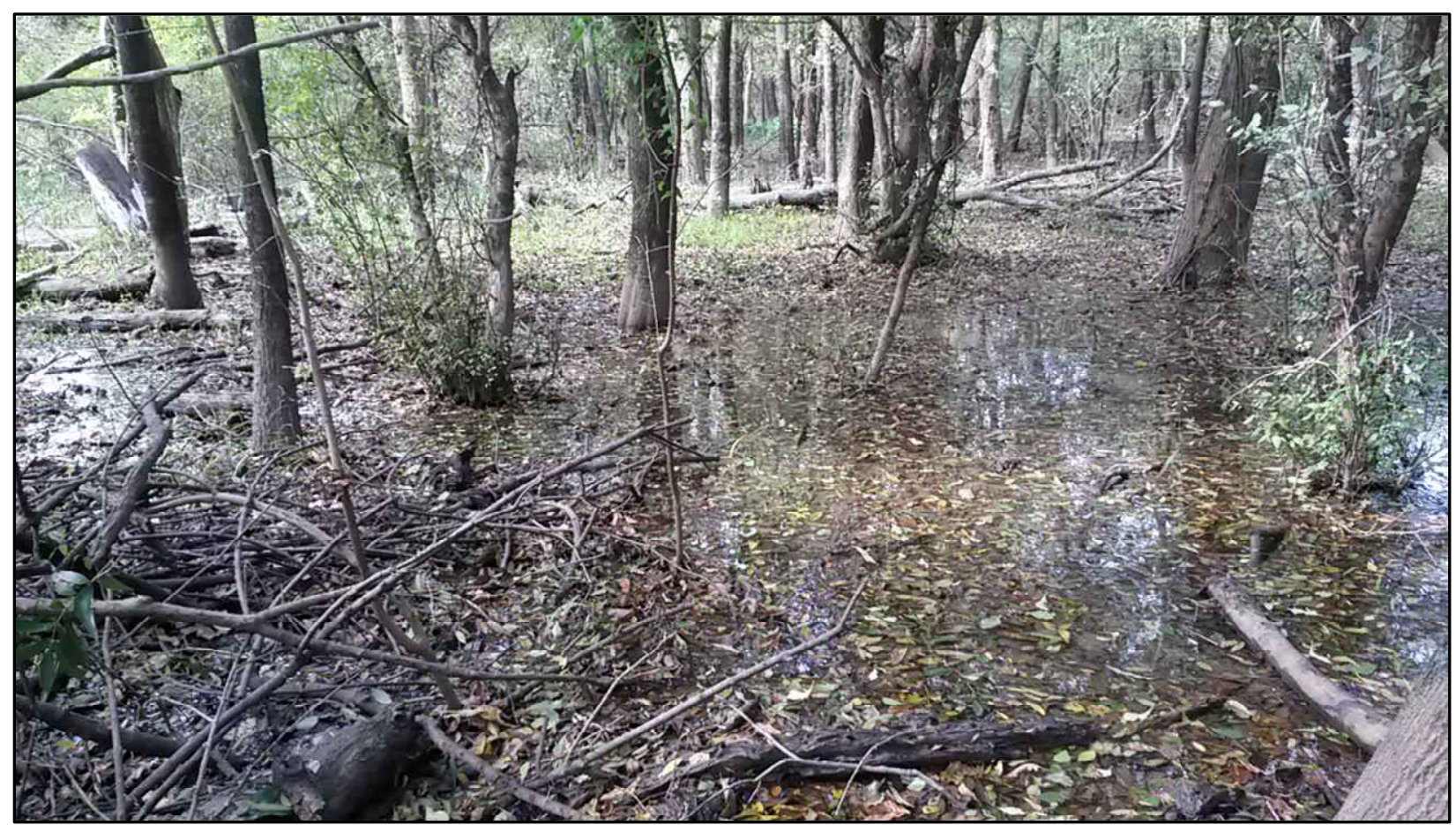

Figure 7. View of inundated areas in Trinity River Bottomlands within Project APE, facing southeast

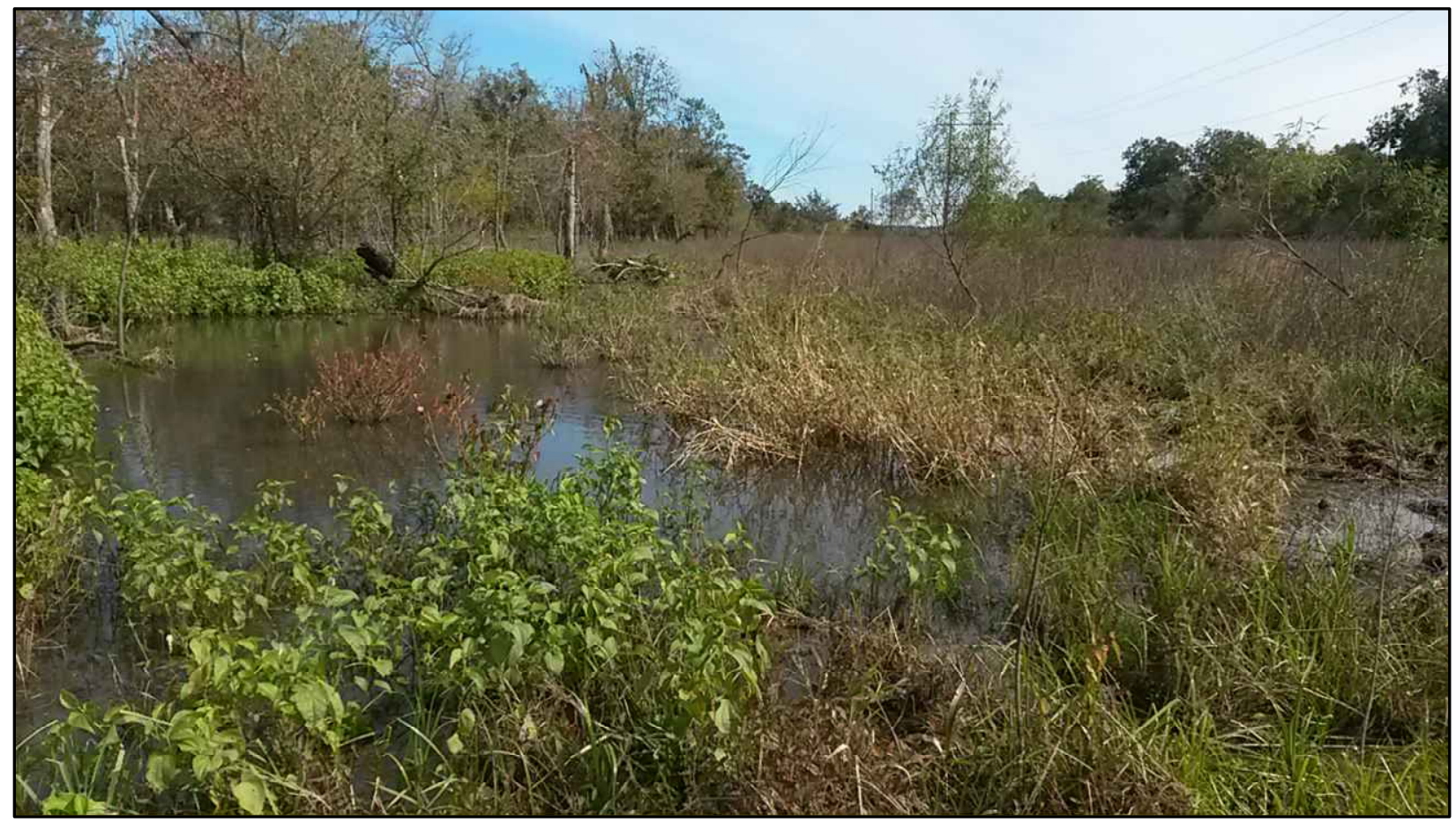

Figure 8. View of inundated areas in Trinity River Bottomlands within Project APE, west 


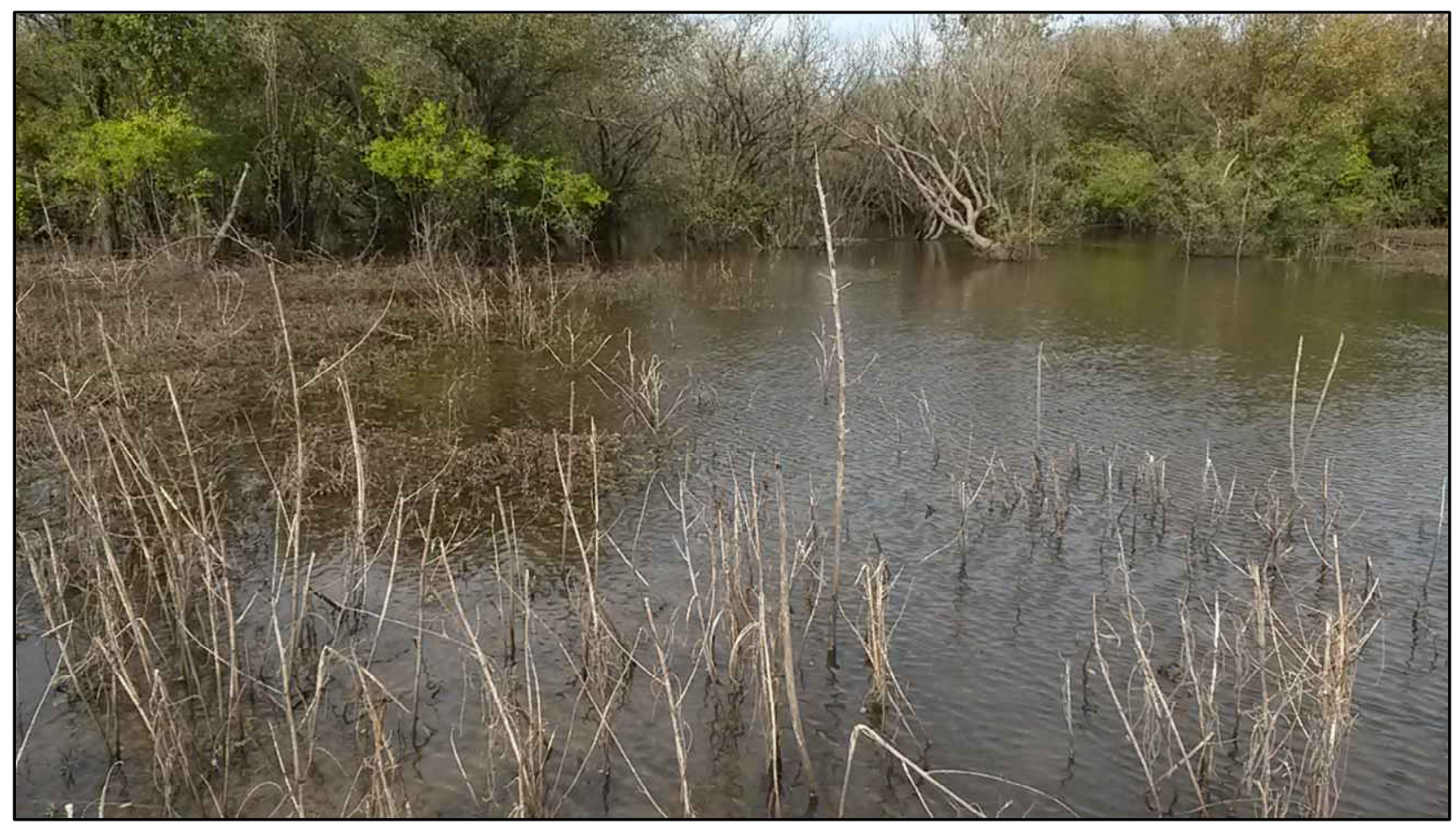

Figure 9. View of inundated areas in Trinity River Bottomlands within Project APE, facing south

The Jefferson County tracts consist of a large sport complex with existing outdoor sport fields and several paved parking lots (two tracts) (Figure 7); a heavily wooded tract with large expansive wetlands; and an existing utility corridor across the southern edge (Figure 8; Table 4;). The City of Beaumont tracts consist of a large, heavily vegetated area with some nature trails present. Large expansive forested and emergent wetlands were present across the entirety of the City of Beaumont tracts (Figure 9), and extensive inundation was present at the time of survey. The DOC - Stiles Units tracts consists of short, dense grasses with large, expansive wetlands present across the property (Figure 10). Generally, ground surface visibility across the Code-permitted tracts was poor.

A total of 66 shovel tests were excavated in non-inundated and undisturbed areas as part of survey efforts for the six Code-permitted tracts, all of which were negative for cultural resources (see Table 4; Figures 11 to 17). The locations of shovel tests within this portion of the Project APE were strategically chosen to avoid any areas with evidence of disturbance, such as existing gravel roads, and large areas of inundation.

The Project APE across the Code-permitted tracts is considered to be within a low probability setting due to underlying soils, geology, and the observed level of disturbance and expansive wetlands. Overall, survey efforts revealed a heavily inundated and disturbed landscape that has a limited capacity to harbor intact cultural resources. 


\begin{tabular}{|c|c|c|c|c|c|}
\hline \multicolumn{6}{|c|}{ Table 4. Survey Data for Code-Permitted Tracts } \\
\hline Tract & Owner & Setting & Acreage & $\begin{array}{c}\text { Shovel } \\
\text { Tests } \\
\text { Excavated }\end{array}$ & Survey Results \\
\hline $\begin{array}{l}\text { TX-JE- } \\
041.402\end{array}$ & $\begin{array}{l}\text { Jefferson } \\
\text { County }\end{array}$ & $\begin{array}{l}\text { Emergent Wetland, } \\
\text { Manicured Lawn, and } \\
\text { Paved Road }\end{array}$ & 2 & 1 & $\begin{array}{c}\text { All surveys negative for } \\
\text { cultural resources - no further } \\
\text { work warranted; Tract bypassed } \\
\text { via bore/HDD }\end{array}$ \\
\hline $\begin{array}{l}\text { TX-JE- } \\
041.406\end{array}$ & $\begin{array}{l}\text { Jefferson } \\
\text { County }\end{array}$ & $\begin{array}{l}\text { Emergent Wetland, Paved } \\
\text { Parking Lot, Sports } \\
\text { Facility, Paved Roads, } \\
\text { and Manicured Lawn } \\
\end{array}$ & 10 & 3 & $\begin{array}{c}\text { All surveys negative for } \\
\text { cultural resources - no further } \\
\text { work warranted; Tract bypassed } \\
\text { via bore/HDD }\end{array}$ \\
\hline $\begin{array}{l}\text { TX-JE- } \\
116.200\end{array}$ & $\begin{array}{l}\text { Jefferson } \\
\text { County }\end{array}$ & $\begin{array}{c}\text { Forested area; disturbed } \\
\text { parking areas }\end{array}$ & 35.6 & 3 & $\begin{array}{l}\text { All surveys negative for } \\
\text { cultural resources - no further } \\
\text { work warranted }\end{array}$ \\
\hline $\begin{array}{l}\text { TX-JE- } \\
077.000\end{array}$ & $\begin{array}{l}\text { City of } \\
\text { Beaumont }\end{array}$ & $\begin{array}{l}\text { Forested and Emergent } \\
\text { Wetlands }\end{array}$ & 21 & 13 & $\begin{array}{c}\text { All surveys negative for } \\
\text { cultural resources - no further } \\
\text { work warranted }\end{array}$ \\
\hline $\begin{array}{l}\text { TX-JE- } \\
079.310\end{array}$ & $\begin{array}{l}\text { City of } \\
\text { Beaumont }\end{array}$ & $\begin{array}{c}\text { Agricultural Fields, } \\
\text { Forested Wetlands, } \\
\text { Emergent Wetlands, } \\
\text { Palustrine Wetlands, } \\
\text { Open Water, and Oil/Gas } \\
\text { Infrastructure } \\
\end{array}$ & 49 & 19 & $\begin{array}{l}\text { All surveys negative for } \\
\text { cultural resources - no further } \\
\text { work warranted }\end{array}$ \\
\hline $\begin{array}{c}\text { TX-JE- } \\
115.220 \\
\text { TX-JE- } \\
115.900 \\
\text { (combined) } \\
\end{array}$ & $\begin{array}{c}\text { Texas } \\
\text { Department } \\
\text { of } \\
\text { Corrections - } \\
\text { Stiles Unit } \\
\end{array}$ & Existing prison facility & 92.6 & 27 & $\begin{array}{l}\text { All surveys negative for } \\
\text { cultural resources - no further } \\
\text { work warranted }\end{array}$ \\
\hline
\end{tabular}




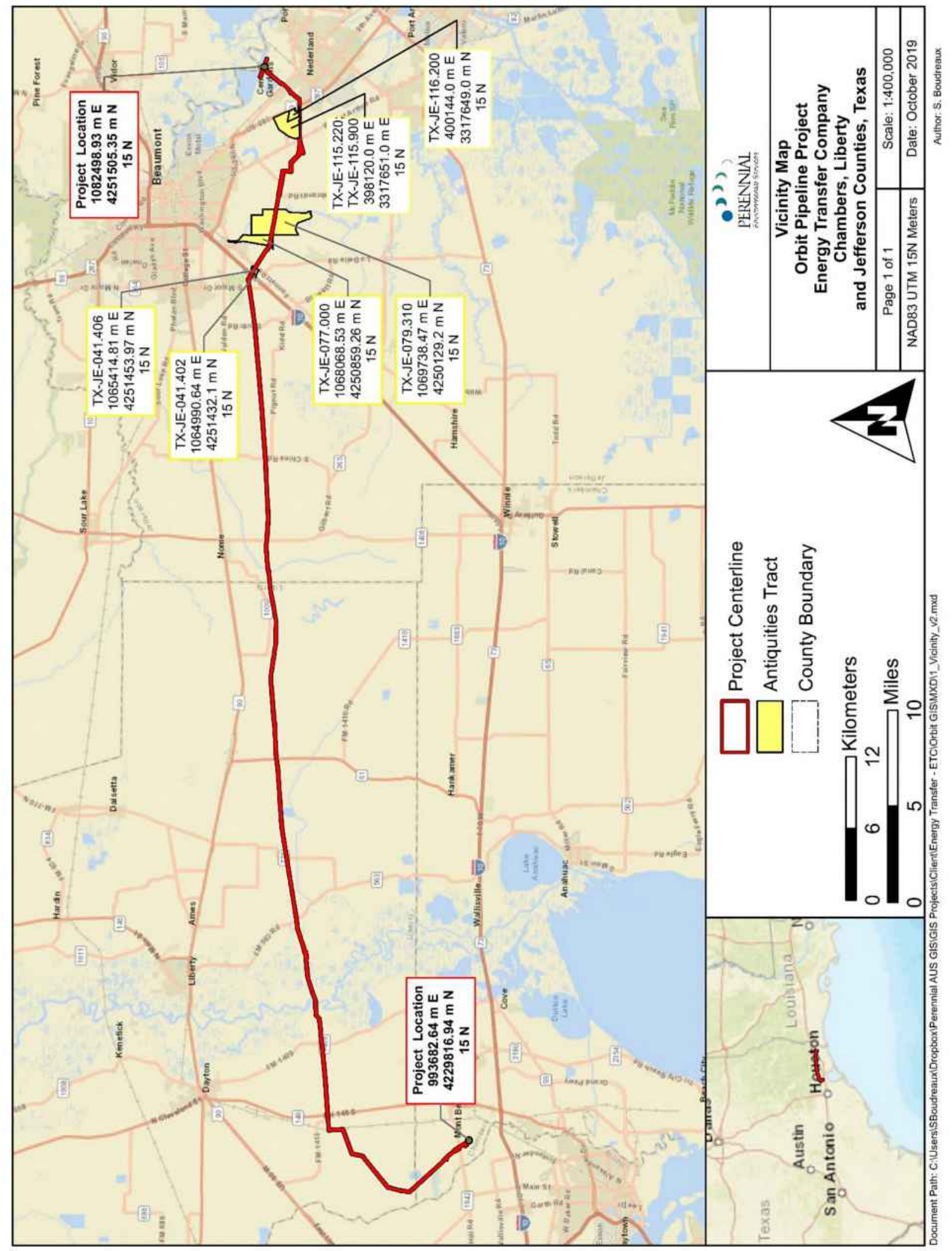

Figure 10. Vicinity Map for Code-Permitted Tracts 


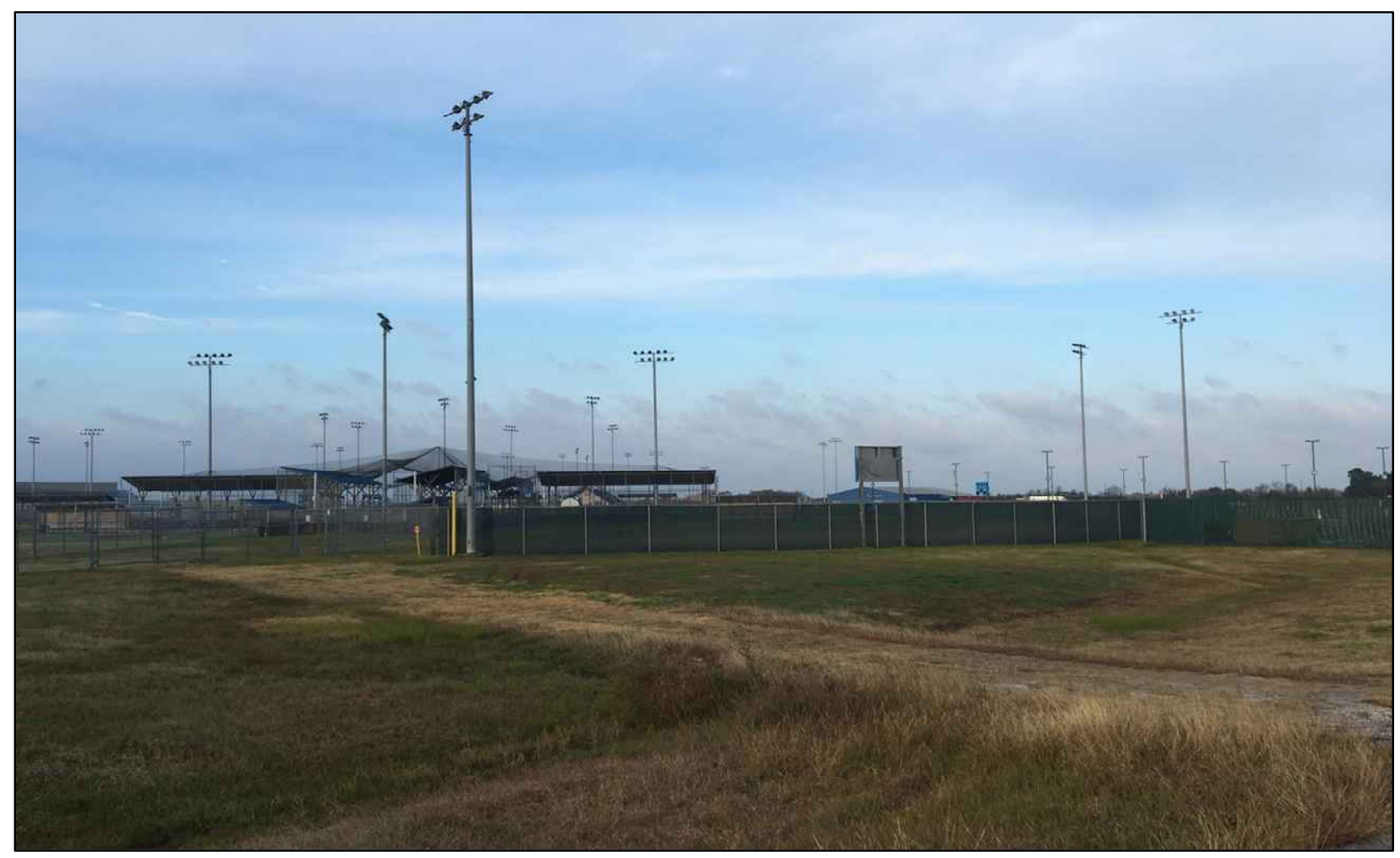

Figure 11. Overview of Jefferson County Tracts, facing northwest

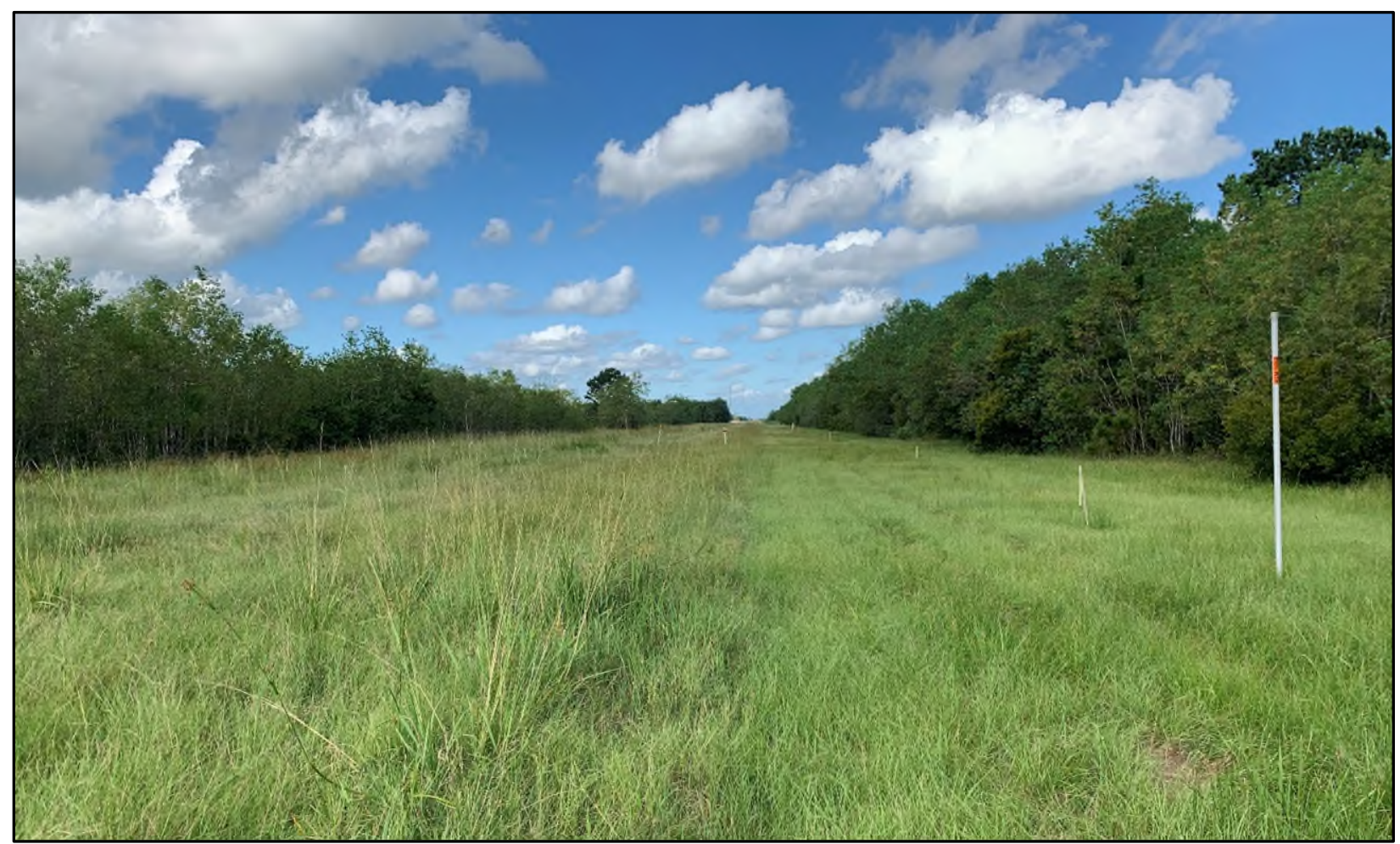

Figure 12. Overview of Jefferson County Tract (adjacent to DOC - Stiles Unit tract), facing west 


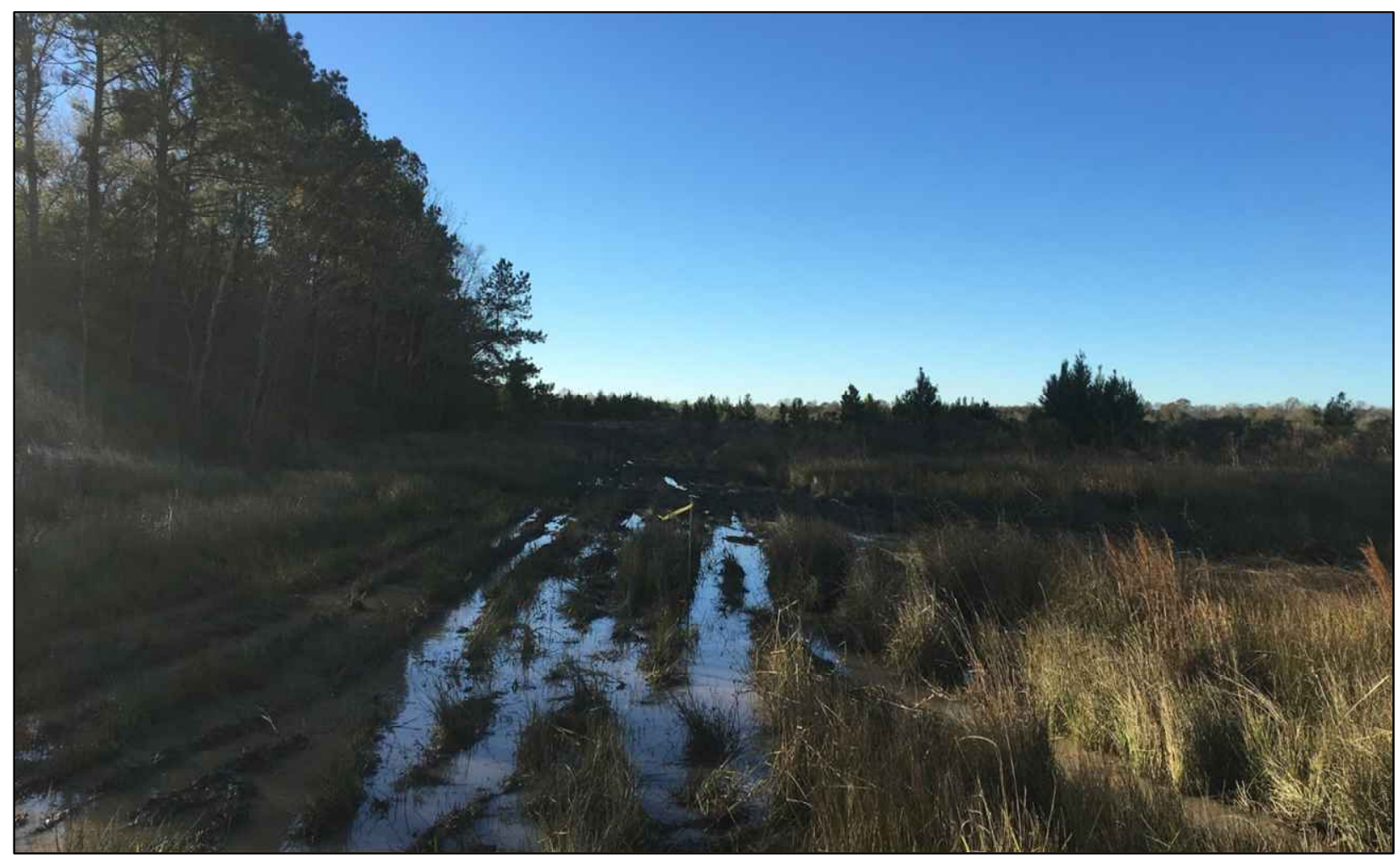

Figure 13. Overview of City of Beaumont Tracts, facing northeast.

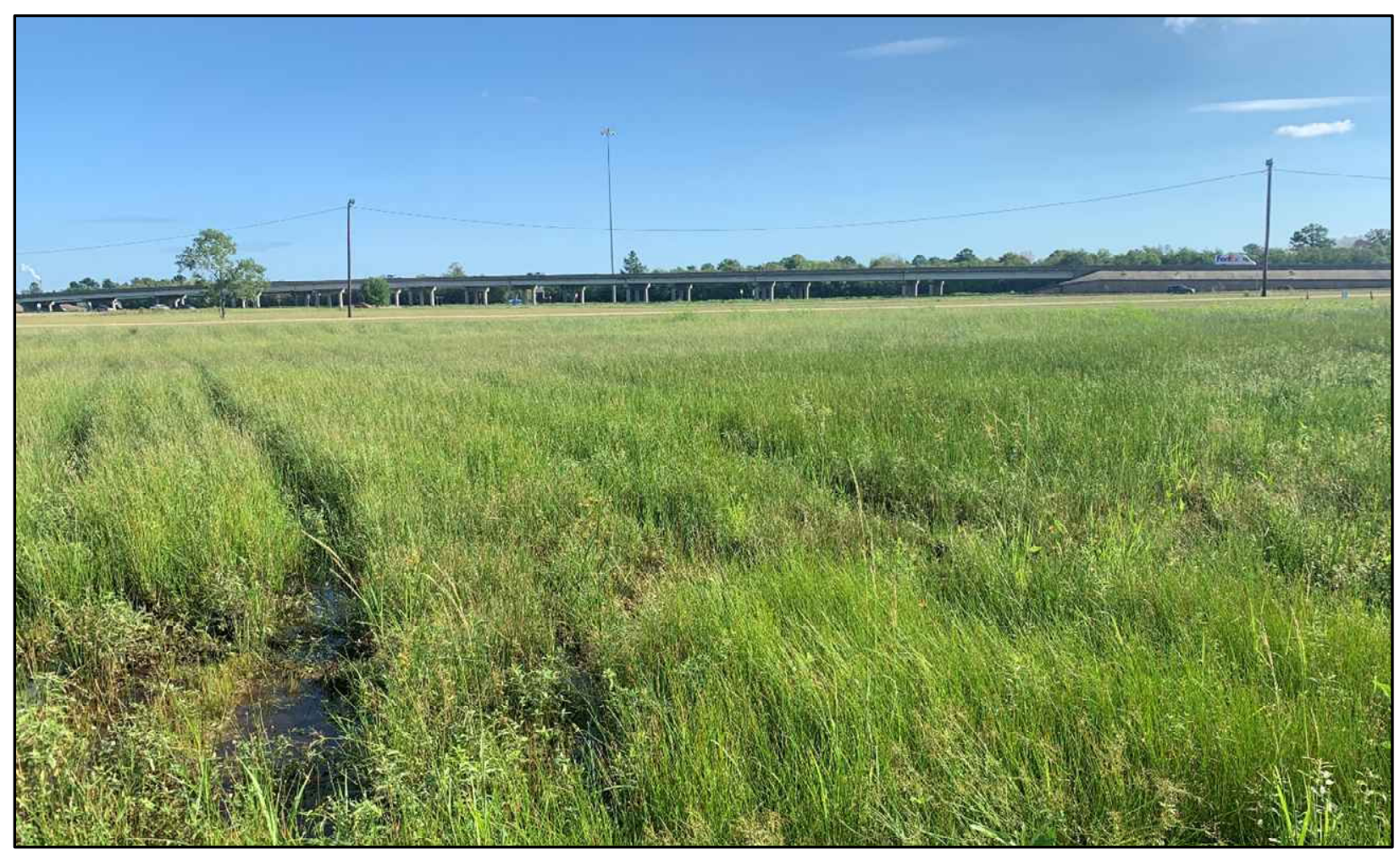

Figure 14. Overview of DOC - Stiles Unit tracts, facing northwest. 


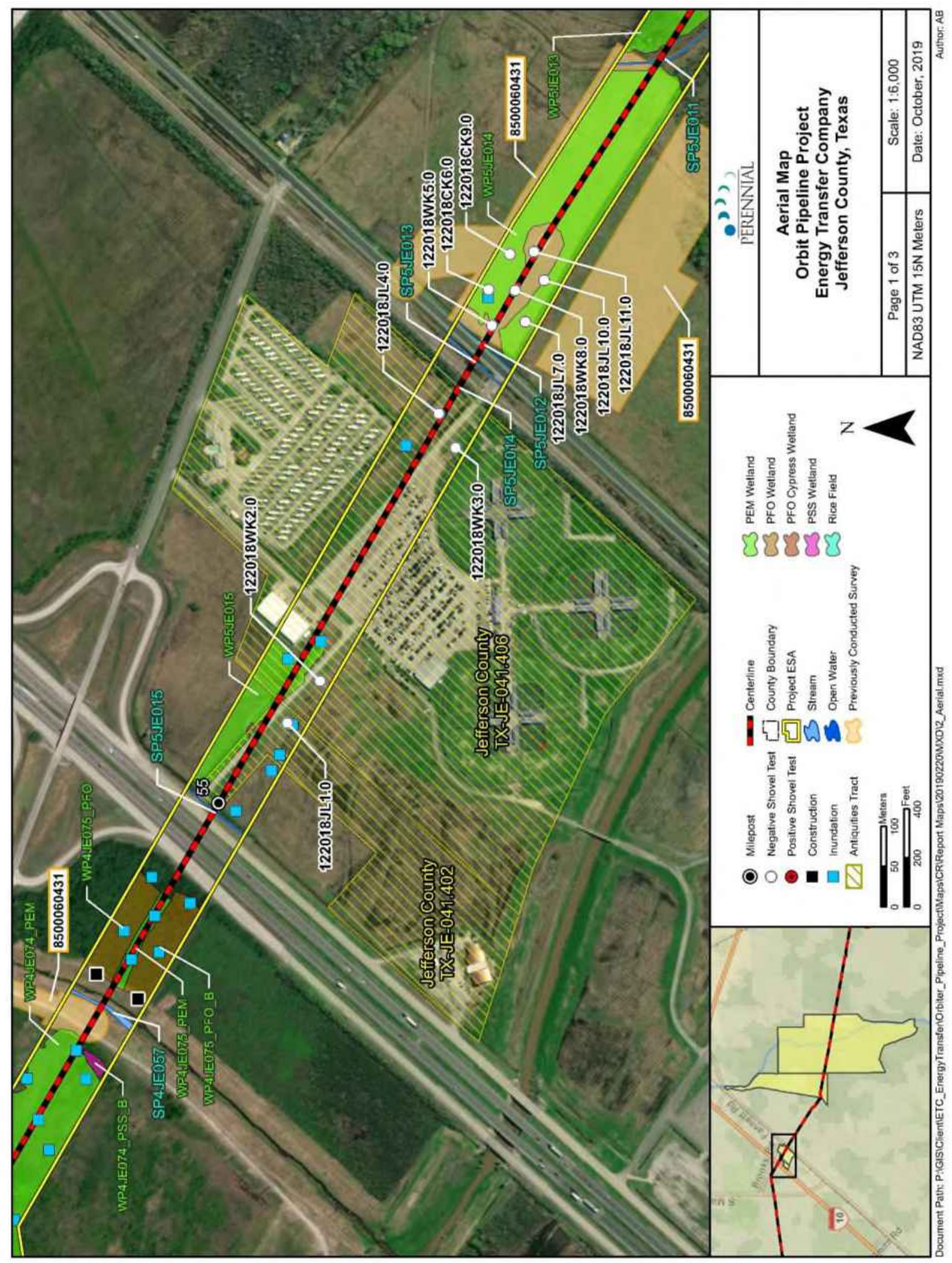

Figure 15. Survey Results for Code-Permitted Tracts - Jefferson County 


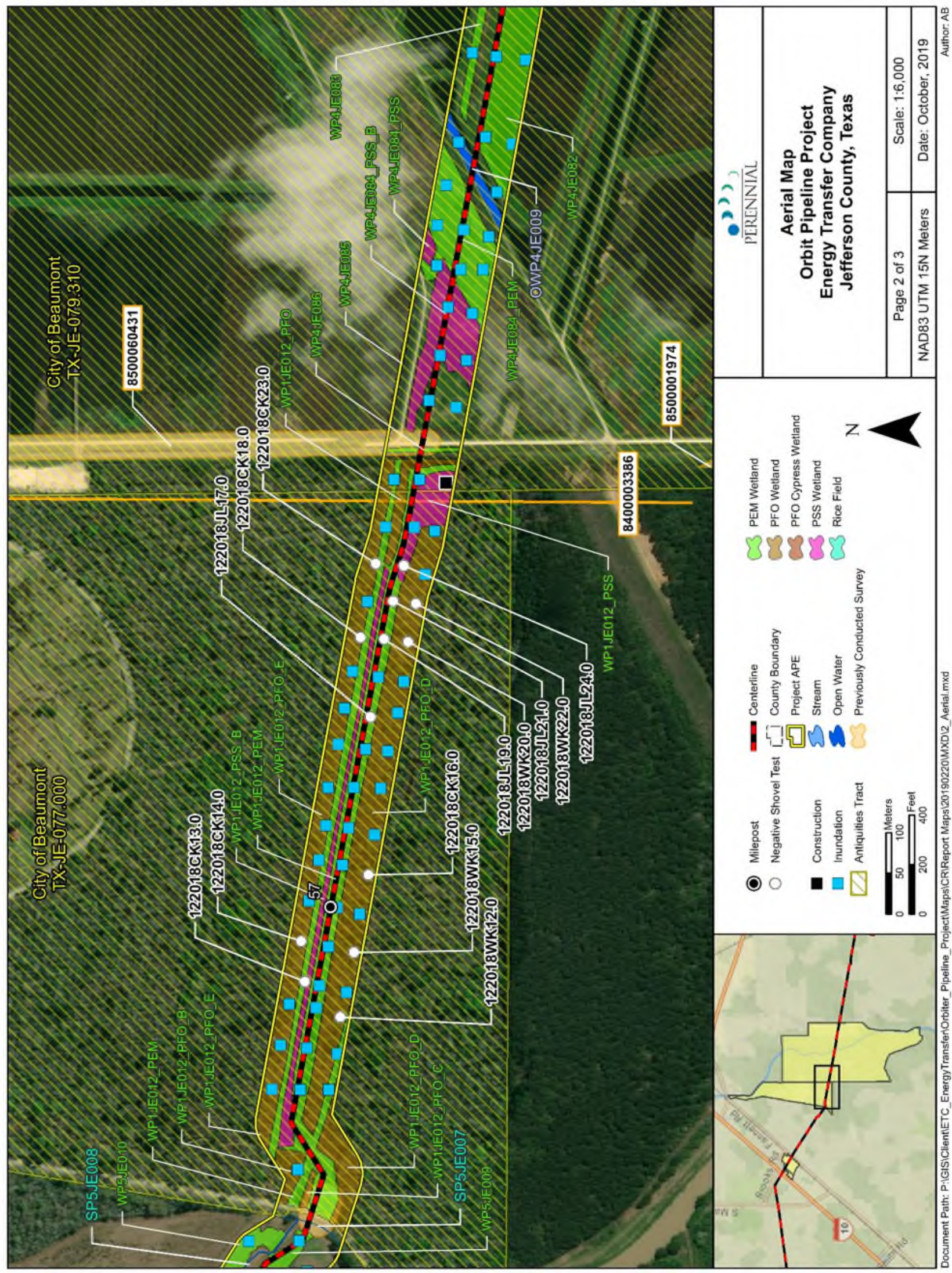

Figure 16. Survey Results for Code-Permitted Tracts - City of Beaumont (continued) 


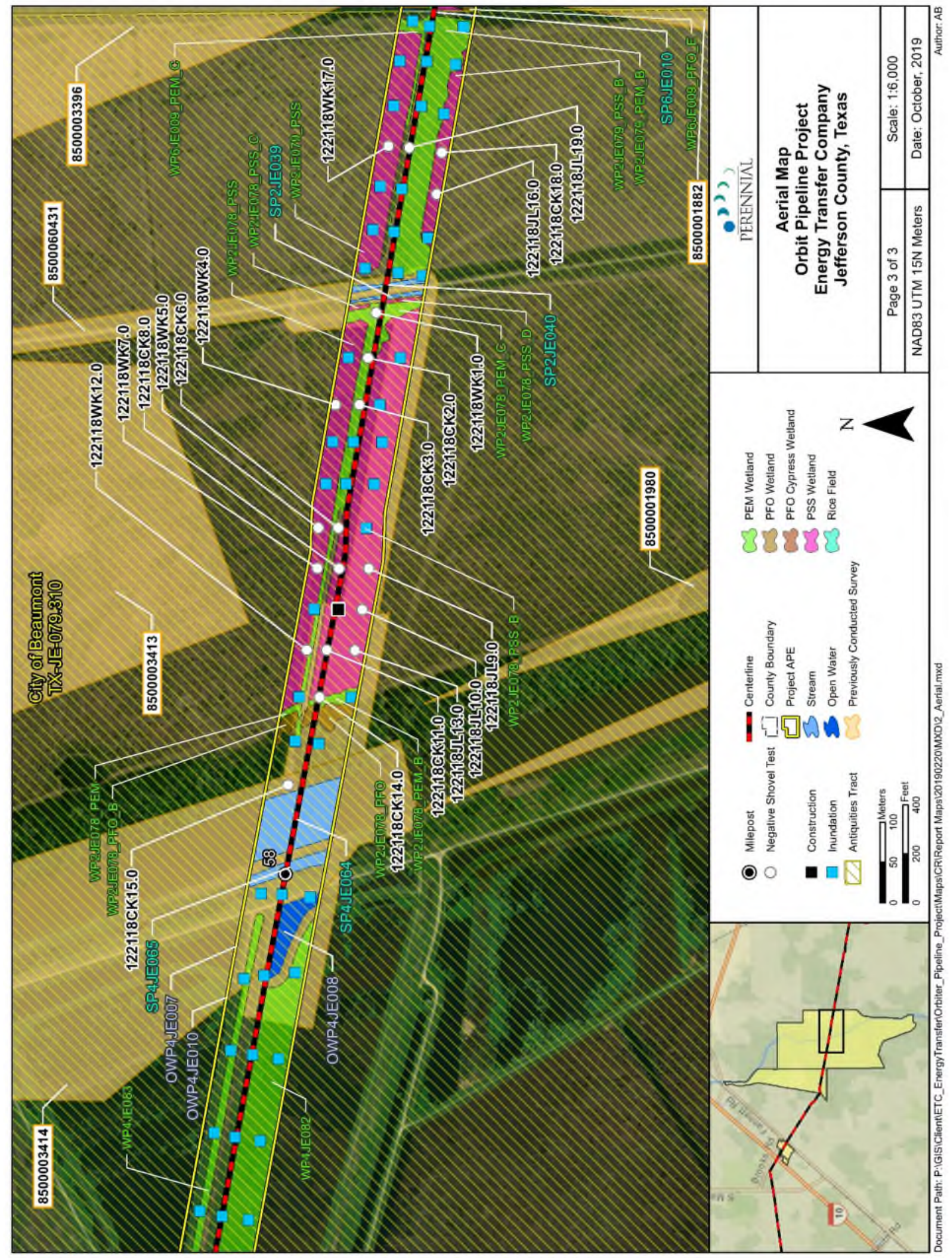

Figure 17. Survey Results for Code-Permitted Tracts - City of Beaumont (continued) 


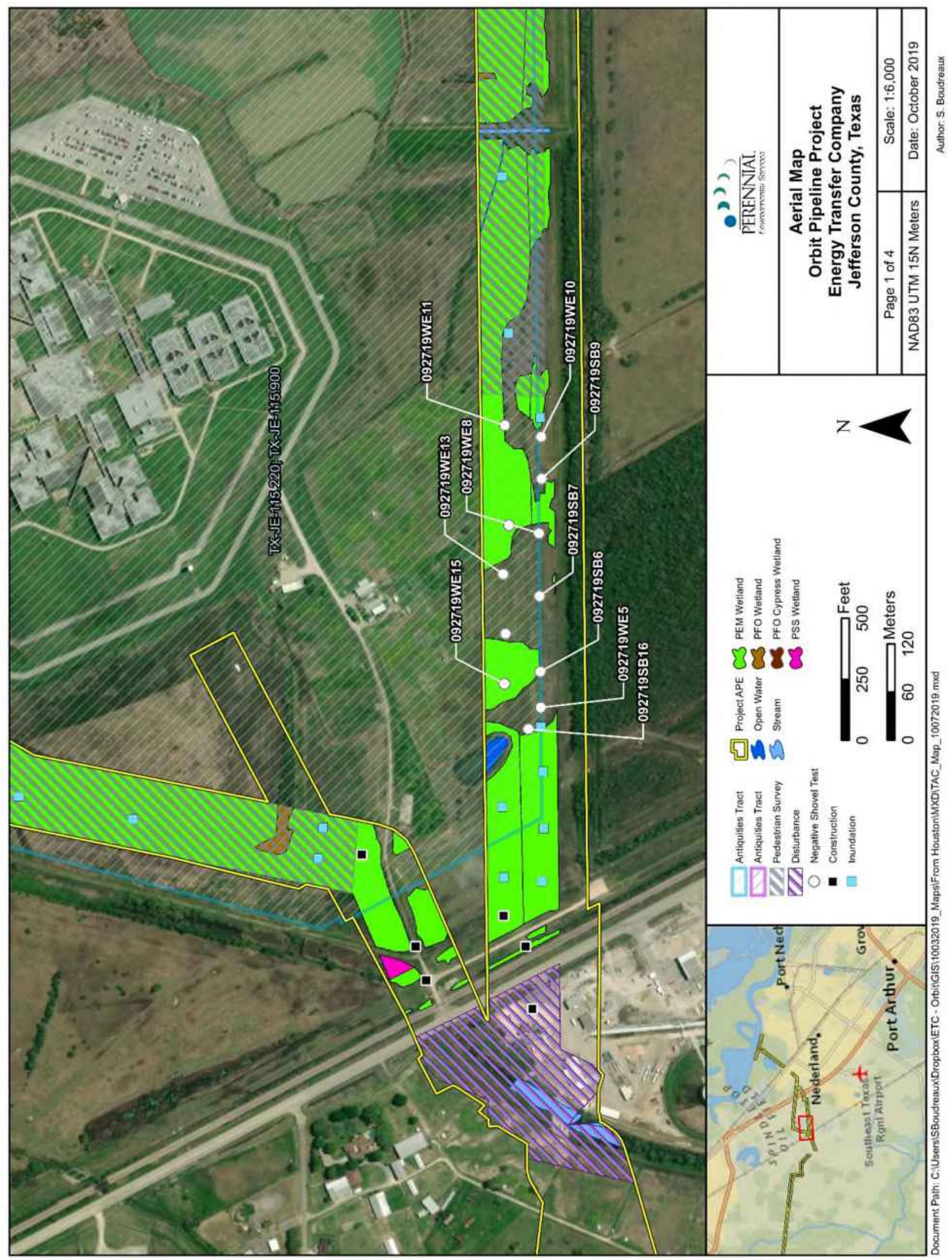

Figure 18. Survey Results for Code-Permitted Tracts, DOC - Stiles Unit and Jefferson County 


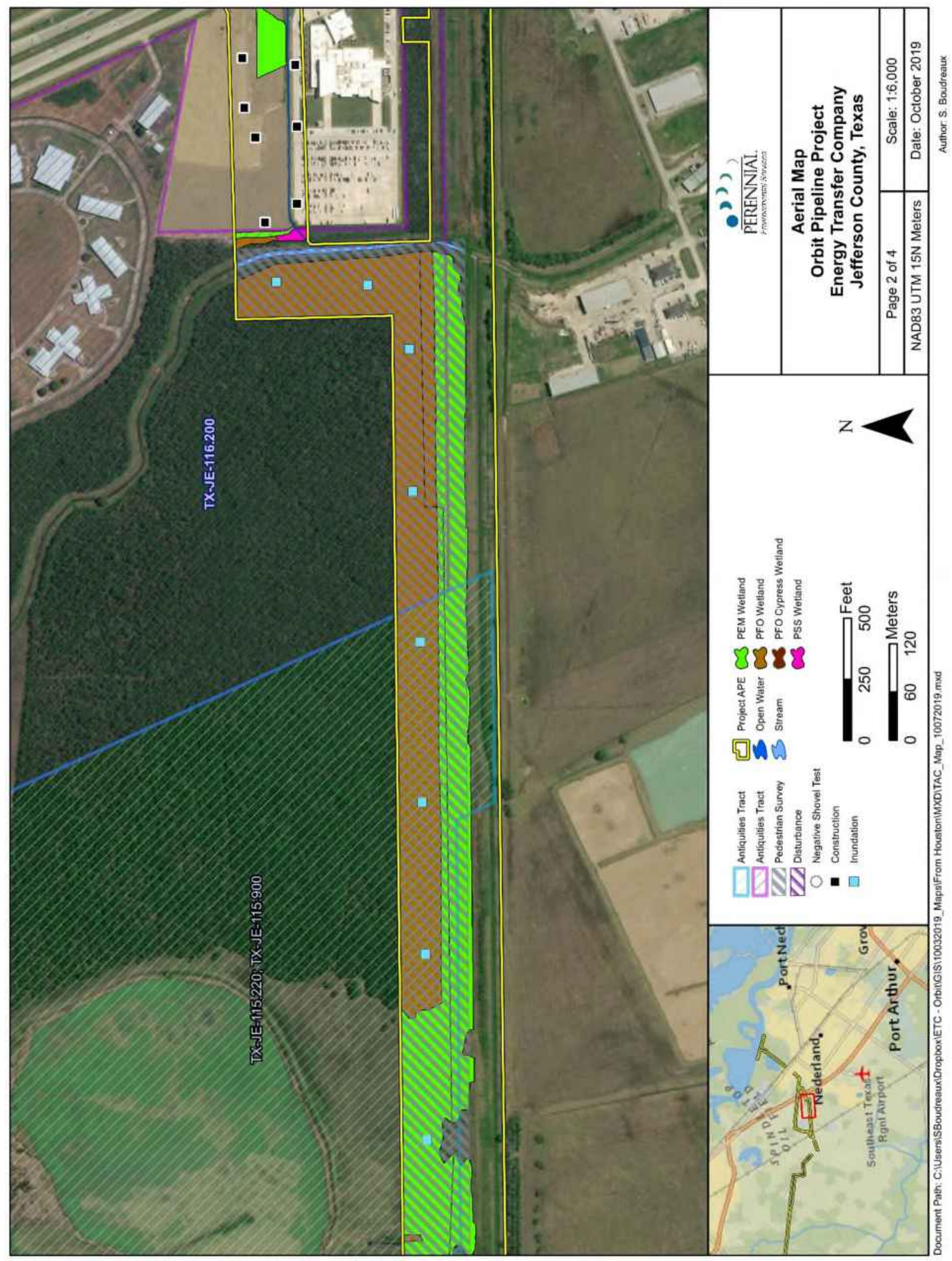

Figure 19. Survey Results for Code-Permitted Tracts, DOC - Stiles Unit and Jefferson County 


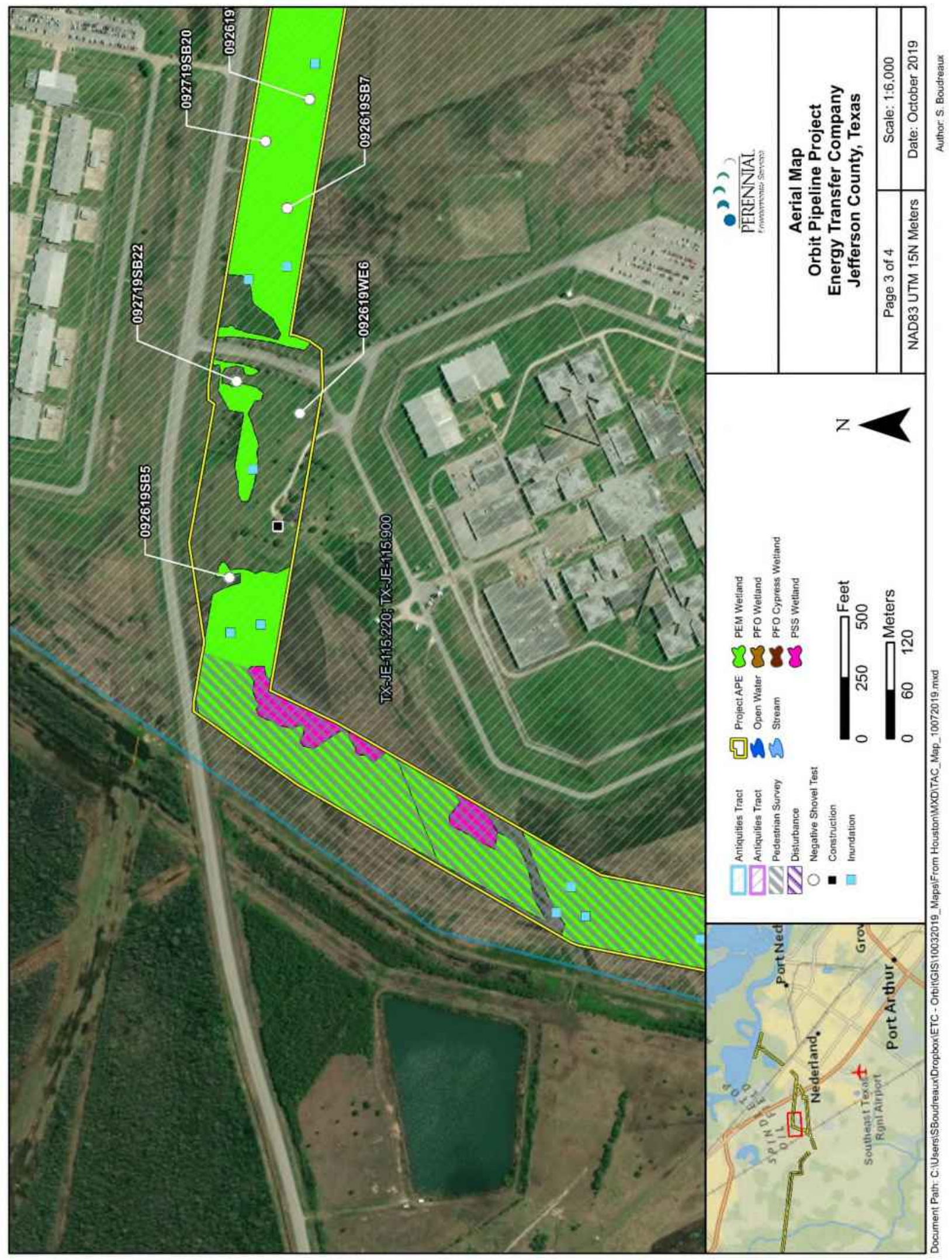

Figure 20. Survey Results for Code-Permitted Tracts, DOC - Stiles Unit and Jefferson County 


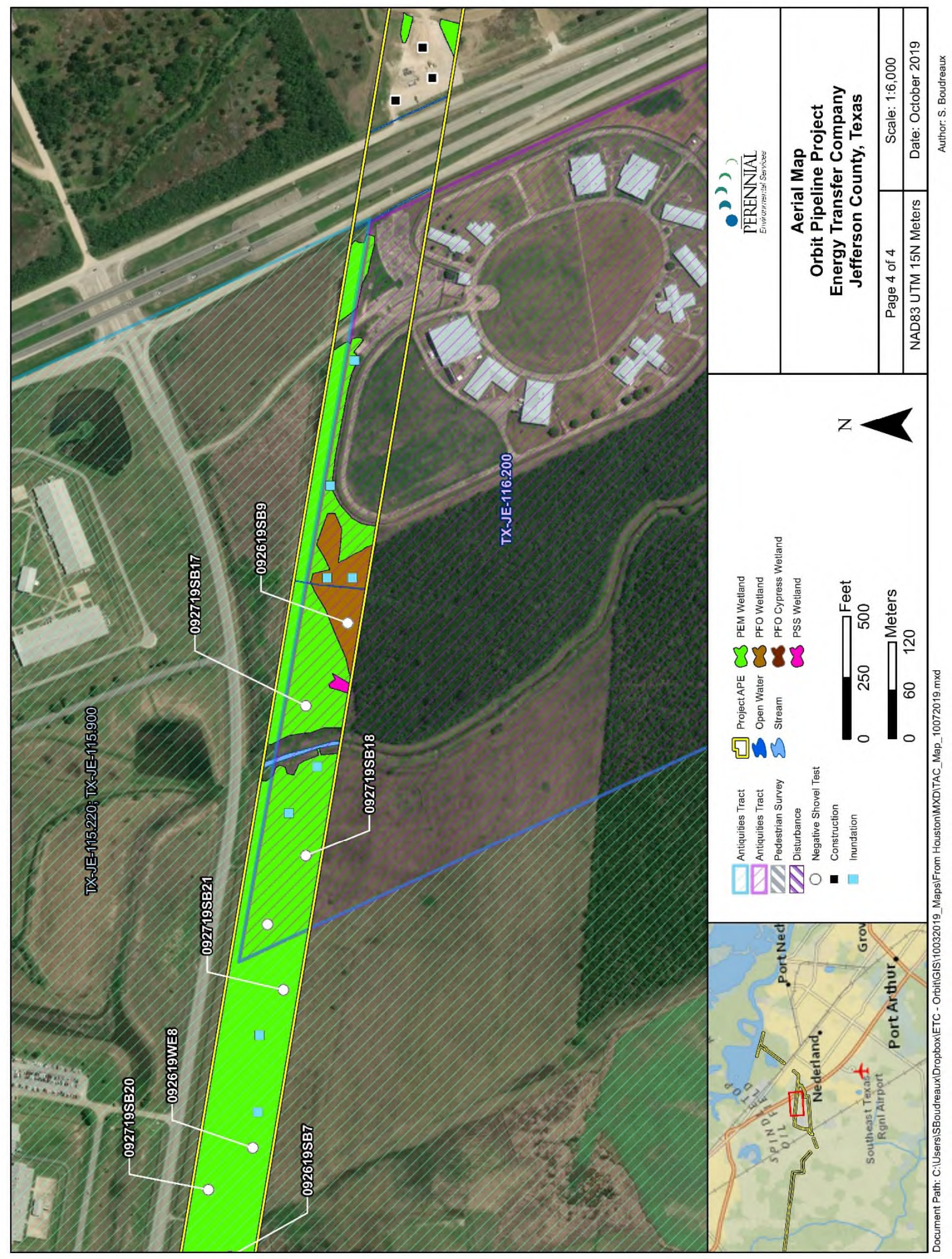

Figure 21. Survey Results for Code-Permitted Tracts, DOC - Stiles Unit and Jefferson County 


\section{CONCLUSION AND RECOMMENDATIONS}

ETC retained Perennial to conduct an intensive Phase I cultural resources investigation for the proposed Project to comply with anticipated USACE and Code permitting requirements. Specifically, archaeological investigations for the Project were conducted in accordance with Section 106 of the NHPA and the Texas SHPO survey standards.

Consistent with the USACE application requirements, the Code, and in accordance with Section 106 of the NHPA of 1966, as amended, the proposed Project must make a reasonable and good faith effort to identify historic properties within the APE and to take into account any direct or indirect effects the proposed undertaking could have on properties listed or considered eligible for listing in the NRHP or for designation as a SAL, as warranted.

As the Project footprint was not finalized at the time of field investigations, survey efforts were concentrated within the vicinity of delineated wetland and waterbody features along the length of the route within a 300.0ft- (91.4-m-) wide ESA. The anticipated depths of impact for the Project will range from 4.0 to $7.0 \mathrm{ft}$. (1.2 to $2.1 \mathrm{~m})$ along the pipeline centerline, with limited deeper impacts at HDD and bore locations, including Cow Island Bayou, Hillebrandt Bayou, Lower Neches Valley River Authority canals, Nolte Canal, the Trinity River, Turtle Bayou, Whites Bayou, Willow Marsh Bayou, and public road crossings. The anticipated depths of impact for temporary workspace areas within the APE corridor would not exceed 0.6 to $1.0 \mathrm{ft}$. (0.1 to $0.3 \mathrm{~m})$. The overall APE for direct effects for the Project measured 2307.19 acres (ac) (933.68 ha), while APE for Code-permitted tracts totaled 210.0 ac $(85.06$ ha). The ESA is coterminous with the Project APE, which is also referred to as the USACE permit areas. Only the areas adjacent to the USACE permit areas and the entirely of Code-permitted areas were surveyed for cultural resources.

Investigations included an archival background review and intensive pedestrian surveys augmented by shovel testing in the vicinity of delineated wetland and waterbody features. Archival research determined that there are no previously recorded sites within, or directly adjacent to the Project APE, and approximately 32.7\% of the Project APE has been previously surveyed for cultural resources. However, many of these surveys are outdated and do not meet modern survey standards. Additionally, many of these previously surveys were conducted for USACE-permitted projects, and the entire Project was not surveyed for the presence of cultural resources. As such, all areas surrounding identified wetland and waterbody for this Project were surveyed.

Perennial biologists delineated a total of 490 wetland areas. Of the 490 wetlands identified within the Project APE, 290 were characterized as palustrine emergent (PEM) wetlands, 106 were characterized as palustrine forested (PFO) wetlands, 4 were classified as PFO-Cypress wetlands, and 90 were characterized as palustrine scrub-shrub (PSS) wetlands. Perennial biologists also identified 270 waterbodies that ranged from perennial streams to ephemeral streams. Of the 270 
waterbodies delineated, 36 were classified as having perennial flow, 66 were classified as having intermittent flow, and 148 were classified as ephemeral flow. Additionally, 20 open water features were delineated and classified as manmade ponds.

Survey efforts were concentrated in the vicinity of these features where land access was voluntarily granted in accordance with a scope of work for the Project approved by the USACE on August 28, 2018, and the Texas Historical Commission (THC) on October 1, 2018. Following the approval of the scope of work, ETC extended the eastern terminus of the Project to the western bank of the Neches River, and other minor reroutes were also implemented. Perennial applied the approved survey methodology to all new Project components.

For Code-permitted tracts, surveys were conducted across the entire length of the Project APE corridor in accordance with a stand-alone scope submitted to the THC on December 14, 2018, and amended August 30, 2019. Additionally, following the receipt of TAP\#8690, the proposed Project was routed onto two additional publicly-owned tracts belonging to the Texas Department of Corrections (DOC) - Stiles Unit and Jefferson County. The TAP \#8690 was revised and amended on August 30, 2019. Following this August 30, 2019, amendment, ETC added additional route options across the DOC - Stiles Unit tract. This additional mileage was surveyed using the same methods as stated in the previously presented revised TAP scope of work for this property.

In all, the survey investigations included the excavation of a total of 1,250 shovel tests of which 1,171 shovel tests were excavated within the Project APE. The remaining 79 shovel tests were excavated outside USACE permit areas but within the Project workspace. For the purposes of this report, only investigations within the Project APE will be discussed.

Survey investigations within the Project APE resulted in entirely negative findings. No archeological sites were encountered within the survey areas reported herein. Additionally, no historic standing structures or landscape features such as historic-age canals were observed with any USACE permit areas. Overall, the surveys documented predominately inundated landscapes with a low probability for intact cultural resources. Numerous existing pipeline corridors and modern canal features are traversed by the Project. While some of the modern canals could be historic in nature or connected to a greater network of irrigation features used historically to supply agricultural crops with water, it is important to note that the majority of the waterways associated with these features will be bypassed via bore/HDD. As such, any impacts to these waterbodies as well as the associated canal structure will be entirely avoided. Additionally, all visual impacts from the proposed pipeline corridor will be temporary in nature.

To date, field surveys have been completed for all accessible wetland and waterbody features along the pipeline route, as well as the total length of the Project survey corridor across all Codepermitted tracts. Prior to the beginning of November 2019, field surveys had not been conducted along the eastern banks and associated bottomlands of the Trinity River due to multiple flooding events that resulted in heavy inundation beginning in September 2018 which prevented access or 
survey investigations of any kind. Additionally, field surveys did not occur along portions of the Project containing denied landowner permissions.

On November 6, 2019, a survey crew was able to access previously inaccessible areas associated with the Trinity River due to several months of normalized weather conditions throughout portions of Eastern Texas. Even under normal conditions, large portions of this area remain constantly inundated due to strong hydrological influences and the geomorphic position of the landscape. However, crews were able to traverse inundated areas by foot to access portions of the Project located immediately adjacent to the Trinity River. While the area located immediately adjacent to the Trinity River was not inundated at the time of survey, wetlands with strong hydrological indicators still dominate the landscape.

Of the 760 delineated wetland and waterbody features, 74 features were originally not surveyed for cultural resources due to restricted land access including denied landowner permissions and significant inundation. Of these 74 features, 38 feature locations ( 27 streams and 42 wetlands) will be bypassed via horizontal directional drill (HDD) or bore trenchless construction methods resulting in no impacts to these features. The remaining five features (including multiple crossing locations of the same feature) are located along the eastern banks of the Trinity River. While these features were surveyed for cultural resources with negative findings, the presence of buried deposits exists within the vicinity of these features. However, access to these features with heavy machinery is not feasible due to the remote location and constant hydrological influences (e.g. inundation and saturation) associated with the floodplain setting of the Trinity River. As such, these five features are proposed to be monitored by a qualified Archeological Monitor during construction efforts. Appendix $\mathbf{C}$ provides each wetland and waterbody feature crossed by the Project with management recommendations and associated comments, while Appendix F provides a Cultural Monitoring Plan to evaluate the five features that will not be avoided during construction efforts. Appendix F also includes a table in response to a letter issued by the USACE Staff Archeologist, Mr. Jerry Androy, on May 17, 2019, indicating that the 74 aforementioned permit areas associated with the Project would require cultural resources investigations. The table lists each permit area, the reason surveys were not originally conducted, and justification for/against the need for monitoring.

Based on the results of the survey effort reported here, no cultural resources will be affected by any construction activities within the Project APE. Aside from Cultural Monitoring at five features (including multiple crossing locations of the same feature) within the Trinity River floodplain, it is Perennial's opinion that no further cultural resources investigations are warranted for the Project. Should archaeological remains be encountered during construction, work in the immediate area will cease, and a qualified archaeologist will be called upon to evaluate the remains and provide recommendations for how to manage the resources under the State's Historic Preservation Plan. 


\section{HUMAN REMAINS}

In the event that human remains are encountered during any part of the Phase I survey effort, work will stop immediately and the appropriate local law enforcement personnel and medical examiner's office will be notified of the discovery. Should the medical examiner determine that the human remains are older than 50 years, personnel will notify the THC and file a notice of existence of a cemetery with the county clerk. Consultation with any concerned parties including landowners, appropriate Tribes, and living descendants will be conducted to ensure compliance with existing state laws. No remains will be removed from the site until jurisdiction has been established and the appropriate permits have been obtained. All activities will adhere to the Texas Health and Safety Code (8 THSC $§ 711.010)$ and the Texas Antiquities Code (13 TAC $\S \S 22.1$ 22.6). If the human remains are determined to be Native American, they will be handled in accordance with (modeled after) the Native American Graves Protection and Repatriation Act (NAGPRA). 


\section{REFERENCES CITED}

Abbott, J.

2001 Houston Area Geoarcheology. A Framework for Archeological Investigation, Interpretation, and Cultural Resources Management in the Houston Highway District. Archeological Studies Program, Report 27. Texas Department of Transportation, Environmental Affairs Division, Austin.

Aten, L.E.

1983 Indians of the Upper Texas Coast. Academic Press, New York

(Atlas) Texas Archaeological Sites Atlas

2019 Texas Archaeological Site Atlas restricted database, Texas Historical Commission. http://nueces.thc. state.tx.us/. Accessed January 8, 2019.

Bousman, C.B., B. W. Baker, and A. C. Kerr

2004 Paleoindian Archeology in Texas. In In The Prehistory of Texas, edited by Timothy K. Perttula. Texas A\&M University Press, College Station.

Brown, K.L.

2004 Ethnographic Analogy, Archaeology, and the African Diaspora: Perspectives from a Tenant Community. Historical Archaeology 38(1):79-89.

Brownlow, R.K., and R. D. Clark

2006 An Intensive Cultural Resources Survey of the USACE Jurisdictional Areas within the Proposed 33-Mile Hull to Mont Belvieu Pipeline Right-of-Way, Liberty County, Texas. Horizon Environmental Services, Inc. Austin

Dillehay, T.D.

1975 Prehistoric Subsistence Exploitation in the Lower Trinity River Delta, Texas. Research Report 51. Austin: Texas Archaeological Survey, University of Texas at Austin.

Ensor, H.B., S. Aronow, MD. Freeman, and J.M. Sanchez

1990 An Archaeological Survey of the Proposed Greens Bayou Stormwater Detention Facility, Greens Bayou, Harris County, Texas. Archaeological Surveys No. 9, Archaeological Research Laboratory, Texas A\&M University, College Station, Texas.

Ensor, H. B. and Robert A. Ricklis

1998 Results of Investigations at the Eagle's Ridge Shell Midden. In Eagle's Ridge: A Stratified Archaic and Clear Lake Period Shell Midden, Wallisville Late Project Area, Chambers County, Texas, edited by H. B. Ensor, pp. 65-236. Wallisville Lake Project Technical Series, Reports of Investigations No. 4. Geo-Marine, Plano TX

Gadus, E.F., and M.A. Howard

1990 Hunter-Fisher-Gatherers on the Upper Texas Coast: Archaeological Investigations at the Peggy Lake Disposal Area, Harris County, Texas. Report of Investigations No. 74. Prewitt and Associates, Inc., Austin. 
Griffith, G.E., S.A. Bryce, J.M. Omernik, J.A. Comstock, A.C. Rogers, B. Harrison, S.L. Hatch, and D. Bezanson.

2007 Ecoregions of Texas. Texas Commission on Environmental Quality, Austin, Texas.

Hall, G.D.

1981 Allens Creek: A Study in the Cultural Prehistory of the Lower Brazos River Valley, Texas. Research Report No. 61. Texas Archeological Survey, The University of Texas at Austin.

Hester, T.R.

1980 A Survey of Paleo-Indian Archeological Remains along the Texas Coast. In Papers on the Archeology of the Texas Coast, edited by L. Highley and T.R. Hester, pp. 1-12. Special Report No. 11. Center for Archaeological Research, The University of Texas at San Antonio.

Kleiner, Diane

2019a Chambers County, Handbook of Texas Online. Electronic Document, http://www.tshaonline.org/handbook/online/articles/hcc09, accessed November 25, 2019.

2019b Jefferson County, Handbook of Texas Online. Electronic Document, http://www.tshaonline.org/handbook/online/articles/hcj05, accessed November 25, 2019.

2019c Liberty County, Handbook of Texas Online. Electronic Document, http://www.tshaonline.org/handbook/online/articles/hcl08, accessed November 25, 2019.

(NRCS) Natural Resources Conservation Service

2019 Web Soil Survey. Online graphic available at

http://websoilsurvey.nrcs.usda.gov/app/WebSoilSurvey.aspx. Accessed on January 8, 2019.

Patterson, L.W.

1980 The Owen Site, 41HR315: A Long Occupation Sequence in Harris County, Texas. Report No. 3. Houston Archeological Society.

Ricklis, Robert A.

1994 Aboriginal Life and Culture on the Upper Texas Coast: Archaeology at the Mitchell Ridge Site, 41GV66, Galveston Island. Coastal Archaeological Research, Inc., Corpus Christi.

2004 The Archeology of the Native American Occupation of Southeast Texas. In The Prehistory of Texas, edited by Timothy K. Perttula. Texas A\&M University Press, College Station.

Ricklis, R.A., and R.A. Weinstein

2005 Sea-Level Rise and Fluctuation on the Central Texas Coast: Exploring Cultural and Ecological Correlates. In Gulf Coast Archaeology: The Southeast United States and Mexico, edited by N.M. White, pp. 68-86. University Press of Florida, Gainesville.

Shafer, H.E., Edward P. Baxter, Thomas B. Stearns, and J. Phil Dering

1975 An Archeological Assessment of the Big Thicket Preserve. Anthropology Laboratory Report 19. Texas A\&M University, College Station. 
Shafer, H. J.

1975 Comments on Woodland Cultures of East Texas. Bulletin of the Texas Archeological Society 46:249-254.

Story, D.A.

1985 Adaptive Strategies of Archaic Cultures of the West Gulf Coastal Plain. In Prehistoric Food Production in North America, edited by R.I. Ford, pp. 19-56. Anthropological Papers No. 75. Museum of Anthropology, University of Michigan, Ann Harbor.

Story, D.A., J.A. Guy, B.A. Burnett, M.D. Freeman, J.C. Rose, D.G. Steele, B.W. Olive, and K.J. Reinhard

1990 The Archeology and Bioarcheology of the Gulf Coastal Plain: Volume 1. Research Series No. 38. Arkansas Archeological Survey, Fayetteville, Arkansas.

Takac, P. R., J. G. Paine and M. B. Collins

2000 Reassessment of Ten Archeological Sites Along the Houston Ship Channel - Morgan's Point to Buffalo Bayou, Harris County, Texas. Studies in Archeology 38. Texas Archeological Research Laboratory, University of Texas, Austin.

(USGS) United States Geological Survey

2019 Mineral Resources Online Spatial Data, United States Department of the Interior. Geologic Maps of US States. http://mrdata.usgs.gov/geology/state, accessed January 8, 2019.

Wilcox, D., M. Chavez, and M. Crow

2007 An Archeological Survey of Portions of the 12" West Texas LPG Pipeline Project in Hardin, Liberty, and Chambers Counties, Texas. SWCA Environmental Consultants, Austin. 
APPENDIX A: AGENCY CORRESPONDENCE 
SCOPE OF WORK

\title{
A Phase I Cultural Resources Survey of the Orbit 20-Inch Ethane Pipeline Project JefFerson, Liberty, AND Chambers Counties, TeXas
}

\author{
Lead Agency: \\ U.S. Army Corps of Engineers
}

Galveston District

Prepared For:

\section{Orbit Gulf Coast NGL Exports, LLC}

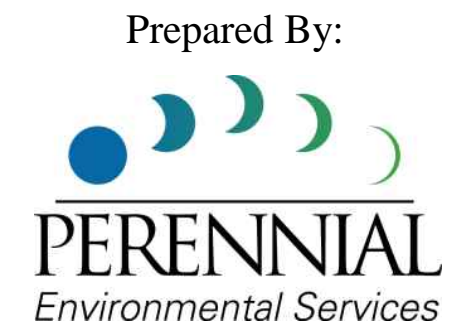

Perennial Environmental Services, LLC 4425 Mopac South, Building II Suite 204

Austin, TX 78735

Principal Investigator:

Heidi Shaw, MSc. 


\section{Introduction}

Orbit Gulf Coast NGL Exports, LLC (Orbit) is proposing to construct approximately 61.5 miles (mi) (107.8 kilometer $[\mathrm{km}]$ ) of new 20.0-inch (50.8-centimeter $[\mathrm{cm}])$-diameter ethane pipeline known as the Orbit 20-inch Ethane Pipeline Project (Project) in Jefferson, Liberty, and Chambers counties, Texas (Figure 1). The U.S. Army Corps of Engineers (USACE) is the lead federal agency and the Project will require a permit pursuant to Sections 10 and 404 of the Clean Water Act. Perennial Environmental Services, LLC (Perennial) has been contracted to conduct the cultural resources survey for the proposed Project.

The following scope of work outlines an intensive Phase I survey plan, including historical inventory and archaeological survey, designed to identify and assess cultural resources within the Project area.

\section{Project Description}

In accordance with permit requirements, Orbit is initiating the pre-application process with the USACE for construction and operation of the Project. Consistent with USACE application requirements, and in accordance with Section 106 of the National Historic Preservation Act (NHPA) of 1966, as amended (36 CFR 800), the proposed Project must make a reasonable and good faith effort to identify historic properties within the Project Area of Potential Effect (APE) and to take into account any potential effects, direct or indirect, the proposed undertaking could have on properties listed or considered eligible for listing in the National Register of Historic Places (NRHP). While the actual APE for direct effects will be limited to the workspace easement (typically not to exceed 120.0feet (ft) [36.6-meters (m)], unless located within additional temporary workspace [ATWS]), survey efforts will cover an expanded 300.0-ft (91.5-m) wide survey corridor, unless otherwise constrained by existing utility infrastructure, to accommodate minor shifts in the pipeline alignment at the discretion of Orbit. The APE for indirect effects at new above-ground facilities will be the area from which any permanent aboveground facilities has the potential to visually diminish or alter the setting of an NRHP-listed or eligible property. Only historic properties within visible range of permanent aboveground Project facilities will be evaluated.

On behalf of Orbit, Perennial is requesting the Texas Historical Commission's (THC) and USACE Galveston District's participation and assistance in determining the need and level of cultural resources investigation necessary for a successful project. It is our hope that by requesting your participation in the process, we can be more effective in protecting important cultural resources. 


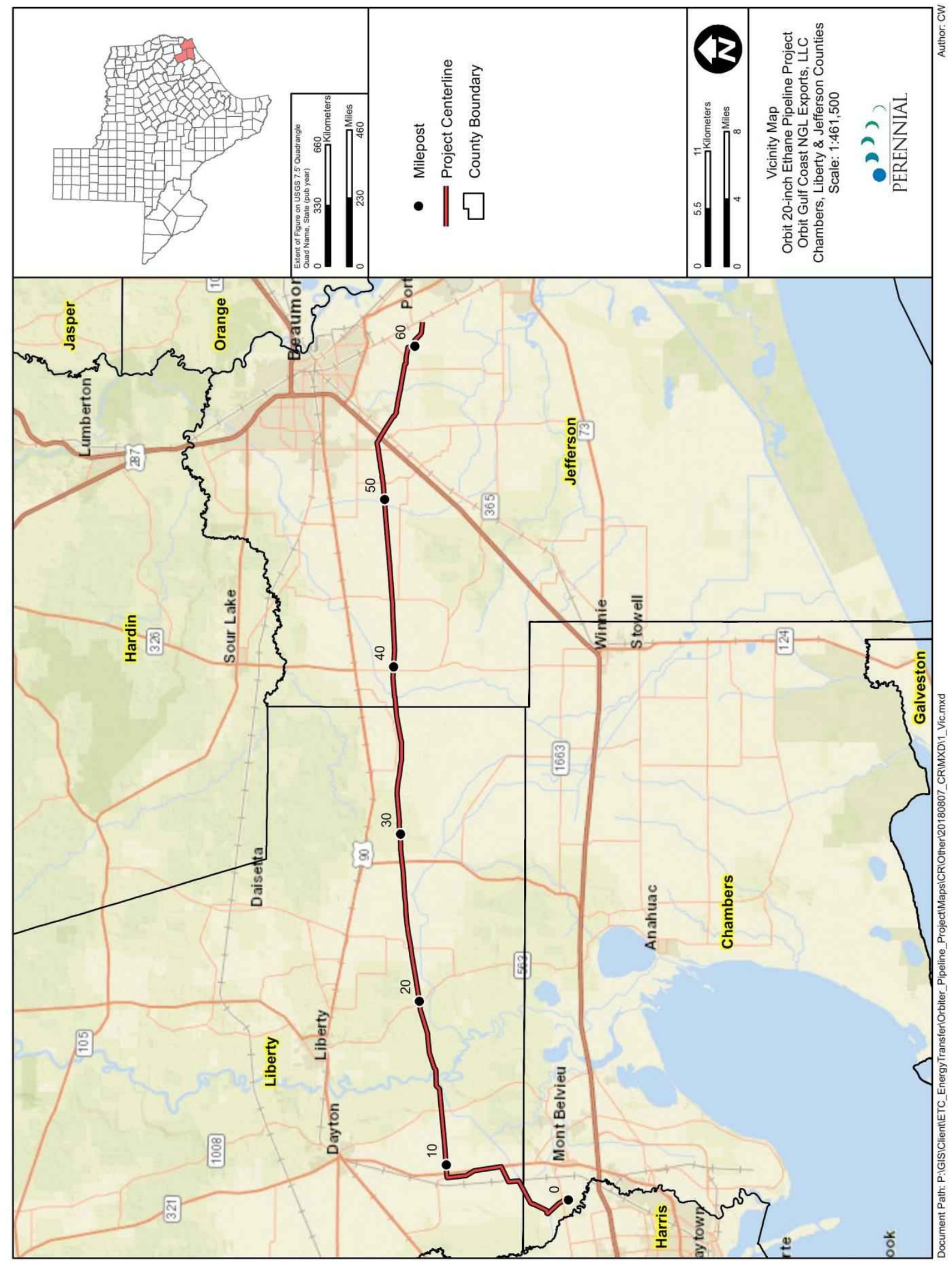

Figure 1. Project location map. 
Cultural resource investigations will be conducted in compliance with Section 106 of the NHPA of 1966 (Public Law [PL] 89-665), as amended; its implementing regulations, "Protection of Historic and Cultural Properties" (36 CFR 800); the National Environmental Policy Act of 1969 (NEPA) (PL 91-190. 83 Stat. 852), and the survey guidelines set forth by the THC and Council of Texas Archeologists (CTA).

The objectives of the survey will be to (1) locate cultural resource sites within the survey corridor within USACE jurisdictional areas; (2) delineate the vertical and horizontal extent of any newly identified sites within the Project boundaries; (3) provide a preliminary evaluation of each site's eligibility for listing in the NRHP; and (4) assess any potential for the Project to directly or indirectly affect historic properties, or other sensitive cultural resources.

To date, Perennial has conducted preliminary research and desktop analyses of the proposed pipeline corridor. The following scope-of-work outlines Perennial's proposed cultural resources survey procedures for the Project.

\section{Previously Recorded Cultural Resources}

Perennial conducted a records and literature review of the THC's Texas Archeological Sites Atlas (Atlas) online database and the NRHP database to identify previously recorded cultural resource sites, historic structures, properties listed in the NRHP, designated historic districts, or State Antiquities Landmarks (SAL) which could potentially be affected by the proposed undertaking. Previously recorded cultural resource site forms, reports of archaeological investigations, general historical documents, and secondary sources concerning the background of the area were reviewed. The records search included a review of all site records and previous surveys on file within $1.0 \mathrm{mi}(1.6 \mathrm{~km})$ of the Project centerline.

In addition to a records and literature search, archaeologists gathered information from secondary sources concerning the prehistoric and historic background of the area. Documents associated with the history of the area were used to model prehistoric and historic settlement patterns in relation to the landscape and terrain characteristics as well as cultural patterns and regional trends. National Resources Conservation Service (NRCS) soil data, US Geological Survey (USGS) 7.5-minute topographic quadrangles, aerial photographs, and contemporary geologic and physiographic features were also examined.

\section{Previously Recorded Sites}

Based upon a background literature review on the THC's Atlas, 3 archaeological sites (Table 1) and 6 cemeteries (Table 2) are within a 1.0-mi (1.6-km) background review 
area (Attachment 1). None of the 3 archaeological sites identified are located within or adjacent to the Project area. These three sites include one prehistoric lithic scatter (41LB121), one historic artifact scatter (41JF97), and a historical farmstead (41JF66). Sites 41LB121 and 41JF97 are not eligible for listing on the NRHP, while Site 41JF66 has an undetermined NRHP eligibility. Site 41JF66 is mapped within $400 \mathrm{ft}$ of the Project ESA and is recorded as a single point on the Atlas, suggesting that the boundaries of the site are not well defined. For this reason, additional archival research and a more intensive shovel testing regime will be implemented in this area to better define the limits and characteristics of the site within the ESA.

Of the six cemeteries identified within the 1-mi background review radius, one cemetery (LC-C070) is recorded as being within $130 \mathrm{ft}$ of the Project ESA. The cemetery appears to be located within a copse of trees within an open pasture. The Project is unlikely to impact the cemetery and the likelihood for unmarked graves within the Project ESA remains low. Nonetheless, if public access to the cemetery is permitted, Perennial will assess the risk of the Project impacting the cemetery and/or unmarked graves during pedestrian survey efforts. Moreover, Perennial is recommending to Orbit to reroute their proposed centerline to better avoid the cemetery delineations. If field efforts indicate a high risk of the Project impacting the cemetery and/or unmarked graves and the client is unable to reroute the centerline, Perennial will recommend mechanical scraping of the topsoil to determine the presence or absence of any human burials within the Project ESA.

\begin{tabular}{|c|c|c|c|c|}
\hline \multicolumn{7}{|c|}{ Sites previously recorded within the 1.0 mi background review radius } \\
\hline Site Trinomial & Site Description & $\begin{array}{c}\text { Year } \\
\text { Recorded }\end{array}$ & $\begin{array}{c}\text { NRHP } \\
\text { Eligibility }\end{array}$ & $\begin{array}{c}\text { Distance from Project } \\
\text { ESA }\end{array}$ \\
\hline 41LB121 & Prehistoric lithic scatter & 2013 & Not eligible & $0.58 \mathrm{mi}$ \\
\hline 41JF66 & $\begin{array}{c}\text { Tyrrell Tenant Farmstead - } \\
\text { Historic, early 20th century }\end{array}$ & 1990 & Undetermined & $360 \mathrm{ft}$ \\
\hline 41JF97 & Historic scatter & 2011 & Not eligible & $0.37 \mathrm{mi}$ \\
\hline
\end{tabular}

\begin{tabular}{|c|c|c|}
\hline \multicolumn{3}{|c|}{ Table 2. } \\
Cemeteries recorded within the 1.0 mi background review radius \\
\hline Cemetery Name & Cemetery Number & Distance from Project ESA \\
\hline Freeman Cemetery & N/A & $0.78 \mathrm{mi}$ \\
\hline Sahara Cemetery & LB-C070 & $130 \mathrm{ft}$ \\
\hline Quarters Cemetery & LB-C075 & $0.43 \mathrm{mi}$ \\
\hline Unknown Cemetery & LB-C067 & $0.93 \mathrm{mi}$ \\
\hline
\end{tabular}


Table 2.

Cemeteries recorded within the $1.0 \mathrm{mi}$ background review radius

\begin{tabular}{|c|c|c|}
\hline Cemetery Name & Cemetery Number & Distance from Project ESA \\
\hline Redmond Field & LB-C132 & $0.76 \mathrm{mi}$ \\
\hline Miller Cemetery & LB-C068 & $0.6 \mathrm{mi}$ \\
\hline
\end{tabular}

\section{Previously Conducted Surveys}

The background review also determined that a total of 37 previous archaeological projects have been conducted within 1-mi $(1.6 \mathrm{~km})$ of the Project centerline (see Attachment 1). The vast majority of these archaeological investigations consist of Phase I surveys for large-scale energy infrastructure projects. The lead agency on these projects is typically either the USACE Galveston District or the Federal Energy Regulatory Commission (FERC). Many of the remaining surveys were conducted in support of municipal transportation projects.

Twenty of the 37 surveys overlap or intersect portions of the Project route. These previous survey investigations constitute an approximately 20 percent overlap with the current Project route. Portions of the Project that have been previously surveyed in accordance with current CTA/THC survey standards will not be resurveyed by Perennial. In areas where the previous survey do not overlap with the proposed Project environmental survey area (ESA), then the area will be surveyed according to the existing standards.

\section{Field Methods}

The cultural resources survey will be conducted by one to two crews comprised of up to three archaeologists each under the supervision of the Principal Investigator who will provide logistical and technical support, as well as oversight of all staff. In anticipation of USACE permitting requirements, Phase I survey investigations will be concentrated in the vicinity of USACE-jurisdictional wetland or waterbody features. In practice, the archeological field crew will utilize data collected by Perennial field biologists to identify jurisdictional feature locations within the proposed survey corridor.

In general, the Texas State Minimum Archeological Survey Standards (TSMASS) require that a minimum of 16 shovel tests be excavated per $1.0 \mathrm{mi}(1.6 \mathrm{~km})$ for rights-of-way (ROWs) measuring up to $100.0 \mathrm{ft}(30.0 \mathrm{~m})$ in width (1 survey transect). For the majority of the Project length, intensive survey investigations will take place within an approximately $300.0 \mathrm{ft}(91.5 \mathrm{~m})$ - wide corridor. Within the vicinity of USACE jurisdictional areas, shovel tests will be excavated along three evenly spaced transects at intervals ranging from 30 to 100 meters, depending on the archaeological sensitivity of the landscape. 
Perennial has been conducted a preliminary probability assessment for the Project ESA utilizing multiple data sources, including location of previously recorded sites, soils and geology, aerial imagery, as well as topographic and historic maps. Overall, the probability classifications are meant to be a guidance tool that serves to inform survey strategy and level of effort while ensuring adherence to state and federal standards and guidelines. The three probability classifications and the characteristics for each probability area are defined as follows:

High Probability Areas (HPAs) will be defined as areas that are likely to contain prehistoric and/or historic-age archaeological sites. HPAs generally include settings along major waterbodies, such as the Trinity River and bayous, and incorporate other high probability settings such as alluvial terraces, Holocene-aged soil deposits, remnant natural levees, benches, and topographic rises in floodplain settings (relatively unaffected by natural or human-induced processes), or proximity to previously recorded sites or historic structures. In HPAs, survey efforts would extend beyond the water feature by a variable distance of 164.0 to $492.0 \mathrm{ft}(50$ to $150 \mathrm{~m}$ ) to include any adjacent prominent landforms, terrace projections, remnant natural levees, benches, topographic rises in floodplain settings, (relatively unaffected by natural or human-induced processes), as well as any previously recorded sites or historic structures. Surveys across HPA settings will include shovel testing intervals ranging from in intervals spaced 82.0 to 164.0 feet (25.0-50.0 meters) along parallel transects. If it is determined that the pipeline will be installed via horizontal directional drill (HDD) under major waterway crossing resulting in no impacts to high probability alluvial settings, then surveys would be concentrated in the HDD entry and exit workspaces.

Medium Probability Areas (MPAs) will be defined as areas that exhibit landscape characteristics of an HPA, but that show evidence of human-induced or natural disturbances that would impact the integrity of any previously unrecorded archaeological deposits. MPAs are also defined as areas in which resources, such as lithic raw materials, may be located. Generally, the remnants of historic structures and artifact scatters are often found in MPAs. In MPAs, survey efforts would extend beyond the water feature by a variable distance of 164.0 to $492.0 \mathrm{ft}(50$ to $150 \mathrm{~m})$ to include any adjacent landscape features and/or potential raw material resources. Surveys across these settings included shovel testing intervals ranging from 165.0 to $330.0 \mathrm{ft}(50.0$ to $75.0 \mathrm{~m})$ along parallel transects

Low Probability Areas (LPAs) will be defined as areas that are inundated lowlands, swamps and sloughs, as well as expansive wetlands within agricultural settings. LPAs will consist of soils that are of Pleistocene-age or older. LPAs will also include locations with significant disturbance from oil and gas, and/or modern development that have created varying levels of landscape modification. Survey efforts in LPAs will be 
conducted within roughly 164.0 to $492.0 \mathrm{ft}(50$ to $150 \mathrm{~m})$ of either side of the delineated feature boundaries. Surveys across these settings included shovel testing intervals ranging from 246.0 to $328.0 \mathrm{ft}$ (75.0 to $100.0 \mathrm{~m})$ along parallel transects

Should wetlands be delineated across large areas with a discontinuous or irregular boundary, then the investigations would consist primarily of pedestrian surveys across feature clusters with shovel testing efforts conducted at the discretion of the Principal Investigator or crew chief.

Portions of the Project that have been previously surveyed in accordance with the current TSMASS will not be resurveyed during the proposed Phase I survey, with the exception of revisiting previously recorded sites. In addition, significant portions of the proposed route are collocated with existing utility corridors with multiple buried pipelines. In such settings, the number of survey transects may be reduced with shovel tests excavated only in greenfield settings. Any deviations from the state mandated survey standards will be thoroughly documented, and disturbances will be photographed.

In general, shovel tests will measure approximately $30-\mathrm{cm}$ (12-in) in diameter and will be excavated by natural strata. Shovel tests will be excavated to a depth commiserate with the project impacts where possible per the THC/CTA survey standards, or to where preHolocene sterile substrates are encountered, if possible, unless manual shovel testing is unable to penetrate hard clay soils. All soil matrices will be sifted through 6.3-millimeter (1/4-inch) mesh hardware cloth unless dominated by clay. Clayey matrix will be finely divided by trowel and visually inspected.

For each of the shovel tests, the following information will be recorded on shovel test logs: location, maximum depth, and the number of soil strata. For each soil stratum, thickness, texture, color, and the presence or absence and nature of cultural materials will be recorded. Perennial will maintain a selective artifact collection survey strategy whereby only representative samples of artifacts by category and diagnostic artifacts will be collected from each newly recorded or revisited site and housed temporarily at Perennial's laboratory for analysis. Any collected artifacts will then be catalogued, analyzed, and prepared for submittal to an approved permanent curatorial facility or returned to the landowner upon request at the completion of the Section 106 consultation process.

The field crew will record all shovel test locations, isolated finds, archaeological sites and associated features using a handheld GPS device. Also, each archaeologist will be equipped with a compass, shovel test and photographic logs, daily journal forms, and appropriate state site forms. The crew will be equipped with topographic maps and aerial 
photographs of the survey corridor, a digital camera, and a cellular telephone to maintain contact with the Principal Investigator (terrain permitting).

If an archaeological site is identified, the appropriate delineation techniques will be systematically applied to identify the horizontal and vertical limits of each site's boundary. Site boundaries may be determined based on both surface artifact density and the presence or lack of subsurface components. For subsurface sites, a series of shovel tests will be excavated radiating in the four cardinal directions or, if more appropriate, along perceived major and minor topographic and site axis. In practice, shovel tests within potential sites will be placed along transects at $10.0-\mathrm{m}(33.0-\mathrm{ft})$ intervals to determine the depth and potential integrity of cultural deposits, and to carefully examine for the presence of intact archaeological features and/or discrete episodes of occupation. In the absence of subsurface deposits, controlled pedestrian surface inspections will be conducted and site boundaries defined based on a marked reduction in surface artifact density. Shovel testing or pedestrian surveys will not be conducted beyond the project right-of-way boundary to ensure no trespassing onto private property occurs.

In addition to shovel testing, a hand-operated or mechanical auger, and/or mechanical trenching may be needed in areas of the Project ESA with deep alluvial sediments that are within HPAs or known archaeological site deposits to probe for deeply buried cultural materials below shovel testing capabilities (i.e., $1 \mathrm{~m}$ ). The method for testing in areas of deep alluvial sediments will be determined at a later date in conjunction with the THC and USACE based on observed field conditions. Should field conditions (i.e., inundation, lack of access, prevalence of buried active utilities) prevent testing of deep alluvial sediments in the Project ESA, then construction monitoring by a qualified archaeologist may be necessary.

\section{Historic Structure Documentation}

All historic-age structures (i.e., older than 50 years) located within the Project survey corridor will be thoroughly documented during the survey efforts. Historic Standing Structure forms will be completed for each resource. The historic structures will be adequately photo-documented, sketch maps will be drawn, and each resource will be plotted with a GPS device. Shovel tests may be excavated in the vicinity of the structures to determine if an associated subsurface archaeological component is present. All data will be reviewed by a qualified architectural historian to determine date of construction, style, integrity, and overall significance and NRHP eligibility.

\section{Viewshed Analysis}

As the majority of the Project will be below-ground, viewshed analysis will only be conducted at designated above-ground facilities. At present, these facilities have not been 
identified. As such, Perennial will assess any indirect effects associated with these individual facilities as they become available. In general, however, most will likely be constructed in high-production areas in close proximity to other similar facilities. In such cases, indirect impacts may have already been incurred and the addition of another similarly-functioning facility would constitute an in-kind element on the landscape.

For all new permanent above ground facilities, investigations will include an initial desktop analysis of the built environment within a 0.5 -mile radius. Using the results of the desktop analysis, features within the built environment that are identified as being historical or potentially historic in age within the viewshed radius will be reviewed through aerial photography, historical topographic maps, and on the ground assessment. If the Principal Investigator determines that the built environmental feature is in fact historical and has the potential to be impacted by the construction of the new facility, an architectural historian will be retained and an official NRHP eligibility assessment rendered.

\section{Reporting}

Following the completion of the field surveys, Perennial will prepare a draft report of the investigations. The format of the report will adhere to review guidelines suitable to the State Historic Preservation Officer (SHPO) in accordance with the THC's Rules of Practice and Procedure, Chapter 26, Section 27, and the CTA Guidelines for Cultural Resources Management Reports. The report will document previous investigations in the area, background cultural setting, the methodology used during the investigations, the general nature and extent of cultural resources encountered during the cultural resource survey, and management recommendations for of any documented cultural resources per all applicable state and federal laws. Once the client approves the report, the draft report will be submitted to the THC and the USACE for review. Following the agency review period, any appropriate edits or comments will be incorporated, and a final draft will be produced and distributed appropriately.

\section{Laboratory Methods}

The artifact collection procedure employed by Perennial is meant to be flexible to accommodate variations in site size, density of artifacts, setting, presence/absence of subsurface assemblages, site type, and feasibility constraints. In general, the artifact collection strategy is designed to procure comprehensive inventory-level information to facilitate NRHP evaluations and avoidance strategies, as well as adhere to property restrictions. Specifically, the artifact collection policy may vary between $100 \%$ collection of observed artifacts, and a representative sample collection strategy. In practice, the $100 \%$ collection strategy would most commonly be applied to sites with subsurface assemblages. For surficial sites, the Principal Investigator in consultation 
with the field supervisor would determine the collection strategy based on the site type and setting. In the event that a sample collection policy is implemented for a site, field staff will inventory and describe the characteristics, material, type, decoration, and other descriptive traits; and photograph all observed artifacts whenever feasible. Meanwhile, Perennial will also collect representative samples of each diagnostic artifact type and variety. All artifacts that are collected will be brought back to Perennial's laboratory to be cleaned, sorted, cataloged, photo-documented, and analyzed. Standard analytical techniques and existing typologies, as appropriate for Southeastern prehistoric and historic archaeological studies, will be employed. Artifacts collected on private land will be curated in Perennial's laboratory in Austin, unless requested otherwise by the landowner.

Laboratory and curation methods for artifacts identified and collected on public lands will be described in a forthcoming Texas Antiquities Committee Permit Application scope-ofwork.

\section{Human Remains}

In the event that human remains are encountered during any part of the Phase I survey effort, work will stop immediately and the appropriate local law enforcement personnel and coroner's office will be notified of the discovery. Should the coroner determine that the human remains are not forensic in nature, then the lead federal agency will claim jurisdiction of the discovery and will commence consultation with any concerned parties including landowners, appropriate tribes, and/or living descendants to ensure compliance with existing state and federal laws. No remains will be removed from the site until jurisdiction has been established and the appropriate permits have been obtained. All activities will adhere to the Texas Health and Safety Code (8 THSC $\S 711.010$ ) and the ACT (13 TAC $\S \S 22.1-22.6$ ). If the human remains are determined to be Native American, they will be handled in accordance with the Native American Graves Protection and Repatriation Act (NAGPRA).

\section{References}

(Atlas) Texas Archaeological Sites Atlas

2018 Texas Archaeological Site Atlas restricted database, Texas Historical Commission. http://nueces.thc. state.tx.us/. Accessed August 1, 2018. 
ATTACHMENT 1 - TOPOGRAPHIC MAPS OF THE PROJECT SURVEY CORRIDOR 
This Page Intentionally Left Blank to Protect Sensitive Cultural Resources 
From:

Sent:

To:

Cc:

Subject:
Androy, Jerry L CIV USARMY CESWG (US) < Jerry.L.Androy@usace.army.mil>

Tuesday, August 28, 2018 3:50 PM

Heidi Shaw

Abby Peyton

RE: Scope of Work submittal for review--Orbit 20-inch Ethane Pipeline Project

Hi Heidi,

The SOW looks fine as submitted. Please make sure the SHPO signs off on the SOW as well.

Jerry Androy

Regulatory Archeologist and Tribal Liaison U.S. Army Corps of Engineers

2000 Fort Point Road

Galveston, TX 77550

(409) 766-3821

Jerry.L.Androy@usace.army.mil

-----Original Message-----

From: Heidi Shaw [mailto:hshaw@perennialenv.com]

Sent: Tuesday, August 21, 2018 12:28 PM

To: Androy, Jerry L CIV USARMY CESWG (US) <Jerry.L.Androy@usace.army.mil>

Cc: Abby Peyton <APeyton@perennialenv.com>

Subject: [Non-DoD Source] Scope of Work submittal for review--Orbit 20-inch Ethane Pipeline Project

Dear Mr Androy,

In anticipation of our client submitting a PCN application for a project in Jefferson, Liberty, and Chambers Counties, we have attached a cultural scope of work for your review. We have concurrently submitted the SOW to the THC for their comments. We estimate that we will be ready to mobilize for fieldwork in mid-September, pending any comments/directions from the ACOE.

If you have any questions or require any clarifications, please feel free to reach out via phone or email.

Kind Regards,

Heidi Shaw

Heidi Shaw, MSc, RPA 
From:

Sent:

To:

Subject: noreply@thc.state.tx.us

Monday, October 01, 2018 9:42 AM

Heidi Shaw; reviews@thc.state.tx.us; Jerry.L.Androy@usace.army.mil

Project Review: 201900720

TEXAS HISTORICAL COMMISSION

real places telling real stories

Re: Project Review under Section 106 of the National Historic Preservation Act and/or the Antiquities Code of Texas

\section{0}

Orbit 20-inch Ethane Pipeline Project

Beaumont,TX

Dear Heidi Petyo:

Thank you for your submittal regarding the above -referenced project. This response represents the comments of the State Historic Preservation Officer, the Executive Director of the Texas Historical Commission (THC), pursuant to review under Section 106 of the National Historic Preservation Act.

The review staff led by Maggie Moore has completed its review and has made the following determinations based on the information submitted for review:

\section{Archeology Comments}

- THC/SHPO concurs with information provided

We look forward to further consultation with your office and hope to maintain a partnership that will foster effective historic preservation. Thank you for your cooperation in this review process, and for your e fforts to preserve the irreplaceable heritage of Texas. If you have any questions concerning our review or if we can be of further assistance, please email the following reviewers: Maggie.Moore@thc.texas.gov.

Sincerely,

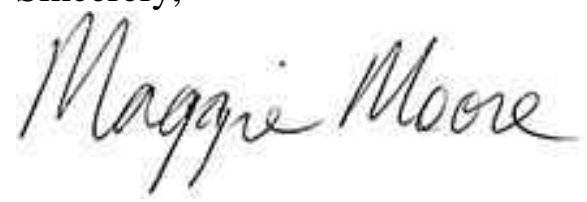

For Mark Wolfe, State Historic Preservation Officer

Executive Director, Texas Historical Commission

\section{Please do not respond to this email.}

cc: Jerry.L.Androy@usace.army.mil 


\section{DEPARTMENT OF THE ARMY \\ U.S. ARMY CORPS OF ENGINEERS, GALVESTON DISTRICT \\ P. O. BOX 1229 \\ GALVESTON, TEXAS $77553-1229$}

May 17, 2019

Evaluation Branch

SUBJECT: Department of the Army Permit Application SWG-2019-00074

Energy Transfer Company

Attn: Jonathan Minton

1300 Main Street

Houston, Texas 77002

Dear Mr. Minton:

The U.S. Army Corps of Engineers, Galveston District (Corps) Staff Archeologist has reviewed the draft report titled, A Phase I Cultural Resources Survey of the Orbit Pipeline Project, Jefferson, Liberty, and Chambers Counties, Texas, prepared for Energy Transfer Company by Perennial Environmental Services, and dated March 2019. Seventy four permit areas have not been investigated for the presence of historic properties (see enclosure 1). A cultural resources investigation is required within these areas to determine if historic properties are present.

The archeologist contracted by the applicant must submit a draft report of the cultural resources investigations for review and approval to both the Corps Staff Archeologist and the SHPO. The Corps Staff Archeologist's approval of the final report and formal concurrence from the SHPO will document completion of the cultural resources review. Your permit application will not be considered complete until the cultural resource review is completed.

Please contact our Staff Archeologist, Mr. Jerry Androy at 409-766-3821 with your Corps Permit Application Number (SWG-2019-00074) for specific instructions regarding the requirements of this investigation. For questions regarding the permit process, please contact Kate Mott at 409-766-3945. Please send a copy of this letter to the archeologist you contract.

Sincerely,

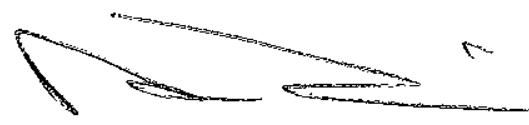

Fon Janet Thomas Botello Chief, Evaluation Branch 
Cc w/Encl:

RD-P - Mr. Jerry Androy

TSHPO - Mr. Mark Wolfe

Perennial Environmental Services, LLC, Attn: Josh Melnar, 13100 Northwest Freeway, Suite 150 , Houston, Texas 77040 


\begin{tabular}{|c|c|c|c|c|}
\hline Feature_ID & Feature_Cl & Crossing_M & Latitude & Longitude \\
\hline WP9CH004_DT & $\mathrm{PFO}$ & $\begin{array}{l}2.7 \text { Workspace } \\
\text { Only b }\end{array}$ & 29.875929 & -94.956216 \\
\hline $\begin{array}{l}\text { WP4LI002_PFO } \\
\text { (First Crossing) }\end{array}$ & $\mathrm{PFO}$ & $\begin{array}{l}\text { 16.9 Workspace } \\
\text { Only b }\end{array}$ & 29.963916 & -94.802633 \\
\hline $\begin{array}{l}\text { WP4LI002_PFO_C } \\
\text { (First Crossing) }\end{array}$ & PFO & $\begin{array}{l}17 \text { Worlkspace } \\
\text { Only b }\end{array}$ & 29.96456 & -94.800863 \\
\hline $\begin{array}{l}\text { WP4LI002_PFO } \\
\text { (Second Crossing) }\end{array}$ & PFO & $\begin{array}{l}17 \text { Workspace } \\
\text { Only b }\end{array}$ & 29.964256 & -94.801752 \\
\hline $\begin{array}{l}\text { WP4LI002_PFO_C } \\
\text { (Second Crossing) }\end{array}$ & $\mathrm{PFO}$ & $\begin{array}{l}\text { 17.2 Workspace } \\
\text { Only b }\end{array}$ & 29.965676 & -94.796901 \\
\hline $\begin{array}{l}\text { WP3LI007_PFO } \\
\text { (First Crossing) }\end{array}$ & $\mathrm{PFO}$ & $\begin{array}{l}\text { 17.8 Workspace } \\
\text { Only b }\end{array}$ & 29.968084 & .94 .788748 \\
\hline $\begin{array}{l}\text { WP3LI007_PFO } \\
\text { (Second Crossing) }\end{array}$ & PFO & $\begin{array}{l}\text { 17.8 Workspace } \\
\text { Only b }\end{array}$ & 29.968249 & -94.78815 \\
\hline $\begin{array}{l}\text { WP3LI007_PFO } \\
\text { (Third Crossing) }\end{array}$ & $\mathrm{PFO}$ & $\begin{array}{l}\text { 17.8 Workspace } \\
\text { Only b }\end{array}$ & 29.968349 & -94.787812 \\
\hline $\begin{array}{l}\text { WP4LI002_PEM } \\
\text { (Second Crossing) }\end{array}$ & PEM & 17.8 Open Cut & 29.965887 & -94.795373 \\
\hline WP3LI007_PEM & PEM & 17.9 Open Cut & 29.968771 & -94.784439 \\
\hline $\begin{array}{l}\text { WP3LI007_PFO } \\
\text { (Fourth Crossing) }\end{array}$ & PFO & $\begin{array}{l}17.9 \text { Workspace } \\
\text { Only b }\end{array}$ & 29.968583 & -94.7873 \\
\hline $\begin{array}{l}\text { WP3LI007_PFO } \\
\text { (Fifth Crossing) }\end{array}$ & PFO & $\begin{array}{l}17.9 \text { Workspace } \\
\text { Only b }\end{array}$ & 29.968669 & -94.786712 \\
\hline $\begin{array}{l}\text { WP3LI007_PFO } \\
\text { (Sixth Crossing) }\end{array}$ & PFO & $\begin{array}{l}18 \text { Workspace } \\
\text { Only b }\end{array}$ & 29.968816 & -94.785516 \\
\hline $\begin{array}{l}\text { WP3LI007_PFO } \\
\text { (Seventh Crossing) }\end{array}$ & PFO & $\begin{array}{l}18 \text { Workspace } \\
\text { Only b }\end{array}$ & 29.968896 & -94.78513 \\
\hline $\begin{array}{l}\text { WP3LI007_PFO } \\
\text { (Eighth Crossing) }\end{array}$ & PFO & $\begin{array}{l}18 \text { Workspace } \\
\text { Only b }\end{array}$ & 29.968951 & -94.784851 \\
\hline $\begin{array}{l}\text { WP3LI007_PFO } \\
\text { (Ninth Crossing) }\end{array}$ & PFO & $\begin{array}{l}\text { 18.1 Workspace } \\
\text { Only b }\end{array}$ & 29.969052 & -94.783928 \\
\hline $\begin{array}{l}\text { WP3LI007_PFO } \\
\text { (Tenth Crossing) }\end{array}$ & PFO & $\begin{array}{l}\text { 18.2 Workspace } \\
\text { Only b }\end{array}$ & 29.969174 & -94.78273 \\
\hline $\begin{array}{l}\text { WP3LI007_PFO } \\
\text { (Eleventh Crossing) }\end{array}$ & $\mathrm{PFO}$ & $\begin{array}{l}\text { 18.3 Workspace } \\
\text { Only b }\end{array}$ & 29.969344 & -94.781038 \\
\hline WP3LI006_PSS & PSS & $\begin{array}{l}\text { 18.3 Workspace } \\
\text { Only b }\end{array}$ & 29.969426 & -94.78005 \\
\hline $\begin{array}{l}\text { WP3LT006_PFO } \\
\text { (First Crossing) }\end{array}$ & $\mathrm{PFO}$ & $\begin{array}{l}\text { 18.3 Workspace } \\
\text { Only b }\end{array}$ & 29.969462 & -94.779828 \\
\hline $\begin{array}{l}\text { WP3LI006_PFO } \\
\text { (Second Crossing) }\end{array}$ & $\mathrm{PFO}$ & $\begin{array}{l}\text { 18.4 Workspace } \\
\text { Only b }\end{array}$ & 29.969477 & -94.779518 \\
\hline WP3LI006_PEM & PEM & 18.4 Open Cut & 29.969788 & -94.774379 \\
\hline $\begin{array}{l}\text { WP3LI006_PFO } \\
\text { (Third Crossing) }\end{array}$ & $\mathrm{PFO}$ & $\begin{array}{l}\text { 18.5 Workspace } \\
\text { Only b }\end{array}$ & 29.969639 & -94.777941 \\
\hline $\begin{array}{l}\text { WP3LI006_PFO } \\
\text { (Fourth Crossing) }\end{array}$ & PFO & $\begin{array}{c}\text { 18.6 Workspace } \\
\text { Only b }\end{array}$ & 29.969816 & -94.776179 \\
\hline $\begin{array}{l}\text { WP3LI006_PFO } \\
\text { (Fifth Crossing) }\end{array}$ & PFO & $\begin{array}{l}\text { 18.6 Workspace } \\
\text { Only b }\end{array}$ & 29.969985 & -94.774364 \\
\hline $\begin{array}{l}\text { WP3LI006_PFO } \\
\text { (Sixth Crossing) }\end{array}$ & $\mathrm{PFO}$ & $\begin{array}{l}\text { 18.8 Workspace } \\
\text { Only b }\end{array}$ & 29.970208 & -94.772384 \\
\hline WP3LI006_PFO & $\mathrm{PFO}$ & $\begin{array}{l}18.9 \text { Workspace } \\
\text { Page } 1 \text { of } 3\end{array}$ & 29.970378 & -94.770791 \\
\hline
\end{tabular}




\begin{tabular}{|c|c|c|c|c|}
\hline Feature_ID & Feature_Cl & Crossing_M & Latitude & ongitude \\
\hline $\begin{array}{l}\text { (Seventh Crossing) } \\
\text { WP3LI002_PEM } \\
\text { (First Crossing) }\end{array}$ & PEM & $\begin{array}{l}\text { Only b } \\
19 \text { HDD }\end{array}$ & 29.970024 & -94.768551 \\
\hline WP3L1002_PSS_B & PSS & 19.1 HDD & 29.970131 & -94.767401 \\
\hline $\begin{array}{l}\text { WP3LI002_PEM } \\
\text { (Second Crossing) }\end{array}$ & PEM & $19.1 \mathrm{HDD}$ & 29.970155 & -94.767593 \\
\hline $\begin{array}{l}\text { WP3LI002_PEM } \\
\text { (Third Crossing) }\end{array}$ & PEM & $19.1 \mathrm{HDD}$ & 29.970164 & -94.767275 \\
\hline WP3LI004 & PSS & $19.1 \mathrm{HDD}$ & 29.970064 & -94.767918 \\
\hline $\begin{array}{l}\text { WP3LI005_PEM } \\
\text { (First Crossing) }\end{array}$ & PEM & $19.1 \mathrm{HDD}$ & 29.970214 & .94 .767066 \\
\hline $\begin{array}{l}\text { WP3LI005_PFO_CY } \\
\text { P }\end{array}$ & PFO - Cypress & 19.2 HDD & 29.970213 & -94.766636 \\
\hline WP4LI020_PSS_B & PSS & $26.5 \mathrm{HDD}$ & 29.990743 & -94.647094 \\
\hline $\begin{array}{l}\text { WP4LI020_PSS } \\
\text { (First Crossing) }\end{array}$ & PSS & 26.5 HDD & 29.990638 & -94.647534 \\
\hline $\begin{array}{l}\text { WP4LI020_PEM } \\
\text { (First Crossing) }\end{array}$ & PEM & $26.5 \mathrm{HDD}$ & 29.990757 & -94.646559 \\
\hline $\begin{array}{l}\text { WP4LI020_PSS } \\
\text { (Second Crossing) }\end{array}$ & PSS & $26.6 \mathrm{HDD}$ & 29.99078 & -94.645972 \\
\hline $\begin{array}{l}\text { WP4LI020_PSS } \\
\text { (Third Crossing) }\end{array}$ & PSS & $26.6 \mathrm{HDD}$ & 29.99085 & -94.645255 \\
\hline $\begin{array}{l}\text { WP4LI020_PEM } \\
\text { (Second Crossing) }\end{array}$ & PEM & $26.7 \mathrm{HDD}$ & 29.991067 & -94.642455 \\
\hline WP4LI021_PSS & PSS & $\begin{array}{l}29.5 \text { Workspace } \\
\text { Only b }\end{array}$ & 29.994985 & -94.597182 \\
\hline WP4LI021_PEM & PEM & $\begin{array}{l}29.5 \text { Workspace } \\
\text { Only b }\end{array}$ & 29.994973 & -94.59725 \\
\hline $\begin{array}{l}\text { WP9JE010_PFO_DT } \\
\text { (First Crossing) }\end{array}$ & PFO & $47.5 \mathrm{HDD}$ & 30.008831 & -94.299479 \\
\hline WP9JE010_PEM_DT & PEM & 47.6 HDD & 30.00881 & -94.29922 \\
\hline $\begin{array}{l}\text { WP9JE010_PFO_DT } \\
\text { (Second Crossing) }\end{array}$ & PFO & $47.6 \mathrm{HDD}$ & 30.008876 & -94.298903 \\
\hline WP4JE060 & PEM & $\begin{array}{l}\text { 47.7 HDD/Open } \\
\text { Cut }\end{array}$ & 30.00908 & .94 .296288 \\
\hline SP9CH002_DT & Intermittent & $2.5 \mathrm{HDD}$ & 29.874287 & -94.954042 \\
\hline SP9CH013_DT & Intermittent & $2.9 \mathrm{HDD}$ & 29.878056 & -94.958084 \\
\hline $\begin{array}{l}\text { SP4LI004 } \\
\text { (First Crossing) }\end{array}$ & Ephemeral & $\begin{array}{l}17 \text { Worlkspace } \\
\text { Onlyb }\end{array}$ & 29.96451 & -94.801017 \\
\hline SP3LT004 & Intermittent & 17.8 Open Cut & 29.968041 & -94.788427 \\
\hline SP3LI003 & Intermittent & 18.3 Open Cut & 29.969306 & -94.78036 \\
\hline $\begin{array}{l}\text { SP3LI001 } \\
\text { (First Crossing) }\end{array}$ & Ephemeral & $\begin{array}{l}19 \text { Workspace } \\
\text { Onlyb }\end{array}$ & 29.969804 & -94.768933 \\
\hline $\begin{array}{l}\text { SP3LT001 } \\
\text { (Second Crossing) }\end{array}$ & Ephemeral & 19 HDD & 29.969993 & -94.768843 \\
\hline $\begin{array}{l}\text { SP3LI002 } \\
\text { (First Crossing) }\end{array}$ & Perennial & $19.1 \mathrm{HDD}$ & 29.970079 & -94.767966 \\
\hline $\begin{array}{l}\text { SP3LI002 } \\
\text { (Second Crossing) }\end{array}$ & Perennial & $19.1 \mathrm{HDD}$ & 29.970163 & -94.767135 \\
\hline SP9LI018_DT & Intermittent & 22.7 Open Cut & 29.982212 & -94.708772 \\
\hline SP9LI016_DT & Intermittent & 23 Open Cut & 29.982905 & -94.70401 \\
\hline
\end{tabular}




\begin{tabular}{|c|c|c|c|c|c|}
\hline Feature_ID & Feature_Cl & Milepost & Crossing_M & Latitude & Longitude \\
\hline SP9LI017_DT & Intermittent & & $23.4 \mathrm{HDD}$ & 29.983716 & -94.698527 \\
\hline SP4LI009 & Perennial & & $26.5 \mathrm{HDD}$ & 29.990659 & -94.647849 \\
\hline SP9JE012_DT & Intermittent & & 46.3 Open Cut & 30.007294 & -94.32033 \\
\hline SP9JE011_DT & Intermittent & & 46.5 Open Cut & 30.007542 & -94.316881 \\
\hline SP9JE010_DT & Intermittent & & 46.9 HDD & 30.008011 & -94.310084 \\
\hline SP9JE009_DT & Intermittent & & $47 \mathrm{HDD}$ & 30.008125 & $-94: 308518$ \\
\hline SP9JE008_DT & Intermittent & & 47.2 Open Cut & 30.008397 & .94 .30514 \\
\hline SP9JE007_DT & Intermittent & & 47.4 HDD & 30.008602 & -94.302008 \\
\hline SP9JE019_DT & Intermittent & & 47.5 HDD & 30.008769 & -94.299814 \\
\hline SP4JE049 & Intermittent & & $47.6 \mathrm{HDD}$ & 30.008875 & -94.298407 \\
\hline SP9JE050_DT & Ephemeral & & 47.8 Open Cut & 30.009144 & -94.295261 \\
\hline $\begin{array}{l}\text { SP9JE001_DT } \\
\text { (First Crossing) }\end{array}$ & Intermittent & & $\begin{array}{l}48.1 \text { Workspace } \\
\text { Onlyb }\end{array}$ & 30.009432 & -94.289942 \\
\hline $\begin{array}{l}\text { SP9JE001_DT } \\
\text { (Second Crossing) }\end{array}$ & Intermittent & & $48.1 \mathrm{HDD}$ & 30.009587 & -94.289724 \\
\hline SP9JE003_DT & Intermittent & & 48.4 HDD & 30.010026 & -94.284569 \\
\hline SP9JE004_DT & Intermittent & & $48.8 \mathrm{HDD}$ & 30.010582 & -94.278097 \\
\hline SP9JE005_DT & Intermittent & & 49 Open Cut & 30.010839 & -94.275431 \\
\hline SP9JE006_DT & Intermittent & & 49.2 Open Cut & 30.011092 & -94.272477 \\
\hline
\end{tabular}


SCOPE OF WORK

\section{A Phase I Cultural Resources Survey of the Orbit Pipeline Project \\ JEFFERSON, LiBERTY, AND CHAMBERS COUNTIES, TEXAS}

Prepared For:

\section{Energy Transfer Company}

Prepared By:

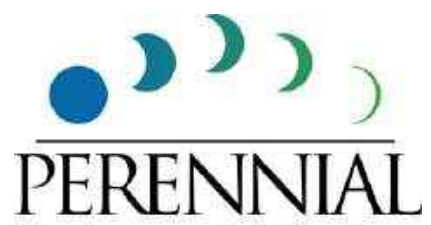

Environmental Services

Perennial Environmental Services, LLC

4425 Mopac South, Building II Suite 204

Austin, TX 78735

Principal Investigator:

Jennifer Cochran MA, RPA

Revised Scope of Work

August 2019 


\section{Introduction}

Energy Transfer Company (ETC) is proposing to construct the Orbit Pipeline Project (Project) located in Jefferson, Liberty, and Chambers counties, Texas. The Project consists of approximately 68.3 miles (mi) (109.9 kilometer [km]) of new 20.0-inch (in) (50.8-centimeter [cm]) diameter pipeline that will be used to transmit ethane and propane (Figure 1 and 2). The U.S. Army Corps of Engineers (USACE) is the lead federal agency and the Project will require a permit pursuant to Sections 10 and 404 of the Clean Water Act. Perennial Environmental Services, LLC (Perennial) has been contracted to conduct the cultural resources survey for the proposed Project.

Consistent with USACE application requirements, and in accordance with Section 106 of the National Historic Preservation Act (NHPA) of 1966, as amended (36 CFR 800), the proposed Project must make a reasonable and good faith effort to identify historic properties within the Project Area of Potential Effects (APE) and to take into account any potential effects, direct or indirect, the proposed undertaking could have on properties listed or considered eligible for listing in the National Register of Historic Places (NRHP). At the time of field investigations, temporary workspaces had not yet been finalized. As such, survey efforts are being expanded to cover a broader Environmental Survey Area (ESA), including a 300-foot (ft) (91.44-meter [m])-wide corridor centered on the pipeline right-of-way that will be considered the APE for the Project.

While the majority of the Project will traverse private property, or fall under the jurisdiction of the USACE, approximately 3.8 cumulative mi $(6.1 \mathrm{~km})$ of the Project route also traverses state-owned properties (Attachment 1). These properties, designated herein as the Permit Areas, are owned by Jefferson County, the City of Beaumont, and Texas Department of Corrections (Stiles Unit) and are considered a political subdivision of the State of Texas (Table 1). As such, the Permit Areas also fall under the jurisdiction of the Antiquities Code of Texas (Code) and the proposed undertaking must take into account any potential effects, direct or indirect, to cultural resources that qualify as State Antiquities Landmarks (SAL). The APE associated with the Permit Areas measures approximately 128.0 acres (ac) (51.8 hectares [ha]) with depths of impact ranging from 4.0 to 6.0 feet (ft) (1.2 to 1.8 meters [m]) below ground surface. The following scope of work outlines the field survey methods, artifact collection and site recordation strategies, and reporting protocols that will be utilized by Perennial for the Permit Areas. 


\begin{tabular}{|c|c|c|c|}
\hline \multicolumn{4}{|c|}{$\begin{array}{l}\text { Table } 1 . \\
\text { Permit Areas }\end{array}$} \\
\hline Tract & Owner & Setting & Acreage \\
\hline TX-JE-041.402 & Jefferson County & $\begin{array}{l}\text { Emergent wetland, manicured lawn, and paved } \\
\text { road }\end{array}$ & 2 \\
\hline TX-JE-041.406 & Jefferson County & $\begin{array}{l}\text { Emergent wetland, paved parking lot, sports } \\
\text { facility, paved roads, and manicured lawn }\end{array}$ & 10 \\
\hline TX-JE-116.200 & Jefferson County & Forested area & 12 \\
\hline TX-JE-077.000 & City of Beaumont & Forested and palustrine wetlands & 21 \\
\hline TX-JE-079.310 & City of Beaumont & $\begin{array}{c}\text { Agricultural fields, forested wetlands, emergent } \\
\text { wetlands, palustrine wetlands, open water, and } \\
\text { oil/gas infrastructure }\end{array}$ & 49 \\
\hline $\begin{array}{l}\text { TX-JE-115.220; TX- } \\
\text { JE-115.900 }\end{array}$ & $\begin{array}{c}\text { Texas Department of } \\
\text { Corrections - Stiles Unit }\end{array}$ & Existing prison facility & 34 \\
\hline
\end{tabular}

\section{Environmental Setting}

The Permit Areas are located along the western edge of the City of Beaumont. According to aerial imagery and U.S. Geological Survey (USGS) topographic maps, the state lands appear to be situated within a disturbed setting, based on the presence of existing utility corridors and recreational facilities. The Permit Areas are located within the Great Plains of the Northern Humid Gulf Coastal Plains ecoregion (Griffith et al. 2004). Locally known as the 'Piney Woods", this ecoregion was once covered by a mix of pine and hardwood forests. Natural vegetation includes loblolly pine, shortleaf pine, southern red oak, post oak, white oak, hickory, and sweetgum, and mid and tall grasses such as yellow Indian grass, pinehill bluestem, narrowleaf woodoats, and panicums. American beautyberry, sumac, greenbriar, and hawthorn are part of the understory (Griffith et al. 2007).

However, much of the region is now covered in loblolly and shortleaf pine plantations with loblolly pine for timber production or converted to improved pasture. (Griffith et al. 2004). Lumber and pulpwood production, livestock grazing, and poultry production are typical land uses. Oil and gas production is also widespread.

Geologically, the Permit Areas are underlain by the Beaumont Formation (Qbs, Qbc) (USGS 2017). Qbs deposits consist of very fine to fine quartz sand, silt, and minor fine gravel that dates to the Pleistocene. Qbc deposits consist of clay and silt intermixed and interbed with lenses of fine sand, decayed organic matter, and calcium carbonate.

Soil survey data for the proposed Project was derived from the National Resources Conservation Service (NRCS 2018). Five soil units are mapped within the Permit Areas including Beaumont 


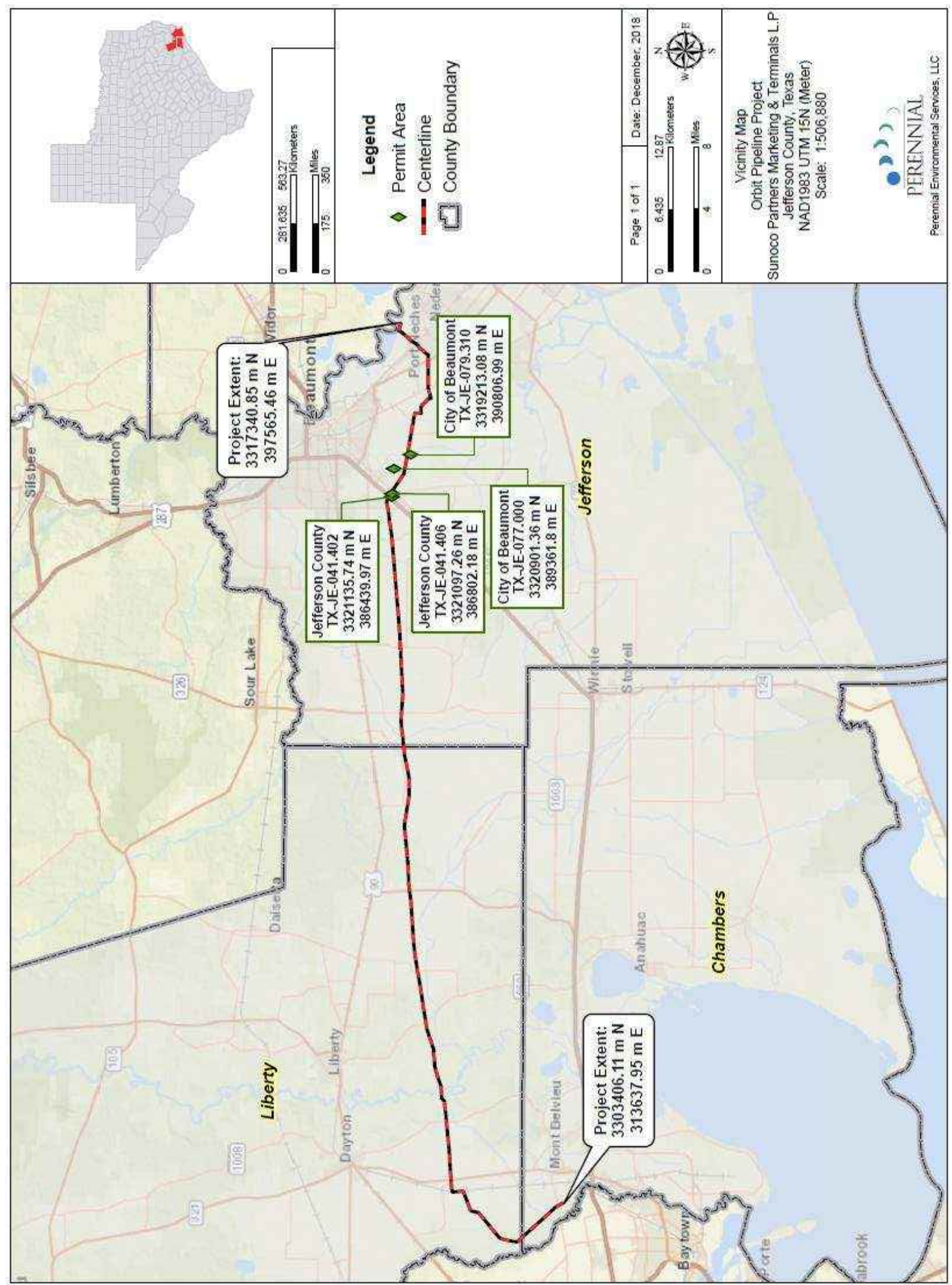

Figure 1 Vicinity Map showing location of original state-owned tracts 


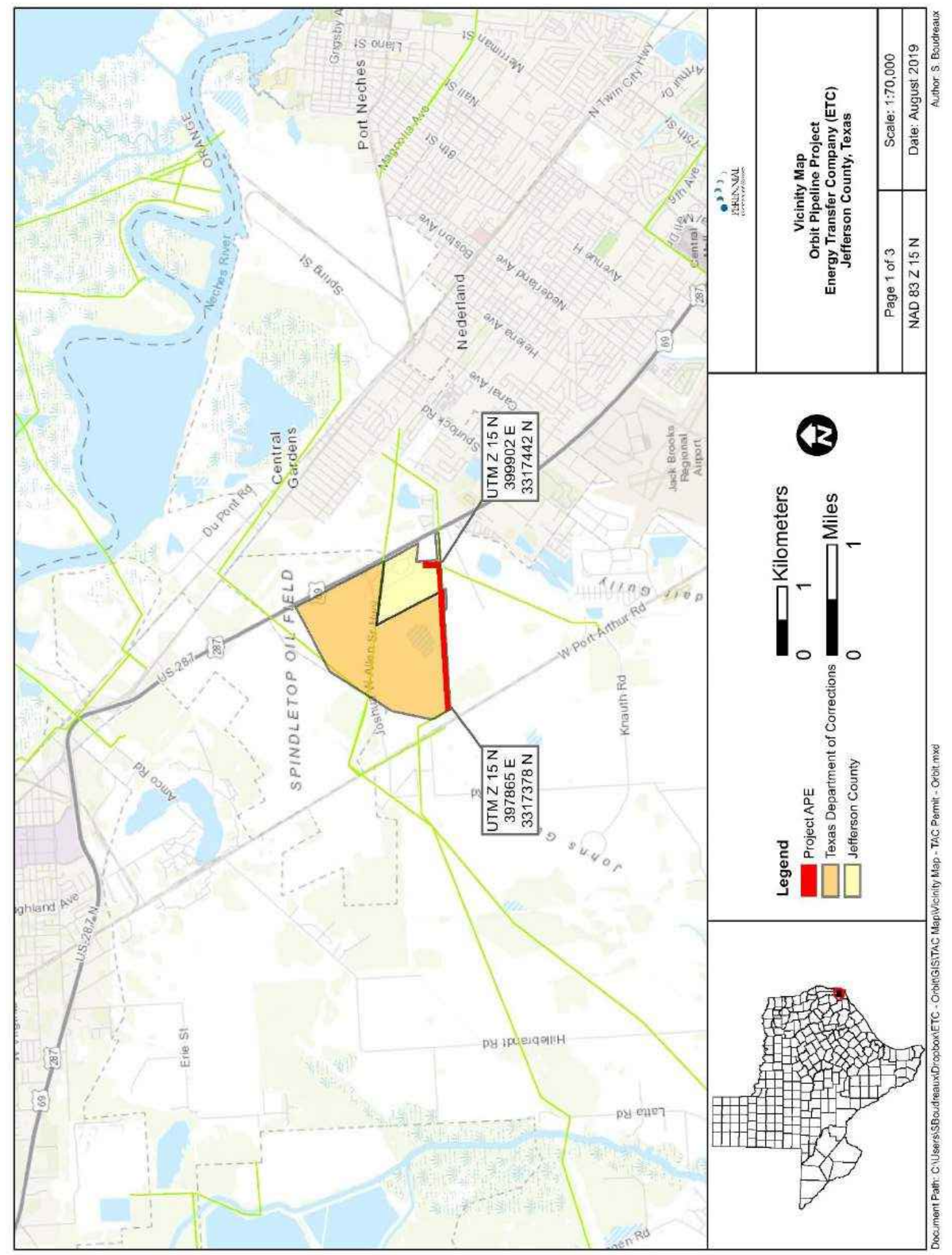

Figure 2 Vicinity Map showing location of recently added state-owned tracts 
clay, 0 to 1 percent slopes; China clay, 0 to 1 percent slopes; Estes clay, 0 to 1 percent clay; League clay, 0 to 1 percent slopes, and Viterbo silty clay loam, 0 to 1 percent slopes. The Beaumont soil series consist of very deep, poorly drained, and very slowly permeable soils on coastal plains. These soils form in clayey sediments on the Beaumont Formation and are of the Pleistocene Age. The China soil series consists of a very deep, somewhat poorly drained, and very slowly permeable soils. These soils form on clayey fluviomarine deposits derived from the Beaumont Formation. The Estes soil series consists of very deep, somewhat poorly drained soils. They form in acid clayey and loamy alluvium in the Coastal Plains. The League soils series consists of very deep, somewhat poorly drained, very slowly permeable soils. These soils form in clayey fluviomarine deposits derived from the Beaumont Formation (NRCS 2019). The Viterbo series consists of very deep, somewhat poorly drained, very slowly permeable soils. These nearly level soils formed in loamy fluviomarine deposits derived from the Beaumont Formation. All of the soils found within the Permit Areas are considered to have a low potential for buried cultural resource deposits.

\section{Cultural Resources Background Review Results}

Perennial conducted a thorough cultural resources and environmental literature search of the Permit Areas. A Perennial archaeologist reviewed the Port Acres, Beaumont East, Beaumont West, and Fannett East, Texas USGS 7.5-minute topographic quadrangle maps and searched the Texas Historical Commission's (THC) Texas Archeological Sites Atlas (Atlas) online database and the NRHP database to identify previously recorded cultural resource sites, historic structures, properties listed in the NRHP, designated historic districts, and SALs, which could potentially be affected by the proposed undertaking. Any previously recorded cultural resource site forms, reports of archaeological investigations, general historical documents, and secondary sources concerning the background of the area were also reviewed. The records search included a review of all previously recorded site forms, cemetery data, and surveys on file within a $1.0-\mathrm{mi}(1.6-\mathrm{km})$ review radius of the Permit Areas.

Based upon the archival review, no previously recorded archeological sites or cemeteries are mapped within the Project Permit Areas. One site is mapped within 1.0-mi (1.6-km) radius of the Permit Areas (Table 2) (Atlas 2019). Site 41JF66 is the Tyrell Tenant Farmstead and is located adjacent to, but distinctly outside of Permit Area TX-JE-041.402. The site is currently recorded as being located across Fannett Road from the Permit Area. Historical imagery from 1938, also corroborate that the historical farmstead was isolated to the southeast side of Fannett Road, opposite the Permit Area. Site 41JF97 also represents a historic farmstead. The site was recorded in 2011. The site has an undetermined NRHP eligibility. However, the site is located approximately $0.49 \mathrm{mi}(0.79 \mathrm{~km})$ northwest of the Permit Area (TX-JE-115.200), and will not be impacted by any project related activities.

A total of 24 surveys have been previously conducted within the 1-0-mi $(1.6-\mathrm{km})$ radius review area for the Permit Areas. Five of the previously conducted surveys cover portions (approximately 30 percent) of the Permit Areas (Table 3). 


\begin{tabular}{|c|c|c|c|c|}
\hline \multicolumn{7}{|c|}{ Table 2. } \\
\hline \multicolumn{2}{|c|}{ Site previously recorded within the 1.0 mi background review radius } \\
\hline Site Trinomial & Site Description & $\begin{array}{c}\text { Year } \\
\text { Recorded }\end{array}$ & $\begin{array}{c}\text { NRHP } \\
\text { Eligibility }\end{array}$ & Distance from Permit Area \\
\hline 41JF66 & $\begin{array}{c}\text { Tyrrell Tenant Farmstead - } \\
\text { Historic, early 20th century }\end{array}$ & 1990 & Undetermined & 600 ft from TX-JE-041.402 \\
\hline 41JF97 & Historic Farmstead & 2011 & Undetermined & $\begin{array}{c}\text { Department of Corrections - } \\
\text { Stiles Unit (TX-JE-115.220, } \\
\text { TX-JE-115.900) }\end{array}$ \\
\hline
\end{tabular}

\begin{tabular}{|c|c|c|c|c|c|}
\hline \multicolumn{6}{|c|}{$\begin{array}{l}\text { Table } 3 \text {. } \\
\text { Surveys previously that overlap with the Permit Areas }\end{array}$} \\
\hline Atlas Number & Survey Type & Year & In APE? & Tract & Acreage of APE \\
\hline 8500060431 & Survey & 2011 & Yes & TX-JE-079.310 & 17.1 \\
\hline 8500003414 & Remote Sensing & 1972 & Yes & TX-JE-079.310 & 5 \\
\hline 8400003386 & Survey & 1990 & Yes & TX-JE-077.000 & Less than $0.5 \mathrm{ac}$ \\
\hline 8500041363 & Survey & 2013 & Yes & $\begin{array}{c}\text { TX-JE-116.200; TX- } \\
\text { JE-115.220, TX-JE- } \\
115.900\end{array}$ & 17.0 \\
\hline 8500001883 & Survey & 1995 & Yes & TX-JE-115.220 & 5.7 \\
\hline
\end{tabular}

\section{Field Methods}

The cultural resources survey will be performed by a team of two archaeologists. The entire 128.0 ac (51.8 ha) of the Permit Areas will be pedestrian surveyed, except in those areas that have been previously surveyed. The objectives of the survey will be to (1) locate cultural resource sites within the portion of the Project APE that corresponds to the Permit Areas; (2) delineate the vertical and horizontal extent of any newly identified sites within the Permit Area portions of the APE; (3) provide a preliminary evaluation of each site's eligibility for listing in the NRHP and as a SAL; and (4) assess any potential for the Project to directly or indirectly affect historic properties, SALs, or other sensitive cultural resources.

In general, the Texas State Minimum Archeological Survey Standards (TSMASS) require that a minimum of 16 shovel tests be excavated per $1.0 \mathrm{mi}(1.6 \mathrm{~km})$ for ROWs measuring up to $100.0 \mathrm{ft}$ $(30.0 \mathrm{~m})$ in width $(1$ survey transect). The proposed ESA for the Permit Areas within the Project 
will measure approximately $100.0 \mathrm{ft}$ to $300.0 \mathrm{ft}$ (32.8 ft to $91.5 \mathrm{~m})$ in width and will require one to three evenly spaced survey transects in order to conform to TSMASS. Overall, the survey will cover 100 percent of the Project length for areas located across the Permit Areas. An intensive pedestrian survey will be conducted at survey intervals no greater than $91.5 \mathrm{ft}$ (30.0 m) apart along the width of the Permit Areas. In areas where the ground visibility is less than 50 percent, shovel tests will be excavated at 164.0 to $328.0-\mathrm{ft}(50.0$ to $100.0-\mathrm{m})$ intervals apart along the length of the Permit Areas. In areas where the surface visibility exceeds 50 percent, shovel testing will be conducted intermittently along areas with increased probability to contain buried cultural deposits, such as areas adjacent to drainages. The number of survey transects may be reduced in areas where the Permit Areas overlaps with existing pipeline corridors or other industrial areas. Additional shovel tests may be excavated in portions of the Permit Areas with distinct landforms. In areas where landforms exhibit moderate-to-extensive disturbances from natural or human-induced processes, such as inundation; land clearing, minor channelization, installation of oil and gas facilities, recreational facilities, pipeline systems, road and utility ROW, and transportation infrastructure; shovel tests were excavated on a discretionary basis. Any deviations from TSMASS will be thoroughly documented, and disturbances will be photographed. Based on the underlying geology and the low potential for buried cultural deposits for soils found within the Permit Areas, it is not anticipated that trenching or mechanical auguring will be warranted in the portions of the Project that fall within the Permit Areas. However, if field investigations encounter areas with the potential for deep deposits, a trenching plan will be coordinated with the THC.

In general, shovel tests will measure approximately 12.0 in $(30.0 \mathrm{~cm})$ in diameter and will be excavated by 3.93 to 7.87 in $(10.0$ to $20.0 \mathrm{~cm})$ levels. Per the Council of Texas Archeologists (CTA)/THC survey standards, shovel tests will be excavated to a depth consistent with the project impacts or to the base of Holocene deposits likely to contain cultural resources unless dense, impenetrable clay soils or inundation prevent the continuation of excavations. All soil matrices will be screened through 6.3 -millimeter (1/4-inch) mesh hardware cloth unless dominated by clay. Clayey matrix will be trowel-sorted and visually inspected.

For each of the shovel tests, the following information will be recorded on shovel test logs: location, maximum depth, reason for termination, and the number of soil strata. Copies of field logs will be provided in the appendices of the Phase I cultural resources report. For each soil stratum, thickness, texture, color, and the presence or absence and nature of cultural materials will be recorded. The field crew will record all shovel test locations, isolated finds, archaeological sites and associated features using a handheld GPS device. Also, each archaeologist will be equipped with a compass, shovel test and photographic logs, daily journal forms, and appropriate THC site forms. The crew will be equipped with topographic maps and aerial photographs of the survey corridor, a digital camera, and a cellular telephone to maintain contact with the home office (terrain permitting). 
If an archaeological site is identified, the appropriate delineation techniques will be systematically applied to identify the horizontal and vertical limits of each site's boundary. Site boundaries may be determined based on both surface artifact density and the presence or lack of subsurface components. For subsurface sites, a series of shovel tests will be excavated radiating in the four cardinal directions or, if more appropriate, along perceived major and minor topographic and site axis. In practice, shovel tests within potential sites will be placed along transects at 33.0-ft (10.0$\mathrm{m}$ ) intervals to determine the depth and potential integrity of cultural deposits, and to carefully examine for the presence of intact archaeological features and/or discrete episodes of occupation. In the absence of subsurface deposits, controlled pedestrian surface inspections will be conducted and site boundaries defined based on a marked reduction in surface artifact density. Shovel testing or pedestrian surveys will not be conducted beyond the Permit Area property boundaries to ensure no trespassing onto private property occurs. Data collected during site recordation will be used to determine whether the site qualifies as a SAL.

\section{Historic Structure Documentation}

All historic-age structures (i.e., older than 50 years) located within the Permit Areas will be thoroughly documented during the survey efforts. Historic Standing Structure forms will be completed for each resource. The historic structures will be adequately photo-documented, sketch maps will be drawn, and each resource will be plotted with a GPS device. Shovel tests may be excavated in the vicinity of the structures to determine if an associated subsurface archaeological component is present. All data will be reviewed by a qualified architectural historian to determine construction date, style, integrity, and overall significance and SAL designation.

\section{Reporting}

Following the completion of the field surveys, Perennial will incorporate findings into the overall report for the Project. Data on any newly recorded sites will be submitted to the Texas Archeological Research Laboratory (TARL) in order to receive trinomial data for reporting. The format of the report will adhere to review guidelines suitable to the State Historic Preservation Officer (SHPO) in accordance with the THC's Rules of Practice and Procedure, Chapter 26, Section 27, and the CTA Guidelines for Cultural Resources Management Reports. The report will document previous investigations in the area, background cultural setting, the methodology used during the investigations, the general nature and extent of cultural resources encountered during the cultural resource survey, and management recommendations for any documented cultural resources per all applicable state and federal laws. Standard analytical techniques and existing typologies, as appropriate for Southeastern prehistoric and historic archaeological studies, will be employed.

\section{Laboratory Methods}

The artifact collection procedure employed by Perennial is meant to be flexible to accommodate variations in site size, density of artifacts, setting, presence/absence of subsurface assemblages, site type, and feasibility constraints. In general, the artifact collection strategy is designed to procure comprehensive inventory-level information to facilitate NRHP evaluations and avoidance 
strategies, as well as adhere to property restrictions. Specifically, the artifact collection policy may vary between $100 \%$ collection of observed artifacts, and a representative sample collection strategy. In practice, the $100 \%$ collection strategy would most commonly be applied to sites with subsurface assemblages. For surficial sites, the Principal Investigator in consultation with the field supervisor would determine the collection strategy based on the site type and setting. In the event that a sample collection policy is implemented for a site, field staff will inventory and describe the characteristics, material, type, decoration, and other descriptive traits; and photograph all observed artifacts whenever feasible. Meanwhile, Perennial will also collect representative samples of each diagnostic artifact type and variety. All artifacts that are collected will be brought back to Perennial's laboratory to be cleaned, sorted, cataloged, photo-documented, and analyzed. All collected material will be curated at the Texas Archaeological Research Laboratory.

Perennial will prepare all paperwork recovered during the study and arrange for the transfer of records to a suitable curatorial facility pursuant to the standards and guidelines set forth by the THC/CTA. An additional ten copies will be distributed to various designated repositories across the state in accordance with the TAC permit obligations.

\section{Human Remains}

In the event that human remains are encountered during any part of the Phase I survey effort, work will stop immediately and the appropriate local law enforcement personnel and medical examiner's office will be notified of the discovery. Should the medical examiner determine that the human remains are older than 50 years, personnel will notify the THC and file a notice of existence of a cemetery with the county clerk. Consultation with any concerned parties including landowners, appropriate Tribes, and living descendants will be conducted to ensure compliance with existing state laws. No remains will be removed from the site until jurisdiction has been established and the appropriate permits have been obtained. All activities will adhere to the Texas Health and Safety Code (8 THSC $\S 711.010)$ and the Texas Antiquities Code (13 TAC $\S \S 22.1$ 22.6).. 


\section{References}

(Atlas) Texas Archaeological Sites Atlas

2019 Texas Archaeological Site Atlas restricted database, Texas Historical Commission. http://nueces.thc. state.tx.us/. Accessed August 18, 2019.

Griffith, G.E., Bryce, S.A., Omernik, J.M., Comstock, J.A., Rogers, A.C., Harrison, B., Hatch, S.L., and Bezanson, D.

2004 Ecoregions of Texas. U.S. Geological Survey, Reston VA (map scale 1:2,500,000)

(NRCS) Natural Resources Conservation Service

2019 Soil Survey Staff, Natural Resources Conservation Service, United States Department of Agriculture. http://websoilsurvey.nrcs.usda.gov. Accessed August 18, 2019.

(USGS) United States Geological Survey

2019 U.S. Geological Survey, United States Department of the Interior. Information and graphics available at http://mrdata.usgs.gov/sgmc/tx.html. Accessed August 18, 2019. 


\section{ATTACHMENT 1 - Permit Area Mapping}


This Page Intentionally Left Blank to Protect Sensitive Cultural Resources 
From:

Sent:

To:

Cc:

Subject:
Maggie Moore <Maggie.Moore@thc.texas.gov>

Friday, August 30, 2019 9:14 AM

Jenn Cochran

Abby Peyton; Laney Fisher

RE: Orbit - Antiquities Permit 8690 - Need to Amend Permit

Hi Jenn,

Your amendment to permit 8690 is approved. Expiration date is still 12/17/2023.

Maggie Moore

Regional Archeologist/Project Reviewer

Texas Historical Commission

Archeology Division

PO Box 12276

Austin, TX 78722-2276

(512) 463-6508

www.thc.texas.gov

TEXAS HISTORICAL COMMISSION

real place telling reat arories

From: Jenn Cochran <JCochran@perennialenv.com>

Sent: Wednesday, August 21, 2019 5:43 PM

To: Maggie Moore <Maggie.Moore@thc.texas.gov>

Cc: Abby Peyton <APeyton@perennialenv.com>; Bill Martin <Bill.Martin@thc.texas.gov>

Subject: RE: Orbit - Antiquities Permit 8690 - Need to Amend Permit

CAUTION: External Email - This email originated from outside the THC email system. Do not click links or open attachments unless you recognize the sender and know the content is safe.

Hi Maggie,

Attached is a revised scope of work to amend new tracts from Jefferson County and the Texas Department of Corrections (DOC) onto Permit \#8690. I have attached a signed permit application for the DOC property. I have already submitted signed permit application for Jefferson County. The tracts will be surveyed using the same methods as for the other TAP properties. The results of the surveys across these tracts will be included in the revised Phase I as well as the comments that you have already provided us. Please let me know if you have any questions or need additional information. We need to survey these tracts soon, so please shoot me an email confirmation as soon as you can.

Thanks,

Jennifer L. Cochran, MA, RPA

Senior Archeologist/ Principal Investigator 
Perennial Environmental

5424 W. US Hwy 290, Suite 208

Austin, TX 78735

512-358-0345 office

512-695-4057 cell

jcochran@perennialenv.com

From: Maggie Moore <Maggie.Moore@thc.texas.gov>

Sent: Friday, July 19, 2019 2:07 PM

To: Jenn Cochran <JCochran@perennialenv.com>

Cc: Abby Peyton <APeyton@perennialenv.com>

Subject: RE: Orbit - Antiquities Permit 8690

Ok, great.

Maggie Moore

Regional Archeologist/Project Reviewer

Texas Historical Commission

Archeology Division

PO Box 12276

Austin, TX 78722-2276

(512) 463-6508

www.thc.texas.gov

TEXAS HISTORICAL COMMISSION

real places tefling reat arories

From: Jenn Cochran <JCochran@perennialenv.com>

Sent: Friday, July 19, 2019 1:53 PM

To: Maggie Moore <Maggie.Moore@thc.texas.gov>

Cc: Abby Peyton <APeyton@perennialenv.com>

Subject: RE: Orbit - Antiquities Permit 8690

CAUTION: External Email - This email originated from outside the THC email system. Do not click links or open attachments unless you recognize the sender and know the content is safe.

Hi Maggie,

Please see attached Permit Transfer letter for TAP \#8690.

Jennifer L. Cochran, MA, RPA

Senior Archeologist/ Principal Investigator 
Perennial Environmental

4425 Mopac South

Building II, Suite 204

Austin, Texas 78735

512-358-0345 office

512-695-4057 cell

jcochran@perennialenv.com

From: Maggie Moore <Maggie.Moore@thc.texas.gov>

Sent: Monday, July 15, 2019 11:36 AM

To: Jenn Cochran <JCochran@perennialenv.com>

Cc: Abby Peyton <APeyton@perennialenv.com>

Subject: RE: Orbit - Antiquities Permit 8690

Ok, because I'm not finding an e-mail about a transfer for this.

\section{Maggie Moore}

Regional Archeologist/Project Reviewer

Texas Historical Commission

Archeology Division

PO Box 12276

Austin, TX 78722-2276

(512) 463-6508

www.thc.texas.gov

\section{TEXAS HISTORICAL COMMISSION}

real place tefling reat urories

From: Jenn Cochran <JCochran@perennialenv.com>

Sent: Monday, July 15, 2019 10:38 AM

To: Maggie Moore <Maggie.Moore@thc.texas.gov>

Cc: Abby Peyton <APeyton@perennialenv.com>

Subject: Re: Orbit - Antiquities Permit 8690

CAUTION: External Email - This email originated from outside the THC email system. Do not click links or open attachments unless you recognize the sender and know the content is safe.

Hi Maggie,

I am pretty sure the Permit was transferred to me. I will look for transfer paperwork once I am back from being in the field.

Thanks,

Jenn C.

Perennial Environmental Services 
On Jul 15, 2019, at 10:31 AM, Maggie Moore <Maggie.Moore@thc.texas.gov> wrote:

Hi Jenn,

A permit revision should be fine. But is this still in Heidi's name? If so, what are your plans on that?

\author{
Maggie Moore \\ Regional Archeologist/Project Reviewer \\ Texas Historical Commission \\ Archeology Division \\ PO Box 12276 \\ Austin, TX 78722-2276 \\ (512) 463-6508 \\ www.thc.texas.gov \\ <image001.png> \\ From: Jenn Cochran <JCochran@perennialenv.com> \\ Sent: Tuesday, June 18, 2019 2:18 PM \\ To: Maggie Moore<Maggie.Moore@thc.texas.gov> \\ Cc: Abby Peyton <APeyton@perennialenv.com> \\ Subject: Orbit - Antiquities Permit 8690
}

CAUTION: External Email - This email originated from outside the THC email system. Do not click links or open attachments unless you recognize the sender and know the content is safe.

HI Maggie,

There is a proposed reroute on the Orbit Project that now crosses another state owned property. Are you ok with me providing any updated SOW, mapping exhibits, and Permit application, so you can amend the existing permit? Or do I need to get a new one?

Thanks,

Jennifer L. Cochran, MA, RPA

Senior Archeologist/ Principal Investigator

Perennial Environmental

4425 Mopac South

Building II, Suite 204

Austin, Texas 78735

512-358-0345 office

512-695-4057 cell

jcochran@perennialenv.com 
From:

Sent:

To:

Subject: noreply@thc.state.tx.us

Friday, December 20, 2019 8:55 AM

Jenn Cochran; reviews@thc.state.tx.us; Jerry.L.Androy@usace.army.mil

Project Review: 202003491

\section{TEXAS HISTORICAL COMMISSION}

real places relliug real stories

Re: Project Review under Section 106 of the National Historic Preservation Act and/or the Antiquities Code of Texas Permit 8690

202003491

Orbit 20-inch Ethane Pipeline Project SWG-2019-00074

intersection of Hatcherville Rd and Crobsy Rd

Mont Belvieu, TX

Dear Jennifer Cochran:

Thank you for your submittal regarding the above-referenced project. This response represents the comments of the State Historic Preservation Officer, the Executive Director of the Texas Historical Commission (THC), pursuant to review under Section 106 of the National Historic Preservation Act and the Antiquities Code of Texas.

The review staff led by Maggie Moore and Caitlin Brashear has completed its review and has made the following determinations based on the information submitted for review:

\section{Above-Ground Resources}

- No historic properties are present or affected by the project as proposed. However, if historic properties are discovered or unanticipated effects on historic properties are found, work should cease in the immediate area; work can continue where no historic properties are present. Please contact the THC\&apos;s History Programs Division at 512-463-5853 to consult on further actions that may be necessary to protect historic properties.

\section{Archeology Comments}

- No historic properties present or affected. However, if buried cultural materials are encountered during construction or disturbance activities, work should cease in the immediate area; work can continue where no cultural materials are present. Please contact the THC\&apos;s Archeology Division at 512-463-6096 to consult on further actions that may be necessary to protect the cultural remains.

- THC/SHPO concurs with information provided.

- Draft report acceptable. Please submit another copy as a final report along with shapefiles showing the area where the archeological work was conducted. Shapefiles should be submitted electronically to Archeological_projects@thc.texas.gov.

We have the following comments: On August 30, 2019, our office approved a Permit Amendment Application adding 12 acres of Jefferson County tract TX-JE0116.200 and 34 acres of Texas Department of Corrections (TDJ) - Stiles Unit tracts TX-JE-115.220 and TX-JE-115.900 to the Antiquities Code-permitted project area. The draft report indicates that substantial additional acreage was added to the project area subsequent to our approval of that amendment. The 
additional 82.2 acres of reroute changes should have been submitted to our office as an additional permit amendment request prior to conducting the fieldwork. To complete the administrative record, a Permit Amendment Application must be submitted for approval before we can accept the final report and close Texas Antiquities Permit. Thank you for addressing our previous comments; please correct the following in the final report. 1) Jerry's last name is misspelled. 2) We recommend showing all shovel tests excavated on this project, including ones outside the USACE permit areas. 3 ) Please clarify the meaning of the Construction dots on the maps (e.g., 1, 59, 63, 67, 72, and elsewhere). Are these GPSed observations, similar to inundation points? Has pipeline construction commenced already? 4) Figure 1 still does not have a meter scale, and the meters on Figure 15 are not labeled. 5) Appendix F Archeological Monitoring: As requested in our $7 / 22 / 2019$ comments on the monitoring SOW, please illustrate where these sites will be on the maps, and add map references to the Appendix $\mathrm{F}$ tables. $\mathrm{B}(2)$ says if the monitor determined that the find constitutes a site, then additional personnel may be required to delineate the find in accordance with THC guidelines. Please insert this prior to that sentence: "All cultural materials found will be fully delineated in accordance with THC guidelines at least within the project area." Our office address is P.O. Box 12276, Austin, TX 78711 (1511 Colorado is not the correct street address either)

We look forward to further consultation with your office and hope to maintain a partnership that will foster effective historic preservation. Thank you for your cooperation in this review process, and for your efforts to preserve the irreplaceable heritage of Texas. If you have any questions concerning our review or if we can be of further assistance, please email the following reviewers: Maggie.Moore@thc.texas.gov, caitlin.brashear@thc.texas.gov.

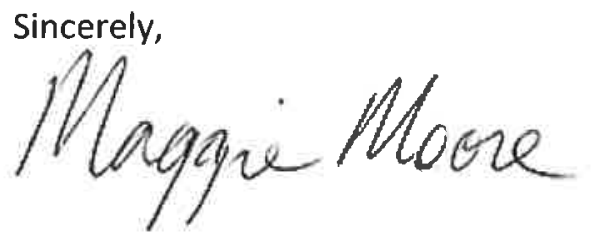

For Mark Wolfe, State Historic Preservation Officer

Executive Director, Texas Historical Commission

\section{Please do not respond to this email.}

cc: Jerry.L.Androy@usace.army.mil 


\section{APPENDIX B: Project MAPPING ExhIBITS}


This Page Intentionally Left Blank to Protect Sensitive Cultural Resources 


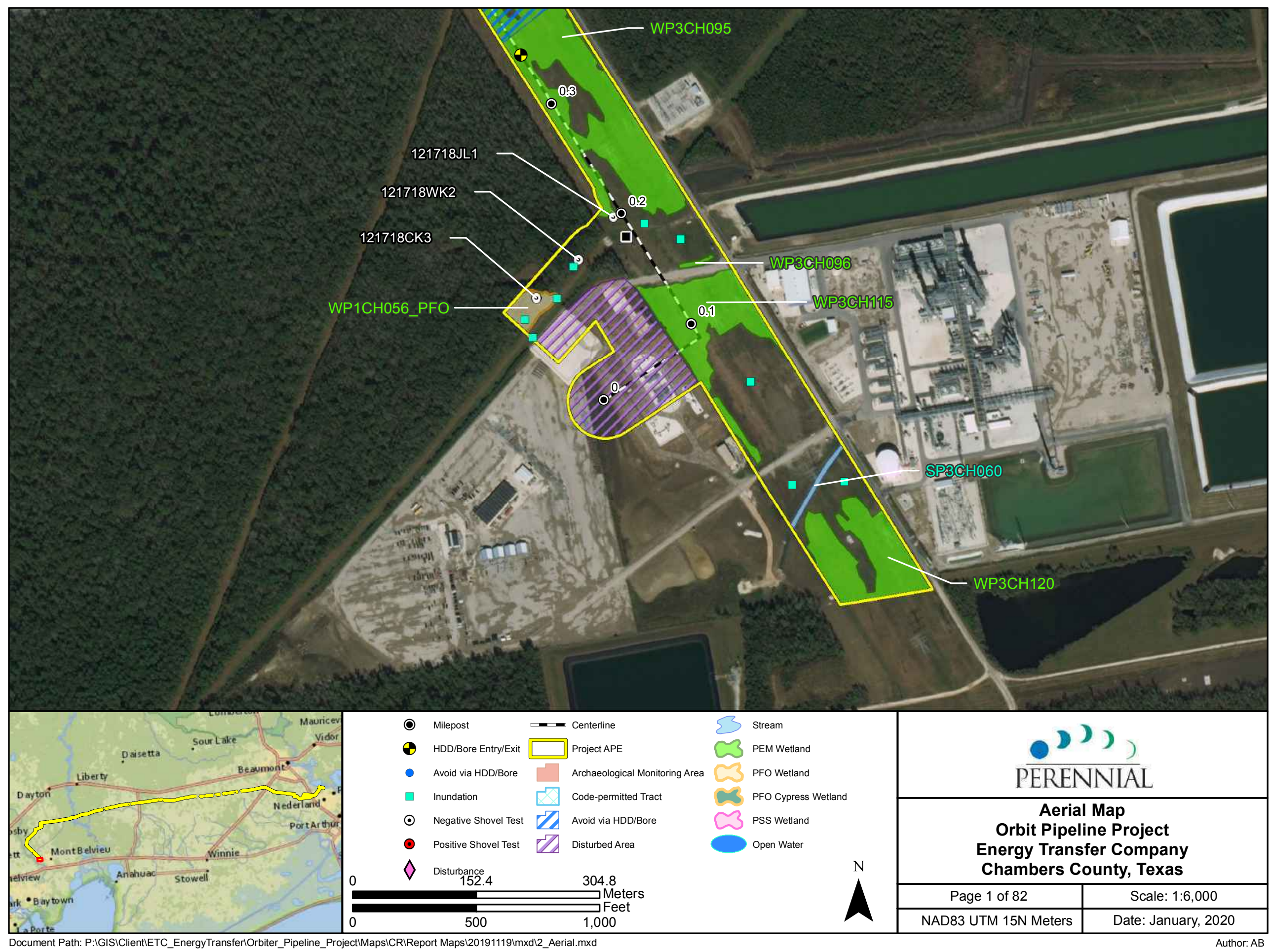




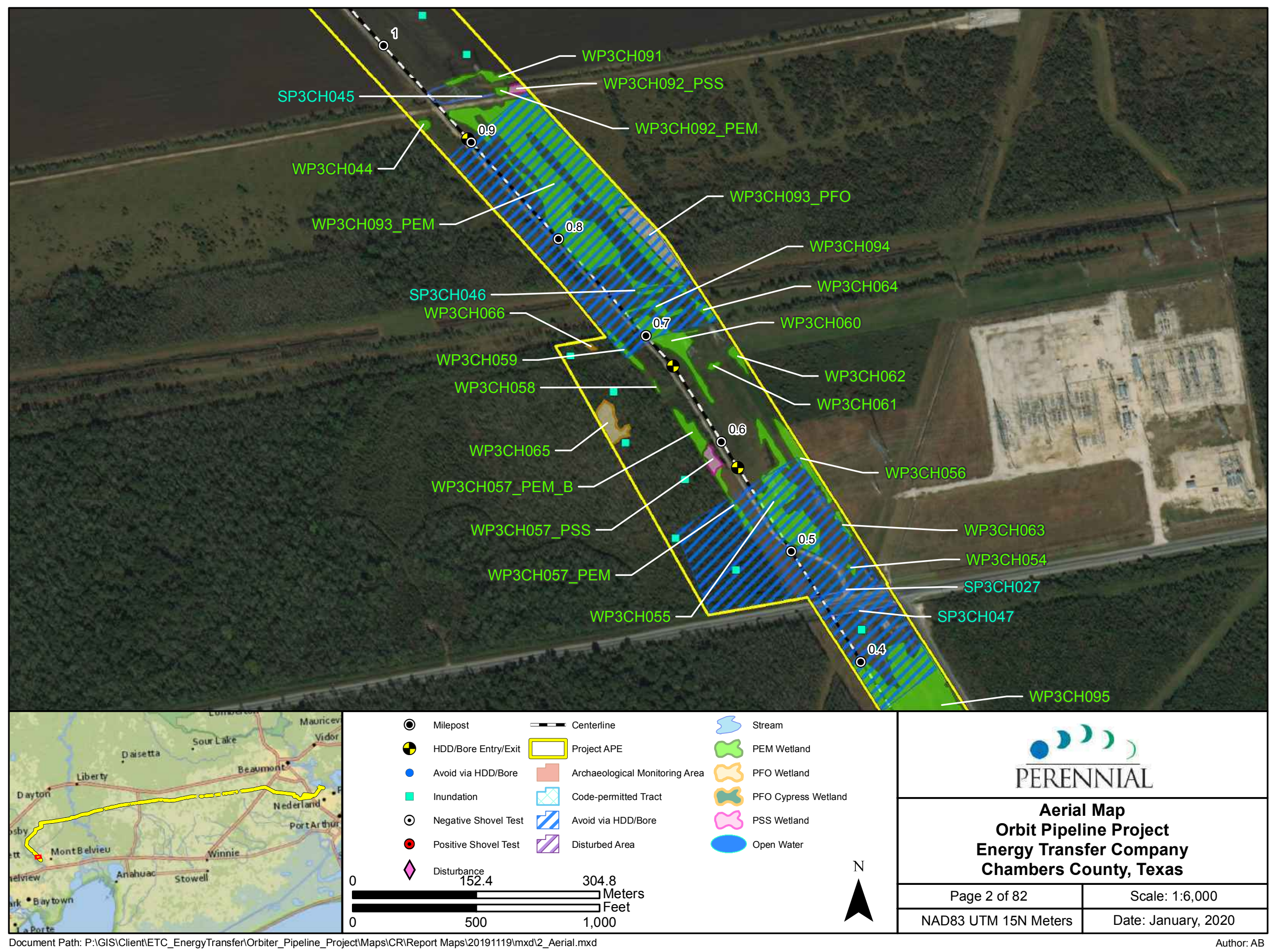




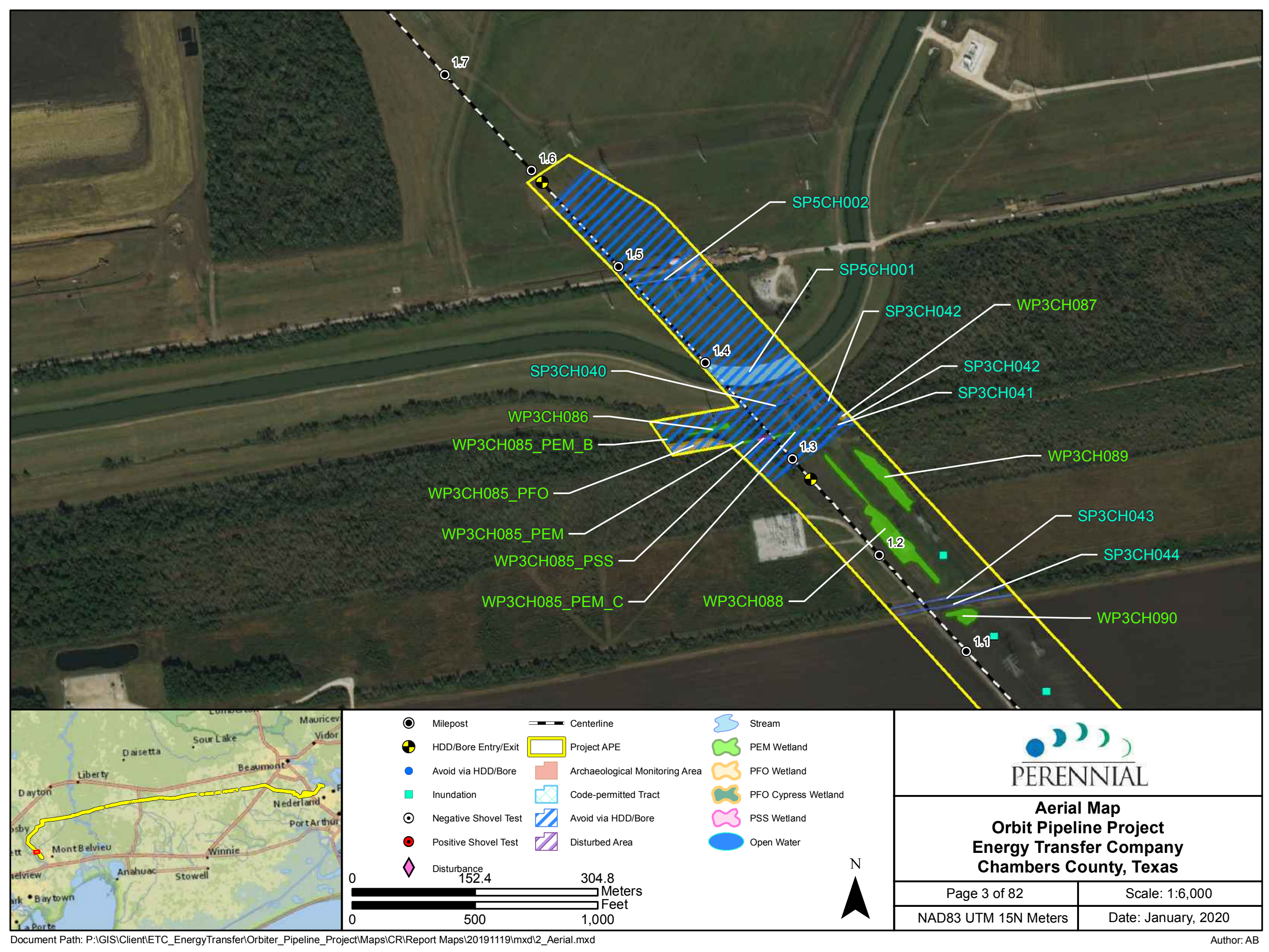




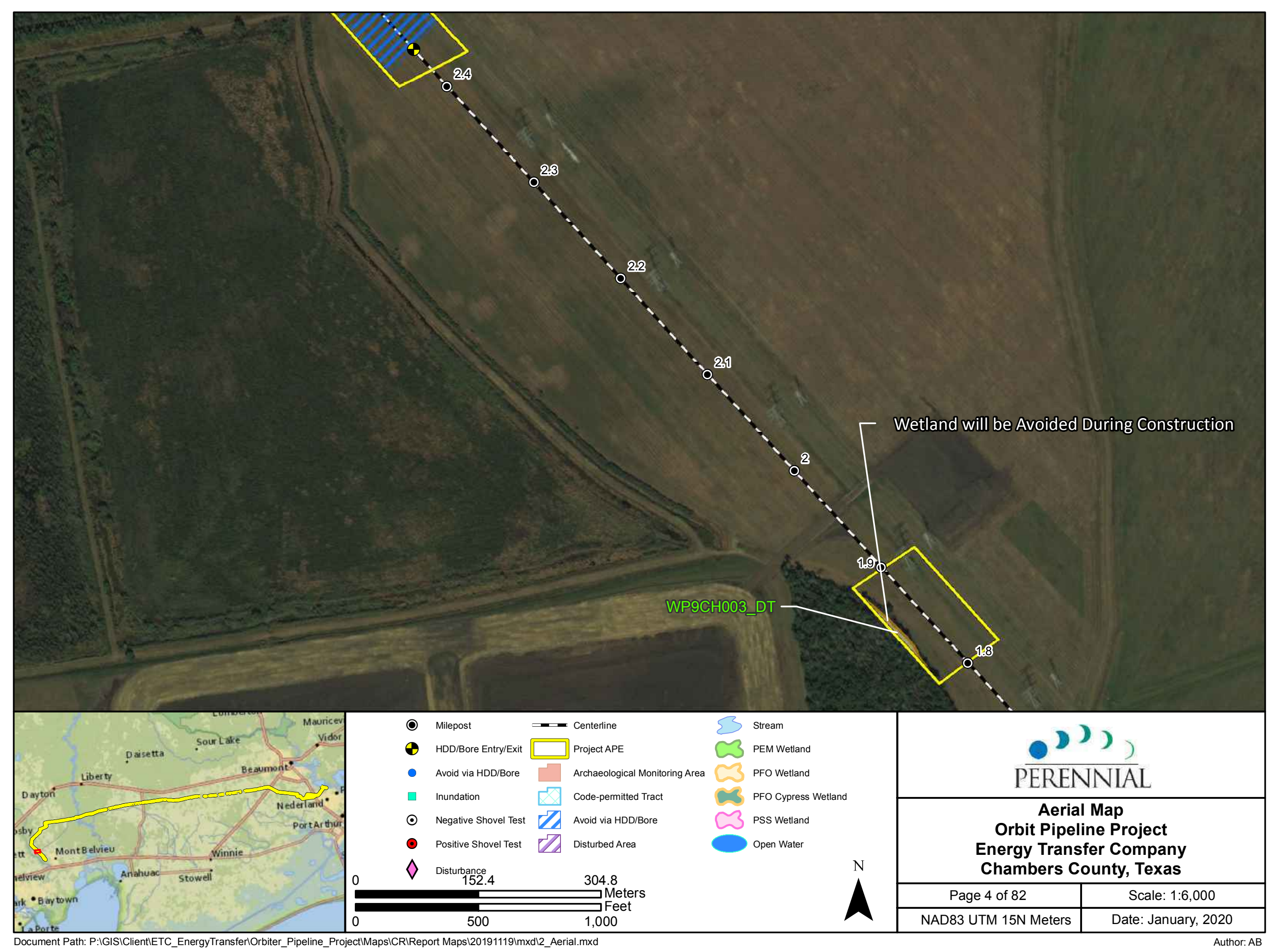




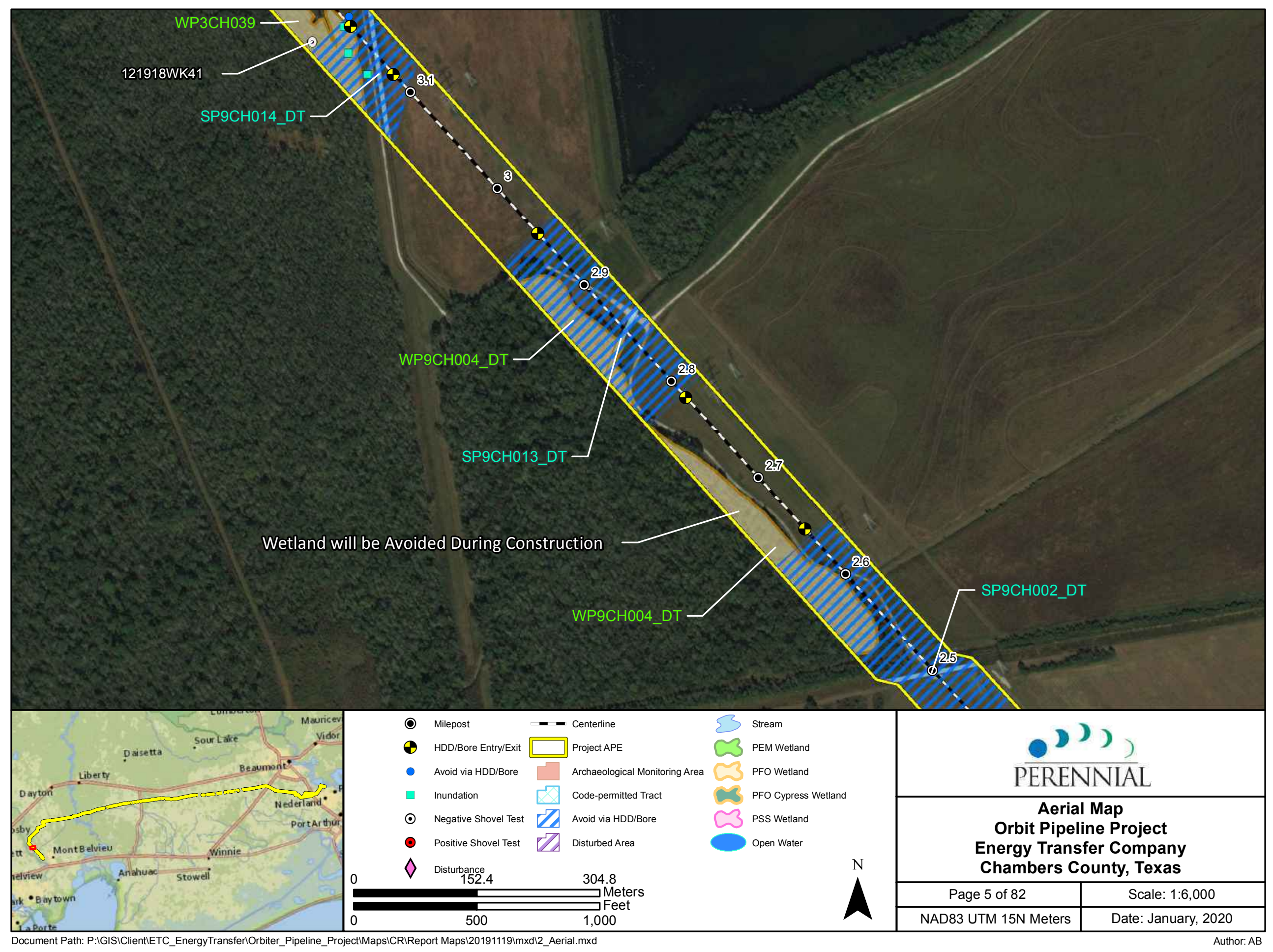




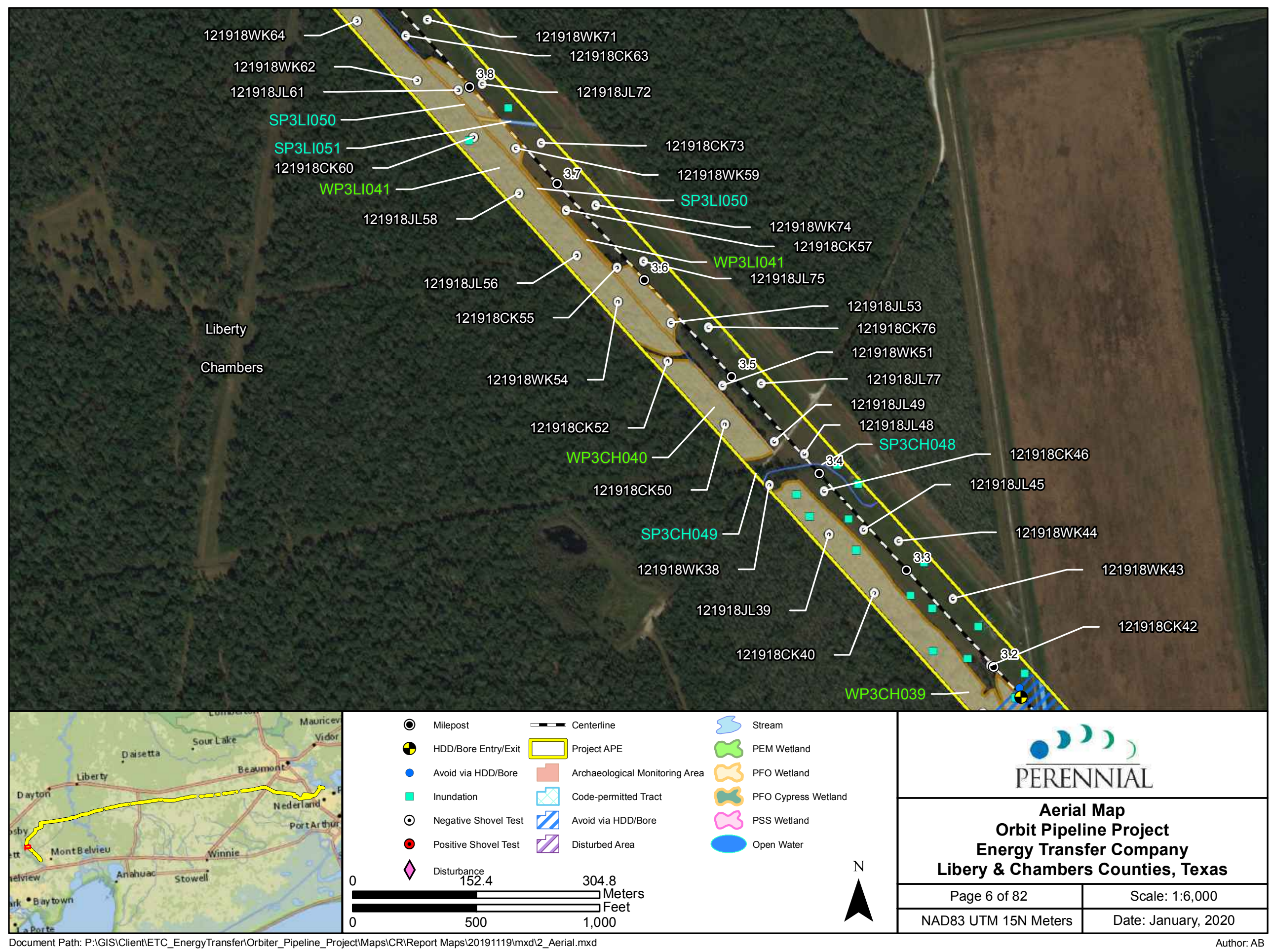




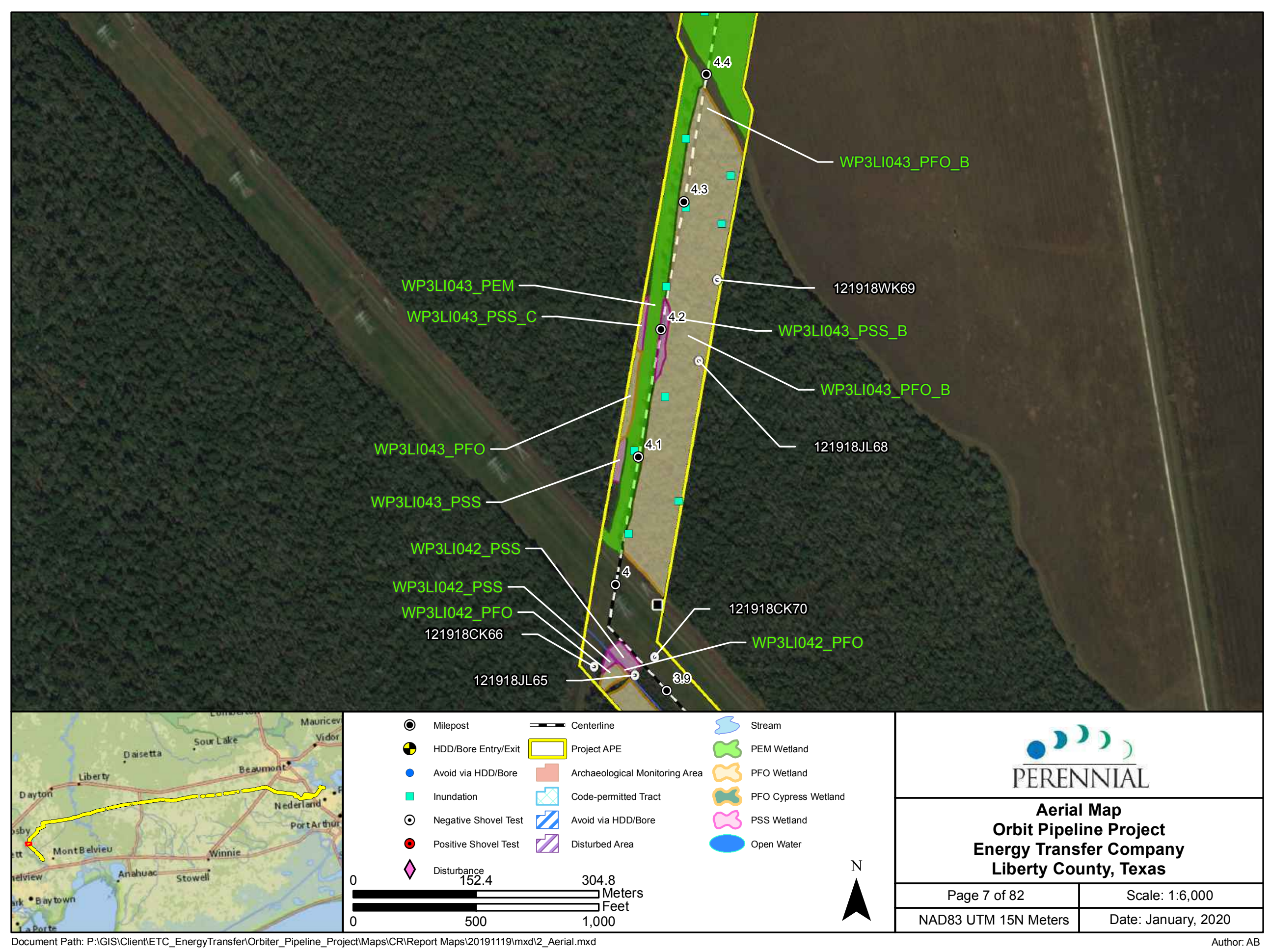




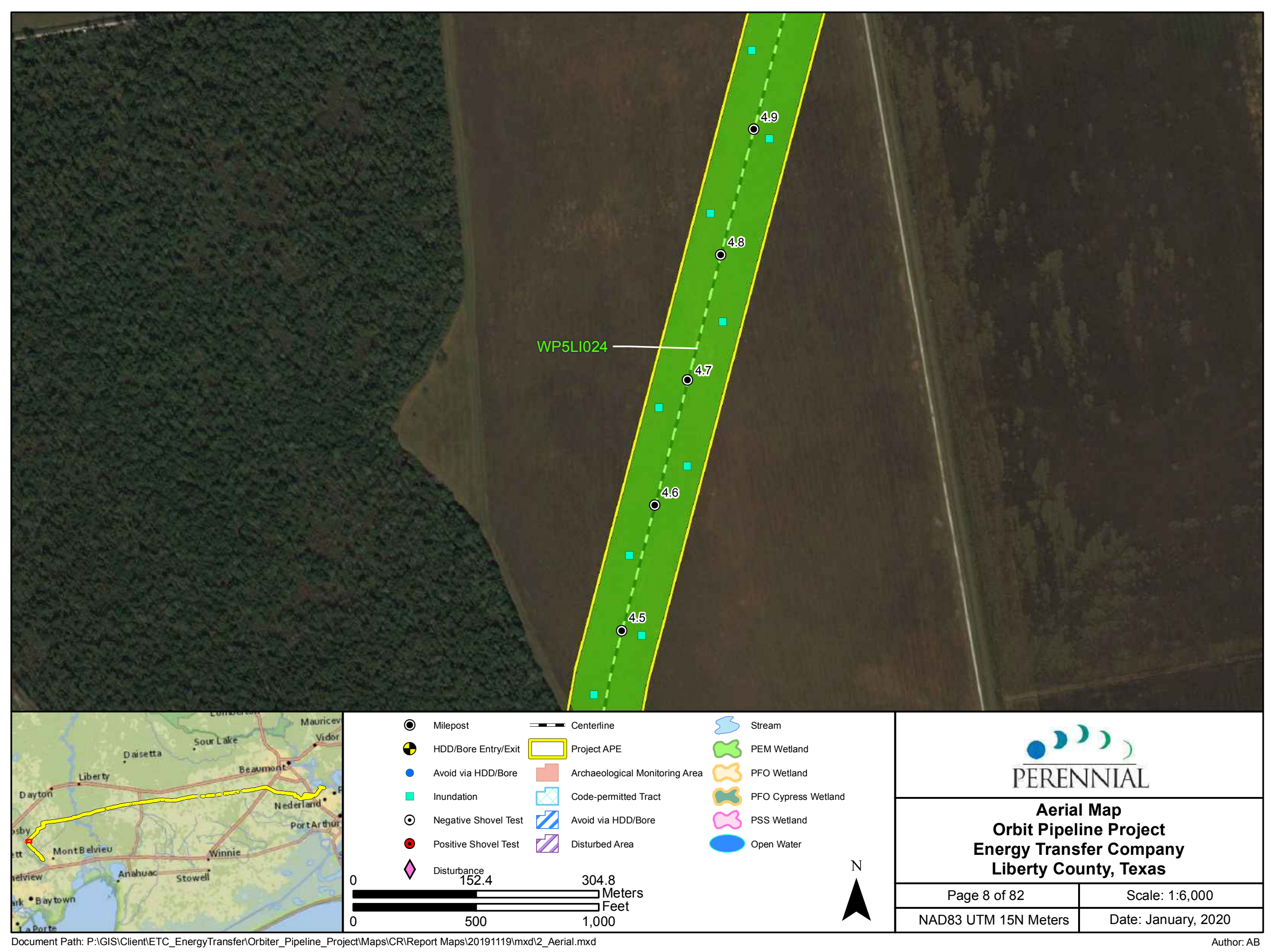




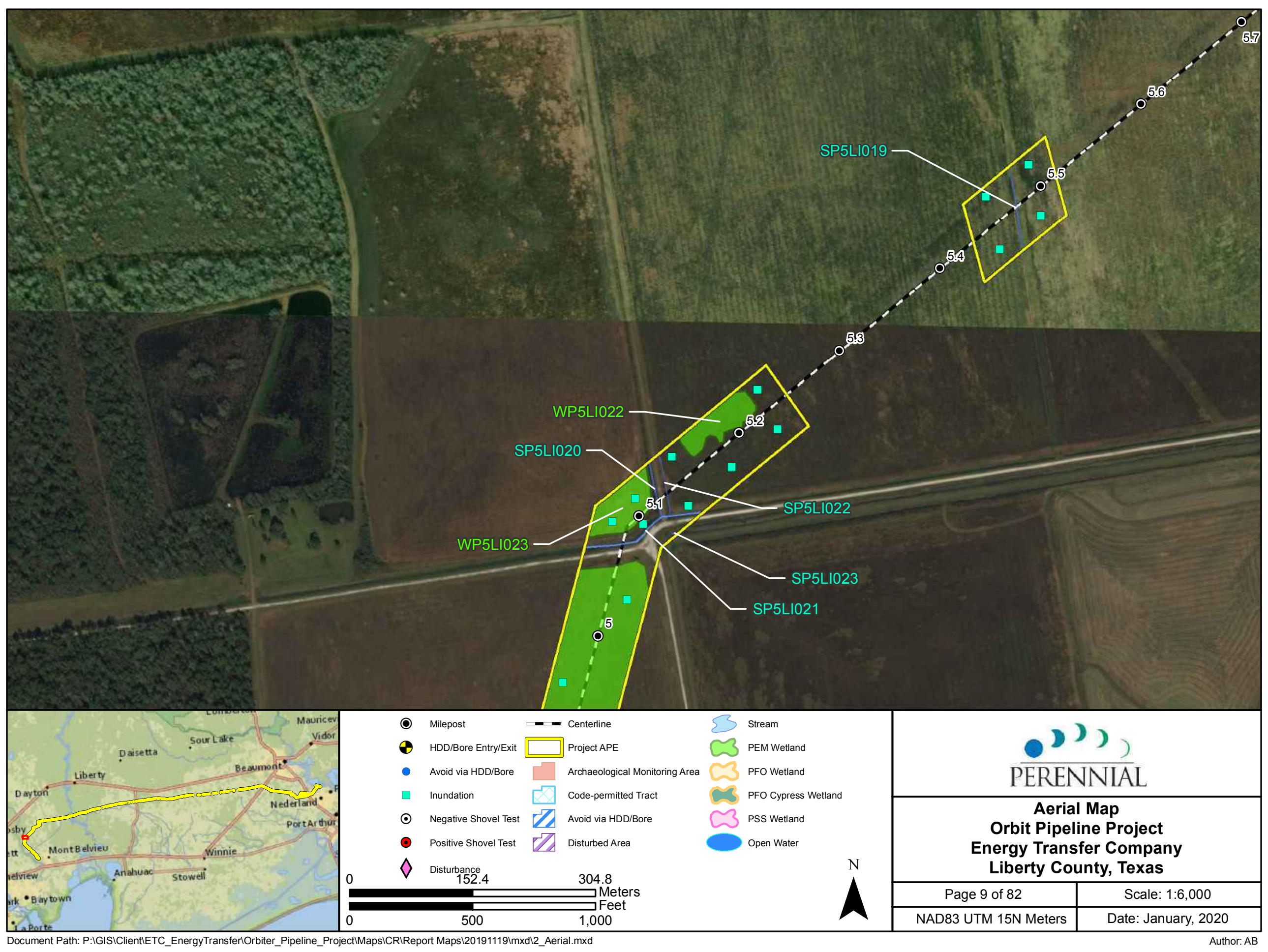




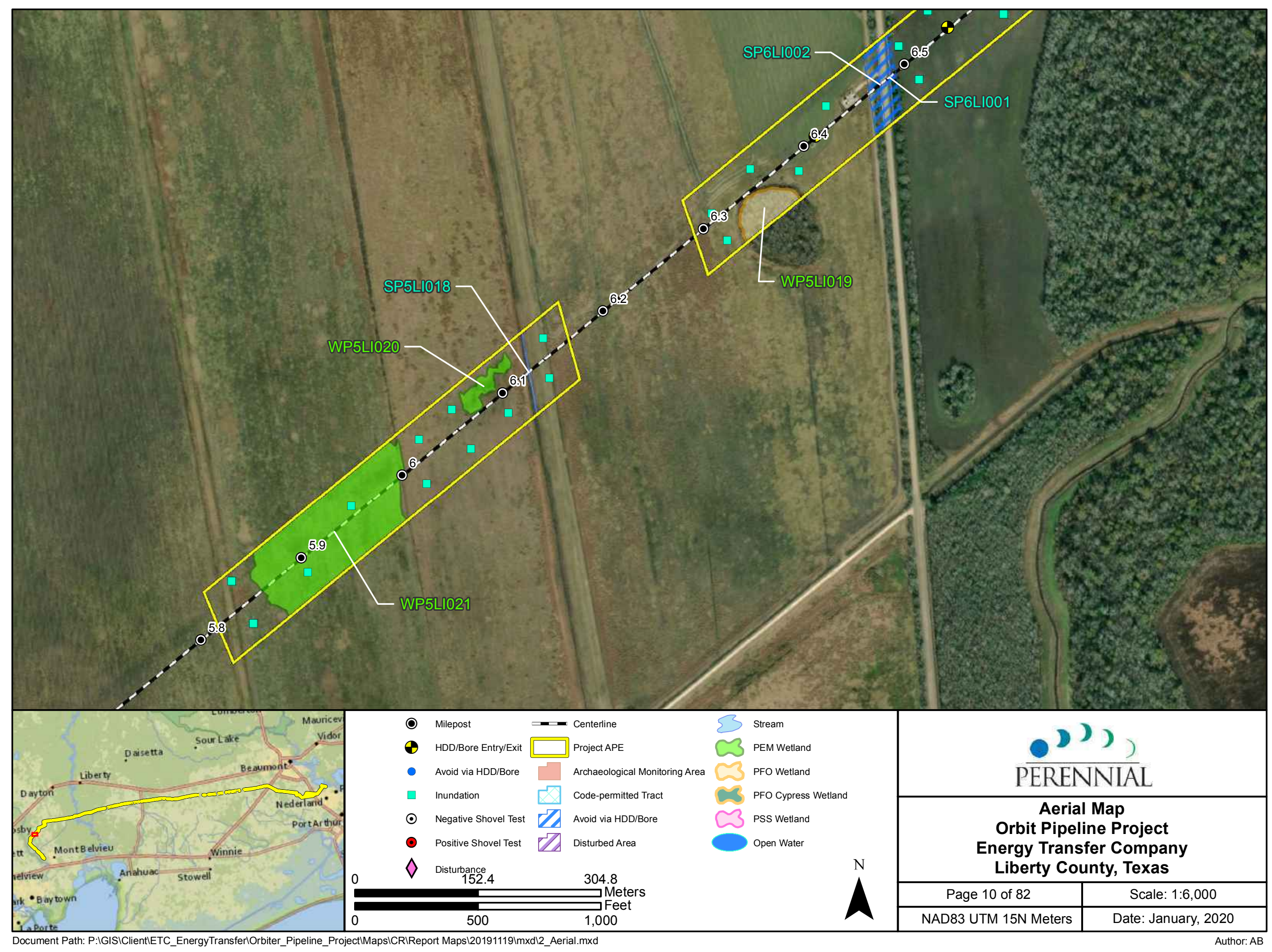




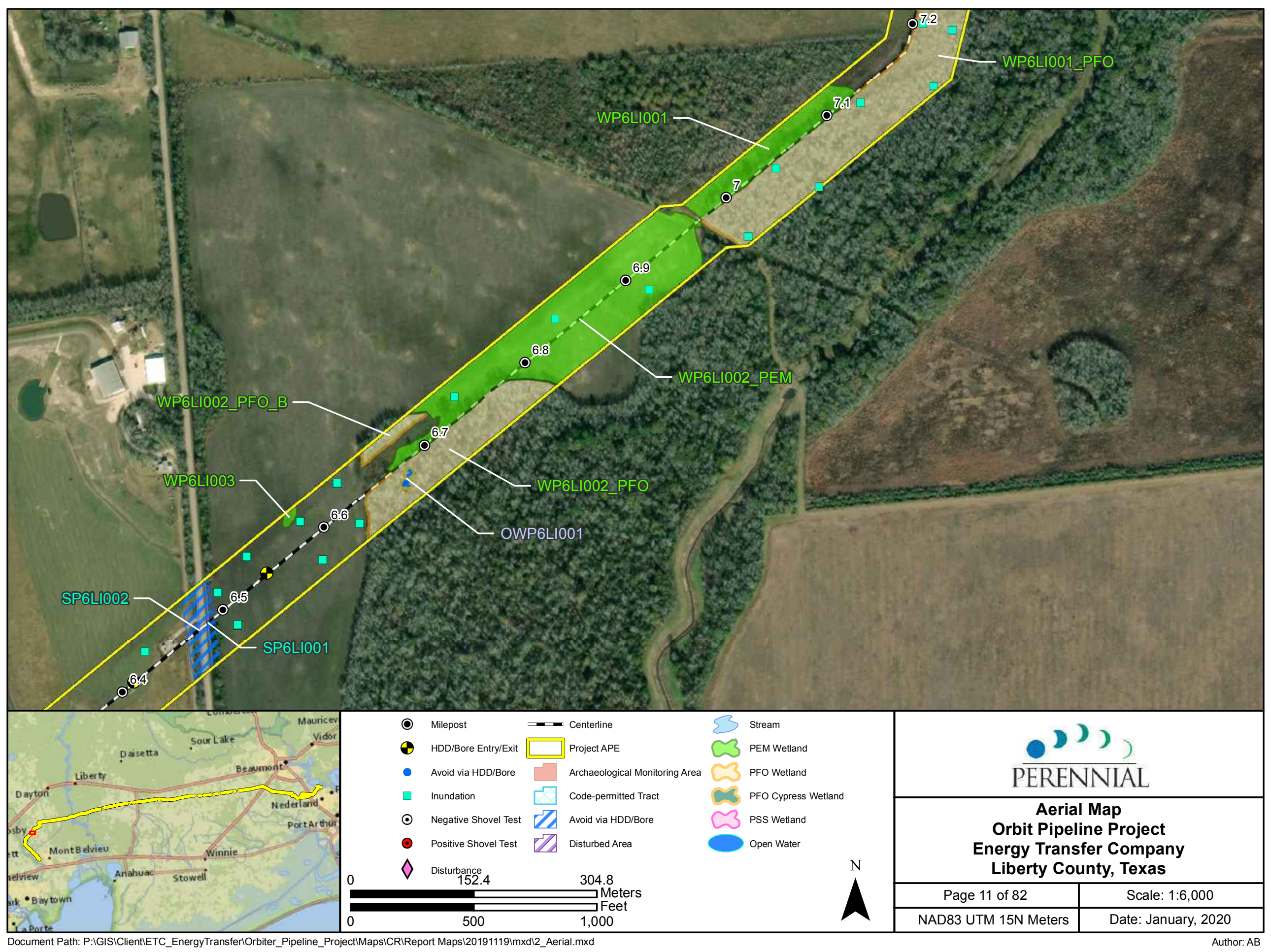




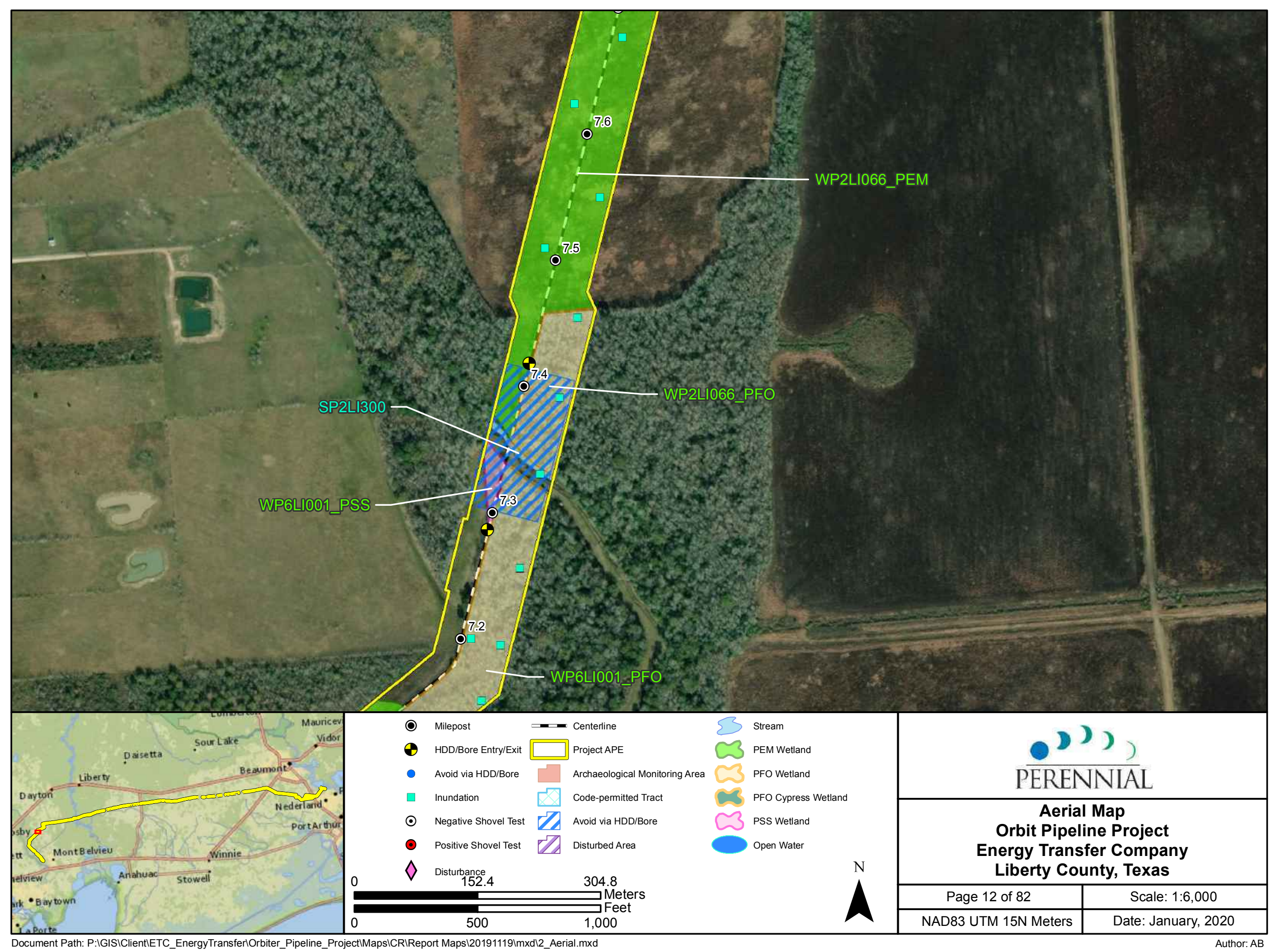




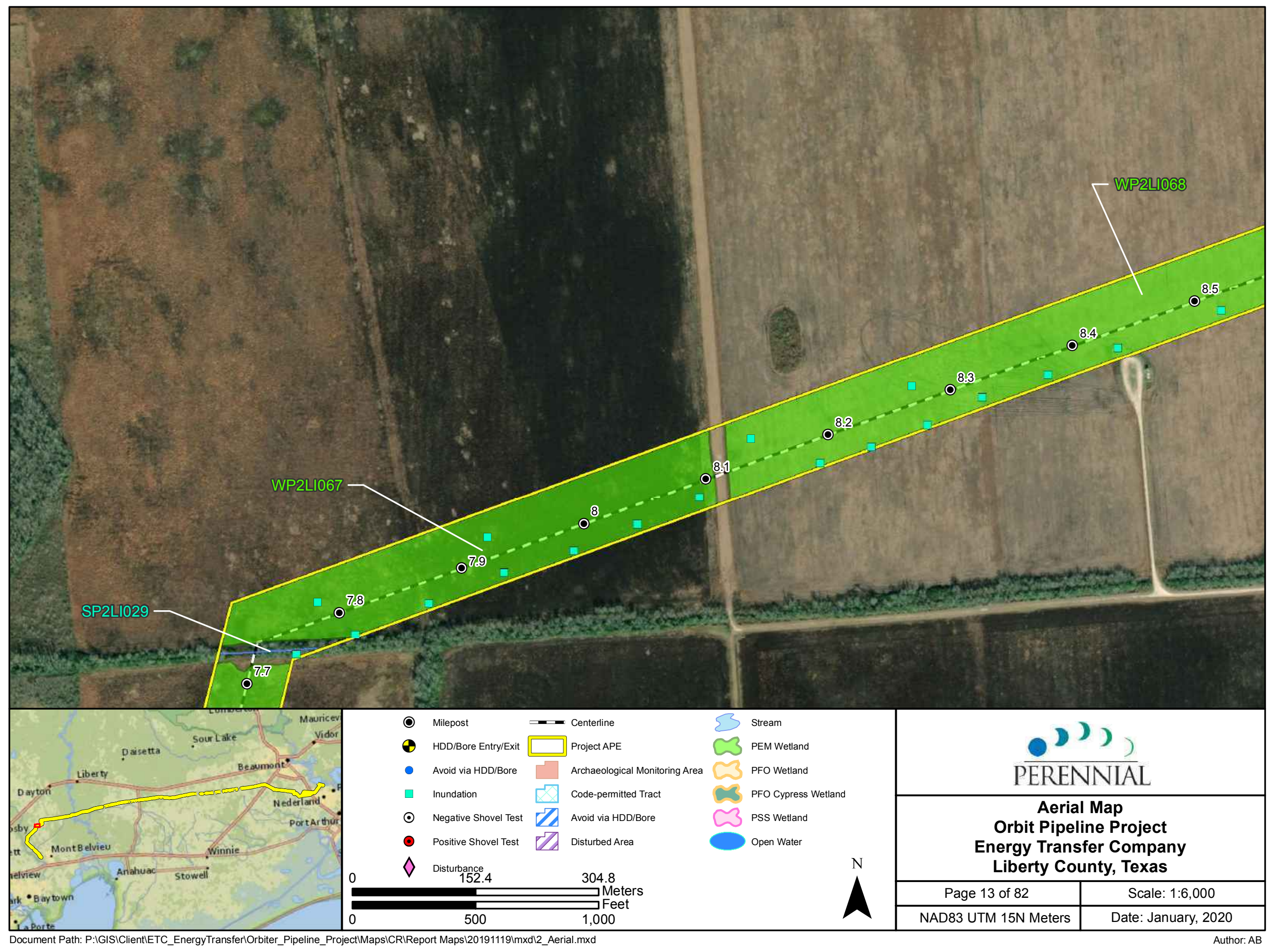




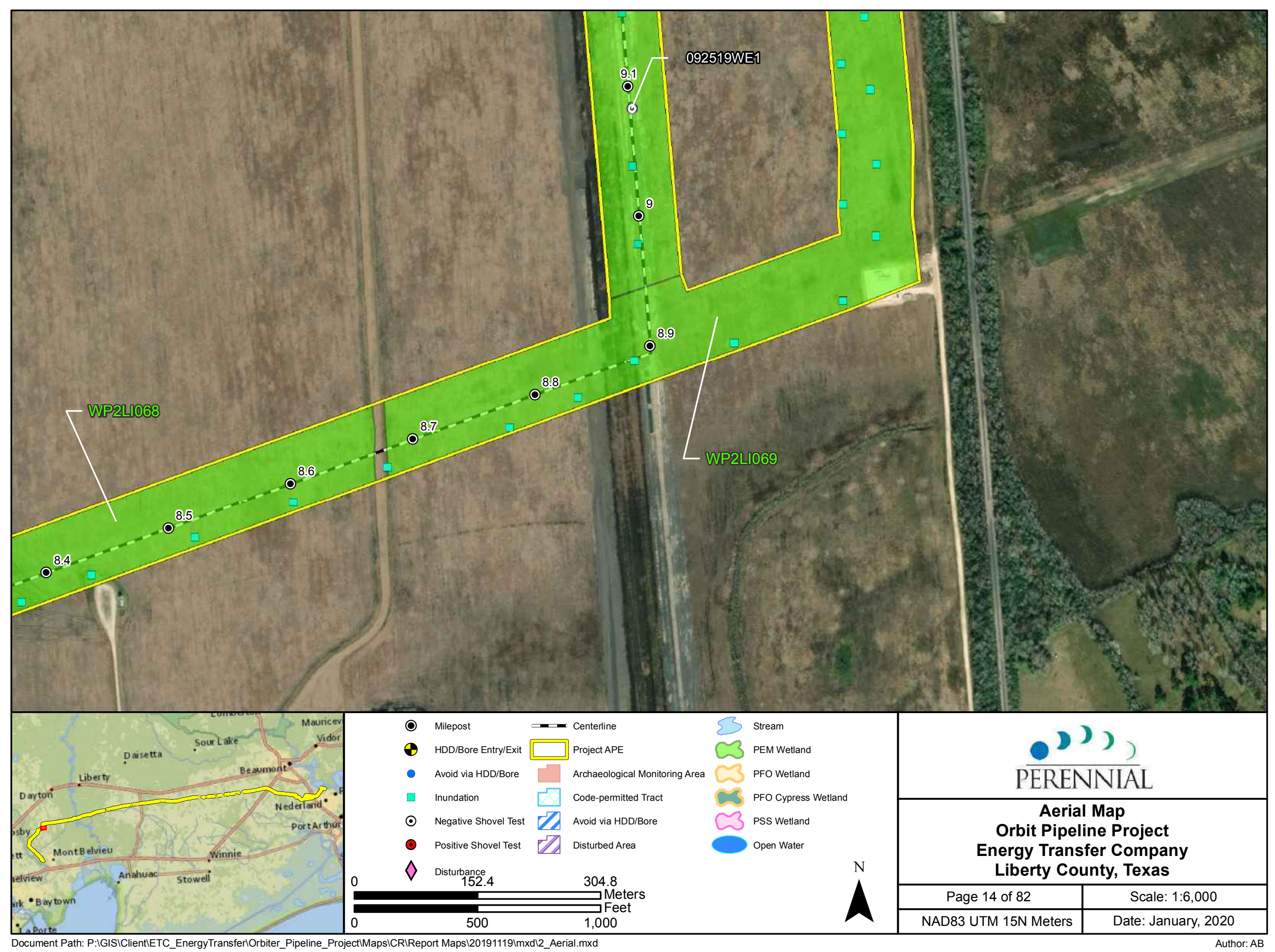




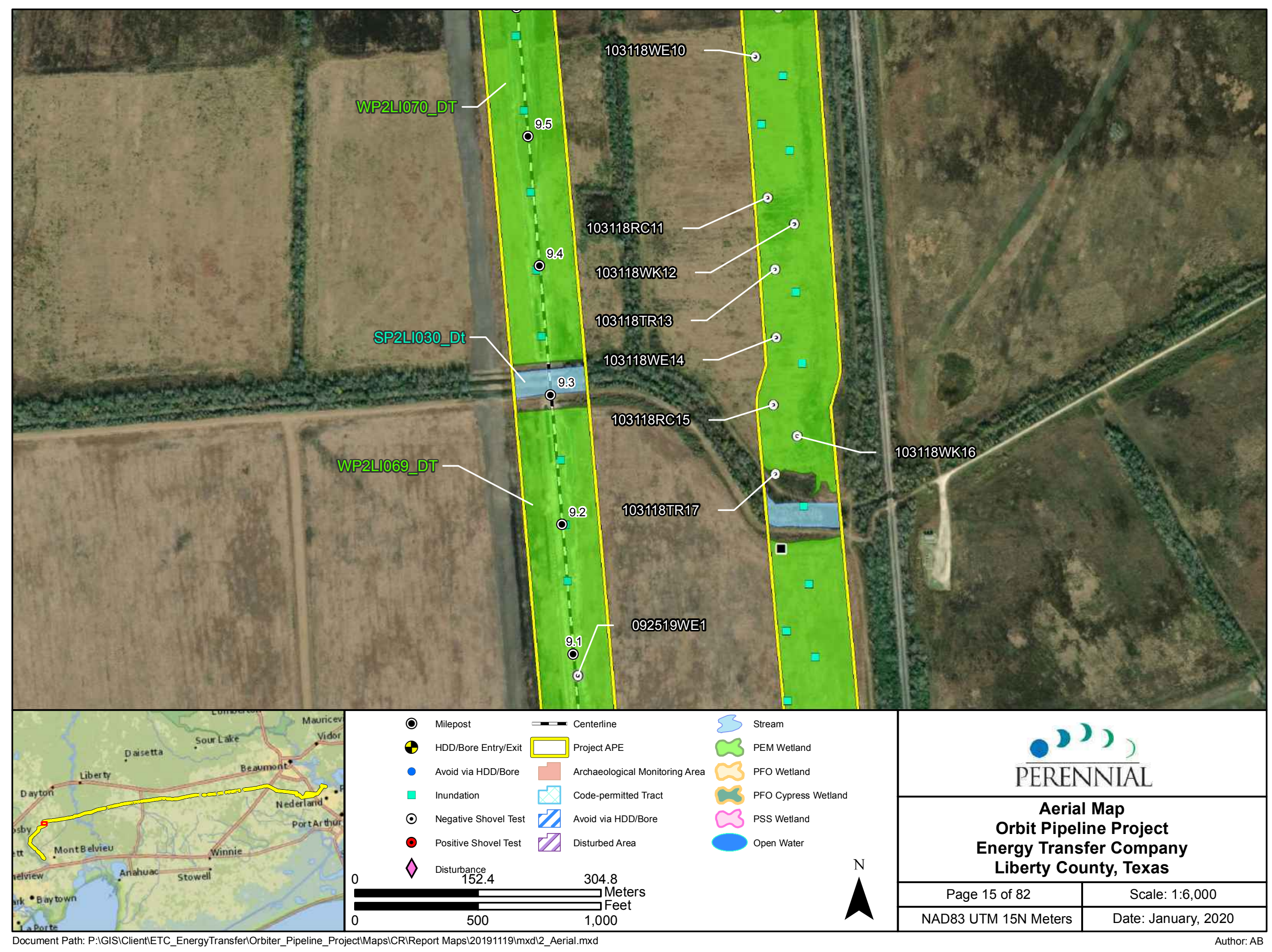




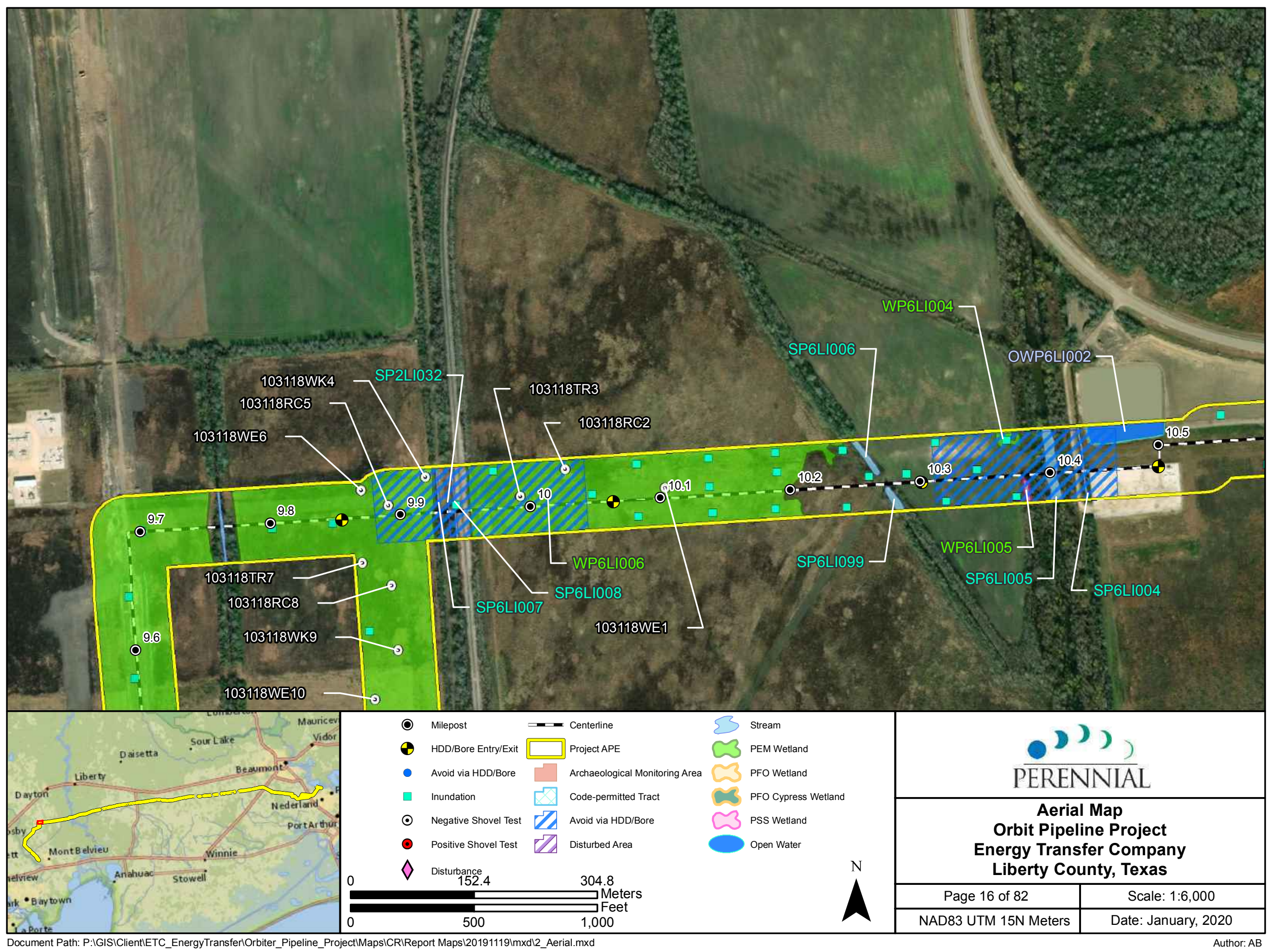




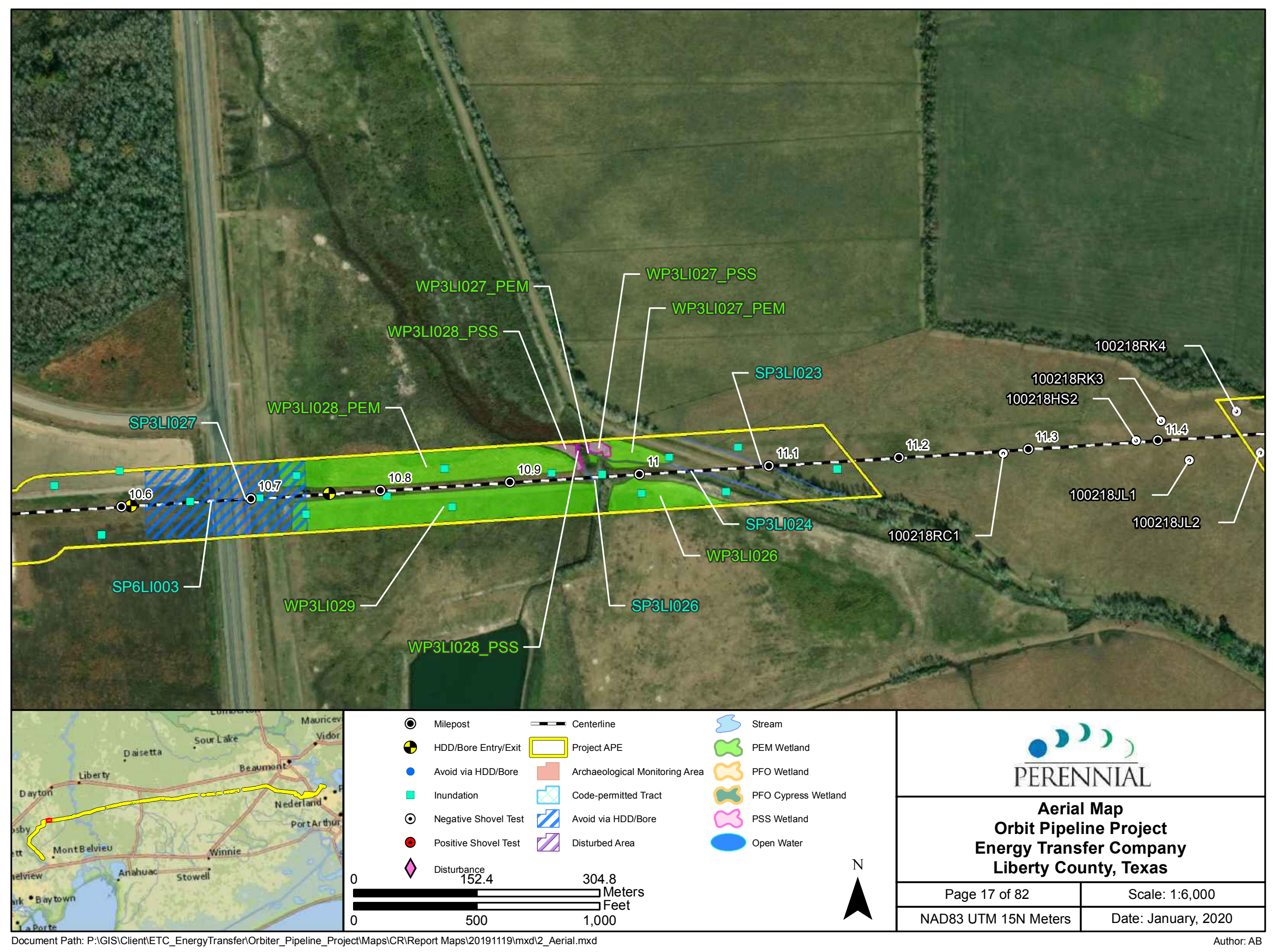




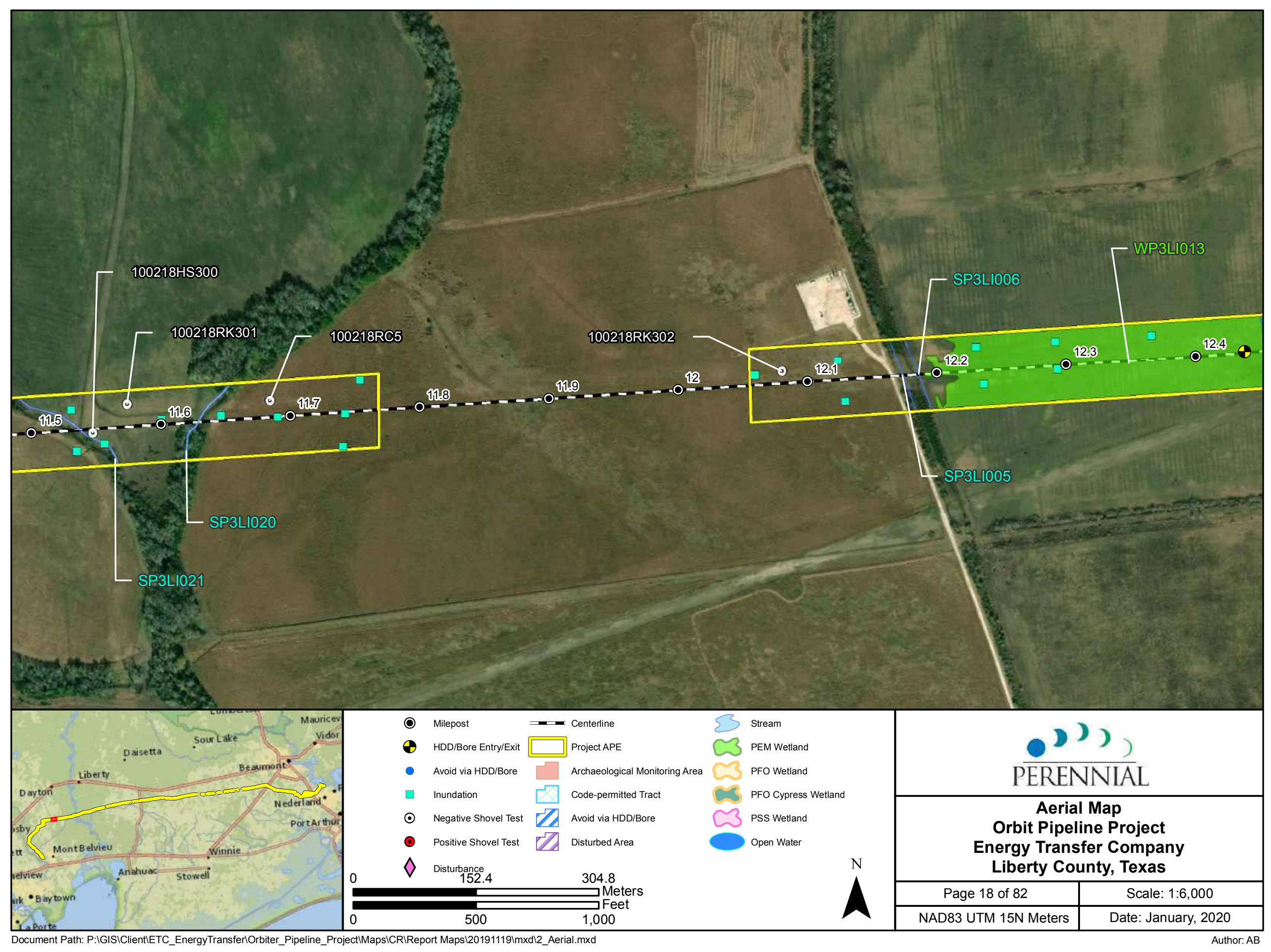




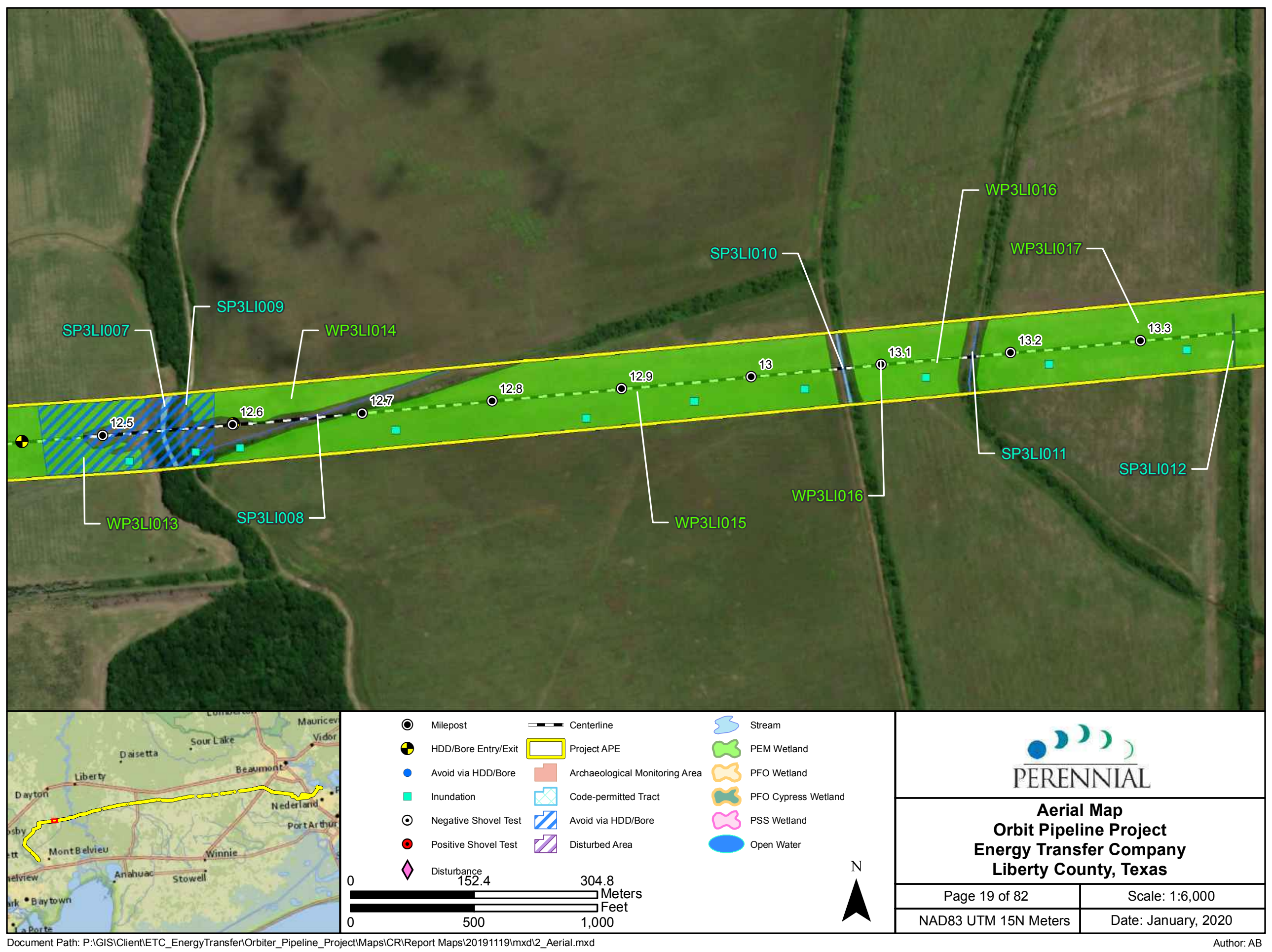




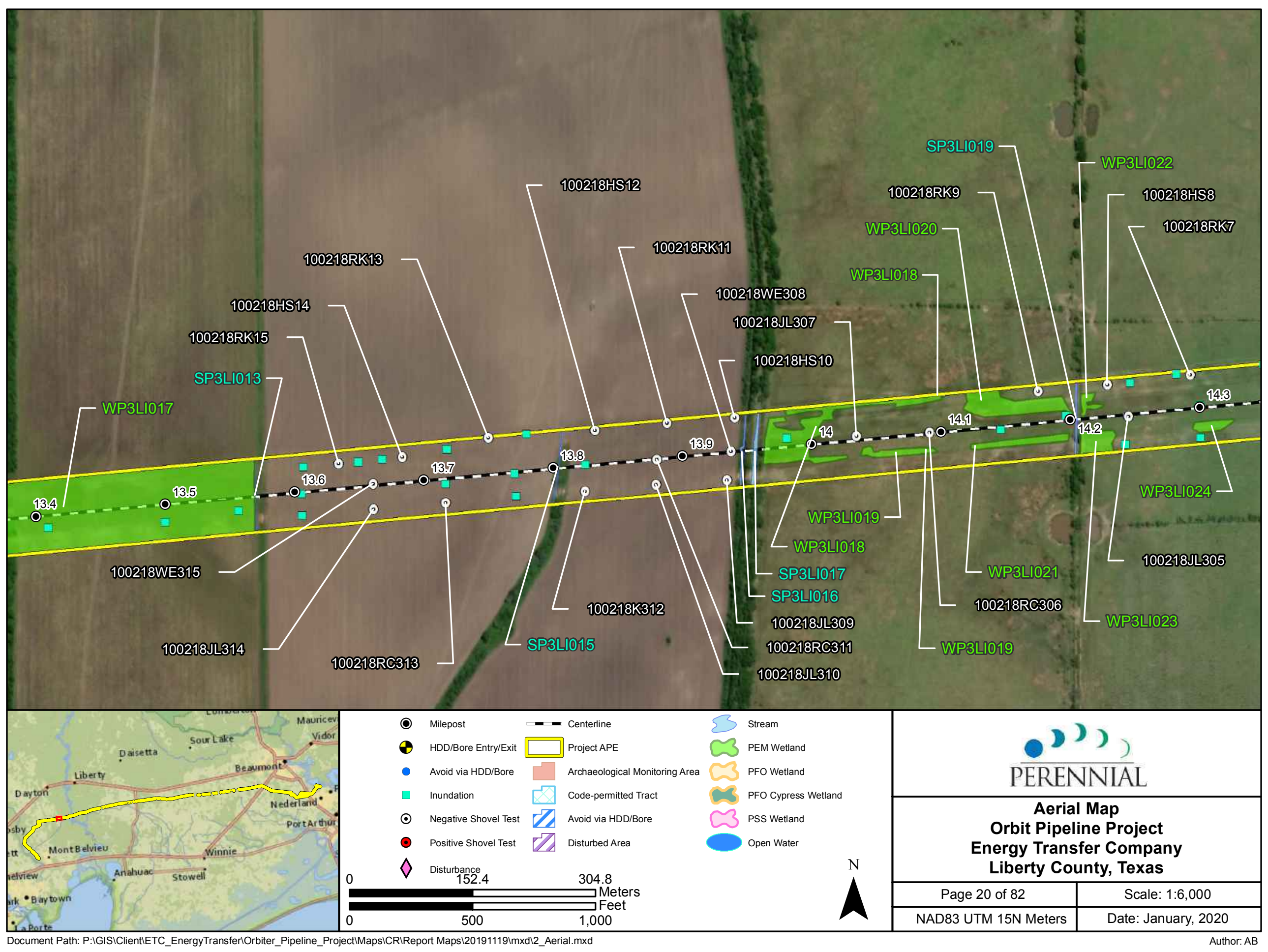




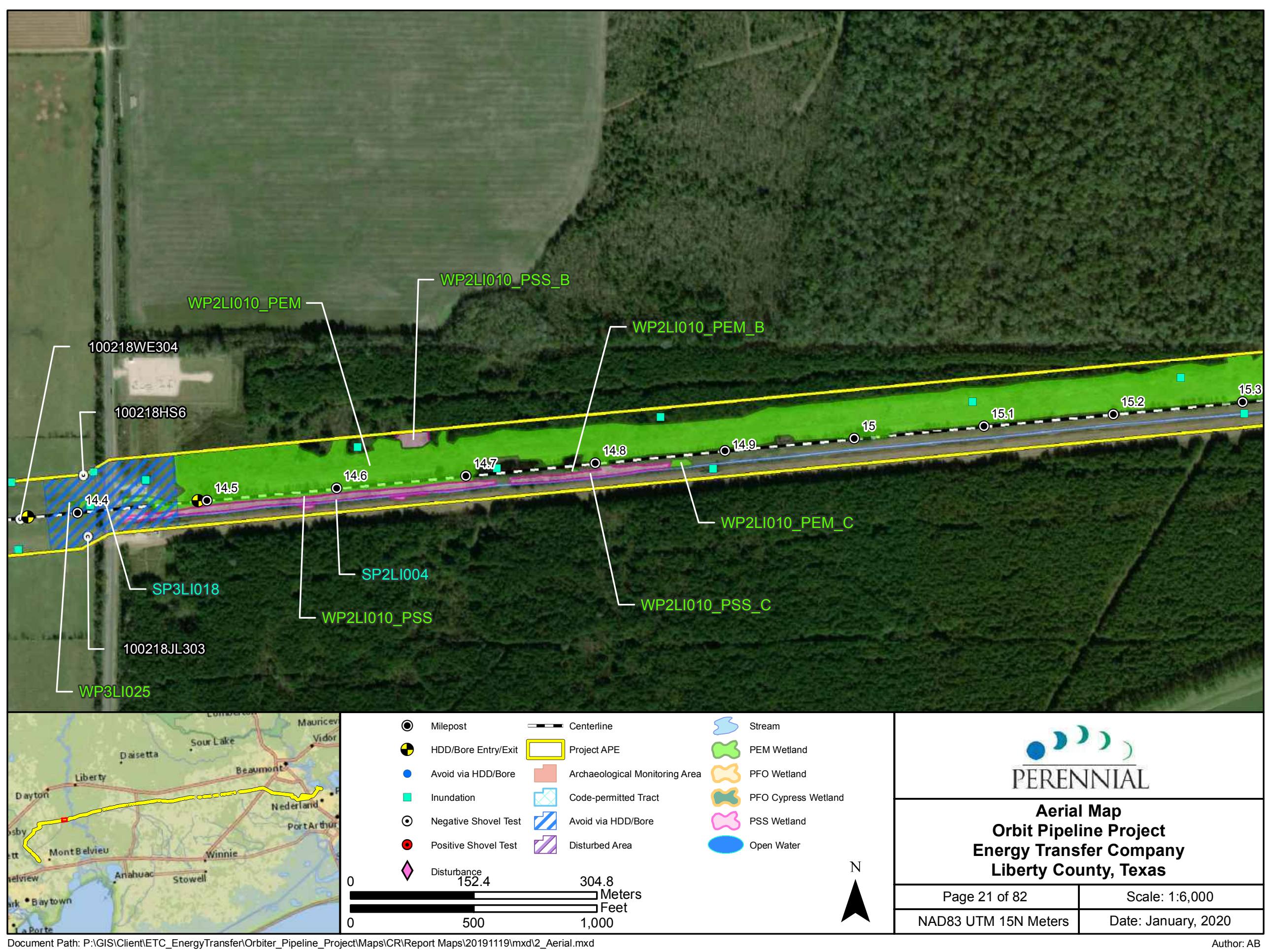




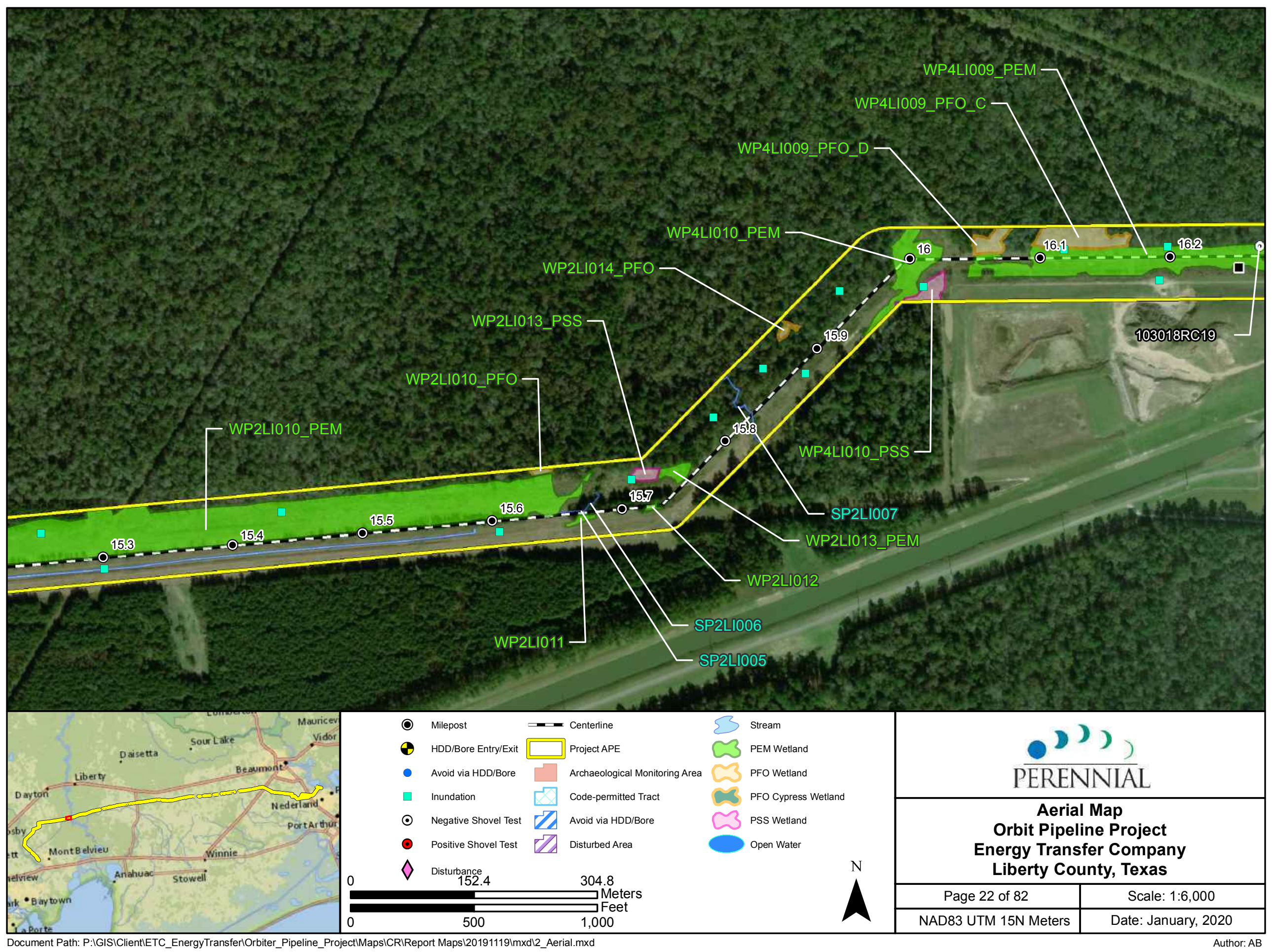




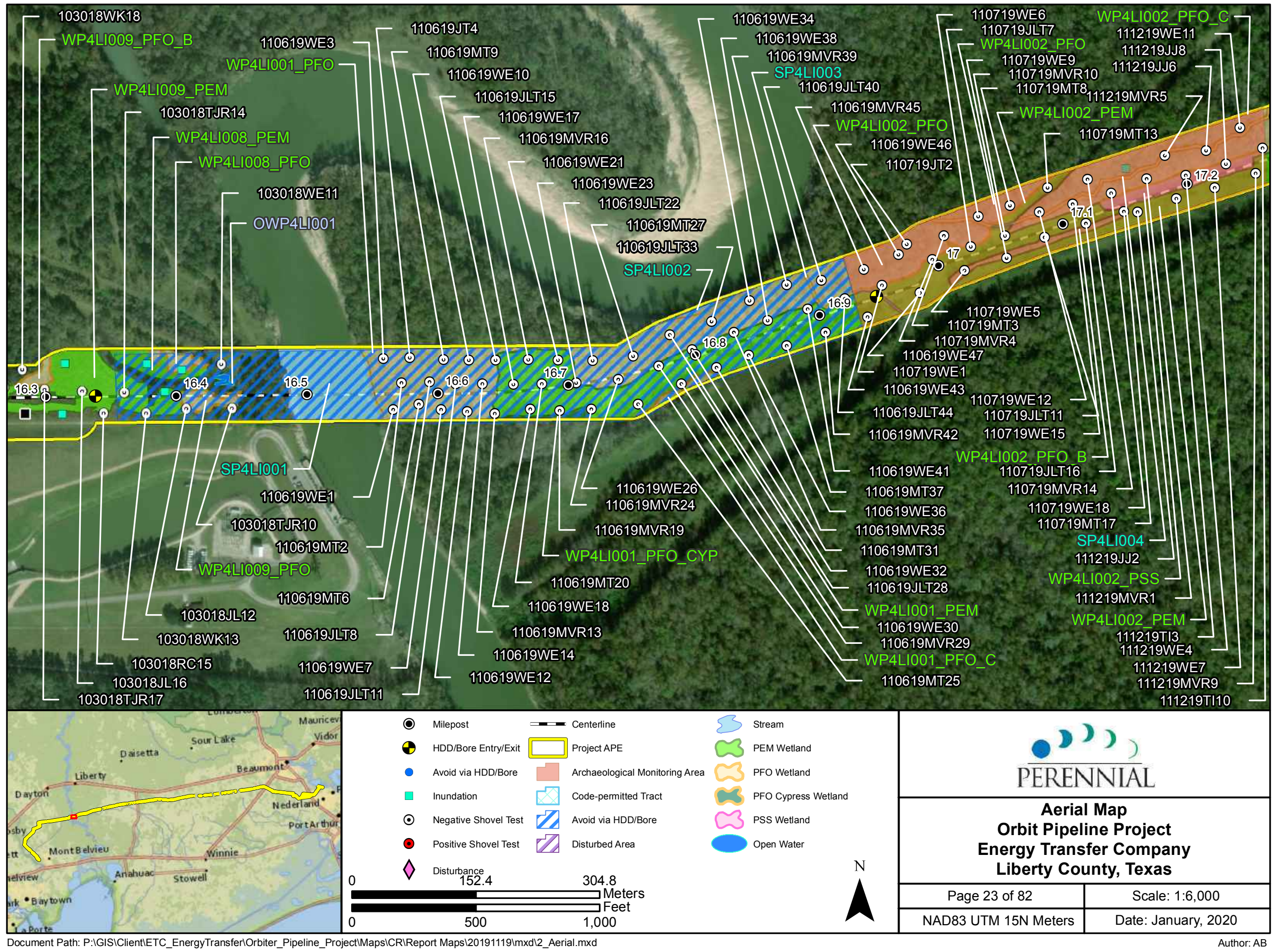




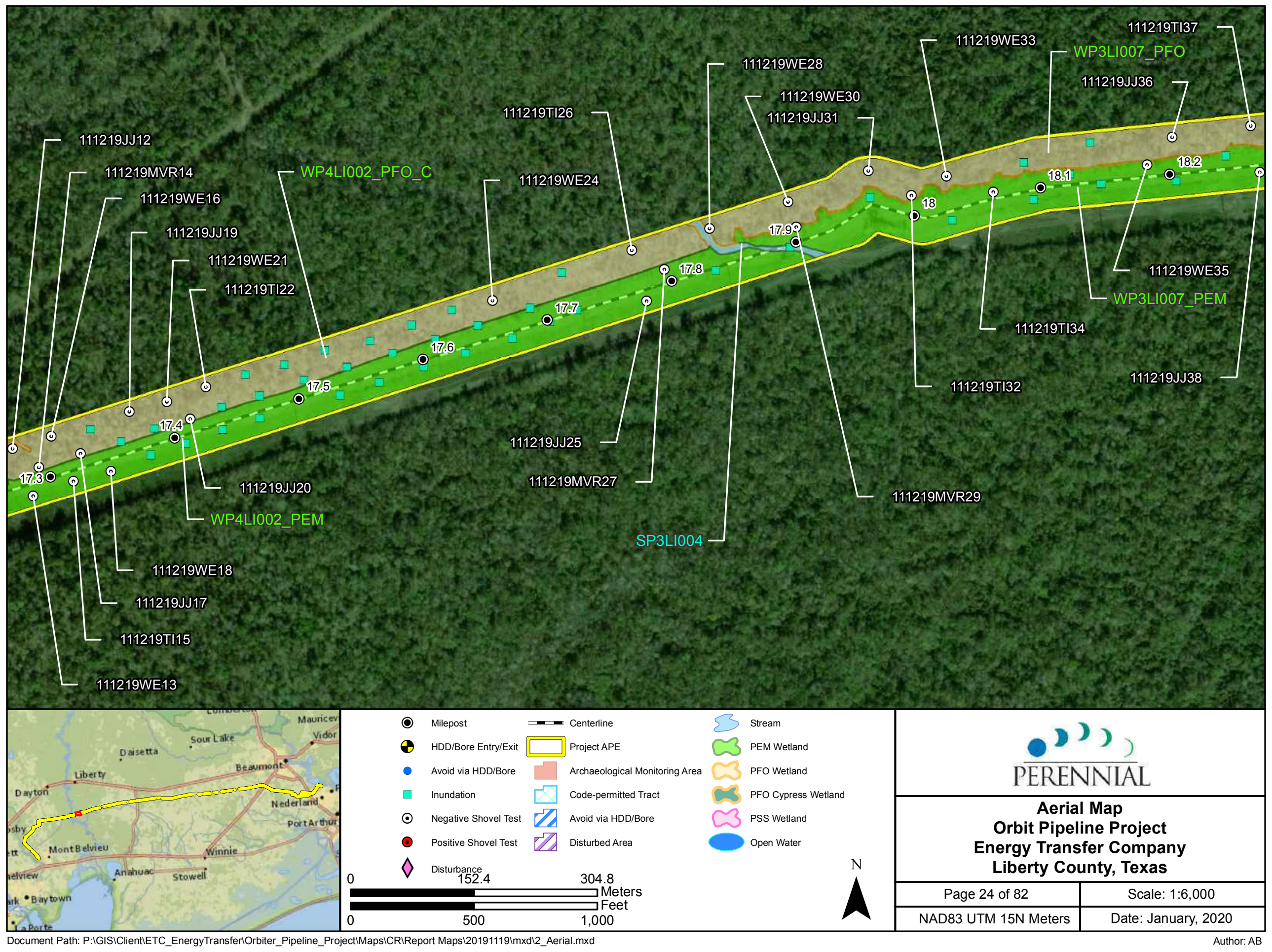




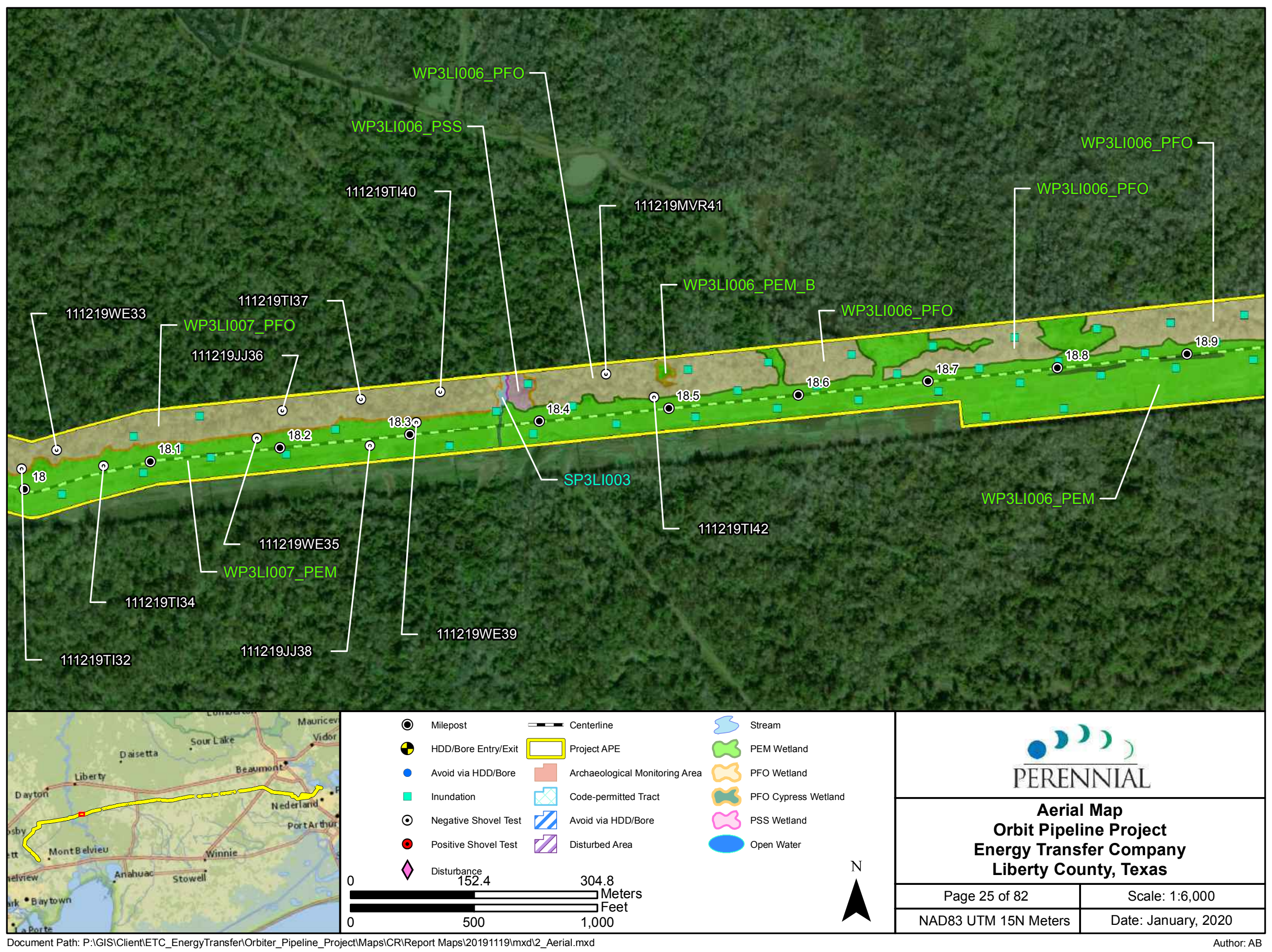




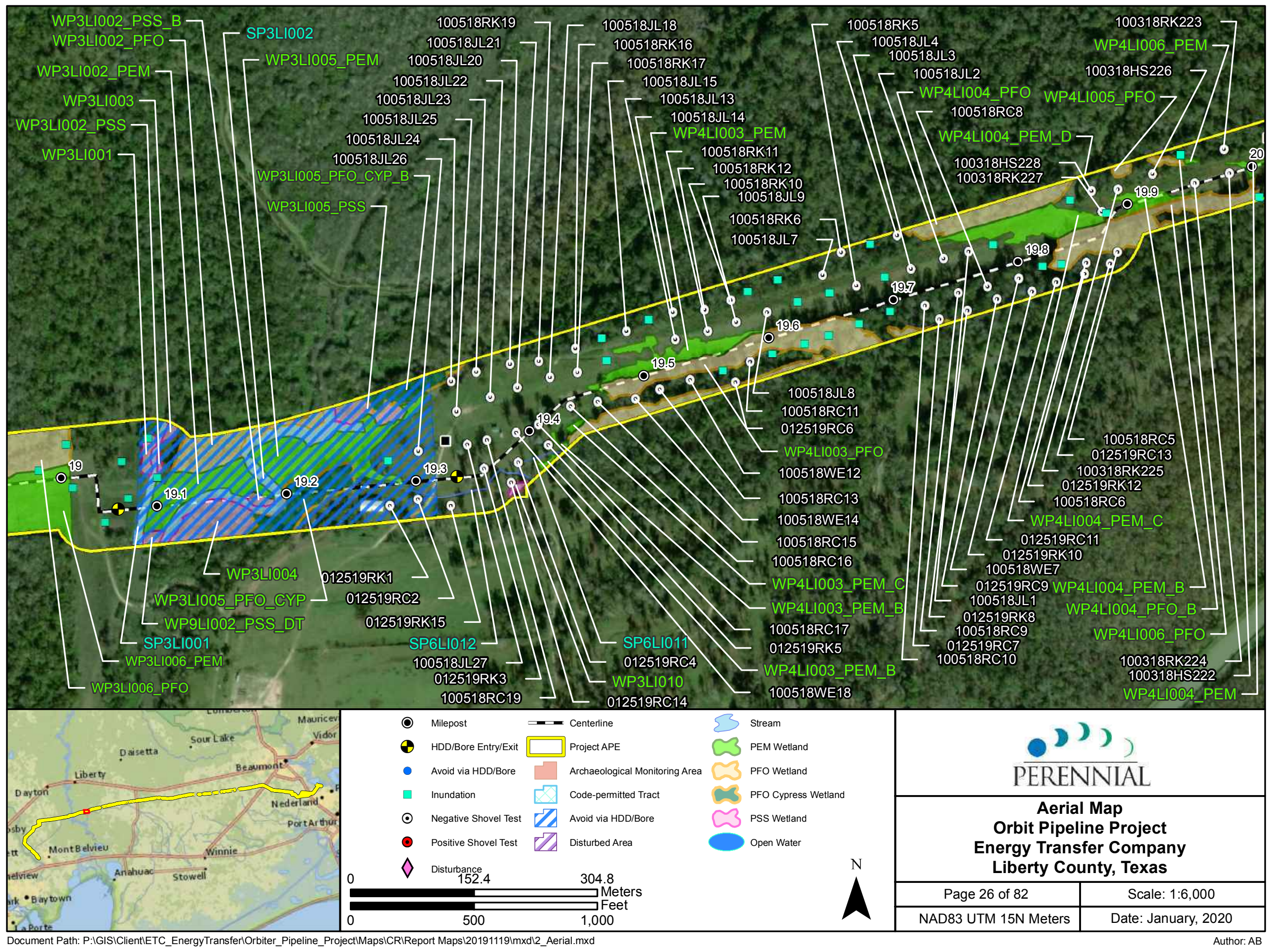




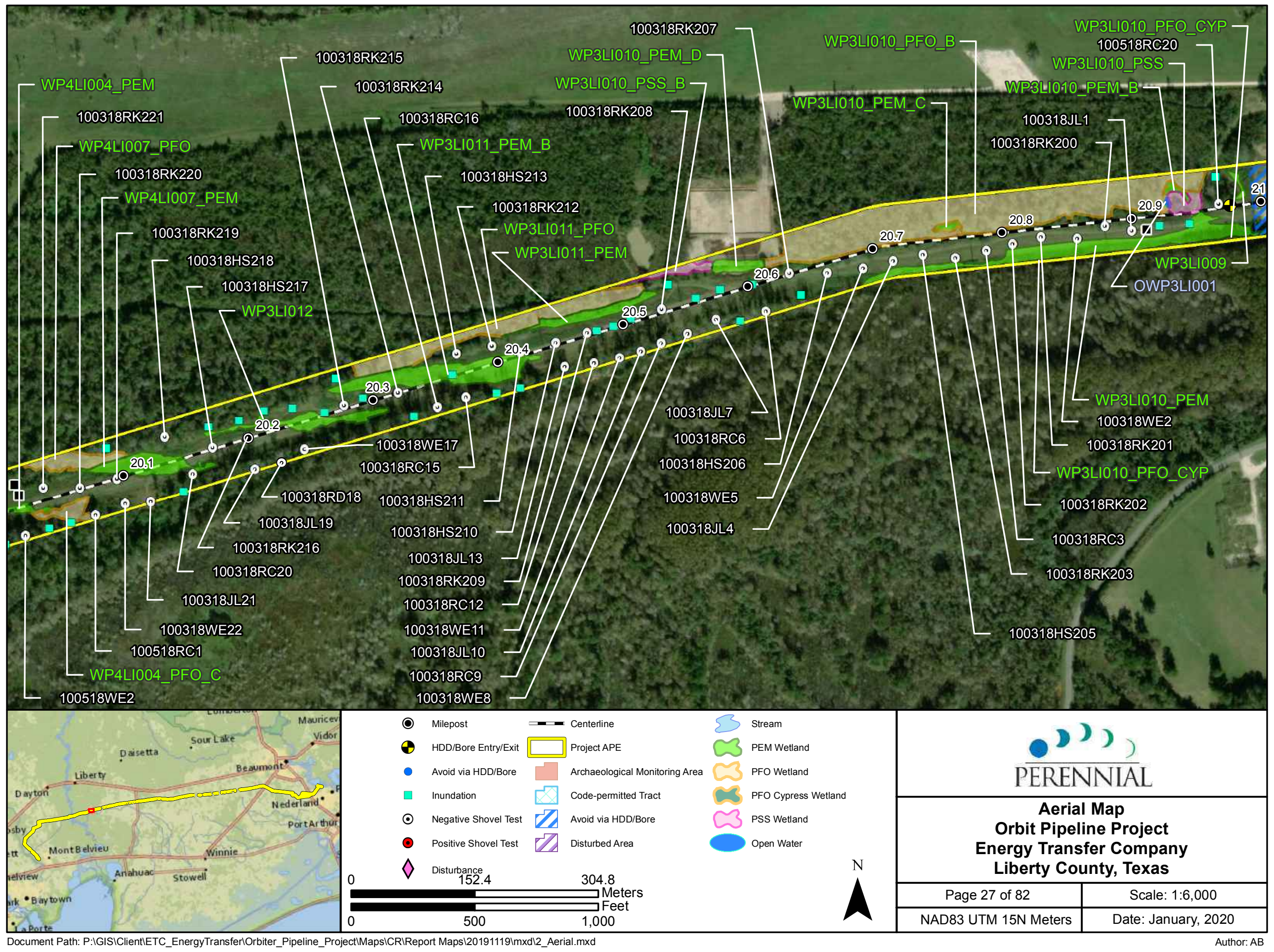




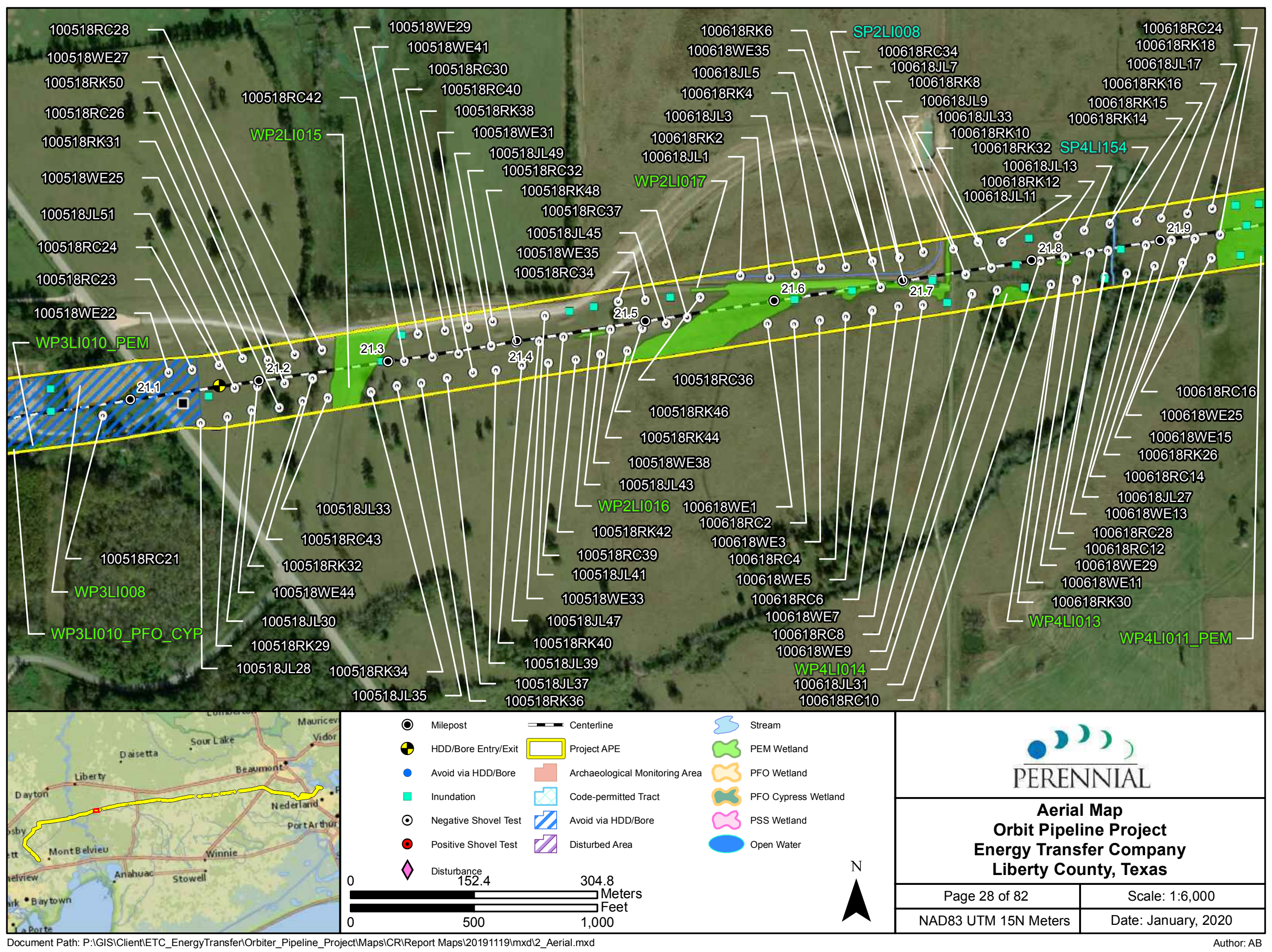




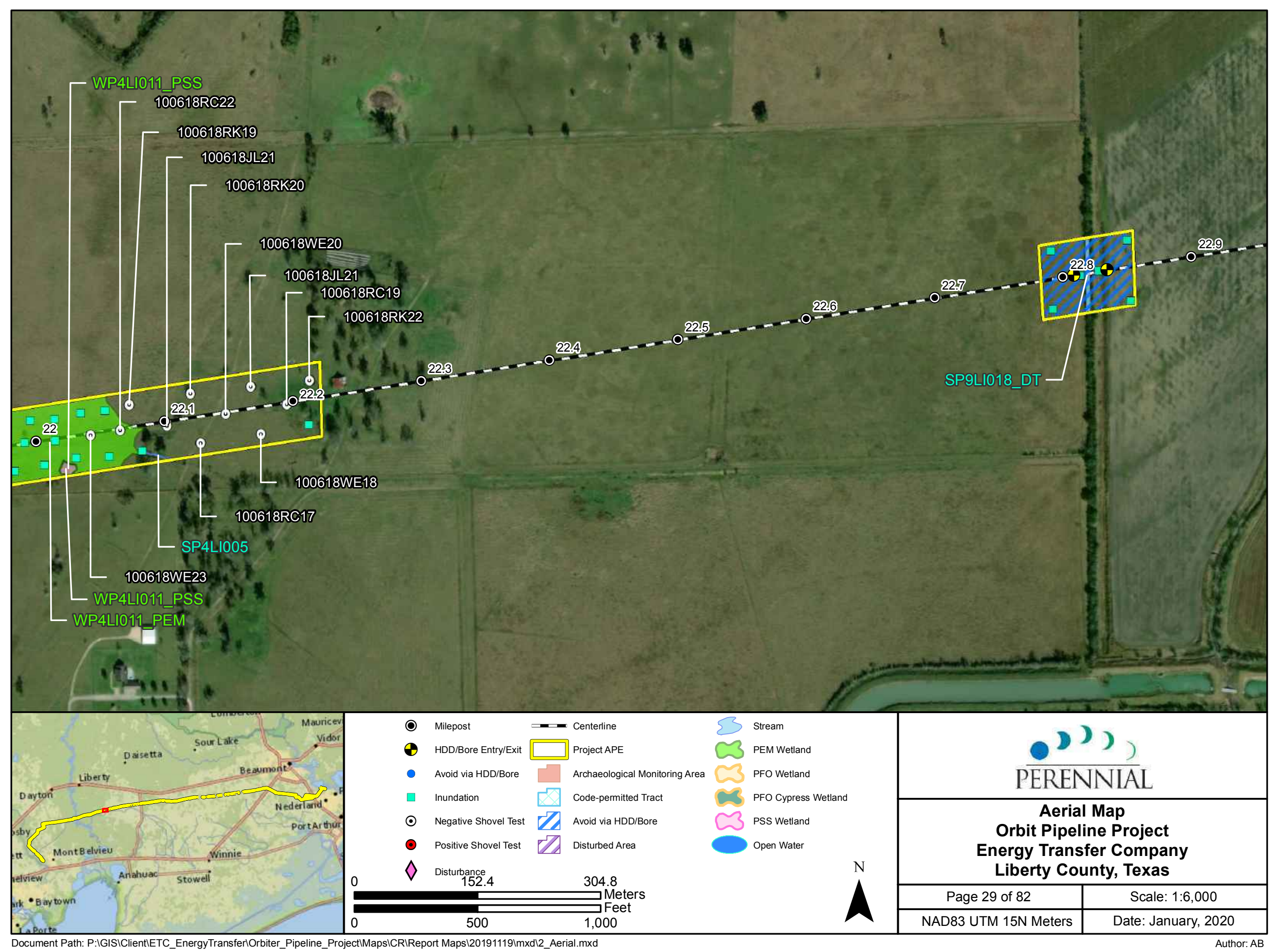




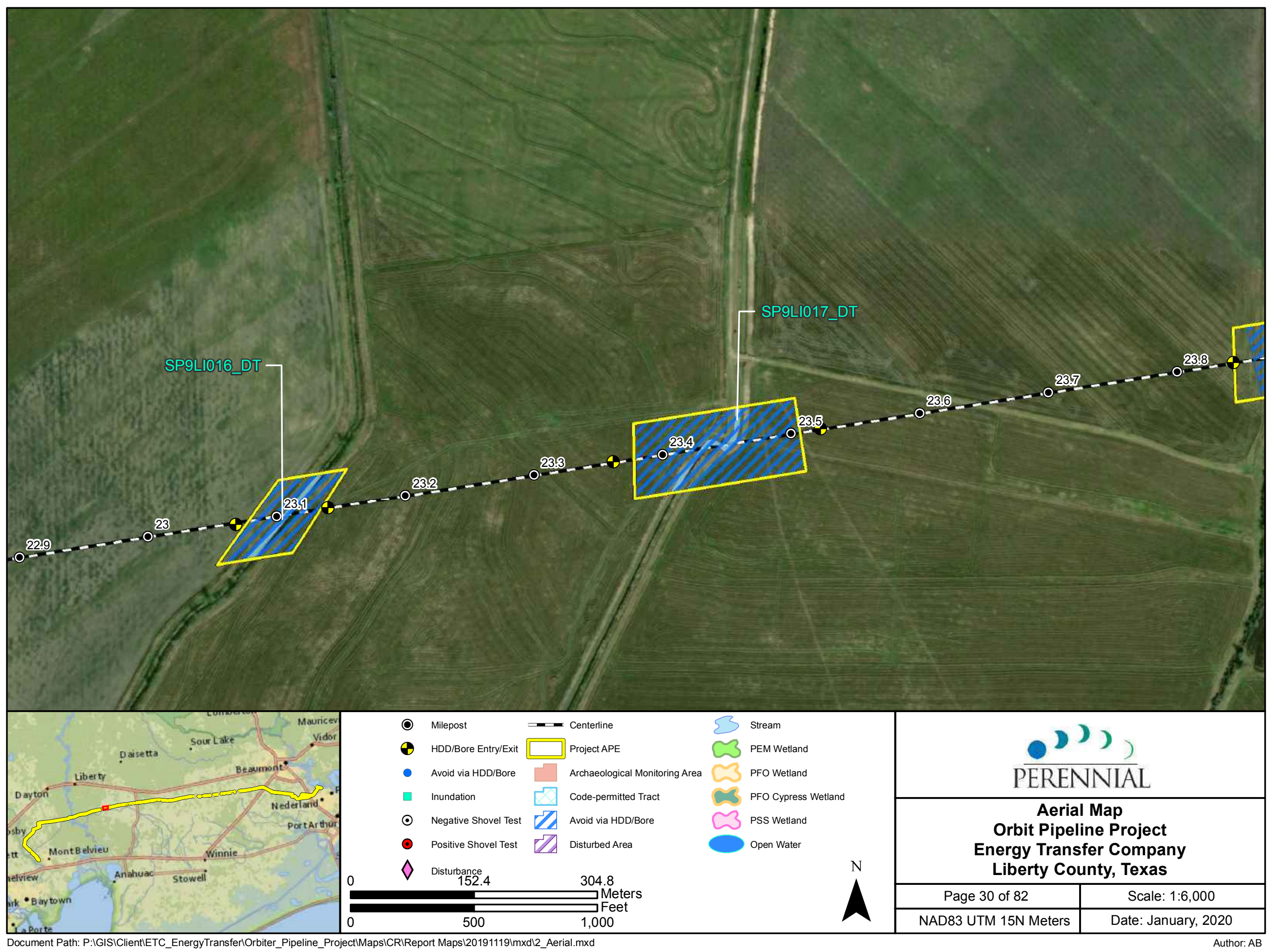




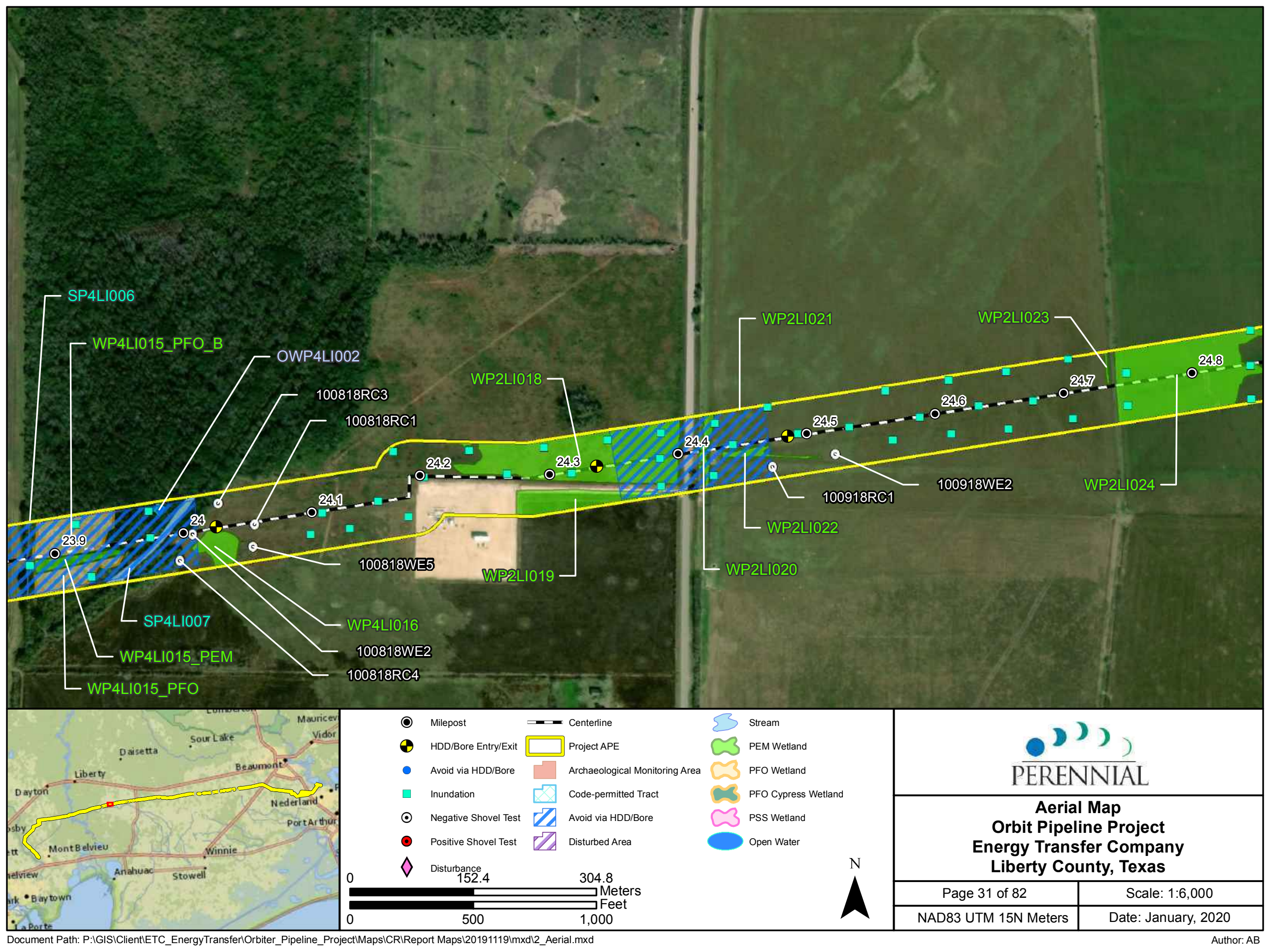




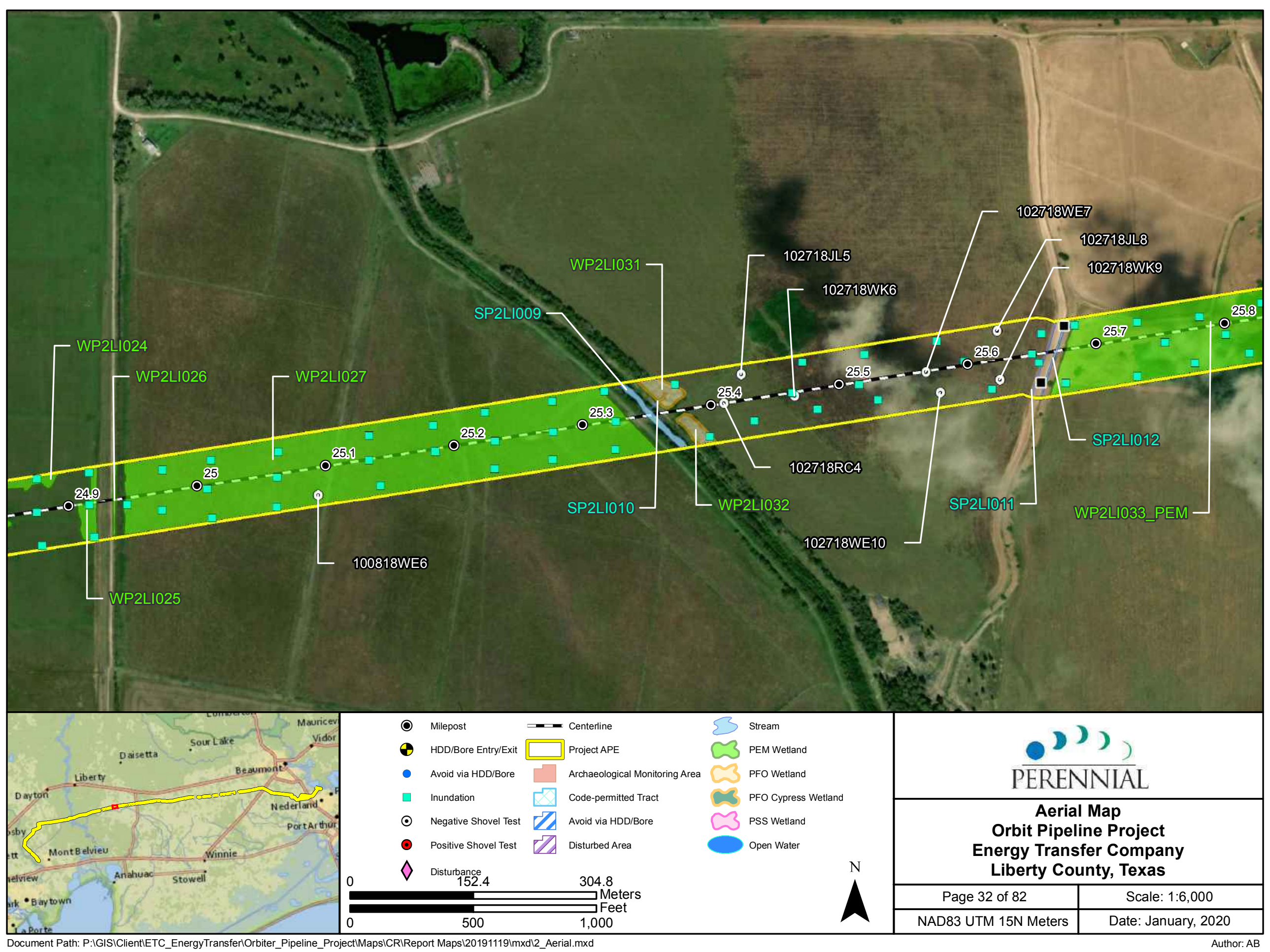




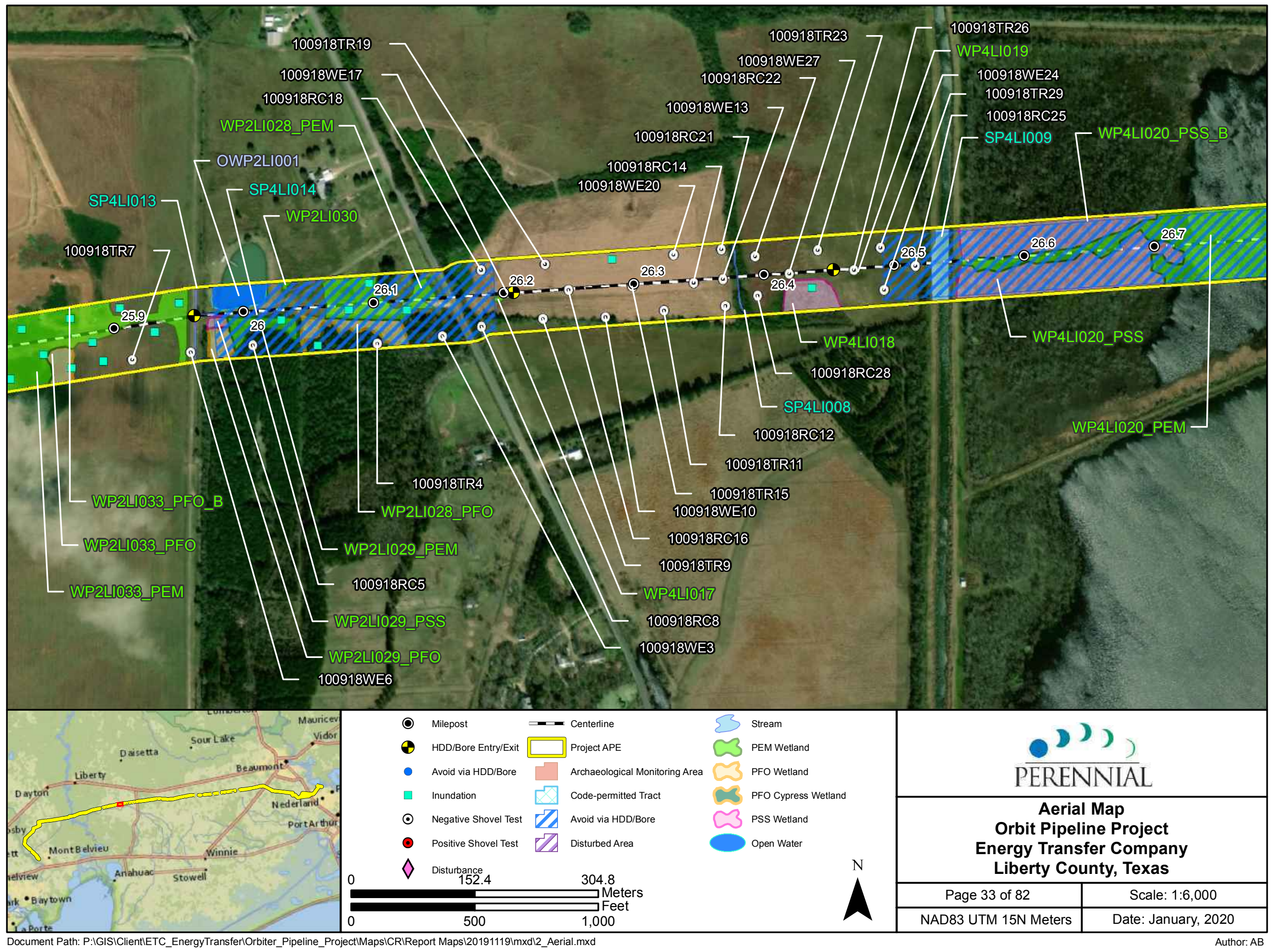




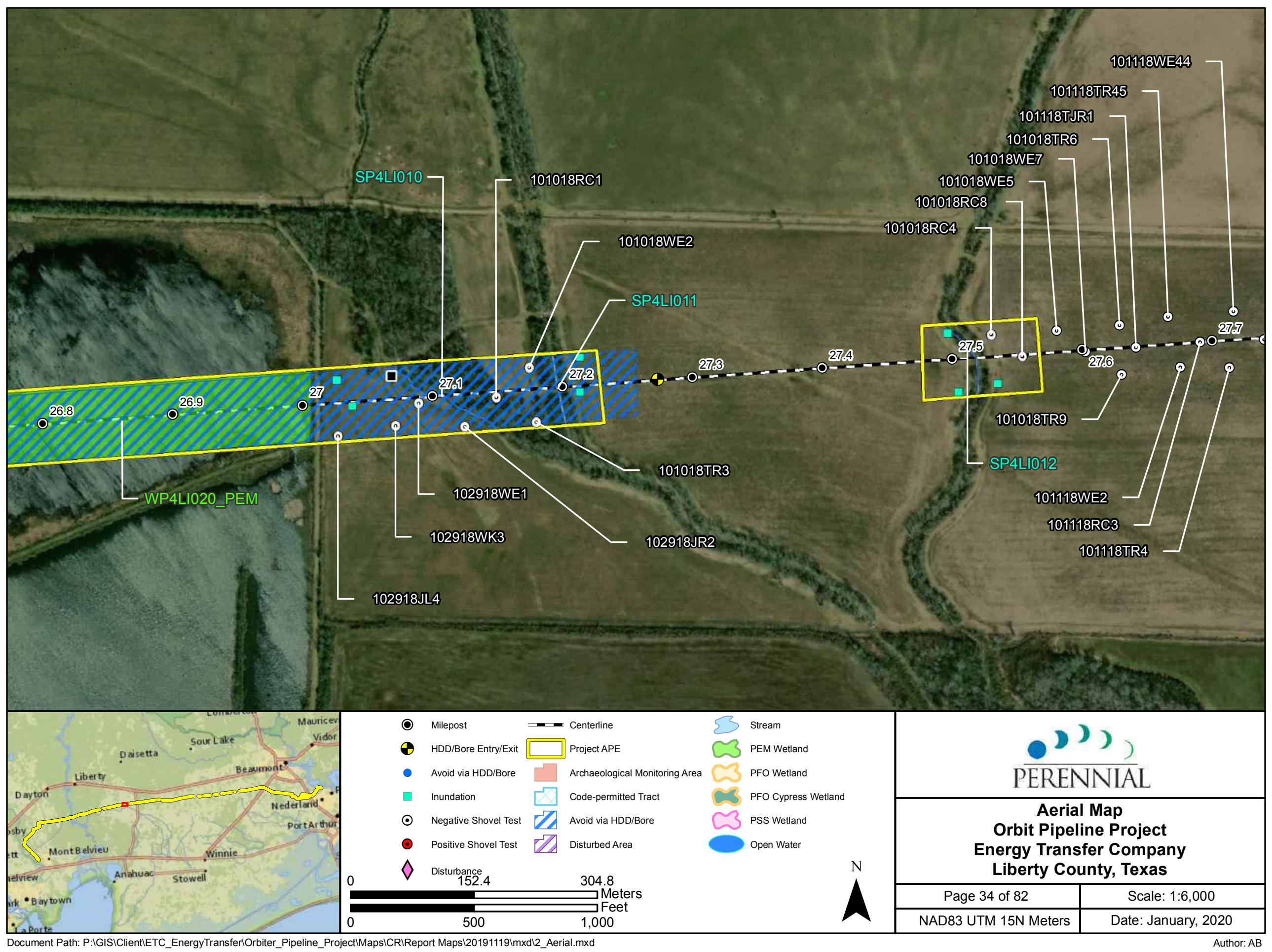




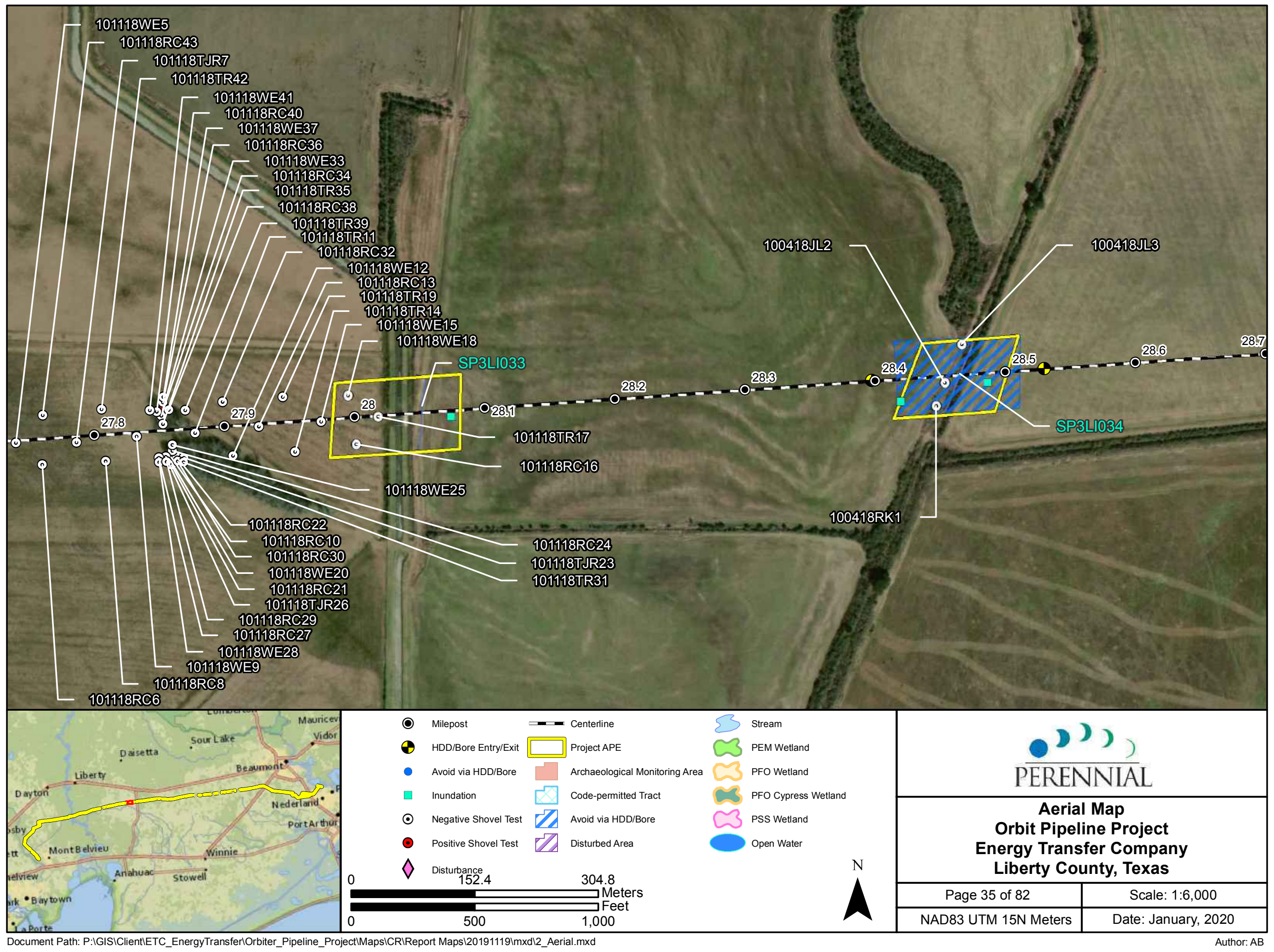




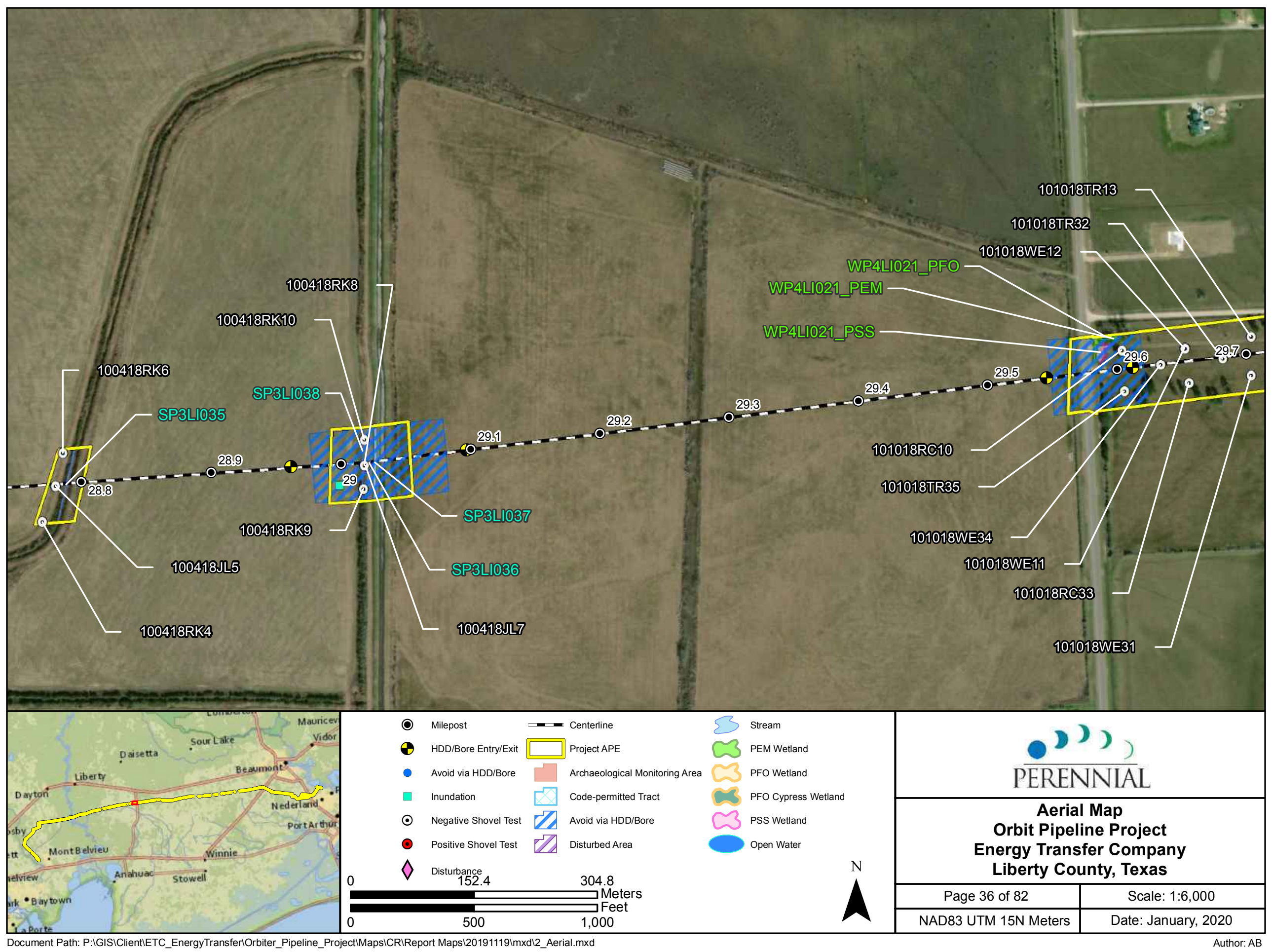




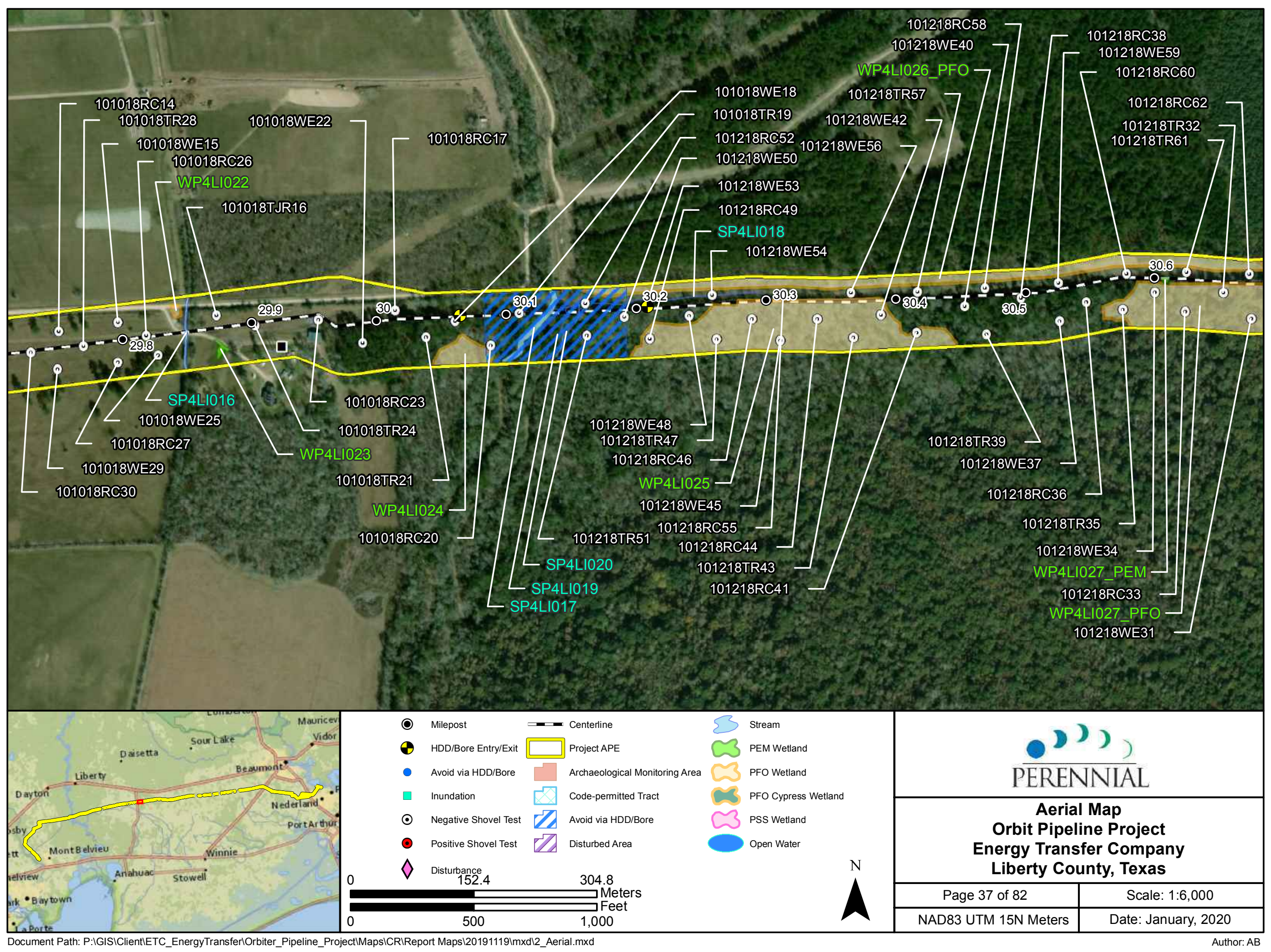




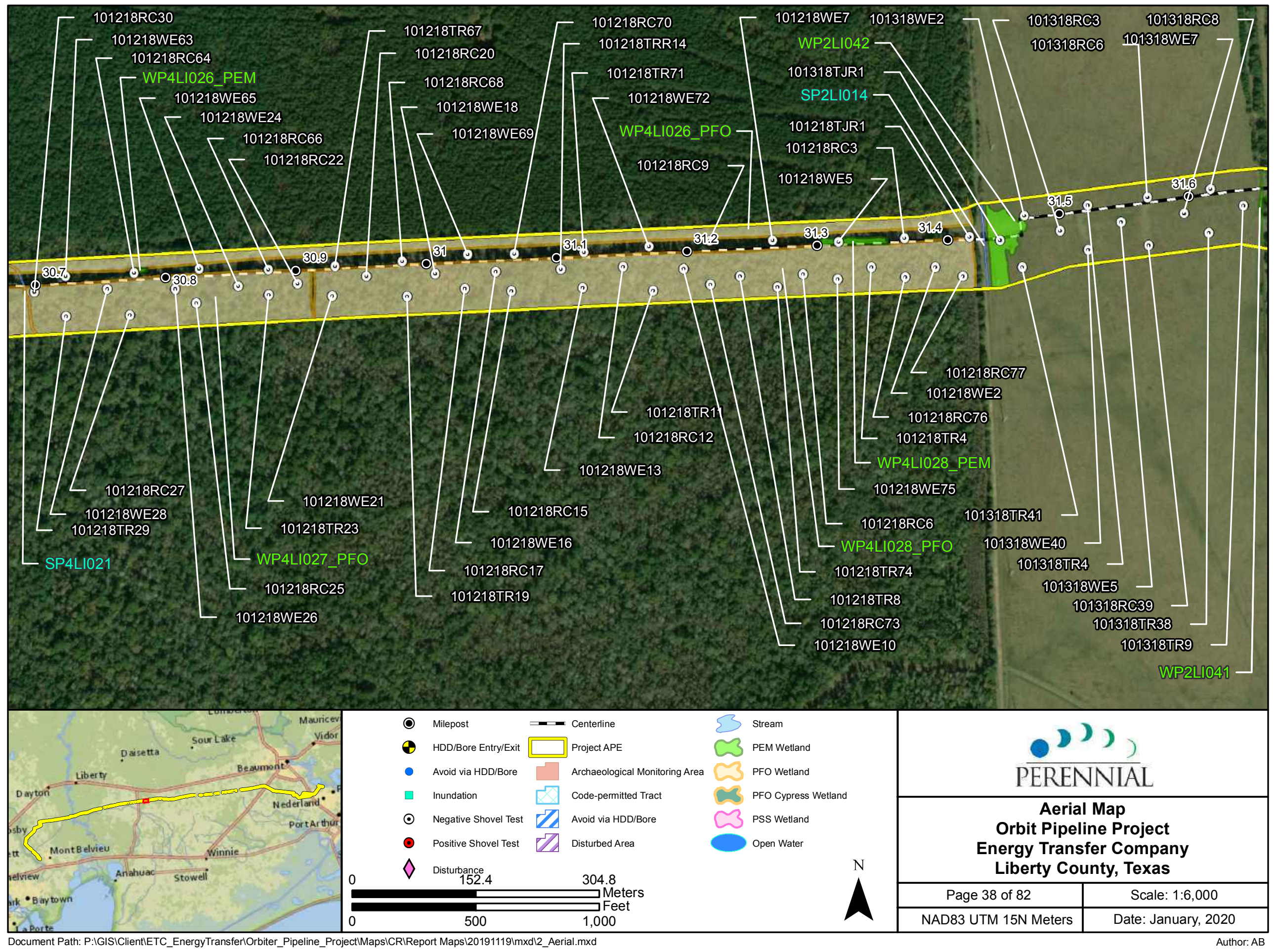




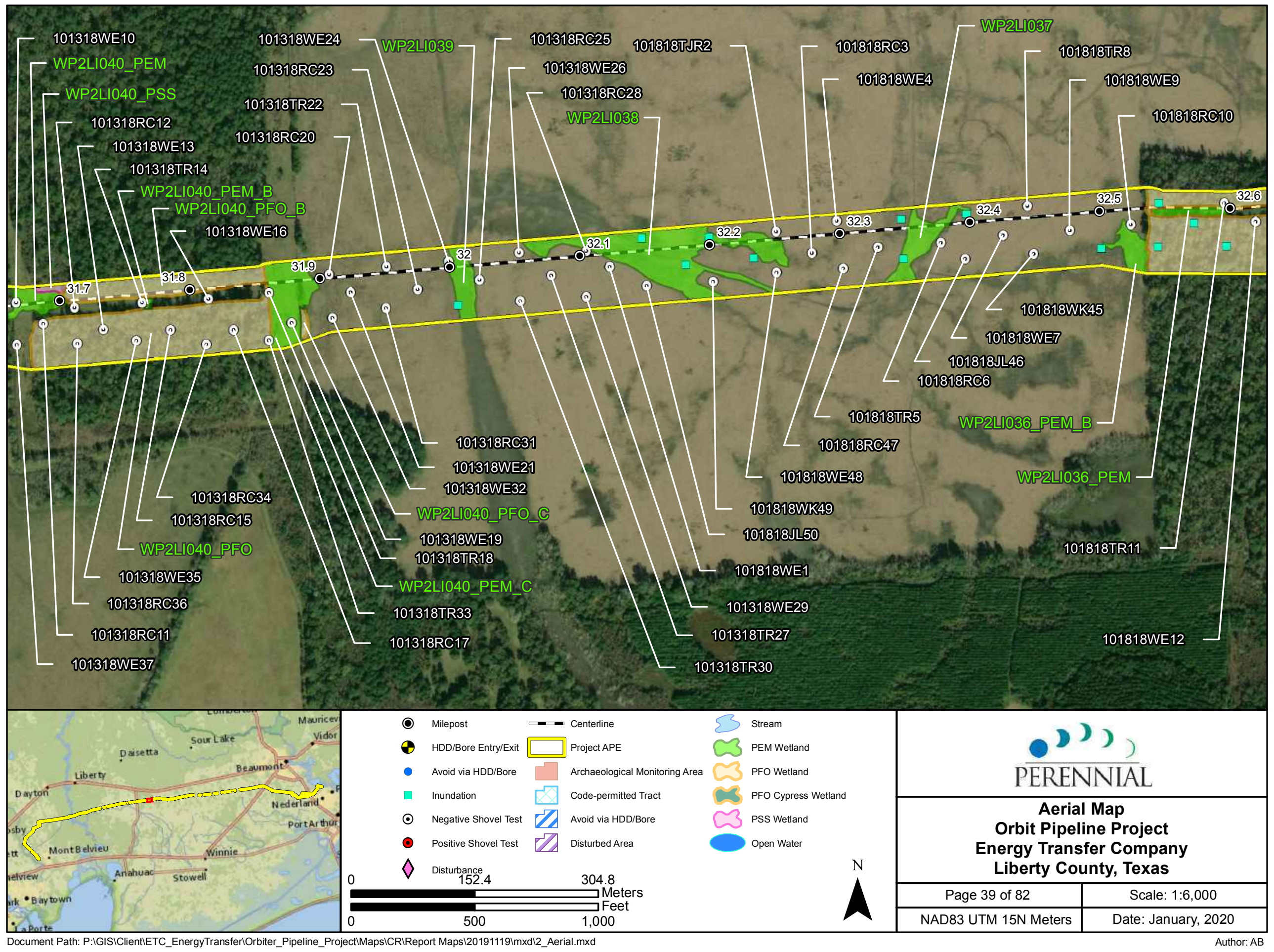




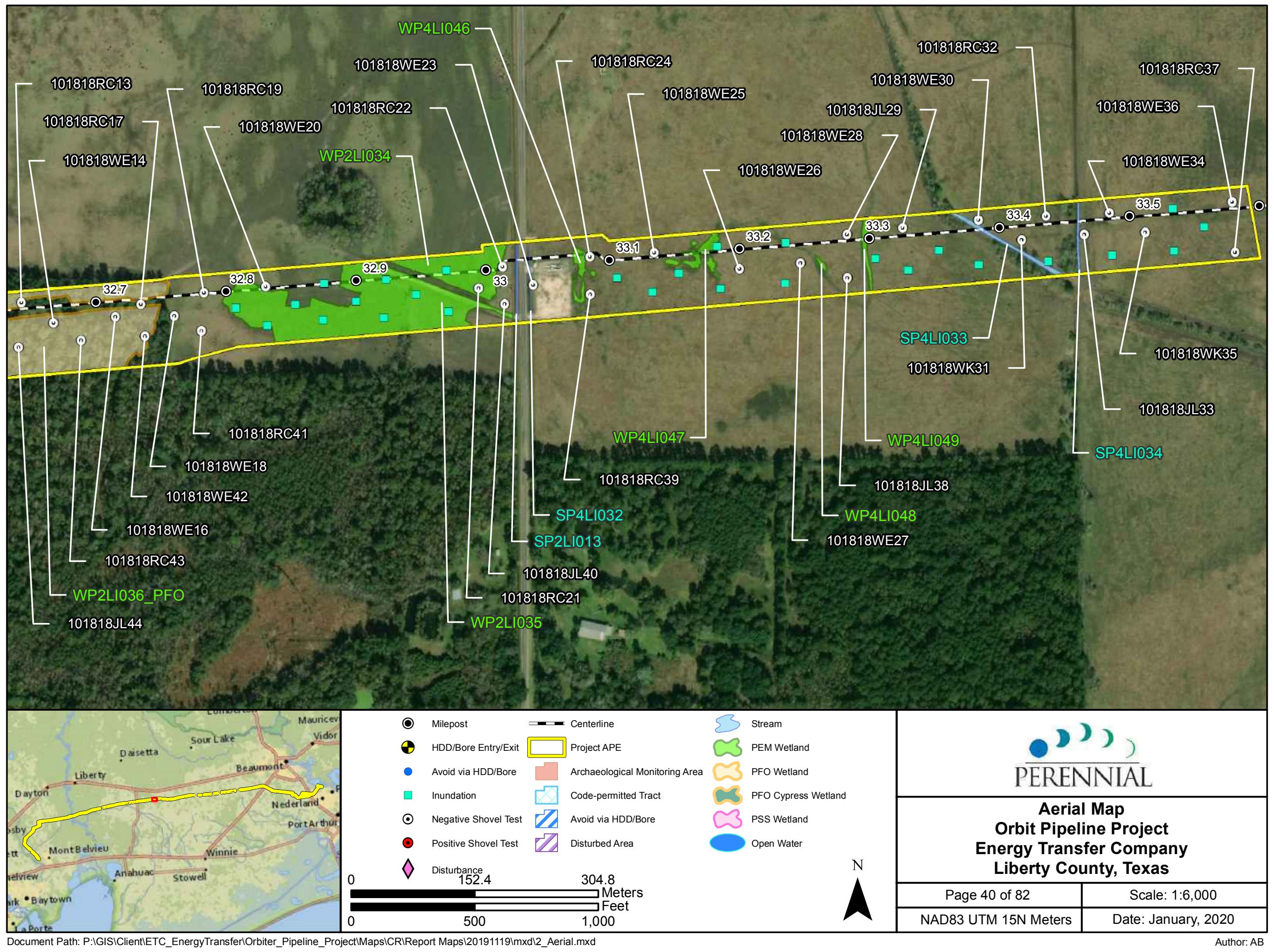




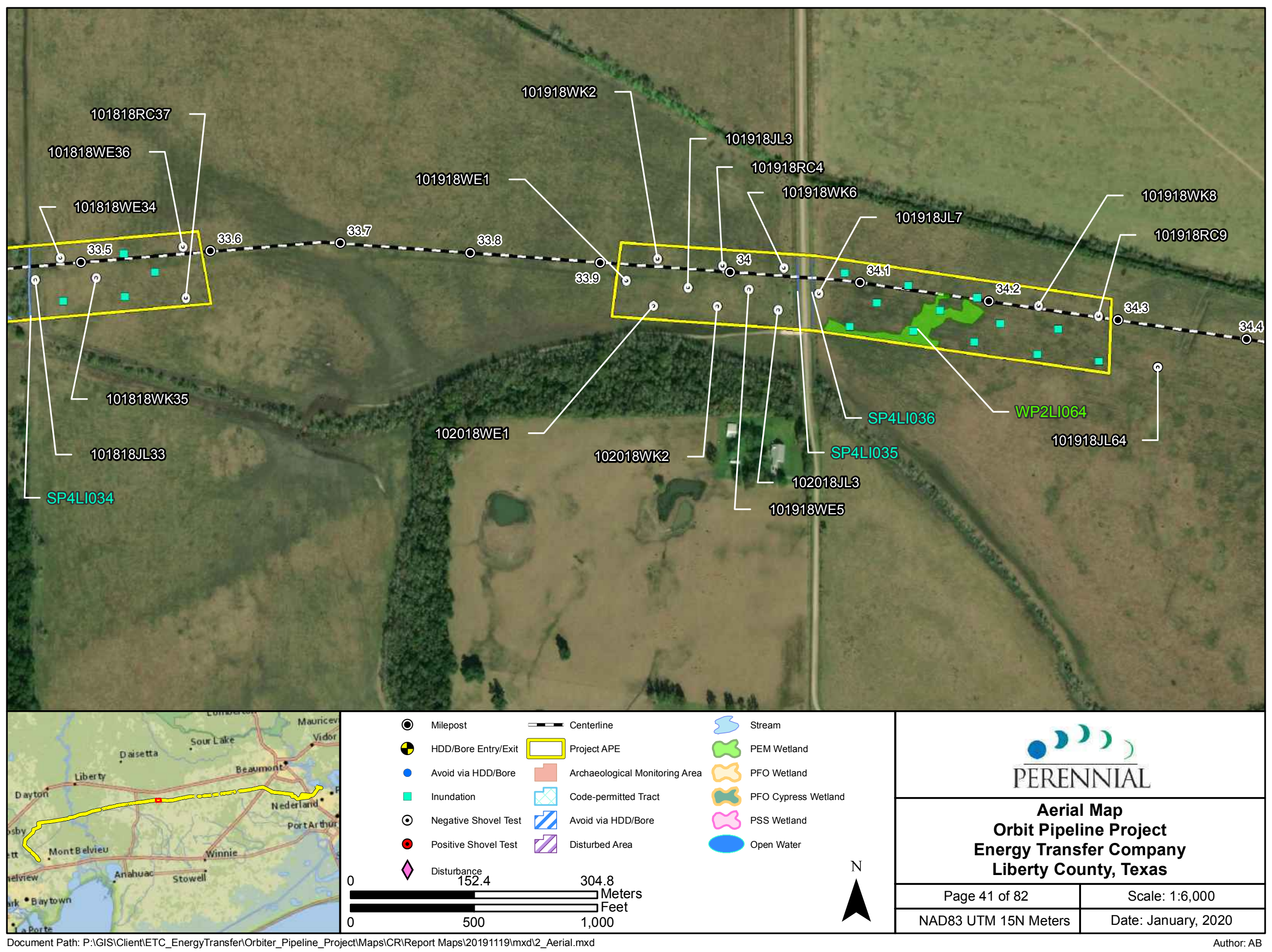




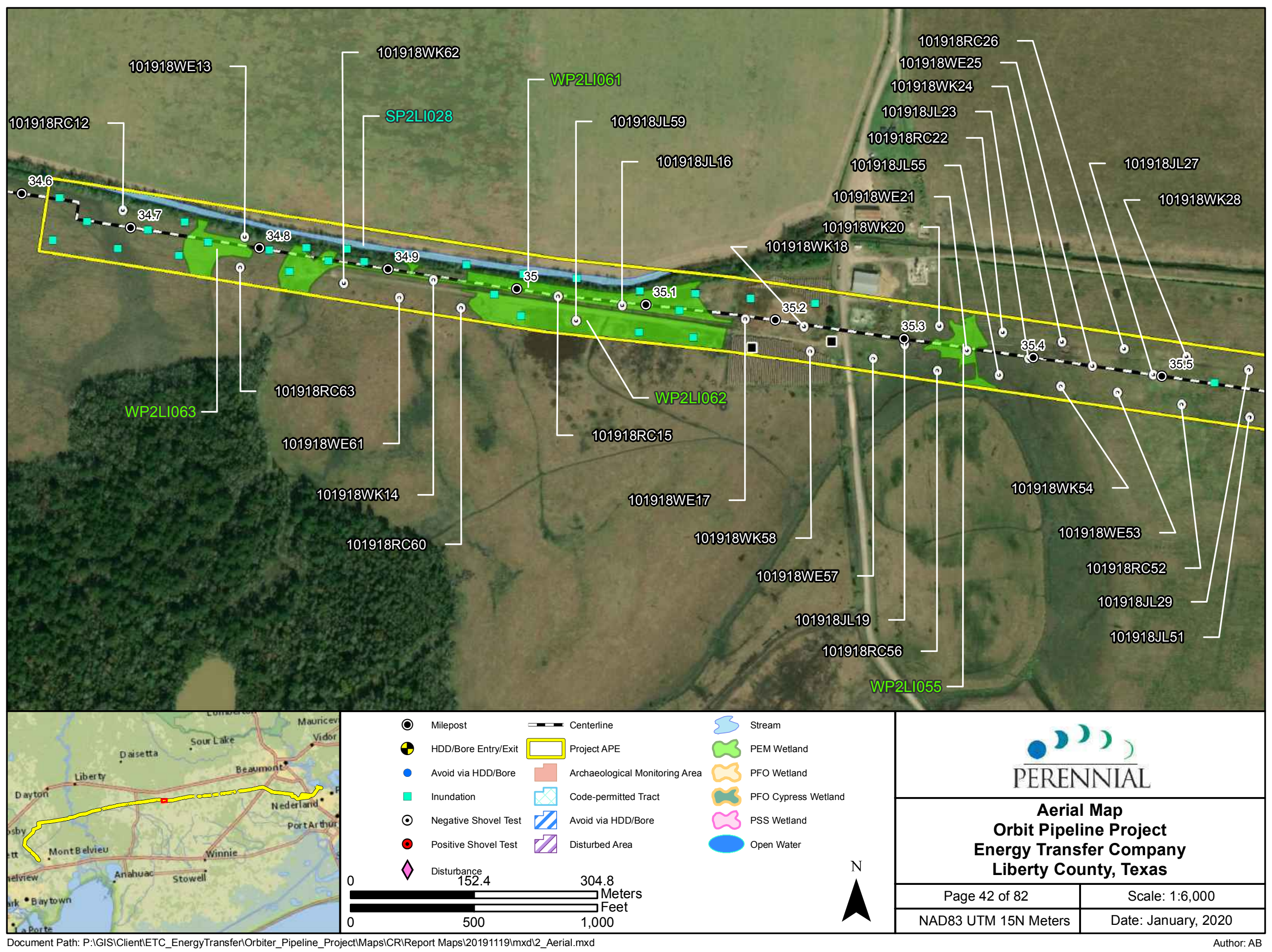




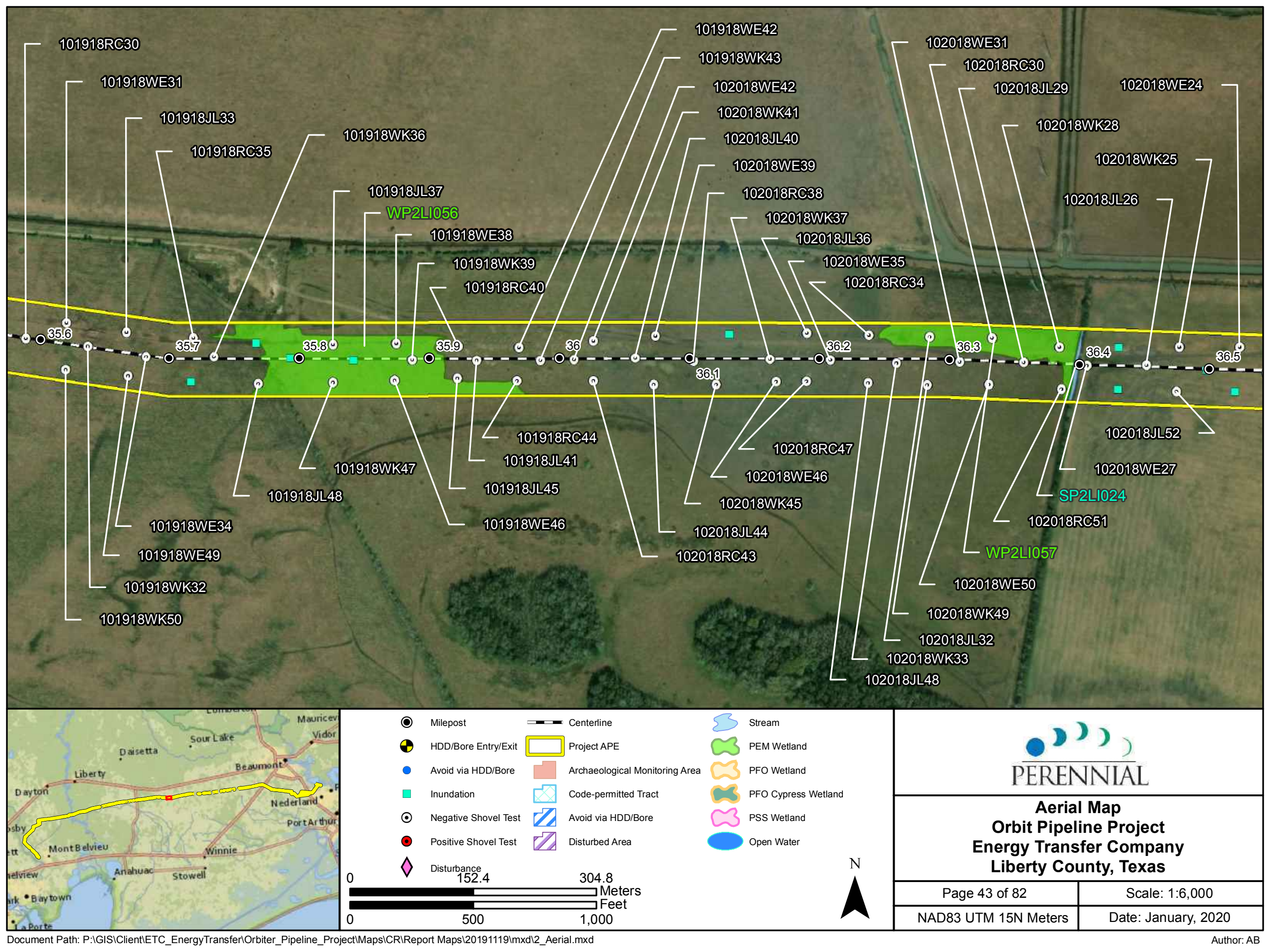




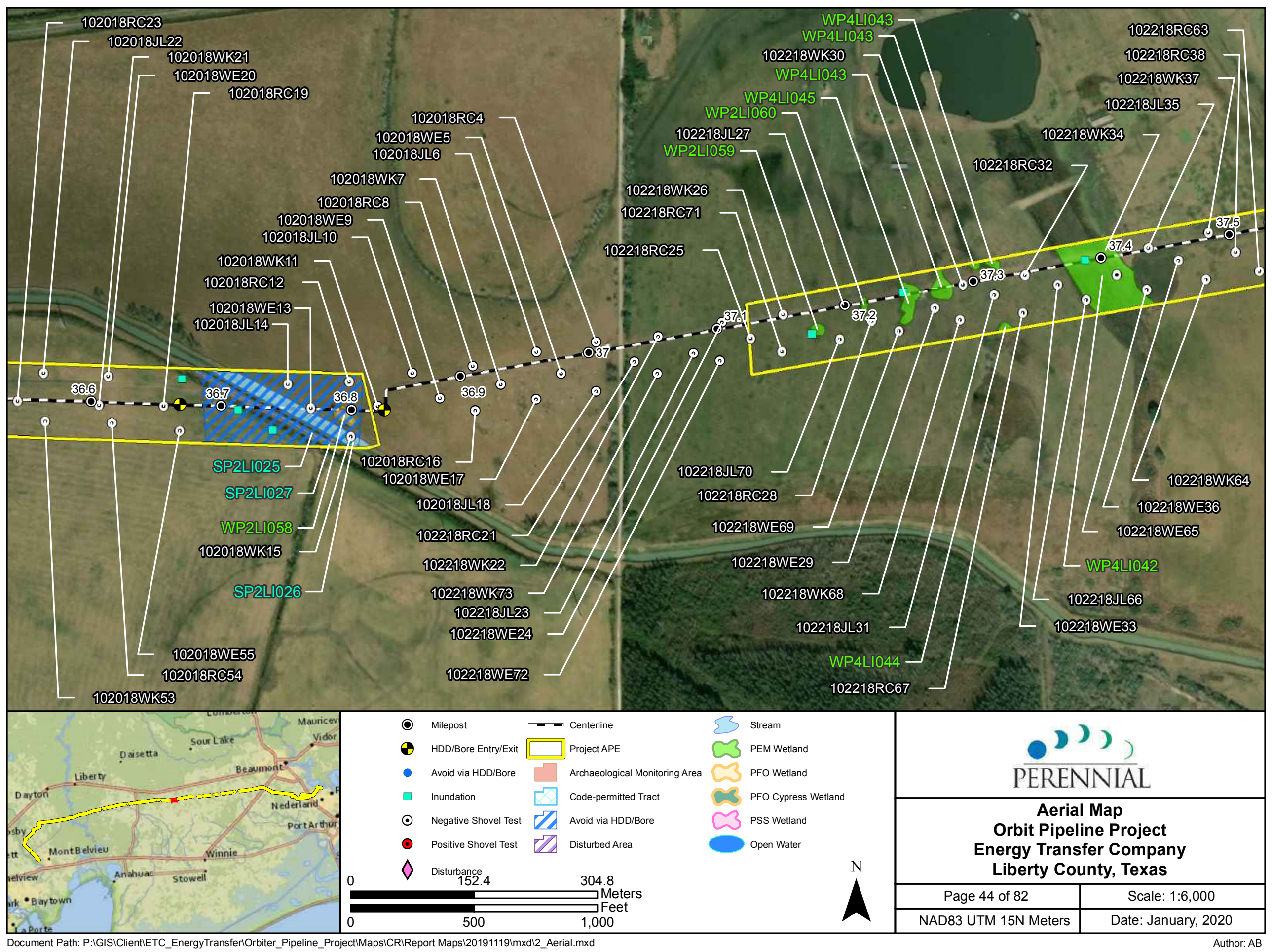




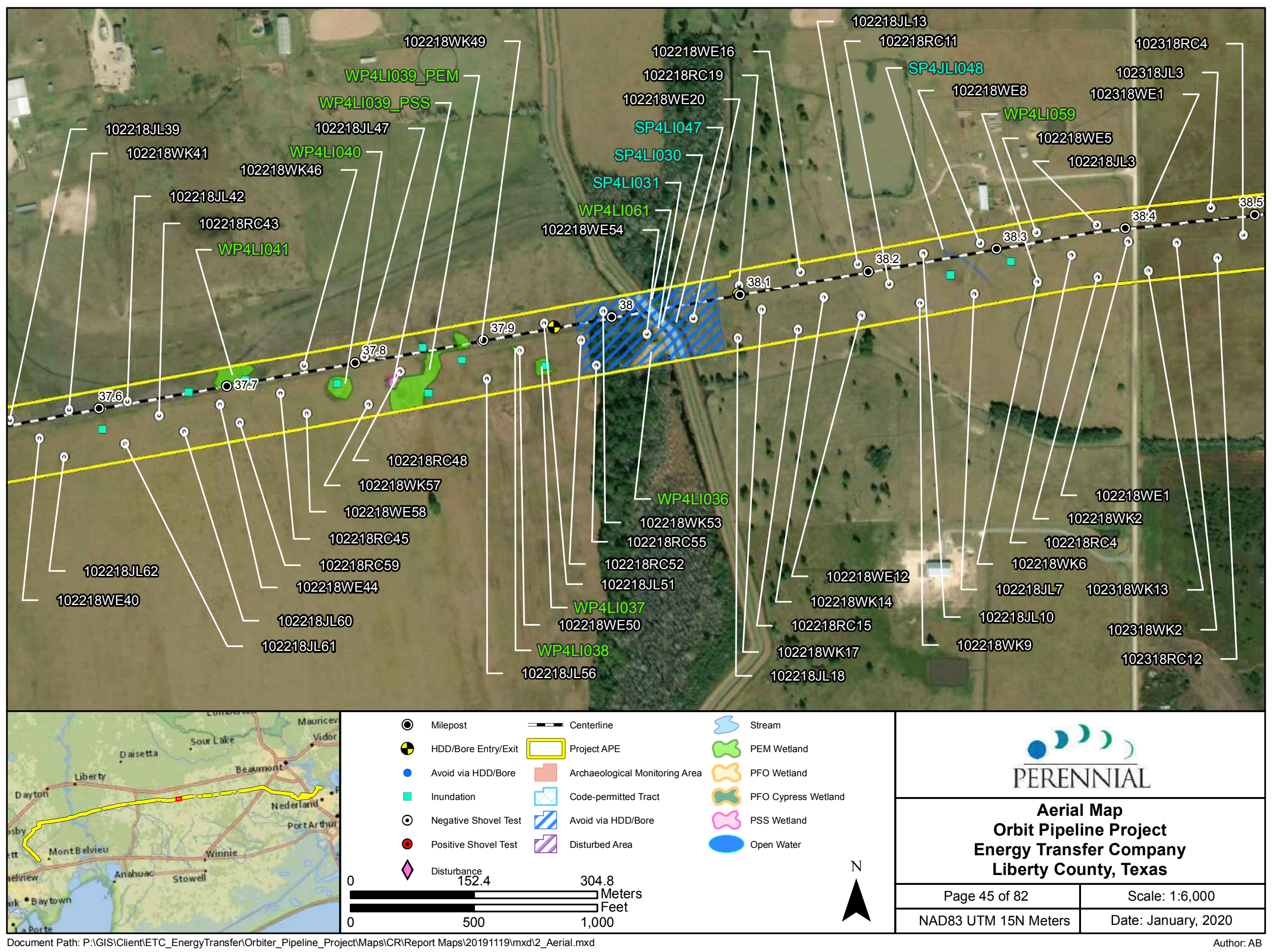




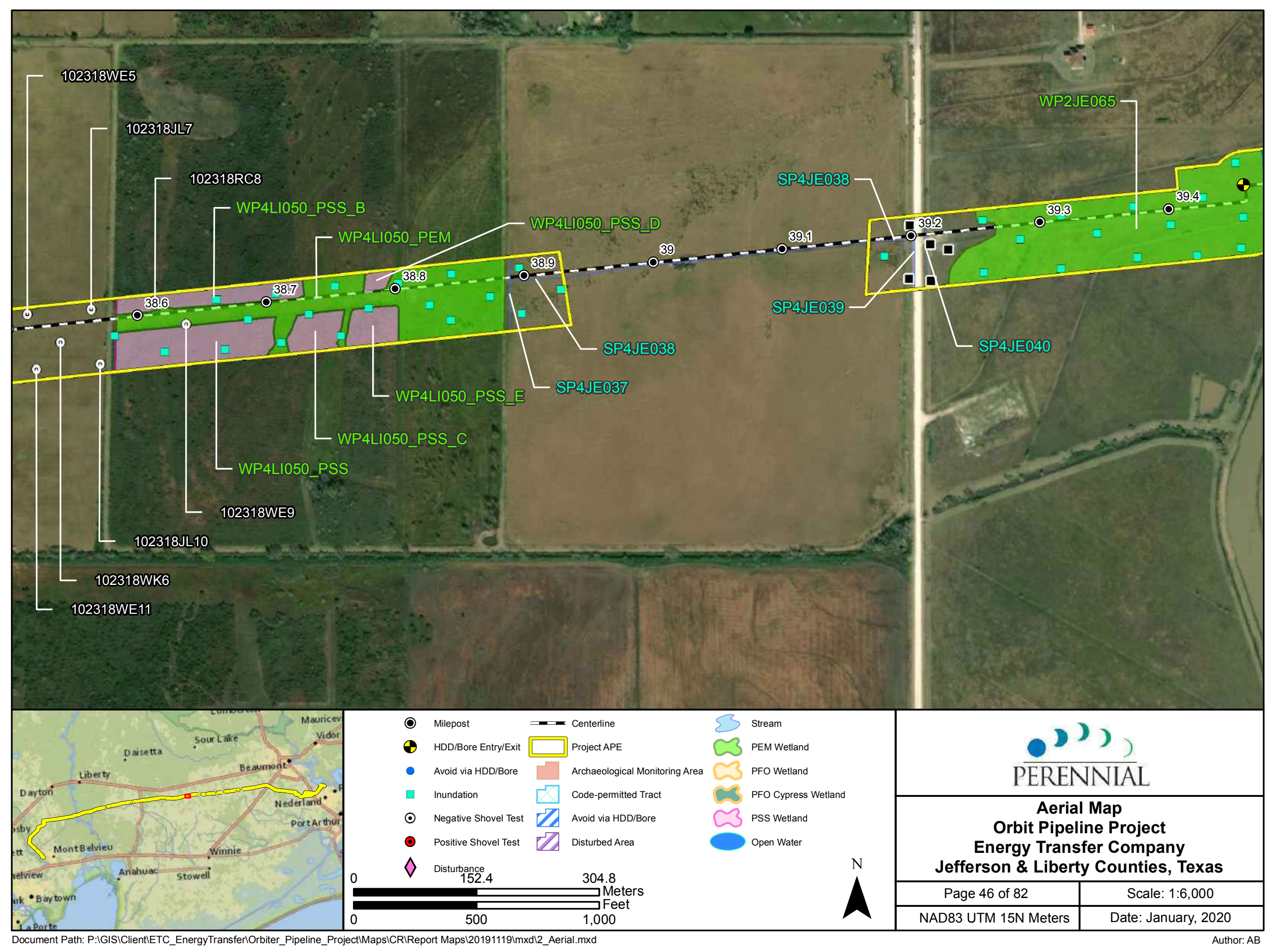




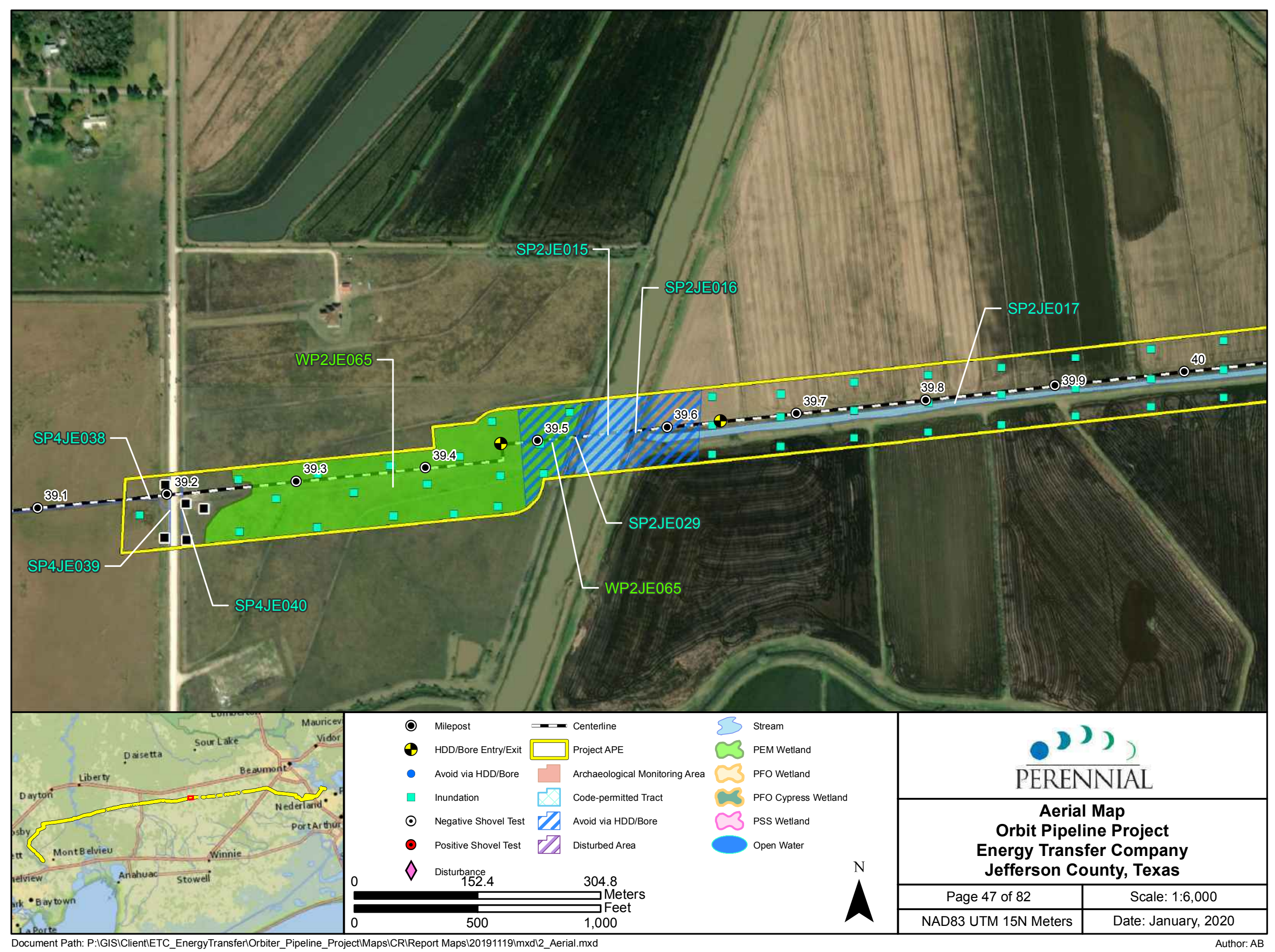




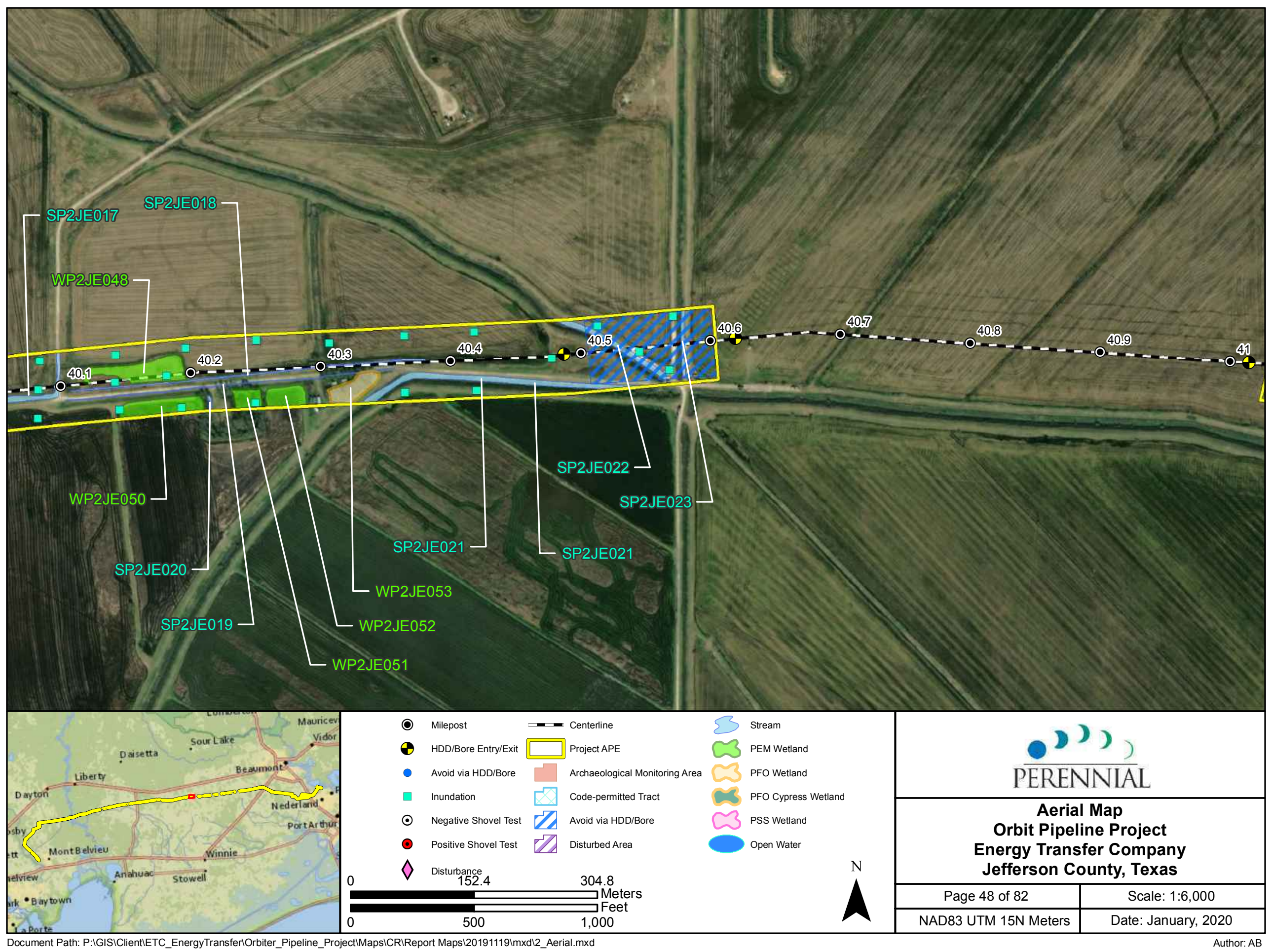




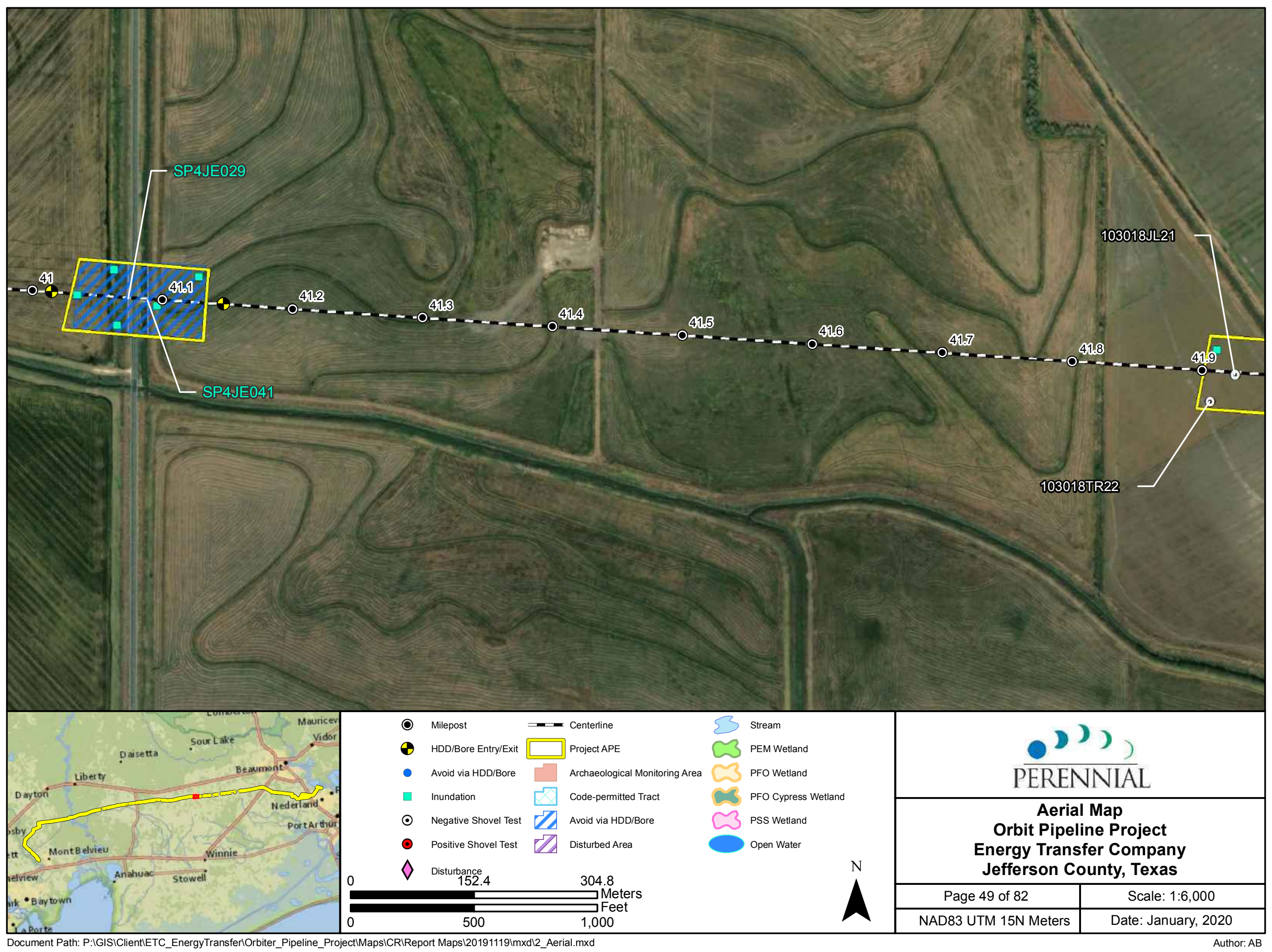




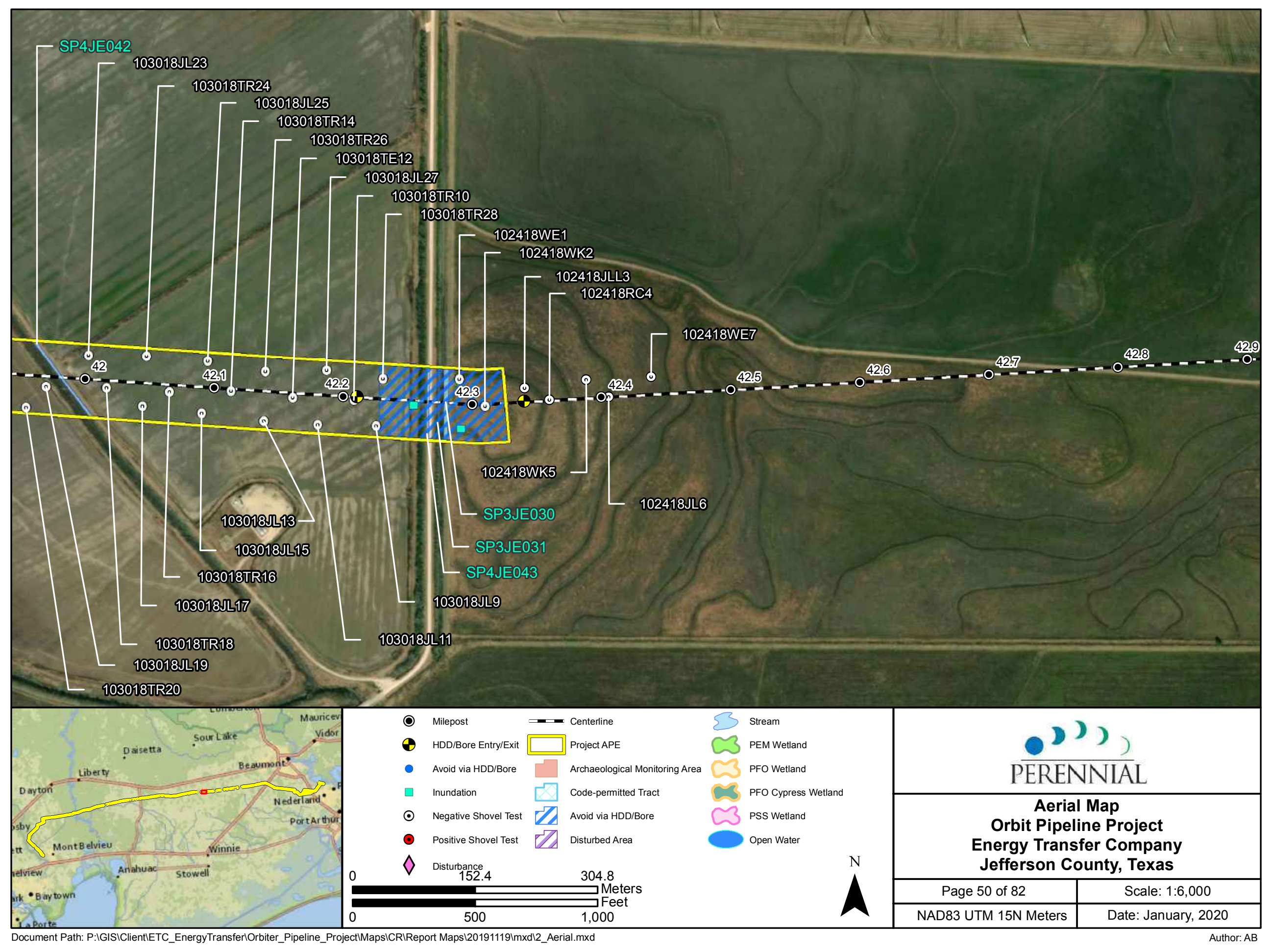




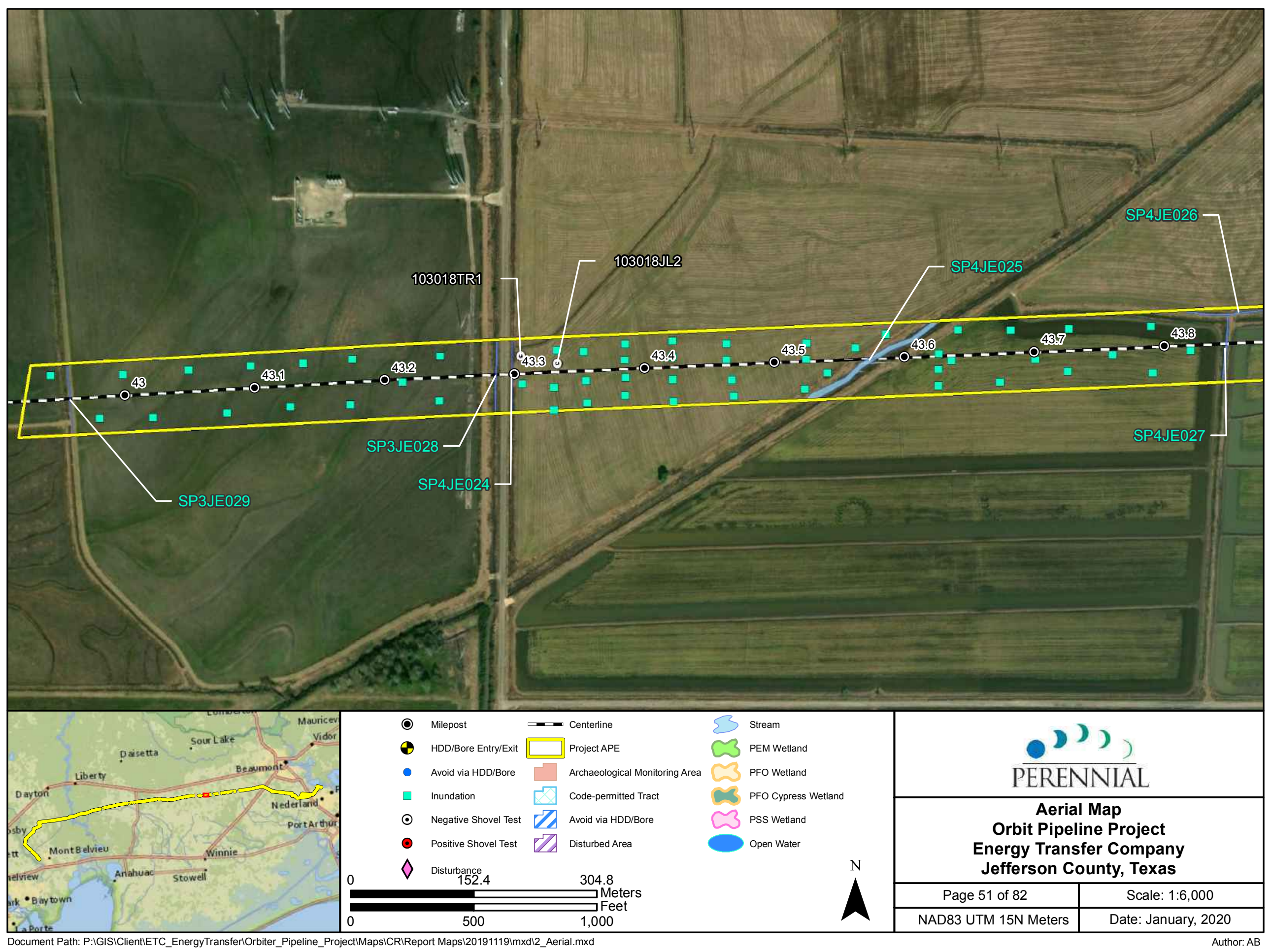




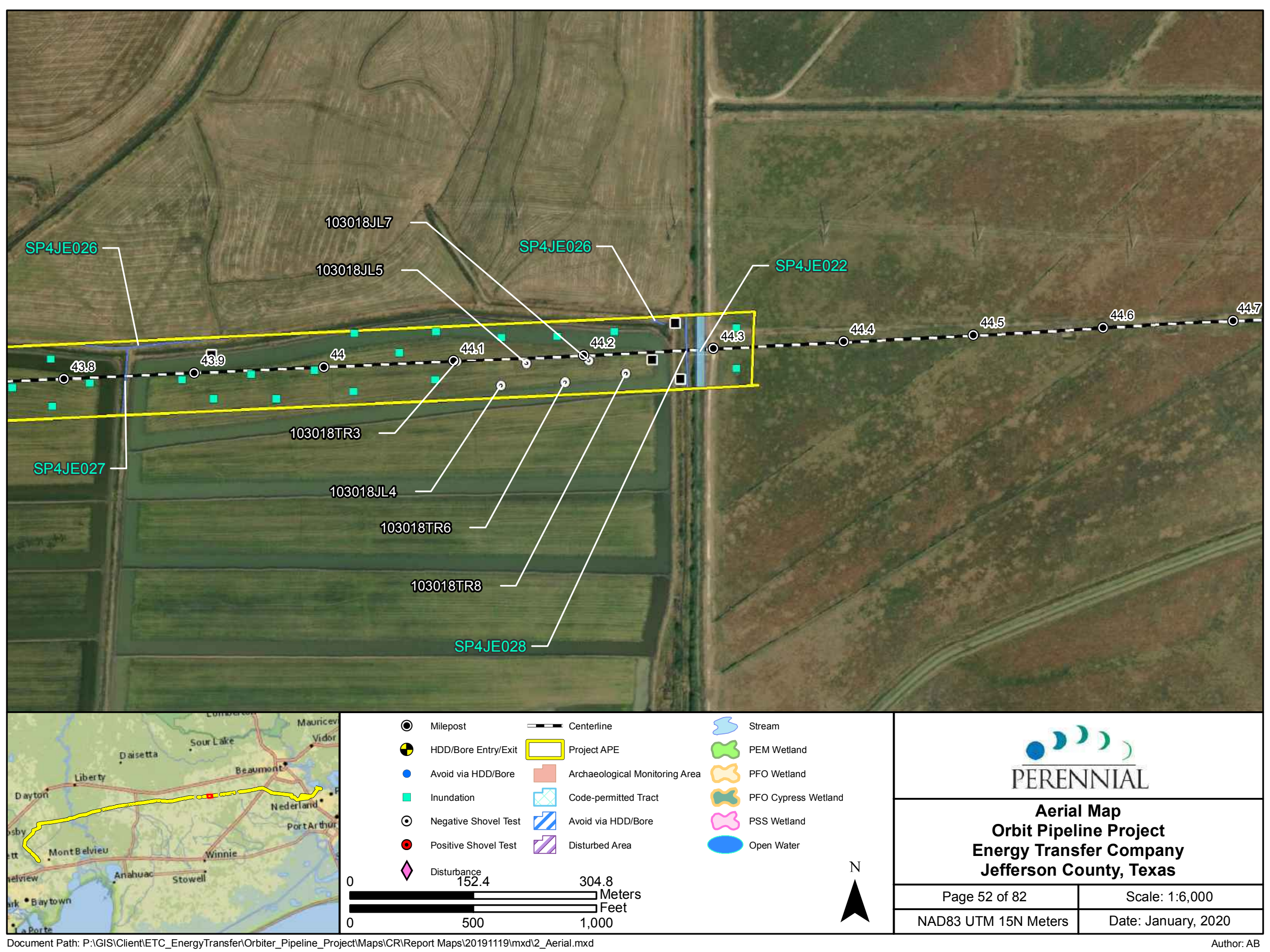




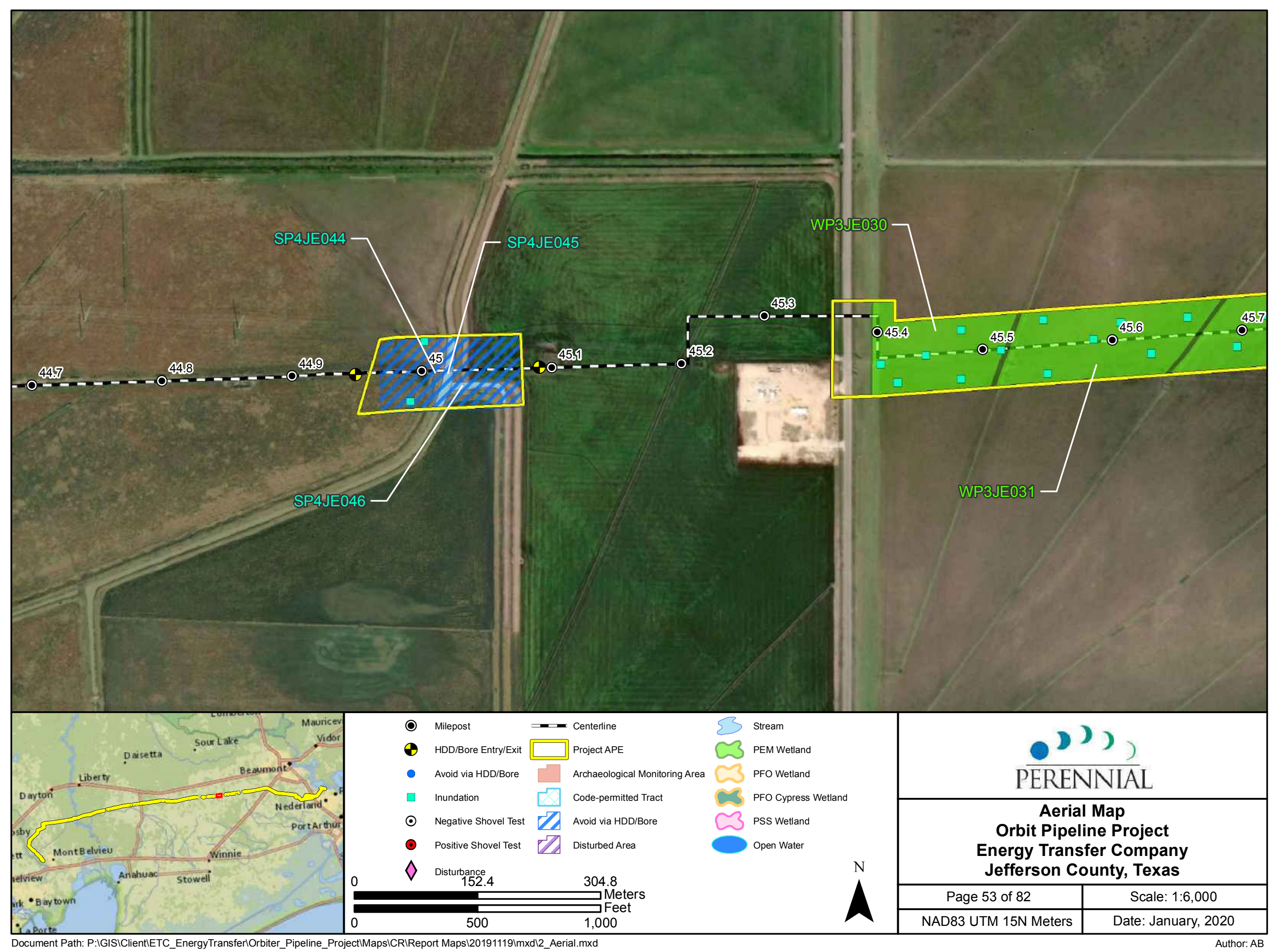




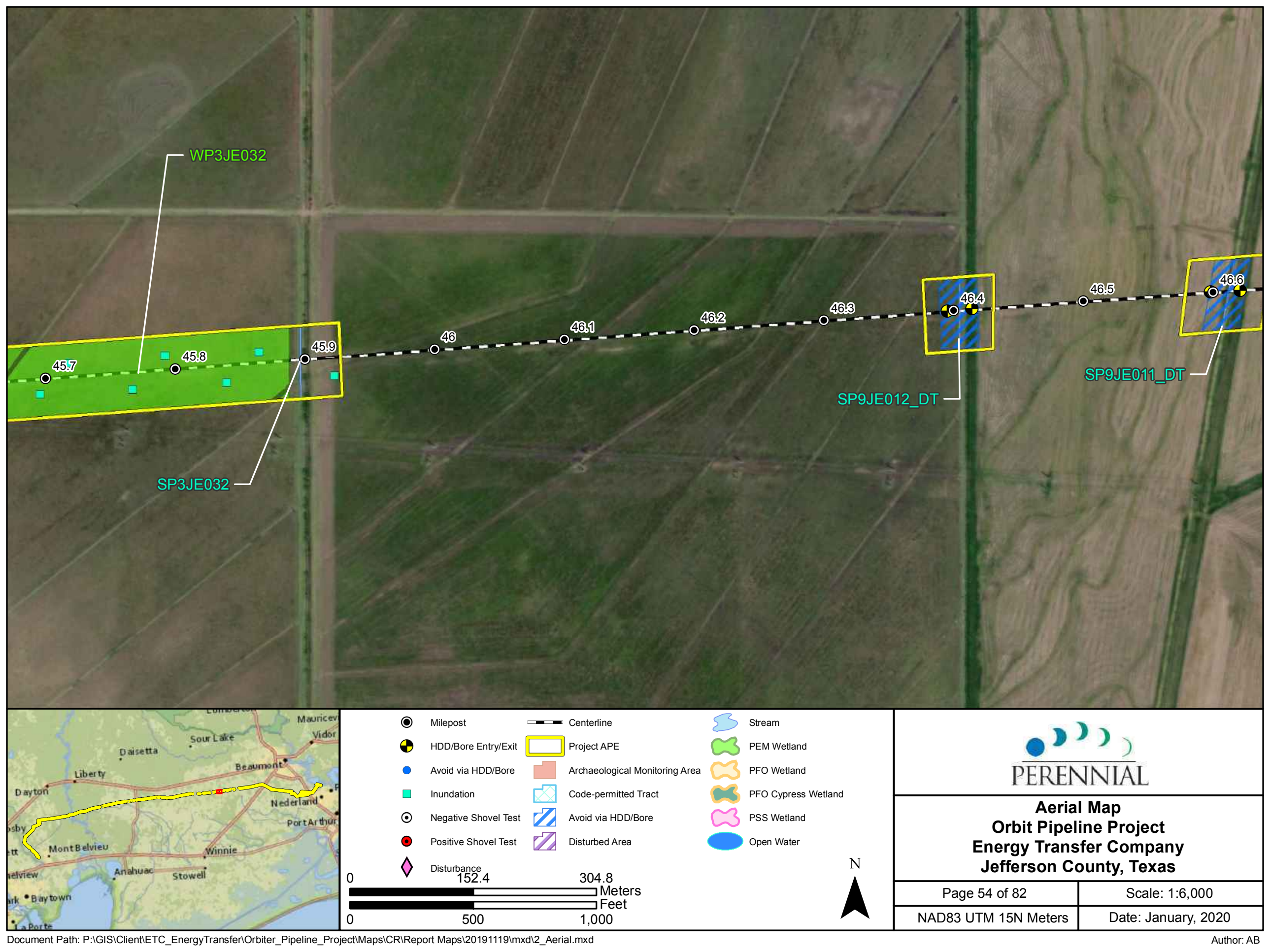




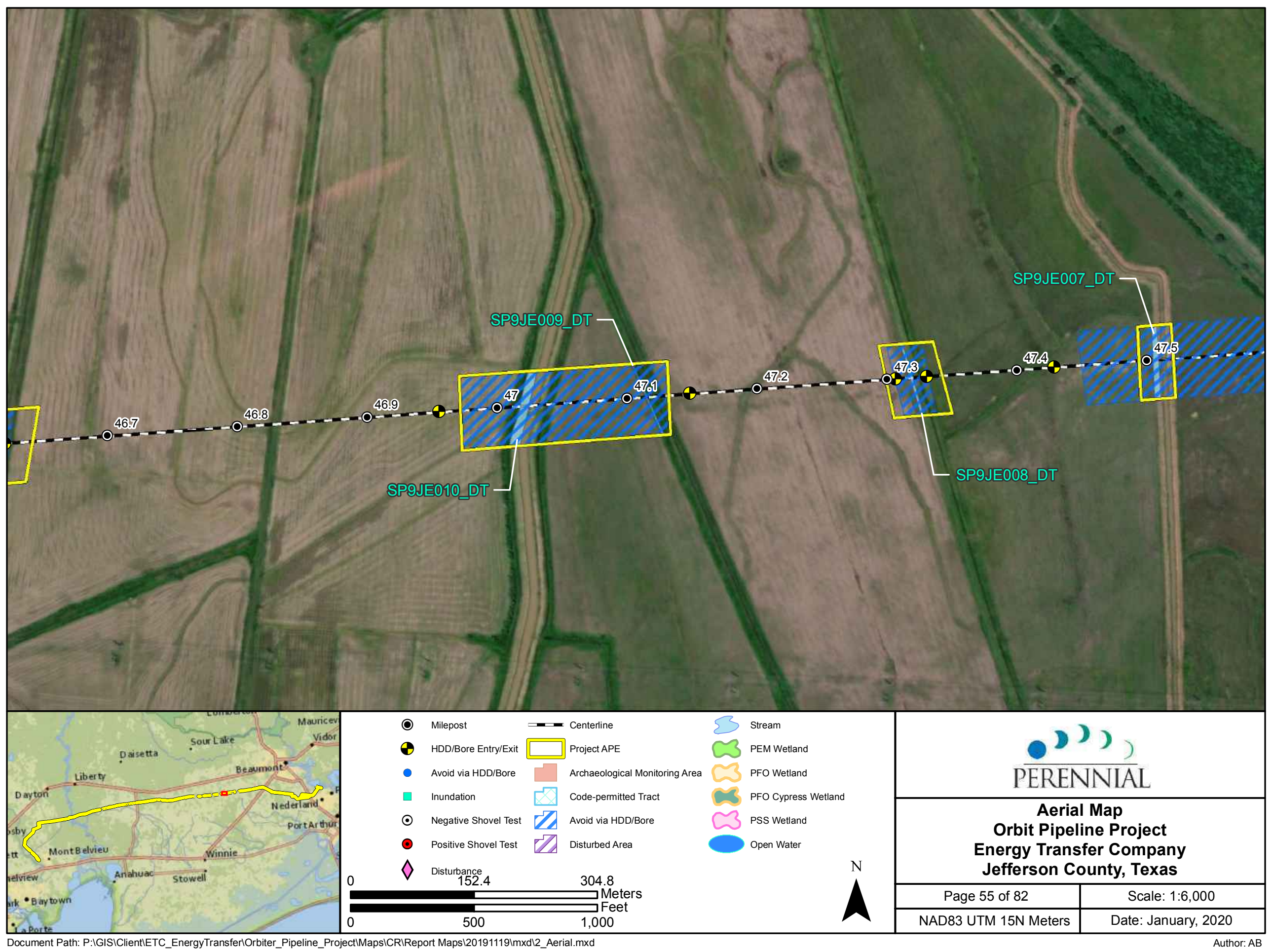




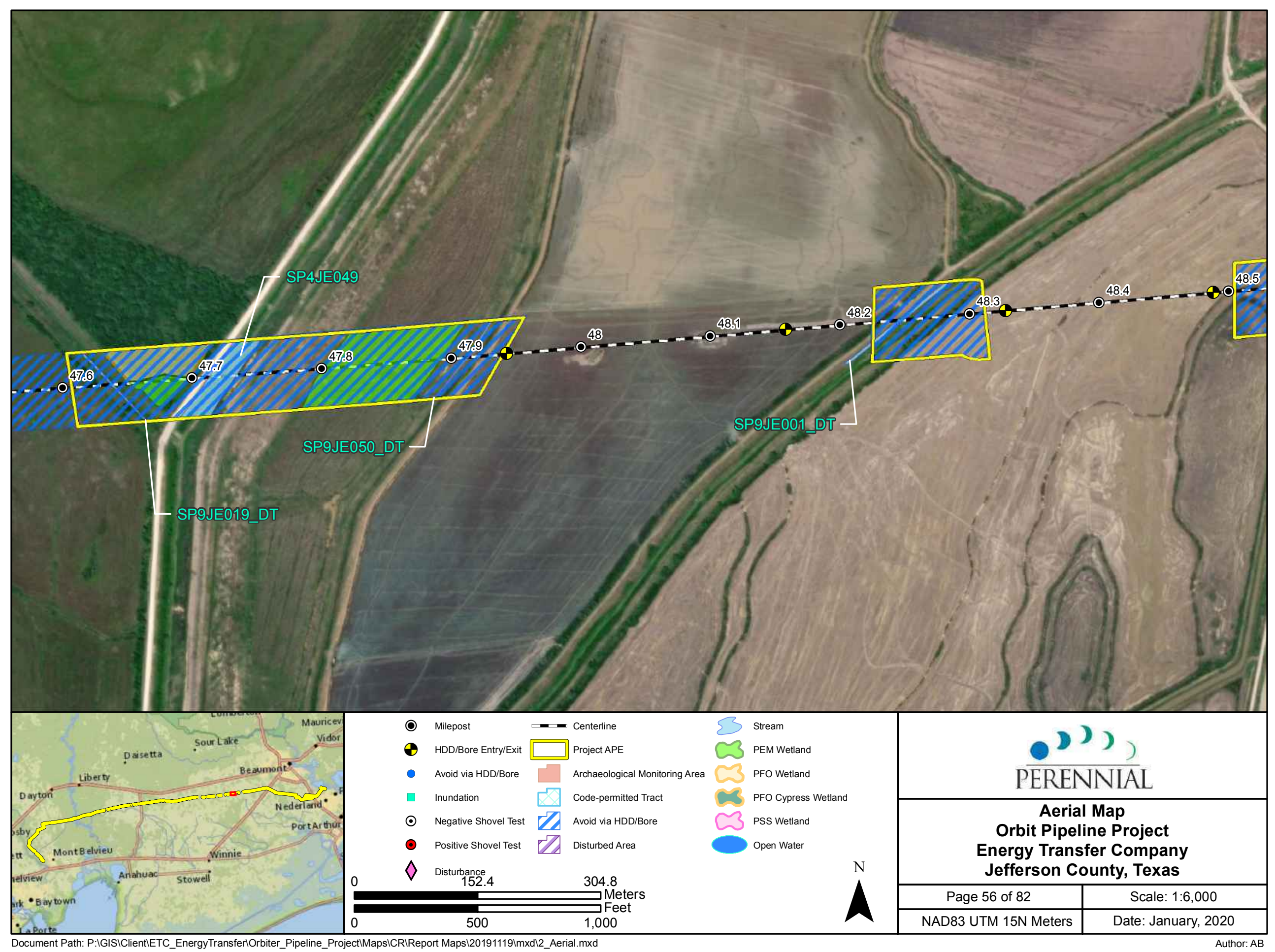




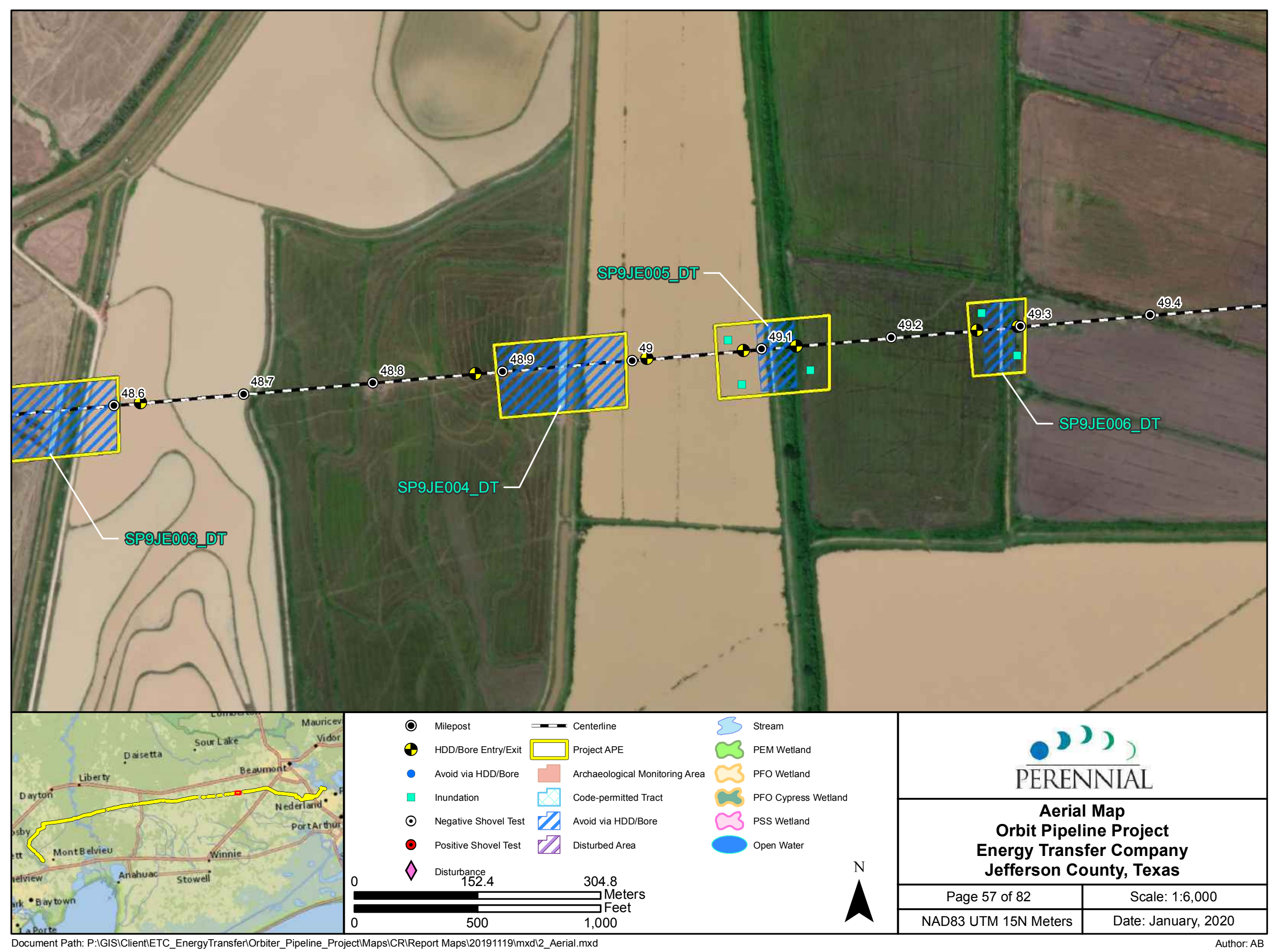




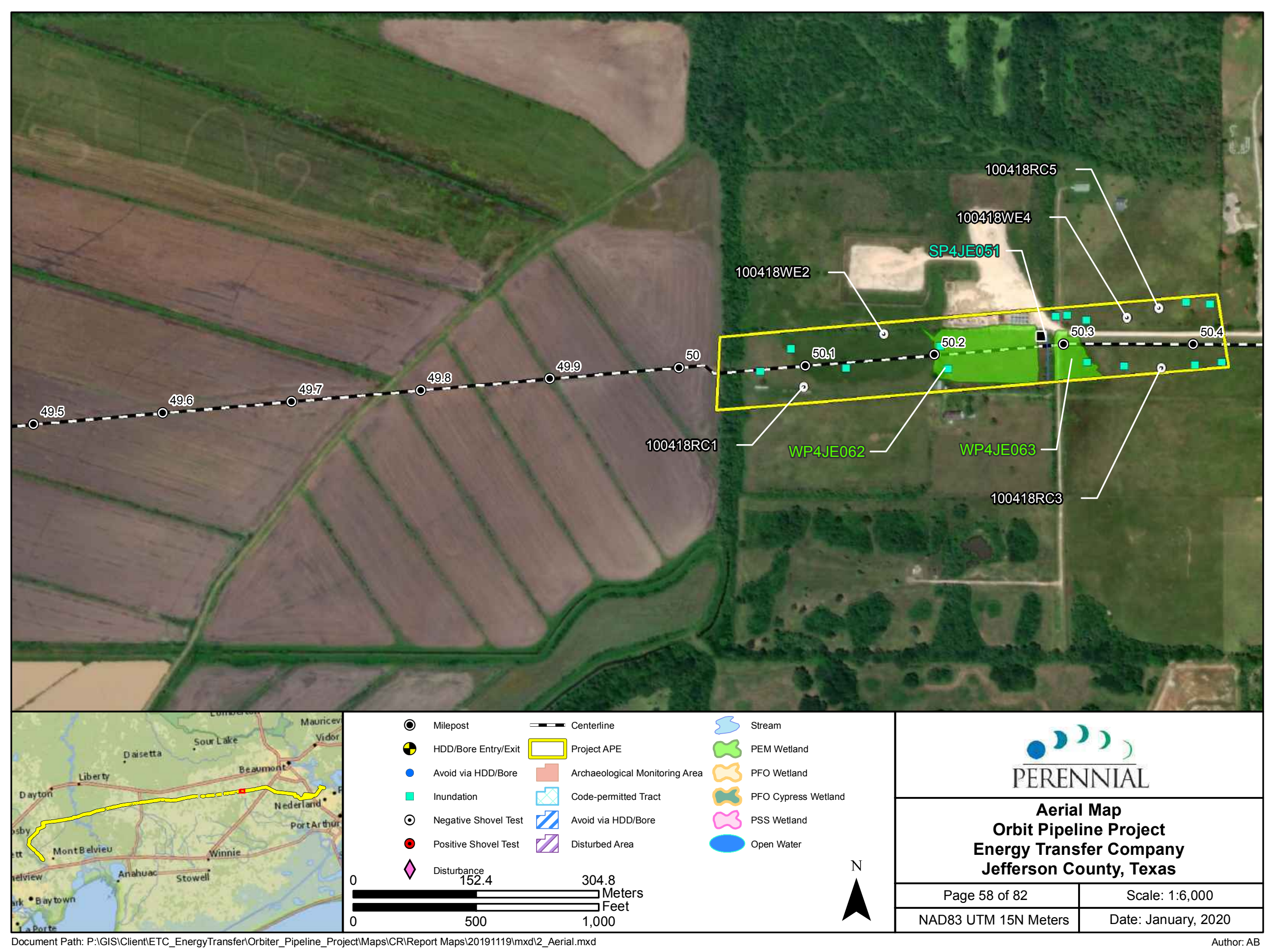




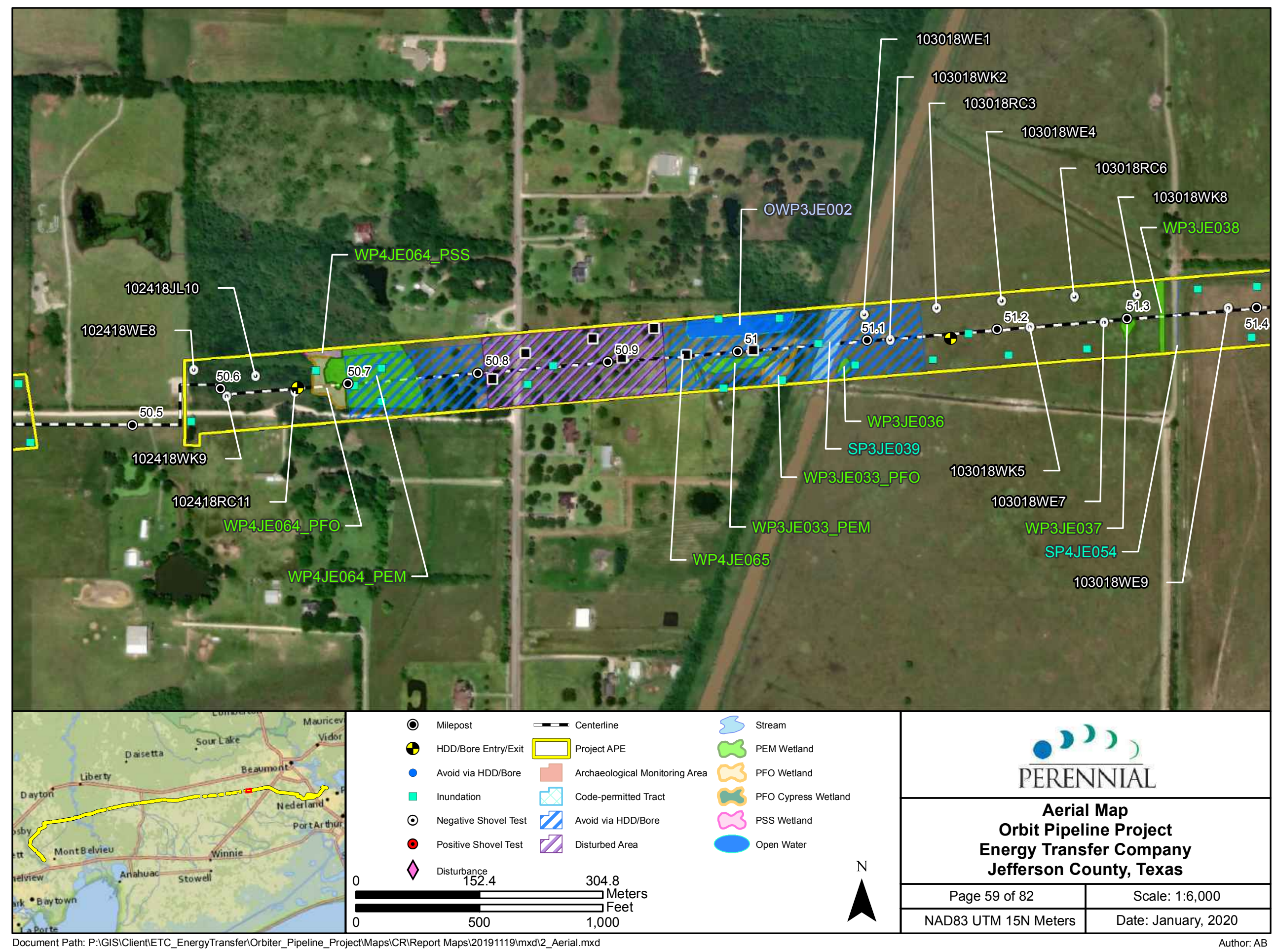




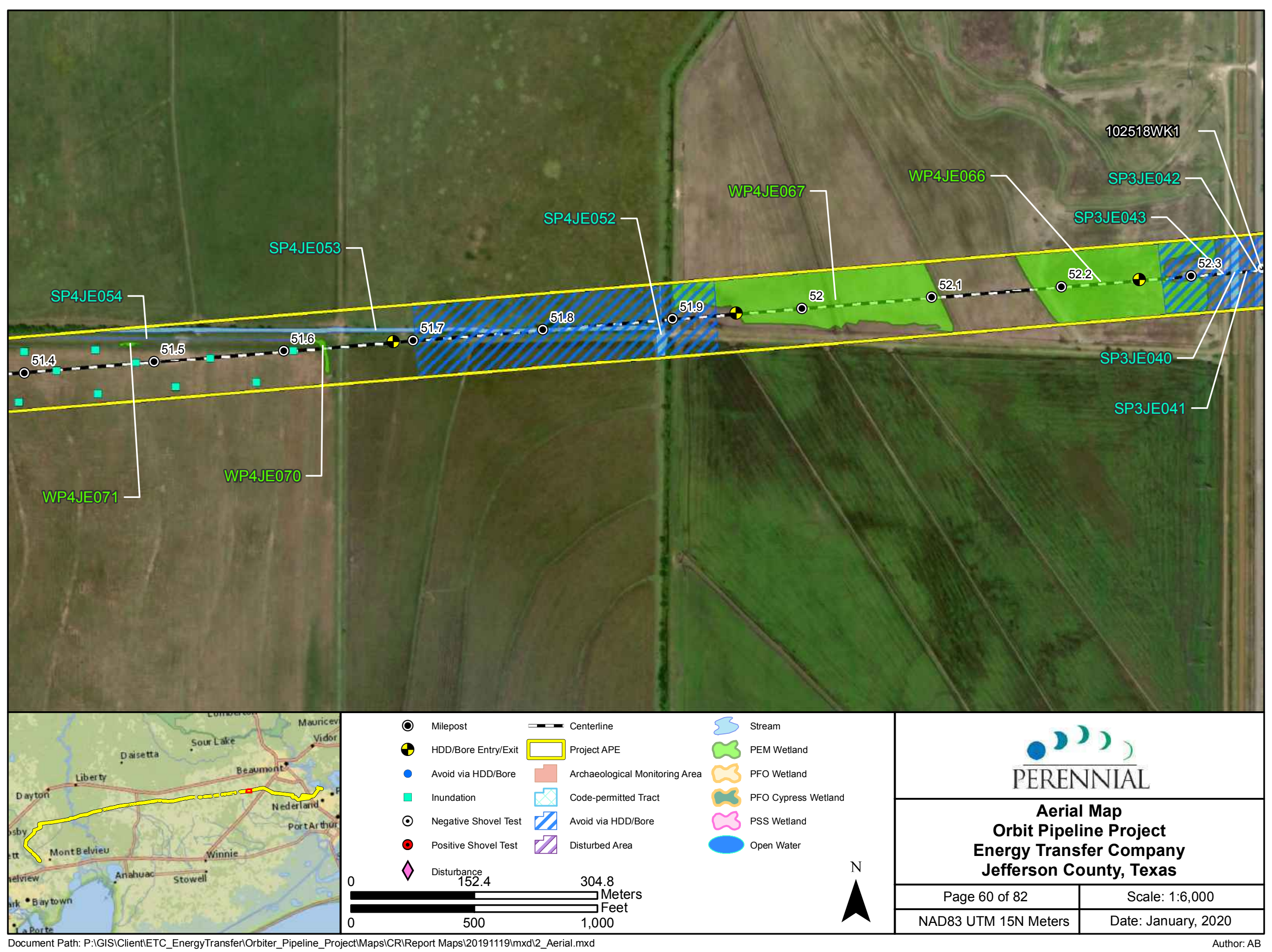




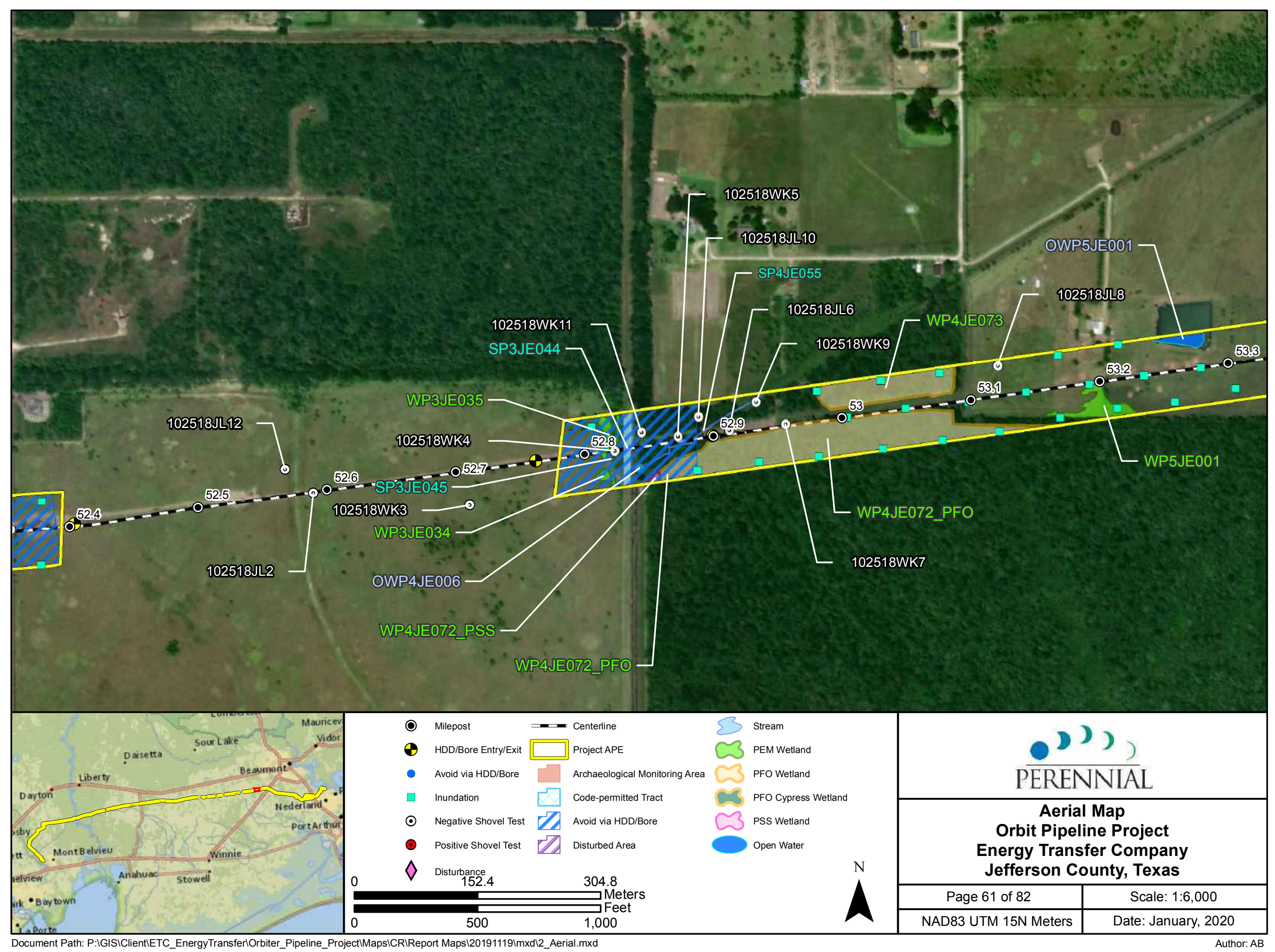









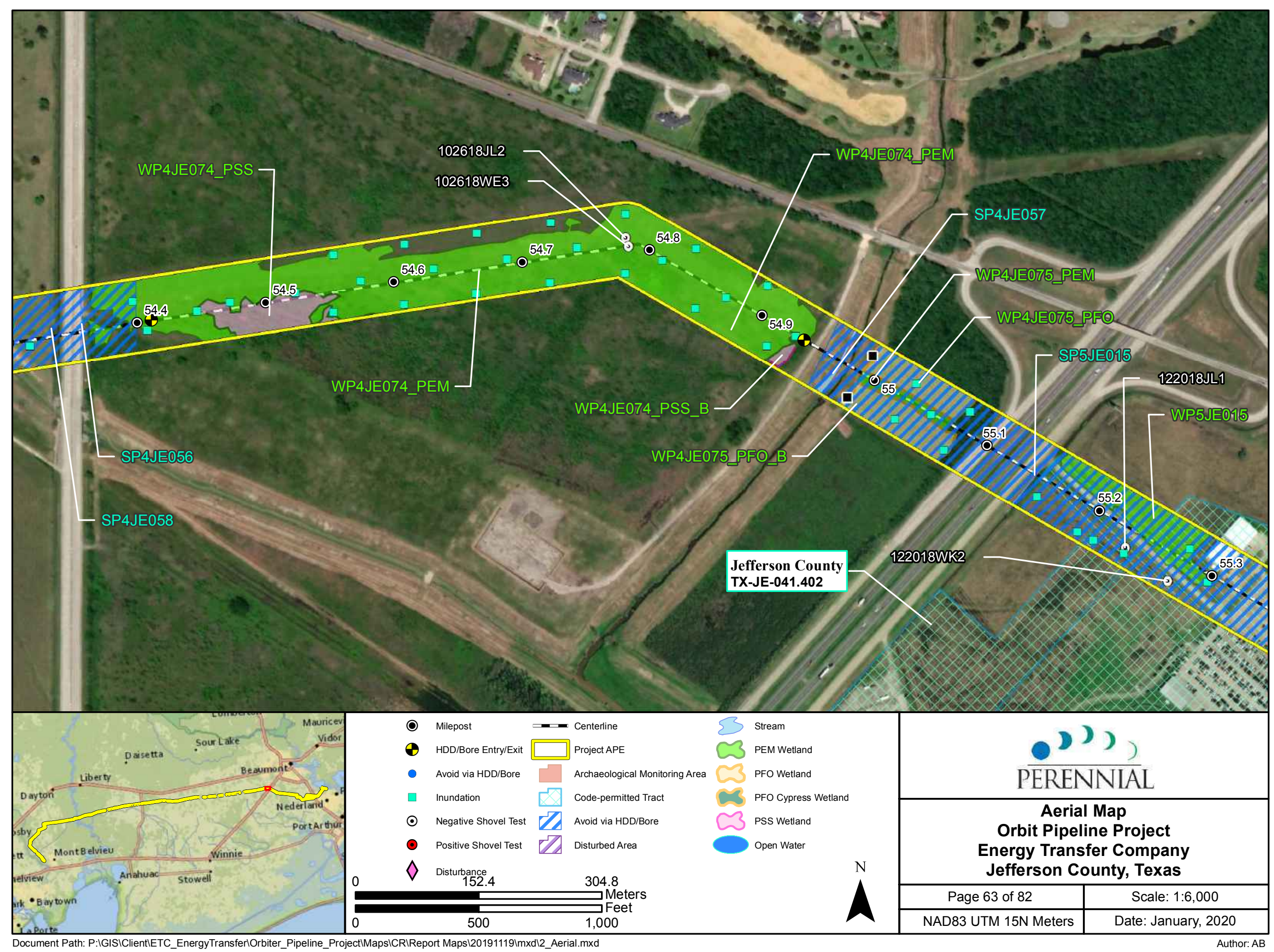




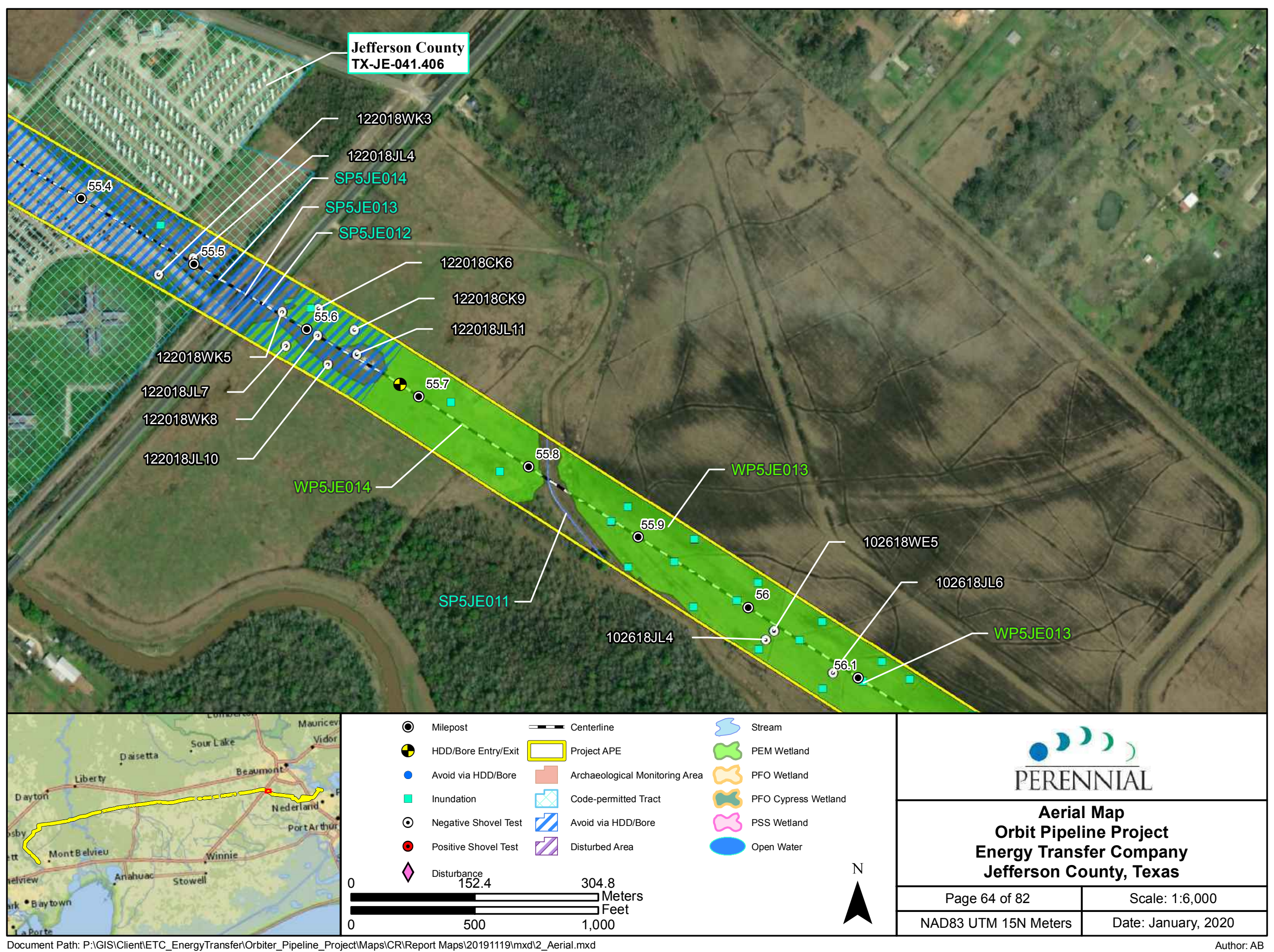




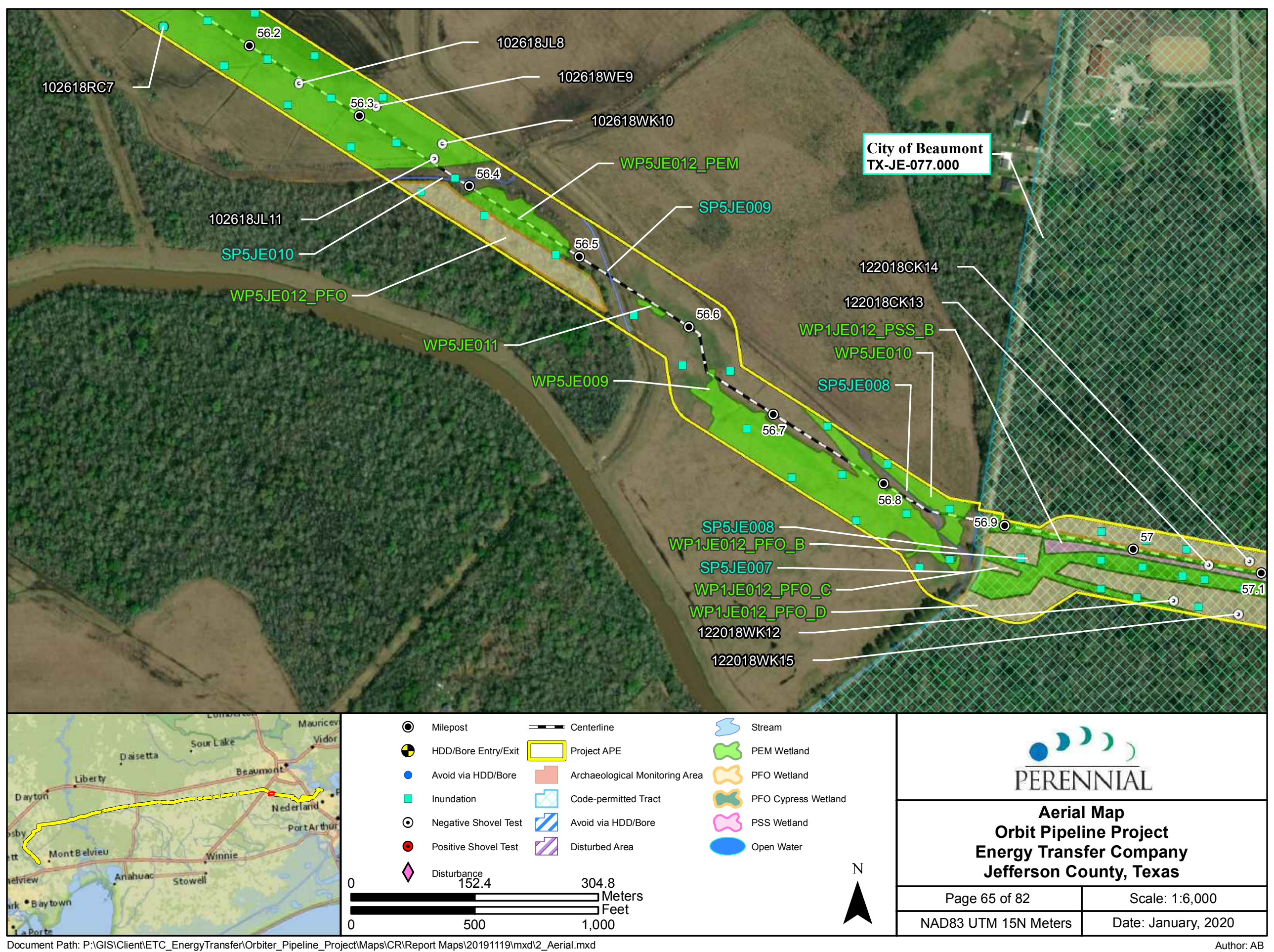




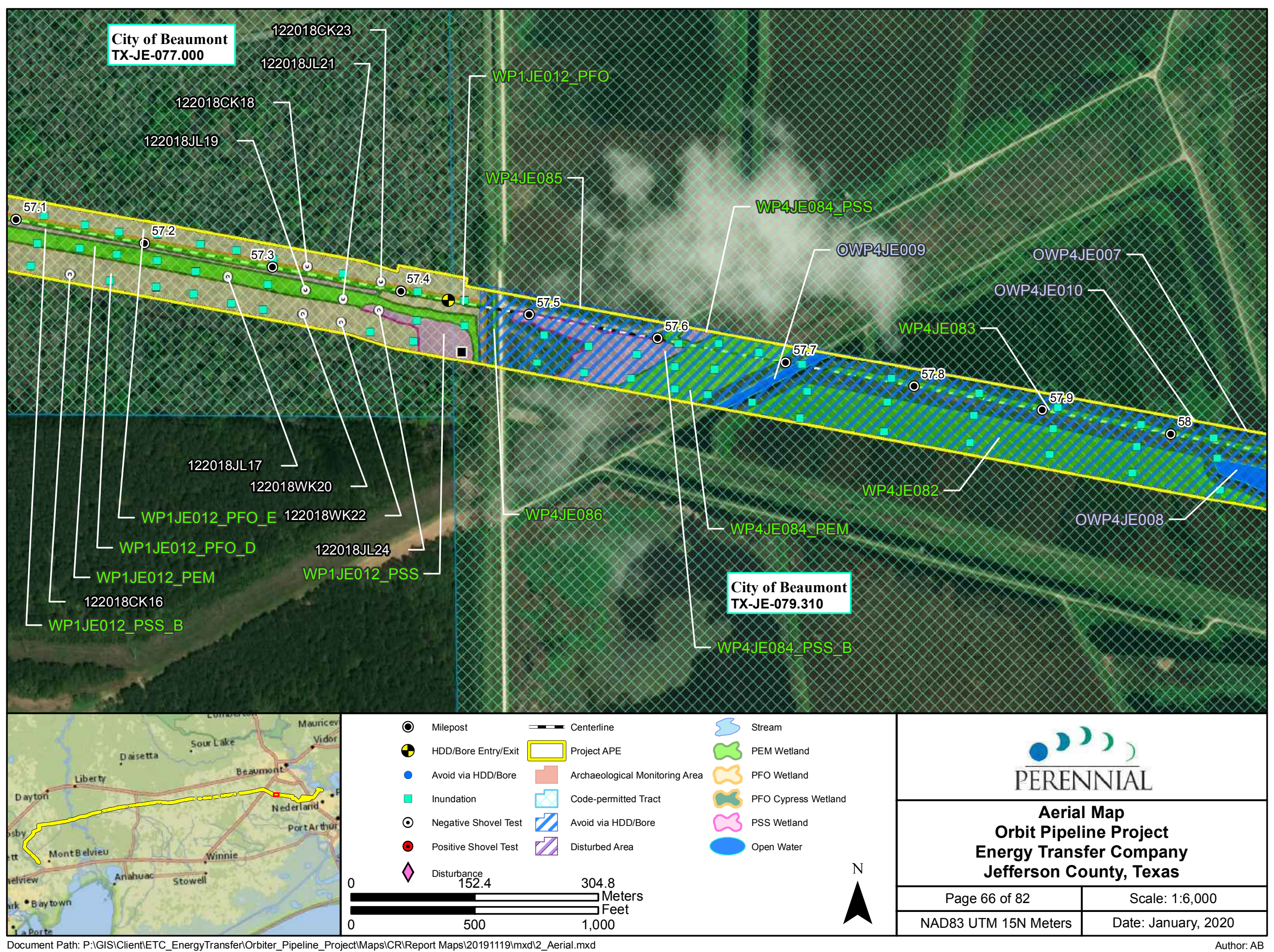




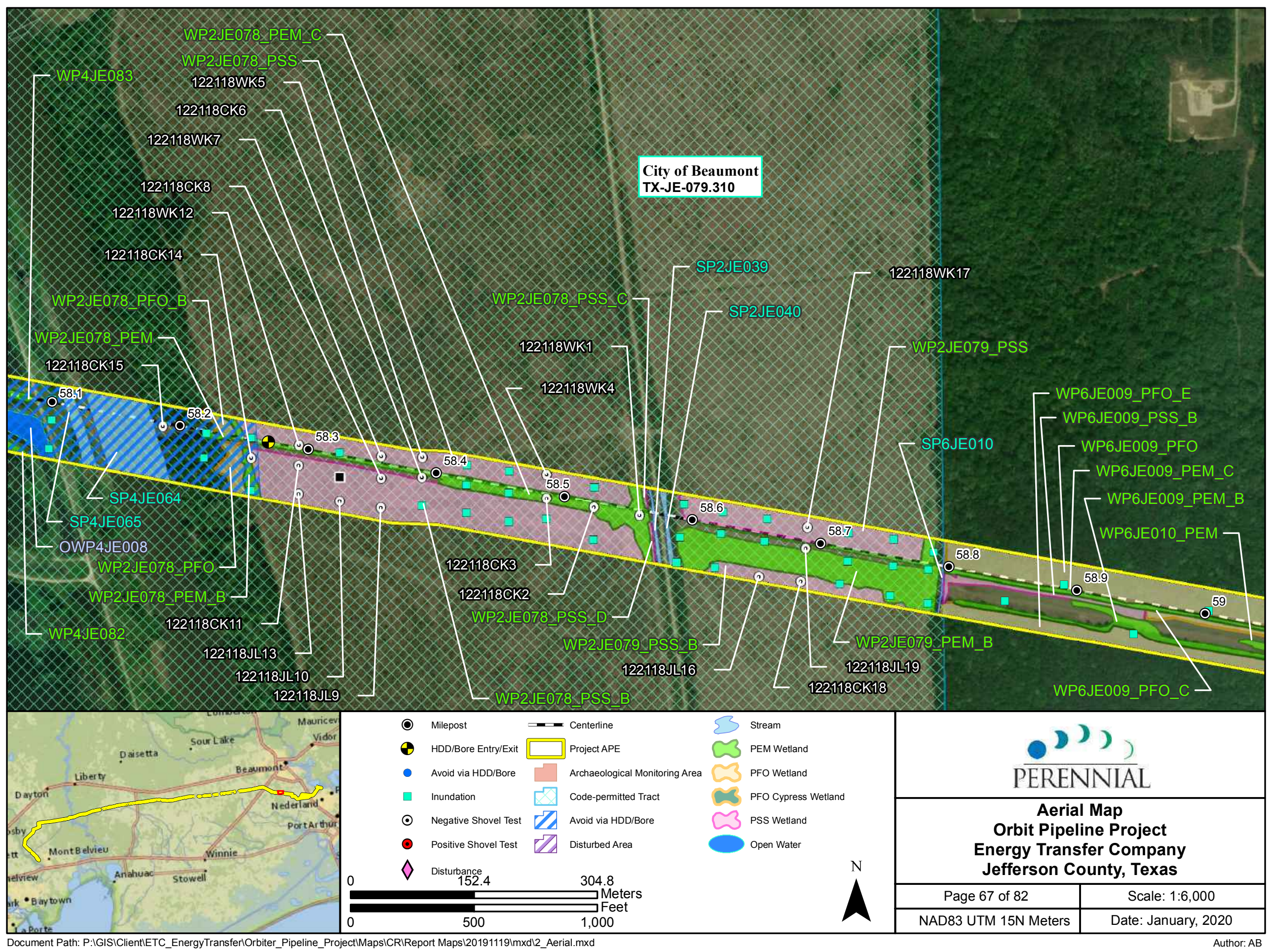




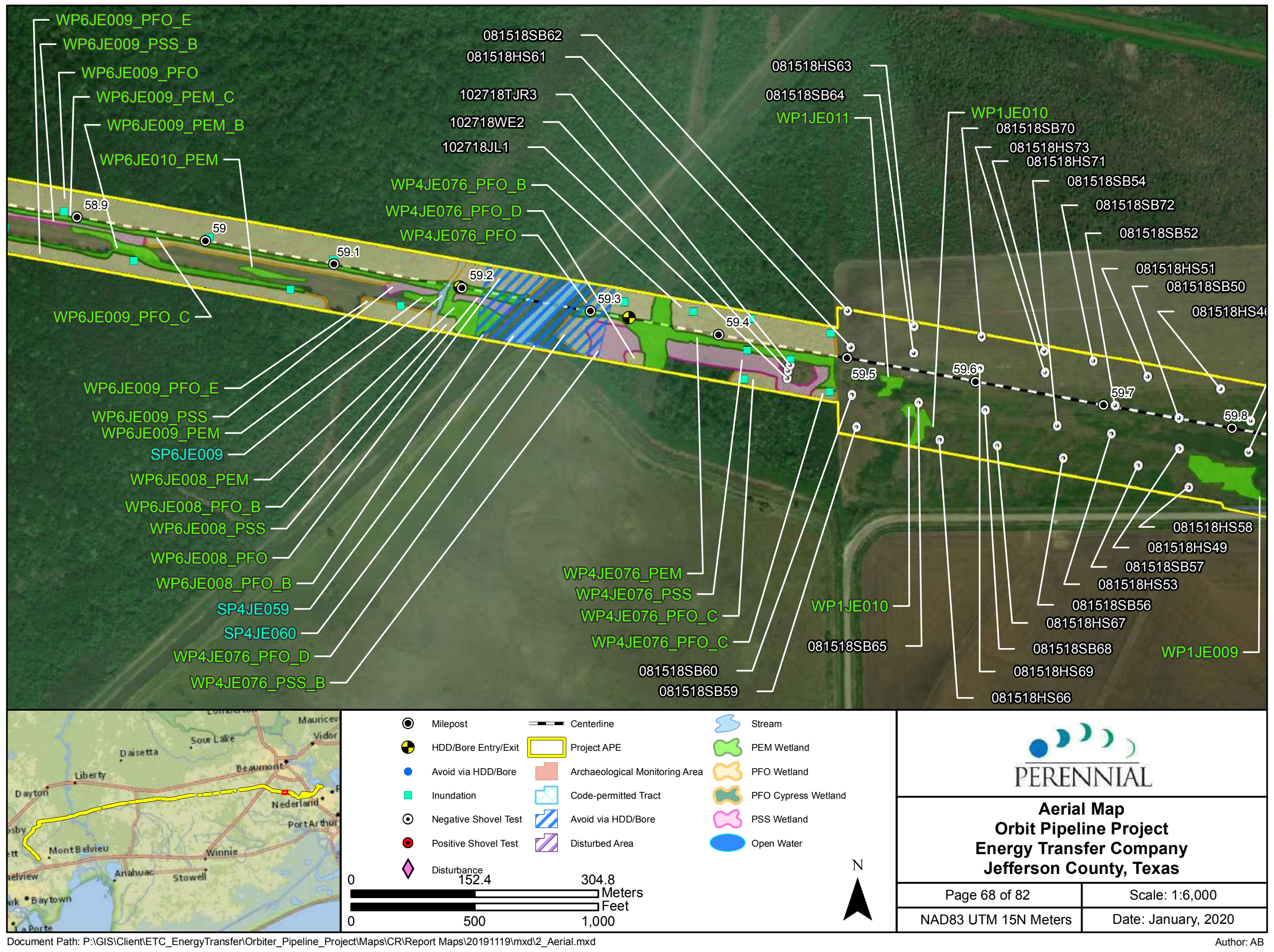




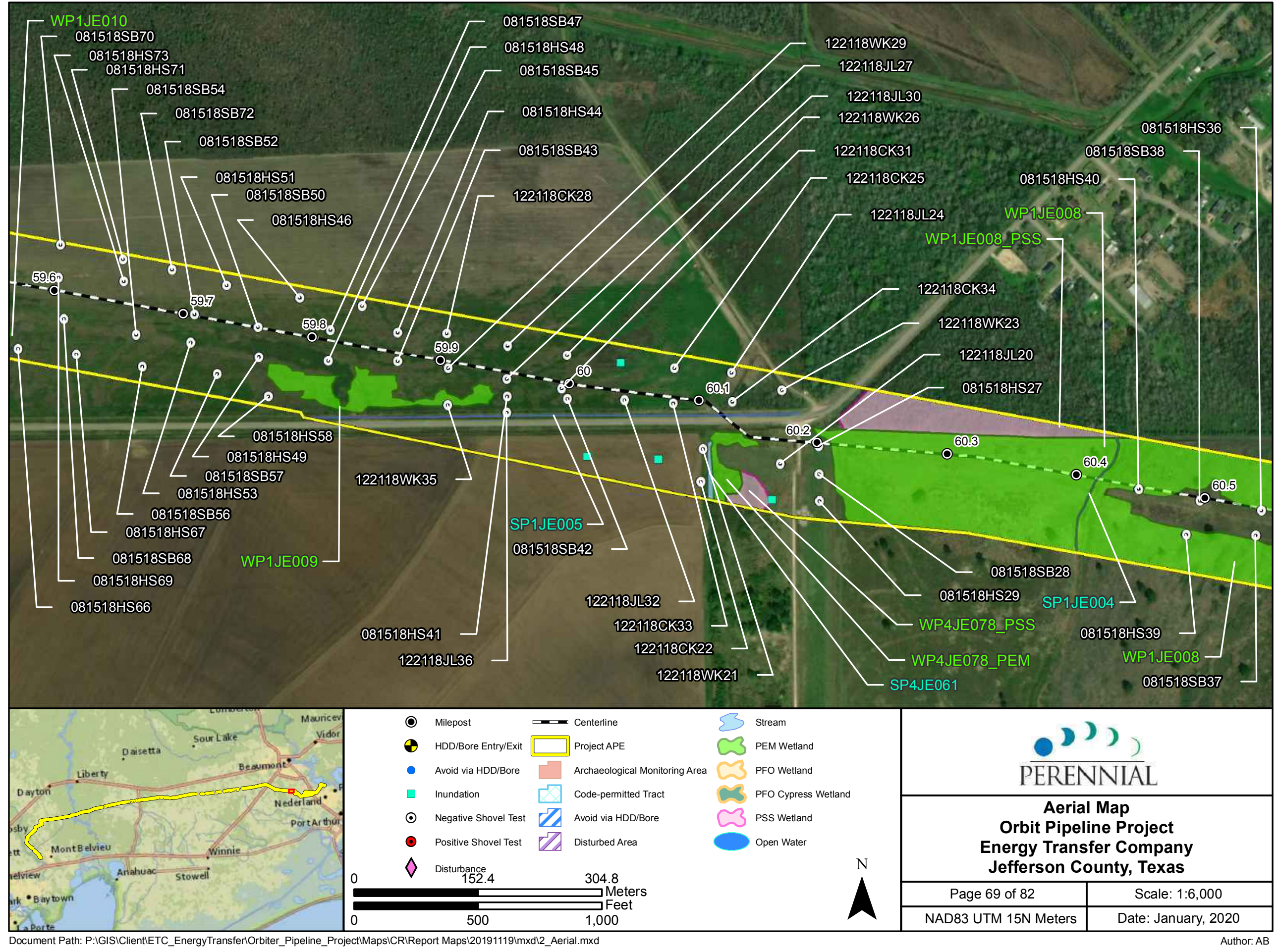




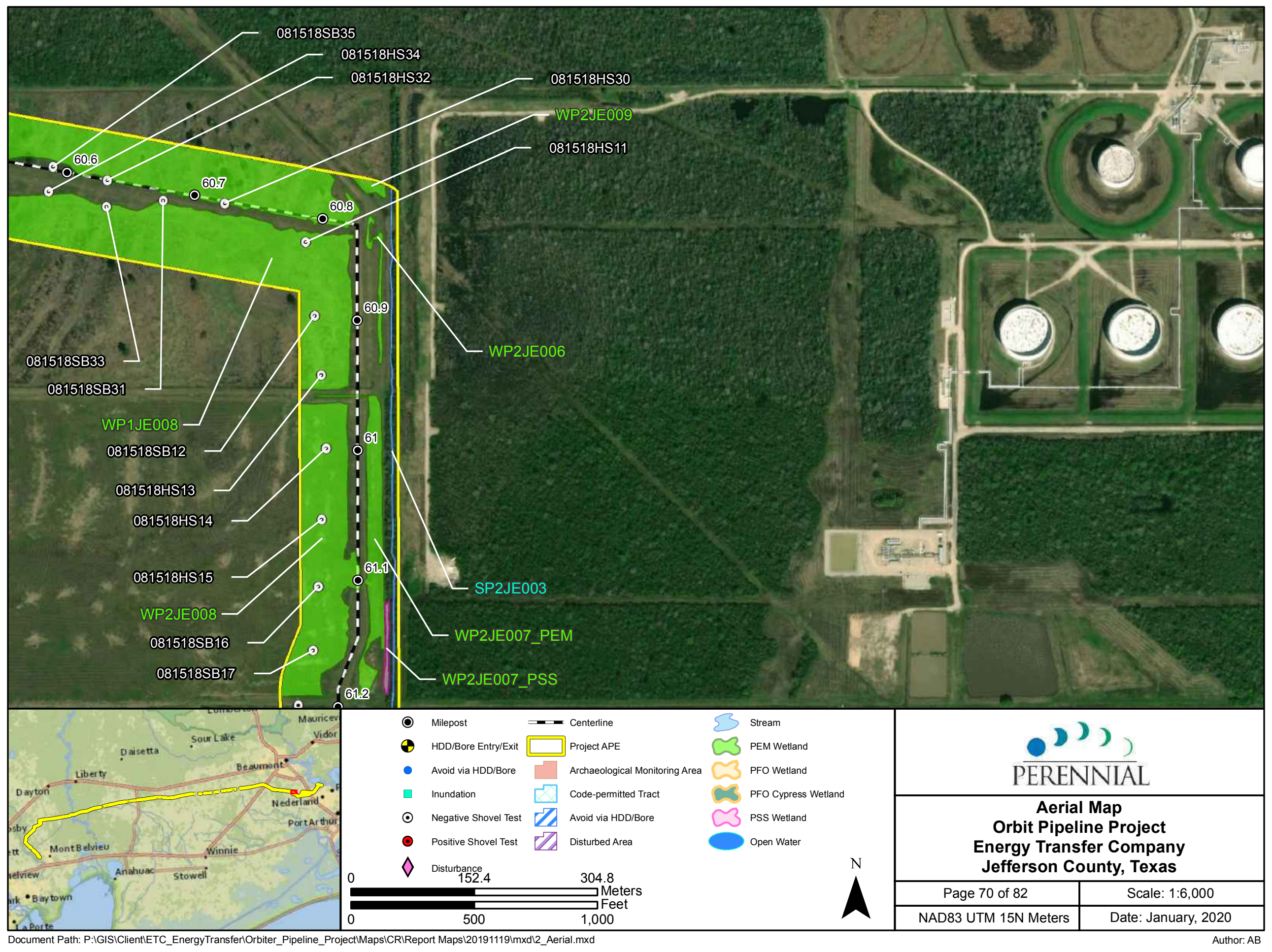




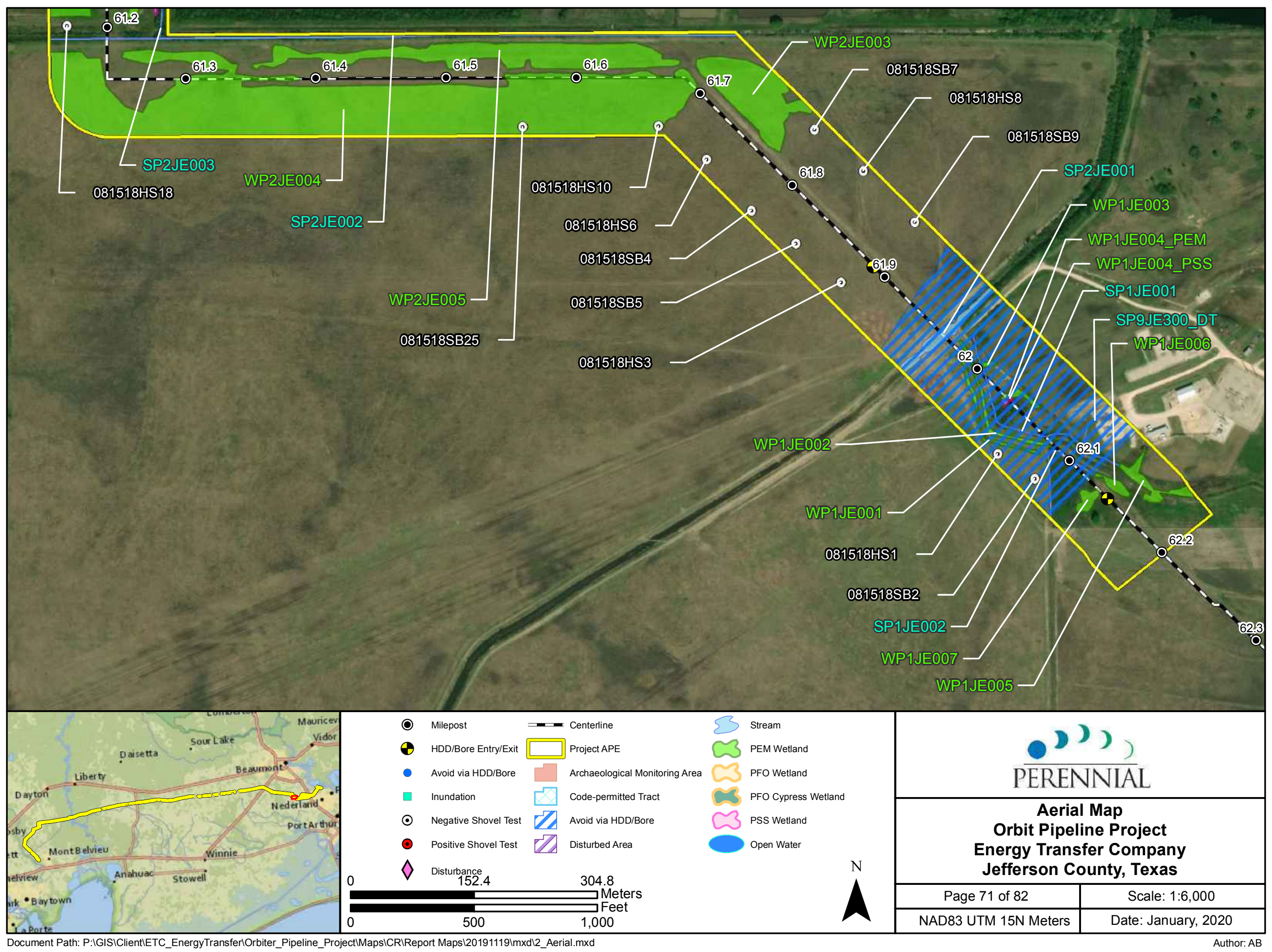




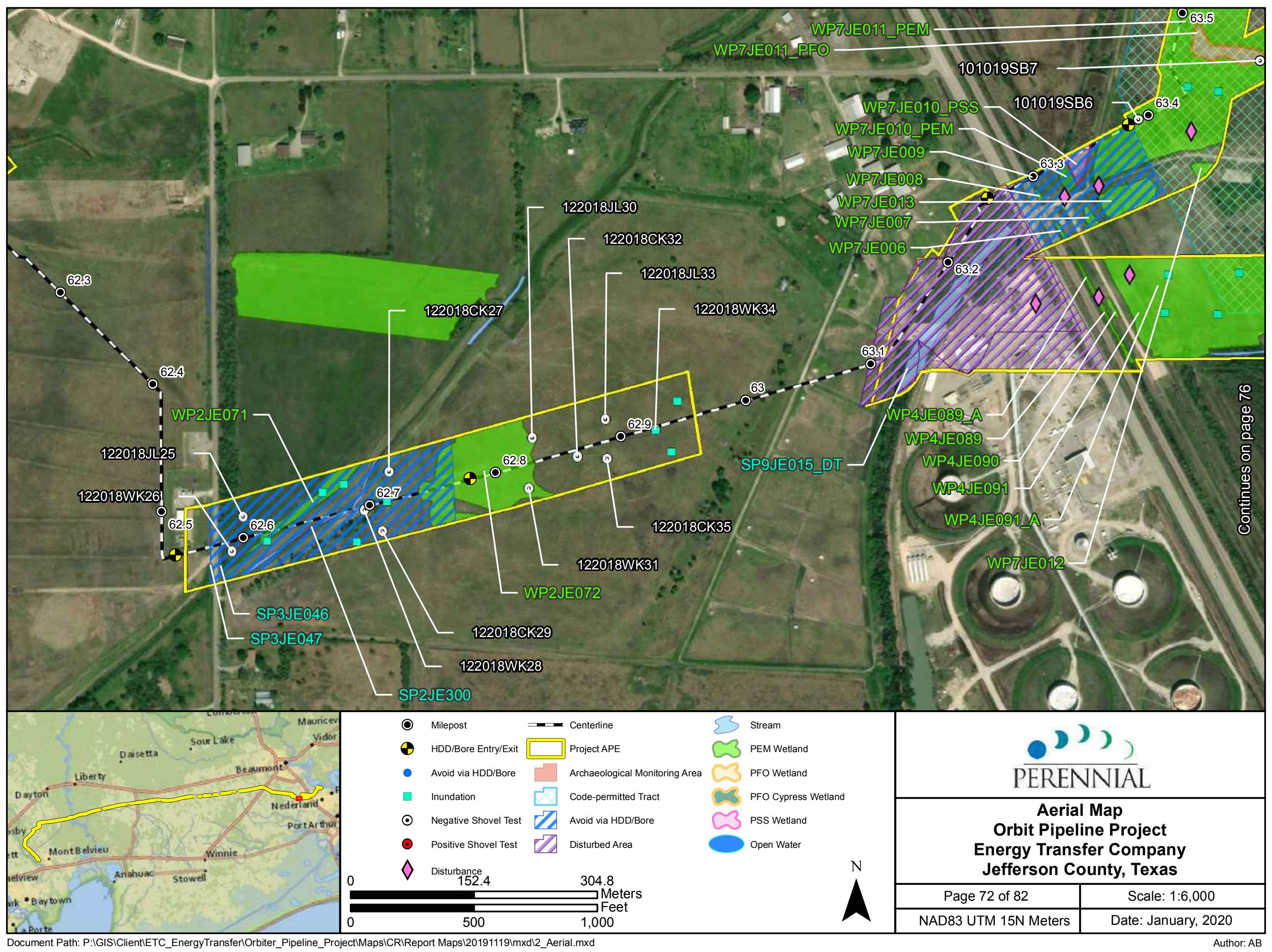




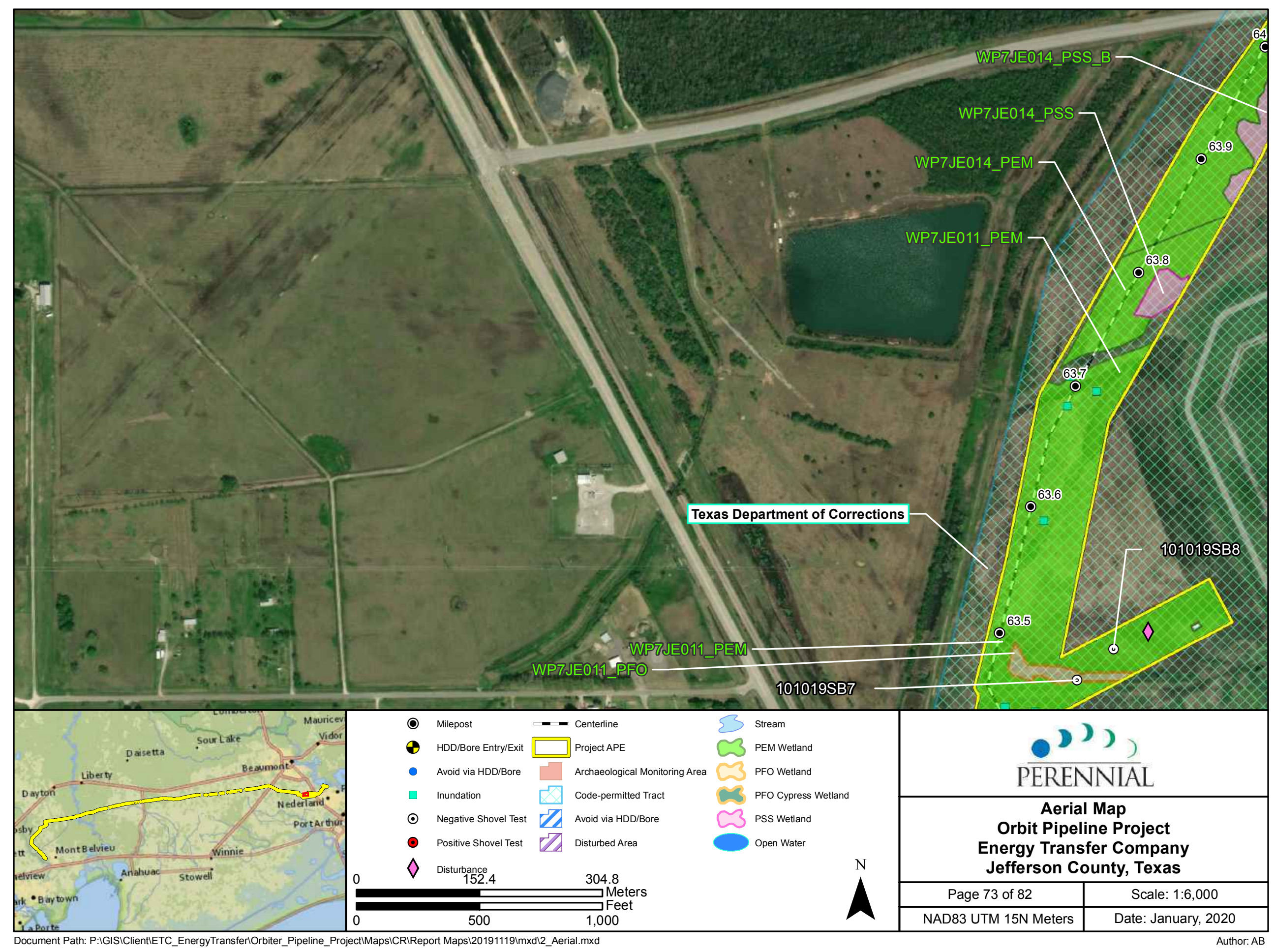




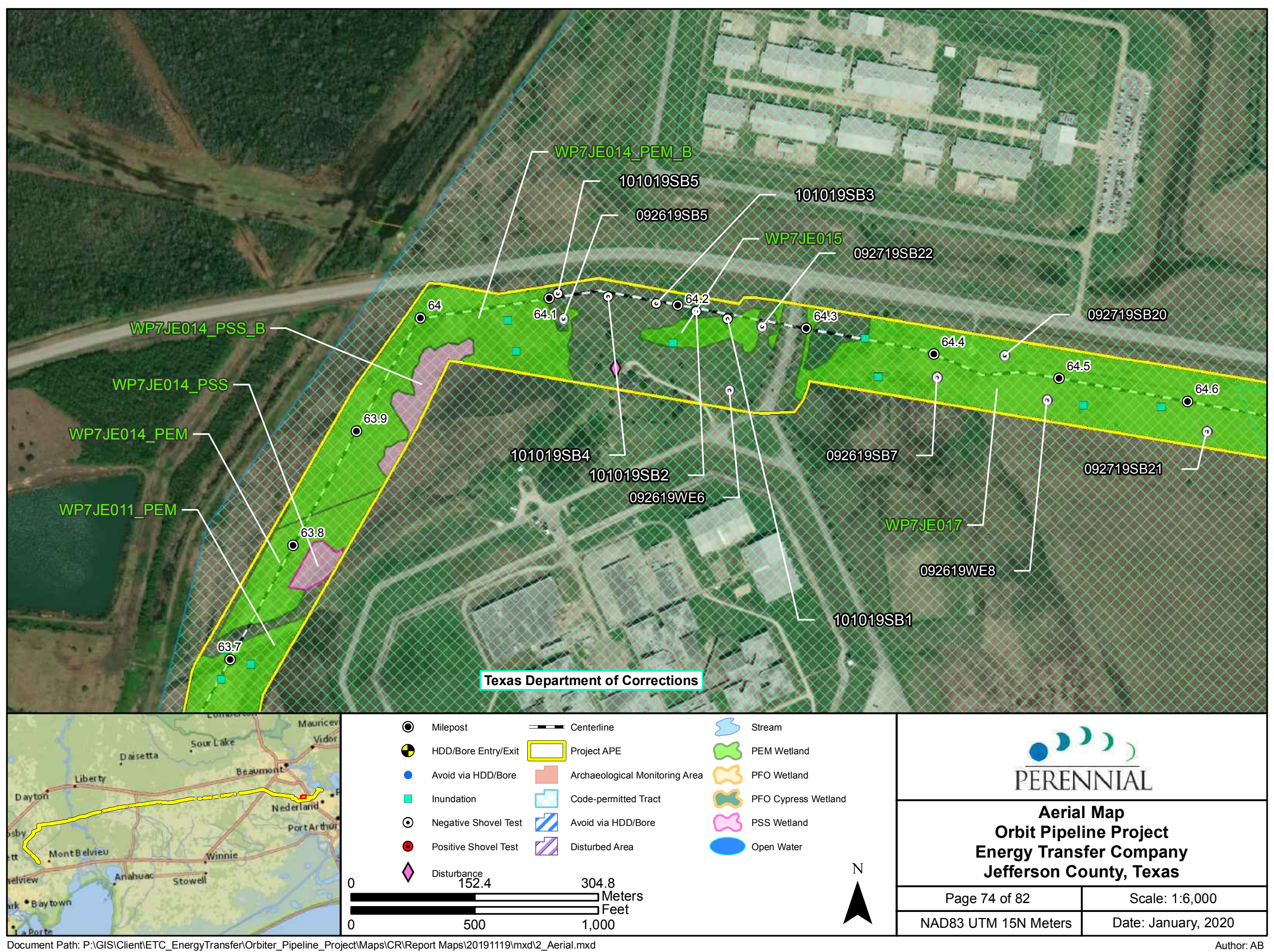




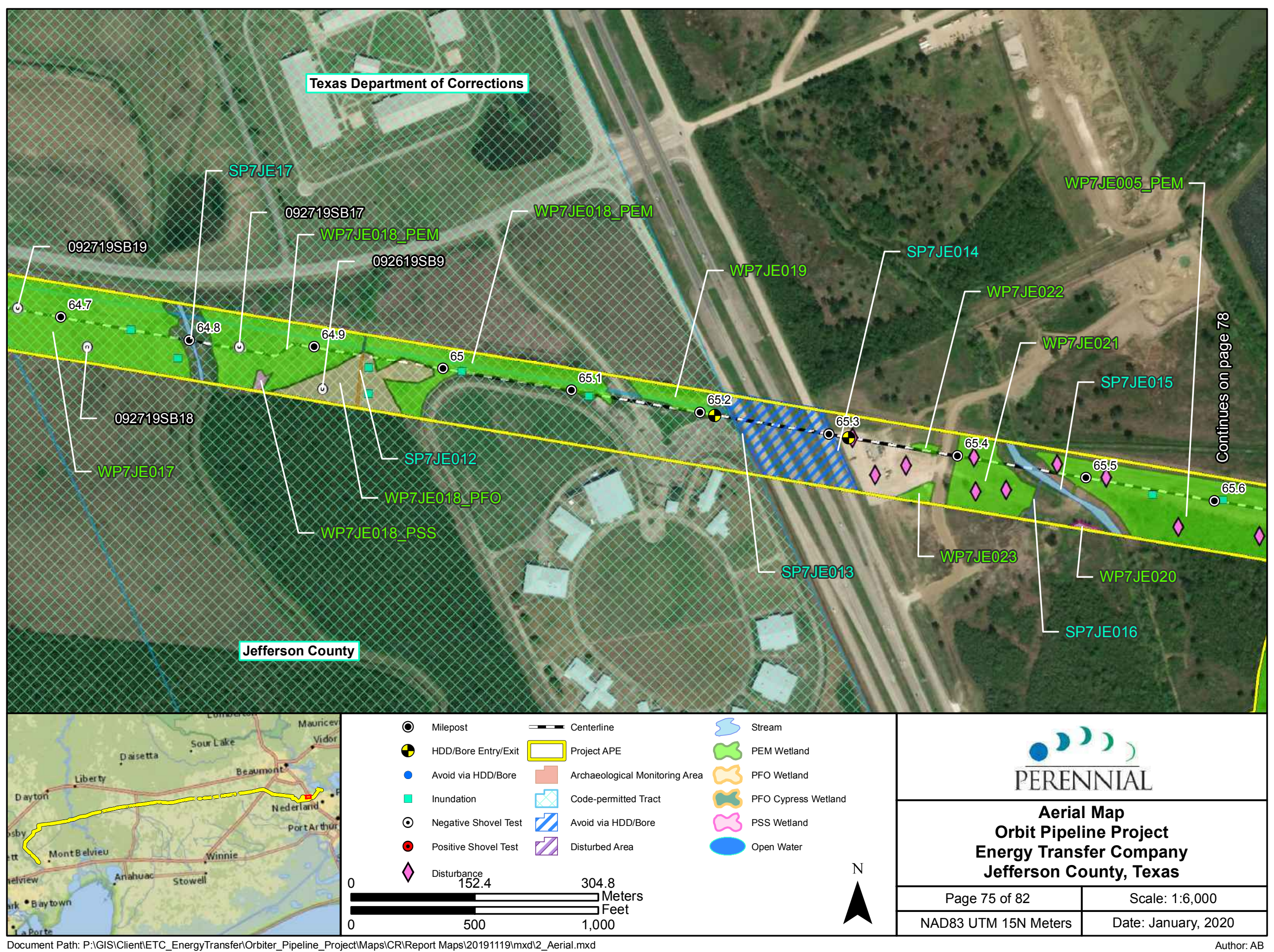




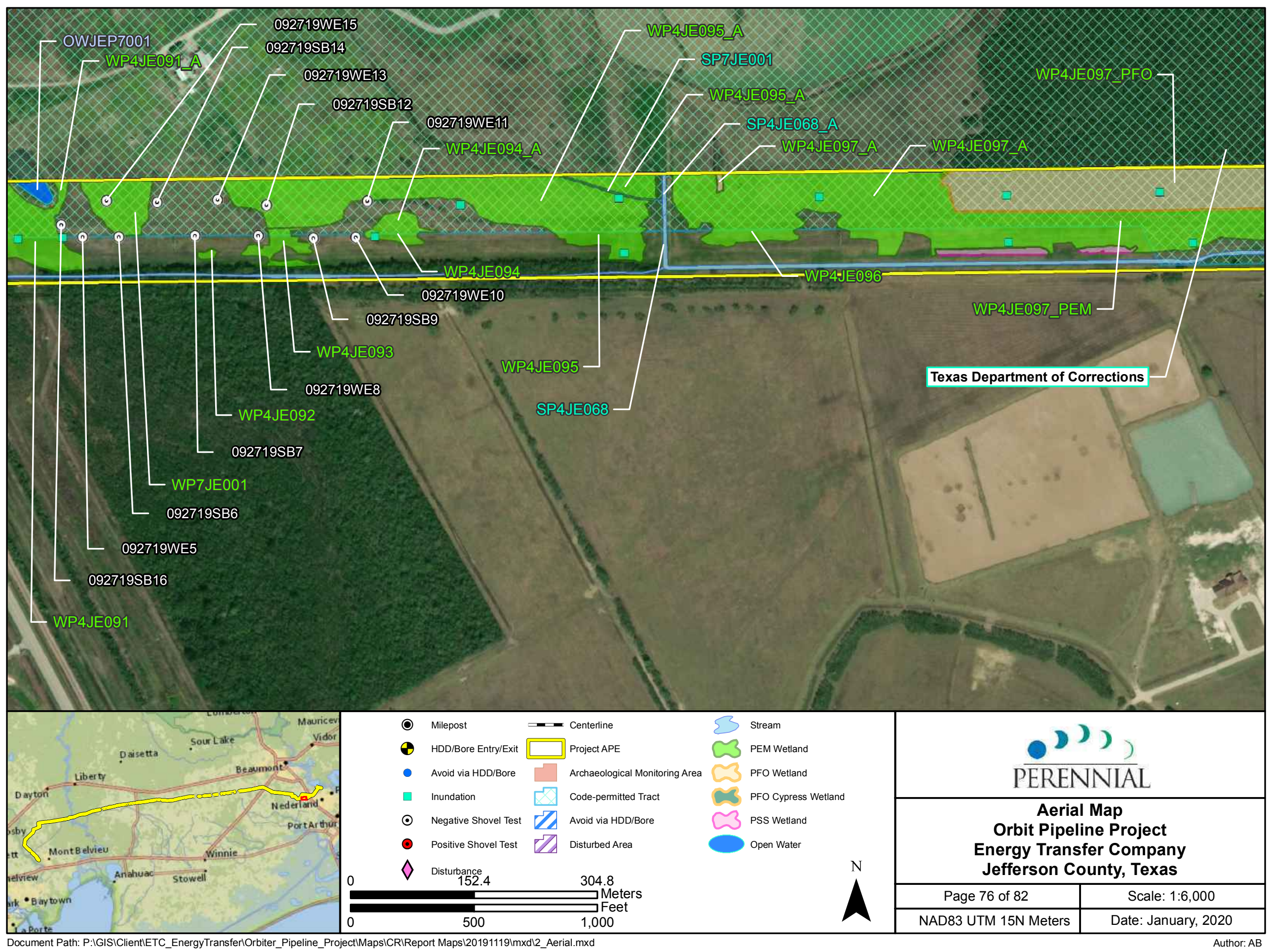




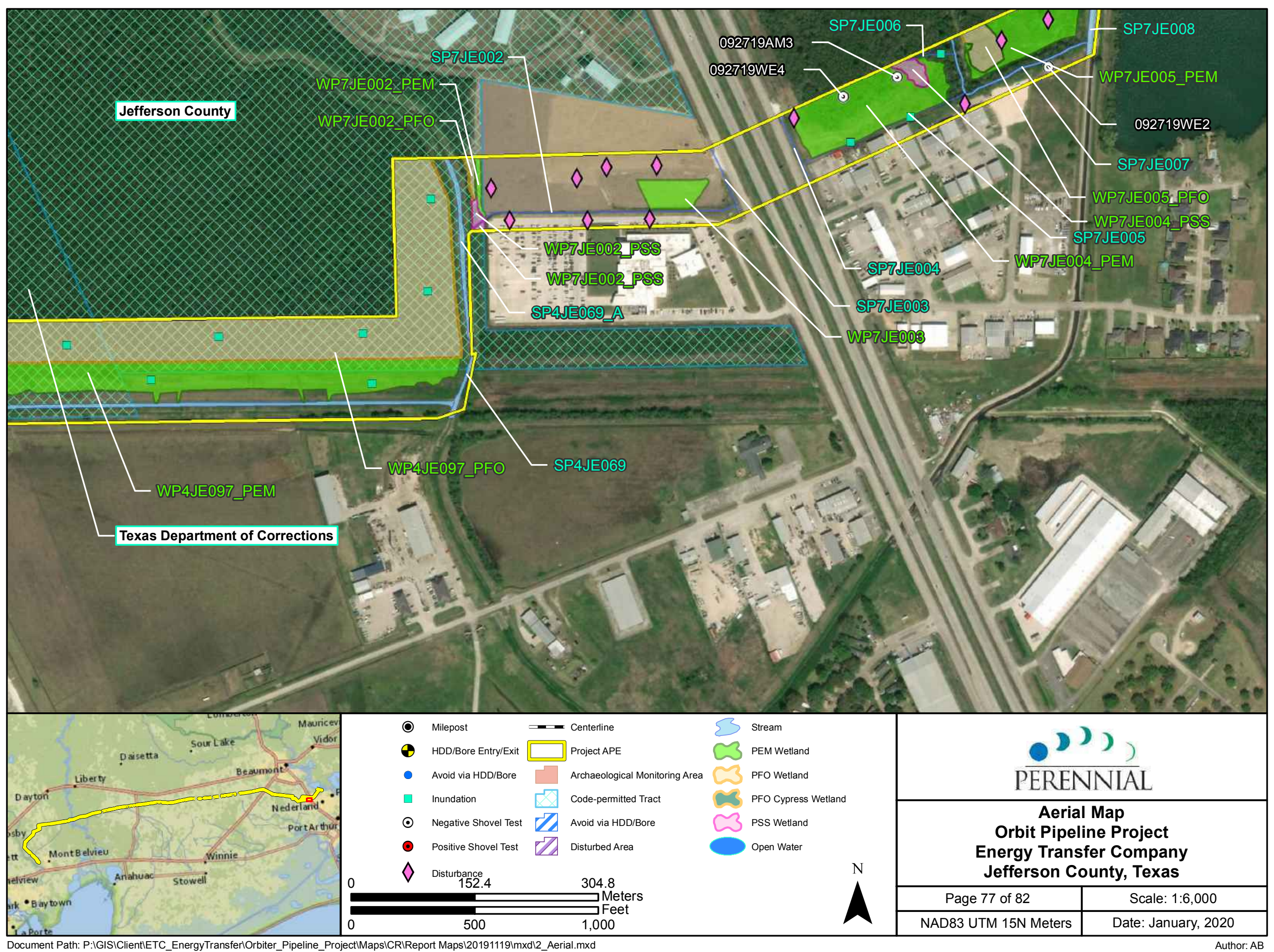




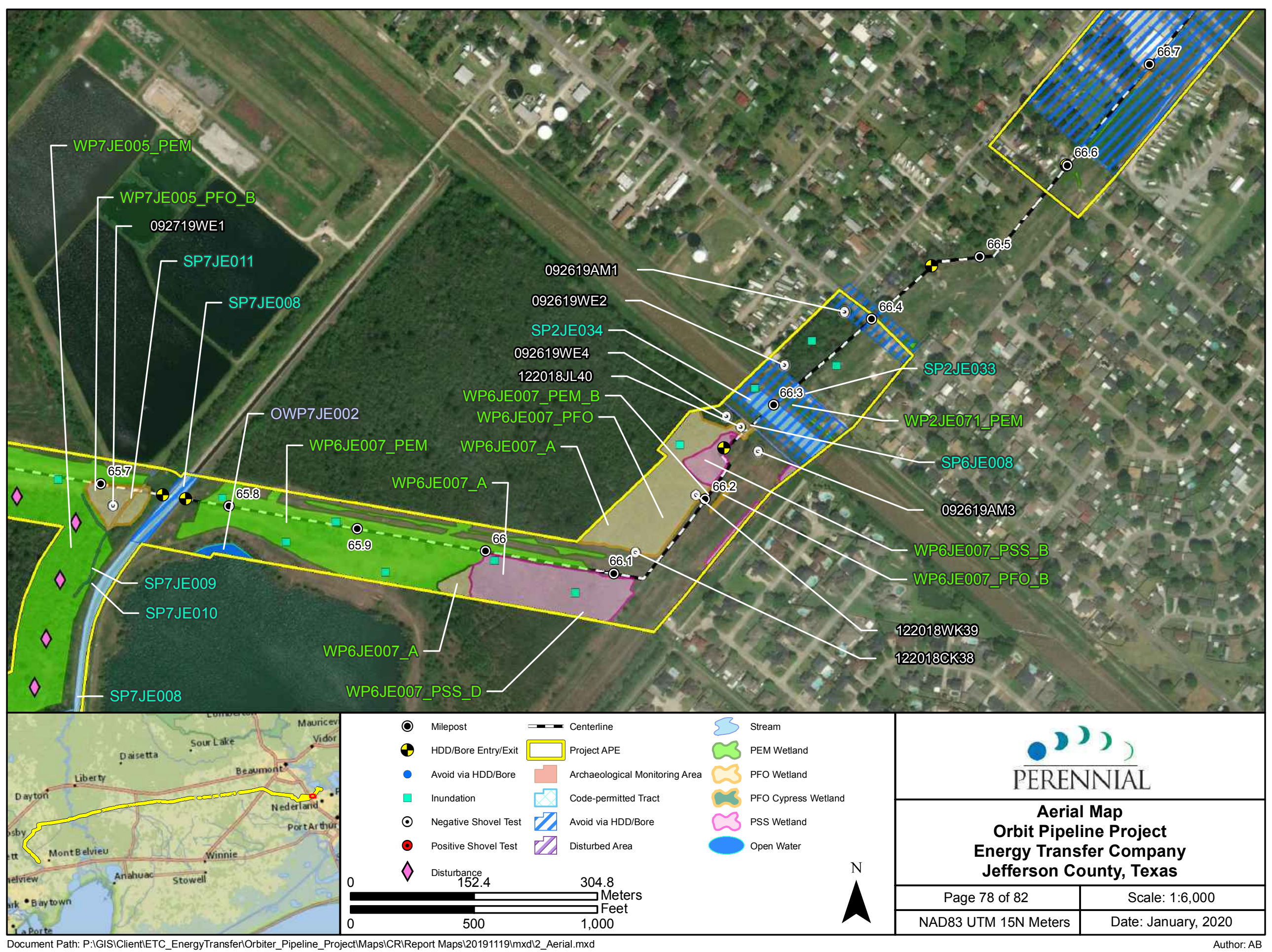




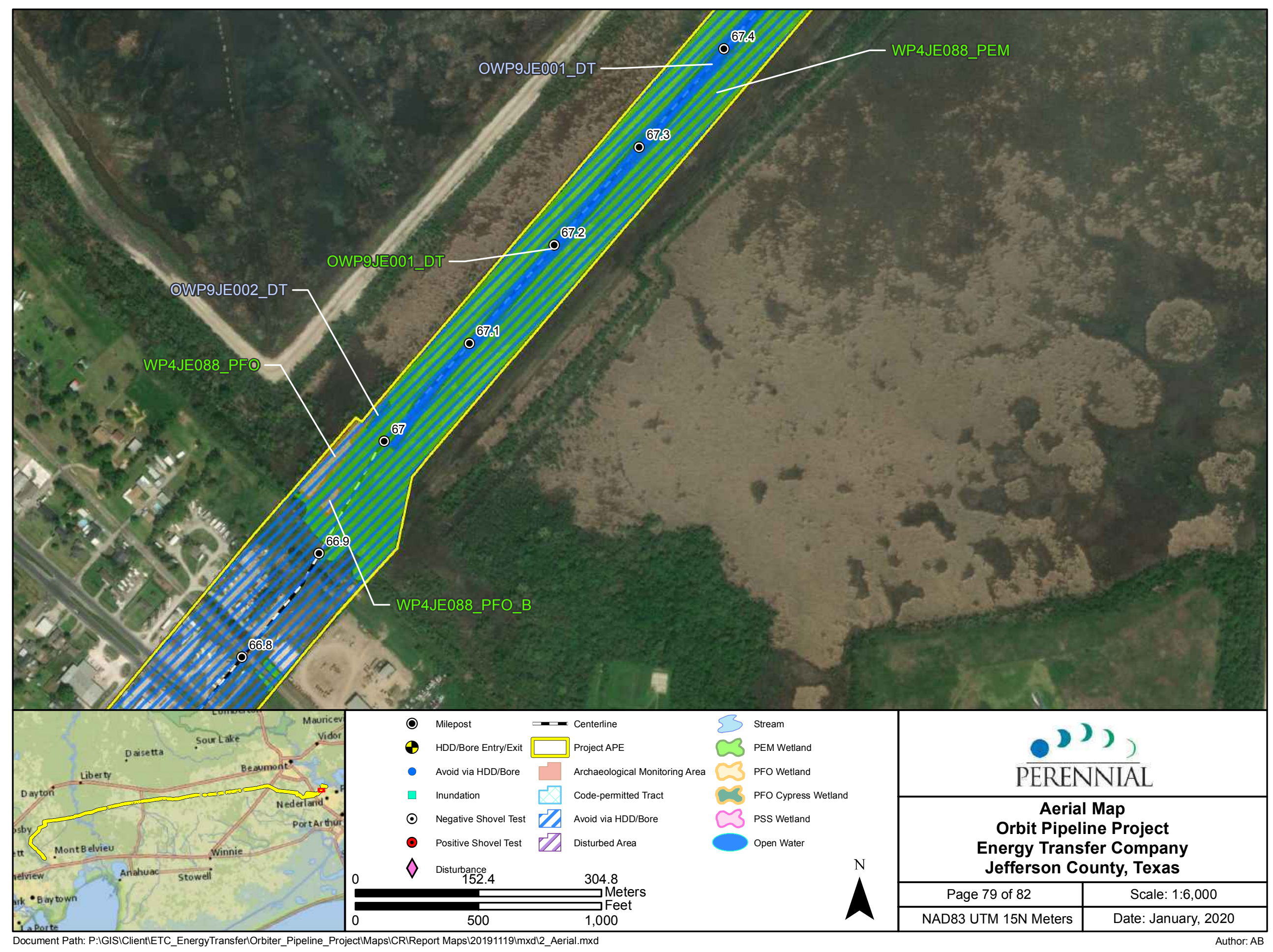




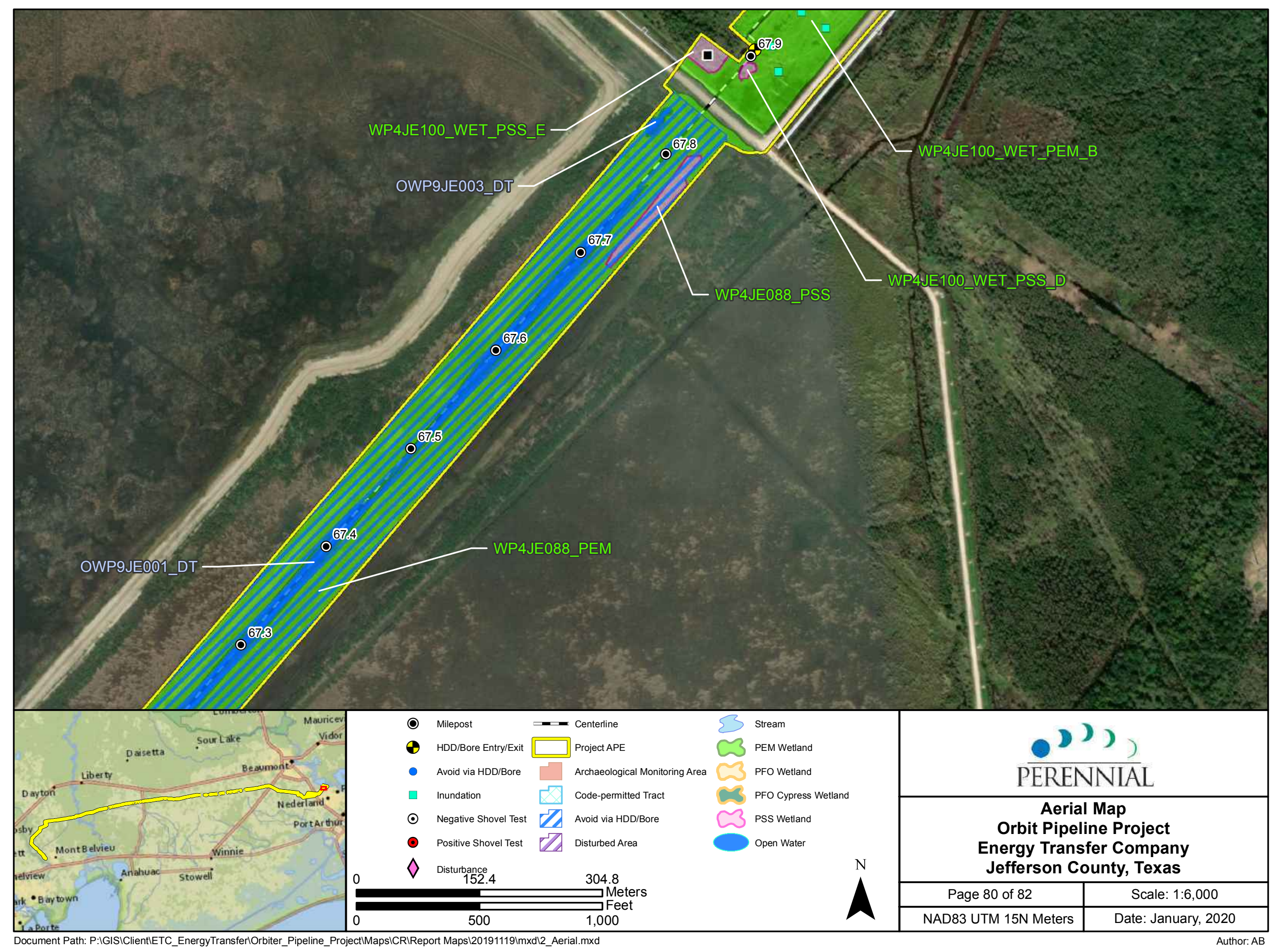




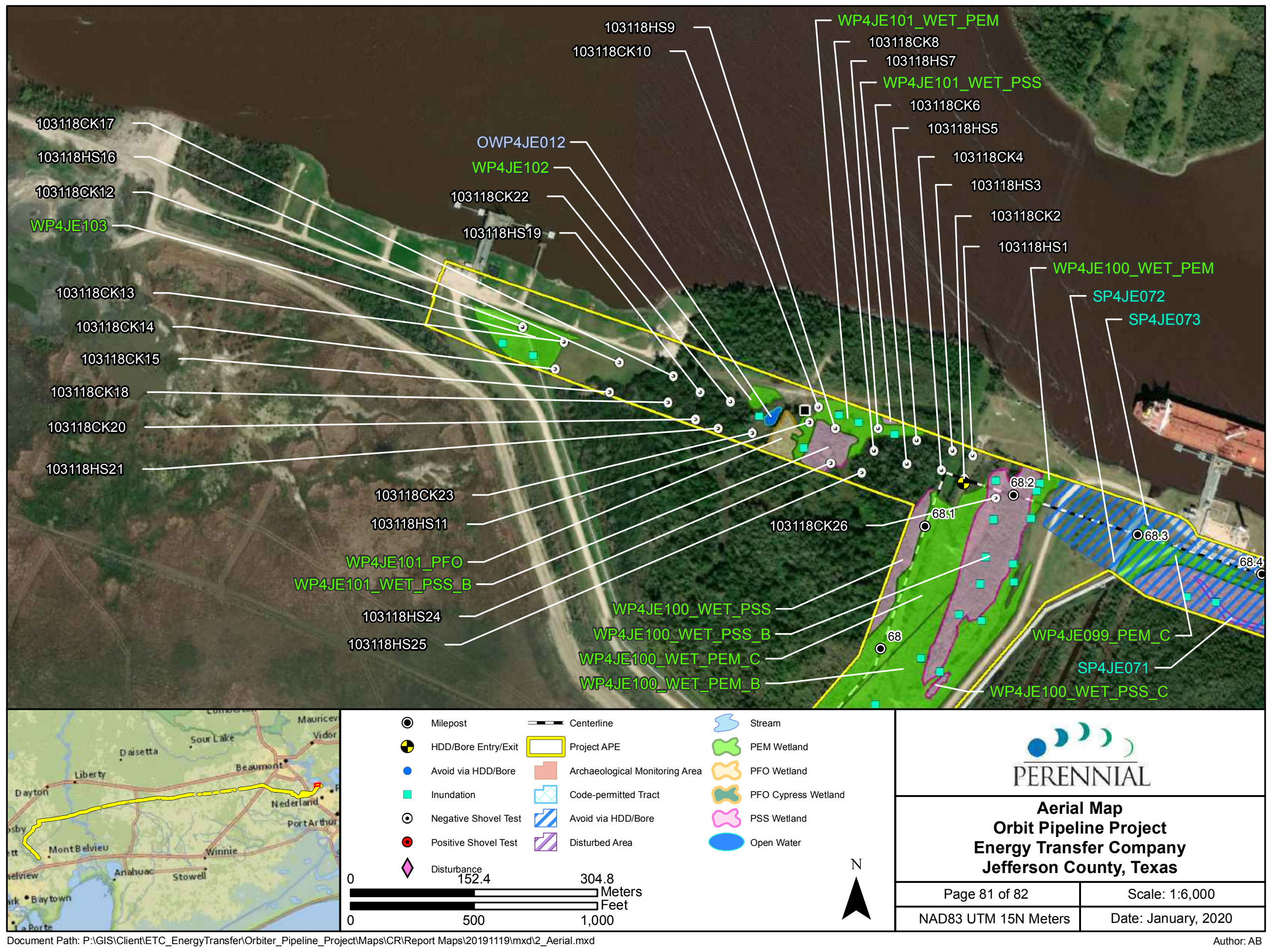



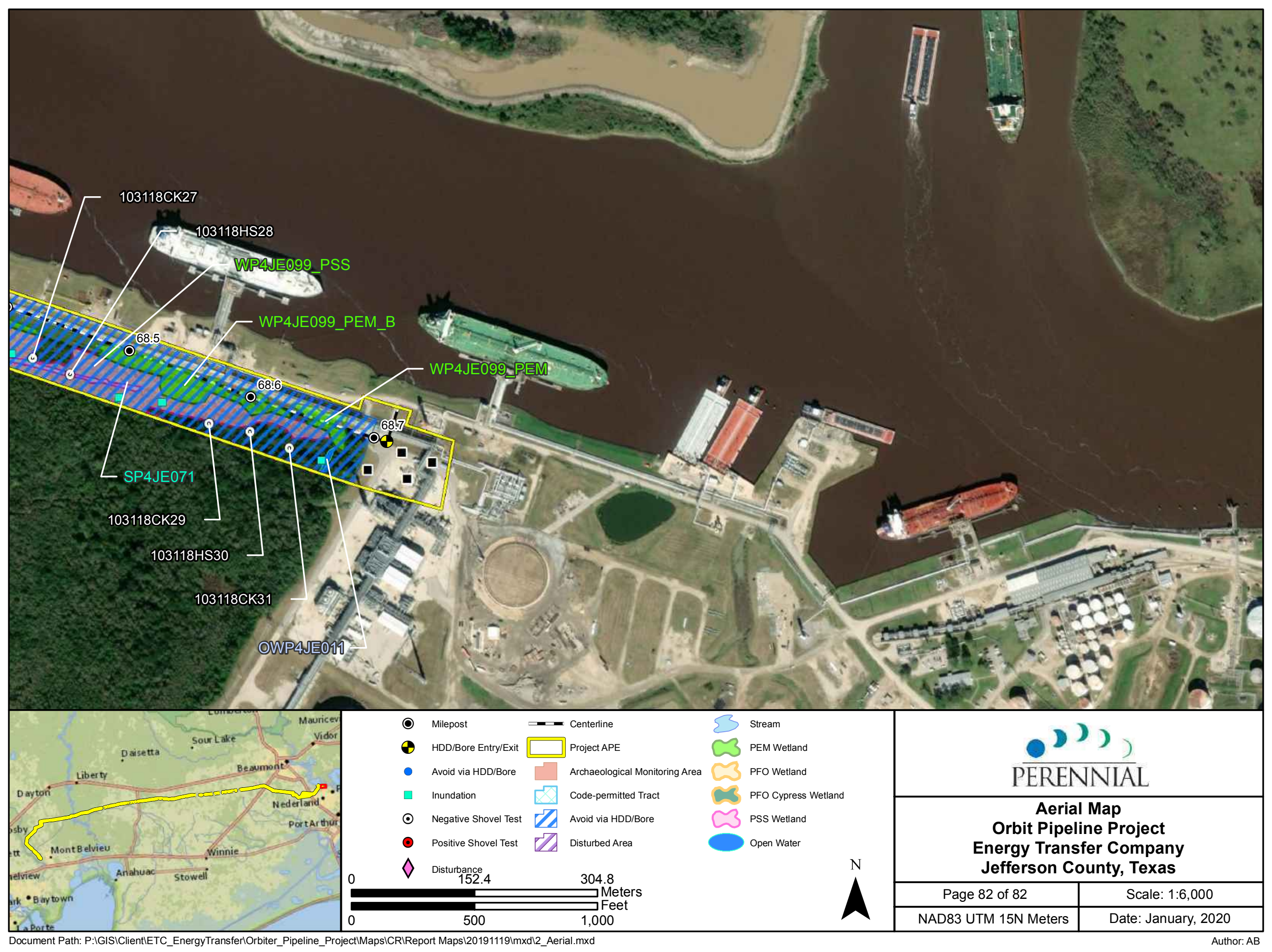
APPENDIX C: WETlaNd AND WATERbody DATA TABLES 
Appendix C 1- Survey Data in the Vicinity of Wetland Features

\begin{tabular}{|c|c|c|c|c|c|c|c|}
\hline \multicolumn{8}{|c|}{ Wetlands Located within the Orbit Project Pipeline APE } \\
\hline Wetland ID & $\begin{array}{c}\text { Milepost } \\
\text { (Approximate) }\end{array}$ & $\begin{array}{l}\text { Wetland } \\
\text { Type }\end{array}$ & Probability & Shovel Tests & $\begin{array}{l}\text { Surveys } \\
\text { Results }\end{array}$ & Recommendations & Comments \\
\hline \multicolumn{8}{|c|}{ Chambers County } \\
\hline WP3CH096 & 0.1 & PEM & Low & None & $\begin{array}{l}\text { No Cultural } \\
\text { Resources }\end{array}$ & $\begin{array}{l}\text { No further work } \\
\text { recommended }\end{array}$ & $\begin{array}{c}\text { Inundated; not located within } \\
\text { workspace }\end{array}$ \\
\hline WP3CH115 & 0.1 & PEM & Low & None & $\begin{array}{l}\text { No Cultural } \\
\text { Resources }\end{array}$ & $\begin{array}{l}\text { No further work } \\
\text { recommended }\end{array}$ & $\begin{array}{c}\text { Inundated; not located within } \\
\text { workspace }\end{array}$ \\
\hline WP3CH120 & 0.1 & PEM & Low & None & $\begin{array}{l}\text { No Cultural } \\
\text { Resources }\end{array}$ & $\begin{array}{l}\text { No further work } \\
\text { recommended }\end{array}$ & $\begin{array}{l}\text { Inundated; not located within } \\
\text { workspace }\end{array}$ \\
\hline WP1CH056_PFO & 0.2 & PFO & Low & 2 & $\begin{array}{l}\text { No Cultural } \\
\text { Resources }\end{array}$ & $\begin{array}{l}\text { No further work } \\
\text { recommended }\end{array}$ & Inundation \\
\hline WP3CH095 & 0.4 & PEM & Low & 1 & $\begin{array}{l}\text { No Cultural } \\
\text { Resources }\end{array}$ & $\begin{array}{l}\text { No further work } \\
\text { recommended }\end{array}$ & $\begin{array}{l}\text { Partially avoided via non- } \\
\text { Section } 10 \mathrm{HDD} / \text { Bore }\end{array}$ \\
\hline WP3CH054 & 0.5 & PEM & Low & None & $\begin{array}{l}\text { No Cultural } \\
\text { Resources }\end{array}$ & $\begin{array}{l}\text { No further work } \\
\text { recommended }\end{array}$ & Inundation \\
\hline WP3CH055 & 0.5 & PEM & Low & None & $\begin{array}{l}\text { No Cultural } \\
\text { Resources }\end{array}$ & $\begin{array}{l}\text { No further work } \\
\text { recommended }\end{array}$ & $\begin{array}{l}\text { Completely avoided via non- } \\
\text { Section } 10 \mathrm{HDD} / \text { Bore }\end{array}$ \\
\hline WP3CH063 & 0.5 & PEM & Low & None & $\begin{array}{l}\text { No Cultural } \\
\text { Resources }\end{array}$ & $\begin{array}{l}\text { No further work } \\
\text { recommended }\end{array}$ & $\begin{array}{l}\text { Located Within Heavily } \\
\text { Inundated Area }\end{array}$ \\
\hline WP3CH056 & 0.6 & PEM & Low & None & $\begin{array}{l}\text { No Cultural } \\
\text { Resources }\end{array}$ & $\begin{array}{l}\text { No further work } \\
\text { recommended }\end{array}$ & $\begin{array}{l}\text { Located Within Heavily } \\
\text { Inundated Area }\end{array}$ \\
\hline WP3CH057_PEM & 0.6 & PEM & Low & None & $\begin{array}{l}\text { No Cultural } \\
\text { Resources }\end{array}$ & $\begin{array}{l}\text { No further work } \\
\text { recommended }\end{array}$ & $\begin{array}{c}\text { Located Within Heavily } \\
\text { Inundated Area }\end{array}$ \\
\hline WP3CH057_PEM_B & 0.6 & PEM & Low & None & $\begin{array}{l}\text { No Cultural } \\
\text { Resources }\end{array}$ & $\begin{array}{l}\text { No further work } \\
\text { recommended }\end{array}$ & $\begin{array}{l}\text { Located Within Heavily } \\
\text { Inundated Area }\end{array}$ \\
\hline WP3CH062 & 0.6 & PEM & Low & None & $\begin{array}{l}\text { No Cultural } \\
\text { Resources }\end{array}$ & $\begin{array}{l}\text { No further work } \\
\text { recommended }\end{array}$ & $\begin{array}{l}\text { Located Within Heavily } \\
\text { Inundated Area }\end{array}$ \\
\hline WP3CH057_PSS & 0.6 & PSS & Low & None & $\begin{array}{l}\text { No Cultural } \\
\text { Resources }\end{array}$ & $\begin{array}{l}\text { No further work } \\
\text { recommended }\end{array}$ & $\begin{array}{l}\text { Located Within Heavily } \\
\text { Inundated Area }\end{array}$ \\
\hline
\end{tabular}


Appendix C 1- Survey Data in the Vicinity of Wetland Features

\begin{tabular}{|c|c|c|c|c|c|c|c|}
\hline Wetland ID & $\begin{array}{c}\text { Milepost } \\
\text { (Approximate) }\end{array}$ & $\begin{array}{l}\text { Wetland } \\
\text { Type }\end{array}$ & Probability & Shovel Tests & $\begin{array}{l}\text { Surveys } \\
\text { Results }\end{array}$ & Recommendations & Comments \\
\hline WP3CH058 & 0.7 & PEM & Low & None & $\begin{array}{l}\text { No Cultural } \\
\text { Resources }\end{array}$ & $\begin{array}{l}\text { No further work } \\
\text { recommended }\end{array}$ & $\begin{array}{c}\text { Located Within Heavily } \\
\text { Inundated Area }\end{array}$ \\
\hline WP3CH059 & 0.7 & PEM & Low & None & $\begin{array}{l}\text { No Cultural } \\
\text { Resources }\end{array}$ & $\begin{array}{l}\text { No further work } \\
\text { recommended }\end{array}$ & Inundation \\
\hline WP3CH060 & 0.7 & PEM & Low & None & $\begin{array}{l}\text { No Cultural } \\
\text { Resources }\end{array}$ & $\begin{array}{l}\text { No further work } \\
\text { recommended }\end{array}$ & $\begin{array}{l}\text { Completely avoided via non- } \\
\text { Section } 10 \mathrm{HDD} / \text { Bore }\end{array}$ \\
\hline WP3CH061 & 0.7 & PEM & Low & None & $\begin{array}{l}\text { No Cultural } \\
\text { Resources }\end{array}$ & $\begin{array}{l}\text { No further work } \\
\text { recommended }\end{array}$ & Inundation \\
\hline WP3CH064 & 0.7 & PEM & Low & None & $\begin{array}{l}\text { No Cultural } \\
\text { Resources }\end{array}$ & $\begin{array}{l}\text { No further work } \\
\text { recommended }\end{array}$ & $\begin{array}{c}\text { Located Within Heavily } \\
\text { Inundated Area }\end{array}$ \\
\hline WP3CH094 & 0.7 & PEM & Low & None & $\begin{array}{l}\text { No Cultural } \\
\text { Resources }\end{array}$ & $\begin{array}{l}\text { No further work } \\
\text { recommended }\end{array}$ & $\begin{array}{l}\text { Partially avoided via non- } \\
\text { Section } 10 \mathrm{HDD} / \text { Bore }\end{array}$ \\
\hline WP3CH065 & 0.7 & $\mathrm{PFO}$ & Low & None & $\begin{array}{l}\text { No Cultural } \\
\text { Resources }\end{array}$ & $\begin{array}{l}\text { No further work } \\
\text { recommended }\end{array}$ & $\begin{array}{c}\text { Located Within Heavily } \\
\text { Inundated Area }\end{array}$ \\
\hline WP3CH066 & 0.7 & PFO & Low & None & $\begin{array}{l}\text { No Cultural } \\
\text { Resources }\end{array}$ & $\begin{array}{l}\text { No further work } \\
\text { recommended }\end{array}$ & $\begin{array}{l}\text { Covered under previously } \\
\text { conducted survey }\end{array}$ \\
\hline WP3CH093_PEM & 0.8 & PEM & Low & None & $\begin{array}{l}\text { No Cultural } \\
\text { Resources }\end{array}$ & $\begin{array}{l}\text { No further work } \\
\text { recommended }\end{array}$ & $\begin{array}{l}\text { Completely avoided via non- } \\
\text { Section } 10 \mathrm{HDD} / \text { Bore }\end{array}$ \\
\hline WP3CH093_PFO & 0.8 & PFO & Low & None & $\begin{array}{l}\text { No Cultural } \\
\text { Resources }\end{array}$ & $\begin{array}{l}\text { No further work } \\
\text { recommended }\end{array}$ & $\begin{array}{c}\text { Located Within Heavily } \\
\text { Inundated Area }\end{array}$ \\
\hline WP3CH044 & 0.9 & PEM & Low & None & $\begin{array}{l}\text { No Cultural } \\
\text { Resources }\end{array}$ & $\begin{array}{l}\text { No further work } \\
\text { recommended }\end{array}$ & $\begin{array}{c}\text { Located Within Heavily } \\
\text { Inundated Area }\end{array}$ \\
\hline WP3CH091 & 0.9 & PEM & Low & None & $\begin{array}{l}\text { No Cultural } \\
\text { Resources }\end{array}$ & $\begin{array}{l}\text { No further work } \\
\text { recommended }\end{array}$ & $\begin{array}{c}\text { Located Within Heavily } \\
\text { Inundated Area }\end{array}$ \\
\hline WP3CH092_PEM & 0.9 & PEM & Low & None & $\begin{array}{l}\text { No Cultural } \\
\text { Resources }\end{array}$ & $\begin{array}{l}\text { No further work } \\
\text { recommended }\end{array}$ & $\begin{array}{c}\text { Located Within Heavily } \\
\text { Inundated Area }\end{array}$ \\
\hline WP3CH092_PSS & 0.9 & PSS & Low & None & $\begin{array}{l}\text { No Cultural } \\
\text { Resources }\end{array}$ & $\begin{array}{l}\text { No further work } \\
\text { recommended }\end{array}$ & $\begin{array}{l}\text { Located Within Heavily } \\
\text { Inundated Area }\end{array}$ \\
\hline WP3CH090 & 1.1 & PEM & Low & None & $\begin{array}{l}\text { No Cultural } \\
\text { Resources }\end{array}$ & $\begin{array}{l}\text { No further work } \\
\text { recommended }\end{array}$ & $\begin{array}{c}\text { Located Within Heavily } \\
\text { Inundated Area }\end{array}$ \\
\hline
\end{tabular}


Appendix C 1- Survey Data in the Vicinity of Wetland Features

\begin{tabular}{|c|c|c|c|c|c|c|c|}
\hline Wetland ID & $\begin{array}{c}\text { Milepost } \\
\text { (Approximate) }\end{array}$ & $\begin{array}{l}\text { Wetland } \\
\text { Type }\end{array}$ & Probability & Shovel Tests & $\begin{array}{l}\text { Surveys } \\
\text { Results }\end{array}$ & Recommendations & Comments \\
\hline WP3CH088 & 1.2 & PEM & Low & None & $\begin{array}{l}\text { No Cultural } \\
\text { Resources }\end{array}$ & $\begin{array}{l}\text { No further work } \\
\text { recommended }\end{array}$ & $\begin{array}{c}\text { Located Within Heavily } \\
\text { Inundated Area }\end{array}$ \\
\hline WP3CH089 & 1.2 & PEM & Low & None & $\begin{array}{l}\text { No Cultural } \\
\text { Resources }\end{array}$ & $\begin{array}{l}\text { No further work } \\
\text { recommended }\end{array}$ & $\begin{array}{l}\text { Located Within Heavily } \\
\text { Inundated Area }\end{array}$ \\
\hline WP3CH085_PEM & 1.3 & PEM & Low & None & $\begin{array}{l}\text { No Cultural } \\
\text { Resources }\end{array}$ & $\begin{array}{l}\text { No further work } \\
\text { recommended }\end{array}$ & Inundation \\
\hline WP3CH085_PEM_C & 1.3 & PEM & Low & None & $\begin{array}{l}\text { No Cultural } \\
\text { Resources }\end{array}$ & $\begin{array}{l}\text { No further work } \\
\text { recommended }\end{array}$ & $\begin{array}{c}\text { Completely avoided via non } \\
\text { Section } 10 \mathrm{HDD} / \text { Bore }\end{array}$ \\
\hline WP3CH085_PFO & 1.3 & $\mathrm{PFO}$ & Low & None & $\begin{array}{l}\text { No Cultural } \\
\text { Resources }\end{array}$ & $\begin{array}{l}\text { No further work } \\
\text { recommended }\end{array}$ & $\begin{array}{c}\text { Completely avoided via non } \\
\text { Section } 10 \mathrm{HDD} / \text { Bore }\end{array}$ \\
\hline WP3CH087 & 1.3 & $\mathrm{PFO}$ & Low & None & $\begin{array}{l}\text { No Cultural } \\
\text { Resources }\end{array}$ & $\begin{array}{l}\text { No further work } \\
\text { recommended }\end{array}$ & $\begin{array}{l}\text { Located Within Heavily } \\
\text { Inundated Area }\end{array}$ \\
\hline WP3CH085_PSS & 1.3 & PSS & Low & None & $\begin{array}{l}\text { No Cultural } \\
\text { Resources }\end{array}$ & $\begin{array}{l}\text { No further work } \\
\text { recommended }\end{array}$ & $\begin{array}{l}\text { Completely avoided via non } \\
\text { Section } 10 \mathrm{HDD} / \text { Bore }\end{array}$ \\
\hline WP3CH085_PEM_B & 1.4 & PEM & Low & None & $\begin{array}{l}\text { No Cultural } \\
\text { Resources }\end{array}$ & $\begin{array}{l}\text { No further work } \\
\text { recommended }\end{array}$ & Mostly inundated \\
\hline WP3CH086 & 1.4 & PEM & Low & None & $\begin{array}{l}\text { No Cultural } \\
\text { Resources }\end{array}$ & $\begin{array}{l}\text { No further work } \\
\text { recommended }\end{array}$ & Mostly inundated \\
\hline WP9CH003_DT ${ }^{a}$ & 1.9 & $\mathrm{PFO}$ & Low & None & $\begin{array}{l}\text { No Cultural } \\
\text { Resources }\end{array}$ & $\begin{array}{l}\text { No further work } \\
\text { recommended }\end{array}$ & $\begin{array}{l}\text { No Impacts, outside of } \\
\text { workspace - Survey } \\
\text { Permission Denied }\end{array}$ \\
\hline WP9CH004_DT ${ }^{\text {a }}$ & 2.7 & $\mathrm{PFO}$ & Low & None & $\begin{array}{l}\text { No Cultural } \\
\text { Resources }\end{array}$ & $\begin{array}{l}\text { No further work } \\
\text { recommended }\end{array}$ & Inundated \\
\hline WP3CH039 & 3.2 & $\mathrm{PFO}$ & Low & 9 & $\begin{array}{l}\text { No Cultural } \\
\text { Resources }\end{array}$ & $\begin{array}{l}\text { No further work } \\
\text { recommended }\end{array}$ & Inundation \\
\hline WP3CH040 & 3.5 & $\mathrm{PFO}$ & Low & 5 & $\begin{array}{l}\text { No Cultural } \\
\text { Resources }\end{array}$ & $\begin{array}{l}\text { No further work } \\
\text { recommended }\end{array}$ & Inundation \\
\hline
\end{tabular}


Appendix C 1- Survey Data in the Vicinity of Wetland Features

\begin{tabular}{|c|c|c|c|c|c|c|c|}
\hline Wetland ID & $\begin{array}{c}\text { Milepost } \\
\text { (Approximate) }\end{array}$ & $\begin{array}{c}\text { Wetland } \\
\text { Type }\end{array}$ & Probability & Shovel Tests & $\begin{array}{l}\text { Surveys } \\
\text { Results }\end{array}$ & Recommendations & Comments \\
\hline WP3LI041 & 3.8 & $\mathrm{PFO}$ & Low & 19 & $\begin{array}{l}\text { No Cultural } \\
\text { Resources }\end{array}$ & $\begin{array}{l}\text { No further work } \\
\text { recommended }\end{array}$ & Inundation \\
\hline WP3LI042_PFO & 3.9 & $\mathrm{PFO}$ & Low & 2 & $\begin{array}{l}\text { No Cultural } \\
\text { Resources }\end{array}$ & $\begin{array}{l}\text { No further work } \\
\text { recommended }\end{array}$ & None \\
\hline WP3LI042_PSS & 3.9 & PSS & Low & 3 & $\begin{array}{l}\text { No Cultural } \\
\text { Resources }\end{array}$ & $\begin{array}{l}\text { No further work } \\
\text { recommended }\end{array}$ & None \\
\hline WP3LI043_PEM & 4.1 & PEM & Low & None & $\begin{array}{l}\text { No Cultural } \\
\text { Resources }\end{array}$ & $\begin{array}{l}\text { No further work } \\
\text { recommended }\end{array}$ & Mostly inundated \\
\hline WP3LI043_PFO & 4.1 & $\mathrm{PFO}$ & Low & None & $\begin{array}{l}\text { No Cultural } \\
\text { Resources }\end{array}$ & $\begin{array}{l}\text { No further work } \\
\text { recommended }\end{array}$ & Mostly inundated \\
\hline WP3LI043_PSS & 4.1 & PSS & Low & None & $\begin{array}{l}\text { No Cultural } \\
\text { Resources }\end{array}$ & $\begin{array}{l}\text { No further work } \\
\text { recommended }\end{array}$ & Mostly inundated \\
\hline WP3LI043_PSS_B & 4.2 & PSS & Low & None & $\begin{array}{l}\text { No Cultural } \\
\text { Resources }\end{array}$ & $\begin{array}{l}\text { No further work } \\
\text { recommended }\end{array}$ & Mostly inundated \\
\hline WP3LI043_PSS_C & 4.2 & PSS & Low & None & $\begin{array}{l}\text { No Cultural } \\
\text { Resources }\end{array}$ & $\begin{array}{l}\text { No further work } \\
\text { recommended }\end{array}$ & Mostly inundated \\
\hline WP3LI043_PFO_B & 4.3 & $\mathrm{PFO}$ & Low & 2 & $\begin{array}{l}\text { No Cultural } \\
\text { Resources }\end{array}$ & $\begin{array}{l}\text { No further work } \\
\text { recommended }\end{array}$ & Mostly inundated \\
\hline WP5LI024 & 4.5 & PEM & Low & None & $\begin{array}{l}\text { No Cultural } \\
\text { Resources }\end{array}$ & $\begin{array}{l}\text { No further work } \\
\text { recommended }\end{array}$ & Mostly inundated \\
\hline WP5LI023 & 5.1 & PEM & Low & None & $\begin{array}{l}\text { No Cultural } \\
\text { Resources }\end{array}$ & $\begin{array}{l}\text { No further work } \\
\text { recommended }\end{array}$ & Mostly inundated \\
\hline WP5LI022 & 5.2 & PEM & Low & None & $\begin{array}{l}\text { No Cultural } \\
\text { Resources }\end{array}$ & $\begin{array}{l}\text { No further work } \\
\text { recommended }\end{array}$ & Mostly inundated \\
\hline WP5LI021 & 5.9 & PEM & Low & None & $\begin{array}{l}\text { No Cultural } \\
\text { Resources }\end{array}$ & $\begin{array}{l}\text { No further work } \\
\text { recommended }\end{array}$ & Mostly inundated \\
\hline WP5LI020 & 6.1 & PEM & Low & None & $\begin{array}{l}\text { No Cultural } \\
\text { Resources }\end{array}$ & $\begin{array}{l}\text { No further work } \\
\text { recommended }\end{array}$ & Mostly inundated \\
\hline WP5LI019 & 6.3 & $\mathrm{PFO}$ & Low & None & $\begin{array}{l}\text { No Cultural } \\
\text { Resources }\end{array}$ & $\begin{array}{l}\text { No further work } \\
\text { recommended }\end{array}$ & Mostly inundated \\
\hline WP6LI003 & 6.6 & PEM & Low & None & $\begin{array}{c}\text { No Cultural } \\
\text { Resources }\end{array}$ & $\begin{array}{l}\text { No further work } \\
\text { recommended }\end{array}$ & Mostly inundated \\
\hline
\end{tabular}


Appendix C 1- Survey Data in the Vicinity of Wetland Features

\begin{tabular}{|c|c|c|c|c|c|c|c|}
\hline Wetland ID & $\begin{array}{c}\text { Milepost } \\
\text { (Approximate) }\end{array}$ & $\begin{array}{l}\text { Wetland } \\
\text { Type }\end{array}$ & Probability & Shovel Tests & $\begin{array}{l}\text { Surveys } \\
\text { Results }\end{array}$ & Recommendations & Comments \\
\hline WP6LI002_PFO & 6.7 & $\mathrm{PFO}$ & Low & None & $\begin{array}{l}\text { No Cultural } \\
\text { Resources }\end{array}$ & $\begin{array}{l}\text { No further work } \\
\text { recommended }\end{array}$ & Mostly inundated \\
\hline WP6LI002_PFO_B & 6.7 & $\mathrm{PFO}$ & Low & None & $\begin{array}{l}\text { No Cultural } \\
\text { Resources }\end{array}$ & $\begin{array}{l}\text { No further work } \\
\text { recommended }\end{array}$ & Mostly inundated \\
\hline WP6LI002_PEM & 6.8 & PEM & Low & None & $\begin{array}{l}\text { No Cultural } \\
\text { Resources }\end{array}$ & $\begin{array}{l}\text { No further work } \\
\text { recommended }\end{array}$ & Mostly inundated \\
\hline WP6LI001 & 7 & PEM & Low & None & $\begin{array}{l}\text { No Cultural } \\
\text { Resources }\end{array}$ & $\begin{array}{l}\text { No further work } \\
\text { recommended }\end{array}$ & Mostly inundated \\
\hline WP6LI001_PFO & 7.3 & $\mathrm{PFO}$ & Low & None & $\begin{array}{l}\text { No Cultural } \\
\text { Resources }\end{array}$ & $\begin{array}{l}\text { No further work } \\
\text { recommended }\end{array}$ & $\begin{array}{l}\text { Partially avoided via non- } \\
\text { Section } 10 \mathrm{HDD} / \text { Bore }\end{array}$ \\
\hline WP6LI001_PSS & 7.3 & PSS & Low & None & $\begin{array}{l}\text { No Cultural } \\
\text { Resources }\end{array}$ & $\begin{array}{l}\text { No further work } \\
\text { recommended }\end{array}$ & $\begin{array}{l}\text { Partially avoided via non- } \\
\text { Section } 10 \text { HDD/Bore }\end{array}$ \\
\hline WP2LI066_PEM & 7.4 & PEM & Low & None & $\begin{array}{l}\text { No Cultural } \\
\text { Resources }\end{array}$ & $\begin{array}{l}\text { No further work } \\
\text { recommended }\end{array}$ & $\begin{array}{l}\text { Partially avoided via non- } \\
\text { Section } 10 \mathrm{HDD} / \text { Bore }\end{array}$ \\
\hline WP2LI066_PFO & 7.4 & $\mathrm{PFO}$ & Low & None & $\begin{array}{l}\text { No Cultural } \\
\text { Resources }\end{array}$ & $\begin{array}{l}\text { No further work } \\
\text { recommended }\end{array}$ & Mostly inundated \\
\hline WP2LI067 & 7.8 & PEM & Low & None & $\begin{array}{l}\text { No Cultural } \\
\text { Resources }\end{array}$ & $\begin{array}{l}\text { No further work } \\
\text { recommended }\end{array}$ & Mostly inundated \\
\hline WP2LI068 & 8.2 & PEM & Low & None & $\begin{array}{l}\text { No Cultural } \\
\text { Resources }\end{array}$ & $\begin{array}{l}\text { No further work } \\
\text { recommended }\end{array}$ & Mostly inundated \\
\hline WP2LI069 & 8.9 & PEM & Low & None & $\begin{array}{l}\text { No Cultural } \\
\text { Resources }\end{array}$ & $\begin{array}{l}\text { No further work } \\
\text { recommended }\end{array}$ & Mostly inundated \\
\hline WP2LI070 & 9.5 & PEM & Low & 14 & $\begin{array}{l}\text { No Cultural } \\
\text { Resources }\end{array}$ & $\begin{array}{l}\text { No further work } \\
\text { recommended }\end{array}$ & Inundation \\
\hline WP6LI006 & 9.9 & PEM & Low & 3 & $\begin{array}{l}\text { No Cultural } \\
\text { Resources }\end{array}$ & $\begin{array}{l}\text { No further work } \\
\text { recommended }\end{array}$ & $\begin{array}{l}\text { Partially avoided via non- } \\
\text { Section } 10 \mathrm{HDD} / \text { Bore }\end{array}$ \\
\hline WP6LI004 & 10.3 & PEM & Low & None & $\begin{array}{l}\text { No Cultural } \\
\text { Resources }\end{array}$ & $\begin{array}{l}\text { No further work } \\
\text { recommended }\end{array}$ & $\begin{array}{c}\text { Located Within Heavily } \\
\text { Inundated Area }\end{array}$ \\
\hline WP6LI005 & 10.3 & PSS & Low & None & $\begin{array}{l}\text { No Cultural } \\
\text { Resources }\end{array}$ & $\begin{array}{l}\text { No further work } \\
\text { recommended }\end{array}$ & $\begin{array}{l}\text { Located Within Heavily } \\
\text { Inundated Area }\end{array}$ \\
\hline WP3LI028_PEM & 10.8 & PEM & Low & None & $\begin{array}{l}\text { No Cultural } \\
\text { Resources }\end{array}$ & $\begin{array}{l}\text { No further work } \\
\text { recommended }\end{array}$ & $\begin{array}{l}\text { Located Within Heavily } \\
\text { Inundated Area }\end{array}$ \\
\hline
\end{tabular}


Appendix C 1- Survey Data in the Vicinity of Wetland Features

\begin{tabular}{|c|c|c|c|c|c|c|c|}
\hline Wetland ID & $\begin{array}{c}\text { Milepost } \\
\text { (Approximate) }\end{array}$ & $\begin{array}{l}\text { Wetland } \\
\text { Type }\end{array}$ & Probability & Shovel Tests & $\begin{array}{l}\text { Surveys } \\
\text { Results }\end{array}$ & Recommendations & Comments \\
\hline WP3LI029 & 10.8 & PEM & Low & None & $\begin{array}{l}\text { No Cultural } \\
\text { Resources }\end{array}$ & $\begin{array}{l}\text { No further work } \\
\text { recommended }\end{array}$ & $\begin{array}{l}\text { Located Within Heavily } \\
\text { Inundated Area }\end{array}$ \\
\hline WP3LI026 & 10.9 & PEM & Low & None & $\begin{array}{l}\text { No Cultural } \\
\text { Resources }\end{array}$ & $\begin{array}{l}\text { No further work } \\
\text { recommended }\end{array}$ & $\begin{array}{l}\text { Located Within Heavily } \\
\text { Inundated Area }\end{array}$ \\
\hline WP3LI027_PEM & 10.9 & PEM & Low & None & $\begin{array}{l}\text { No Cultural } \\
\text { Resources }\end{array}$ & $\begin{array}{l}\text { No further work } \\
\text { recommended }\end{array}$ & $\begin{array}{l}\text { Located Within Heavily } \\
\text { Inundated Area }\end{array}$ \\
\hline WP3LI027_PSS & 10.9 & PSS & Low & None & $\begin{array}{l}\text { No Cultural } \\
\text { Resources }\end{array}$ & $\begin{array}{l}\text { No further work } \\
\text { recommended }\end{array}$ & $\begin{array}{c}\text { Located Within Heavily } \\
\text { Inundated Area }\end{array}$ \\
\hline WP3LI028_PSS & 10.9 & PSS & Low & None & $\begin{array}{c}\text { No Cultural } \\
\text { Resources }\end{array}$ & $\begin{array}{l}\text { No further work } \\
\text { recommended }\end{array}$ & $\begin{array}{c}\text { Located Within Heavily } \\
\text { Inundated Area }\end{array}$ \\
\hline WP3LI013 & 12.5 & PEM & Low & None & $\begin{array}{l}\text { No Cultural } \\
\text { Resources }\end{array}$ & $\begin{array}{l}\text { No further work } \\
\text { recommended }\end{array}$ & $\begin{array}{l}\text { Partially avoided via non- } \\
\text { Section } 10 \mathrm{HDD} / \text { Bore }\end{array}$ \\
\hline WP3LI014 & 12.5 & PEM & Low & None & $\begin{array}{l}\text { No Cultural } \\
\text { Resources }\end{array}$ & $\begin{array}{l}\text { No further work } \\
\text { recommended }\end{array}$ & $\begin{array}{c}\text { conducted survey and mostly } \\
\text { inundated }\end{array}$ \\
\hline WP3LI015 & 12.7 & PEM & Low & None & $\begin{array}{l}\text { No Cultural } \\
\text { Resources }\end{array}$ & $\begin{array}{l}\text { No further work } \\
\text { recommended }\end{array}$ & $\begin{array}{l}\text { Located Within Heavily } \\
\text { Inundated Area }\end{array}$ \\
\hline WP3LI016 & 13 & PEM & Low & None & $\begin{array}{l}\text { No Cultural } \\
\text { Resources }\end{array}$ & $\begin{array}{l}\text { No further work } \\
\text { recommended }\end{array}$ & $\begin{array}{l}\text { Located Within Heavily } \\
\text { Inundated Area }\end{array}$ \\
\hline WP3LI017 & 13.3 & PEM & Low & None & $\begin{array}{l}\text { No Cultural } \\
\text { Resources }\end{array}$ & $\begin{array}{l}\text { No further work } \\
\text { recommended }\end{array}$ & $\begin{array}{l}\text { Located Within Heavily } \\
\text { Inundated Area }\end{array}$ \\
\hline WP3LI018 & 13.9 & PEM & Low & 2 & $\begin{array}{l}\text { No Cultural } \\
\text { Resources }\end{array}$ & $\begin{array}{l}\text { No further work } \\
\text { recommended }\end{array}$ & Inundation \\
\hline WP3LI019 & 14 & PEM & Low & 2 & $\begin{array}{l}\text { No Cultural } \\
\text { Resources }\end{array}$ & $\begin{array}{l}\text { No further work } \\
\text { recommended }\end{array}$ & None \\
\hline WP3LI020 & 14.1 & PEM & Low & 1 & $\begin{array}{l}\text { No Cultural } \\
\text { Resources }\end{array}$ & $\begin{array}{l}\text { No further work } \\
\text { recommended }\end{array}$ & None \\
\hline WP3LI021 & 14.1 & PEM & Low & None & $\begin{array}{c}\text { No Cultural } \\
\text { Resources }\end{array}$ & $\begin{array}{l}\text { No further work } \\
\text { recommended }\end{array}$ & $\begin{array}{c}\text { Located Within Heavily } \\
\text { Inundated Area }\end{array}$ \\
\hline WP3LI022 & 14.1 & PEM & Low & 1 & $\begin{array}{l}\text { No Cultural } \\
\text { Resources }\end{array}$ & $\begin{array}{l}\text { No further work } \\
\text { recommended }\end{array}$ & None \\
\hline WP3LI023 & 14.1 & PEM & Low & 1 & $\begin{array}{l}\text { No Cultural } \\
\text { Resources }\end{array}$ & $\begin{array}{l}\text { No further work } \\
\text { recommended }\end{array}$ & None \\
\hline
\end{tabular}


Appendix C 1- Survey Data in the Vicinity of Wetland Features

\begin{tabular}{|c|c|c|c|c|c|c|c|}
\hline Wetland ID & $\begin{array}{c}\text { Milepost } \\
\text { (Approximate) }\end{array}$ & $\begin{array}{l}\text { Wetland } \\
\text { Type }\end{array}$ & Probability & Shovel Tests & $\begin{array}{l}\text { Surveys } \\
\text { Results }\end{array}$ & Recommendations & Comments \\
\hline WP3LI024 & 14.2 & PEM & Low & None & $\begin{array}{l}\text { No Cultural } \\
\text { Resources }\end{array}$ & $\begin{array}{l}\text { No further work } \\
\text { recommended }\end{array}$ & $\begin{array}{c}\text { Located Within Heavily } \\
\text { Inundated Area }\end{array}$ \\
\hline WP3LI025 & 14.3 & PEM & Low & None & $\begin{array}{l}\text { No Cultural } \\
\text { Resources }\end{array}$ & $\begin{array}{l}\text { No further work } \\
\text { recommended }\end{array}$ & $\begin{array}{l}\text { Completely avoided via non- } \\
\text { Section } 10 \text { HDD/Bore }\end{array}$ \\
\hline WP2LI010_PEM & 14.5 & PEM & Low & None & $\begin{array}{l}\text { No Cultural } \\
\text { Resources }\end{array}$ & $\begin{array}{l}\text { No further work } \\
\text { recommended }\end{array}$ & $\begin{array}{l}\text { Partially avoided via non- } \\
\text { Section } 10 \text { HDD/Bore }\end{array}$ \\
\hline WP2LI010_PSS & 14.5 & PSS & Low & None & $\begin{array}{l}\text { No Cultural } \\
\text { Resources }\end{array}$ & $\begin{array}{l}\text { No further work } \\
\text { recommended }\end{array}$ & $\begin{array}{c}\text { Located Within Heavily } \\
\text { Inundated Area }\end{array}$ \\
\hline WP2LI010_PSS_B & 14.6 & PSS & Low & None & $\begin{array}{l}\text { No Cultural } \\
\text { Resources }\end{array}$ & $\begin{array}{l}\text { No further work } \\
\text { recommended }\end{array}$ & $\begin{array}{l}\text { Located Within Heavily } \\
\text { Inundated Area }\end{array}$ \\
\hline WP2LI010_PEM_B & 14.7 & PEM & Low & None & $\begin{array}{l}\text { No Cultural } \\
\text { Resources }\end{array}$ & $\begin{array}{l}\text { No further work } \\
\text { recommended }\end{array}$ & $\begin{array}{l}\text { Located Within Heavily } \\
\text { Inundated Area }\end{array}$ \\
\hline WP2LI010_PSS_C & 14.7 & PSS & Low & None & $\begin{array}{l}\text { No Cultural } \\
\text { Resources }\end{array}$ & $\begin{array}{l}\text { No further work } \\
\text { recommended }\end{array}$ & $\begin{array}{l}\text { Located Within Heavily } \\
\text { Inundated Area }\end{array}$ \\
\hline WP2LI010_PEM_C & 14.8 & PEM & Low & None & $\begin{array}{l}\text { No Cultural } \\
\text { Resources }\end{array}$ & $\begin{array}{l}\text { No further work } \\
\text { recommended }\end{array}$ & Mostly inundated \\
\hline WP2LI011 & 15.6 & PEM & Low & None & $\begin{array}{l}\text { No Cultural } \\
\text { Resources }\end{array}$ & $\begin{array}{l}\text { No further work } \\
\text { recommended }\end{array}$ & Mostly inundated \\
\hline WP2LI010_PFO & 15.6 & $\mathrm{PFO}$ & Low & None & $\begin{array}{l}\text { No Cultural } \\
\text { Resources }\end{array}$ & $\begin{array}{l}\text { No further work } \\
\text { recommended }\end{array}$ & Mostly inundated \\
\hline WP2LI012 & 15.7 & PEM & Low & None & $\begin{array}{l}\text { No Cultural } \\
\text { Resources }\end{array}$ & $\begin{array}{l}\text { No further work } \\
\text { recommended }\end{array}$ & Mostly inundated \\
\hline WP2LI013_PEM & 15.7 & PEM & Low & None & $\begin{array}{c}\text { No Cultural } \\
\text { Resources }\end{array}$ & $\begin{array}{l}\text { No further work } \\
\text { recommended }\end{array}$ & Mostly inundated \\
\hline WP2LI013_PSS & 15.7 & PSS & Low & None & $\begin{array}{c}\text { No Cultural } \\
\text { Resources }\end{array}$ & $\begin{array}{l}\text { No further work } \\
\text { recommended }\end{array}$ & Mostly inundated \\
\hline WP2LI014_PFO & 15.8 & $\mathrm{PFO}$ & Low & None & $\begin{array}{c}\text { No Cultural } \\
\text { Resources }\end{array}$ & $\begin{array}{l}\text { No further work } \\
\text { recommended }\end{array}$ & Mostly inundated \\
\hline WP4LI010_PEM & 15.9 & PEM & Low & None & $\begin{array}{c}\text { No Cultural } \\
\text { Resources }\end{array}$ & $\begin{array}{l}\text { No further work } \\
\text { recommended }\end{array}$ & Mostly inundated \\
\hline
\end{tabular}


Appendix C 1- Survey Data in the Vicinity of Wetland Features

\begin{tabular}{|c|c|c|c|c|c|c|c|}
\hline Wetland ID & $\begin{array}{c}\text { Milepost } \\
\text { (Approximate) }\end{array}$ & $\begin{array}{l}\text { Wetland } \\
\text { Type }\end{array}$ & Probability & Shovel Tests & $\begin{array}{l}\text { Surveys } \\
\text { Results }\end{array}$ & Recommendations & Comments \\
\hline WP4LI010_PSS & 15.9 & PSS & Low & None & $\begin{array}{l}\text { No Cultural } \\
\text { Resources }\end{array}$ & $\begin{array}{l}\text { No further work } \\
\text { recommended }\end{array}$ & Mostly inundated \\
\hline WP4LI009_PFO_D & 16 & PFO & Low & None & $\begin{array}{l}\text { No Cultural } \\
\text { Resources }\end{array}$ & $\begin{array}{l}\text { No further work } \\
\text { recommended }\end{array}$ & Mostly inundated \\
\hline WP4LI009_PEM & 16.3 & PEM & Medium & 8 & $\begin{array}{l}\text { No Cultural } \\
\text { Resources }\end{array}$ & $\begin{array}{l}\text { No further work } \\
\text { recommended }\end{array}$ & $\begin{array}{l}\text { Completely avoided via non- } \\
\text { Section } 10 \mathrm{HDD} / \text { Bore }\end{array}$ \\
\hline WP4LI009_PFO_C & 16.1 & PFO & Medium & None & $\begin{array}{l}\text { No Cultural } \\
\text { Resources }\end{array}$ & $\begin{array}{l}\text { No further work } \\
\text { recommended }\end{array}$ & Mostly inundated \\
\hline WP4LI009_PFO_B & 16.2 & PFO & Medium & 3 & $\begin{array}{l}\text { No Cultural } \\
\text { Resources }\end{array}$ & $\begin{array}{l}\text { No further work } \\
\text { recommended }\end{array}$ & None \\
\hline WP4LI008_PEM & 16.3 & PEM & Medium & 1 & $\begin{array}{l}\text { No Cultural } \\
\text { Resources }\end{array}$ & $\begin{array}{l}\text { No further work } \\
\text { recommended }\end{array}$ & $\begin{array}{l}\text { No Impacts - Completely } \\
\text { avoided via Section } 10 \\
\text { HDD/Bore }\end{array}$ \\
\hline WP4LI008_PFO & 16.3 & $\mathrm{PFO}$ & Medium & 1 & $\begin{array}{l}\text { No Cultural } \\
\text { Resources }\end{array}$ & $\begin{array}{l}\text { No further work } \\
\text { recommended }\end{array}$ & $\begin{array}{l}\text { No Impacts - Completely } \\
\text { avoided via Section } 10 \\
\text { HDD/Bore }\end{array}$ \\
\hline WP4LI009_PFO & 16.4 & PFO & Medium & 2 & $\begin{array}{l}\text { No Cultural } \\
\text { Resources }\end{array}$ & $\begin{array}{l}\text { No further work } \\
\text { recommended }\end{array}$ & $\begin{array}{c}\text { No Impacts - Completely } \\
\text { avoided via Section } 10 \\
\text { HDD/Bore }\end{array}$ \\
\hline WP4LI001_PFO & 16.6 & PFO & High & 34 & $\begin{array}{l}\text { No Cultural } \\
\text { Resources }\end{array}$ & $\begin{array}{l}\text { No further work } \\
\text { recommended }\end{array}$ & $\begin{array}{c}\text { No Impacts - Completely } \\
\text { avoided via Section } 10 \\
\text { HDD/Bore }\end{array}$ \\
\hline WP4LI001_PEM & 16.7 & PEM & High & 24 & $\begin{array}{l}\text { No Cultural } \\
\text { Resources }\end{array}$ & $\begin{array}{l}\text { No further work } \\
\text { recommended }\end{array}$ & $\begin{array}{c}\text { Aocated in Heavily Inundated } \\
\text { Area within Trinity River } \\
\text { Floodplain }\end{array}$ \\
\hline WP4LI001_PFO_CYP & 16.6 & PFO_CYP & High & 4 & $\begin{array}{l}\text { No Cultural } \\
\text { Resources }\end{array}$ & $\begin{array}{l}\text { No further work } \\
\text { recommended }\end{array}$ & $\begin{array}{l}\text { No Impacts - Completely } \\
\text { avoided via Section } 10 \\
\text { HDD/Bore }\end{array}$ \\
\hline WP4LI001_PFO_C & 16.7 & PFO & High & 3 & $\begin{array}{l}\text { No Cultural } \\
\text { Resources }\end{array}$ & $\begin{array}{l}\text { No further work } \\
\text { recommended }\end{array}$ & $\begin{array}{c}\text { No Impacts - Completely } \\
\text { avoided via Section } 10 \\
\text { HDD/Bore }\end{array}$ \\
\hline
\end{tabular}


Appendix C 1- Survey Data in the Vicinity of Wetland Features

\begin{tabular}{|c|c|c|c|c|c|c|c|}
\hline Wetland ID & $\begin{array}{c}\text { Milepost } \\
\text { (Approximate) }\end{array}$ & $\begin{array}{l}\text { Wetland } \\
\text { Type }\end{array}$ & Probability & Shovel Tests & $\begin{array}{l}\text { Surveys } \\
\text { Results }\end{array}$ & Recommendations & Comments \\
\hline WP4LI002_PFO & 16.9 & $\mathrm{PFO}$ & High & 7 & $\begin{array}{l}\text { No Cultural } \\
\text { Resources }\end{array}$ & $\begin{array}{c}\text { Archeological Monitor } \\
\text { Needed }\end{array}$ & $\begin{array}{c}\text { Located in Heavily Inundated } \\
\text { Area within Trinity River } \\
\text { Floodplain }\end{array}$ \\
\hline WP4LI002_PFO_B & 17 & $\mathrm{PFO}$ & High & 6 & $\begin{array}{l}\text { No Cultural } \\
\text { Resources }\end{array}$ & $\begin{array}{l}\text { No further work } \\
\text { recommended }\end{array}$ & $\begin{array}{c}\text { Located in Heavily Inundated } \\
\text { Area within Trinity River } \\
\text { Floodplain }\end{array}$ \\
\hline WP4LI002_PSS & 17.1 & PSS & High & 9 & $\begin{array}{l}\text { No Cultural } \\
\text { Resources }\end{array}$ & $\begin{array}{l}\text { No further work } \\
\text { recommended }\end{array}$ & $\begin{array}{c}\text { Located in Heavily Inundated } \\
\text { Area within Trinity River } \\
\text { Floodplain }\end{array}$ \\
\hline WP4LI002_PFO_C & 17.2 & $\mathrm{PFO}$ & High & 26 & $\begin{array}{l}\text { No Cultural } \\
\text { Resources }\end{array}$ & $\begin{array}{c}\text { Archeological Monitor } \\
\text { Needed }\end{array}$ & $\begin{array}{c}\text { Located in Heavily Inundated } \\
\text { Area within Trinity River } \\
\text { Floodplain }\end{array}$ \\
\hline WP4LI002_PEM & 17.8 & PEM & High & 23 & $\begin{array}{l}\text { No Cultural } \\
\text { Resources }\end{array}$ & $\begin{array}{l}\text { No further work } \\
\text { recommended }\end{array}$ & $\begin{array}{c}\text { Located in Heavily Inundated } \\
\text { Area within Trinity River } \\
\text { Floodplain }\end{array}$ \\
\hline WP3LI007_PEM & 17.9 & PEM & High & 6 & $\begin{array}{l}\text { No Cultural } \\
\text { Resources }\end{array}$ & $\begin{array}{l}\text { No further work } \\
\text { recommended }\end{array}$ & $\begin{array}{c}\text { Located in Heavily Inundated } \\
\text { Area within Trinity River } \\
\text { Floodplain }\end{array}$ \\
\hline WP3LI007_PFO & 17.9 & PFO & High & 13 & $\begin{array}{l}\text { No Cultural } \\
\text { Resources }\end{array}$ & $\begin{array}{l}\text { No further work } \\
\text { recommended }\end{array}$ & $\begin{array}{c}\text { Area within Trinity River } \\
\text { Floodplain }\end{array}$ \\
\hline WP3LI006_PSS & 18.3 & PSS & High & None & $\begin{array}{l}\text { No Cultural } \\
\text { Resources }\end{array}$ & $\begin{array}{l}\text { No further work } \\
\text { recommended }\end{array}$ & Heavily Inundated \\
\hline WP3LI006_PEM & 18.4 & PEM & High & None & $\begin{array}{l}\text { No Cultural } \\
\text { Resources }\end{array}$ & $\begin{array}{l}\text { No further work } \\
\text { recommended }\end{array}$ & $\begin{array}{c}\text { Area within Trinity River } \\
\text { Floodplain }\end{array}$ \\
\hline WP3LI006_PEM_B & 18.4 & PEM & High & 1 & $\begin{array}{l}\text { No Cultural } \\
\text { Resources }\end{array}$ & $\begin{array}{l}\text { No further work } \\
\text { recommended }\end{array}$ & $\begin{array}{c}\text { Located in Heavily Inundated } \\
\text { Area within Trinity River } \\
\text { Floodplain }\end{array}$ \\
\hline WP3LI006_PFO & 18.6 & PFO & High & 2 & $\begin{array}{l}\text { No Cultural } \\
\text { Resources }\end{array}$ & $\begin{array}{l}\text { No further work } \\
\text { recommended }\end{array}$ & $\begin{array}{c}\text { Located in Heavily Inundated } \\
\text { Area within Trinity River } \\
\text { Floodplain }\end{array}$ \\
\hline
\end{tabular}


Appendix C 1- Survey Data in the Vicinity of Wetland Features

\begin{tabular}{|c|c|c|c|c|c|c|c|}
\hline Wetland ID & $\begin{array}{c}\text { Milepost } \\
\text { (Approximate) }\end{array}$ & $\begin{array}{c}\text { Wetland } \\
\text { Type }\end{array}$ & Probability & Shovel Tests & $\begin{array}{l}\text { Surveys } \\
\text { Results }\end{array}$ & Recommendations & Comments \\
\hline WP3LI001 & 19 & PSS & High & None & $\begin{array}{l}\text { No Cultural } \\
\text { Resources }\end{array}$ & $\begin{array}{l}\text { No further work } \\
\text { recommended }\end{array}$ & $\begin{array}{l}\text { Completely avoided via non- } \\
\text { Section } 10 \mathrm{HDD} / \text { Bore; } \\
\text { Outside of workspace }\end{array}$ \\
\hline WP3LI002_PSS & 19 & PSS & High & None & $\begin{array}{c}\text { No Cultural } \\
\text { Resources }\end{array}$ & $\begin{array}{l}\text { No further work } \\
\text { recommended }\end{array}$ & $\begin{array}{l}\text { Completely avoided via non- } \\
\text { Section } 10 \mathrm{HDD} / \text { Bore }\end{array}$ \\
\hline WP3LI003 & 19 & PSS & High & None & $\begin{array}{l}\text { No Cultural } \\
\text { Resources }\end{array}$ & $\begin{array}{l}\text { No further work } \\
\text { recommended }\end{array}$ & $\begin{array}{l}\text { Completely avoided via non- } \\
\text { Section } 10 \text { HDD/Bore; } \\
\text { Outside of workspace }\end{array}$ \\
\hline WP9LI002_PSS_DT ${ }^{\text {a }}$ & 19 & PSS & High & None & $\begin{array}{c}\text { No Cultural } \\
\text { Resources }\end{array}$ & $\begin{array}{l}\text { No further work } \\
\text { recommended }\end{array}$ & $\begin{array}{l}\text { Completely avoided via non- } \\
\text { Section } 10 \text { HDD/Bore; } \\
\text { Outside of workspace }\end{array}$ \\
\hline WP3LI002_PEM & 19.1 & PEM & High & None & $\begin{array}{c}\text { No Cultural } \\
\text { Resources }\end{array}$ & $\begin{array}{l}\text { No further work } \\
\text { recommended }\end{array}$ & $\begin{array}{l}\text { Completely avoided via non- } \\
\text { Section } 10 \mathrm{HDD} / \text { Bore }\end{array}$ \\
\hline WP3LI002_PFO & 19.1 & $\mathrm{PFO}$ & High & None & $\begin{array}{l}\text { No Cultural } \\
\text { Resources }\end{array}$ & $\begin{array}{l}\text { No further work } \\
\text { recommended }\end{array}$ & $\begin{array}{l}\text { Completely avolded via non } \\
\text { Section } 10 \text { HDD/Bore; } \\
\text { Outside of workspace }\end{array}$ \\
\hline WP3LI005_PFO_CYP & 19.1 & PFO_CYP & High & None & $\begin{array}{l}\text { No Cultural } \\
\text { Resources }\end{array}$ & $\begin{array}{l}\text { No further work } \\
\text { recommended }\end{array}$ & $\begin{array}{l}\text { Completely avoided via non- } \\
\text { Section } 10 \mathrm{HDD} / \text { Bore }\end{array}$ \\
\hline WP3LI002_PSS_B & 19.1 & PSS & High & None & $\begin{array}{l}\text { No Cultural } \\
\text { Resources }\end{array}$ & $\begin{array}{l}\text { No further work } \\
\text { recommended }\end{array}$ & $\begin{array}{l}\text { Completely avoided via non- } \\
\text { Section } 10 \mathrm{HDD} / \text { Bore }\end{array}$ \\
\hline WP3LI004 & 19.1 & PSS & High & None & $\begin{array}{l}\text { No Cultural } \\
\text { Resources }\end{array}$ & $\begin{array}{l}\text { No further work } \\
\text { recommended }\end{array}$ & $\begin{array}{l}\text { Completely avoided via non- } \\
\text { Section } 10 \mathrm{HDD} / \text { Bore }\end{array}$ \\
\hline WP3LI005_PEM & 19.2 & PEM & High & None & $\begin{array}{l}\text { No Cultural } \\
\text { Resources }\end{array}$ & $\begin{array}{l}\text { No further work } \\
\text { recommended }\end{array}$ & $\begin{array}{l}\text { Completely avoided via non- } \\
\text { Section } 10 \mathrm{HDD} / \text { Bore }\end{array}$ \\
\hline $\begin{array}{c}\text { WP3LI005_PFO_CYP } \\
\text { _B }\end{array}$ & 19.2 & PFO_CYP & High & 2 & $\begin{array}{l}\text { No Cultural } \\
\text { Resources }\end{array}$ & $\begin{array}{l}\text { No further work } \\
\text { recommended }\end{array}$ & $\begin{array}{l}\text { Completely avoided via non- } \\
\text { Section } 10 \mathrm{HDD} / \text { Bore }\end{array}$ \\
\hline WP3LI005_PSS & 19.2 & PSS & High & None & $\begin{array}{c}\text { No Cultural } \\
\text { Resources }\end{array}$ & $\begin{array}{l}\text { No further work } \\
\text { recommended }\end{array}$ & $\begin{array}{l}\text { Section } 10 \text { HDD/Bore; } \\
\text { Outside of workspace }\end{array}$ \\
\hline WP4LI003_PEM_B & 19.3 & PEM & High & None & $\begin{array}{l}\text { No Cultural } \\
\text { Resources }\end{array}$ & $\begin{array}{l}\text { No further work } \\
\text { recommended }\end{array}$ & $\begin{array}{l}\text { Completely avoided via non- } \\
\text { Section } 10 \mathrm{HDD} / \text { Bore }\end{array}$ \\
\hline WP3LI010 & 19.3 & PSS & High & 1 & $\begin{array}{c}\text { No Cultural } \\
\text { Resources }\end{array}$ & $\begin{array}{l}\text { No further work } \\
\text { recommended }\end{array}$ & None \\
\hline
\end{tabular}


Appendix C 1- Survey Data in the Vicinity of Wetland Features

\begin{tabular}{|c|c|c|c|c|c|c|c|}
\hline Wetland ID & $\begin{array}{c}\text { Milepost } \\
\text { (Approximate) }\end{array}$ & $\begin{array}{c}\text { Wetland } \\
\text { Type }\end{array}$ & Probability & Shovel Tests & $\begin{array}{l}\text { Surveys } \\
\text { Results }\end{array}$ & Recommendations & Comments \\
\hline WP4LI003_PEM_C & 19.4 & PEM & High & 2 & $\begin{array}{l}\text { No Cultural } \\
\text { Resources }\end{array}$ & $\begin{array}{l}\text { No further work } \\
\text { recommended }\end{array}$ & None \\
\hline WP4LI003_PFO & 19.4 & $\mathrm{PFO}$ & High & 5 & $\begin{array}{l}\text { No Cultural } \\
\text { Resources }\end{array}$ & $\begin{array}{l}\text { No further work } \\
\text { recommended }\end{array}$ & None \\
\hline WP4LI003_PEM & 19.5 & PEM & High & 8 & $\begin{array}{l}\text { No Cultural } \\
\text { Resources }\end{array}$ & $\begin{array}{l}\text { No further work } \\
\text { recommended }\end{array}$ & None \\
\hline WP4LI004_PFO & 19.7 & $\mathrm{PFO}$ & High & 2 & $\begin{array}{l}\text { No Cultural } \\
\text { Resources }\end{array}$ & $\begin{array}{l}\text { No further work } \\
\text { recommended }\end{array}$ & None \\
\hline WP4LI004_PEM_B & 19.8 & PEM & High & 5 & $\begin{array}{l}\text { No Cultural } \\
\text { Resources }\end{array}$ & $\begin{array}{l}\text { No further work } \\
\text { recommended }\end{array}$ & None \\
\hline WP4LI004_PEM_C & 19.8 & PEM & High & 4 & $\begin{array}{l}\text { No Cultural } \\
\text { Resources }\end{array}$ & $\begin{array}{l}\text { No further work } \\
\text { recommended }\end{array}$ & None \\
\hline WP4LI004_PEM_D & 19.8 & PEM & High & 3 & $\begin{array}{l}\text { No Cultural } \\
\text { Resources }\end{array}$ & $\begin{array}{l}\text { No further work } \\
\text { recommended }\end{array}$ & None \\
\hline WP4LI004_PFO_B & 19.8 & $\mathrm{PFO}$ & High & 7 & $\begin{array}{l}\text { No Cultural } \\
\text { Resources }\end{array}$ & $\begin{array}{l}\text { No further work } \\
\text { recommended }\end{array}$ & Inundation \\
\hline WP4LI005_PFO & 19.8 & $\mathrm{PFO}$ & High & 3 & $\begin{array}{l}\text { No Cultural } \\
\text { Resources }\end{array}$ & $\begin{array}{l}\text { No further work } \\
\text { recommended }\end{array}$ & None \\
\hline WP4LI004_PEM & 19.9 & PEM & High & 2 & $\begin{array}{l}\text { No Cultural } \\
\text { Resources }\end{array}$ & $\begin{array}{l}\text { No further work } \\
\text { recommended }\end{array}$ & None \\
\hline WP4LI006_PEM & 19.9 & PEM & High & 3 & $\begin{array}{l}\text { No Cultural } \\
\text { Resources }\end{array}$ & $\begin{array}{l}\text { No further work } \\
\text { recommended }\end{array}$ & None \\
\hline WP4LI006_PFO & 19.9 & $\mathrm{PFO}$ & High & 2 & $\begin{array}{l}\text { No Cultural } \\
\text { Resources }\end{array}$ & $\begin{array}{l}\text { No further work } \\
\text { recommended }\end{array}$ & None \\
\hline WP4LI004_PFO_C & 20 & $\mathrm{PFO}$ & High & 3 & $\begin{array}{l}\text { No Cultural } \\
\text { Resources }\end{array}$ & $\begin{array}{l}\text { No further work } \\
\text { recommended }\end{array}$ & None \\
\hline WP4LI007_PFO & 20 & $\mathrm{PFO}$ & High & 1 & $\begin{array}{l}\text { No Cultural } \\
\text { Resources }\end{array}$ & $\begin{array}{l}\text { No further work } \\
\text { recommended }\end{array}$ & None \\
\hline WP4LI007_PEM & 20.1 & PEM & High & 4 & $\begin{array}{l}\text { No Cultural } \\
\text { Resources }\end{array}$ & $\begin{array}{l}\text { No further work } \\
\text { recommended }\end{array}$ & None \\
\hline WP3LI012 & 20.2 & PEM & High & 6 & $\begin{array}{l}\text { No Cultural } \\
\text { Resources }\end{array}$ & $\begin{array}{l}\text { No further work } \\
\text { recommended }\end{array}$ & None \\
\hline
\end{tabular}


Appendix C 1- Survey Data in the Vicinity of Wetland Features

\begin{tabular}{|c|c|c|c|c|c|c|c|}
\hline Wetland ID & $\begin{array}{c}\text { Milepost } \\
\text { (Approximate) }\end{array}$ & $\begin{array}{l}\text { Wetland } \\
\text { Type }\end{array}$ & Probability & Shovel Tests & $\begin{array}{l}\text { Surveys } \\
\text { Results }\end{array}$ & Recommendations & Comments \\
\hline WP3LI011_PEM_B & 20.3 & PEM & High & 7 & $\begin{array}{l}\text { No Cultural } \\
\text { Resources }\end{array}$ & $\begin{array}{l}\text { No further work } \\
\text { recommended }\end{array}$ & Inundation \\
\hline WP3LI011_PEM & 20.4 & PEM & High & 3 & $\begin{array}{l}\text { No Cultural } \\
\text { Resources }\end{array}$ & $\begin{array}{l}\text { No further work } \\
\text { recommended }\end{array}$ & None \\
\hline WP3LI011_PFO & 20.4 & PFO & High & 6 & $\begin{array}{l}\text { No Cultural } \\
\text { Resources }\end{array}$ & $\begin{array}{l}\text { No further work } \\
\text { recommended }\end{array}$ & None \\
\hline WP3LI010_PEM_D & 20.5 & PEM & Medium & None & $\begin{array}{l}\text { No Cultural } \\
\text { Resources }\end{array}$ & $\begin{array}{l}\text { No further work } \\
\text { recommended }\end{array}$ & $\begin{array}{c}\text { Located Within Heavily } \\
\text { Inundated Area }\end{array}$ \\
\hline WP3LI010_PSS_B & 20.5 & PSS & Medium & 1 & $\begin{array}{l}\text { No Cultural } \\
\text { Resources }\end{array}$ & $\begin{array}{l}\text { No further work } \\
\text { recommended }\end{array}$ & None \\
\hline WP3LI010_PFO_B & 20.6 & PFO & Medium & 13 & $\begin{array}{l}\text { No Cultural } \\
\text { Resources }\end{array}$ & $\begin{array}{l}\text { No further work } \\
\text { recommended }\end{array}$ & None \\
\hline WP3LI010_PEM_C & 20.7 & PEM & Medium & 3 & $\begin{array}{l}\text { No Cultural } \\
\text { Resources }\end{array}$ & $\begin{array}{l}\text { No further work } \\
\text { recommended }\end{array}$ & None \\
\hline WP3LI009 & 20.9 & PEM & Medium & 1 & $\begin{array}{l}\text { No Cultural } \\
\text { Resources }\end{array}$ & $\begin{array}{l}\text { No further work } \\
\text { recommended }\end{array}$ & None \\
\hline WP3LI010_PEM & 20.9 & PEM & Medium & 10 & $\begin{array}{l}\text { No Cultural } \\
\text { Resources }\end{array}$ & $\begin{array}{l}\text { No further work } \\
\text { recommended }\end{array}$ & None \\
\hline WP3LI010_PEM_B & 20.9 & PEM & Medium & 1 & $\begin{array}{l}\text { No Cultural } \\
\text { Resources }\end{array}$ & $\begin{array}{l}\text { No further work } \\
\text { recommended }\end{array}$ & None \\
\hline WP3LI010_PFO_CYP & 20.9 & PFO_CYP & Medium & 10 & $\begin{array}{l}\text { No Cultural } \\
\text { Resources }\end{array}$ & $\begin{array}{l}\text { No further work } \\
\text { recommended }\end{array}$ & None \\
\hline WP3LI010_PSS & 20.9 & PSS & Medium & 1 & $\begin{array}{l}\text { No Cultural } \\
\text { Resources }\end{array}$ & $\begin{array}{l}\text { No further work } \\
\text { recommended }\end{array}$ & None \\
\hline WP3LI008 & 21 & PFO & Medium & 1 & $\begin{array}{l}\text { No Cultural } \\
\text { Resources }\end{array}$ & $\begin{array}{l}\text { No further work } \\
\text { recommended }\end{array}$ & $\begin{array}{l}\text { Completely avoided via non- } \\
\text { Section } 10 \mathrm{HDD} / \text { Bore }\end{array}$ \\
\hline WP2LI015 & 21.2 & PEM & High & 31 & $\begin{array}{l}\text { No Cultural } \\
\text { Resources }\end{array}$ & $\begin{array}{l}\text { No further work } \\
\text { recommended }\end{array}$ & None \\
\hline WP2LI016 & 21.4 & PEM & High & 11 & $\begin{array}{l}\text { No Cultural } \\
\text { Resources }\end{array}$ & $\begin{array}{l}\text { No further work } \\
\text { recommended }\end{array}$ & None \\
\hline
\end{tabular}


Appendix C 1- Survey Data in the Vicinity of Wetland Features

\begin{tabular}{|c|c|c|c|c|c|c|c|}
\hline Wetland ID & $\begin{array}{c}\text { Milepost } \\
\text { (Approximate) }\end{array}$ & $\begin{array}{l}\text { Wetland } \\
\text { Type }\end{array}$ & Probability & Shovel Tests & $\begin{array}{l}\text { Surveys } \\
\text { Results }\end{array}$ & Recommendations & Comments \\
\hline WP2LI017 & 21.5 & PEM & High & 33 & $\begin{array}{l}\text { No Cultural } \\
\text { Resources }\end{array}$ & $\begin{array}{l}\text { No further work } \\
\text { recommended }\end{array}$ & Inundation \\
\hline WP4LI014 & 21.7 & PEM & High & 6 & $\begin{array}{l}\text { No Cultural } \\
\text { Resources }\end{array}$ & $\begin{array}{l}\text { No further work } \\
\text { recommended }\end{array}$ & None \\
\hline WP4LI013 & 21.8 & PEM & High & 8 & $\begin{array}{l}\text { No Cultural } \\
\text { Resources }\end{array}$ & $\begin{array}{l}\text { No further work } \\
\text { recommended }\end{array}$ & None \\
\hline WP4LI011_PEM & 22 & PEM & High & 23 & $\begin{array}{l}\text { No Cultural } \\
\text { Resources }\end{array}$ & $\begin{array}{l}\text { No further work } \\
\text { recommended }\end{array}$ & Inundation \\
\hline WP4LI011_PSS & 22 & PSS & High & None & $\begin{array}{l}\text { No Cultural } \\
\text { Resources }\end{array}$ & $\begin{array}{l}\text { No further work } \\
\text { recommended }\end{array}$ & $\begin{array}{l}\text { Located Within Heavily } \\
\text { Inundated Area }\end{array}$ \\
\hline WP4LI015_PEM & 23.8 & PEM & Low & None & $\begin{array}{l}\text { No Cultural } \\
\text { Resources }\end{array}$ & $\begin{array}{l}\text { No further work } \\
\text { recommended }\end{array}$ & $\begin{array}{c}\text { Completely avoided via non } \\
\text { Section } 10 \mathrm{HDD} / \text { Bore }\end{array}$ \\
\hline WP4LI015_PFO & 23.8 & $\mathrm{PFO}$ & Low & None & $\begin{array}{l}\text { No Cultural } \\
\text { Resources }\end{array}$ & $\begin{array}{l}\text { No further work } \\
\text { recommended }\end{array}$ & $\begin{array}{l}\text { Located Within Heavily } \\
\text { Inundated Area }\end{array}$ \\
\hline WP4LI015_PFO_B & 23.8 & PFO & Low & None & $\begin{array}{l}\text { No Cultural } \\
\text { Resources }\end{array}$ & $\begin{array}{l}\text { No further work } \\
\text { recommended }\end{array}$ & $\begin{array}{l}\text { Completely avoided via non } \\
\text { Section } 10 \mathrm{HDD} / \text { Bore }\end{array}$ \\
\hline WP4LI016 & 24 & PEM & Low & 5 & $\begin{array}{l}\text { No Cultural } \\
\text { Resources }\end{array}$ & $\begin{array}{l}\text { No further work } \\
\text { recommended }\end{array}$ & Inundation \\
\hline WP2LI019 & 24.2 & PEM & Low & None & $\begin{array}{l}\text { No Cultural } \\
\text { Resources }\end{array}$ & $\begin{array}{l}\text { No further work } \\
\text { recommended }\end{array}$ & $\begin{array}{l}\text { Located Within Heavily } \\
\text { Inundated Area }\end{array}$ \\
\hline WP2LI018 & 24.3 & PEM & Low & None & $\begin{array}{l}\text { No Cultural } \\
\text { Resources }\end{array}$ & $\begin{array}{l}\text { No further work } \\
\text { recommended }\end{array}$ & $\begin{array}{l}\text { Completely avoided via non } \\
\text { Section } 10 \mathrm{HDD} / \text { Bore }\end{array}$ \\
\hline WP2LI020 & 24.3 & PEM & Low & None & $\begin{array}{l}\text { No Cultural } \\
\text { Resources }\end{array}$ & $\begin{array}{l}\text { No further work } \\
\text { recommended }\end{array}$ & $\begin{array}{l}\text { Completely avoided via non } \\
\text { Section } 10 \mathrm{HDD} / \text { Bore }\end{array}$ \\
\hline WP2LI021 & 24.4 & PEM & Low & None & $\begin{array}{l}\text { No Cultural } \\
\text { Resources }\end{array}$ & $\begin{array}{l}\text { No further work } \\
\text { recommended }\end{array}$ & $\begin{array}{l}\text { Located Within Heavily } \\
\text { Inundated Area }\end{array}$ \\
\hline
\end{tabular}


Appendix C 1- Survey Data in the Vicinity of Wetland Features

\begin{tabular}{|c|c|c|c|c|c|c|c|}
\hline Wetland ID & $\begin{array}{c}\text { Milepost } \\
\text { (Approximate) }\end{array}$ & $\begin{array}{l}\text { Wetland } \\
\text { Type }\end{array}$ & Probability & Shovel Tests & $\begin{array}{l}\text { Surveys } \\
\text { Results }\end{array}$ & Recommendations & Comments \\
\hline WP2LI022 & 24.4 & PEM & Low & 2 & $\begin{array}{l}\text { No Cultural } \\
\text { Resources }\end{array}$ & $\begin{array}{l}\text { No further work } \\
\text { recommended }\end{array}$ & $\begin{array}{l}\text { Completely avoided via non- } \\
\text { Section } 10 \mathrm{HDD} / \text { Bore }\end{array}$ \\
\hline WP2LI023 & 24.7 & PEM & Low & None & $\begin{array}{l}\text { No Cultural } \\
\text { Resources }\end{array}$ & $\begin{array}{l}\text { No further work } \\
\text { recommended }\end{array}$ & $\begin{array}{l}\text { Located Within Heavily } \\
\text { Inundated Area }\end{array}$ \\
\hline WP2LI024 & 24.7 & PEM & Low & None & $\begin{array}{l}\text { No Cultural } \\
\text { Resources }\end{array}$ & $\begin{array}{l}\text { No further work } \\
\text { recommended }\end{array}$ & $\begin{array}{l}\text { Located Within Heavily } \\
\text { Inundated Area }\end{array}$ \\
\hline WP2LI025 & 24.8 & PEM & Low & None & $\begin{array}{l}\text { No Cultural } \\
\text { Resources }\end{array}$ & $\begin{array}{l}\text { No further work } \\
\text { recommended }\end{array}$ & $\begin{array}{l}\text { Located Within Heavily } \\
\text { Inundated Area }\end{array}$ \\
\hline WP2LI026 & 24.9 & PEM & Low & None & $\begin{array}{l}\text { No Cultural } \\
\text { Resources }\end{array}$ & $\begin{array}{l}\text { No further work } \\
\text { recommended }\end{array}$ & $\begin{array}{l}\text { Located Within Heavily } \\
\text { Inundated Area }\end{array}$ \\
\hline WP2LI027 & 25.2 & PEM & Low & 1 & $\begin{array}{l}\text { No Cultural } \\
\text { Resources }\end{array}$ & $\begin{array}{l}\text { No further work } \\
\text { recommended }\end{array}$ & Inundation \\
\hline WP2LI031 & 25.3 & $\mathrm{PFO}$ & Low & None & $\begin{array}{l}\text { No Cultural } \\
\text { Resources }\end{array}$ & $\begin{array}{l}\text { No further work } \\
\text { recommended }\end{array}$ & $\begin{array}{l}\text { Located Within Heavily } \\
\text { Inundated Area }\end{array}$ \\
\hline WP2LI032 & 25.3 & $\mathrm{PFO}$ & Low & 3 & $\begin{array}{l}\text { No Cultural } \\
\text { Resources }\end{array}$ & $\begin{array}{l}\text { No further work } \\
\text { recommended }\end{array}$ & $\begin{array}{l}\text { Located Within Heavily } \\
\text { Inundated Area }\end{array}$ \\
\hline WP2LI033_PEM & 25.7 & PEM & Low & 1 & $\begin{array}{l}\text { No Cultural } \\
\text { Resources }\end{array}$ & $\begin{array}{l}\text { No further work } \\
\text { recommended }\end{array}$ & $\begin{array}{l}\text { Located Within Heavily } \\
\text { Inundated Area }\end{array}$ \\
\hline WP2LI033_PFO & 25.8 & $\mathrm{PFO}$ & Low & None & $\begin{array}{l}\text { No Cultural } \\
\text { Resources }\end{array}$ & $\begin{array}{l}\text { No further work } \\
\text { recommended }\end{array}$ & $\begin{array}{l}\text { Located Within Heavily } \\
\text { Inundated Area }\end{array}$ \\
\hline WP2LI033_PFO_B & 25.8 & $\mathrm{PFO}$ & Low & None & $\begin{array}{l}\text { No Cultural } \\
\text { Resources }\end{array}$ & $\begin{array}{l}\text { No further work } \\
\text { recommended }\end{array}$ & $\begin{array}{l}\text { Located Within Heavily } \\
\text { Inundated Area }\end{array}$ \\
\hline WP2LI029_PEM & 25.9 & PEM & Low & 1 & $\begin{array}{l}\text { No Cultural } \\
\text { Resources }\end{array}$ & $\begin{array}{l}\text { No further work } \\
\text { recommended }\end{array}$ & $\begin{array}{l}\text { Completely avoided via non- } \\
\text { Section } 10 \mathrm{HDD} / \text { Bore }\end{array}$ \\
\hline WP2LI029_PFO & 25.9 & $\mathrm{PFO}$ & Low & 1 & $\begin{array}{l}\text { No Cultural } \\
\text { Resources }\end{array}$ & $\begin{array}{l}\text { No further work } \\
\text { recommended }\end{array}$ & None \\
\hline WP2LI029_PSS & 25.9 & PSS & Low & 1 & $\begin{array}{l}\text { No Cultural } \\
\text { Resources }\end{array}$ & $\begin{array}{l}\text { No further work } \\
\text { recommended }\end{array}$ & $\begin{array}{l}\text { Partially avoided via non- } \\
\text { Section } 10 \mathrm{HDD} / \text { Bore }\end{array}$ \\
\hline
\end{tabular}


Appendix C 1- Survey Data in the Vicinity of Wetland Features

\begin{tabular}{|c|c|c|c|c|c|c|c|}
\hline Wetland ID & $\begin{array}{c}\text { Milepost } \\
\text { (Approximate) }\end{array}$ & $\begin{array}{l}\text { Wetland } \\
\text { Type }\end{array}$ & Probability & Shovel Tests & $\begin{array}{l}\text { Surveys } \\
\text { Results }\end{array}$ & Recommendations & Comments \\
\hline WP2LI028_PEM & 26 & PEM & Low & None & $\begin{array}{l}\text { No Cultural } \\
\text { Resources }\end{array}$ & $\begin{array}{l}\text { No further work } \\
\text { recommended }\end{array}$ & $\begin{array}{l}\text { Completely avoided via non- } \\
\text { Section } 10 \mathrm{HDD} / \text { Bore }\end{array}$ \\
\hline WP2LI030 & 26 & PEM & Low & None & $\begin{array}{l}\text { No Cultural } \\
\text { Resources }\end{array}$ & $\begin{array}{l}\text { No further work } \\
\text { recommended }\end{array}$ & $\begin{array}{c}\text { Located Within Heavily } \\
\text { Inundated Area }\end{array}$ \\
\hline WP2LI028_PFO & 26 & $\mathrm{PFO}$ & Low & 2 & $\begin{array}{l}\text { No Cultural } \\
\text { Resources }\end{array}$ & $\begin{array}{l}\text { No further work } \\
\text { recommended }\end{array}$ & Inundation \\
\hline WP4LI017 & 26.2 & PEM & Low & 3 & $\begin{array}{l}\text { No Cultural } \\
\text { Resources }\end{array}$ & $\begin{array}{l}\text { No further work } \\
\text { recommended }\end{array}$ & $\begin{array}{l}\text { Partially avoided via non- } \\
\text { Section } 10 \text { HDD/Bore }\end{array}$ \\
\hline WP4LI019 & 26.4 & PEM & Low & 4 & $\begin{array}{l}\text { No Cultural } \\
\text { Resources }\end{array}$ & $\begin{array}{l}\text { No further work } \\
\text { recommended }\end{array}$ & None \\
\hline WP4LI018 & 26.4 & PSS & Low & 5 & $\begin{array}{l}\text { No Cultural } \\
\text { Resources }\end{array}$ & $\begin{array}{l}\text { No further work } \\
\text { recommended }\end{array}$ & None \\
\hline WP4LI020_PEM & 26.5 & PEM & Low & 1 & $\begin{array}{l}\text { No Cultural } \\
\text { Resources }\end{array}$ & $\begin{array}{l}\text { No further work } \\
\text { recommended }\end{array}$ & $\begin{array}{l}\text { Completely avoided via non- } \\
\text { Section } 10 \mathrm{HDD} / \text { Bore }\end{array}$ \\
\hline WP4LI020_PSS_B & 26.5 & PSS & Low & None & $\begin{array}{l}\text { No Cultural } \\
\text { Resources }\end{array}$ & $\begin{array}{l}\text { No further work } \\
\text { recommended }\end{array}$ & $\begin{array}{l}\text { Completely avoided via non- } \\
\text { Section } 10 \mathrm{HDD} / \text { Bore }\end{array}$ \\
\hline WP4LI020_PSS & 26.5 & PSS & Low & None & $\begin{array}{l}\text { No Cultural } \\
\text { Resources }\end{array}$ & $\begin{array}{l}\text { No further work } \\
\text { recommended }\end{array}$ & $\begin{array}{l}\text { Completely avoided via non- } \\
\text { Section } 10 \mathrm{HDD} / \text { Bore }\end{array}$ \\
\hline WP4LI021_PEM & 29.5 & PEM & Low & 2 & $\begin{array}{l}\text { No Cultural } \\
\text { Resources }\end{array}$ & $\begin{array}{l}\text { No further work } \\
\text { recommended }\end{array}$ & $\begin{array}{l}\text { Completely avoided via non- } \\
\text { Section } 10 \mathrm{HDD} / \text { Bore }\end{array}$ \\
\hline WP4LI021_PFO & 29.5 & $\mathrm{PFO}$ & Low & 2 & $\begin{array}{l}\text { No Cultural } \\
\text { Resources }\end{array}$ & $\begin{array}{l}\text { No further work } \\
\text { recommended }\end{array}$ & $\begin{array}{l}\text { Completely avoided via non- } \\
\text { Section } 10 \mathrm{HDD} / \text { Bore }\end{array}$ \\
\hline WP4LI021_PSS & 29.5 & PSS & Low & 2 & $\begin{array}{l}\text { No Cultural } \\
\text { Resources }\end{array}$ & $\begin{array}{c}\text { No further work } \\
\text { recommended }\end{array}$ & $\begin{array}{l}\text { Completely avoided via non- } \\
\text { Section } 10 \mathrm{HDD} / \text { Bore }\end{array}$ \\
\hline WP4LI023 & 29.8 & PEM & Low & 2 & $\begin{array}{l}\text { No Cultural } \\
\text { Resources }\end{array}$ & $\begin{array}{l}\text { No further work } \\
\text { recommended }\end{array}$ & None \\
\hline WP4LI022 & 29.8 & $\mathrm{PFO}$ & Low & 2 & $\begin{array}{l}\text { No Cultural } \\
\text { Resources }\end{array}$ & $\begin{array}{l}\text { No further work } \\
\text { recommended }\end{array}$ & None \\
\hline
\end{tabular}


Appendix C 1- Survey Data in the Vicinity of Wetland Features

\begin{tabular}{|c|c|c|c|c|c|c|c|}
\hline Wetland ID & $\begin{array}{c}\text { Milepost } \\
\text { (Approximate) }\end{array}$ & $\begin{array}{c}\text { Wetland } \\
\text { Type }\end{array}$ & Probability & Shovel Tests & $\begin{array}{l}\text { Surveys } \\
\text { Results }\end{array}$ & Recommendations & Comments \\
\hline WP4LI024 & 30 & $\mathrm{PFO}$ & Low & 4 & $\begin{array}{l}\text { No Cultural } \\
\text { Resources }\end{array}$ & $\begin{array}{l}\text { No further work } \\
\text { recommended }\end{array}$ & None \\
\hline WP4LI025 & 30.3 & $\mathrm{PFO}$ & Low & 16 & $\begin{array}{l}\text { No Cultural } \\
\text { Resources }\end{array}$ & $\begin{array}{l}\text { No further work } \\
\text { recommended }\end{array}$ & Inundation \\
\hline WP4LI027_PEM & 30.5 & PEM & Low & 2 & $\begin{array}{l}\text { No Cultural } \\
\text { Resources }\end{array}$ & $\begin{array}{l}\text { No further work } \\
\text { recommended }\end{array}$ & None \\
\hline WP4LI027_PFO & 30.6 & $\mathrm{PFO}$ & Low & 21 & $\begin{array}{l}\text { No Cultural } \\
\text { Resources }\end{array}$ & $\begin{array}{l}\text { No further work } \\
\text { recommended }\end{array}$ & None \\
\hline WP4LI026_PEM & 30.7 & PEM & Low & 1 & $\begin{array}{l}\text { No Cultural } \\
\text { Resources }\end{array}$ & $\begin{array}{l}\text { No further work } \\
\text { recommended }\end{array}$ & None \\
\hline WP4LI026_PFO & 30.7 & $\mathrm{PFO}$ & Low & 26 & $\begin{array}{l}\text { No Cultural } \\
\text { Resources }\end{array}$ & $\begin{array}{l}\text { No further work } \\
\text { recommended }\end{array}$ & None \\
\hline WP4LI028_PFO & 31.1 & $\mathrm{PFO}$ & Low & 32 & $\begin{array}{l}\text { No Cultural } \\
\text { Resources }\end{array}$ & $\begin{array}{l}\text { No further work } \\
\text { recommended }\end{array}$ & None \\
\hline WP4LI028_PEM & 31.3 & PEM & Low & 2 & $\begin{array}{l}\text { No Cultural } \\
\text { Resources }\end{array}$ & $\begin{array}{l}\text { No further work } \\
\text { recommended }\end{array}$ & None \\
\hline WP2LI042 & 31.4 & PEM & Low & 4 & $\begin{array}{l}\text { No Cultural } \\
\text { Resources }\end{array}$ & $\begin{array}{l}\text { No further work } \\
\text { recommended }\end{array}$ & None \\
\hline WP2LI040_PEM & 31.6 & PEM & Low & 3 & $\begin{array}{l}\text { No Cultural } \\
\text { Resources }\end{array}$ & $\begin{array}{l}\text { No further work } \\
\text { recommended }\end{array}$ & None \\
\hline WP2LI041 & 31.6 & PEM & Low & 3 & $\begin{array}{l}\text { No Cultural } \\
\text { Resources }\end{array}$ & $\begin{array}{l}\text { No further work } \\
\text { recommended }\end{array}$ & None \\
\hline WP2LI040_PSS & 31.6 & PSS & Low & 2 & $\begin{array}{l}\text { No Cultural } \\
\text { Resources }\end{array}$ & $\begin{array}{l}\text { No further work } \\
\text { recommended }\end{array}$ & None \\
\hline WP2LI040_PEM_B & 31.7 & PEM & Low & 1 & $\begin{array}{l}\text { No Cultural } \\
\text { Resources }\end{array}$ & $\begin{array}{l}\text { No further work } \\
\text { recommended }\end{array}$ & None \\
\hline WP2LI040_PFO & 31.7 & $\mathrm{PFO}$ & Low & 13 & $\begin{array}{l}\text { No Cultural } \\
\text { Resources }\end{array}$ & $\begin{array}{l}\text { No further work } \\
\text { recommended }\end{array}$ & None \\
\hline WP2LI040_PFO_B & 31.7 & $\mathrm{PFO}$ & Low & 4 & $\begin{array}{l}\text { No Cultural } \\
\text { Resources }\end{array}$ & $\begin{array}{l}\text { No further work } \\
\text { recommended }\end{array}$ & None \\
\hline WP2LI040_PEM_C & 31.8 & PEM & Low & 6 & $\begin{array}{c}\text { No Cultural } \\
\text { Resources }\end{array}$ & $\begin{array}{l}\text { No further work } \\
\text { recommended }\end{array}$ & None \\
\hline
\end{tabular}


Appendix C 1- Survey Data in the Vicinity of Wetland Features

\begin{tabular}{|c|c|c|c|c|c|c|c|}
\hline Wetland ID & $\begin{array}{c}\text { Milepost } \\
\text { (Approximate) }\end{array}$ & $\begin{array}{c}\text { Wetland } \\
\text { Type }\end{array}$ & Probability & Shovel Tests & $\begin{array}{l}\text { Surveys } \\
\text { Results }\end{array}$ & Recommendations & Comments \\
\hline WP2LI040_PFO_C & 31.8 & $\mathrm{PFO}$ & Low & 6 & $\begin{array}{l}\text { No Cultural } \\
\text { Resources }\end{array}$ & $\begin{array}{l}\text { No further work } \\
\text { recommended }\end{array}$ & None \\
\hline WP2LI039 & 31.9 & PEM & Low & 6 & $\begin{array}{l}\text { No Cultural } \\
\text { Resources }\end{array}$ & $\begin{array}{l}\text { No further work } \\
\text { recommended }\end{array}$ & Inundation \\
\hline WP2LI038 & 32.1 & PEM & Low & 10 & $\begin{array}{l}\text { No Cultural } \\
\text { Resources }\end{array}$ & $\begin{array}{l}\text { No further work } \\
\text { recommended }\end{array}$ & Inundation \\
\hline WP2LI037 & 32.3 & PEM & Low & 6 & $\begin{array}{l}\text { No Cultural } \\
\text { Resources }\end{array}$ & $\begin{array}{l}\text { No further work } \\
\text { recommended }\end{array}$ & Inundation \\
\hline WP2LI036_PEM_B & 32.4 & PEM & Low & 1 & $\begin{array}{l}\text { No Cultural } \\
\text { Resources }\end{array}$ & $\begin{array}{l}\text { No further work } \\
\text { recommended }\end{array}$ & Inundation \\
\hline WP2LI036_PEM & 32.5 & PEM & Low & 1 & $\begin{array}{l}\text { No Cultural } \\
\text { Resources }\end{array}$ & $\begin{array}{l}\text { No further work } \\
\text { recommended }\end{array}$ & None \\
\hline WP2LI036_PFO & 32.5 & $\mathrm{PFO}$ & Low & 11 & $\begin{array}{l}\text { No Cultural } \\
\text { Resources }\end{array}$ & $\begin{array}{l}\text { No further work } \\
\text { recommended }\end{array}$ & Inundation \\
\hline WP2LI035 & 32.8 & PEM & Low & 3 & $\begin{array}{l}\text { No Cultural } \\
\text { Resources }\end{array}$ & $\begin{array}{l}\text { No further work } \\
\text { recommended }\end{array}$ & Inundation \\
\hline WP2LI034 & 32.9 & PEM & Low & 2 & $\begin{array}{l}\text { No Cultural } \\
\text { Resources }\end{array}$ & $\begin{array}{l}\text { No further work } \\
\text { recommended }\end{array}$ & Inundation \\
\hline WP4LI046 & 33 & PEM & Low & 2 & $\begin{array}{l}\text { No Cultural } \\
\text { Resources }\end{array}$ & $\begin{array}{l}\text { No further work } \\
\text { recommended }\end{array}$ & None \\
\hline WP4LI047 & 33.1 & PEM & Low & 2 & $\begin{array}{l}\text { No Cultural } \\
\text { Resources }\end{array}$ & $\begin{array}{l}\text { No further work } \\
\text { recommended }\end{array}$ & None \\
\hline WP4LI048 & 33.2 & PEM & Low & 2 & $\begin{array}{l}\text { No Cultural } \\
\text { Resources }\end{array}$ & $\begin{array}{l}\text { No further work } \\
\text { recommended }\end{array}$ & None \\
\hline WP4LI049 & 33.2 & PEM & Low & 2 & $\begin{array}{l}\text { No Cultural } \\
\text { Resources }\end{array}$ & $\begin{array}{l}\text { No further work } \\
\text { recommended }\end{array}$ & None \\
\hline WP2LI064 & 34.1 & PEM & Low & None & $\begin{array}{l}\text { No Cultural } \\
\text { Resources }\end{array}$ & $\begin{array}{l}\text { No further work } \\
\text { recommended }\end{array}$ & $\begin{array}{l}\text { Located Within Heavily } \\
\text { Inundated Area }\end{array}$ \\
\hline WP2LI063 & 34.7 & PEM & Low & 2 & $\begin{array}{l}\text { No Cultural } \\
\text { Resources }\end{array}$ & $\begin{array}{l}\text { No further work } \\
\text { recommended }\end{array}$ & Inundation \\
\hline WP2LI061 & 34.9 & PEM & Low & 4 & $\begin{array}{l}\text { No Cultural } \\
\text { Resources }\end{array}$ & $\begin{array}{l}\text { No further work } \\
\text { recommended }\end{array}$ & Inundation \\
\hline
\end{tabular}


Appendix C 1- Survey Data in the Vicinity of Wetland Features

\begin{tabular}{|c|c|c|c|c|c|c|c|}
\hline Wetland ID & $\begin{array}{c}\text { Milepost } \\
\text { (Approximate) }\end{array}$ & $\begin{array}{l}\text { Wetland } \\
\text { Type }\end{array}$ & Probability & Shovel Tests & $\begin{array}{l}\text { Surveys } \\
\text { Results }\end{array}$ & Recommendations & Comments \\
\hline WP2LI062 & 34.9 & PEM & Low & 6 & $\begin{array}{l}\text { No Cultural } \\
\text { Resources }\end{array}$ & $\begin{array}{l}\text { No further work } \\
\text { recommended }\end{array}$ & Inundation \\
\hline WP2LI055 & 35.3 & PEM & Low & 11 & $\begin{array}{l}\text { No Cultural } \\
\text { Resources }\end{array}$ & $\begin{array}{l}\text { No further work } \\
\text { recommended }\end{array}$ & None \\
\hline WP2LI056 & 35.7 & PEM & Low & 11 & $\begin{array}{l}\text { No Cultural } \\
\text { Resources }\end{array}$ & $\begin{array}{l}\text { No further work } \\
\text { recommended }\end{array}$ & Inundation \\
\hline WP2LI057 & 36.3 & PEM & Low & 12 & $\begin{array}{l}\text { No Cultural } \\
\text { Resources }\end{array}$ & $\begin{array}{l}\text { No further work } \\
\text { recommended }\end{array}$ & None \\
\hline WP2LI058 & 36.7 & $\mathrm{PFO}$ & Low & 4 & $\begin{array}{l}\text { No Cultural } \\
\text { Resources }\end{array}$ & $\begin{array}{l}\text { No further work } \\
\text { recommended }\end{array}$ & $\begin{array}{l}\text { Completely avoided via non- } \\
\text { Section } 10 \mathrm{HDD} / \text { Bore }\end{array}$ \\
\hline WP2LI059 & 37.1 & PEM & Low & 2 & $\begin{array}{l}\text { No Cultural } \\
\text { Resources }\end{array}$ & $\begin{array}{l}\text { No further work } \\
\text { recommended }\end{array}$ & Inundation \\
\hline WP2LI060 & 37.1 & PEM & Low & 2 & $\begin{array}{l}\text { No Cultural } \\
\text { Resources }\end{array}$ & $\begin{array}{l}\text { No further work } \\
\text { recommended }\end{array}$ & Inundation \\
\hline WP4LI043 & 37.2 & PEM & Low & 3 & $\begin{array}{l}\text { No Cultural } \\
\text { Resources }\end{array}$ & $\begin{array}{l}\text { No further work } \\
\text { recommended }\end{array}$ & None \\
\hline WP4LI044 & 37.2 & PEM & Low & 2 & $\begin{array}{l}\text { No Cultural } \\
\text { Resources }\end{array}$ & $\begin{array}{l}\text { No further work } \\
\text { recommended }\end{array}$ & None \\
\hline WP4LI045 & 37.2 & PEM & Low & 2 & $\begin{array}{l}\text { No Cultural } \\
\text { Resources }\end{array}$ & $\begin{array}{l}\text { No further work } \\
\text { recommended }\end{array}$ & Inundation \\
\hline WP4LI042 & 37.3 & PEM & Low & 5 & $\begin{array}{l}\text { No Cultural } \\
\text { Resources }\end{array}$ & $\begin{array}{l}\text { No further work } \\
\text { recommended }\end{array}$ & Inundation \\
\hline WP4LI041 & 37.6 & PEM & Low & 2 & $\begin{array}{l}\text { No Cultural } \\
\text { Resources }\end{array}$ & $\begin{array}{l}\text { No further work } \\
\text { recommended }\end{array}$ & Inundation \\
\hline WP4LI040 & 37.7 & PEM & Low & 4 & $\begin{array}{l}\text { No Cultural } \\
\text { Resources }\end{array}$ & $\begin{array}{l}\text { No further work } \\
\text { recommended }\end{array}$ & Inundation \\
\hline WP4LI039_PEM & 37.8 & PEM & Low & 2 & $\begin{array}{l}\text { No Cultural } \\
\text { Resources }\end{array}$ & $\begin{array}{l}\text { No further work } \\
\text { recommended }\end{array}$ & None \\
\hline WP4LI039_PSS & 37.8 & PSS & Low & 2 & $\begin{array}{l}\text { No Cultural } \\
\text { Resources }\end{array}$ & $\begin{array}{l}\text { No further work } \\
\text { recommended }\end{array}$ & None \\
\hline
\end{tabular}


Appendix C 1- Survey Data in the Vicinity of Wetland Features

\begin{tabular}{|c|c|c|c|c|c|c|c|}
\hline Wetland ID & $\begin{array}{c}\text { Milepost } \\
\text { (Approximate) }\end{array}$ & $\begin{array}{c}\text { Wetland } \\
\text { Type }\end{array}$ & Probability & Shovel Tests & $\begin{array}{l}\text { Surveys } \\
\text { Results }\end{array}$ & Recommendations & Comments \\
\hline WP4LI037 & 37.9 & PEM & Low & 4 & $\begin{array}{c}\text { No Cultural } \\
\text { Resources }\end{array}$ & $\begin{array}{l}\text { No further work } \\
\text { recommended }\end{array}$ & Inundation \\
\hline WP4LI038 & 37.9 & PEM & Low & 1 & $\begin{array}{c}\text { No Cultural } \\
\text { Resources }\end{array}$ & $\begin{array}{l}\text { No further work } \\
\text { recommended }\end{array}$ & None \\
\hline WP4LI061 & 38 & PEM & Low & 2 & $\begin{array}{l}\text { No Cultural } \\
\text { Resources }\end{array}$ & $\begin{array}{l}\text { No further work } \\
\text { recommended }\end{array}$ & $\begin{array}{c}\text { Completely avoided via non } \\
\text { Section } 10 \mathrm{HDD} / \text { Bore }\end{array}$ \\
\hline WP4LI036 & 38 & $\mathrm{PFO}$ & Low & 1 & $\begin{array}{c}\text { No Cultural } \\
\text { Resources }\end{array}$ & $\begin{array}{l}\text { No further work } \\
\text { recommended }\end{array}$ & None \\
\hline WP4LI059 & 38.3 & PEM & Low & 1 & $\begin{array}{l}\text { No Cultural } \\
\text { Resources }\end{array}$ & $\begin{array}{l}\text { No further work } \\
\text { recommended }\end{array}$ & None \\
\hline WP4LI050_PEM & 38.6 & PEM & Low & 2 & $\begin{array}{l}\text { No Cultural } \\
\text { Resources }\end{array}$ & $\begin{array}{l}\text { No further work } \\
\text { recommended }\end{array}$ & $\begin{array}{l}\text { Located Within Heavily } \\
\text { Inundated Area }\end{array}$ \\
\hline WP4LI050_PSS & 38.6 & PSS & Low & 2 & $\begin{array}{l}\text { No Cultural } \\
\text { Resources }\end{array}$ & $\begin{array}{l}\text { No further work } \\
\text { recommended }\end{array}$ & $\begin{array}{c}\text { Located Within Heavily } \\
\text { Inundated Area }\end{array}$ \\
\hline WP4LI050_PSS_B & 38.6 & PSS & Low & 3 & $\begin{array}{l}\text { No Cultural } \\
\text { Resources }\end{array}$ & $\begin{array}{l}\text { No further work } \\
\text { recommended }\end{array}$ & $\begin{array}{c}\text { Located Within Heavily } \\
\text { Inundated Area }\end{array}$ \\
\hline WP4LI050_PSS_C & 38.7 & PSS & Low & None & $\begin{array}{c}\text { No Cultural } \\
\text { Resources }\end{array}$ & $\begin{array}{l}\text { No further work } \\
\text { recommended }\end{array}$ & $\begin{array}{c}\text { Located Within Heavily } \\
\text { Inundated Area }\end{array}$ \\
\hline WP4LI050_PSS_D & 38.7 & PSS & Low & None & $\begin{array}{c}\text { No Cultural } \\
\text { Resources }\end{array}$ & $\begin{array}{l}\text { No further work } \\
\text { recommended }\end{array}$ & $\begin{array}{c}\text { Located Within Heavily } \\
\text { Inundated Area }\end{array}$ \\
\hline WP4LI050_PSS_E & 38.7 & PSS & Low & None & $\begin{array}{c}\text { No Cultural } \\
\text { Resources }\end{array}$ & $\begin{array}{l}\text { No further work } \\
\text { recommended }\end{array}$ & $\begin{array}{l}\text { Located Within Heavily } \\
\text { Inundated Area }\end{array}$ \\
\hline \multicolumn{8}{|c|}{ Jefferson County, Texas } \\
\hline WP2JE065 & 39.2 & PEM & Low & None & $\begin{array}{c}\text { No Cultural } \\
\text { Resources }\end{array}$ & $\begin{array}{l}\text { No further work } \\
\text { recommended }\end{array}$ & $\begin{array}{l}\text { Partially avoided via non- } \\
\text { Section } 10 \mathrm{HDD} / \text { Bore }\end{array}$ \\
\hline WP2JE048 & 40.1 & PEM & Low & None & $\begin{array}{l}\text { No Cultural } \\
\text { Resources }\end{array}$ & $\begin{array}{l}\text { No further work } \\
\text { recommended }\end{array}$ & $\begin{array}{l}\text { Located Within Heavily } \\
\text { Inundated Area }\end{array}$ \\
\hline WP2JE050 & 40.1 & PEM & Low & None & $\begin{array}{l}\text { No Cultural } \\
\text { Resources }\end{array}$ & $\begin{array}{l}\text { No further work } \\
\text { recommended }\end{array}$ & $\begin{array}{l}\text { Located Within Heavily } \\
\text { Inundated Area }\end{array}$ \\
\hline
\end{tabular}


Appendix C 1- Survey Data in the Vicinity of Wetland Features

\begin{tabular}{|c|c|c|c|c|c|c|c|}
\hline Wetland ID & $\begin{array}{c}\text { Milepost } \\
\text { (Approximate) }\end{array}$ & $\begin{array}{c}\text { Wetland } \\
\text { Type }\end{array}$ & Probability & Shovel Tests & $\begin{array}{l}\text { Surveys } \\
\text { Results }\end{array}$ & Recommendations & Comments \\
\hline WP2JE051 & 40.2 & PEM & Low & None & $\begin{array}{c}\text { No Cultural } \\
\text { Resources }\end{array}$ & $\begin{array}{l}\text { No further work } \\
\text { recommended }\end{array}$ & $\begin{array}{c}\text { Located Within Heavily } \\
\text { Inundated Area }\end{array}$ \\
\hline WP2JE052 & 40.2 & PEM & Low & None & $\begin{array}{l}\text { No Cultural } \\
\text { Resources }\end{array}$ & $\begin{array}{l}\text { No further work } \\
\text { recommended }\end{array}$ & $\begin{array}{l}\text { Located Within Heavily } \\
\text { Inundated Area }\end{array}$ \\
\hline WP2JE053 & 40.3 & $\mathrm{PFO}$ & Low & None & $\begin{array}{l}\text { No Cultural } \\
\text { Resources }\end{array}$ & $\begin{array}{l}\text { No further work } \\
\text { recommended }\end{array}$ & $\begin{array}{l}\text { Located Within Heavily } \\
\text { Inundated Area }\end{array}$ \\
\hline WP3JE030 & 45.3 & PEM & Low & None & $\begin{array}{l}\text { No Cultural } \\
\text { Resources }\end{array}$ & $\begin{array}{l}\text { No further work } \\
\text { recommended }\end{array}$ & $\begin{array}{c}\text { Located Within Heavily } \\
\text { Inundated Area }\end{array}$ \\
\hline WP3JE031 & 45.5 & PEM & Low & None & $\begin{array}{l}\text { No Cultural } \\
\text { Resources }\end{array}$ & $\begin{array}{l}\text { No further work } \\
\text { recommended }\end{array}$ & $\begin{array}{l}\text { Located Within Heavily } \\
\text { Inundated Area }\end{array}$ \\
\hline WP3JE032 & 45.6 & PEM & Low & None & $\begin{array}{c}\text { No Cultural } \\
\text { Resources }\end{array}$ & $\begin{array}{l}\text { No further work } \\
\text { recommended }\end{array}$ & $\begin{array}{c}\text { Located Within Heavily } \\
\text { Inundated Area }\end{array}$ \\
\hline WP9JE010_PEM_DT ${ }^{\text {a }}$ & 47.6 & PEM & Low & None & $\begin{array}{c}\text { No Cultural } \\
\text { Resources }\end{array}$ & $\begin{array}{l}\text { No further work } \\
\text { recommended }\end{array}$ & $\begin{array}{l}\text { Completely avoided via non- } \\
\text { Section } 10 \mathrm{HDD} / \text { Bore }\end{array}$ \\
\hline WP9JE010_PFO_DT ${ }^{\text {a }}$ & 47.5 & $\mathrm{PFO}$ & Low & None & $\begin{array}{c}\text { No Cultural } \\
\text { Resources }\end{array}$ & $\begin{array}{l}\text { No further work } \\
\text { recommended }\end{array}$ & $\begin{array}{l}\text { Completely avoided via non- } \\
\text { Section } 10 \mathrm{HDD} / \text { Bore }\end{array}$ \\
\hline WP4JE060 & 47.7 & PEM & Low & None & $\begin{array}{l}\text { No Cultural } \\
\text { Resources }\end{array}$ & $\begin{array}{l}\text { No further work } \\
\text { recommended }\end{array}$ & $\begin{array}{l}\text { Completely avoided via non- } \\
\text { Section } 10 \mathrm{HDD} / \text { Bore }\end{array}$ \\
\hline WP4JE062 & 50.1 & PEM & Low & None & $\begin{array}{l}\text { No Cultural } \\
\text { Resources }\end{array}$ & $\begin{array}{l}\text { No further work } \\
\text { recommended }\end{array}$ & $\begin{array}{c}\text { Located Within Heavily } \\
\text { Inundated Area }\end{array}$ \\
\hline WP4JE063 & 50.2 & PEM & Low & None & $\begin{array}{l}\text { No Cultural } \\
\text { Resources }\end{array}$ & $\begin{array}{l}\text { No further work } \\
\text { recommended }\end{array}$ & Inundation \\
\hline WP4JE064_PFO & 50.5 & $\mathrm{PFO}$ & Low & None & $\begin{array}{c}\text { No Cultural } \\
\text { Resources }\end{array}$ & $\begin{array}{c}\text { No further work } \\
\text { recommended }\end{array}$ & $\begin{array}{l}\text { Partially avoided via non- } \\
\text { Section } 10 \mathrm{HDD} / \text { Bore }\end{array}$ \\
\hline WP4JE064_PSS & 50.5 & PSS & Low & None & $\begin{array}{l}\text { No Cultural } \\
\text { Resources }\end{array}$ & $\begin{array}{l}\text { No further work } \\
\text { recommended }\end{array}$ & None \\
\hline WP4JE064_PEM & 50.6 & PEM & Low & None & $\begin{array}{l}\text { No Cultural } \\
\text { Resources }\end{array}$ & $\begin{array}{l}\text { No further work } \\
\text { recommended }\end{array}$ & $\begin{array}{l}\text { Partially avoided via non- } \\
\text { Section } 10 \mathrm{HDD} / \text { Bore }\end{array}$ \\
\hline WP3JE033_PEM & 50.8 & PEM & Low & None & $\begin{array}{l}\text { No Cultural } \\
\text { Resources }\end{array}$ & $\begin{array}{l}\text { No further work } \\
\text { recommended }\end{array}$ & $\begin{array}{l}\text { Completely avoided via non- } \\
\text { Section } 10 \mathrm{HDD} / \text { Bore }\end{array}$ \\
\hline
\end{tabular}


Appendix C 1- Survey Data in the Vicinity of Wetland Features

\begin{tabular}{|c|c|c|c|c|c|c|c|}
\hline Wetland ID & $\begin{array}{c}\text { Milepost } \\
\text { (Approximate) }\end{array}$ & $\begin{array}{l}\text { Wetland } \\
\text { Type }\end{array}$ & Probability & Shovel Tests & $\begin{array}{l}\text { Surveys } \\
\text { Results }\end{array}$ & Recommendations & Comments \\
\hline WP4JE065 & 50.8 & PEM & Low & None & $\begin{array}{c}\text { No Cultural } \\
\text { Resources }\end{array}$ & $\begin{array}{l}\text { No further work } \\
\text { recommended }\end{array}$ & $\begin{array}{l}\text { Completely avoided via non- } \\
\text { Section } 10 \mathrm{HDD} / \text { Bore }\end{array}$ \\
\hline WP3JE036 & 50.9 & PEM & Low & None & $\begin{array}{l}\text { No Cultural } \\
\text { Resources }\end{array}$ & $\begin{array}{l}\text { No further work } \\
\text { recommended }\end{array}$ & None \\
\hline WP3JE033_PFO & 50.9 & $\mathrm{PFO}$ & Low & None & $\begin{array}{c}\text { No Cultural } \\
\text { Resources }\end{array}$ & $\begin{array}{l}\text { No further work } \\
\text { recommended }\end{array}$ & $\begin{array}{l}\text { Completely avoided via non- } \\
\text { Section } 10 \mathrm{HDD} / \text { Bore }\end{array}$ \\
\hline WP3JE037 & 51.1 & PEM & Low & None & $\begin{array}{l}\text { No Cultural } \\
\text { Resources }\end{array}$ & $\begin{array}{l}\text { No further work } \\
\text { recommended }\end{array}$ & None \\
\hline WP3JE038 & 51.2 & PEM & Low & None & $\begin{array}{c}\text { No Cultural } \\
\text { Resources }\end{array}$ & $\begin{array}{l}\text { No further work } \\
\text { recommended }\end{array}$ & None \\
\hline WP4JE071 & 51.3 & PEM & Low & None & $\begin{array}{l}\text { No Cultural } \\
\text { Resources }\end{array}$ & $\begin{array}{l}\text { No further work } \\
\text { recommended }\end{array}$ & $\begin{array}{c}\text { Located Within Heavily } \\
\text { Inundated Area }\end{array}$ \\
\hline WP4JE070 & 51.5 & PEM & Low & None & $\begin{array}{l}\text { No Cultural } \\
\text { Resources }\end{array}$ & $\begin{array}{l}\text { No further work } \\
\text { recommended }\end{array}$ & $\begin{array}{c}\text { Located Within Heavily } \\
\text { Inundated Area }\end{array}$ \\
\hline WP4JE067 & 51.8 & PEM & Low & None & $\begin{array}{l}\text { No Cultural } \\
\text { Resources }\end{array}$ & $\begin{array}{l}\text { No further work } \\
\text { recommended }\end{array}$ & $\begin{array}{c}\text { Located Within Heavily } \\
\text { Inundated Area }\end{array}$ \\
\hline WP4JE066 & 52.1 & PEM & Low & None & $\begin{array}{l}\text { No Cultural } \\
\text { Resources }\end{array}$ & $\begin{array}{l}\text { No further work } \\
\text { recommended }\end{array}$ & $\begin{array}{l}\text { Partially avoided via non- } \\
\text { Section } 10 \mathrm{HDD} / \text { Bore }\end{array}$ \\
\hline WP3JE034 & 52.7 & PEM & Low & None & $\begin{array}{l}\text { No Cultural } \\
\text { Resources }\end{array}$ & $\begin{array}{l}\text { No further work } \\
\text { recommended }\end{array}$ & None \\
\hline WP3JE035 & 52.7 & PEM & Low & None & $\begin{array}{l}\text { No Cultural } \\
\text { Resources }\end{array}$ & $\begin{array}{l}\text { No further work } \\
\text { recommended }\end{array}$ & $\begin{array}{l}\text { Completely avoided via non- } \\
\text { Section } 10 \mathrm{HDD} / \text { Bore }\end{array}$ \\
\hline WP4JE072_PSS & 52.7 & PSS & Low & None & $\begin{array}{c}\text { No Cultural } \\
\text { Resources }\end{array}$ & $\begin{array}{l}\text { No further work } \\
\text { recommended }\end{array}$ & $\begin{array}{c}\text { Located Within Heavily } \\
\text { Inundated Area }\end{array}$ \\
\hline WP4JE072_PFO & 52.8 & PFO & Low & None & $\begin{array}{l}\text { No Cultural } \\
\text { Resources }\end{array}$ & $\begin{array}{l}\text { No further work } \\
\text { recommended }\end{array}$ & Inundation \\
\hline WP4JE073 & 52.9 & $\mathrm{PFO}$ & Low & None & $\begin{array}{l}\text { No Cultural } \\
\text { Resources }\end{array}$ & $\begin{array}{l}\text { No further work } \\
\text { recommended }\end{array}$ & $\begin{array}{c}\text { Located Within Heavily } \\
\text { Inundated Area }\end{array}$ \\
\hline WP5JE001 & 53 & PEM & Low & None & $\begin{array}{l}\text { No Cultural } \\
\text { Resources }\end{array}$ & $\begin{array}{l}\text { No further work } \\
\text { recommended }\end{array}$ & $\begin{array}{c}\text { Located Within Heavily } \\
\text { Inundated Area }\end{array}$ \\
\hline
\end{tabular}


Appendix C 1- Survey Data in the Vicinity of Wetland Features

\begin{tabular}{|c|c|c|c|c|c|c|c|}
\hline Wetland ID & $\begin{array}{c}\text { Milepost } \\
\text { (Approximate) }\end{array}$ & $\begin{array}{l}\text { Wetland } \\
\text { Type }\end{array}$ & Probability & Shovel Tests & $\begin{array}{l}\text { Surveys } \\
\text { Results }\end{array}$ & Recommendations & Comments \\
\hline WP5JE002 & 53.3 & PEM & Low & None & $\begin{array}{l}\text { No Cultural } \\
\text { Resources }\end{array}$ & $\begin{array}{l}\text { No further work } \\
\text { recommended }\end{array}$ & $\begin{array}{l}\text { Located Within Heavily } \\
\text { Inundated Area }\end{array}$ \\
\hline WP5JE003 & 53.4 & PEM & Low & None & $\begin{array}{l}\text { No Cultural } \\
\text { Resources }\end{array}$ & $\begin{array}{l}\text { No further work } \\
\text { recommended }\end{array}$ & $\begin{array}{l}\text { Located Within Heavily } \\
\text { Inundated Area }\end{array}$ \\
\hline WP5JE004 & 53.6 & PEM & Low & None & $\begin{array}{l}\text { No Cultural } \\
\text { Resources }\end{array}$ & $\begin{array}{l}\text { No further work } \\
\text { recommended }\end{array}$ & $\begin{array}{l}\text { Located Within Heavily } \\
\text { Inundated Area }\end{array}$ \\
\hline WP5JE005 & 53.7 & PEM & Low & None & $\begin{array}{l}\text { No Cultural } \\
\text { Resources }\end{array}$ & $\begin{array}{l}\text { No further work } \\
\text { recommended }\end{array}$ & $\begin{array}{l}\text { Located Within Heavily } \\
\text { Inundated Area }\end{array}$ \\
\hline WP5JE006 & 53.9 & PEM & Low & 1 & $\begin{array}{l}\text { No Cultural } \\
\text { Resources }\end{array}$ & $\begin{array}{l}\text { No further work } \\
\text { recommended }\end{array}$ & Inundation \\
\hline WP5JE007 & 54.1 & PEM & Low & None & $\begin{array}{l}\text { No Cultural } \\
\text { Resources }\end{array}$ & $\begin{array}{l}\text { No further work } \\
\text { recommended }\end{array}$ & $\begin{array}{l}\text { Completely avoided via non- } \\
\text { Section } 10 \mathrm{HDD} / \text { Bore }\end{array}$ \\
\hline WP4JE074_PSS & 54.3 & PSS & Low & None & $\begin{array}{l}\text { No Cultural } \\
\text { Resources }\end{array}$ & $\begin{array}{l}\text { No further work } \\
\text { recommended }\end{array}$ & $\begin{array}{l}\text { Located Within Heavily } \\
\text { Inundated Area }\end{array}$ \\
\hline WP4JE074_PEM & 54.4 & PEM & Low & 2 & $\begin{array}{l}\text { No Cultural } \\
\text { Resources }\end{array}$ & $\begin{array}{l}\text { No further work } \\
\text { recommended }\end{array}$ & $\begin{array}{l}\text { Completely avoided via non- } \\
\text { Section } 10 \mathrm{HDD} / \text { Bore }\end{array}$ \\
\hline WP4JE074_PSS_B & 54.8 & PSS & Low & None & $\begin{array}{l}\text { No Cultural } \\
\text { Resources }\end{array}$ & $\begin{array}{l}\text { No further work } \\
\text { recommended }\end{array}$ & $\begin{array}{l}\text { Located Within Heavily } \\
\text { Inundated Area }\end{array}$ \\
\hline WP4JE075_PEM & 54.9 & PEM & Low & None & $\begin{array}{l}\text { No Cultural } \\
\text { Resources }\end{array}$ & $\begin{array}{l}\text { No further work } \\
\text { recommended }\end{array}$ & $\begin{array}{l}\text { Completely avoided via non- } \\
\text { Section } 10 \mathrm{HDD} / \text { Bore }\end{array}$ \\
\hline WP4JE075_PFO & 54.9 & $\mathrm{PFO}$ & Low & None & $\begin{array}{l}\text { No Cultural } \\
\text { Resources }\end{array}$ & $\begin{array}{l}\text { No further work } \\
\text { recommended }\end{array}$ & $\begin{array}{l}\text { Completely avoided via non- } \\
\text { Section } 10 \mathrm{HDD} / \text { Bore }\end{array}$ \\
\hline WP4JE075_PFO_B & 54.9 & $\mathrm{PFO}$ & Low & None & $\begin{array}{l}\text { No Cultural } \\
\text { Resources }\end{array}$ & $\begin{array}{l}\text { No further work } \\
\text { recommended }\end{array}$ & $\begin{array}{l}\text { Completely avoided via non- } \\
\text { Section } 10 \mathrm{HDD} / \text { Bore }\end{array}$ \\
\hline WP5JE015 & 55.1 & PEM & Low & 2 & $\begin{array}{l}\text { No Cultural } \\
\text { Resources }\end{array}$ & $\begin{array}{l}\text { No further work } \\
\text { recommended }\end{array}$ & $\begin{array}{l}\text { Completely avoided via non- } \\
\text { Section } 10 \mathrm{HDD} / \text { Bore }\end{array}$ \\
\hline
\end{tabular}


Appendix C 1- Survey Data in the Vicinity of Wetland Features

\begin{tabular}{|c|c|c|c|c|c|c|c|}
\hline Wetland ID & $\begin{array}{c}\text { Milepost } \\
\text { (Approximate) }\end{array}$ & $\begin{array}{l}\text { Wetland } \\
\text { Type }\end{array}$ & Probability & Shovel Tests & $\begin{array}{l}\text { Surveys } \\
\text { Results }\end{array}$ & Recommendations & Comments \\
\hline WP5JE014 & 55.6 & PEM & Low & 8 & $\begin{array}{l}\text { No Cultural } \\
\text { Resources }\end{array}$ & $\begin{array}{l}\text { No further work } \\
\text { recommended }\end{array}$ & $\begin{array}{l}\text { Completely avoided via non- } \\
\text { Section } 10 \mathrm{HDD} / \text { Bore }\end{array}$ \\
\hline WP5JE013 & 56.2 & PEM & Low & 8 & $\begin{array}{l}\text { No Cultural } \\
\text { Resources }\end{array}$ & $\begin{array}{l}\text { No further work } \\
\text { recommended }\end{array}$ & Inundation \\
\hline WP5JE012_PEM & 56.3 & PEM & Low & None & $\begin{array}{l}\text { No Cultural } \\
\text { Resources }\end{array}$ & $\begin{array}{l}\text { No further work } \\
\text { recommended }\end{array}$ & Inundation \\
\hline WP5JE012_PFO & 56.3 & PFO & Low & None & $\begin{array}{l}\text { No Cultural } \\
\text { Resources }\end{array}$ & $\begin{array}{l}\text { No further work } \\
\text { recommended }\end{array}$ & Inundation \\
\hline WP5JE011 & 56.4 & PEM & Low & None & $\begin{array}{l}\text { No Cultural } \\
\text { Resources }\end{array}$ & $\begin{array}{l}\text { No further work } \\
\text { recommended }\end{array}$ & Inundation \\
\hline WP5JE009 & 56.5 & PEM & Low & None & $\begin{array}{l}\text { No Cultural } \\
\text { Resources }\end{array}$ & $\begin{array}{l}\text { No further work } \\
\text { recommended }\end{array}$ & $\begin{array}{l}\text { Located Within Heavily } \\
\text { Inundated Area }\end{array}$ \\
\hline WP5JE010 & 56.7 & PEM & Low & None & $\begin{array}{l}\text { No Cultural } \\
\text { Resources }\end{array}$ & $\begin{array}{l}\text { No further work } \\
\text { recommended }\end{array}$ & $\begin{array}{l}\text { Located Within Heavily } \\
\text { Inundated Area }\end{array}$ \\
\hline WP1JE012_PEM & 56.8 & PEM & Low & 3 & $\begin{array}{l}\text { No Cultural } \\
\text { Resources }\end{array}$ & $\begin{array}{l}\text { No further work } \\
\text { recommended }\end{array}$ & $\begin{array}{l}\text { Partially avoided via non- } \\
\text { Section } 10 \text { HDD/Bore }\end{array}$ \\
\hline WP1JE012_PFO_B & 56.8 & PFO & Low & None & $\begin{array}{l}\text { No Cultural } \\
\text { Resources }\end{array}$ & $\begin{array}{l}\text { No further work } \\
\text { recommended }\end{array}$ & $\begin{array}{l}\text { Located Within Heavily } \\
\text { Inundated Area }\end{array}$ \\
\hline WP1JE012_PFO_C & 56.8 & PFO & Low & None & $\begin{array}{l}\text { No Cultural } \\
\text { Resources }\end{array}$ & $\begin{array}{l}\text { No further work } \\
\text { recommended }\end{array}$ & $\begin{array}{l}\text { Located Within Heavily } \\
\text { Inundated Area }\end{array}$ \\
\hline WP1JE012_PFO_E & 56.9 & PFO & Low & 4 & $\begin{array}{l}\text { No Cultural } \\
\text { Resources }\end{array}$ & $\begin{array}{l}\text { No further work } \\
\text { recommended }\end{array}$ & Inundation \\
\hline WP1JE012_PFO_D & 57 & PFO & Low & 9 & $\begin{array}{l}\text { No Cultural } \\
\text { Resources }\end{array}$ & $\begin{array}{l}\text { No further work } \\
\text { recommended }\end{array}$ & Inundation \\
\hline WP1JE012_PSS_B & 57.2 & PSS & Low & 3 & $\begin{array}{l}\text { No Cultural } \\
\text { Resources }\end{array}$ & $\begin{array}{l}\text { No further work } \\
\text { recommended }\end{array}$ & None \\
\hline WP1JE012_PFO & 57.3 & PFO & Low & 2 & $\begin{array}{l}\text { No Cultural } \\
\text { Resources }\end{array}$ & $\begin{array}{l}\text { No further work } \\
\text { recommended }\end{array}$ & $\begin{array}{l}\text { Partially avoided via non- } \\
\text { Section } 10 \mathrm{HDD} / \text { Bore }\end{array}$ \\
\hline WP1JE012_PSS & 57.3 & PSS & Low & 1 & $\begin{array}{l}\text { No Cultural } \\
\text { Resources }\end{array}$ & $\begin{array}{l}\text { No further work } \\
\text { recommended }\end{array}$ & Inundation \\
\hline
\end{tabular}


Appendix C 1- Survey Data in the Vicinity of Wetland Features

\begin{tabular}{|c|c|c|c|c|c|c|c|}
\hline Wetland ID & $\begin{array}{c}\text { Milepost } \\
\text { (Approximate) }\end{array}$ & $\begin{array}{l}\text { Wetland } \\
\text { Type }\end{array}$ & Probability & Shovel Tests & $\begin{array}{l}\text { Surveys } \\
\text { Results }\end{array}$ & Recommendations & Comments \\
\hline WP4JE085 & 57.4 & PEM & Low & None & $\begin{array}{l}\text { No Cultural } \\
\text { Resources }\end{array}$ & $\begin{array}{l}\text { No further work } \\
\text { recommended }\end{array}$ & $\begin{array}{l}\text { Located Within Heavily } \\
\text { Inundated Area }\end{array}$ \\
\hline WP4JE086 & 57.4 & PEM & Low & None & $\begin{array}{l}\text { No Cultural } \\
\text { Resources }\end{array}$ & $\begin{array}{l}\text { No further work } \\
\text { recommended }\end{array}$ & $\begin{array}{l}\text { Completely avoided via non- } \\
\text { Section } 10 \mathrm{HDD} / \text { Bore }\end{array}$ \\
\hline WP4JE084_PEM & 57.5 & PEM & Low & None & $\begin{array}{l}\text { No Cultural } \\
\text { Resources }\end{array}$ & $\begin{array}{l}\text { No further work } \\
\text { recommended }\end{array}$ & $\begin{array}{c}\text { Located Within Heavily } \\
\text { Inundated Area }\end{array}$ \\
\hline WP4JE084_PSS & 57.5 & PSS & Low & None & $\begin{array}{l}\text { No Cultural } \\
\text { Resources }\end{array}$ & $\begin{array}{l}\text { No further work } \\
\text { recommended }\end{array}$ & $\begin{array}{l}\text { Completely avoided via non- } \\
\text { Section } 10 \mathrm{HDD} / \text { Bore }\end{array}$ \\
\hline WP4JE084_PSS_B & 57.5 & PSS & Low & None & $\begin{array}{l}\text { No Cultural } \\
\text { Resources }\end{array}$ & $\begin{array}{l}\text { No further work } \\
\text { recommended }\end{array}$ & $\begin{array}{l}\text { Completely avoided via non- } \\
\text { Section } 10 \mathrm{HDD} / \text { Bore }\end{array}$ \\
\hline WP4JE082 & 57.7 & PEM & Low & None & $\begin{array}{l}\text { No Cultural } \\
\text { Resources }\end{array}$ & $\begin{array}{l}\text { No further work } \\
\text { recommended }\end{array}$ & $\begin{array}{l}\text { Located Within Heavily } \\
\text { Inundated Area }\end{array}$ \\
\hline WP4JE083 & 57.9 & PEM & Low & None & $\begin{array}{l}\text { No Cultural } \\
\text { Resources }\end{array}$ & $\begin{array}{l}\text { No further work } \\
\text { recommended }\end{array}$ & $\begin{array}{l}\text { Completely avoided via non- } \\
\text { Section } 10 \mathrm{HDD} / \text { Bore }\end{array}$ \\
\hline WP2JE078_PEM & 58.1 & PEM & Low & 1 & $\begin{array}{l}\text { No Cultural } \\
\text { Resources }\end{array}$ & $\begin{array}{l}\text { No further work } \\
\text { recommended }\end{array}$ & $\begin{array}{l}\text { Completely avoided via non- } \\
\text { Section } 10 \mathrm{HDD} / \text { Bore }\end{array}$ \\
\hline WP2JE078_PEM_B & 58.1 & PEM & Low & 1 & $\begin{array}{l}\text { No Cultural } \\
\text { Resources }\end{array}$ & $\begin{array}{l}\text { No further work } \\
\text { recommended }\end{array}$ & None \\
\hline WP2JE078_PFO & 58.1 & PFO & Low & 1 & $\begin{array}{l}\text { No Cultural } \\
\text { Resources }\end{array}$ & $\begin{array}{l}\text { No further work } \\
\text { recommended }\end{array}$ & None \\
\hline WP2JE078_PFO_B & 58.1 & $\mathrm{PFO}$ & Low & None & $\begin{array}{l}\text { No Cultural } \\
\text { Resources }\end{array}$ & $\begin{array}{l}\text { No further work } \\
\text { recommended }\end{array}$ & $\begin{array}{l}\text { Completely avoided via non- } \\
\text { Section } 10 \mathrm{HDD} / \text { Bore }\end{array}$ \\
\hline WP2JE078_PSS_B & 58.2 & PSS & Low & 6 & $\begin{array}{l}\text { No Cultural } \\
\text { Resources }\end{array}$ & $\begin{array}{l}\text { No further work } \\
\text { recommended }\end{array}$ & Inundation \\
\hline WP2JE078_PEM_C & 58.4 & PEM & Low & 7 & $\begin{array}{l}\text { No Cultural } \\
\text { Resources }\end{array}$ & $\begin{array}{l}\text { No further work } \\
\text { recommended }\end{array}$ & Inundation \\
\hline
\end{tabular}


Appendix C 1- Survey Data in the Vicinity of Wetland Features

\begin{tabular}{|c|c|c|c|c|c|c|c|}
\hline Wetland ID & $\begin{array}{c}\text { Milepost } \\
\text { (Approximate) }\end{array}$ & $\begin{array}{c}\text { Wetland } \\
\text { Type }\end{array}$ & Probability & Shovel Tests & $\begin{array}{l}\text { Surveys } \\
\text { Results }\end{array}$ & Recommendations & Comments \\
\hline WP2JE078_PSS & 58.4 & PSS & Low & 1 & $\begin{array}{l}\text { No Cultural } \\
\text { Resources }\end{array}$ & $\begin{array}{l}\text { No further work } \\
\text { recommended }\end{array}$ & Inundation \\
\hline WP2JE078_PSS_C & 58.4 & PSS & Low & 3 & $\begin{array}{l}\text { No Cultural } \\
\text { Resources }\end{array}$ & $\begin{array}{l}\text { No further work } \\
\text { recommended }\end{array}$ & None \\
\hline WP2JE079_PEM_B & 58.5 & PEM & Low & None & $\begin{array}{l}\text { No Cultural } \\
\text { Resources }\end{array}$ & $\begin{array}{l}\text { No further work } \\
\text { recommended }\end{array}$ & $\begin{array}{l}\text { Located Within Heavily } \\
\text { Inundated Area }\end{array}$ \\
\hline WP2JE078_PSS_D & 58.5 & PSS & Low & 2 & $\begin{array}{l}\text { No Cultural } \\
\text { Resources }\end{array}$ & $\begin{array}{l}\text { No further work } \\
\text { recommended }\end{array}$ & Inundation \\
\hline WP2JE079_PSS & 58.6 & PSS & Low & 2 & $\begin{array}{l}\text { No Cultural } \\
\text { Resources }\end{array}$ & $\begin{array}{l}\text { No further work } \\
\text { recommended }\end{array}$ & Inundation \\
\hline WP2JE079_PSS_B & 58.6 & PSS & Low & 2 & $\begin{array}{l}\text { No Cultural } \\
\text { Resources }\end{array}$ & $\begin{array}{l}\text { No further work } \\
\text { recommended }\end{array}$ & Inundation \\
\hline WP6JE009_PSS_B & 58.7 & PSS & Low & None & $\begin{array}{l}\text { No Cultural } \\
\text { Resources }\end{array}$ & $\begin{array}{l}\text { No further work } \\
\text { recommended }\end{array}$ & Inundation \\
\hline WP6JE009_PEM_B & 58.8 & PEM & Low & None & $\begin{array}{l}\text { No Cultural } \\
\text { Resources }\end{array}$ & $\begin{array}{l}\text { No further work } \\
\text { recommended }\end{array}$ & Inundation \\
\hline WP6JE010_PEM & 58.9 & PEM & Low & None & $\begin{array}{l}\text { No Cultural } \\
\text { Resources }\end{array}$ & $\begin{array}{l}\text { No further work } \\
\text { recommended }\end{array}$ & Inundation \\
\hline WP6JE009_PFO & 58.9 & $\mathrm{PFO}$ & Low & None & $\begin{array}{l}\text { No Cultural } \\
\text { Resources }\end{array}$ & $\begin{array}{l}\text { No further work } \\
\text { recommended }\end{array}$ & Inundation \\
\hline WP6JE009_PFO_C & 58.9 & $\mathrm{PFO}$ & Low & None & $\begin{array}{l}\text { No Cultural } \\
\text { Resources }\end{array}$ & $\begin{array}{l}\text { No further work } \\
\text { recommended }\end{array}$ & Inundation \\
\hline WP6JE009_PEM_C & 59 & PEM & Low & None & $\begin{array}{l}\text { No Cultural } \\
\text { Resources }\end{array}$ & $\begin{array}{l}\text { No further work } \\
\text { recommended }\end{array}$ & Inundation \\
\hline WP6JE009_PFO_E & 59 & $\mathrm{PFO}$ & Low & None & $\begin{array}{l}\text { No Cultural } \\
\text { Resources }\end{array}$ & $\begin{array}{l}\text { No further work } \\
\text { recommended }\end{array}$ & Inundation \\
\hline WP6JE009_PSS & 59 & PSS & Low & None & $\begin{array}{l}\text { No Cultural } \\
\text { Resources }\end{array}$ & $\begin{array}{l}\text { No further work } \\
\text { recommended }\end{array}$ & Inundation \\
\hline WP6JE008_PEM & 59.1 & PEM & Low & None & $\begin{array}{l}\text { No Cultural } \\
\text { Resources }\end{array}$ & $\begin{array}{l}\text { No further work } \\
\text { recommended }\end{array}$ & Inundation \\
\hline WP6JE009_PEM & 59.1 & PEM & Low & None & $\begin{array}{l}\text { No Cultural } \\
\text { Resources }\end{array}$ & $\begin{array}{l}\text { No further work } \\
\text { recommended }\end{array}$ & Inundation \\
\hline
\end{tabular}


Appendix C 1- Survey Data in the Vicinity of Wetland Features

\begin{tabular}{|c|c|c|c|c|c|c|c|}
\hline Wetland ID & $\begin{array}{c}\text { Milepost } \\
\text { (Approximate) }\end{array}$ & $\begin{array}{l}\text { Wetland } \\
\text { Type }\end{array}$ & Probability & Shovel Tests & $\begin{array}{l}\text { Surveys } \\
\text { Results }\end{array}$ & Recommendations & Comments \\
\hline WP6JE008_PFO & 59.1 & $\mathrm{PFO}$ & Low & None & $\begin{array}{l}\text { No Cultural } \\
\text { Resources }\end{array}$ & $\begin{array}{l}\text { No further work } \\
\text { recommended }\end{array}$ & $\begin{array}{l}\text { Partially avoided via non- } \\
\text { Section } 10 \mathrm{HDD} / \text { Bore }\end{array}$ \\
\hline WP6JE008_PFO_B & 59.1 & $\mathrm{PFO}$ & Low & None & $\begin{array}{l}\text { No Cultural } \\
\text { Resources }\end{array}$ & $\begin{array}{l}\text { No further work } \\
\text { recommended }\end{array}$ & Inundation \\
\hline WP6JE008_PSS & 59.1 & PSS & Low & None & $\begin{array}{l}\text { No Cultural } \\
\text { Resources }\end{array}$ & $\begin{array}{l}\text { No further work } \\
\text { recommended }\end{array}$ & $\begin{array}{l}\text { Partially avoided via non- } \\
\text { Section } 10 \mathrm{HDD} / \text { Bore }\end{array}$ \\
\hline WP4JE076_PEM & 59.2 & PEM & Low & None & $\begin{array}{l}\text { No Cultural } \\
\text { Resources }\end{array}$ & $\begin{array}{l}\text { No further work } \\
\text { recommended }\end{array}$ & $\begin{array}{l}\text { Partially avoided via non- } \\
\text { Section } 10 \mathrm{HDD} / \text { Bore }\end{array}$ \\
\hline WP4JE076_PFO & 59.2 & $\mathrm{PFO}$ & Low & None & $\begin{array}{l}\text { No Cultural } \\
\text { Resources }\end{array}$ & $\begin{array}{l}\text { No further work } \\
\text { recommended }\end{array}$ & $\begin{array}{l}\text { Partially avoided via non- } \\
\text { Section } 10 \mathrm{HDD} / \text { Bore }\end{array}$ \\
\hline WP4JE076_PFO_D & 59.2 & $\mathrm{PFO}$ & Low & None & $\begin{array}{l}\text { No Cultural } \\
\text { Resources }\end{array}$ & $\begin{array}{l}\text { No further work } \\
\text { recommended }\end{array}$ & $\begin{array}{l}\text { Partially avoided via non- } \\
\text { Section } 10 \text { HDD/Bore }\end{array}$ \\
\hline WP4JE076_PSS_B & 59.2 & PSS & Low & None & $\begin{array}{l}\text { No Cultural } \\
\text { Resources }\end{array}$ & $\begin{array}{l}\text { No further work } \\
\text { recommended }\end{array}$ & $\begin{array}{c}\text { Located Within Heavily } \\
\text { Inundated Area }\end{array}$ \\
\hline WP4JE076_PFO_B & 59.3 & $\mathrm{PFO}$ & Low & None & $\begin{array}{l}\text { No Cultural } \\
\text { Resources }\end{array}$ & $\begin{array}{l}\text { No further work } \\
\text { recommended }\end{array}$ & $\begin{array}{l}\text { Located Within Heavily } \\
\text { Inundated Area }\end{array}$ \\
\hline WP4JE076_PFO_C & 59.3 & $\mathrm{PFO}$ & Low & None & $\begin{array}{l}\text { No Cultural } \\
\text { Resources }\end{array}$ & $\begin{array}{l}\text { No further work } \\
\text { recommended }\end{array}$ & $\begin{array}{l}\text { Located Within Heavily } \\
\text { Inundated Area }\end{array}$ \\
\hline WP4JE076_PSS & 59.3 & PSS & Low & 3 & $\begin{array}{l}\text { No Cultural } \\
\text { Resources }\end{array}$ & $\begin{array}{l}\text { No further work } \\
\text { recommended }\end{array}$ & None \\
\hline WP1JE010 & 59.4 & PEM & Low & 2 & $\begin{array}{l}\text { No Cultural } \\
\text { Resources }\end{array}$ & $\begin{array}{l}\text { No further work } \\
\text { recommended }\end{array}$ & None \\
\hline WP1JE011 & 59.4 & PEM & Low & 2 & $\begin{array}{l}\text { No Cultural } \\
\text { Resources }\end{array}$ & $\begin{array}{l}\text { No further work } \\
\text { recommended }\end{array}$ & None \\
\hline WP1JE009 & 59.8 & PEM & Low & 16 & $\begin{array}{l}\text { No Cultural } \\
\text { Resources }\end{array}$ & $\begin{array}{l}\text { No further work } \\
\text { recommended }\end{array}$ & None \\
\hline WP4JE078_PEM & 60 & PEM & Low & 2 & $\begin{array}{l}\text { No Cultural } \\
\text { Resources }\end{array}$ & $\begin{array}{l}\text { No further work } \\
\text { recommended }\end{array}$ & None \\
\hline WP4JE078_PSS & 60 & PSS & Low & None & $\begin{array}{l}\text { No Cultural } \\
\text { Resources }\end{array}$ & $\begin{array}{l}\text { No further work } \\
\text { recommended }\end{array}$ & $\begin{array}{l}\text { Located Within Heavily } \\
\text { Inundated Area }\end{array}$ \\
\hline WP1JE008_PSS & 60.1 & PSS & Low & None & $\begin{array}{l}\text { No Cultural } \\
\text { Resources }\end{array}$ & $\begin{array}{l}\text { No further work } \\
\text { recommended }\end{array}$ & $\begin{array}{l}\text { Located Within Heavily } \\
\text { Inundated Area }\end{array}$ \\
\hline
\end{tabular}


Appendix C 1- Survey Data in the Vicinity of Wetland Features

\begin{tabular}{|c|c|c|c|c|c|c|c|}
\hline Wetland ID & $\begin{array}{c}\text { Milepost } \\
\text { (Approximate) }\end{array}$ & $\begin{array}{c}\text { Wetland } \\
\text { Type }\end{array}$ & Probability & Shovel Tests & $\begin{array}{l}\text { Surveys } \\
\text { Results }\end{array}$ & Recommendations & Comments \\
\hline WP1JE008 & 60.6 & PEM & Low & 14 & $\begin{array}{l}\text { No Cultural } \\
\text { Resources }\end{array}$ & $\begin{array}{l}\text { No further work } \\
\text { recommended }\end{array}$ & None \\
\hline WP2JE009 & 60.7 & PEM & Low & None & $\begin{array}{l}\text { No Cultural } \\
\text { Resources }\end{array}$ & $\begin{array}{l}\text { No further work } \\
\text { recommended }\end{array}$ & $\begin{array}{l}\text { Located Within Heavily } \\
\text { Inundated Area }\end{array}$ \\
\hline WP2JE006 & 60.8 & PEM & Low & None & $\begin{array}{l}\text { No Cultural } \\
\text { Resources }\end{array}$ & $\begin{array}{l}\text { No further work } \\
\text { recommended }\end{array}$ & $\begin{array}{l}\text { Located Within Heavily } \\
\text { Inundated Area }\end{array}$ \\
\hline WP2JE007_PEM & 60.9 & PEM & Low & None & $\begin{array}{l}\text { No Cultural } \\
\text { Resources }\end{array}$ & $\begin{array}{l}\text { No further work } \\
\text { recommended }\end{array}$ & $\begin{array}{l}\text { Located Within Heavily } \\
\text { Inundated Area }\end{array}$ \\
\hline WP2JE008 & 61 & PEM & Low & 5 & $\begin{array}{l}\text { No Cultural } \\
\text { Resources }\end{array}$ & $\begin{array}{l}\text { No further work } \\
\text { recommended }\end{array}$ & None \\
\hline WP2JE007_PSS & 61 & PSS & Low & None & $\begin{array}{l}\text { No Cultural } \\
\text { Resources }\end{array}$ & $\begin{array}{l}\text { No further work } \\
\text { recommended }\end{array}$ & $\begin{array}{l}\text { Located Within Heavily } \\
\text { Inundated Area }\end{array}$ \\
\hline WP2JE004 & 61.2 & PEM & Low & 3 & $\begin{array}{l}\text { No Cultural } \\
\text { Resources }\end{array}$ & $\begin{array}{l}\text { No further work } \\
\text { recommended }\end{array}$ & None \\
\hline WP2JE005 & 61.5 & PEM & Low & None & $\begin{array}{l}\text { No Cultural } \\
\text { Resources }\end{array}$ & $\begin{array}{l}\text { No further work } \\
\text { recommended }\end{array}$ & $\begin{array}{l}\text { Located Within Heavily } \\
\text { Inundated Area }\end{array}$ \\
\hline WP2JE003 & 61.6 & PEM & Low & None & $\begin{array}{l}\text { No Cultural } \\
\text { Resources }\end{array}$ & $\begin{array}{l}\text { No further work } \\
\text { recommended }\end{array}$ & $\begin{array}{l}\text { Located Within Heavily } \\
\text { Inundated Area }\end{array}$ \\
\hline WP1JE001 & 61.9 & PEM & Low & 1 & $\begin{array}{l}\text { No Cultural } \\
\text { Resources }\end{array}$ & $\begin{array}{l}\text { No further work } \\
\text { recommended }\end{array}$ & $\begin{array}{l}\text { Completely avoided via non- } \\
\text { Section } 10 \mathrm{HDD} / \text { Bore }\end{array}$ \\
\hline WP1JE002 & 61.9 & PEM & Low & 1 & $\begin{array}{l}\text { No Cultural } \\
\text { Resources }\end{array}$ & $\begin{array}{l}\text { No further work } \\
\text { recommended }\end{array}$ & $\begin{array}{l}\text { Completely avoided via non- } \\
\text { Section } 10 \mathrm{HDD} / \text { Bore }\end{array}$ \\
\hline WP1JE003 & 61.9 & PEM & Low & 1 & $\begin{array}{l}\text { No Cultural } \\
\text { Resources }\end{array}$ & $\begin{array}{l}\text { No further work } \\
\text { recommended }\end{array}$ & None \\
\hline WP1JE004_PEM & 61.9 & PEM & Low & None & $\begin{array}{l}\text { No Cultural } \\
\text { Resources }\end{array}$ & $\begin{array}{l}\text { No further work } \\
\text { recommended }\end{array}$ & $\begin{array}{l}\text { Completely avoided via non- } \\
\text { Section } 10 \mathrm{HDD} / \text { Bore }\end{array}$ \\
\hline
\end{tabular}


Appendix C 1- Survey Data in the Vicinity of Wetland Features

\begin{tabular}{|c|c|c|c|c|c|c|c|}
\hline Wetland ID & $\begin{array}{c}\text { Milepost } \\
\text { (Approximate) }\end{array}$ & $\begin{array}{l}\text { Wetland } \\
\text { Type }\end{array}$ & Probability & Shovel Tests & $\begin{array}{l}\text { Surveys } \\
\text { Results }\end{array}$ & Recommendations & Comments \\
\hline WP1JE004_PSS & 61.9 & PSS & Low & None & $\begin{array}{l}\text { No Cultural } \\
\text { Resources }\end{array}$ & $\begin{array}{l}\text { No further work } \\
\text { recommended }\end{array}$ & $\begin{array}{l}\text { Completely avoided via non- } \\
\text { Section } 10 \mathrm{HDD} / \text { Bore }\end{array}$ \\
\hline WP1JE005 & 62 & PEM & Low & None & $\begin{array}{l}\text { No Cultural } \\
\text { Resources }\end{array}$ & $\begin{array}{l}\text { No further work } \\
\text { recommended }\end{array}$ & Inundation \\
\hline WP1JE006 & 62 & PEM & Low & None & $\begin{array}{l}\text { No Cultural } \\
\text { Resources }\end{array}$ & $\begin{array}{l}\text { No further work } \\
\text { recommended }\end{array}$ & Inundation \\
\hline WP1JE007 & 62 & PEM & Low & None & $\begin{array}{l}\text { No Cultural } \\
\text { Resources }\end{array}$ & $\begin{array}{l}\text { No further work } \\
\text { recommended }\end{array}$ & Inundation \\
\hline WP4JE087 & 62.3 & PEM & Low & None & $\begin{array}{l}\text { No Cultural } \\
\text { Resources }\end{array}$ & $\begin{array}{l}\text { No further work } \\
\text { recommended }\end{array}$ & Inundation \\
\hline WP2JE071 & 62.5 & PEM & Low & None & $\begin{array}{l}\text { No Cultural } \\
\text { Resources }\end{array}$ & $\begin{array}{l}\text { No further work } \\
\text { recommended }\end{array}$ & $\begin{array}{l}\text { Completely avoided via non- } \\
\text { Section } 10 \mathrm{HDD} / \text { Bore }\end{array}$ \\
\hline WP2JE072 & 62.7 & PEM & Low & 6 & $\begin{array}{l}\text { No Cultural } \\
\text { Resources }\end{array}$ & $\begin{array}{l}\text { No further work } \\
\text { recommended }\end{array}$ & $\begin{array}{l}\text { Partially avoided via non- } \\
\text { Section } 10 \mathrm{HDD} / \text { Bore }\end{array}$ \\
\hline WP7JE009 & 63.2 & PEM & Low & None & $\begin{array}{l}\text { No Cultural } \\
\text { Resources }\end{array}$ & $\begin{array}{l}\text { No further work } \\
\text { recommended }\end{array}$ & $\begin{array}{l}\text { Completely avoided via non- } \\
\text { Section } 10 \mathrm{HDD} / \text { Bore }\end{array}$ \\
\hline WP7JE008 & 63.2 & PEM & Low & None & $\begin{array}{l}\text { No Cultural } \\
\text { Resources }\end{array}$ & $\begin{array}{l}\text { No further work } \\
\text { recommended }\end{array}$ & $\begin{array}{l}\text { Completely avoided via non- } \\
\text { Section } 10 \mathrm{HDD} / \text { Bore }\end{array}$ \\
\hline WP7JE006 & 63.2 & PEM & Low & None & $\begin{array}{l}\text { No Cultural } \\
\text { Resources }\end{array}$ & $\begin{array}{l}\text { No further work } \\
\text { recommended }\end{array}$ & $\begin{array}{l}\text { Completely avoided via non- } \\
\text { Section } 10 \mathrm{HDD} / \text { Bore }\end{array}$ \\
\hline WP7JE010_PEM & 63.2 & PEM & Low & None & $\begin{array}{l}\text { No Cultural } \\
\text { Resources }\end{array}$ & $\begin{array}{l}\text { No further work } \\
\text { recommended }\end{array}$ & $\begin{array}{l}\text { Completely avoided via non- } \\
\text { Section } 10 \mathrm{HDD} / \text { Bore }\end{array}$ \\
\hline WP7JE010_PSS & 63.2 & PEM & Low & None & $\begin{array}{l}\text { No Cultural } \\
\text { Resources }\end{array}$ & $\begin{array}{l}\text { No further work } \\
\text { recommended }\end{array}$ & $\begin{array}{l}\text { Completely avoided via non- } \\
\text { Section } 10 \mathrm{HDD} / \text { Bore }\end{array}$ \\
\hline
\end{tabular}


Appendix C 1- Survey Data in the Vicinity of Wetland Features

\begin{tabular}{|c|c|c|c|c|c|c|c|}
\hline Wetland ID & $\begin{array}{c}\text { Milepost } \\
\text { (Approximate) }\end{array}$ & $\begin{array}{l}\text { Wetland } \\
\text { Type }\end{array}$ & Probability & Shovel Tests & $\begin{array}{l}\text { Surveys } \\
\text { Results }\end{array}$ & Recommendations & Comments \\
\hline WP7JE007 & 63.2 & PEM & Low & None & $\begin{array}{l}\text { No Cultural } \\
\text { Resources }\end{array}$ & $\begin{array}{l}\text { No further work } \\
\text { recommended }\end{array}$ & $\begin{array}{l}\text { Completely avoided via non- } \\
\text { Section } 10 \mathrm{HDD} / \text { Bore }\end{array}$ \\
\hline WP4JE089 & 63.2 & PEM & Low & None & $\begin{array}{l}\text { No Cultural } \\
\text { Resources }\end{array}$ & $\begin{array}{l}\text { No further work } \\
\text { recommended }\end{array}$ & Inundation \\
\hline WP4JE090 & 63.2 & PEM & Low & None & $\begin{array}{l}\text { No Cultural } \\
\text { Resources }\end{array}$ & $\begin{array}{l}\text { No further work } \\
\text { recommended }\end{array}$ & Inundation \\
\hline WP7JE013 & 63.2 & PEM & Low & None & $\begin{array}{l}\text { No Cultural } \\
\text { Resources }\end{array}$ & $\begin{array}{l}\text { No further work } \\
\text { recommended }\end{array}$ & $\begin{array}{l}\text { Completely avoided via non- } \\
\text { Section } 10 \mathrm{HDD} / \text { Bore }\end{array}$ \\
\hline WP7JE012 & 63.2 & PEM & Low & None & $\begin{array}{l}\text { No Cultural } \\
\text { Resources }\end{array}$ & $\begin{array}{l}\text { No further work } \\
\text { recommended }\end{array}$ & Inundation \\
\hline WP4JE091 & 63.2 & PEM & Low & None & $\begin{array}{l}\text { No Cultural } \\
\text { Resources }\end{array}$ & $\begin{array}{l}\text { No further work } \\
\text { recommended }\end{array}$ & Inundation \\
\hline WP7JE011_PEM & 63.2 & PEM & Low & None & $\begin{array}{l}\text { No Cultural } \\
\text { Resources }\end{array}$ & $\begin{array}{l}\text { No further work } \\
\text { recommended }\end{array}$ & $\begin{array}{l}\text { Partially avoided via non- } \\
\text { Section } 10 \mathrm{HDD} / \text { Bore }\end{array}$ \\
\hline WP7JE011_PFO & 63.2 & $\mathrm{PFO}$ & Low & 1 & $\begin{array}{l}\text { No Cultural } \\
\text { Resources }\end{array}$ & $\begin{array}{l}\text { No further work } \\
\text { recommended }\end{array}$ & Inundation \\
\hline WP7JE014_PEM & 63.4 & PEM & Low & None & $\begin{array}{l}\text { No Cultural } \\
\text { Resources }\end{array}$ & $\begin{array}{l}\text { No further work } \\
\text { recommended }\end{array}$ & Inundation \\
\hline WP7JE014_PSS & 63.9 & PSS & Low & None & $\begin{array}{l}\text { No Cultural } \\
\text { Resources }\end{array}$ & $\begin{array}{l}\text { No further work } \\
\text { recommended }\end{array}$ & Inundation \\
\hline WP7JE001 & 63.9 & PEM & Low & 5 & $\begin{array}{l}\text { No Cultural } \\
\text { Resources }\end{array}$ & $\begin{array}{l}\text { No further work } \\
\text { recommended }\end{array}$ & Inundation \\
\hline WP7JE014_PSS_B & 63.9 & PSS & Low & None & $\begin{array}{l}\text { No Cultural } \\
\text { Resources }\end{array}$ & $\begin{array}{l}\text { No further work } \\
\text { recommended }\end{array}$ & Inundation \\
\hline WP7JE014_PEM_B & 63.9 & PEM & Low & None & $\begin{array}{l}\text { No Cultural } \\
\text { Resources }\end{array}$ & $\begin{array}{l}\text { No further work } \\
\text { recommended }\end{array}$ & Inundation \\
\hline WP4JE092 & $\mathrm{n} / \mathrm{a}$ & PEM & Low & None & $\begin{array}{l}\text { No Cultural } \\
\text { Resources }\end{array}$ & $\begin{array}{l}\text { No further work } \\
\text { recommended }\end{array}$ & Mostly inundated \\
\hline WP4JE093 & $\mathrm{n} / \mathrm{a}$ & PEM & Low & None & $\begin{array}{l}\text { No Cultural } \\
\text { Resources }\end{array}$ & $\begin{array}{l}\text { No further work } \\
\text { recommended }\end{array}$ & Mostly inundated \\
\hline
\end{tabular}


Appendix C 1- Survey Data in the Vicinity of Wetland Features

\begin{tabular}{|c|c|c|c|c|c|c|c|}
\hline Wetland ID & $\begin{array}{c}\text { Milepost } \\
\text { (Approximate) }\end{array}$ & $\begin{array}{c}\text { Wetland } \\
\text { Type }\end{array}$ & Probability & Shovel Tests & $\begin{array}{l}\text { Surveys } \\
\text { Results }\end{array}$ & Recommendations & Comments \\
\hline WP4JE094 & $\mathrm{n} / \mathrm{a}$ & PEM & Low & None & $\begin{array}{l}\text { No Cultural } \\
\text { Resources }\end{array}$ & $\begin{array}{l}\text { No further work } \\
\text { recommended }\end{array}$ & Mostly inundated \\
\hline WP4JE095 & $\mathrm{n} / \mathrm{a}$ & PEM & Low & None & $\begin{array}{l}\text { No Cultural } \\
\text { Resources }\end{array}$ & $\begin{array}{l}\text { No further work } \\
\text { recommended }\end{array}$ & Mostly inundated \\
\hline WP7JE015 & $n / a$ & PEM & Low & 4 & $\begin{array}{l}\text { No Cultural } \\
\text { Resources }\end{array}$ & $\begin{array}{l}\text { No further work } \\
\text { recommended }\end{array}$ & Mostly inundated \\
\hline WP4JE097_PFO_B & $\mathrm{n} / \mathrm{a}$ & $\mathrm{PFO}$ & Low & None & $\begin{array}{l}\text { No Cultural } \\
\text { Resources }\end{array}$ & $\begin{array}{l}\text { No further work } \\
\text { recommended }\end{array}$ & Mostly inundated \\
\hline WP7JE017 & $\mathrm{n} / \mathrm{a}$ & PEM & Low & None & $\begin{array}{l}\text { No Cultural } \\
\text { Resources }\end{array}$ & $\begin{array}{l}\text { No further work } \\
\text { recommended }\end{array}$ & Mostly inundated \\
\hline WP4JE096 & 63.9 & PEM & Low & None & $\begin{array}{l}\text { No Cultural } \\
\text { Resources }\end{array}$ & $\begin{array}{l}\text { No further work } \\
\text { recommended }\end{array}$ & Inundation \\
\hline WP4JE097_PEM & 64.1 & PEM & Low & None & $\begin{array}{l}\text { No Cultural } \\
\text { Resources }\end{array}$ & $\begin{array}{l}\text { No further work } \\
\text { recommended }\end{array}$ & Inundation \\
\hline WP4JE097_PSS & 64.1 & PSS & Low & None & $\begin{array}{l}\text { No Cultural } \\
\text { Resources }\end{array}$ & $\begin{array}{l}\text { No further work } \\
\text { recommended }\end{array}$ & Inundation \\
\hline WP4JE097_PSS_B & 64.1 & PSS & Low & None & $\begin{array}{l}\text { No Cultural } \\
\text { Resources }\end{array}$ & $\begin{array}{l}\text { No further work } \\
\text { recommended }\end{array}$ & Inundation \\
\hline WP7JE018_PSS & $\mathrm{n} / \mathrm{a}$ & PSS & Low & 2 & $\begin{array}{l}\text { No Cultural } \\
\text { Resources }\end{array}$ & $\begin{array}{l}\text { No further work } \\
\text { recommended }\end{array}$ & Inundation \\
\hline WP4JE097_PFO & 64.5 & $\mathrm{PFO}$ & Low & None & $\begin{array}{l}\text { No Cultural } \\
\text { Resources }\end{array}$ & $\begin{array}{l}\text { No further work } \\
\text { recommended }\end{array}$ & Inundation \\
\hline WP7JE018_PFO & $\mathrm{n} / \mathrm{a}$ & $\mathrm{PFO}$ & Low & 1 & $\begin{array}{l}\text { No Cultural } \\
\text { Resources }\end{array}$ & $\begin{array}{l}\text { No further work } \\
\text { recommended }\end{array}$ & Inundation \\
\hline WP7JE018_PEM & $\mathrm{n} / \mathrm{a}$ & PEM & Low & None & $\begin{array}{l}\text { No Cultural } \\
\text { Resources }\end{array}$ & $\begin{array}{l}\text { No further work } \\
\text { recommended }\end{array}$ & Inundation \\
\hline WP7JE019 & $\mathrm{n} / \mathrm{a}$ & PEM & Low & None & $\begin{array}{l}\text { No Cultural } \\
\text { Resources }\end{array}$ & $\begin{array}{l}\text { No further work } \\
\text { recommended }\end{array}$ & Inundation \\
\hline WP7JE002_PFO & $\mathrm{n} / \mathrm{a}$ & $\mathrm{PFO}$ & Low & None & $\begin{array}{l}\text { No Cultural } \\
\text { Resources }\end{array}$ & $\begin{array}{l}\text { No further work } \\
\text { recommended }\end{array}$ & Inundation \\
\hline WP7JE002_PSS & $\mathrm{n} / \mathrm{a}$ & PSS & Low & None & $\begin{array}{c}\text { No Cultural } \\
\text { Resources }\end{array}$ & $\begin{array}{l}\text { No further work } \\
\text { recommended }\end{array}$ & Inundation \\
\hline
\end{tabular}


Appendix C 1- Survey Data in the Vicinity of Wetland Features

\begin{tabular}{|c|c|c|c|c|c|c|c|}
\hline Wetland ID & $\begin{array}{c}\text { Milepost } \\
\text { (Approximate) }\end{array}$ & $\begin{array}{l}\text { Wetland } \\
\text { Type }\end{array}$ & Probability & Shovel Tests & $\begin{array}{l}\text { Surveys } \\
\text { Results }\end{array}$ & Recommendations & Comments \\
\hline WP7JE002_PEM & $\mathrm{n} / \mathrm{a}$ & PEM & Low & None & $\begin{array}{l}\text { No Cultural } \\
\text { Resources }\end{array}$ & $\begin{array}{l}\text { No further work } \\
\text { recommended }\end{array}$ & Inundation \\
\hline WP4JE098_PSS & 64.7 & PSS & Low & None & $\begin{array}{l}\text { No Cultural } \\
\text { Resources }\end{array}$ & $\begin{array}{l}\text { No further work } \\
\text { recommended }\end{array}$ & Inundation \\
\hline WP4JE098_PEM & 64.8 & PEM & Low & None & $\begin{array}{l}\text { No Cultural } \\
\text { Resources }\end{array}$ & $\begin{array}{l}\text { No further work } \\
\text { recommended }\end{array}$ & Inundation \\
\hline WP4JE097_WET_PSS & 64.8 & PSS & Low & None & $\begin{array}{l}\text { No Cultural } \\
\text { Resources }\end{array}$ & $\begin{array}{l}\text { No further work } \\
\text { recommended }\end{array}$ & Inundation \\
\hline WP7JE003 & $\mathrm{n} / \mathrm{a}$ & PEM & Low & None & $\begin{array}{l}\text { No Cultural } \\
\text { Resources }\end{array}$ & $\begin{array}{l}\text { No further work } \\
\text { recommended }\end{array}$ & Inundation \\
\hline WP7JE023 & $\mathrm{n} / \mathrm{a}$ & PEM & Low & None & $\begin{array}{l}\text { No Cultural } \\
\text { Resources }\end{array}$ & $\begin{array}{c}\text { No further work } \\
\text { recommended }\end{array}$ & Inundation \\
\hline WP7JE022 & $\mathrm{n} / \mathrm{a}$ & PEM & Low & None & $\begin{array}{l}\text { No Cultural } \\
\text { Resources }\end{array}$ & $\begin{array}{l}\text { No further work } \\
\text { recommended }\end{array}$ & Inundation \\
\hline WP7JE021 & $\mathrm{n} / \mathrm{a}$ & PEM & Low & None & $\begin{array}{l}\text { No Cultural } \\
\text { Resources }\end{array}$ & $\begin{array}{l}\text { No further work } \\
\text { recommended }\end{array}$ & Inundation \\
\hline WP7JE020 & $\mathrm{n} / \mathrm{a}$ & PSS & Low & None & $\begin{array}{l}\text { No Cultural } \\
\text { Resources }\end{array}$ & $\begin{array}{l}\text { No further work } \\
\text { recommended }\end{array}$ & Inundation \\
\hline WP7JE004_PEM & $\mathrm{n} / \mathrm{a}$ & PEM & Low & None & $\begin{array}{l}\text { No Cultural } \\
\text { Resources }\end{array}$ & $\begin{array}{l}\text { No further work } \\
\text { recommended }\end{array}$ & Inundation \\
\hline WP7JE004_PSS & $\mathrm{n} / \mathrm{a}$ & PSS & Low & None & $\begin{array}{l}\text { No Cultural } \\
\text { Resources }\end{array}$ & $\begin{array}{l}\text { No further work } \\
\text { recommended }\end{array}$ & Inundation \\
\hline WP7JE005_PFO & 65.8 & $\mathrm{PFO}$ & Low & None & $\begin{array}{l}\text { No Cultural } \\
\text { Resources }\end{array}$ & $\begin{array}{l}\text { No further work } \\
\text { recommended }\end{array}$ & Inundation \\
\hline WP7JE005_PEM & 65.8 & PEM & Low & None & $\begin{array}{l}\text { No Cultural } \\
\text { Resources }\end{array}$ & $\begin{array}{l}\text { No further work } \\
\text { recommended }\end{array}$ & Inundation \\
\hline WP6JE007_PFO_D & 65.1 & $\mathrm{PFO}$ & Low & None & $\begin{array}{l}\text { No Cultural } \\
\text { Resources }\end{array}$ & $\begin{array}{l}\text { No further work } \\
\text { recommended }\end{array}$ & Inundation \\
\hline WP7JE005_PFO_B & 65.8 & $\mathrm{PFO}$ & Low & 1 & $\begin{array}{l}\text { No Cultural } \\
\text { Resources }\end{array}$ & $\begin{array}{l}\text { No further work } \\
\text { recommended }\end{array}$ & Inundation \\
\hline WP6JE007_PFO_C & 65.2 & $\mathrm{PFO}$ & Low & None & $\begin{array}{c}\text { No Cultural } \\
\text { Resources }\end{array}$ & $\begin{array}{l}\text { No further work } \\
\text { recommended }\end{array}$ & Inundation \\
\hline
\end{tabular}


Appendix C 1- Survey Data in the Vicinity of Wetland Features

\begin{tabular}{|c|c|c|c|c|c|c|c|}
\hline Wetland ID & $\begin{array}{c}\text { Milepost } \\
\text { (Approximate) }\end{array}$ & $\begin{array}{l}\text { Wetland } \\
\text { Type }\end{array}$ & Probability & Shovel Tests & $\begin{array}{l}\text { Surveys } \\
\text { Results }\end{array}$ & Recommendations & Comments \\
\hline WP6JE007_PSS_F & 65.2 & PSS & Low & None & $\begin{array}{l}\text { No Cultural } \\
\text { Resources }\end{array}$ & $\begin{array}{l}\text { No further work } \\
\text { recommended }\end{array}$ & Inundation \\
\hline WP6JE007_PEM & 66.1 & PEM & Low & None & $\begin{array}{l}\text { No Cultural } \\
\text { Resources }\end{array}$ & $\begin{array}{l}\text { No further work } \\
\text { recommended }\end{array}$ & Inundation \\
\hline WP6JE007_PFO_E & 66.1 & $\mathrm{PFO}$ & Low & None & $\begin{array}{l}\text { No Cultural } \\
\text { Resources }\end{array}$ & $\begin{array}{l}\text { No further work } \\
\text { recommended }\end{array}$ & Inundation \\
\hline WP6JE007_PSS_E & 65.6 & PSS & Low & None & $\begin{array}{l}\text { No Cultural } \\
\text { Resources }\end{array}$ & $\begin{array}{l}\text { No further work } \\
\text { recommended }\end{array}$ & Inundation \\
\hline WP6JE007_PEM_B & 65.7 & PEM & Low & 3 & $\begin{array}{l}\text { No Cultural } \\
\text { Resources }\end{array}$ & $\begin{array}{l}\text { No further work } \\
\text { recommended }\end{array}$ & Inundation \\
\hline WP6JE007_PSS_D & 65.7 & PSS & Low & None & $\begin{array}{l}\text { No Cultural } \\
\text { Resources }\end{array}$ & $\begin{array}{l}\text { No further work } \\
\text { recommended }\end{array}$ & $\begin{array}{c}\text { Located Within Heavily } \\
\text { Inundated Area }\end{array}$ \\
\hline WP6JE007_PFO & 65.8 & $\mathrm{PFO}$ & Low & 3 & $\begin{array}{l}\text { No Cultural } \\
\text { Resources }\end{array}$ & $\begin{array}{l}\text { No further work } \\
\text { recommended }\end{array}$ & None \\
\hline WP6JE007_PFO_B & 65.8 & $\mathrm{PFO}$ & Low & None & $\begin{array}{l}\text { No Cultural } \\
\text { Resources }\end{array}$ & $\begin{array}{l}\text { No further work } \\
\text { recommended }\end{array}$ & Inundation \\
\hline WP6JE007_PSS_B & 65.8 & PSS & Low & None & $\begin{array}{l}\text { No Cultural } \\
\text { Resources }\end{array}$ & $\begin{array}{l}\text { No further work } \\
\text { recommended }\end{array}$ & Inundation \\
\hline WP6JE007_PSS_C & 65.8 & PSS & Low & None & $\begin{array}{l}\text { No Cultural } \\
\text { Resources }\end{array}$ & $\begin{array}{l}\text { No further work } \\
\text { recommended }\end{array}$ & Inundation \\
\hline WP2JE071_PEM & 65.9 & PEM & Low & None & $\begin{array}{l}\text { No Cultural } \\
\text { Resources }\end{array}$ & $\begin{array}{l}\text { No further work } \\
\text { recommended }\end{array}$ & Inundation \\
\hline WP6JE007_PSS & 65.9 & PSS & Low & None & $\begin{array}{l}\text { No Cultural } \\
\text { Resources }\end{array}$ & $\begin{array}{l}\text { No further work } \\
\text { recommended }\end{array}$ & Inundation \\
\hline WP2JE073_PEM & 66 & PEM & Low & None & $\begin{array}{l}\text { No Cultural } \\
\text { Resources }\end{array}$ & $\begin{array}{l}\text { No further work } \\
\text { recommended }\end{array}$ & Inundation \\
\hline WP2JE072_PEM & 66.2 & PEM & Low & None & $\begin{array}{l}\text { No Cultural } \\
\text { Resources }\end{array}$ & $\begin{array}{l}\text { No further work } \\
\text { recommended }\end{array}$ & Inundation \\
\hline WP2JE074_PEM & 66.2 & PEM & Low & None & $\begin{array}{l}\text { No Cultural } \\
\text { Resources }\end{array}$ & $\begin{array}{l}\text { No further work } \\
\text { recommended }\end{array}$ & $\begin{array}{l}\text { Completely avoided via non- } \\
\text { Section } 10 \mathrm{HDD} / \text { Bore }\end{array}$ \\
\hline
\end{tabular}


Appendix C 1- Survey Data in the Vicinity of Wetland Features

\begin{tabular}{|c|c|c|c|c|c|c|c|}
\hline Wetland ID & $\begin{array}{c}\text { Milepost } \\
\text { (Approximate) }\end{array}$ & $\begin{array}{l}\text { Wetland } \\
\text { Type }\end{array}$ & Probability & Shovel Tests & $\begin{array}{l}\text { Surveys } \\
\text { Results }\end{array}$ & Recommendations & Comments \\
\hline WP2JE074_PFO & 66.3 & $\mathrm{PFO}$ & Low & None & $\begin{array}{l}\text { No Cultural } \\
\text { Resources }\end{array}$ & $\begin{array}{l}\text { No further work } \\
\text { recommended }\end{array}$ & $\begin{array}{l}\text { Completely avoided via non- } \\
\text { Section } 10 \text { HDD/Bore }\end{array}$ \\
\hline WP2JE075_PEM & 66.4 & PEM & Low & None & $\begin{array}{l}\text { No Cultural } \\
\text { Resources }\end{array}$ & $\begin{array}{l}\text { No further work } \\
\text { recommended }\end{array}$ & Inundation \\
\hline WP2JE076_PEM & 66.4 & PEM & Low & None & $\begin{array}{l}\text { No Cultural } \\
\text { Resources }\end{array}$ & $\begin{array}{l}\text { No further work } \\
\text { recommended }\end{array}$ & Inundation \\
\hline WP4JE088_PFO_B & 66.5 & $\mathrm{PFO}$ & Low & None & $\begin{array}{l}\text { No Cultural } \\
\text { Resources }\end{array}$ & $\begin{array}{l}\text { No further work } \\
\text { recommended }\end{array}$ & Inundation \\
\hline WP4JE088_PFO & 66.6 & $\mathrm{PFO}$ & Low & None & $\begin{array}{l}\text { No Cultural } \\
\text { Resources }\end{array}$ & $\begin{array}{l}\text { No further work } \\
\text { recommended }\end{array}$ & Inundation \\
\hline WP4JE088_PEM & 67.4 & PEM & Low & None & $\begin{array}{c}\text { No Cultural } \\
\text { Resources }\end{array}$ & $\begin{array}{l}\text { No further work } \\
\text { recommended }\end{array}$ & $\begin{array}{c}\text { Completely avoided via non- } \\
\text { Section } 10 \mathrm{HDD} / \text { Bore }\end{array}$ \\
\hline WP4JE088_PSS & 67.4 & PSS & Low & None & $\begin{array}{l}\text { No Cultural } \\
\text { Resources }\end{array}$ & $\begin{array}{l}\text { No further work } \\
\text { recommended }\end{array}$ & Inundation \\
\hline $\begin{array}{c}\text { WP4JE100_WET_PE } \\
\text { M_B }\end{array}$ & 67.5 & PEM & Low & None & $\begin{array}{c}\text { No Cultural } \\
\text { Resources }\end{array}$ & $\begin{array}{l}\text { No further work } \\
\text { recommended }\end{array}$ & Inundation \\
\hline $\begin{array}{c}\text { WP4JE100_WET_PSS } \\
\text { _D }\end{array}$ & 67.5 & PSS & Low & None & $\begin{array}{c}\text { No Cultural } \\
\text { Resources }\end{array}$ & $\begin{array}{l}\text { No further work } \\
\text { recommended }\end{array}$ & $\begin{array}{c}\text { Located Within Heavily } \\
\text { Inundated Area }\end{array}$ \\
\hline $\begin{array}{c}\text { WP4JE100_WET_PSS } \\
\text { E }\end{array}$ & 67.5 & PSS & Low & None & $\begin{array}{c}\text { No Cultural } \\
\text { Resources }\end{array}$ & $\begin{array}{l}\text { No further work } \\
\text { recommended }\end{array}$ & $\begin{array}{c}\text { Located Within Heavily } \\
\text { Inundated Area }\end{array}$ \\
\hline $\begin{array}{c}\text { WP4JE100_WET_PE } \\
\text { M_C }\end{array}$ & 67.6 & PEM & Low & None & $\begin{array}{c}\text { No Cultural } \\
\text { Resources }\end{array}$ & $\begin{array}{l}\text { No further work } \\
\text { recommended }\end{array}$ & $\begin{array}{c}\text { Located Within Heavily } \\
\text { Inundated Area }\end{array}$ \\
\hline $\begin{array}{c}\text { WP4JE100_WET_PSS } \\
\text { _C }\end{array}$ & 67.6 & PSS & Low & None & $\begin{array}{c}\text { No Cultural } \\
\text { Resources }\end{array}$ & $\begin{array}{l}\text { No further work } \\
\text { recommended }\end{array}$ & Inundation \\
\hline $\begin{array}{c}\text { WP4JE101_WET_PE } \\
\text { M }\end{array}$ & 67.7 & PEM & Low & 4 & $\begin{array}{c}\text { No Cultural } \\
\text { Resources }\end{array}$ & $\begin{array}{l}\text { No further work } \\
\text { recommended }\end{array}$ & Inundation \\
\hline WP4JE102 & 67.7 & PEM & Low & 3 & $\begin{array}{l}\text { No Cultural } \\
\text { Resources }\end{array}$ & $\begin{array}{l}\text { No further work } \\
\text { recommended }\end{array}$ & None \\
\hline WP4JE101_PFO & 67.7 & $\mathrm{PFO}$ & Low & 3 & $\begin{array}{l}\text { No Cultural } \\
\text { Resources }\end{array}$ & $\begin{array}{l}\text { No further work } \\
\text { recommended }\end{array}$ & None \\
\hline
\end{tabular}


Appendix C 1- Survey Data in the Vicinity of Wetland Features

\begin{tabular}{|c|c|c|c|c|c|c|c|}
\hline Wetland ID & $\begin{array}{c}\text { Milepost } \\
\text { (Approximate) }\end{array}$ & $\begin{array}{l}\text { Wetland } \\
\text { Type }\end{array}$ & Probability & Shovel Tests & $\begin{array}{l}\text { Surveys } \\
\text { Results }\end{array}$ & Recommendations & Comments \\
\hline WP4JE100_WET_PSS & 67.7 & PSS & Low & None & $\begin{array}{l}\text { No Cultural } \\
\text { Resources }\end{array}$ & $\begin{array}{l}\text { No further work } \\
\text { recommended }\end{array}$ & $\begin{array}{l}\text { Located Within Heavily } \\
\text { Inundated Area }\end{array}$ \\
\hline WP4JE101_WET_PSS & 67.7 & PSS & Low & 1 & $\begin{array}{l}\text { No Cultural } \\
\text { Resources }\end{array}$ & $\begin{array}{l}\text { No further work } \\
\text { recommended }\end{array}$ & $\begin{array}{l}\text { Located Within Heavily } \\
\text { Inundated Area }\end{array}$ \\
\hline $\begin{array}{c}\text { WP4JE101_WET_PSS } \\
\text { _B }\end{array}$ & 67.7 & PSS & Low & 1 & $\begin{array}{l}\text { No Cultural } \\
\text { Resources }\end{array}$ & $\begin{array}{l}\text { No further work } \\
\text { recommended }\end{array}$ & None \\
\hline $\begin{array}{c}\text { WP4JE100_WET_PE } \\
M\end{array}$ & 67.8 & PEM & Low & None & $\begin{array}{l}\text { No Cultural } \\
\text { Resources }\end{array}$ & $\begin{array}{l}\text { No further work } \\
\text { recommended }\end{array}$ & $\begin{array}{l}\text { Located Within Heavily } \\
\text { Inundated Area }\end{array}$ \\
\hline $\begin{array}{c}\text { WP4JE100_WET_PSS } \\
\text { _B }\end{array}$ & 67.8 & PSS & Low & 1 & $\begin{array}{l}\text { No Cultural } \\
\text { Resources }\end{array}$ & $\begin{array}{l}\text { No further work } \\
\text { recommended }\end{array}$ & Inundation \\
\hline WP4JE099_PEM_C & 68.3 & PEM & Low & None & $\begin{array}{l}\text { No Cultural } \\
\text { Resources }\end{array}$ & $\begin{array}{l}\text { No further work } \\
\text { recommended }\end{array}$ & $\begin{array}{l}\text { Completely avoided via non- } \\
\text { Section } 10 \mathrm{HDD} / \text { Bore }\end{array}$ \\
\hline WP4JE099_PSS & 68 & PSS & Low & 5 & $\begin{array}{l}\text { No Cultural } \\
\text { Resources }\end{array}$ & $\begin{array}{l}\text { No further work } \\
\text { recommended }\end{array}$ & Inundation \\
\hline WP4JE099_PEM_B & 68.6 & PEM & Low & None & $\begin{array}{l}\text { No Cultural } \\
\text { Resources }\end{array}$ & $\begin{array}{l}\text { No further work } \\
\text { recommended }\end{array}$ & $\begin{array}{l}\text { Completely avoided via non- } \\
\text { Section } 10 \mathrm{HDD} / \text { Bore }\end{array}$ \\
\hline WP4JE099_PEM & 68.7 & PEM & Low & None & $\begin{array}{l}\text { No Cultural } \\
\text { Resources }\end{array}$ & $\begin{array}{l}\text { No further work } \\
\text { recommended }\end{array}$ & $\begin{array}{l}\text { Completely avoided via non- } \\
\text { Section } 10 \mathrm{HDD} / \text { Bore }\end{array}$ \\
\hline WP4JE103 & 0 & PEM & Low & 3 & $\begin{array}{l}\text { No Cultural } \\
\text { Resources }\end{array}$ & $\begin{array}{l}\text { No further work } \\
\text { recommended }\end{array}$ & Inundation \\
\hline
\end{tabular}


Appendix C2 - Survey Data in the Vicinity of Waterbody Features

\begin{tabular}{|c|c|c|c|c|c|c|c|c|}
\hline Waterbody ID & $\begin{array}{c}\text { Milepost } \\
\text { (Approximate) }\end{array}$ & Waterbody Name & $\begin{array}{l}\text { Waterbody } \\
\text { Type }\end{array}$ & Probability & $\begin{array}{c}\text { Shovel } \\
\text { Tests }\end{array}$ & Surveys Results & Recommendations & Comments \\
\hline \multicolumn{9}{|c|}{ Chambers County } \\
\hline SP3CH060 & 0.1 & $\begin{array}{c}\text { UT to Cedar } \\
\text { Bayou }\end{array}$ & Intermittent & Low & None & $\begin{array}{c}\text { No Cultural } \\
\text { Resources }\end{array}$ & $\begin{array}{c}\text { No further work } \\
\text { recommended }\end{array}$ & Inundation \\
\hline SP3CH047 & 0.4 & $\begin{array}{c}\text { UT to Cedar } \\
\text { Bayou }\end{array}$ & Ephemeral & Low & None & $\begin{array}{c}\text { No Cultural } \\
\text { Resources }\end{array}$ & $\begin{array}{l}\text { No further work } \\
\text { recommended }\end{array}$ & $\begin{array}{l}\text { Completely avoided via non } \\
\text { Section } 10 \mathrm{HDD} / \text { Bore }\end{array}$ \\
\hline SP3CH027 & 0.5 & $\begin{array}{c}\text { UT to Cedar } \\
\text { Bayou }\end{array}$ & Ephemeral & Low & None & $\begin{array}{c}\text { No Cultural } \\
\text { Resources }\end{array}$ & $\begin{array}{l}\text { No further work } \\
\text { recommended }\end{array}$ & $\begin{array}{l}\text { Completely avoided via non } \\
\text { Section } 10 \mathrm{HDD} / \text { Bore }\end{array}$ \\
\hline SP3CH046 & 0.7 & $\begin{array}{l}\text { UT to Cedar } \\
\text { Bayou }\end{array}$ & Perennial & Low & None & $\begin{array}{c}\text { No Cultural } \\
\text { Resources }\end{array}$ & $\begin{array}{l}\text { No further work } \\
\text { recommended }\end{array}$ & $\begin{array}{c}\text { Completely avoided via non } \\
\text { Section } 10 \mathrm{HDD} / \text { Bore }\end{array}$ \\
\hline SP3CH045 & 0.9 & $\begin{array}{c}\text { UT to Cedar } \\
\text { Bayou }\end{array}$ & Ephemeral & Low & None & $\begin{array}{c}\text { No Cultural } \\
\text { Resources }\end{array}$ & $\begin{array}{l}\text { No further work } \\
\text { recommended }\end{array}$ & Inundation \\
\hline SP3CH044 & 1.1 & $\begin{array}{c}\text { UT to Cedar } \\
\text { Bayou }\end{array}$ & Ephemeral & Low & None & $\begin{array}{c}\text { No Cultural } \\
\text { Resources } \\
\end{array}$ & $\begin{array}{l}\text { No further work } \\
\text { recommended }\end{array}$ & Inundation \\
\hline SP3CH043 & 1.2 & $\begin{array}{c}\text { UT to Cedar } \\
\text { Bayou }\end{array}$ & Ephemeral & Low & None & $\begin{array}{c}\text { No Cultural } \\
\text { Resources }\end{array}$ & $\begin{array}{l}\text { No further work } \\
\text { recommended }\end{array}$ & Inundation \\
\hline SP3CH041 & 1.3 & $\begin{array}{c}\text { UT to Cedar } \\
\text { Bayou }\end{array}$ & Ephemeral & Low & None & $\begin{array}{c}\text { No Cultural } \\
\text { Resources }\end{array}$ & $\begin{array}{l}\text { No further work } \\
\text { recommended }\end{array}$ & Inundation \\
\hline SP3CH042 & 1.3 & $\begin{array}{c}\text { UT to Cedar } \\
\text { Bayou }\end{array}$ & Ephemeral & Low & None & $\begin{array}{c}\text { No Cultural } \\
\text { Resources }\end{array}$ & $\begin{array}{l}\text { No further work } \\
\text { recommended }\end{array}$ & Inundation \\
\hline SP5CH001 & 1.4 & $\begin{array}{c}\text { Water Authority } \\
\text { Canal }\end{array}$ & Perennial & Low & None & $\begin{array}{c}\text { No Cultural } \\
\text { Resources } \\
\end{array}$ & $\begin{array}{l}\text { No further work } \\
\text { recommended }\end{array}$ & $\begin{array}{c}\text { Completely avoided via non } \\
\text { Section } 10 \mathrm{HDD} / \text { Bore }\end{array}$ \\
\hline $\mathrm{SP} 3 \mathrm{CH} 040$ & 1.4 & $\begin{array}{c}\text { UT to Cedar } \\
\text { Bayou }\end{array}$ & Intermittent & Low & None & $\begin{array}{c}\text { No Cultural } \\
\text { Resources }\end{array}$ & $\begin{array}{l}\text { No further work } \\
\text { recommended }\end{array}$ & $\begin{array}{c}\text { Completely avoided via non } \\
\text { Section } 10 \mathrm{HDD} / \text { Bore }\end{array}$ \\
\hline SP5CH002 & 1.5 & $\begin{array}{l}\text { UT to Cedar } \\
\text { Bayou }\end{array}$ & Ephemeral & Low & None & $\begin{array}{c}\text { No Cultural } \\
\text { Resources }\end{array}$ & $\begin{array}{l}\text { No further work } \\
\text { recommended }\end{array}$ & $\begin{array}{c}\text { Completely avoided via non } \\
\text { Section } 10 \mathrm{HDD} / \text { Bore }\end{array}$ \\
\hline SP9CH002_DT & 2.5 & $\begin{array}{l}\text { UT to Hickory } \\
\text { Island Gully }\end{array}$ & Intermittent & Low & None & $\begin{array}{c}\text { No Cultural } \\
\text { Resources }\end{array}$ & $\begin{array}{l}\text { No further work } \\
\text { recommended }\end{array}$ & $\begin{array}{l}\text { Completely avoided via non } \\
\text { Section } 10 \mathrm{HDD} / \text { Bore }\end{array}$ \\
\hline SP9CH013_DT & 2.9 & $\begin{array}{l}\text { Hickory Island } \\
\text { Gully } \\
\end{array}$ & Intermittent & Low & None & $\begin{array}{c}\text { No Cultural } \\
\text { Resources }\end{array}$ & $\begin{array}{l}\text { No further work } \\
\text { recommended }\end{array}$ & $\begin{array}{l}\text { Completely avoided via non } \\
\text { Section } 10 \mathrm{HDD} / \text { Bore }\end{array}$ \\
\hline SP9CH014_DT & 3.1 & $\begin{array}{l}\text { UT to Hickory } \\
\text { Island Gully }\end{array}$ & Intermittent & Low & 2 & $\begin{array}{c}\text { No Cultural } \\
\text { Resources }\end{array}$ & $\begin{array}{l}\text { No further work } \\
\text { recommended }\end{array}$ & Inundation \\
\hline SP3CH048 & 3.4 & $\begin{array}{l}\text { UT to Hickory } \\
\text { Island Gully } \\
\end{array}$ & Ephemeral & Low & 10 & $\begin{array}{c}\text { No Cultural } \\
\text { Resources }\end{array}$ & $\begin{array}{l}\text { No further work } \\
\text { recommended }\end{array}$ & Inundation \\
\hline SP3CH049 & 3.4 & $\begin{array}{l}\text { UT to Hickory } \\
\text { Island Gully }\end{array}$ & Ephemeral & Low & 6 & $\begin{array}{l}\text { No Cultural } \\
\text { Resources }\end{array}$ & $\begin{array}{l}\text { No further work } \\
\text { recommended }\end{array}$ & Inundation \\
\hline \multicolumn{9}{|c|}{ Liberty County, Texas } \\
\hline
\end{tabular}


Appendix C2 - Survey Data in the Vicinity of Waterbody Features

\begin{tabular}{|c|c|c|c|c|c|c|c|c|}
\hline Waterbody ID & $\begin{array}{c}\text { Milepost } \\
\text { (Approximate) }\end{array}$ & Waterbody Name & $\begin{array}{l}\text { Waterbody } \\
\text { Type }\end{array}$ & Probability & $\begin{array}{l}\text { Shovel } \\
\text { Tests }\end{array}$ & Surveys Results & Recommendations & Comments \\
\hline SP3LI050 & 3.8 & $\begin{array}{l}\text { UT to Hickory } \\
\text { Island Gully }\end{array}$ & Ephemeral & Low & 28 & $\begin{array}{l}\text { No Cultural } \\
\text { Resources }\end{array}$ & $\begin{array}{l}\text { No further work } \\
\text { recommended }\end{array}$ & Inundation \\
\hline SP3LI051 & 3.8 & $\begin{array}{l}\text { UT to Hickory } \\
\text { Island Gully }\end{array}$ & Ephemeral & Low & 6 & $\begin{array}{l}\text { No Cultural } \\
\text { Resources }\end{array}$ & $\begin{array}{l}\text { No further work } \\
\text { recommended }\end{array}$ & Inundation \\
\hline SP5LI020 & 5.1 & $\begin{array}{l}\text { UT to Hickory } \\
\text { Island Gully }\end{array}$ & Ephemeral & Low & None & $\begin{array}{c}\text { No Cultural } \\
\text { Resources }\end{array}$ & $\begin{array}{l}\text { No further work } \\
\text { recommended }\end{array}$ & Inundation \\
\hline SP5LI021 & 5.1 & $\begin{array}{l}\text { UT to Hickory } \\
\text { Island Gully }\end{array}$ & Ephemeral & Low & None & $\begin{array}{l}\text { No Cultural } \\
\text { Resources }\end{array}$ & $\begin{array}{l}\text { No further work } \\
\text { recommended }\end{array}$ & Inundation \\
\hline SP5LI022 & 5.1 & $\begin{array}{l}\text { UT to Hickory } \\
\text { Island Gully }\end{array}$ & Ephemeral & Low & None & $\begin{array}{l}\text { No Cultural } \\
\text { Resources }\end{array}$ & $\begin{array}{l}\text { No further work } \\
\text { recommended }\end{array}$ & Inundation \\
\hline SP5LI023 & 5.1 & $\begin{array}{l}\text { UT to Hickory } \\
\text { Island Gully }\end{array}$ & Ephemeral & Low & None & $\begin{array}{l}\text { No Cultural } \\
\text { Resources }\end{array}$ & $\begin{array}{l}\text { No further work } \\
\text { recommended }\end{array}$ & Inundation \\
\hline SP5LI019 & 5.5 & $\begin{array}{l}\text { UT to Hickory } \\
\text { Island Gully }\end{array}$ & Ephemeral & Low & None & $\begin{array}{l}\text { No Cultural } \\
\text { Resources }\end{array}$ & $\begin{array}{l}\text { No further work } \\
\text { recommended }\end{array}$ & Inundation \\
\hline SP5LI018 & 6.1 & $\begin{array}{l}\text { UT to Hickory } \\
\text { Island Gully }\end{array}$ & Ephemeral & Low & None & $\begin{array}{l}\text { No Cultural } \\
\text { Resources }\end{array}$ & $\begin{array}{l}\text { No further work } \\
\text { recommended }\end{array}$ & Inundation \\
\hline SP6LI002 & 6.5 & $\begin{array}{l}\text { UT to Hickory } \\
\text { Island Gully }\end{array}$ & Ephemeral & Low & None & $\begin{array}{l}\text { No Cultural } \\
\text { Resources }\end{array}$ & $\begin{array}{l}\text { No further work } \\
\text { recommended }\end{array}$ & $\begin{array}{l}\text { Completely avoided via non } \\
\text { Section } 10 \mathrm{HDD} / \text { Bore }\end{array}$ \\
\hline SP6LI001 & 6.5 & $\begin{array}{l}\text { UT to Hickory } \\
\text { Island Gully }\end{array}$ & Ephemeral & Low & None & $\begin{array}{l}\text { No Cultural } \\
\text { Resources }\end{array}$ & $\begin{array}{l}\text { No further work } \\
\text { recommended }\end{array}$ & $\begin{array}{l}\text { Completely avoided via non } \\
\text { Section } 10 \mathrm{HDD} / \text { Bore }\end{array}$ \\
\hline OWP6LI001 & 6.7 & N/A & Manmade Pond & Low & None & $\begin{array}{l}\text { No Cultural } \\
\text { Resources }\end{array}$ & $\begin{array}{l}\text { No further work } \\
\text { recommended }\end{array}$ & Mostly Inundated \\
\hline SP2LI300 & 7.4 & $\begin{array}{l}\text { Hickory Island } \\
\text { Gully }\end{array}$ & Intermittent & Low & None & $\begin{array}{l}\text { No Cultural } \\
\text { Resources }\end{array}$ & $\begin{array}{l}\text { No further work } \\
\text { recommended }\end{array}$ & $\begin{array}{c}\text { Located Within Heavily Inundated } \\
\text { Area }\end{array}$ \\
\hline SP2LI029 & 7.7 & $\begin{array}{l}\text { UT to Hickory } \\
\text { Island Gully }\end{array}$ & Ephemeral & Low & None & $\begin{array}{l}\text { No Cultural } \\
\text { Resources }\end{array}$ & $\begin{array}{l}\text { No further work } \\
\text { recommended }\end{array}$ & $\begin{array}{c}\text { Located Within Heavily Inundated } \\
\text { Area } \\
\end{array}$ \\
\hline SP2LI030_DT & 9.3 & $\begin{array}{l}\text { UT to Dayton } \\
\text { Canal }\end{array}$ & Perennial & Low & None & $\begin{array}{l}\text { No Cultural } \\
\text { Resources }\end{array}$ & $\begin{array}{l}\text { No further work } \\
\text { recommended }\end{array}$ & Inundation \\
\hline SP2LI033_DT & 9.7 & $\begin{array}{l}\text { UT to Dayton } \\
\text { Canal }\end{array}$ & Intermittent & Low & None & $\begin{array}{l}\text { No Cultural } \\
\text { Resources }\end{array}$ & $\begin{array}{l}\text { No further work } \\
\text { recommended }\end{array}$ & Inundation \\
\hline SP2LI030 & 9.3 & Dayton Canal & Perennial & Low & 6 & $\begin{array}{l}\text { No Cultural } \\
\text { Resources }\end{array}$ & $\begin{array}{l}\text { No further work } \\
\text { recommended }\end{array}$ & Inundation \\
\hline SP2LI032 & 9.9 & $\begin{array}{l}\text { UT to Dayton } \\
\text { Canal }\end{array}$ & Ephemeral & Low & 10 & $\begin{array}{l}\text { No Cultural } \\
\text { Resources }\end{array}$ & $\begin{array}{l}\text { No further work } \\
\text { recommended }\end{array}$ & $\begin{array}{l}\text { Completely avoided via non } \\
\text { Section } 10 \mathrm{HDD} / \text { Bore }\end{array}$ \\
\hline SP6LI008 & 9.9 & $\begin{array}{l}\text { UT to Dayton } \\
\text { Canal }\end{array}$ & Ephemeral & Low & 10 & $\begin{array}{l}\text { No Cultural } \\
\text { Resources }\end{array}$ & $\begin{array}{l}\text { No further work } \\
\text { recommended }\end{array}$ & $\begin{array}{l}\text { Completely avoided via non } \\
\text { Section } 10 \mathrm{HDD} / \text { Bore }\end{array}$ \\
\hline
\end{tabular}


Appendix C2 - Survey Data in the Vicinity of Waterbody Features

\begin{tabular}{|c|c|c|c|c|c|c|c|c|}
\hline Waterbody ID & $\begin{array}{c}\text { Milepost } \\
\text { (Approximate) }\end{array}$ & Waterbody Name & $\begin{array}{l}\text { Waterbody } \\
\text { Type }\end{array}$ & Probability & $\begin{array}{l}\text { Shovel } \\
\text { Tests }\end{array}$ & Surveys Results & Recommendations & Comments \\
\hline SP6LI007 & 9.9 & $\begin{array}{l}\text { UT to Dayton } \\
\text { Canal }\end{array}$ & Ephemeral & Low & 10 & $\begin{array}{l}\text { No Cultural } \\
\text { Resources }\end{array}$ & $\begin{array}{l}\text { No further work } \\
\text { recommended }\end{array}$ & $\begin{array}{l}\text { Completely avoided via non } \\
\text { Section } 10 \text { HDD/Bore }\end{array}$ \\
\hline SP6LI006 & 10.2 & $\begin{array}{l}\text { UT to Dayton } \\
\text { Canal }\end{array}$ & Ephemeral & Low & None & $\begin{array}{l}\text { No Cultural } \\
\text { Resources }\end{array}$ & $\begin{array}{l}\text { No further work } \\
\text { recommended }\end{array}$ & Inundation \\
\hline SP6LI099 & 10.2 & $\begin{array}{l}\text { UT to Dayton } \\
\text { Canal }\end{array}$ & Ephemeral & Low & None & $\begin{array}{c}\text { No Cultural } \\
\text { Resources }\end{array}$ & $\begin{array}{l}\text { No further work } \\
\text { recommended }\end{array}$ & Inundation \\
\hline SP6LI005 & 10.3 & Dayton Canal & Intermittent & Low & None & $\begin{array}{l}\text { No Cultural } \\
\text { Resources }\end{array}$ & $\begin{array}{l}\text { No further work } \\
\text { recommended }\end{array}$ & $\begin{array}{l}\text { Completely avoided via non } \\
\text { Section } 10 \text { HDD/Bore }\end{array}$ \\
\hline SP6LI004 & 10.4 & $\begin{array}{l}\text { UT to Dayton } \\
\text { Canal }\end{array}$ & Ephemeral & Low & None & $\begin{array}{l}\text { No Cultural } \\
\text { Resources }\end{array}$ & $\begin{array}{l}\text { No further work } \\
\text { recommended }\end{array}$ & $\begin{array}{l}\text { Completely avoided via non } \\
\text { Section } 10 \text { HDD/Bore }\end{array}$ \\
\hline OWP6LI002 & 10.4 & N/A & Manmade Pond & Low & None & $\begin{array}{l}\text { No Cultural } \\
\text { Resources }\end{array}$ & $\begin{array}{l}\text { No further work } \\
\text { recommended }\end{array}$ & Inundation \\
\hline SP3LI027 & 10.6 & UT to West Prong & Ephemeral & Low & None & $\begin{array}{l}\text { No Cultural } \\
\text { Resources }\end{array}$ & $\begin{array}{l}\text { No further work } \\
\text { recommended }\end{array}$ & $\begin{array}{l}\text { Completely avoided via non } \\
\text { Section } 10 \text { HDD/Bore }\end{array}$ \\
\hline SP6LI003 & 10.6 & UT to West Prong & Ephemeral & Low & None & $\begin{array}{l}\text { No Cultural } \\
\text { Resources }\end{array}$ & $\begin{array}{l}\text { No further work } \\
\text { recommended }\end{array}$ & $\begin{array}{c}\text { Completely avoided via non } \\
\text { Section } 10 \mathrm{HDD} / \text { Bore }\end{array}$ \\
\hline SP3LI026 & 10.9 & West Prong & Ephemeral & Low & None & $\begin{array}{l}\text { No Cultural } \\
\text { Resources }\end{array}$ & $\begin{array}{l}\text { No further work } \\
\text { recommended }\end{array}$ & Inundation \\
\hline SP3LI024 & 11 & UT to Old River & Ephemeral & Low & None & $\begin{array}{l}\text { No Cultural } \\
\text { Resources }\end{array}$ & $\begin{array}{l}\text { No further work } \\
\text { recommended }\end{array}$ & Inundation \\
\hline SP3LI023 & 11 & UT to Old River & Ephemeral & Low & None & $\begin{array}{l}\text { No Cultural } \\
\text { Resources }\end{array}$ & $\begin{array}{l}\text { No further work } \\
\text { recommended }\end{array}$ & Inundation \\
\hline SP3LI021 & 11.5 & UT to Old River & Ephemeral & Low & 7 & $\begin{array}{l}\text { No Cultural } \\
\text { Resources }\end{array}$ & $\begin{array}{l}\text { No further work } \\
\text { recommended }\end{array}$ & Inundation \\
\hline SP3LI020 & 11.6 & East Prong & Ephemeral & Low & 3 & $\begin{array}{l}\text { No Cultural } \\
\text { Resources }\end{array}$ & $\begin{array}{l}\text { No further work } \\
\text { recommended }\end{array}$ & Inundation \\
\hline SP3LI005 & 12.1 & UT to Old River & Ephemeral & Low & 1 & $\begin{array}{l}\text { No Cultural } \\
\text { Resources }\end{array}$ & $\begin{array}{l}\text { No further work } \\
\text { recommended }\end{array}$ & Inundation \\
\hline SP3LI006 & 12.1 & UT to Old River & Ephemeral & Low & 1 & $\begin{array}{l}\text { No Cultural } \\
\text { Resources }\end{array}$ & $\begin{array}{l}\text { No further work } \\
\text { recommended }\end{array}$ & Inundation \\
\hline SP3LI009 & 12.5 & Old River Drain & Ephemeral & Low & None & $\begin{array}{l}\text { No Cultural } \\
\text { Resources }\end{array}$ & $\begin{array}{l}\text { No further work } \\
\text { recommended }\end{array}$ & $\begin{array}{c}\text { Completely avoided via non } \\
\text { Section } 10 \text { HDD/Bore }\end{array}$ \\
\hline SP3LI007 & 12.5 & Old River Drain & Ephemeral & Low & None & $\begin{array}{l}\text { No Cultural } \\
\text { Resources }\end{array}$ & $\begin{array}{l}\text { No further work } \\
\text { recommended }\end{array}$ & $\begin{array}{l}\text { Completely avoided via non } \\
\text { Section } 10 \text { HDD/Bore }\end{array}$ \\
\hline SP3LI008 & 12.6 & Old River Drain & Ephemeral & Low & None & $\begin{array}{l}\text { No Cultural } \\
\text { Resources }\end{array}$ & $\begin{array}{l}\text { No further work } \\
\text { recommended }\end{array}$ & Inundation \\
\hline
\end{tabular}


Appendix C2 - Survey Data in the Vicinity of Waterbody Features

\begin{tabular}{|c|c|c|c|c|c|c|c|c|}
\hline Waterbody ID & $\begin{array}{c}\text { Milepost } \\
\text { (Approximate) }\end{array}$ & Waterbody Name & $\begin{array}{l}\text { Waterbody } \\
\text { Type }\end{array}$ & Probability & $\begin{array}{l}\text { Shovel } \\
\text { Tests }\end{array}$ & Surveys Results & Recommendations & Comments \\
\hline SP3LI010 & 13 & UT to Old River & Ephemeral & Low & None & $\begin{array}{c}\text { No Cultural } \\
\text { Resources }\end{array}$ & $\begin{array}{l}\text { No further work } \\
\text { recommended }\end{array}$ & Inundation \\
\hline SP3LI011 & 13.1 & $\begin{array}{c}\text { UT to Coastal } \\
\text { Water Authority } \\
\text { Canal }\end{array}$ & Ephemeral & Low & None & $\begin{array}{l}\text { No Cultural } \\
\text { Resources }\end{array}$ & $\begin{array}{l}\text { No further work } \\
\text { recommended }\end{array}$ & Inundation \\
\hline SP3LI012 & 13.3 & $\begin{array}{c}\text { UT to Coastal } \\
\text { Water Authority } \\
\text { Canal }\end{array}$ & Ephemeral & Low & None & $\begin{array}{l}\text { No Cultural } \\
\text { Resources }\end{array}$ & $\begin{array}{l}\text { No further work } \\
\text { recommended }\end{array}$ & Inundation \\
\hline SP3LI013 & 13.5 & $\begin{array}{c}\text { UT to Coastal } \\
\text { Water Authority } \\
\text { Canal }\end{array}$ & Ephemeral & Low & 4 & $\begin{array}{l}\text { No Cultural } \\
\text { Resources }\end{array}$ & $\begin{array}{l}\text { No further work } \\
\text { recommended }\end{array}$ & $\begin{array}{l}\text { Completely avoided via non } \\
\text { Section } 10 \mathrm{HDD} / \text { Bore }\end{array}$ \\
\hline SP3LI015 & 13.7 & $\begin{array}{c}\text { UT to Coastal } \\
\text { Water Authority } \\
\text { Canal }\end{array}$ & Ephemeral & Low & 6 & $\begin{array}{l}\text { No Cultural } \\
\text { Resources }\end{array}$ & $\begin{array}{l}\text { No further work } \\
\text { recommended }\end{array}$ & Inundation \\
\hline SP3LI016 & 13.9 & $\begin{array}{c}\text { UT to Coastal } \\
\text { Water Authority } \\
\text { Canal }\end{array}$ & Ephemeral & Low & 7 & $\begin{array}{l}\text { No Cultural } \\
\text { Resources }\end{array}$ & $\begin{array}{l}\text { No further work } \\
\text { recommended }\end{array}$ & Inundation \\
\hline SP3LI017 & 13.9 & $\begin{array}{c}\text { UT to Coastal } \\
\text { Water Authority } \\
\text { Canal }\end{array}$ & Ephemeral & Low & 7 & $\begin{array}{l}\text { No Cultural } \\
\text { Resources }\end{array}$ & $\begin{array}{l}\text { No further work } \\
\text { recommended }\end{array}$ & Inundation \\
\hline SP3LI019 & 14.1 & $\begin{array}{c}\text { UT to Coastal } \\
\text { Water Authority } \\
\text { Canal }\end{array}$ & Ephemeral & Low & 5 & $\begin{array}{l}\text { No Cultural } \\
\text { Resources }\end{array}$ & $\begin{array}{l}\text { No further work } \\
\text { recommended }\end{array}$ & Inundation \\
\hline SP3LI018 & 14.4 & $\begin{array}{c}\text { UT to Coastal } \\
\text { Water Authority } \\
\text { Canal }\end{array}$ & Ephemeral & Low & 6 & $\begin{array}{l}\text { No Cultural } \\
\text { Resources }\end{array}$ & $\begin{array}{l}\text { No further work } \\
\text { recommended }\end{array}$ & $\begin{array}{l}\text { Completely avoided via non } \\
\text { Section } 10 \mathrm{HDD} / \text { Bore }\end{array}$ \\
\hline SP2LI004 & 15.3 & $\begin{array}{c}\text { UT to Coastal } \\
\text { Water Authority } \\
\text { Canal }\end{array}$ & Intermittent & Low & 4 & $\begin{array}{l}\text { No Cultural } \\
\text { Resources }\end{array}$ & $\begin{array}{l}\text { No further work } \\
\text { recommended }\end{array}$ & Inundation \\
\hline SP2LI005 & 15.6 & $\begin{array}{c}\text { UT to Coastal } \\
\text { Water Authority } \\
\text { Canal }\end{array}$ & Ephemeral & Low & None & $\begin{array}{l}\text { No Cultural } \\
\text { Resources }\end{array}$ & $\begin{array}{l}\text { No further work } \\
\text { recommended }\end{array}$ & Inundation \\
\hline SP2LI006 & 15.6 & $\begin{array}{c}\text { UT to Coastal } \\
\text { Water Authority } \\
\text { Canal }\end{array}$ & Ephemeral & Low & None & $\begin{array}{l}\text { No Cultural } \\
\text { Resources }\end{array}$ & $\begin{array}{l}\text { No further work } \\
\text { recommended }\end{array}$ & Inundation \\
\hline
\end{tabular}


Appendix C2 - Survey Data in the Vicinity of Waterbody Features

\begin{tabular}{|c|c|c|c|c|c|c|c|c|}
\hline Waterbody ID & $\begin{array}{c}\text { Milepost } \\
\text { (Approximate) }\end{array}$ & Waterbody Name & $\begin{array}{l}\text { Waterbody } \\
\text { Type }\end{array}$ & Probability & $\begin{array}{l}\text { Shovel } \\
\text { Tests }\end{array}$ & Surveys Results & Recommendations & Comments \\
\hline SP2LI007 & 15.8 & $\begin{array}{c}\text { UT to Coastal } \\
\text { Water Authority } \\
\text { Canal }\end{array}$ & Intermittent & Low & None & $\begin{array}{l}\text { No Cultural } \\
\text { Resources }\end{array}$ & $\begin{array}{l}\text { No further work } \\
\text { recommended }\end{array}$ & Inundation \\
\hline SP4LI001 & 16.4 & Trinity River & Perennial & High & 50 & $\begin{array}{l}\text { No Cultural } \\
\text { Resources }\end{array}$ & $\begin{array}{l}\text { No further work } \\
\text { recommended }\end{array}$ & $\begin{array}{c}\text { Located in Heavily Inundated } \\
\text { Area within Trinity River } \\
\text { Floodplain }\end{array}$ \\
\hline OWP4LI001 & 16.4 & N/A & Manmade Pond & Medium & 10 & $\begin{array}{l}\text { No Cultural } \\
\text { Resources }\end{array}$ & $\begin{array}{l}\text { No further work } \\
\text { recommended }\end{array}$ & $\begin{array}{l}\text { No Impacts - Completely avoided } \\
\text { via Section } 10 \mathrm{HDD} / \text { Bore }\end{array}$ \\
\hline SP4LI002 & 16.7 & Trinity River & Perennial & High & 9 & $\begin{array}{l}\text { No Cultural } \\
\text { Resources }\end{array}$ & $\begin{array}{l}\text { No further work } \\
\text { recommended }\end{array}$ & No - Avoided via HDD/Bore \\
\hline SP4LI003 & 16.9 & $\begin{array}{l}\text { UT to Trinity } \\
\text { River }\end{array}$ & Ephemeral & High & 1 & $\begin{array}{l}\text { No Cultural } \\
\text { Resources }\end{array}$ & $\begin{array}{l}\text { No further work } \\
\text { recommended }\end{array}$ & $\begin{array}{c}\text { Located in Heavily Inundated } \\
\text { Area within Trinity River } \\
\text { Floodplain }\end{array}$ \\
\hline SP4LI004 & 17 & $\begin{array}{l}\text { UT to Trinity } \\
\text { River }\end{array}$ & Ephemeral & High & 1 & $\begin{array}{l}\text { No Cultural } \\
\text { Resources }\end{array}$ & $\begin{array}{l}\text { Archeological } \\
\text { Monitor Needed }\end{array}$ & $\begin{array}{c}\text { Located in Heavily Inundated } \\
\text { Area within Trinity River } \\
\text { Floodplain }\end{array}$ \\
\hline SP3LI004 & 17.8 & $\begin{array}{l}\text { UT to Trinity } \\
\text { River }\end{array}$ & Intermittent & High & 6 & $\begin{array}{l}\text { No Cultural } \\
\text { Resources }\end{array}$ & $\begin{array}{l}\text { No further work } \\
\text { recommended }\end{array}$ & $\begin{array}{c}\text { Located in Heavily Inundated } \\
\text { Area within Trinity River } \\
\text { Floodplain }\end{array}$ \\
\hline SP3LI003 & 18.3 & $\begin{array}{l}\text { UT to Trinity } \\
\text { River }\end{array}$ & Intermittent & High & 3 & $\begin{array}{l}\text { No Cultural } \\
\text { Resources }\end{array}$ & $\begin{array}{l}\text { No further work } \\
\text { recommended }\end{array}$ & $\begin{array}{c}\text { Located in Heavily Inundated } \\
\text { Area within Trinity River } \\
\text { Floodplain }\end{array}$ \\
\hline SP3LI001 & 19 & Self Bayou & Ephemeral & High & None & $\begin{array}{l}\text { No Cultural } \\
\text { Resources }\end{array}$ & $\begin{array}{l}\text { No further work } \\
\text { recommended }\end{array}$ & $\begin{array}{l}\text { Completely avoided via non } \\
\text { Section } 10 \mathrm{HDD} / \text { Bore }\end{array}$ \\
\hline SP3LI002 & 19.1 & Self Bayou & Perennial & High & 17 & $\begin{array}{l}\text { No Cultural } \\
\text { Resources }\end{array}$ & $\begin{array}{l}\text { No further work } \\
\text { recommended }\end{array}$ & $\begin{array}{l}\text { Completely avoided via non } \\
\text { Section } 10 \mathrm{HDD} / \text { Bore } \\
\end{array}$ \\
\hline SP6LI012 & 19.3 & UT to Self Bayou & Ephemeral & High & 17 & $\begin{array}{l}\text { No Cultural } \\
\text { Resources }\end{array}$ & $\begin{array}{l}\text { No further work } \\
\text { recommended }\end{array}$ & Inundation \\
\hline SP6LI011 & 19.3 & UT to Black Gully & Ephemeral & High & 17 & $\begin{array}{l}\text { No Cultural } \\
\text { Resources }\end{array}$ & $\begin{array}{l}\text { No further work } \\
\text { recommended }\end{array}$ & Inundation \\
\hline OWP3LI001 & 20.9 & N/A & Manmade Pond & High & 8 & $\begin{array}{l}\text { No Cultural } \\
\text { Resources }\end{array}$ & $\begin{array}{l}\text { No further work } \\
\text { recommended }\end{array}$ & Inundation \\
\hline SP2LI008 & 21.6 & UT to Black Gully & Ephemeral & High & 43 & $\begin{array}{l}\text { No Cultural } \\
\text { Resources }\end{array}$ & $\begin{array}{l}\text { No further work } \\
\text { recommended }\end{array}$ & Inundation \\
\hline
\end{tabular}


Appendix C2 - Survey Data in the Vicinity of Waterbody Features

\begin{tabular}{|c|c|c|c|c|c|c|c|c|}
\hline Waterbody ID & $\begin{array}{c}\text { Milepost } \\
\text { (Approximate) }\end{array}$ & Waterbody Name & $\begin{array}{l}\text { Waterbody } \\
\text { Type }\end{array}$ & Probability & $\begin{array}{l}\text { Shovel } \\
\text { Tests }\end{array}$ & Surveys Results & Recommendations & Comments \\
\hline SP4LI154 & 21.8 & Black Gully & Perennial & High & 29 & $\begin{array}{c}\text { No Cultural } \\
\text { Resources }\end{array}$ & $\begin{array}{l}\text { No further work } \\
\text { recommended }\end{array}$ & Inundation \\
\hline SP4LI005 & 22 & UT to Black Gully & Intermittent & High & 11 & $\begin{array}{c}\text { No Cultural } \\
\text { Resources }\end{array}$ & $\begin{array}{l}\text { No further work } \\
\text { recommended }\end{array}$ & Inundation \\
\hline SP9LI018_DT & 22.7 & $\begin{array}{c}\text { UT to West } \\
\text { Branch Devers } \\
\text { Canal }\end{array}$ & Intermittent & Low & None & $\begin{array}{l}\text { No Cultural } \\
\text { Resources }\end{array}$ & $\begin{array}{l}\text { No further work } \\
\text { recommended }\end{array}$ & $\begin{array}{l}\text { Completely avoided via non } \\
\text { Section } 10 \text { HDD/Bore }\end{array}$ \\
\hline SP9LI016_DT & 23 & Pignut Gully & Intermittent & Low & None & $\begin{array}{c}\text { No Cultural } \\
\text { Resources }\end{array}$ & $\begin{array}{l}\text { No further work } \\
\text { recommended }\end{array}$ & $\begin{array}{l}\text { Completely avoided via non } \\
\text { Section } 10 \text { HDD/Bore }\end{array}$ \\
\hline SP9LI017_DT & 23.4 & $\begin{array}{c}\text { UT to West } \\
\text { Branch Devers } \\
\text { Canal }\end{array}$ & Intermittent & Low & None & $\begin{array}{l}\text { No Cultural } \\
\text { Resources }\end{array}$ & $\begin{array}{l}\text { No further work } \\
\text { recommended }\end{array}$ & $\begin{array}{l}\text { Completely avoided via non } \\
\text { Section } 10 \text { HDD/Bore }\end{array}$ \\
\hline SP4LI006 & 23.8 & $\begin{array}{c}\text { UT to West } \\
\text { Branch Devers } \\
\text { Canal }\end{array}$ & Intermittent & Low & None & $\begin{array}{l}\text { No Cultural } \\
\text { Resources }\end{array}$ & $\begin{array}{l}\text { No further work } \\
\text { recommended }\end{array}$ & $\begin{array}{l}\text { Completely avoided via non } \\
\text { Section } 10 \text { HDD/Bore }\end{array}$ \\
\hline SP4LI007 & 23.9 & $\begin{array}{c}\text { UT to West } \\
\text { Branch Devers } \\
\text { Canal }\end{array}$ & Perennial & Low & 5 & $\begin{array}{l}\text { No Cultural } \\
\text { Resources }\end{array}$ & $\begin{array}{l}\text { No further work } \\
\text { recommended }\end{array}$ & $\begin{array}{l}\text { Completely avoided via non } \\
\text { Section } 10 \text { HDD/Bore }\end{array}$ \\
\hline OWP4LI002 & 23.9 & N/A & Manmade Pond & Low & 5 & $\begin{array}{l}\text { No Cultural } \\
\text { Resources }\end{array}$ & $\begin{array}{l}\text { No further work } \\
\text { recommended }\end{array}$ & Inundation \\
\hline SP2LI010 & 25.3 & $\begin{array}{c}\text { UT to West } \\
\text { Branch Devers } \\
\text { Canal }\end{array}$ & Ephemeral & Low & 3 & $\begin{array}{l}\text { No Cultural } \\
\text { Resources }\end{array}$ & $\begin{array}{l}\text { No further work } \\
\text { recommended }\end{array}$ & Inundation \\
\hline SP2LI009 & 25.3 & Turtle Bayou & Perennial & Low & 4 & $\begin{array}{c}\text { No Cultural } \\
\text { Resources }\end{array}$ & $\begin{array}{l}\text { No further work } \\
\text { recommended }\end{array}$ & $\begin{array}{l}\text { Completely avoided via non } \\
\text { Section } 10 \mathrm{HDD} / \text { Bore }\end{array}$ \\
\hline SP2LI011 & 25.6 & $\begin{array}{l}\text { UT to Turtle } \\
\text { Bayou }\end{array}$ & Ephemeral & Low & 4 & $\begin{array}{l}\text { No Cultural } \\
\text { Resources }\end{array}$ & $\begin{array}{l}\text { No further work } \\
\text { recommended }\end{array}$ & $\begin{array}{l}\text { Completely avoided via non } \\
\text { Section } 10 \mathrm{HDD} / \text { Bore }\end{array}$ \\
\hline SP2LI012 & 25.6 & $\begin{array}{l}\text { UT to Turtle } \\
\text { Bayou }\end{array}$ & Intermittent & Low & 4 & $\begin{array}{c}\text { No Cultural } \\
\text { Resources }\end{array}$ & $\begin{array}{l}\text { No further work } \\
\text { recommended }\end{array}$ & $\begin{array}{l}\text { Completely avoided via non } \\
\text { Section } 10 \text { HDD/Bore }\end{array}$ \\
\hline SP4LI013 & 25.9 & $\begin{array}{c}\text { UT to Turtle } \\
\text { Bayou }\end{array}$ & Ephemeral & Low & 3 & $\begin{array}{l}\text { No Cultural } \\
\text { Resources }\end{array}$ & $\begin{array}{l}\text { No further work } \\
\text { recommended }\end{array}$ & Inundation \\
\hline SP4LI014 & 26 & $\begin{array}{l}\text { UT to Turtle } \\
\text { Bayou }\end{array}$ & Ephemeral & Low & 5 & $\begin{array}{c}\text { No Cultural } \\
\text { Resources }\end{array}$ & $\begin{array}{l}\text { No further work } \\
\text { recommended }\end{array}$ & $\begin{array}{c}\text { Partially avoided via non Section } \\
10 \mathrm{HDD} / \text { Bore } \\
\end{array}$ \\
\hline OWP2LI001 & 25.9 & N/A & Manmade Pond & Low & 5 & $\begin{array}{c}\text { No Cultural } \\
\text { Resources }\end{array}$ & $\begin{array}{l}\text { No further work } \\
\text { recommended }\end{array}$ & Inundation \\
\hline
\end{tabular}


Appendix C2 - Survey Data in the Vicinity of Waterbody Features

\begin{tabular}{|c|c|c|c|c|c|c|c|c|}
\hline Waterbody ID & $\begin{array}{c}\text { Milepost } \\
\text { (Approximate) }\end{array}$ & Waterbody Name & $\begin{array}{l}\text { Waterbody } \\
\text { Type }\end{array}$ & Probability & $\begin{array}{l}\text { Shovel } \\
\text { Tests }\end{array}$ & Surveys Results & Recommendations & Comments \\
\hline SP4LI008 & 26.3 & $\begin{array}{c}\text { UT to West } \\
\text { Branch Devers } \\
\text { Canal }\end{array}$ & Intermittent & Low & 17 & $\begin{array}{l}\text { No Cultural } \\
\text { Resources }\end{array}$ & $\begin{array}{l}\text { No further work } \\
\text { recommended }\end{array}$ & Inundation \\
\hline SP4LI009 & 26.5 & $\begin{array}{l}\text { West Branch } \\
\text { Devers Canal }\end{array}$ & Perennial & Low & 8 & $\begin{array}{l}\text { No Cultural } \\
\text { Resources }\end{array}$ & $\begin{array}{l}\text { No further work } \\
\text { recommended }\end{array}$ & $\begin{array}{l}\text { Completely avoided via non } \\
\text { Section } 10 \text { HDD/Bore }\end{array}$ \\
\hline SP4LI010 & 27 & $\begin{array}{c}\text { UT to West } \\
\text { Branch Devers } \\
\text { Canal }\end{array}$ & Ephemeral & Low & 7 & $\begin{array}{l}\text { No Cultural } \\
\text { Resources }\end{array}$ & $\begin{array}{l}\text { No further work } \\
\text { recommended }\end{array}$ & Inundation \\
\hline SP4LI011 & 27.1 & $\begin{array}{c}\text { UT to West } \\
\text { Branch Devers } \\
\text { Canal }\end{array}$ & Ephemeral & Low & 7 & $\begin{array}{l}\text { No Cultural } \\
\text { Resources }\end{array}$ & $\begin{array}{l}\text { No further work } \\
\text { recommended }\end{array}$ & Inundation \\
\hline SP4LI012 & 27.4 & $\begin{array}{c}\text { UT to West } \\
\text { Branch Devers } \\
\text { Canal }\end{array}$ & Intermittent & Low & 6 & $\begin{array}{l}\text { No Cultural } \\
\text { Resources }\end{array}$ & $\begin{array}{l}\text { No further work } \\
\text { recommended }\end{array}$ & Inundation \\
\hline SP3LI033 & 28 & $\begin{array}{c}\text { UT to West } \\
\text { Branch Devers } \\
\text { Canal }\end{array}$ & Ephemeral & Low & 7 & $\begin{array}{l}\text { No Cultural } \\
\text { Resources }\end{array}$ & $\begin{array}{l}\text { No further work } \\
\text { recommended }\end{array}$ & Inundation \\
\hline SP3LI034 & 28.4 & $\begin{array}{c}\text { UT to West } \\
\text { Branch Devers } \\
\text { Canal }\end{array}$ & Ephemeral & Low & 3 & $\begin{array}{l}\text { No Cultural } \\
\text { Resources }\end{array}$ & $\begin{array}{l}\text { No further work } \\
\text { recommended }\end{array}$ & $\begin{array}{l}\text { Completely avoided via non } \\
\text { Section } 10 \text { HDD/Bore }\end{array}$ \\
\hline SP3LI035 & 28.7 & $\begin{array}{c}\text { UT to West } \\
\text { Branch Devers } \\
\text { Canal }\end{array}$ & Ephemeral & Low & 3 & $\begin{array}{l}\text { No Cultural } \\
\text { Resources }\end{array}$ & $\begin{array}{l}\text { No further work } \\
\text { recommended }\end{array}$ & Inundation \\
\hline SP3LI038 & 28.9 & $\begin{array}{c}\text { UT to West } \\
\text { Branch Devers } \\
\text { Canal }\end{array}$ & Ephemeral & Low & 3 & $\begin{array}{l}\text { No Cultural } \\
\text { Resources }\end{array}$ & $\begin{array}{l}\text { No further work } \\
\text { recommended }\end{array}$ & $\begin{array}{l}\text { Completely avoided via non } \\
\text { Section } 10 \text { HDD/Bore }\end{array}$ \\
\hline SP3LI037 & 29 & $\begin{array}{c}\text { UT to West } \\
\text { Branch Devers } \\
\text { Canal }\end{array}$ & Intermittent & Low & 3 & $\begin{array}{l}\text { No Cultural } \\
\text { Resources }\end{array}$ & $\begin{array}{l}\text { No further work } \\
\text { recommended }\end{array}$ & $\begin{array}{l}\text { Completely avoided via non } \\
\text { Section } 10 \mathrm{HDD} / \text { Bore }\end{array}$ \\
\hline SP3LI036 & 29 & $\begin{array}{c}\text { UT to West } \\
\text { Branch Devers } \\
\text { Canal }\end{array}$ & Ephemeral & Low & 3 & $\begin{array}{l}\text { No Cultural } \\
\text { Resources }\end{array}$ & $\begin{array}{l}\text { No further work } \\
\text { recommended }\end{array}$ & $\begin{array}{l}\text { Completely avoided via non } \\
\text { Section } 10 \mathrm{HDD} / \text { Bore }\end{array}$ \\
\hline SP4LI016 & 29.8 & $\begin{array}{l}\text { UT to Whites } \\
\text { Bayou }\end{array}$ & Intermittent & Low & 24 & $\begin{array}{l}\text { No Cultural } \\
\text { Resources }\end{array}$ & $\begin{array}{l}\text { No further work } \\
\text { recommended }\end{array}$ & Inundation \\
\hline SP4LI017 & 30.1 & Whites Bayou & Perennial & Low & 22 & $\begin{array}{l}\text { No Cultural } \\
\text { Resources }\end{array}$ & $\begin{array}{l}\text { No further work } \\
\text { recommended }\end{array}$ & $\begin{array}{c}\text { Completely avoided via non } \\
\text { Section } 10 \mathrm{HDD} / \text { Bore }\end{array}$ \\
\hline
\end{tabular}


Appendix C2 - Survey Data in the Vicinity of Waterbody Features

\begin{tabular}{|c|c|c|c|c|c|c|c|c|}
\hline Waterbody ID & $\begin{array}{c}\text { Milepost } \\
\text { (Approximate) }\end{array}$ & Waterbody Name & $\begin{array}{l}\text { Waterbody } \\
\text { Type }\end{array}$ & Probability & $\begin{array}{l}\text { Shovel } \\
\text { Tests }\end{array}$ & Surveys Results & Recommendations & Comments \\
\hline SP4LI020 & 30.1 & $\begin{array}{l}\text { UT to Whites } \\
\text { Bayou }\end{array}$ & Ephemeral & Low & 22 & $\begin{array}{c}\text { No Cultural } \\
\text { Resources }\end{array}$ & $\begin{array}{l}\text { No further work } \\
\text { recommended }\end{array}$ & $\begin{array}{l}\text { Completely avoided via non } \\
\text { Section } 10 \text { HDD/Bore }\end{array}$ \\
\hline SP4LI019 & 30.1 & $\begin{array}{l}\text { UT to Whites } \\
\text { Bayou }\end{array}$ & Ephemeral & Low & 22 & $\begin{array}{l}\text { No Cultural } \\
\text { Resources }\end{array}$ & $\begin{array}{l}\text { No further work } \\
\text { recommended }\end{array}$ & $\begin{array}{l}\text { Completely avoided via non } \\
\text { Section } 10 \text { HDD/Bore }\end{array}$ \\
\hline SP4LI018 & 30.3 & $\begin{array}{l}\text { UT to Whites } \\
\text { Bayou }\end{array}$ & Ephemeral & Low & 26 & $\begin{array}{c}\text { No Cultural } \\
\text { Resources }\end{array}$ & $\begin{array}{l}\text { No further work } \\
\text { recommended }\end{array}$ & $\begin{array}{c}\text { Completely avoided via non } \\
\text { Section } 10 \mathrm{HDD} / \text { Bore }\end{array}$ \\
\hline SP4LI021 & 30.6 & $\begin{array}{l}\text { UT to Whites } \\
\text { Bayou }\end{array}$ & Ephemeral & Low & 19 & $\begin{array}{l}\text { No Cultural } \\
\text { Resources }\end{array}$ & $\begin{array}{l}\text { No further work } \\
\text { recommended }\end{array}$ & Inundation \\
\hline SP2LI014 & 31.4 & $\begin{array}{l}\text { UT to Whites } \\
\text { Bayou }\end{array}$ & Ephemeral & Low & 22 & $\begin{array}{c}\text { No Cultural } \\
\text { Resources }\end{array}$ & $\begin{array}{l}\text { No further work } \\
\text { recommended }\end{array}$ & Inundation \\
\hline SP4LI032 & 33 & $\begin{array}{l}\text { UT to East Branch } \\
\text { Devers Canal }\end{array}$ & Ephemeral & Low & 8 & $\begin{array}{l}\text { No Cultural } \\
\text { Resources }\end{array}$ & $\begin{array}{l}\text { No further work } \\
\text { recommended }\end{array}$ & Inundation \\
\hline SP2LI013 & 33 & $\begin{array}{l}\text { UT to Willis } \\
\text { Marsh }\end{array}$ & Ephemeral & Low & 8 & $\begin{array}{l}\text { No Cultural } \\
\text { Resources }\end{array}$ & $\begin{array}{l}\text { No further work } \\
\text { recommended }\end{array}$ & Inundation \\
\hline SP4LI033 & 33.3 & $\begin{array}{l}\text { UT to Willis } \\
\text { Marsh }\end{array}$ & Intermittent & Low & 10 & $\begin{array}{l}\text { No Cultural } \\
\text { Resources }\end{array}$ & $\begin{array}{l}\text { No further work } \\
\text { recommended }\end{array}$ & Inundation \\
\hline SP4LI034 & 33.4 & $\begin{array}{l}\text { UT to Willis } \\
\text { Marsh }\end{array}$ & Intermittent & Low & 9 & $\begin{array}{l}\text { No Cultural } \\
\text { Resources }\end{array}$ & $\begin{array}{l}\text { No further work } \\
\text { recommended }\end{array}$ & Inundation \\
\hline SP4LI035 & 34 & $\begin{array}{l}\text { UT to Willis } \\
\text { Marsh }\end{array}$ & Ephemeral & Low & 10 & $\begin{array}{l}\text { No Cultural } \\
\text { Resources }\end{array}$ & $\begin{array}{l}\text { No further work } \\
\text { recommended }\end{array}$ & Inundation \\
\hline SP4LI036 & 34 & $\begin{array}{l}\text { UT to Willis } \\
\text { Marsh }\end{array}$ & Ephemeral & Low & 10 & $\begin{array}{l}\text { No Cultural } \\
\text { Resources }\end{array}$ & $\begin{array}{l}\text { No further work } \\
\text { recommended }\end{array}$ & Inundation \\
\hline SP2LI028 & 35 & $\begin{array}{l}\text { UT to Willis } \\
\text { Marsh }\end{array}$ & Perennial & Low & 14 & $\begin{array}{l}\text { No Cultural } \\
\text { Resources }\end{array}$ & $\begin{array}{l}\text { No further work } \\
\text { recommended }\end{array}$ & Inundation \\
\hline SP2LI024 & 36.3 & $\begin{array}{l}\text { UT to Willis } \\
\text { Marsh }\end{array}$ & Intermittent & Low & 27 & $\begin{array}{c}\text { No Cultural } \\
\text { Resources }\end{array}$ & $\begin{array}{l}\text { No further work } \\
\text { recommended }\end{array}$ & Inundation \\
\hline SP2LI026 & 36.7 & Nolte Canal & Perennial & Low & 21 & $\begin{array}{l}\text { No Cultural } \\
\text { Resources }\end{array}$ & $\begin{array}{l}\text { No further work } \\
\text { recommended }\end{array}$ & $\begin{array}{l}\text { Completely avoided via non } \\
\text { Section } 10 \text { HDD/Bore }\end{array}$ \\
\hline SP2LI025 & 36.8 & Nolte Canal & Intermittent & Low & 21 & $\begin{array}{l}\text { No Cultural } \\
\text { Resources }\end{array}$ & $\begin{array}{l}\text { No further work } \\
\text { recommended }\end{array}$ & $\begin{array}{l}\text { Completely avoided via non } \\
\text { Section } 10 \mathrm{HDD} / \text { Bore }\end{array}$ \\
\hline SP2LI027 & 36.7 & UT to Nolty Canal & Intermittent & Low & 21 & $\begin{array}{l}\text { No Cultural } \\
\text { Resources }\end{array}$ & $\begin{array}{l}\text { No further work } \\
\text { recommended }\end{array}$ & Inundation \\
\hline SP4LI047 & 38 & UT to Nolty Canal & Ephemeral & Low & 15 & $\begin{array}{l}\text { No Cultural } \\
\text { Resources }\end{array}$ & $\begin{array}{l}\text { No further work } \\
\text { recommended }\end{array}$ & $\begin{array}{l}\text { Completely avoided via non } \\
\text { Section } 10 \text { HDD/Bore }\end{array}$ \\
\hline
\end{tabular}


Appendix C2 - Survey Data in the Vicinity of Waterbody Features

\begin{tabular}{|c|c|c|c|c|c|c|c|c|}
\hline Waterbody ID & $\begin{array}{c}\text { Milepost } \\
\text { (Approximate) }\end{array}$ & Waterbody Name & $\begin{array}{l}\text { Waterbody } \\
\text { Type }\end{array}$ & Probability & $\begin{array}{l}\text { Shovel } \\
\text { Tests }\end{array}$ & Surveys Results & Recommendations & Comments \\
\hline SP4LI031 & 38 & UT to Nolty Canal & Ephemeral & Low & 15 & $\begin{array}{l}\text { No Cultural } \\
\text { Resources }\end{array}$ & $\begin{array}{l}\text { No further work } \\
\text { recommended }\end{array}$ & $\begin{array}{l}\text { Completely avoided via non } \\
\text { Section } 10 \mathrm{HDD} / \text { Bore }\end{array}$ \\
\hline SP4LI030 & 38 & Nolte Canal & Perennial & Low & 15 & $\begin{array}{l}\text { No Cultural } \\
\text { Resources }\end{array}$ & $\begin{array}{l}\text { No further work } \\
\text { recommended }\end{array}$ & $\begin{array}{l}\text { Completely avoided via non } \\
\text { Section } 10 \mathrm{HDD} / \text { Bore }\end{array}$ \\
\hline SP4JLI048 & 38.2 & UT to Nolty Canal & Ephemeral & Low & 13 & $\begin{array}{l}\text { No Cultural } \\
\text { Resources }\end{array}$ & $\begin{array}{l}\text { No further work } \\
\text { recommended }\end{array}$ & Inundation \\
\hline \multicolumn{9}{|c|}{ Jefferson County, Texas } \\
\hline SP4JE037 & 38.8 & UT to Nolty Canal & Ephemeral & Low & None & $\begin{array}{l}\text { No Cultural } \\
\text { Resources }\end{array}$ & $\begin{array}{l}\text { No further work } \\
\text { recommended }\end{array}$ & $\begin{array}{c}\text { Located Within Heavily Inundated } \\
\text { Area }\end{array}$ \\
\hline SP4JE038 & 39 & UT to Nolty Canal & Ephemeral & Low & None & $\begin{array}{l}\text { No Cultural } \\
\text { Resources }\end{array}$ & $\begin{array}{l}\text { No further work } \\
\text { recommended }\end{array}$ & $\begin{array}{c}\text { Located Within Heavily Inundated } \\
\text { Area }\end{array}$ \\
\hline SP4JE039 & 39.1 & UT to Nolty Canal & Ephemeral & Low & None & $\begin{array}{l}\text { No Cultural } \\
\text { Resources }\end{array}$ & $\begin{array}{l}\text { No further work } \\
\text { recommended }\end{array}$ & $\begin{array}{c}\text { Located within or adjacent to Rice } \\
\text { Field }\end{array}$ \\
\hline SP4JE040 & 39.1 & UT to Nolty Canal & Ephemeral & Low & None & $\begin{array}{l}\text { No Cultural } \\
\text { Resources }\end{array}$ & $\begin{array}{l}\text { No further work } \\
\text { recommended }\end{array}$ & $\begin{array}{c}\text { Located within or adjacent to Rice } \\
\text { Field }\end{array}$ \\
\hline SP2JE029 & 39.5 & $\begin{array}{c}\text { UT to Lower } \\
\text { Neches Valley } \\
\text { Authority Canal }\end{array}$ & Ephemeral & Low & None & $\begin{array}{l}\text { No Cultural } \\
\text { Resources }\end{array}$ & $\begin{array}{l}\text { No further work } \\
\text { recommended }\end{array}$ & $\begin{array}{l}\text { Completely avoided via non } \\
\text { Section } 10 \mathrm{HDD} / \text { Bore }\end{array}$ \\
\hline SP2JE015 & 39.5 & $\begin{array}{c}\text { Lower Neches } \\
\text { Valley Authority } \\
\text { Canal }\end{array}$ & Perennial & Low & None & $\begin{array}{l}\text { No Cultural } \\
\text { Resources }\end{array}$ & $\begin{array}{l}\text { No further work } \\
\text { recommended }\end{array}$ & $\begin{array}{l}\text { Completely avoided via non } \\
\text { Section } 10 \mathrm{HDD} / \text { Bore }\end{array}$ \\
\hline SP2JE016 & 39.5 & $\begin{array}{c}\text { UT to Lower } \\
\text { Neches Valley } \\
\text { Authority Canal }\end{array}$ & Intermittent & Low & None & $\begin{array}{l}\text { No Cultural } \\
\text { Resources }\end{array}$ & $\begin{array}{l}\text { No further work } \\
\text { recommended }\end{array}$ & $\begin{array}{l}\text { Completely avoided via non } \\
\text { Section } 10 \mathrm{HDD} / \text { Bore }\end{array}$ \\
\hline SP2JE017 & 39.8 & $\begin{array}{c}\text { UT to Lower } \\
\text { Neches Valley } \\
\text { Authority Canal }\end{array}$ & Perennial & Low & None & $\begin{array}{l}\text { No Cultural } \\
\text { Resources }\end{array}$ & $\begin{array}{l}\text { No further work } \\
\text { recommended }\end{array}$ & $\begin{array}{l}\text { Completely avoided via non } \\
\text { Section } 10 \mathrm{HDD} / \text { Bore }\end{array}$ \\
\hline SP2JE020 & 40.1 & $\begin{array}{c}\text { UT to Lower } \\
\text { Neches Valley } \\
\text { Authority Canal }\end{array}$ & Intermittent & Low & None & $\begin{array}{l}\text { No Cultural } \\
\text { Resources }\end{array}$ & $\begin{array}{l}\text { No further work } \\
\text { recommended }\end{array}$ & $\begin{array}{l}\text { Located within or adjacent to Rice } \\
\text { Field }\end{array}$ \\
\hline SP2JE019 & 40.2 & $\begin{array}{c}\text { UT to Lower } \\
\text { Neches Valley } \\
\text { Authority Canal }\end{array}$ & Ephemeral & Low & None & $\begin{array}{l}\text { No Cultural } \\
\text { Resources }\end{array}$ & $\begin{array}{l}\text { No further work } \\
\text { recommended }\end{array}$ & $\begin{array}{l}\text { Located within or adjacent to Rice } \\
\text { Field }\end{array}$ \\
\hline SP2JE018 & 40.3 & $\begin{array}{l}\text { UT to Lower } \\
\text { Neches Valley } \\
\text { Authority Canal }\end{array}$ & Ephemeral & Low & None & $\begin{array}{l}\text { No Cultural } \\
\text { Resources }\end{array}$ & $\begin{array}{l}\text { No further work } \\
\text { recommended }\end{array}$ & $\begin{array}{l}\text { Located within or adjacent to Rice } \\
\text { Field }\end{array}$ \\
\hline
\end{tabular}


Appendix C2 - Survey Data in the Vicinity of Waterbody Features

\begin{tabular}{|c|c|c|c|c|c|c|c|c|}
\hline Waterbody ID & $\begin{array}{c}\text { Milepost } \\
\text { (Approximate) }\end{array}$ & Waterbody Name & $\begin{array}{l}\text { Waterbody } \\
\text { Type }\end{array}$ & Probability & $\begin{array}{l}\text { Shovel } \\
\text { Tests }\end{array}$ & Surveys Results & Recommendations & Comments \\
\hline SP2JE021 & 40.3 & $\begin{array}{l}\text { UT to Lower } \\
\text { Neches Valley } \\
\text { Authority Canal }\end{array}$ & Intermittent & Low & None & $\begin{array}{l}\text { No Cultural } \\
\text { Resources }\end{array}$ & $\begin{array}{l}\text { No further work } \\
\text { recommended }\end{array}$ & $\begin{array}{l}\text { Located within or adjacent to Rice } \\
\text { Field }\end{array}$ \\
\hline SP2JE022 & 40.5 & $\begin{array}{c}\text { UT to Lower } \\
\text { Neches Valley } \\
\text { Authority Canal }\end{array}$ & Perennial & Low & None & $\begin{array}{l}\text { No Cultural } \\
\text { Resources }\end{array}$ & $\begin{array}{l}\text { No further work } \\
\text { recommended }\end{array}$ & $\begin{array}{l}\text { Completely avoided via non } \\
\text { Section } 10 \mathrm{HDD} / \text { Bore }\end{array}$ \\
\hline SP2JE023 & 40.5 & $\begin{array}{c}\text { UT to Lower } \\
\text { Neches Valley } \\
\text { Authority Canal }\end{array}$ & Perennial & Low & None & $\begin{array}{l}\text { No Cultural } \\
\text { Resources }\end{array}$ & $\begin{array}{l}\text { No further work } \\
\text { recommended }\end{array}$ & $\begin{array}{l}\text { Completely avoided via non } \\
\text { Section } 10 \mathrm{HDD} / \text { Bore }\end{array}$ \\
\hline SP4JE041 & 41 & $\begin{array}{c}\text { UT to Lower } \\
\text { Neches Valley } \\
\text { Authority Canal }\end{array}$ & Ephemeral & Low & None & $\begin{array}{l}\text { No Cultural } \\
\text { Resources }\end{array}$ & $\begin{array}{l}\text { No further work } \\
\text { recommended }\end{array}$ & $\begin{array}{l}\text { Completely avoided via non } \\
\text { Section } 10 \mathrm{HDD} / \text { Bore }\end{array}$ \\
\hline SP4JE029 & 41 & $\begin{array}{c}\text { UT to Lower } \\
\text { Neches Valley } \\
\text { Authority Canal }\end{array}$ & Ephemeral & Low & None & $\begin{array}{l}\text { No Cultural } \\
\text { Resources }\end{array}$ & $\begin{array}{l}\text { No further work } \\
\text { recommended }\end{array}$ & $\begin{array}{l}\text { Completely avoided via non } \\
\text { Section } 10 \mathrm{HDD} / \text { Bore }\end{array}$ \\
\hline SP4JE042 & 41.9 & $\begin{array}{c}\text { UT to Lower } \\
\text { Neches Valley } \\
\text { Authority Canal }\end{array}$ & Intermittent & Low & 18 & $\begin{array}{l}\text { No Cultural } \\
\text { Resources }\end{array}$ & $\begin{array}{l}\text { No further work } \\
\text { recommended }\end{array}$ & Inundation \\
\hline SP4JE043 & 42.2 & $\begin{array}{l}\text { UT to Lower } \\
\text { Neches Valley } \\
\text { Authority Canal }\end{array}$ & Ephemeral & Low & 17 & $\begin{array}{l}\text { No Cultural } \\
\text { Resources }\end{array}$ & $\begin{array}{l}\text { No further work } \\
\text { recommended }\end{array}$ & $\begin{array}{l}\text { Completely avoided via non } \\
\text { Section } 10 \mathrm{HDD} / \text { Bore }\end{array}$ \\
\hline SP3JE031 & 42.2 & $\begin{array}{c}\text { UT to Lower } \\
\text { Neches Valley } \\
\text { Authority Canal }\end{array}$ & Ephemeral & Low & 17 & $\begin{array}{l}\text { No Cultural } \\
\text { Resources }\end{array}$ & $\begin{array}{l}\text { No further work } \\
\text { recommended }\end{array}$ & $\begin{array}{l}\text { Completely avoided via non } \\
\text { Section } 10 \mathrm{HDD} / \text { Bore }\end{array}$ \\
\hline SP3JE030 & 42.2 & $\begin{array}{c}\text { UT to Lower } \\
\text { Neches Valley } \\
\text { Authority Canal }\end{array}$ & Ephemeral & Low & 17 & $\begin{array}{l}\text { No Cultural } \\
\text { Resources }\end{array}$ & $\begin{array}{l}\text { No further work } \\
\text { recommended }\end{array}$ & $\begin{array}{l}\text { Completely avoided via non } \\
\text { Section } 10 \mathrm{HDD} / \text { Bore }\end{array}$ \\
\hline SP3JE029 & 42.9 & $\begin{array}{c}\text { UT to Lower } \\
\text { Neches Valley } \\
\text { Authority Canal }\end{array}$ & Ephemeral & Low & None & $\begin{array}{l}\text { No Cultural } \\
\text { Resources }\end{array}$ & $\begin{array}{l}\text { No further work } \\
\text { recommended }\end{array}$ & $\begin{array}{l}\text { Located within or adjacent to Rice } \\
\text { Field }\end{array}$ \\
\hline SP3JE028 & 43.2 & $\begin{array}{l}\text { UT to Pignut } \\
\text { Gully }\end{array}$ & Ephemeral & Low & 2 & $\begin{array}{l}\text { No Cultural } \\
\text { Resources }\end{array}$ & $\begin{array}{l}\text { No further work } \\
\text { recommended }\end{array}$ & Inundation \\
\hline SP4JE024 & 43.2 & $\begin{array}{l}\text { UT to Pignut } \\
\text { Gully } \\
\end{array}$ & Ephemeral & Low & 2 & $\begin{array}{l}\text { No Cultural } \\
\text { Resources }\end{array}$ & $\begin{array}{l}\text { No further work } \\
\text { recommended }\end{array}$ & Inundation \\
\hline SP4JE025 & 43.5 & $\begin{array}{l}\text { UT to Lower } \\
\text { Neches Valley } \\
\text { Authority Canal }\end{array}$ & Ephemeral & Low & None & $\begin{array}{l}\text { No Cultural } \\
\text { Resources }\end{array}$ & $\begin{array}{l}\text { No further work } \\
\text { recommended }\end{array}$ & $\begin{array}{l}\text { Located within or adjacent to Rice } \\
\text { Field }\end{array}$ \\
\hline
\end{tabular}


Appendix C2 - Survey Data in the Vicinity of Waterbody Features

\begin{tabular}{|c|c|c|c|c|c|c|c|c|}
\hline Waterbody ID & $\begin{array}{c}\text { Milepost } \\
\text { (Approximate) }\end{array}$ & Waterbody Name & $\begin{array}{l}\text { Waterbody } \\
\text { Type }\end{array}$ & Probability & $\begin{array}{l}\text { Shovel } \\
\text { Tests }\end{array}$ & Surveys Results & Recommendations & Comments \\
\hline SP4JE027 & 43.8 & $\begin{array}{l}\text { UT to Pignut } \\
\text { Gully }\end{array}$ & Ephemeral & Low & None & $\begin{array}{l}\text { No Cultural } \\
\text { Resources }\end{array}$ & $\begin{array}{l}\text { No further work } \\
\text { recommended }\end{array}$ & $\begin{array}{c}\text { Located within or adjacent to Rice } \\
\text { Field }\end{array}$ \\
\hline SP4JE026 & 44.2 & $\begin{array}{l}\text { UT to Pignut } \\
\text { Gully }\end{array}$ & Ephemeral & Low & 6 & $\begin{array}{l}\text { No Cultural } \\
\text { Resources }\end{array}$ & $\begin{array}{l}\text { No further work } \\
\text { recommended }\end{array}$ & Inundation \\
\hline SP4JE028 & 44.2 & $\begin{array}{l}\text { UT to Pignut } \\
\text { Gully }\end{array}$ & Ephemeral & Low & 6 & $\begin{array}{l}\text { No Cultural } \\
\text { Resources }\end{array}$ & $\begin{array}{l}\text { No further work } \\
\text { recommended }\end{array}$ & Inundation \\
\hline SP4JE022 & 44.2 & $\begin{array}{l}\text { UT to Pignut } \\
\text { Gully }\end{array}$ & Ephemeral & Low & 6 & $\begin{array}{l}\text { No Cultural } \\
\text { Resources }\end{array}$ & $\begin{array}{l}\text { No further work } \\
\text { recommended }\end{array}$ & Inundation \\
\hline SP4JE044 & 44.9 & $\begin{array}{l}\text { UT to Pignut } \\
\text { Gully }\end{array}$ & Ephemeral & Low & None & $\begin{array}{l}\text { No Cultural } \\
\text { Resources }\end{array}$ & $\begin{array}{l}\text { No further work } \\
\text { recommended }\end{array}$ & $\begin{array}{l}\text { Completely avoided via non } \\
\text { Section } 10 \mathrm{HDD} / \text { Bore }\end{array}$ \\
\hline SP4JE045 & 45 & $\begin{array}{l}\text { UT to Pignut } \\
\text { Gully }\end{array}$ & Perennial & Low & None & $\begin{array}{l}\text { No Cultural } \\
\text { Resources }\end{array}$ & $\begin{array}{l}\text { No further work } \\
\text { recommended }\end{array}$ & $\begin{array}{l}\text { Completely avoided via non } \\
\text { Section } 10 \mathrm{HDD} / \text { Bore }\end{array}$ \\
\hline SP4JE046 & 45 & $\begin{array}{l}\text { UT to Pignut } \\
\text { Gully }\end{array}$ & Perennial & Low & None & $\begin{array}{c}\text { No Cultural } \\
\text { Resources }\end{array}$ & $\begin{array}{l}\text { No further work } \\
\text { recommended }\end{array}$ & $\begin{array}{c}\text { Located within or adjacent to Rice } \\
\text { Field } \\
\end{array}$ \\
\hline SP3JE032 & 45.8 & $\begin{array}{l}\text { UT to Pignut } \\
\text { Gully }\end{array}$ & Ephemeral & Low & None & $\begin{array}{l}\text { No Cultural } \\
\text { Resources }\end{array}$ & $\begin{array}{l}\text { No further work } \\
\text { recommended }\end{array}$ & $\begin{array}{c}\text { Located within or adjacent to Rice } \\
\text { Field }\end{array}$ \\
\hline SP9JE012_DT & 46.3 & UT to Pond Gully & Intermittent & Low & None & $\begin{array}{l}\text { No Cultural } \\
\text { Resources }\end{array}$ & $\begin{array}{l}\text { No further work } \\
\text { recommended }\end{array}$ & $\begin{array}{l}\text { Completely avoided via non } \\
\text { Section } 10 \mathrm{HDD} / \text { Bore }\end{array}$ \\
\hline SP9JE011_DT & 46.5 & UT to Pond Gully & Intermittent & Low & None & $\begin{array}{l}\text { No Cultural } \\
\text { Resources }\end{array}$ & $\begin{array}{l}\text { No further work } \\
\text { recommended }\end{array}$ & $\begin{array}{l}\text { Completely avoided via non } \\
\text { Section } 10 \mathrm{HDD} / \text { Bore }\end{array}$ \\
\hline SP9JE010_DT & 46.9 & UT to Pond Gully & Intermittent & Low & None & $\begin{array}{l}\text { No Cultural } \\
\text { Resources }\end{array}$ & $\begin{array}{l}\text { No further work } \\
\text { recommended }\end{array}$ & $\begin{array}{l}\text { Completely avoided via non } \\
\text { Section } 10 \mathrm{HDD} / \text { Bore }\end{array}$ \\
\hline SP9JE009_DT & 47 & UT to Pond Gully & Intermittent & Low & None & $\begin{array}{l}\text { No Cultural } \\
\text { Resources }\end{array}$ & $\begin{array}{l}\text { No further work } \\
\text { recommended }\end{array}$ & $\begin{array}{l}\text { Completely avoided via non } \\
\text { Section } 10 \mathrm{HDD} / \text { Bore }\end{array}$ \\
\hline SP9JE008_DT & 47.2 & UT to Pond Gully & Intermittent & Low & None & $\begin{array}{l}\text { No Cultural } \\
\text { Resources }\end{array}$ & $\begin{array}{l}\text { No further work } \\
\text { recommended }\end{array}$ & $\begin{array}{l}\text { Completely avoided via non } \\
\text { Section } 10 \mathrm{HDD} / \text { Bore } \\
\end{array}$ \\
\hline SP9JE007_DT & 47.4 & UT to Pond Gully & Intermittent & Low & None & $\begin{array}{l}\text { No Cultural } \\
\text { Resources }\end{array}$ & $\begin{array}{l}\text { No further work } \\
\text { recommended }\end{array}$ & $\begin{array}{l}\text { Completely avoided via non } \\
\text { Section } 10 \mathrm{HDD} / \text { Bore }\end{array}$ \\
\hline SP9JE019_DT & 47.5 & $\begin{array}{c}\text { UT to Green Pond } \\
\text { Gully }\end{array}$ & Intermittent & Low & None & $\begin{array}{l}\text { No Cultural } \\
\text { Resources }\end{array}$ & $\begin{array}{l}\text { No further work } \\
\text { recommended }\end{array}$ & $\begin{array}{l}\text { Completely avoided via non } \\
\text { Section } 10 \mathrm{HDD} / \text { Bore }\end{array}$ \\
\hline SP4JE049 & 47.6 & $\begin{array}{l}\text { UT to Green Pond } \\
\text { Gully }\end{array}$ & Intermittent & Low & None & $\begin{array}{l}\text { No Cultural } \\
\text { Resources }\end{array}$ & $\begin{array}{l}\text { No further work } \\
\text { recommended }\end{array}$ & $\begin{array}{l}\text { Completely avoided via non } \\
\text { Section } 10 \mathrm{HDD} / \text { Bore } \\
\end{array}$ \\
\hline SP9JE050_DT & 47.8 & $\begin{array}{l}\text { UT to Green Pond } \\
\text { Gully }\end{array}$ & Ephemeral & Low & None & $\begin{array}{l}\text { No Cultural } \\
\text { Resources }\end{array}$ & $\begin{array}{l}\text { No further work } \\
\text { recommended }\end{array}$ & $\begin{array}{l}\text { Completely avoided via non } \\
\text { Section } 10 \mathrm{HDD} / \text { Bore }\end{array}$ \\
\hline SP9JE001_DT & 48.1 & Green Pond Gully & Intermittent & Low & None & $\begin{array}{l}\text { No Cultural } \\
\text { Resources }\end{array}$ & $\begin{array}{l}\text { No further work } \\
\text { recommended }\end{array}$ & $\begin{array}{l}\text { Completely avoided via non } \\
\text { Section } 10 \mathrm{HDD} / \text { Bore }\end{array}$ \\
\hline
\end{tabular}


Appendix C2 - Survey Data in the Vicinity of Waterbody Features

\begin{tabular}{|c|c|c|c|c|c|c|c|c|}
\hline Waterbody ID & $\begin{array}{c}\text { Milepost } \\
\text { (Approximate) }\end{array}$ & Waterbody Name & $\begin{array}{l}\text { Waterbody } \\
\text { Type }\end{array}$ & Probability & $\begin{array}{l}\text { Shovel } \\
\text { Tests }\end{array}$ & Surveys Results & Recommendations & Comments \\
\hline SP9JE003_DT & 48.4 & UT to Pond Gully & Intermittent & Low & None & $\begin{array}{c}\text { No Cultural } \\
\text { Resources }\end{array}$ & $\begin{array}{l}\text { No further work } \\
\text { recommended }\end{array}$ & $\begin{array}{l}\text { Completely avoided via non } \\
\text { Section } 10 \text { HDD/Bore }\end{array}$ \\
\hline SP9JE004_DT & 48.8 & UT to Pond Gully & Intermittent & Low & None & $\begin{array}{c}\text { No Cultural } \\
\text { Resources }\end{array}$ & $\begin{array}{l}\text { No further work } \\
\text { recommended }\end{array}$ & $\begin{array}{l}\text { Completely avoided via non } \\
\text { Section } 10 \mathrm{HDD} / \text { Bore }\end{array}$ \\
\hline SP9JE005_DT & 49 & UT to Pond Gully & Intermittent & Low & None & $\begin{array}{c}\text { No Cultural } \\
\text { Resources }\end{array}$ & $\begin{array}{l}\text { No further work } \\
\text { recommended }\end{array}$ & $\begin{array}{l}\text { Completely avoided via non } \\
\text { Section } 10 \text { HDD/Bore }\end{array}$ \\
\hline SP9JE006_DT & 49.2 & UT to Pond Gully & Intermittent & Low & None & $\begin{array}{c}\text { No Cultural } \\
\text { Resources }\end{array}$ & $\begin{array}{l}\text { No further work } \\
\text { recommended }\end{array}$ & $\begin{array}{l}\text { Completely avoided via non } \\
\text { Section } 10 \text { HDD/Bore }\end{array}$ \\
\hline SP4JE051 & 50.2 & UT to Bayou Din & Ephemeral & Low & 5 & $\begin{array}{c}\text { No Cultural } \\
\text { Resources }\end{array}$ & $\begin{array}{l}\text { No further work } \\
\text { recommended }\end{array}$ & Inundation \\
\hline SP3JE039 & 50.9 & Gallier Canal & Perennial & Low & 4 & $\begin{array}{l}\text { No Cultural } \\
\text { Resources }\end{array}$ & $\begin{array}{l}\text { No further work } \\
\text { recommended }\end{array}$ & $\begin{array}{l}\text { Completely avoided via non } \\
\text { Section } 10 \mathrm{HDD} / \text { Bore }\end{array}$ \\
\hline OWP3JE002 & 50.9 & N/A & Manmade Pond & Low & 3 & $\begin{array}{c}\text { No Cultural } \\
\text { Resources }\end{array}$ & $\begin{array}{l}\text { No further work } \\
\text { recommended }\end{array}$ & Inundation \\
\hline SP4JE054 & 51.2 & UT to Kid Gully & Ephemeral & Low & 1 & $\begin{array}{c}\text { No Cultural } \\
\text { Resources }\end{array}$ & $\begin{array}{l}\text { No further work } \\
\text { recommended }\end{array}$ & $\begin{array}{l}\text { Completely avoided via non } \\
\text { Section } 10 \mathrm{HDD} / \text { Bore }\end{array}$ \\
\hline SP4JE053 & 51.7 & UT to Kid Gully & Perennial & Low & 1 & $\begin{array}{l}\text { No Cultural } \\
\text { Resources }\end{array}$ & $\begin{array}{l}\text { No further work } \\
\text { recommended }\end{array}$ & $\begin{array}{l}\text { Completely avoided via non } \\
\text { Section } 10 \mathrm{HDD} / \text { Bore }\end{array}$ \\
\hline SP4JE052 & 51.7 & Kid Gully & Intermittent & Low & None & $\begin{array}{c}\text { No Cultural } \\
\text { Resources }\end{array}$ & $\begin{array}{l}\text { No further work } \\
\text { recommended }\end{array}$ & $\begin{array}{l}\text { Completely avoided via non } \\
\text { Section } 10 \mathrm{HDD} / \text { Bore }\end{array}$ \\
\hline SP3JE043 & 52.2 & $\begin{array}{l}\text { UT to Smith } \\
\text { Levee }\end{array}$ & Ephemeral & Low & 1 & $\begin{array}{c}\text { No Cultural } \\
\text { Resources }\end{array}$ & $\begin{array}{l}\text { No further work } \\
\text { recommended }\end{array}$ & $\begin{array}{l}\text { Completely avoided via non } \\
\text { Section } 10 \mathrm{HDD} / \text { Bore }\end{array}$ \\
\hline SP3JE042 & 52.2 & $\begin{array}{l}\text { UT to Smith } \\
\text { Levee }\end{array}$ & Ephemeral & Low & 1 & $\begin{array}{c}\text { No Cultural } \\
\text { Resources }\end{array}$ & $\begin{array}{l}\text { No further work } \\
\text { recommended }\end{array}$ & $\begin{array}{l}\text { Completely avoided via non } \\
\text { Section } 10 \mathrm{HDD} / \text { Bore }\end{array}$ \\
\hline SP3JE041 & 52.2 & $\begin{array}{l}\text { UT to Smith } \\
\text { Levee }\end{array}$ & Ephemeral & Low & 1 & $\begin{array}{l}\text { No Cultural } \\
\text { Resources }\end{array}$ & $\begin{array}{l}\text { No further work } \\
\text { recommended }\end{array}$ & $\begin{array}{l}\text { Completely avoided via non } \\
\text { Section } 10 \mathrm{HDD} / \text { Bore } \\
\end{array}$ \\
\hline SP3JE040 & 52.2 & Smith Levee & Intermittent & Low & 1 & $\begin{array}{l}\text { No Cultural } \\
\text { Resources }\end{array}$ & $\begin{array}{l}\text { No further work } \\
\text { recommended }\end{array}$ & $\begin{array}{c}\text { Completely avoided via non } \\
\text { Section } 10 \text { HDD/Bore }\end{array}$ \\
\hline SP4JE055 & 52.7 & $\begin{array}{l}\text { UT to Pevitot } \\
\text { Gully }\end{array}$ & Ephemeral & Low & 7 & $\begin{array}{c}\text { No Cultural } \\
\text { Resources }\end{array}$ & $\begin{array}{l}\text { No further work } \\
\text { recommended }\end{array}$ & $\begin{array}{c}\text { Partially avoided via non Section } \\
10 \mathrm{HDD} / \text { Bore }\end{array}$ \\
\hline SP3JE045 & 52.7 & $\begin{array}{l}\text { UT to Pevitot } \\
\text { Gully }\end{array}$ & Ephemeral & Low & 7 & $\begin{array}{c}\text { No Cultural } \\
\text { Resources }\end{array}$ & $\begin{array}{l}\text { No further work } \\
\text { recommended }\end{array}$ & Inundation \\
\hline SP3JE044 & 52.7 & $\begin{array}{l}\text { UT to Pevitot } \\
\text { Gully }\end{array}$ & Perennial & Low & 7 & $\begin{array}{c}\text { No Cultural } \\
\text { Resources }\end{array}$ & $\begin{array}{l}\text { No further work } \\
\text { recommended }\end{array}$ & $\begin{array}{c}\text { Completely avoided via non } \\
\text { Section } 10 \mathrm{HDD} / \text { Bore }\end{array}$ \\
\hline OWP4JE006 & 52.7 & N/A & Manmade Pond & Low & 7 & $\begin{array}{l}\text { No Cultural } \\
\text { Resources }\end{array}$ & $\begin{array}{l}\text { No further work } \\
\text { recommended }\end{array}$ & Inundation \\
\hline
\end{tabular}


Appendix C2 - Survey Data in the Vicinity of Waterbody Features

\begin{tabular}{|c|c|c|c|c|c|c|c|c|}
\hline Waterbody ID & $\begin{array}{c}\text { Milepost } \\
\text { (Approximate) }\end{array}$ & Waterbody Name & $\begin{array}{l}\text { Waterbody } \\
\text { Type }\end{array}$ & Probability & $\begin{array}{l}\text { Shovel } \\
\text { Tests }\end{array}$ & Surveys Results & Recommendations & Comments \\
\hline OWP5JE001 & 53.1 & N/A & Manmade Pond & Low & None & $\begin{array}{l}\text { No Cultural } \\
\text { Resources }\end{array}$ & $\begin{array}{l}\text { No further work } \\
\text { recommended }\end{array}$ & $\begin{array}{c}\text { Located Within Heavily Inundated } \\
\text { Area }\end{array}$ \\
\hline SP5JE001 & 53.2 & $\begin{array}{l}\text { UT to Pevitot } \\
\text { Gully }\end{array}$ & Ephemeral & Low & None & $\begin{array}{l}\text { No Cultural } \\
\text { Resources }\end{array}$ & $\begin{array}{l}\text { No further work } \\
\text { recommended }\end{array}$ & $\begin{array}{c}\text { Located Within Heavily Inundated } \\
\text { Area }\end{array}$ \\
\hline SP5JE002 & 53.5 & $\begin{array}{l}\text { UT to Pevitot } \\
\text { Gully }\end{array}$ & Intermittent & Low & None & $\begin{array}{l}\text { No Cultural } \\
\text { Resources }\end{array}$ & $\begin{array}{l}\text { No further work } \\
\text { recommended }\end{array}$ & $\begin{array}{c}\text { Located Within Heavily Inundated } \\
\text { Area }\end{array}$ \\
\hline SP5JE004 & 53.6 & $\begin{array}{l}\text { UT to Pevitot } \\
\text { Gully }\end{array}$ & Ephemeral & Low & None & $\begin{array}{l}\text { No Cultural } \\
\text { Resources }\end{array}$ & $\begin{array}{l}\text { No further work } \\
\text { recommended }\end{array}$ & $\begin{array}{c}\text { Located Within Heavily Inundated } \\
\text { Area }\end{array}$ \\
\hline SP5JE003 & 53.6 & $\begin{array}{l}\text { UT to Pevitot } \\
\text { Gully }\end{array}$ & Ephemeral & Low & None & $\begin{array}{l}\text { No Cultural } \\
\text { Resources }\end{array}$ & $\begin{array}{l}\text { No further work } \\
\text { recommended }\end{array}$ & $\begin{array}{c}\text { Located Within Heavily Inundated } \\
\text { Area }\end{array}$ \\
\hline SP5JE005 & 53.6 & $\begin{array}{l}\text { UT to Pevitot } \\
\text { Gully }\end{array}$ & Perennial & Low & None & $\begin{array}{l}\text { No Cultural } \\
\text { Resources }\end{array}$ & $\begin{array}{l}\text { No further work } \\
\text { recommended }\end{array}$ & $\begin{array}{c}\text { Located Within Heavily Inundated } \\
\text { Area }\end{array}$ \\
\hline SP5JE006 & 54.1 & $\begin{array}{c}\text { Willow Marsh } \\
\text { Bayou } \\
\end{array}$ & Perennial & Low & None & $\begin{array}{c}\text { No Cultural } \\
\text { Resources }\end{array}$ & $\begin{array}{l}\text { No further work } \\
\text { recommended }\end{array}$ & $\begin{array}{c}\text { Completely avoided via non } \\
\text { Section } 10 \mathrm{HDD} / \text { Bore } \\
\end{array}$ \\
\hline SP4JE058 & 54.2 & $\begin{array}{l}\text { UT to Willow } \\
\text { Marsh Bayou }\end{array}$ & Ephemeral & Low & None & $\begin{array}{l}\text { No Cultural } \\
\text { Resources }\end{array}$ & $\begin{array}{l}\text { No further work } \\
\text { recommended }\end{array}$ & $\begin{array}{l}\text { Completely avoided via non } \\
\text { Section } 10 \mathrm{HDD} / \text { Bore }\end{array}$ \\
\hline SP4JE056 & 54.2 & $\begin{array}{l}\text { UT to Willow } \\
\text { Marsh Bayou }\end{array}$ & Ephemeral & Low & None & $\begin{array}{l}\text { No Cultural } \\
\text { Resources }\end{array}$ & $\begin{array}{l}\text { No further work } \\
\text { recommended }\end{array}$ & $\begin{array}{l}\text { Completely avoided via non } \\
\text { Section } 10 \mathrm{HDD} / \text { Bore }\end{array}$ \\
\hline SP4JE057 & 54.8 & Spoil Bank & Intermittent & Low & None & $\begin{array}{l}\text { No Cultural } \\
\text { Resources }\end{array}$ & $\begin{array}{l}\text { No further work } \\
\text { recommended }\end{array}$ & $\begin{array}{c}\text { Located Within Heavily Inundated } \\
\text { Area }\end{array}$ \\
\hline SP5JE015 & 55 & $\begin{array}{l}\text { UT to Willow } \\
\text { Marsh Bayou }\end{array}$ & Intermittent & Low & 2 & $\begin{array}{l}\text { No Cultural } \\
\text { Resources }\end{array}$ & $\begin{array}{l}\text { No further work } \\
\text { recommended }\end{array}$ & $\begin{array}{c}\text { Completely avoided via non } \\
\text { Section } 10 \text { HDD/Bore }\end{array}$ \\
\hline SP5JE012 & 55.4 & $\begin{array}{l}\text { UT to Willow } \\
\text { Marsh Bayou }\end{array}$ & Ephemeral & Medium & 9 & $\begin{array}{l}\text { No Cultural } \\
\text { Resources }\end{array}$ & $\begin{array}{l}\text { No further work } \\
\text { recommended }\end{array}$ & $\begin{array}{l}\text { Completely avoided via non } \\
\text { Section } 10 \mathrm{HDD} / \text { Bore }\end{array}$ \\
\hline SP5JE013 & 55.4 & $\begin{array}{l}\text { UT to Willow } \\
\text { Marsh Bayou }\end{array}$ & Ephemeral & Medium & 9 & $\begin{array}{l}\text { No Cultural } \\
\text { Resources }\end{array}$ & $\begin{array}{l}\text { No further work } \\
\text { recommended }\end{array}$ & $\begin{array}{l}\text { Completely avoided via non } \\
\text { Section } 10 \mathrm{HDD} / \text { Bore } \\
\end{array}$ \\
\hline SP5JE014 & 55.4 & $\begin{array}{l}\text { UT to Willow } \\
\text { Marsh Bayou }\end{array}$ & Ephemeral & Medium & 9 & $\begin{array}{l}\text { No Cultural } \\
\text { Resources }\end{array}$ & $\begin{array}{l}\text { No further work } \\
\text { recommended }\end{array}$ & $\begin{array}{l}\text { Completely avoided via non } \\
\text { Section } 10 \mathrm{HDD} / \text { Bore }\end{array}$ \\
\hline SP5JE011 & 55.7 & $\begin{array}{l}\text { UT to Willow } \\
\text { Marsh Bayou }\end{array}$ & Intermittent & Low & 2 & $\begin{array}{l}\text { No Cultural } \\
\text { Resources }\end{array}$ & $\begin{array}{l}\text { No further work } \\
\text { recommended }\end{array}$ & Inundation \\
\hline SP5JE010 & 56.2 & $\begin{array}{l}\text { UT to Willow } \\
\text { Marsh Bayou }\end{array}$ & Ephemeral & Low & 4 & $\begin{array}{l}\text { No Cultural } \\
\text { Resources }\end{array}$ & $\begin{array}{l}\text { No further work } \\
\text { recommended }\end{array}$ & Inundation \\
\hline SP5JE009 & 56.4 & $\begin{array}{l}\text { UT to Willow } \\
\text { Marsh Bayou }\end{array}$ & Perennial & Low & None & $\begin{array}{l}\text { No Cultural } \\
\text { Resources }\end{array}$ & $\begin{array}{l}\text { No further work } \\
\text { recommended }\end{array}$ & $\begin{array}{c}\text { Covered under previously } \\
\text { conducted survey }\end{array}$ \\
\hline SP5JE007 & 56.7 & $\begin{array}{l}\text { UT to Willow } \\
\text { Marsh Bayou }\end{array}$ & Ephemeral & Low & None & $\begin{array}{l}\text { No Cultural } \\
\text { Resources }\end{array}$ & $\begin{array}{l}\text { No further work } \\
\text { recommended }\end{array}$ & $\begin{array}{c}\text { Located Within Heavily Inundated } \\
\text { Area }\end{array}$ \\
\hline
\end{tabular}


Appendix C2 - Survey Data in the Vicinity of Waterbody Features

\begin{tabular}{|c|c|c|c|c|c|c|c|c|}
\hline Waterbody ID & $\begin{array}{c}\text { Milepost } \\
\text { (Approximate) }\end{array}$ & Waterbody Name & $\begin{array}{l}\text { Waterbody } \\
\text { Type }\end{array}$ & Probability & $\begin{array}{l}\text { Shovel } \\
\text { Tests }\end{array}$ & Surveys Results & Recommendations & Comments \\
\hline SP5JE008 & 56.7 & $\begin{array}{l}\text { UT to Willow } \\
\text { Marsh Bayou }\end{array}$ & Intermittent & Low & None & $\begin{array}{l}\text { No Cultural } \\
\text { Resources }\end{array}$ & $\begin{array}{l}\text { No further work } \\
\text { recommended }\end{array}$ & $\begin{array}{c}\text { Located Within Heavily Inundated } \\
\text { Area }\end{array}$ \\
\hline OWP4JE009 & 57.6 & N/A & Manmade Pond & Low & None & $\begin{array}{l}\text { No Cultural } \\
\text { Resources }\end{array}$ & $\begin{array}{l}\text { No further work } \\
\text { recommended }\end{array}$ & $\begin{array}{l}\text { Completely avoided via non } \\
\text { Section } 10 \mathrm{HDD} / \text { Bore }\end{array}$ \\
\hline OWP4JE007 & 57.9 & N/A & Manmade Pond & Low & None & $\begin{array}{c}\text { No Cultural } \\
\text { Resources }\end{array}$ & $\begin{array}{l}\text { No further work } \\
\text { recommended }\end{array}$ & $\begin{array}{c}\text { Located Within Heavily Inundated } \\
\text { Area }\end{array}$ \\
\hline OWP4JE008 & 57.9 & N/A & Manmade Pond & Low & None & $\begin{array}{l}\text { No Cultural } \\
\text { Resources }\end{array}$ & $\begin{array}{l}\text { No further work } \\
\text { recommended }\end{array}$ & $\begin{array}{c}\text { Located Within Heavily Inundated } \\
\text { Area }\end{array}$ \\
\hline OWP4JE010 & 57.9 & N/A & Manmade Pond & Low & None & $\begin{array}{l}\text { No Cultural } \\
\text { Resources }\end{array}$ & $\begin{array}{l}\text { No further work } \\
\text { recommended }\end{array}$ & $\begin{array}{c}\text { Located Within Heavily Inundated } \\
\text { Area }\end{array}$ \\
\hline SP4JE064 & 58 & $\begin{array}{l}\text { Hilderbrandt } \\
\text { Bayou }\end{array}$ & Perennial & Low & 9 & $\begin{array}{l}\text { No Cultural } \\
\text { Resources }\end{array}$ & $\begin{array}{l}\text { No further work } \\
\text { recommended }\end{array}$ & $\begin{array}{l}\text { Completely avoided via non } \\
\text { Section } 10 \text { HDD/Bore }\end{array}$ \\
\hline SP4JE065 & 58 & $\begin{array}{l}\text { Hilderbrandt } \\
\text { Bayou }\end{array}$ & Intermittent & Low & None & $\begin{array}{c}\text { No Cultural } \\
\text { Resources }\end{array}$ & $\begin{array}{l}\text { No further work } \\
\text { recommended }\end{array}$ & $\begin{array}{l}\text { Completely avoided via non } \\
\text { Section } 10 \mathrm{HDD} / \text { Bore }\end{array}$ \\
\hline SP2JE039 & 58.5 & $\begin{array}{c}\text { UT to } \\
\text { Hilderbrandt } \\
\text { Bayou }\end{array}$ & Intermittent & Low & 8 & $\begin{array}{l}\text { No Cultural } \\
\text { Resources }\end{array}$ & $\begin{array}{l}\text { No further work } \\
\text { recommended }\end{array}$ & Inundation \\
\hline SP2JE040 & 58.5 & $\begin{array}{c}\text { UT to } \\
\text { Hilderbrandt } \\
\text { Bayou }\end{array}$ & Intermittent & Low & 8 & $\begin{array}{l}\text { No Cultural } \\
\text { Resources }\end{array}$ & $\begin{array}{l}\text { No further work } \\
\text { recommended }\end{array}$ & Inundation \\
\hline SP6JE010 & 58.7 & $\begin{array}{c}\text { UT to } \\
\text { Hilderbrandt } \\
\text { Bayou }\end{array}$ & Ephemeral & Low & 4 & $\begin{array}{l}\text { No Cultural } \\
\text { Resources }\end{array}$ & $\begin{array}{l}\text { No further work } \\
\text { recommended }\end{array}$ & Inundation \\
\hline SP4JE060 & 59.1 & $\begin{array}{c}\text { UT to } \\
\text { Hilderbrandt } \\
\text { Bayou }\end{array}$ & Perennial & Low & None & $\begin{array}{l}\text { No Cultural } \\
\text { Resources }\end{array}$ & $\begin{array}{l}\text { No further work } \\
\text { recommended }\end{array}$ & Inundation \\
\hline SP6JE009 & 59.1 & $\begin{array}{c}\text { UT to } \\
\text { Hilderbrandt } \\
\text { Bayou }\end{array}$ & Intermittent & Low & None & $\begin{array}{l}\text { No Cultural } \\
\text { Resources }\end{array}$ & $\begin{array}{l}\text { No further work } \\
\text { recommended }\end{array}$ & Inundation \\
\hline SP4JE059 & 59.2 & $\begin{array}{l}\text { Hilderbrandt } \\
\text { Bayou }\end{array}$ & Perennial & Low & None & $\begin{array}{l}\text { No Cultural } \\
\text { Resources }\end{array}$ & $\begin{array}{l}\text { No further work } \\
\text { recommended }\end{array}$ & $\begin{array}{l}\text { Completely avoided via non } \\
\text { Section } 10 \mathrm{HDD} / \text { Bore }\end{array}$ \\
\hline SP4JE061 & 60 & $\begin{array}{l}\text { UT to } \\
\text { Hilderbrandt } \\
\text { Bayou }\end{array}$ & Perennial & Low & 17 & $\begin{array}{l}\text { No Cultural } \\
\text { Resources }\end{array}$ & $\begin{array}{l}\text { No further work } \\
\text { recommended }\end{array}$ & Inundation \\
\hline SP1JE005 & 60 & $\begin{array}{c}\text { UT to } \\
\text { Hilderbrandt } \\
\text { Bayou }\end{array}$ & Ephemeral & Low & 31 & $\begin{array}{l}\text { No Cultural } \\
\text { Resources }\end{array}$ & $\begin{array}{l}\text { No further work } \\
\text { recommended }\end{array}$ & Inundation \\
\hline
\end{tabular}


Appendix C2 - Survey Data in the Vicinity of Waterbody Features

\begin{tabular}{|c|c|c|c|c|c|c|c|c|}
\hline Waterbody ID & $\begin{array}{c}\text { Milepost } \\
\text { (Approximate) }\end{array}$ & Waterbody Name & $\begin{array}{l}\text { Waterbody } \\
\text { Type }\end{array}$ & Probability & $\begin{array}{l}\text { Shovel } \\
\text { Tests }\end{array}$ & Surveys Results & Recommendations & Comments \\
\hline SP1JE004 & 60.3 & $\begin{array}{c}\text { UT to } \\
\text { Hilderbrandt } \\
\text { Bayou }\end{array}$ & Ephemeral & Low & 5 & $\begin{array}{l}\text { No Cultural } \\
\text { Resources }\end{array}$ & $\begin{array}{l}\text { No further work } \\
\text { recommended }\end{array}$ & Inundation \\
\hline SP2JE003 & 60.8 & $\begin{array}{c}\text { UT to } \\
\text { Hilderbrandt } \\
\text { Bayou }\end{array}$ & Ephemeral & Low & 7 & $\begin{array}{l}\text { No Cultural } \\
\text { Resources }\end{array}$ & $\begin{array}{l}\text { No further work } \\
\text { recommended }\end{array}$ & Inundation \\
\hline SP2JE002 & 61.1 & $\begin{array}{c}\text { UT to } \\
\text { Hilderbrandt } \\
\text { Bayou }\end{array}$ & Ephemeral & Low & 11 & $\begin{array}{l}\text { No Cultural } \\
\text { Resources }\end{array}$ & $\begin{array}{l}\text { No further work } \\
\text { recommended }\end{array}$ & $\begin{array}{l}\text { Completely avoided via non } \\
\text { Section } 10 \mathrm{HDD} / \text { Bore }\end{array}$ \\
\hline SP2JE001 & 61.8 & $\begin{array}{c}\text { UT to } \\
\text { Hilderbrandt } \\
\text { Bayou }\end{array}$ & Ephemeral & Low & 10 & $\begin{array}{l}\text { No Cultural } \\
\text { Resources }\end{array}$ & $\begin{array}{l}\text { No further work } \\
\text { recommended }\end{array}$ & $\begin{array}{l}\text { Completely avoided via non } \\
\text { Section } 10 \mathrm{HDD} / \text { Bore }\end{array}$ \\
\hline SP1JE001 & 61.9 & $\begin{array}{c}\text { UT to } \\
\text { Hilderbrandt } \\
\text { Bayou }\end{array}$ & Ephemeral & Low & 10 & $\begin{array}{l}\text { No Cultural } \\
\text { Resources }\end{array}$ & $\begin{array}{l}\text { No further work } \\
\text { recommended }\end{array}$ & Inundation \\
\hline SP1JE002 & 62 & $\begin{array}{c}\text { UT to } \\
\text { Hilderbrandt } \\
\text { Bayou }\end{array}$ & Ephemeral & Low & 6 & $\begin{array}{l}\text { No Cultural } \\
\text { Resources }\end{array}$ & $\begin{array}{l}\text { No further work } \\
\text { recommended }\end{array}$ & Inundation \\
\hline SP9JE300_DT ${ }^{\text {a }}$ & 62 & $\begin{array}{c}\text { UT to } \\
\text { Hilderbrandt } \\
\text { Bayou }\end{array}$ & Intermittent & Low & 6 & $\begin{array}{l}\text { No Cultural } \\
\text { Resources }\end{array}$ & $\begin{array}{l}\text { No further work } \\
\text { recommended }\end{array}$ & Inundation \\
\hline SP1JE003 & 62.3 & UT to Johns Gully & Ephemeral & Low & None & $\begin{array}{l}\text { No Cultural } \\
\text { Resources }\end{array}$ & $\begin{array}{l}\text { No further work } \\
\text { recommended }\end{array}$ & $\begin{array}{c}\text { Located Within Heavily Inundated } \\
\text { Area }\end{array}$ \\
\hline SP3JE047 & 62.4 & UT to Johns Gully & Ephemeral & Low & 7 & $\begin{array}{l}\text { No Cultural } \\
\text { Resources }\end{array}$ & $\begin{array}{l}\text { No further work } \\
\text { recommended }\end{array}$ & $\begin{array}{c}\text { Completely avoided via non } \\
\text { Section } 10 \mathrm{HDD} / \text { Bore }\end{array}$ \\
\hline SP3JE046 & 62.5 & UT to Johns Gully & Ephemeral & Low & 7 & $\begin{array}{l}\text { No Cultural } \\
\text { Resources }\end{array}$ & $\begin{array}{l}\text { No further work } \\
\text { recommended }\end{array}$ & Inundation \\
\hline SP2JE300 & 62.5 & Johns Gully & Intermittent & Low & 7 & $\begin{array}{l}\text { No Cultural } \\
\text { Resources }\end{array}$ & $\begin{array}{l}\text { No further work } \\
\text { recommended }\end{array}$ & $\begin{array}{l}\text { Completely avoided via non } \\
\text { Section } 10 \mathrm{HDD} / \text { Bore }\end{array}$ \\
\hline SP4JE066 & 62.7 & Johns Gully & Intermittent & Low & None & $\begin{array}{l}\text { No Cultural } \\
\text { Resources }\end{array}$ & $\begin{array}{l}\text { No further work } \\
\text { recommended }\end{array}$ & Inundation \\
\hline SP9JE015_DT & 63 & $\begin{array}{l}\text { UT to Rhodair } \\
\text { Gully }\end{array}$ & Ephemeral & Low & None & $\begin{array}{l}\text { No Cultural } \\
\text { Resources }\end{array}$ & $\begin{array}{l}\text { No further work } \\
\text { recommended }\end{array}$ & Inundation \\
\hline SP4JE067 & 63.3 & $\begin{array}{l}\text { UT to Rhodair } \\
\text { Gully }\end{array}$ & Intermittent & Low & None & $\begin{array}{l}\text { No Cultural } \\
\text { Resources }\end{array}$ & $\begin{array}{l}\text { No further work } \\
\text { recommended }\end{array}$ & Inundation \\
\hline SP4JE068 & 64.6 & $\begin{array}{l}\text { UT to Rhodair } \\
\text { Gully }\end{array}$ & Intermittent & Low & None & $\begin{array}{l}\text { No Cultural } \\
\text { Resources }\end{array}$ & $\begin{array}{l}\text { No further work } \\
\text { recommended }\end{array}$ & Inundation \\
\hline
\end{tabular}


Appendix C2 - Survey Data in the Vicinity of Waterbody Features

\begin{tabular}{|c|c|c|c|c|c|c|c|c|}
\hline Waterbody ID & $\begin{array}{c}\text { Milepost } \\
\text { (Approximate) }\end{array}$ & Waterbody Name & $\begin{array}{c}\text { Waterbody } \\
\text { Type }\end{array}$ & Probability & $\begin{array}{l}\text { Shovel } \\
\text { Tests }\end{array}$ & Surveys Results & Recommendations & Comments \\
\hline SP7JE17 & & $\begin{array}{l}\text { UT to Rhodair } \\
\text { Gully }\end{array}$ & Intermittent & Low & None & $\begin{array}{c}\text { No Cultural } \\
\text { Resources }\end{array}$ & $\begin{array}{l}\text { No further work } \\
\text { recommended }\end{array}$ & Inundation \\
\hline SP7JE012 & 65.8 & $\begin{array}{l}\text { UT to Rhodair } \\
\text { Gully }\end{array}$ & Ephemeral & Low & 1 & $\begin{array}{l}\text { No Cultural } \\
\text { Resources }\end{array}$ & $\begin{array}{l}\text { No further work } \\
\text { recommended }\end{array}$ & Inundation \\
\hline SP4JE069 & 64.6 & Rhodair Gully & Intermittent & Low & None & $\begin{array}{c}\text { No Cultural } \\
\text { Resources }\end{array}$ & $\begin{array}{l}\text { No further work } \\
\text { recommended }\end{array}$ & Inundation \\
\hline SP7JE013 & 65.2 & $\begin{array}{l}\text { UT to Rhodair } \\
\text { Gully }\end{array}$ & Ephemeral & Low & None & $\begin{array}{l}\text { No Cultural } \\
\text { Resources }\end{array}$ & $\begin{array}{l}\text { No further work } \\
\text { recommended }\end{array}$ & Inundation \\
\hline SP7JE002 & 64.9 & $\begin{array}{l}\text { UT to Rhodair } \\
\text { Gully }\end{array}$ & Ephemeral & Low & None & $\begin{array}{l}\text { No Cultural } \\
\text { Resources }\end{array}$ & $\begin{array}{l}\text { No further work } \\
\text { recommended }\end{array}$ & Inundation \\
\hline SP7JE014 & 65.3 & $\begin{array}{l}\text { UT to Rhodair } \\
\text { Gully }\end{array}$ & Ephemeral & Low & None & $\begin{array}{l}\text { No Cultural } \\
\text { Resources }\end{array}$ & $\begin{array}{l}\text { No further work } \\
\text { recommended }\end{array}$ & $\begin{array}{l}\text { Completely avoided via non } \\
\text { Section } 10 \mathrm{HDD} / \text { Bore }\end{array}$ \\
\hline SP7JE003 & $\mathrm{n} / \mathrm{a}$ & $\begin{array}{l}\text { UT to Rhodair } \\
\text { Gully }\end{array}$ & Ephemeral & Low & None & $\begin{array}{l}\text { No Cultural } \\
\text { Resources }\end{array}$ & $\begin{array}{l}\text { No further work } \\
\text { recommended }\end{array}$ & Inundation \\
\hline SP7JE004 & $\mathrm{n} / \mathrm{a}$ & $\begin{array}{l}\text { UT to Rhodair } \\
\text { Gully }\end{array}$ & Ephemeral & Low & None & $\begin{array}{l}\text { No Cultural } \\
\text { Resources }\end{array}$ & $\begin{array}{l}\text { No further work } \\
\text { recommended }\end{array}$ & Inundation \\
\hline SP7JE016 & 65.4 & $\begin{array}{l}\text { UT to Rhodair } \\
\text { Gully }\end{array}$ & Ephemeral & Low & None & $\begin{array}{c}\text { No Cultural } \\
\text { Resources }\end{array}$ & $\begin{array}{l}\text { No further work } \\
\text { recommended }\end{array}$ & Inundation \\
\hline SP4JE070 & 64.9 & $\begin{array}{l}\text { UT to Rhodair } \\
\text { Gully }\end{array}$ & Ephemeral & Low & None & $\begin{array}{l}\text { No Cultural } \\
\text { Resources }\end{array}$ & $\begin{array}{l}\text { No further work } \\
\text { recommended }\end{array}$ & Inundation \\
\hline SP7JE015 & 65.4 & $\begin{array}{l}\text { UT to Rhodair } \\
\text { Gully }\end{array}$ & Intermittent & Low & None & $\begin{array}{l}\text { No Cultural } \\
\text { Resources }\end{array}$ & $\begin{array}{l}\text { No further work } \\
\text { recommended }\end{array}$ & Inundation \\
\hline SP7JE005 & $\mathrm{n} / \mathrm{a}$ & $\begin{array}{l}\text { UT to Rhodair } \\
\text { Gully }\end{array}$ & Ephemeral & Low & None & $\begin{array}{l}\text { No Cultural } \\
\text { Resources }\end{array}$ & $\begin{array}{l}\text { No further work } \\
\text { recommended }\end{array}$ & Inundation \\
\hline SP7JE006 & $\mathrm{n} / \mathrm{a}$ & $\begin{array}{c}\text { UT to Rhodair } \\
\text { Gully }\end{array}$ & Ephemeral & Low & None & $\begin{array}{l}\text { No Cultural } \\
\text { Resources }\end{array}$ & $\begin{array}{l}\text { No further work } \\
\text { recommended }\end{array}$ & Inundation \\
\hline SP7JE007 & $\mathrm{n} / \mathrm{a}$ & $\begin{array}{l}\text { UT to Rhodair } \\
\text { Gully }\end{array}$ & Intermittent & Low & 1 & $\begin{array}{l}\text { No Cultural } \\
\text { Resources }\end{array}$ & $\begin{array}{l}\text { No further work } \\
\text { recommended }\end{array}$ & Inundation \\
\hline SP2JE037 & 65.1 & Rhodair Gully & Perennial & Low & None & $\begin{array}{l}\text { No Cultural } \\
\text { Resources }\end{array}$ & $\begin{array}{l}\text { No further work } \\
\text { recommended }\end{array}$ & Inundation \\
\hline SP2JE038 & 65.1 & $\begin{array}{c}\text { UT to Rhodair } \\
\text { Gully }\end{array}$ & Intermittent & Low & None & $\begin{array}{l}\text { No Cultural } \\
\text { Resources }\end{array}$ & $\begin{array}{l}\text { No further work } \\
\text { recommended }\end{array}$ & Inundation \\
\hline SP7JE009 & 65.8 & $\begin{array}{c}\text { UT to Port Arthur } \\
\text { Canal }\end{array}$ & Ephemeral & Low & None & $\begin{array}{l}\text { No Cultural } \\
\text { Resources }\end{array}$ & $\begin{array}{l}\text { No further work } \\
\text { recommended }\end{array}$ & Inundation \\
\hline SP7JE010 & 65.8 & $\begin{array}{c}\text { UT to Port Arthur } \\
\text { Canal }\end{array}$ & Ephemeral & Low & None & $\begin{array}{l}\text { No Cultural } \\
\text { Resources }\end{array}$ & $\begin{array}{l}\text { No further work } \\
\text { recommended }\end{array}$ & Inundation \\
\hline
\end{tabular}


Appendix C2 - Survey Data in the Vicinity of Waterbody Features

\begin{tabular}{|c|c|c|c|c|c|c|c|c|}
\hline Waterbody ID & $\begin{array}{c}\text { Milepost } \\
\text { (Approximate) }\end{array}$ & Waterbody Name & $\begin{array}{l}\text { Waterbody } \\
\text { Type }\end{array}$ & Probability & $\begin{array}{l}\text { Shovel } \\
\text { Tests }\end{array}$ & Surveys Results & Recommendations & Comments \\
\hline SP7JE008 & 65.8 & $\begin{array}{c}\text { UT to Port Arthur } \\
\text { Canal }\end{array}$ & Perennial & Low & None & $\begin{array}{l}\text { No Cultural } \\
\text { Resources }\end{array}$ & $\begin{array}{l}\text { No further work } \\
\text { recommended }\end{array}$ & $\begin{array}{l}\text { Completely avoided via non } \\
\text { Section } 10 \mathrm{HDD} / \text { Bore }\end{array}$ \\
\hline SP7JE011 & 65.8 & $\begin{array}{c}\text { UT to Port Arthur } \\
\text { Canal }\end{array}$ & Ephemeral & Low & 1 & $\begin{array}{l}\text { No Cultural } \\
\text { Resources }\end{array}$ & $\begin{array}{l}\text { No further work } \\
\text { recommended }\end{array}$ & Inundation \\
\hline SP2JE034 & 65.9 & Port Arthur Canal & Perennial & Low & 3 & $\begin{array}{l}\text { No Cultural } \\
\text { Resources }\end{array}$ & $\begin{array}{l}\text { No further work } \\
\text { recommended }\end{array}$ & $\begin{array}{l}\text { Completely avoided via non } \\
\text { Section } 10 \mathrm{HDD} / \text { Bore }\end{array}$ \\
\hline SP2JE033 & 65.9 & $\begin{array}{c}\text { UT to Port Arthur } \\
\text { Canal }\end{array}$ & Ephemeral & Low & 3 & $\begin{array}{l}\text { No Cultural } \\
\text { Resources }\end{array}$ & $\begin{array}{l}\text { No further work } \\
\text { recommended }\end{array}$ & $\begin{array}{l}\text { Completely avoided via non } \\
\text { Section } 10 \mathrm{HDD} / \text { Bore }\end{array}$ \\
\hline SP6JE008 & 65.9 & $\begin{array}{l}\text { UT to Port Arthur } \\
\text { Canal }\end{array}$ & Ephemeral & Low & 3 & $\begin{array}{l}\text { No Cultural } \\
\text { Resources }\end{array}$ & $\begin{array}{l}\text { No further work } \\
\text { recommended }\end{array}$ & $\begin{array}{l}\text { Completely avoided via non } \\
\text { Section } 10 \mathrm{HDD} / \text { Bore }\end{array}$ \\
\hline SP2JE036 & 66.3 & $\begin{array}{l}\text { UT to Port Arthur } \\
\text { Canal }\end{array}$ & Ephemeral & Low & None & $\begin{array}{l}\text { No Cultural } \\
\text { Resources }\end{array}$ & $\begin{array}{l}\text { No further work } \\
\text { recommended }\end{array}$ & $\begin{array}{l}\text { Completely avoided via non } \\
\text { Section } 10 \mathrm{HDD} / \text { Bore }\end{array}$ \\
\hline SP2JE035 & 66.3 & $\begin{array}{c}\text { UT to Port Arthur } \\
\text { Canal }\end{array}$ & Ephemeral & Low & None & $\begin{array}{l}\text { No Cultural } \\
\text { Resources }\end{array}$ & $\begin{array}{l}\text { No further work } \\
\text { recommended }\end{array}$ & Inundation \\
\hline OWP9JE002_DT & 66.6 & N/A & Manmade Pond & Low & None & $\begin{array}{l}\text { No Cultural } \\
\text { Resources }\end{array}$ & $\begin{array}{l}\text { No further work } \\
\text { recommended }\end{array}$ & Inundation \\
\hline OWP9JE001_DT & 66.9 & N/A & Manmade Pond & Low & None & $\begin{array}{l}\text { No Cultural } \\
\text { Resources }\end{array}$ & $\begin{array}{l}\text { No further work } \\
\text { recommended }\end{array}$ & $\begin{array}{l}\text { Completely avoided via non } \\
\text { Section } 10 \mathrm{HDD} / \text { Bore }\end{array}$ \\
\hline OWP9JE003_DT & 67.4 & N/A & Manmade Pond & Low & None & $\begin{array}{l}\text { No Cultural } \\
\text { Resources }\end{array}$ & $\begin{array}{l}\text { No further work } \\
\text { recommended }\end{array}$ & Inundation \\
\hline OWP4JE012 & 67.7 & N/A & Manmade Pond & Low & 22 & $\begin{array}{l}\text { No Cultural } \\
\text { Resources }\end{array}$ & $\begin{array}{l}\text { No further work } \\
\text { recommended }\end{array}$ & Inundation \\
\hline SP4JE072 & 67.9 & UT to Smith Bluff & Perennial & Low & 9 & $\begin{array}{l}\text { No Cultural } \\
\text { Resources }\end{array}$ & $\begin{array}{l}\text { No further work } \\
\text { recommended }\end{array}$ & $\begin{array}{l}\text { Completely avoided via non } \\
\text { Section } 10 \text { HDD/Bore }\end{array}$ \\
\hline SP4JE073 & 67.9 & UT to Smith Bluff & Perennial & Low & 9 & $\begin{array}{l}\text { No Cultural } \\
\text { Resources }\end{array}$ & $\begin{array}{l}\text { No further work } \\
\text { recommended }\end{array}$ & Inundation \\
\hline SP4JE071 & 68.1 & UT to Smith Bluff & Intermittent & Low & 5 & $\begin{array}{l}\text { No Cultural } \\
\text { Resources }\end{array}$ & $\begin{array}{l}\text { No further work } \\
\text { recommended }\end{array}$ & Inundation \\
\hline OWP4JE011 & 68.3 & N/A & Manmade Pond & Low & 3 & $\begin{array}{l}\text { No Cultural } \\
\text { Resources }\end{array}$ & $\begin{array}{l}\text { No further work } \\
\text { recommended }\end{array}$ & Inundation \\
\hline
\end{tabular}


APPENDIX D: Shovel TeSt DATA For USACE PERMit AReaS 


\begin{tabular}{|c|c|c|c|c|c|c|c|c|}
\hline Shovel Test \# & $\begin{array}{l}\text { Level } \\
\text { (Strat) }\end{array}$ & $\begin{array}{l}\text { Depth } \\
\text { (cmbs) }\end{array}$ & GSV \% & $\begin{array}{l}\begin{array}{l}\text { Positive or } \\
\text { Negative }\end{array} \\
\end{array}$ & $\begin{array}{c}\text { Munsell } \\
\text { Color }\end{array}$ & Soil Texture Description & Description (Area; Vegetation) & Reason for Termination \\
\hline 0815HS01 & 1 & $0-20$ & 10 & Negative & 10YR $4 / 3$ & Loam & In grassy field; beside Stream; redoxed; very compact soil; no A horizon; hard to firm soil & Subsoil \\
\hline $0815 \mathrm{HSO3}$ & 1 & $0-15$ & 10 & Negative & 10YR $3 / 1$ & Loam & In grassy field; cow pasture; friable soil & Soil change \\
\hline $0815 \mathrm{HSO3}$ & 2 & $15-30$ & 10 & Negative & 10YR $3 / 1$ & Clay loam & In grassy field; cow pasture; firm soil & Subsoil \\
\hline 0815HS06 & 1 & $0-15$ & 10 & Negative & $10 Y R 3 / 1$ & Loam & In grassy field; cow pasture; friable soil & Soil change \\
\hline 0815HS06 & 2 & $15-30$ & 10 & Negative & $10 \mathrm{YR} 3 / 1$ & Clay loam & In grassy field; cow pasture; friable soil & Subsoil \\
\hline $0815 \mathrm{HS} 08$ & 1 & $0-15$ & 10 & Negative & 10YR $3 / 1$ & Loam & In grassy field; cow pasture; friable soil & Soil change \\
\hline 0815HS08 & 2 & $15-30$ & 10 & Negative & 10YR $3 / 1$ & Clay loam & In grassy field; cow pasture; firm soil & Subsoil \\
\hline $0815 \mathrm{HS} 10$ & 1 & $0-15$ & 10 & Negative & 10YR 3/1 & Loam & In grassy field; cow pasture; friable soil & Soil change \\
\hline 0815HS10 & 2 & $15-30$ & 10 & Negative & 10YR 3/1 & Clay loam & In grassy field; cow pasture; firm soil & Subsoil \\
\hline 0815HS11 & 1 & $0-15$ & 10 & Negative & 10YR $4 / 1$ & Clay & In grassy field with high grass; wetland feature; previously used as a sugarcane field & Soil change \\
\hline 0815HS11 & 2 & $15-30$ & 10 & Negative & 10YR $3 / 1$ & Clay & In grassy field with high grass; wetland feature; previously used as a sugarcane field & Subsoil \\
\hline $0815 \mathrm{HS} 13$ & 1 & $0-15$ & 10 & Negative & 10YR $4 / 1$ & Clay & In grassy field with high grass; wetland feature; previously used as a sugarcane field & Soil change \\
\hline $0815 \mathrm{HS} 13$ & 2 & $15-30$ & 10 & Negative & 10YR 3/1 & Clay & In grassy field with high grass; wetland feature; previously used as a sugarcane field & Subsoil \\
\hline 0815HS14 & 1 & $0-15$ & 10 & Negative & 10YR 4/1 & Clay & In grassy field with high grass; wetland feature; previously used as a sugarcane field & Soil change \\
\hline 0815HS14 & 2 & $15-30$ & 10 & Negative & 10YR $3 / 1$ & Clay & In grassy field with high grass; wetland feature; previously used as a sugarcane field & Subsoil \\
\hline 0815HS15 & 1 & $0-15$ & 10 & Negative & 10YR 4/1 & Clay & In grassy field with high grass; wetland feature; previously used as a sugarcane field & Soil change \\
\hline $0815 \mathrm{HS} 15$ & 2 & $15-30$ & 10 & Negative & 10YR $3 / 1$ & Clay & In grassy field with high grass; wetland feature; previously used as a sugarcane field & Subsoil \\
\hline 0815HS18 & 1 & $0-15$ & 10 & Negative & 10YR 4/1 & Clay & In grassy field with high grass; wetland feature; previously used as a sugarcane field & Soil change \\
\hline 0815HS18 & 2 & $15-30$ & 10 & Negative & 10YR 3/1 & Clay & In grassy field with high grass; wetland feature; previously used as a sugarcane field & Subsoil \\
\hline 0815HS27 & 1 & $0-15$ & 10 & Negative & 10YR 4/1 & Clay & In grassy area beside wetland and gravel road & Soil change \\
\hline 0815HS27 & 2 & $15-30$ & 10 & Negative & 10YR $3 / 1$ & Clay & In grassy area beside wetland and gravel road & Subsoil \\
\hline 081518HS73 & 1 & $0-20$ & 10 & Negative & 10YR $4 / 1$ & Clay & In grassy field; cow pasture; friable soil & Soil change \\
\hline 081518HS73 & 2 & $20-50$ & 10 & Negative & 10YR $3 / 1$ & Clay & In grassy field; cow pasture; friable soil & Subsoil \\
\hline 081518HS71 & 1 & $0-20$ & 10 & Negative & 10YR $4 / 1$ & Clay & In grassy field; cow pasture; friable soil & Soil change \\
\hline 081518HS71 & 2 & $20-30$ & 10 & Negative & 10YR $3 / 1$ & Clay & In grassy field; cow pasture; friable soil & Subsoil \\
\hline 081518HS69 & 1 & $0-15$ & 10 & Negative & 10YR $4 / 1$ & Clay & In grassy field; cow pasture; friable soil & Soil change \\
\hline 081518HS69 & 2 & $15-20$ & 10 & Negative & 10YR $3 / 1$ & Clay & In grassy field; cow pasture; friable soil & Subsoil \\
\hline 081518HS67 & 1 & $0-20$ & 10 & Negative & 10YR $4 / 1$ & Clay & In grassy field; cow pasture; friable soil & Soil change \\
\hline 081518HS67 & 2 & $20-25$ & 10 & Negative & 10YR $3 / 1$ & Clay & In grassy field; cow pasture; friable soil & Subsoil \\
\hline 081518HS66 & 1 & $0-20$ & 10 & Negative & 10YR $4 / 1$ & Clay & In grassy field; cow pasture; friable soil & Soil change \\
\hline 081518HS66 & 2 & $20-50$ & 10 & Negative & 10YR $3 / 1$ & Clay & In grassy field; cow pasture; friable soil & Subsoil \\
\hline 081518HS63 & 1 & $0-20$ & 10 & Negative & 10YR $4 / 1$ & Clay & In grassy field; cow pasture; friable soil & Soil change \\
\hline 081518HS63 & 2 & $20-30$ & 10 & Negative & 10YR $3 / 1$ & Clay & In grassy field; cow pasture; friable soil & Subsoil \\
\hline 081518HS61 & 1 & $0-15$ & 10 & Negative & 10YR $4 / 1$ & Clay & In grassy field; cow pasture; friable soil & Soil change \\
\hline 081518HS61 & 2 & $15-20$ & 10 & Negative & 10YR $3 / 1$ & Clay & In grassy field; cow pasture; friable soil & Subsoil \\
\hline 081518HS58 & 1 & $0-20$ & 10 & Negative & 10YR $4 / 1$ & Clay & In grassy field; cow pasture; friable soil & Soil change \\
\hline 081518HS58 & 2 & $20-25$ & 10 & Negative & 10YR $3 / 1$ & Clay & In grassy field; cow pasture; friable soil & Subsoil \\
\hline 081518HS53 & 1 & $0-15$ & 10 & Negative & 10YR $4 / 1$ & Clay & In grassy field; cow pasture; friable soil & Soil change \\
\hline
\end{tabular}




\begin{tabular}{|c|c|c|c|c|c|c|c|c|}
\hline Shovel Test \# & $\begin{array}{l}\begin{array}{l}\text { Level } \\
\text { (Strat) }\end{array} \\
\end{array}$ & $\begin{array}{l}\text { Depth } \\
\text { (cmbs) }\end{array}$ & GSV \% & $\begin{array}{c}\begin{array}{c}\text { Positive or } \\
\text { Negative }\end{array} \\
\end{array}$ & $\begin{array}{c}\begin{array}{c}\text { Munsell } \\
\text { Color }\end{array} \\
\end{array}$ & Soil Texture Description & Description (Area; Vegetation) & Reason for Termination \\
\hline 081518HS53 & 2 & $15-30$ & 10 & Negative & 10YR $3 / 1$ & Clay & In grassy field; cow pasture; friable soil & Subsoil \\
\hline 081518HS51 & 1 & $0-15$ & 10 & Negative & 10YR $4 / 1$ & Clay & In grassy field; cow pasture; friable soil & Soil change \\
\hline 081518HS51 & 2 & $15-30$ & 10 & Negative & 10YR $3 / 1$ & Clay & In grassy field; cow pasture; friable soil & Subsoil \\
\hline 081518HS49 & 1 & $0-15$ & 10 & Negative & 10YR $4 / 1$ & Clay & In grassy field; cow pasture; friable soil & Soil change \\
\hline 081518HS49 & 2 & $15-30$ & 10 & Negative & 10YR $3 / 1$ & Clay & In grassy field; cow pasture; friable soil & Subsoil \\
\hline 081518HS48 & 1 & $0-15$ & 10 & Negative & 10YR $4 / 1$ & Clay & In grassy field; cow pasture; friable soil & Soil change \\
\hline 081518HS48 & 2 & $0-20$ & 10 & Negative & 10YR $3 / 1$ & Clay & In grassy field; cow pasture; friable soil & Subsoil \\
\hline 081518HS46 & 1 & $20-25$ & 10 & Negative & 10YR $4 / 1$ & Clay & In grassy field; cow pasture; friable soil & Soil change \\
\hline 081518HS46 & 2 & $0-20$ & 10 & Negative & 10YR $3 / 1$ & Clay & In grassy field; cow pasture; friable soil & Subsoil \\
\hline 081518HS44 & 1 & $20-50$ & 10 & Negative & 10YR $4 / 1$ & Clay & In grassy field; cow pasture; friable soil & Soil change \\
\hline 081518HS44 & 2 & $0-20$ & 10 & Negative & 10YR $3 / 1$ & Clay & In grassy field; cow pasture; friable soil & Subsoil \\
\hline 081518HS41 & 1 & $0-20$ & 10 & Negative & 10YR $4 / 1$ & Clay & In grassy field; cow pasture; friable soil & Soil change \\
\hline 081518HS41 & 2 & $20-25$ & 10 & Negative & 10YR $3 / 1$ & Clay & In grassy field; cow pasture; friable soil & Subsoil \\
\hline 081518HS40 & 1 & $0-20$ & 10 & Negative & 10YR $4 / 1$ & Clay & In grassy field; cow pasture; friable soil & Soil change \\
\hline 081518HS40 & 2 & $20-50$ & 10 & Negative & 10YR $3 / 1$ & Clay & In grassy field; cow pasture; friable soil & Subsoil \\
\hline 081518HS39 & 1 & $0-20$ & 10 & Negative & 10YR $4 / 1$ & Clay & In grassy field; cow pasture; friable soil & Soil change \\
\hline 081518HS39 & 2 & $20-30$ & 10 & Negative & 10YR $3 / 1$ & Clay & In grassy field; cow pasture; friable soil & Subsoil \\
\hline 081518HS36 & 1 & $0-15$ & 10 & Negative & 10YR $4 / 1$ & Clay & In grassy field; cow pasture; friable soil & Soil change \\
\hline 081518HS36 & 2 & $15-20$ & 10 & Negative & 10YR $3 / 1$ & Clay & In grassy field; cow pasture; friable soil & Subsoil \\
\hline 081518HS34 & 1 & $0-20$ & 10 & Negative & 10YR $4 / 1$ & Clay & In grassy field; cow pasture; friable soil & Soil change \\
\hline 081518HS34 & 2 & $20-25$ & 10 & Negative & 10YR $3 / 1$ & Clay & In grassy field; cow pasture; friable soil & Subsoil \\
\hline 081518HS32 & 1 & $0-15$ & 10 & Negative & 10YR $4 / 1$ & Clay & In grassy field; cow pasture; friable soil & Soil change \\
\hline 081518HS32 & 2 & $15-30$ & 10 & Negative & 10YR $3 / 1$ & Clay & In grassy field; cow pasture; friable soil & Subsoil \\
\hline 081518HS30 & 1 & $0-15$ & 10 & Negative & 10YR $4 / 1$ & Clay & In grassy field; cow pasture; friable soil & Soil change \\
\hline 081518HS30 & 2 & $15-30$ & 10 & Negative & 10YR $3 / 1$ & Clay & In grassy field; cow pasture; friable soil & Subsoil \\
\hline 081518HS29 & 1 & $0-15$ & 10 & Negative & 10YR $4 / 1$ & Clay & In grassy field; cow pasture; friable soil & Soil change \\
\hline 081518HS29 & 2 & $15-30$ & 10 & Negative & 10YR $3 / 1$ & Clay & In grassy field; cow pasture; friable soil & Subsoil \\
\hline $1002 J L 02$ & 1 & $0-20$ & 10 & Negative & 10YR 4/1 & Silty clay & Knee high grass; mostly inundated & Soil change \\
\hline 1002JL02 & 2 & $20-30$ & 10 & Negative & 10YR $3 / 1$ & Clay loam & Knee high grass; mostly inundated & Subsoil \\
\hline $1002 J L 303$ & 1 & $0-15$ & 3 & Negative & Disturbed & Disturbed & Next to gate opening onto highway; semi-inundated & Disturbed \\
\hline
\end{tabular}




\begin{tabular}{|c|c|c|c|c|c|c|c|c|}
\hline Shovel Test \# & $\begin{array}{l}\text { Level } \\
\text { (Strat) }\end{array}$ & $\begin{array}{l}\text { Depth } \\
\text { (cmbs) }\end{array}$ & GSV \% & $\begin{array}{l}\text { Positive or } \\
\text { Negative }\end{array}$ & $\begin{array}{l}\text { Munsell } \\
\text { Color }\end{array}$ & Soil Texture Description & Description (Area; Vegetation) & Reason for Termination \\
\hline 1002JL305 & 1 & $0-8$ & 10 & Negative & 10YR $4 / 1$ & Silty clay & In cow pasture; mostly inundated & Inundation \\
\hline 1002JL307 & 1 & $0-20$ & 10 & Negative & 10YR $4 / 1$ & Silt loam & In cow pasture; mostly inundated & Soil change \\
\hline $1002 J L 307$ & 2 & $20-50$ & 10 & Negative & 10YR $3 / 1$ & Silty clay & In cow pasture; mostly inundated & Subsoil \\
\hline 1002JL309 & 1 & $0-20$ & 100 & Negative & 10YR 4/1 & Clay & Next to soybean field and canal; surface gravel & Soil change \\
\hline 1002JL309 & 2 & $20-50$ & 100 & Negative & 10YR $3 / 1$ & Clay & Next to soybean field and canal; surface gravel & Subsoil \\
\hline $1002 J L 310$ & 1 & $0-20$ & 100 & Negative & 10YR $4 / 1$ & Clay & Next to soybean field; iron deposits at $20 \mathrm{~cm}$ & Soil change \\
\hline $1002 J L 310$ & 2 & $20-50$ & 100 & Negative & 10YR $3 / 1$ & Clay & Next to soybean field; $20 \%$ iron deposits & Subsoil \\
\hline $1002 J L 312$ & 1 & $0-20$ & 100 & Negative & 10YR $4 / 1$ & Clay & Next to soybean field; $20 \%$ iron deposits & Soil change \\
\hline $1002 J \mathrm{~J} 312$ & 2 & $20-50$ & 100 & Negative & 10YR $3 / 1$ & Clay & Next to soybean field; $20 \%$ iron deposits & Subsoil \\
\hline $1002 J L 314$ & 1 & $0-20$ & 100 & Negative & 10YR $4 / 1$ & Clay & In between two soybean rows; $10 \%$ iron deposits & Soil change \\
\hline $1002 J L 314$ & 2 & $20-30$ & 100 & Negative & 10YR $3 / 1$ & Clay & In between two soybean rows; $10 \%$ iron deposits & Subsoil \\
\hline 1002WE304 & 1 & $0-15$ & $2-5$ & Negative & 10YR $3 / 2$ & Clay loam & Open cow pasture; short grass; trees around fence line; scattered inundations; friable soil & Soil change \\
\hline 1002WE304 & 2 & $15-20$ & $2-5$ & Negative & 10YR $3 / 4$ & Clay loam & Open cow pasture; short grass; trees around fence line; scattered inundations; friable soil & Subsoil \\
\hline 1002WE308 & 1 & $0-20$ & 0 & Negative & $10 Y R 5 / 2$ & Clay & Soy bean field; muddy; adjacent to fence line and ditch & Soil change \\
\hline 1002WE308 & 2 & $20-25$ & 0 & Negative & 10YR $5 / 2$ & Clay loam & Soy bean field; muddy; adjacent to fence line and ditch & Subsoil \\
\hline 1002WE315 & 1 & 0 & 0 & Negative & 0 & 0 & Inundation & Inundation \\
\hline 1002RK04 & 1 & $0-25$ & 0 & Negative & 10YR $3 / 1$ & Silty clay & In tall grass on high ground; soil is very firm with plants; hit water at $25 \mathrm{cmbs}$ & Soil change \\
\hline 1002RK04 & 2 & $25-35$ & 0 & Negative & 10YR $4 / 1$ & Silty clay & In tall grass on high ground; soil is very firm with plants; hit water at $25 \mathrm{cmbs}$ & Subsoil \\
\hline 1002RK301 & 1 & $0-25$ & 0 & Negative & 10YR $3 / 1$ & Silty clay & In short grass & Soil change \\
\hline 1002RK301 & 2 & $25-35$ & 0 & Negative & 10YR $4 / 1$ & Silty clay & In short grass & Subsoil \\
\hline 1002RK302 & 1 & $0-20$ & 0 & Negative & $\begin{array}{c}10 Y R \\
3 / 1 ; 6 / 1 \\
\end{array}$ & Silty clay & In high grass on high ground & Soil change \\
\hline 1002RK302 & 2 & $20-30$ & 0 & Negative & 10YR $4 / 1$ & Silty clay & In high grass on high ground & Subsoil \\
\hline 1002RK07 & 1 & $0-25$ & 0 & Negative & 10YR $3 / 2$ & Silty loam & In short grass & Soil change \\
\hline 1002RK07 & 2 & $25-35$ & 0 & Negative & 10YR $5 / 4$ & Silty clay & In short grass & Subsoil \\
\hline 1002RK09 & 1 & $0-25$ & 0 & Negative & 10YR $3 / 2$ & Silty loam & In short grass & Soil change \\
\hline 1002RK09 & 2 & $25-35$ & 0 & Negative & 10YR $5 / 4$ & Silty clay & In short grass & Subsoil \\
\hline 1002RK11 & 1 & $0-30$ & 40 & Negative & $\begin{array}{c}10 Y R \\
4 / 1 ; 3 / 1 \\
\end{array}$ & Clay & Disturbed by soy bean field & Soil change \\
\hline 1002RK11 & 2 & $0-30$ & 40 & Negative & $\begin{array}{c}10 Y R \\
4 / 1 ; 3 / 1\end{array}$ & Clay & Disturbed by soy bean field & Subsoil \\
\hline 1002RK13 & 1 & $0-30$ & 40 & Negative & $\begin{array}{c}10 Y R \\
4 / 1 ; 3 / 1 \\
\end{array}$ & Clay & Disturbed by soy bean field & Soil change \\
\hline 1002RK13 & 2 & $0-30$ & 40 & Negative & $\begin{array}{c}10 Y R \\
4 / 1 ; 3 / 1 \\
\end{array}$ & Clay & Disturbed by soy bean field & Subsoil \\
\hline 1002RK15 & 1 & $0-30$ & 40 & Negative & $\begin{array}{c}10 Y R \\
4 / 1 ; 3 / 1 \\
\end{array}$ & Clay & Disturbed by soy bean field & Soil change \\
\hline 1002RK15 & 2 & $0-30$ & 40 & Negative & $\begin{array}{c}10 Y R \\
4 / 1 ; 3 / 1 \\
\end{array}$ & Clay & Disturbed by soy bean field & Subsoil \\
\hline $1002 \mathrm{HS} 300$ & 1 & $0-20$ & 10 & Negative & 10YR $3 / 1$ & Silty clay & Open grassy field; in row corridor & Soil change \\
\hline $1002 \mathrm{HS} 300$ & 2 & $20-30$ & 10 & Negative & 10YR $3 / 1$ & Silty clay loam & Open grassy field; in row corridor; massive soil & Subsoil \\
\hline 100218RC313 & 1 & $0-25$ & 10 & Negative & 10YR $4 / 1$ & Sandy clay loam & Grassy pasture; very inundated; some disturbance evident in row corridor & Soil change \\
\hline $1002 \mathrm{HSO6}$ & 1 & $0-25$ & 10 & Negative & 10YR $4 / 1$ & Sandy clay loam & Grassy pasture; very inundated; some disturbance evident in row corridor & Soil change \\
\hline $1002 \mathrm{HSO6}$ & 2 & $25-40$ & 10 & Negative & 10YR $3 / 1$ & Clay loam & Grassy pasture; very inundated; some disturbance evident in row corridor & Subsoil \\
\hline $1002 \mathrm{HS} 08$ & 1 & $0-15$ & 90 & Negative & 10YR $4 / 1$ & Clay & In soy bean field; very inundated; mixed A and B & Soil change \\
\hline $1002 \mathrm{HS} 08$ & 2 & $15-25$ & 90 & Negative & 10YR $3 / 1$ & Clay & In soy bean field; very inundated; mixed A and $B$ & Subsoil \\
\hline $1002 \mathrm{HS} 10$ & 1 & $0-15$ & 90 & Negative & 10YR $4 / 1$ & Clay & In soy bean field; very inundated; mixed A and $B$ & Soil change \\
\hline $1002 \mathrm{HS} 10$ & 2 & $15-25$ & 90 & Negative & 10YR $3 / 1$ & Clay & In soy bean field; very inundated; mixed A and $B$ & Subsoil \\
\hline $1002 \mathrm{HS} 12$ & 1 & $0-10$ & 90 & Negative & 10YR 4/1 & Clay & In soy bean field; very inundated; mixed $A$ and $B$ & Inundation \\
\hline $1002 \mathrm{HS} 14$ & 1 & $0-10$ & 90 & Negative & 10YR $3 / 1$ & Clay & In soy bean field; very inundated; mixed $A$ and $B$ & Inundation \\
\hline 1002RC05 & 1 & $0-10$ & 0 & Negative & 10YR $3 / 1$ & Clay & Tall grass; wetland; trees along property line; cow pasture & Subsoil \\
\hline $1002 \mathrm{RC} 05$ & 2 & $10-15$ & 0 & Negative & $10 Y R 3 / 2$ & Clay loam & Tall grass; wetland; trees along property line; cow pasture & Subsoil \\
\hline 1002RC305 & 1 & $0-30$ & 0 & Negative & $10 Y R 7 / 4$ & Loam & Open cow pasture; trees along fence line; short grass & Subsoil \\
\hline 1002RC305 & 2 & $30-60$ & 0 & Negative & $10 Y R 7 / 2$ & Silty loam & Open cow pasture; trees along fence line; short grass & Subsoil \\
\hline
\end{tabular}




\begin{tabular}{|c|c|c|c|c|c|c|c|c|}
\hline Shovel Test \# & $\begin{array}{l}\text { Level } \\
\text { (Strat) }\end{array}$ & $\begin{array}{l}\text { Depth } \\
\text { (cmbs) }\end{array}$ & GSV \% & $\begin{array}{l}\text { Positive or } \\
\text { Negative }\end{array}$ & $\begin{array}{l}\text { Munsell } \\
\text { Color }\end{array}$ & Soil Texture Description & Description (Area; Vegetation) & Reason for Termination \\
\hline 1002RC311 & 1 & $0-10$ & 0 & Negative & 10YR $5 / 2$ & Clay loam & Soybean field; mature growth; trees along fence line & Subsoil \\
\hline 1002RC311 & 2 & $10-20$ & 0 & Negative & 10YR 5/1 & Clay & Soybean field; mature growth; trees along fence line & Subsoil \\
\hline 1003RK200 & 1 & $0-20$ & 5 & Negative & 10YR $3 / 1$ & Clay & In cleared corridor; mostly grasses; moist; upland & Soil change \\
\hline 1003RK200 & 2 & $20-30$ & 5 & Negative & 10YR $3 / 1$ & Clay & In cleared corridor; mostly grasses; moist; upland; In transmission+utility row & Impenetrable \\
\hline 1003RK201 & 1 & $0-20$ & 5 & Negative & $10 Y R 3 / 1$ & Clay & In cleared corridor; mostly grasses; moist; upland pea gravel inclusions & Disturbed \\
\hline 1003RK202 & 1 & $0-30$ & 5 & Negative & $10 Y R 3 / 1$ & Clay & In cleared corridor; mostly grasses; moist; upland & Impenetrable \\
\hline 1003RK203 & 1 & $0-25$ & 5 & Negative & $10 Y R 3 / 1$ & Clay & In cleared corridor; mostly grasses; moist; upland; mixed A and B horizons & Disturbed \\
\hline $1003 \mathrm{HS} 205$ & 1 & $0-30$ & 5 & Negative & $10 \mathrm{YR} 3 / 1$ & Silty clay loam & In cleared corridor; mostly grasses; moist; upland; In transmission+utility row & Soil change \\
\hline $1003 \mathrm{HS} 205$ & 2 & $30-40$ & 5 & Negative & $10 \mathrm{YR} 3 / 1$ & Clay & In cleared corridor; mostly grasses; moist; upland; In transmission+utility row & Subsoil \\
\hline 1003 HS206 & 1 & $0-20$ & 5 & Negative & 10YR $3 / 1$ & Clay & In cleared corridor; mostly grasses; moist; upland; pea gravel inclusions & Disturbed \\
\hline $1003 \mathrm{HS} 207$ & 1 & $0-20$ & 5 & Negative & 10YR $3 / 1$ & Silty clay & In cleared corridor; mostly grasses; moist; upland; pea gravel inclusions & Disturbed \\
\hline 1003RK208 & 1 & $0-20$ & 5 & Negative & 10YR 3/1 & Silty clay & In cleared corridor; mostly grasses; moist; upland; mixed A and B horizons & Disturbed \\
\hline 1003RK209 & 1 & $0-30$ & 5 & Negative & 10YR 4/2 & Silty clay loam & In cleared corridor; mostly grasses; moist; upland; mixed A and B horizons & Disturbed \\
\hline 1003 HS210 & 1 & $0-20$ & 5 & Negative & $10 \mathrm{YR} 4 / 2$ & Silty clay loam & In cleared corridor; mostly grasses; moist; upland; mixed A and B horizons & Disturbed \\
\hline $1003 \mathrm{HS} 211$ & 1 & $0-30$ & 5 & Negative & 10YR $4 / 2$ & Silty clay loam & $\begin{array}{l}\text { In cleared corridor; mostly grasses; moist; upland; In transmission+utility row; Pieces of plastic at } \\
\qquad 30 \mathrm{cmbs} \text {; sand }\end{array}$ & Disturbed \\
\hline 1003RK212 & 1 & $0-20$ & 5 & Negative & 1OYR $4 / 2$ & Silty clay loam & In cleared corridor; mostly grasses; moist; upland; sand & Disturbed \\
\hline $1003 \mathrm{HS} 213$ & 1 & $0-20$ & 5 & Negative & $10 \mathrm{Yr} 4 / 2$ & Silty clay loam & In cleared corridor; mostly grasses; moist; upland; sand & Disturbed \\
\hline $1003 \mathrm{HS} 214$ & 1 & $0-20$ & 5 & Negative & $10 \mathrm{YR} 4 / 2$ & Silty clay loam & In cleared corridor; mostly grasses; moist; upland; sand & Disturbed \\
\hline 1003 HS215 & 1 & $0-60$ & 5 & Negative & 1OYR $4 / 2$ & Sandy loam & In cleared corridor; mostly grasses; moist; upland; very deep disturbed sandy deposit & Disturbed \\
\hline $1003 \mathrm{HS} 216$ & 1 & $0-60$ & 5 & Negative & $10 \mathrm{YR} 4 / 2$ & Sandy loam & In cleared corridor; mostly grasses; moist; upland & Disturbed \\
\hline 1003 HS217 & 1 & $0-60$ & 10 & Negative & $10 Y R 4 / 2$ & Sandy loam & Very deep sandy deposit & Disturbed \\
\hline 1003 HS218 & 1 & $0-30$ & 5 & Negative & $10 Y R 4 / 2$ & Sandy loam & Very deep sandy deposit & Disturbed \\
\hline 1003 HS219 & 1 & $0-30$ & 5 & Negative & $10 Y R 4 / 2$ & Sandy loam & Very deep sandy deposit & Disturbed \\
\hline 1003 HS220 & 1 & $0-30$ & 5 & Negative & $10 \mathrm{YYR} 4 / 2$ & Sandy loam & Very deep sandy deposit & Disturbed \\
\hline 1003RK221 & 1 & $0-30$ & 5 & Negative & $10 \mathrm{YR} 4 / 2$ & Sandy loam & Very deep sandy deposit & Disturbed \\
\hline 1003RK222 & 1 & $0-30$ & 5 & Negative & 10YR $4 / 2$ & Sandy loam & Very deep sandy deposit & Disturbed \\
\hline $1003 \mathrm{HS} 223$ & 1 & $0-30$ & 5 & Negative & $10 \mathrm{YR} 4 / 2$ & Sandy loam & Very deep sandy deposit & Disturbed \\
\hline 1003RK224 & 1 & $0-30$ & 5 & Negative & 10YR $4 / 2$ & Sandy loam & Very deep sandy deposit & Disturbed \\
\hline 1003RK225 & 1 & $0-30$ & 5 & Negative & 10YR $4 / 2$ & Sandy loam & Very deep sandy deposit & Disturbed \\
\hline 1003 HS226 & 1 & $0-30$ & 5 & Negative & $10 \mathrm{YYR} 4 / 2$ & Sandy loam & Very deep sandy deposit & Disturbed \\
\hline 1003RK227 & 1 & $0-30$ & 5 & Negative & $10 Y R 4 / 2$ & Sandy loam & Very deep sandy deposit & Disturbed \\
\hline 1003 HS228 & 1 & $0-30$ & 5 & Negative & $10 \mathrm{YR} 4 / 2$ & Sandy loam & Very deep sandy deposit & Disturbed \\
\hline 1003WE02 & 1 & $0-20$ & 0 & Negative & $10 Y R 5 / 2$ & Clay loam & Wetland; tall grass; within utility corridor; trees lining corridor & Soil change \\
\hline 1003WE02 & 2 & $20-25$ & 0 & Negative & $10 Y R 5 / 2$ & Clay loam & Wetland; tall grass; within utility corridor; trees lining corridor & Subsoil \\
\hline 1003WE05 & 1 & $0-10$ & 0 & Negative & 10YR $5 / 2$ & Clay loam & Wetland; tall grass; within utility corridor; trees lining corridor & Impenetrable \\
\hline 1003WE05 & 2 & $10-40$ & 0 & Negative & 10YR $5 / 2$ & Clay loam & Wetland; tall grass; within utility corridor; trees lining corridor & Impenetrable \\
\hline 1003WE08 & 1 & $0-10$ & 0 & Negative & $10 \mathrm{YYR} 3 / 1$ & Loam & Wetland; tall grass and brush; south of corridor & Impenetrable \\
\hline 1003WE08 & 2 & $10-15$ & 0 & Negative & $10 Y R 3 / 1$ & Loam & Wetland; tall grass and brush; south of corridor & Impenetrable \\
\hline 1003WE11 & 1 & $0-15$ & 0 & Negative & 10YR 3/1 & Loam & Wetland; tall grass & Subsoil \\
\hline 1003WE11 & 2 & $15-20$ & 0 & Negative & 10YR $3 / 1$ & Loam & Wetland; tall grass & Subsoil \\
\hline 1003WE17 & 1 & $0-10$ & 10 & Negative & $10 \mathrm{YR} 3 / 1$ & Loam & Aspen forest; many roots; muddy & Subsoil \\
\hline 1003WE17 & 2 & $10-15$ & 10 & Negative & 10YR $3 / 1$ & Loam & Aspen forest; many roots; muddy & Subsoil \\
\hline 1003WE22 & 1 & $0-2$ & 0 & Negative & 10YR $3 / 1$ & Loam & Wetland; edge of trees; tall brush and grass & Subsoil \\
\hline 1003WE22 & 2 & $2-5$ & 0 & Negative & 10YR $3 / 1$ & Loam & Wetland; edge of trees; tall brush and grass & Subsoil \\
\hline 1003RC03 & 1 & $0-50$ & 0 & Negative & 10YR $3 / 1$ & Clay & Wetland; tall grass; large inundated patches; briars; brush; thick forest cover heavy vegetation. & Impenetrable \\
\hline $1003 \mathrm{RCO3}$ & 2 & $50-60$ & 0 & Negative & 10YR $3 / 2$ & Clay loam & Wetland; tall grass; large inundated patches; briars; brush; thick forest cover heavy vegetation. & Impenetrable \\
\hline $1003 R C 06$ & 1 & $0-50$ & 0 & Negative & 10YR $3 / 1$ & Loamy clay & Wetland; tall grass; large inundated patches; briars; brush; thick forest cover heavy vegetation. & Impenetrable \\
\hline 1003RC09 & 1 & $0-50$ & 0 & Negative & 10YR $3 / 1$ & Loamy clay & Wetland; tall grass; large inundated patches; briars; brush; thick forest cover heavy vegetation. & Impenetrable \\
\hline 1003RC12 & 1 & $0-50$ & 0 & Negative & 10YR $3 / 1$ & Loamy clay & Wetland; tall grass; large inundated patches; briars; brush; thick forest cover heavy vegetation. & Impenetrable \\
\hline 1003RC15 & 1 & $0-50$ & 0 & Negative & 10YR $3 / 1$ & Loamy clay & Wetland; tall grass; large inundated patches; briars; brush; thick forest cover heavy vegetation. & Impenetrable \\
\hline
\end{tabular}




\begin{tabular}{|c|c|c|c|c|c|c|c|c|}
\hline Shovel Test \# & $\begin{array}{l}\begin{array}{l}\text { Level } \\
\text { (Strat) }\end{array} \\
\end{array}$ & $\begin{array}{l}\text { Depth } \\
\text { (cmbs) }\end{array}$ & GSV \% & $\begin{array}{c}\begin{array}{c}\text { Positive or } \\
\text { Negative }\end{array} \\
\end{array}$ & $\begin{array}{c}\text { Munsell } \\
\text { Color }\end{array}$ & Soil Texture Description & Description (Area; Vegetation) & Reason for Termination \\
\hline 1003 RC18 & 1 & $0-50$ & 0 & Negative & 10YR $3 / 1$ & Loamy clay & Wetland; tall grass; large inundated patches; briars; brush; thick forest cover heavy vegetation. & Impenetrable \\
\hline 1003RC20 & 1 & $0-50$ & 0 & Negative & 10YR $3 / 1$ & Loamy clay & Wetland; tall grass; large inundated patches; briars; brush; thick forest cover heavy vegetation. & Impenetrable \\
\hline 1003JL01 & 1 & $0-15$ & 0 & Negative & 10YR 3/1 & Clay & Off the two-track; dense brush/rose thorns; wetland & Soil change \\
\hline 1003JL01 & 2 & $15-20$ & 0 & Negative & $10 \mathrm{YR} 2 / 1$ & Clay & Off the two-track; dense brush/rose thorns; wetland & Soil change \\
\hline 1003JL04 & 1 & $0-10$ & 0 & Negative & 10YR 3/1 & Clay & Off the two-track; tall grass; wetland & Soil change \\
\hline 1003JL04 & 2 & $10-15$ & 0 & Negative & 10YR $2 / 1$ & Clay & Off the two-track; tall grass; wetland & Soil change \\
\hline 1003JL07 & 1 & $0-2$ & 0 & Negative & $10 \mathrm{Yr} 3 / 1$ & Clay & In dense brush; wetland & Soil change \\
\hline 1003JL07 & 2 & $2-5$ & 0 & Negative & 10YR $2 / 1$ & Clay & In dense brush; wetland & Soil change \\
\hline 1003JL10 & 1 & $0-15$ & 0 & Negative & $10 \mathrm{YR} 3 / 1$ & Clay & In dense brush; wetland & Soil change \\
\hline 1003JL10 & 2 & $15-20$ & 0 & Negative & 10YR $2 / 1$ & Clay & In dense brush; wetland & Soil change \\
\hline 1003JL13 & 1 & $0-10$ & 0 & Negative & 10YR 3/1 & Clay & Semi dense brush; wetland & Soil change \\
\hline 1003JL13 & 2 & $10-15$ & 0 & Negative & 10YR 2/1 & Clay & Semi dense brush; wetland & Soil change \\
\hline 1003JL19 & 1 & $0-2$ & 0 & Negative & 10YR $3 / 1$ & Clay & Semi dense brush; wetland & Soil change \\
\hline 1003JL19 & 2 & $2-5$ & 0 & Negative & $10 \mathrm{YrR} 2 / 1$ & Clay & Semi dense brush; wetland & Soil change \\
\hline 1003JL21 & 1 & $0-50$ & 0 & Negative & Disturbed & clay & Knee high grass; next to wooded area & Disturbed \\
\hline $1003 J$ L21 & 2 & $50-60$ & 0 & Negative & Disturbed & clay & Knee high grass; next to wooded area & Disturbed \\
\hline 1004RK01 & 1 & $0-30$ & 5 & Negative & 10YR 3/1 & Clay & Berm and soils disturbed & Disturbed \\
\hline $1004 J \mathrm{JL}$ & 1 & $0-10$ & 100 & Negative & $10 \mathrm{YR} 3 / 1$ & Clay & Berm and soils disturbed & Disturbed \\
\hline 1004JL04 & 1 & $0-10$ & 100 & Negative & 10YR 3/1 & Clay & Berm and soils disturbed & Disturbed \\
\hline 1004JL06 & 1 & $0-10$ & 100 & Negative & 10YR 3/1 & Clay & Berm and soils disturbed & Disturbed \\
\hline 1004JL08 & 1 & $0-10$ & 100 & Negative & 10YR 3/1 & Clay & Berm and soils disturbed & Disturbed \\
\hline 1004JL10 & 1 & $0-10$ & 100 & Negative & 10YR $3 / 1$ & Clay & Berm and soils disturbed & Disturbed \\
\hline 1004RK04 & 1 & $0-30$ & 5 & Negative & 10YR $3 / 1$ & Clay & Berm and soils disturbed & Disturbed \\
\hline 1004RK06 & 1 & $0-30$ & 5 & Negative & 10YR $3 / 1$ & Clay & Berm and soils disturbed & Disturbed \\
\hline 1004RK08 & 1 & $0-30$ & 5 & Negative & 10YR $3 / 1$ & Clay & Berm and soils disturbed & Disturbed \\
\hline 1004RK09 & 1 & $0-30$ & 5 & Negative & $10 \mathrm{YR} 3 / 1$ & Clay & Berm and soils disturbed & Disturbed \\
\hline 1004WE02 & 1 & $0-15$ & 0 & Negative & $10 \mathrm{YR} 3 / 2$ & Clay & Upland; tall grass; areas of inundation & Inundation \\
\hline 1004WE04 & 1 & $0-20$ & 0 & Negative & 10YR 4/3 & Silty clay & Upland; horse pasture; areas of inundation & Inundation \\
\hline 1004RC01 & 1 & $0-20$ & 0 & Negative & 10YR 3/1 & Clay loam & Open field; tall grass; partially inundated; trees along fence line & Inundation \\
\hline 1004RC03 & 1 & $0-20$ & 0 & Negative & $10 \mathrm{YR} 3 / 2$ & Clay loam & Open field; tall grass; partially inundated; trees along fence line & Inundation \\
\hline 1004RC05 & 1 & $0-30$ & 0 & Negative & $10 \mathrm{YR} 3 / 1$ & Clay loam & Open field; tall grass; partially inundated; trees along fence line & Inundation \\
\hline $1005 \mathrm{RC} 01$ & 1 & $0-30$ & 0 & Negative & $10 \mathrm{Yr} 3 / 1$ & Silty loam & Heavy vegetation; wetland; large inundated areas; forest cover; vines; brush & Impenetrable \\
\hline $1005 \mathrm{RC} 05$ & 1 & $0-30$ & 0 & Negative & $10 \mathrm{Yr} 3 / 1$ & Loam & Heavy vegetation; wetland; large inundated areas; forest cover; vines; brush & Impenetrable \\
\hline 1005RC08 & 1 & $0-40$ & 0 & Negative & 10YR $3 / 1$ & Loam & Heavy vegetation; wetland; large inundated areas; forest cover; vines; brush & Subsoil \\
\hline 1005 RC10 & 1 & $0-30$ & 0 & Negative & 10YR $3 / 1$ & Loam & Heavy vegetation; wetland; large inundated areas; forest cover; vines; brush & Subsoil \\
\hline 1005RC11 & 1 & $0-40$ & 0 & Negative & $10 \mathrm{YYR} 3 / 1$ & Loam & Heavy vegetation; wetland; large inundated areas; forest cover; vines; brush & Subsoil \\
\hline 1005RC13 & 1 & $0-40$ & 0 & Negative & 10YR $3 / 1$ & Loam & Heavy vegetation; wetland; large inundated areas; forest cover; vines; brush & Subsoil \\
\hline 1005RC15 & 1 & $0-30$ & 0 & Negative & $10 \mathrm{YYR} 3 / 1$ & Silty loam & Heavy vegetation; wetland; large inundated areas; forest cover; vines; brush & Subsoil \\
\hline 1005RC17 & 1 & $0-40$ & 0 & Negative & 1OYR $2 / 1$ & Silty loam & open field; wetland; forest cover & Subsoil \\
\hline 1005 RC19 & 1 & $0-30$ & 0 & Negative & $10 \mathrm{Yr} 3 / 1$ & Silty loam & open field; wetland; forest cover & Subsoil \\
\hline 1005 RC20 & 1 & $0-30$ & 0 & Negative & 10YR $3 / 1$ & Silty loam & open field; wetland; forest cover & Subsoil \\
\hline 1005RC21 & 1 & $0-30$ & 0 & Negative & 10YR $3 / 1$ & Silty loam & open field; wetland; forest cover & Subsoil \\
\hline 1005 RC23 & 1 & $0-10$ & 0 & Negative & 10YR $2 / 1$ & Clay loam & Cow pasture; some wetland; tall grass & Inundation \\
\hline 1005RC24 & 1 & $0-10$ & 0 & Negative & 10YR $2 / 1$ & Clay loam & Cow pasture; some wetland; tall grass & Inundation \\
\hline 1005 RC26 & 1 & $0-15$ & 0 & Negative & 10YR 2/1 & Clay loam & Cow pasture; some wetland; tall grass & Inundation \\
\hline 1005 RC28 & 1 & $0-15$ & 0 & Negative & 10YR $2 / 1$ & Clay loam & Cow pasture; some wetland; tall grass & Inundation \\
\hline 1005 RC30 & 1 & $0-30$ & 0 & Negative & 10YR $2 / 1$ & Clay & Cow pasture; tall grass; scattered Trees & Impenetrable \\
\hline 1005RC32 & 1 & $0-20$ & 0 & Negative & 10YR $2 / 1$ & Clay & Cow pasture; tall grass; scattered Trees & Impenetrable \\
\hline 1005RC34 & 1 & $0-20$ & 0 & Negative & 10YR $2 / 1$ & Clay & Cow pasture; tall grass; scattered Trees & Impenetrable \\
\hline 1005 RC36 & 1 & $0-30$ & 0 & Negative & $10 \mathrm{Yr} 2 / 1$ & Clay & Cow pasture; tall grass; scattered Trees & Impenetrable \\
\hline 1005RC37 & 1 & $0-30$ & 0 & Negative & 10YR $2 / 1$ & Clay & Cow pasture; tall grass; scattered Trees & Impenetrable \\
\hline 1005RC39 & 1 & $0-30$ & 0 & Negative & 10YR $2 / 1$ & Clay & Cow pasture; tall grass; scattered Trees & Impenetrable \\
\hline 1005 RC40 & 1 & $0-30$ & 0 & Negative & 10YR $2 / 1$ & Clay & Cow pasture; tall grass; scattered Trees & Impenetrable \\
\hline 1005 RC42 & 1 & $0-30$ & 0 & Negative & 10YR $2 / 1$ & Clay & Cow pasture; tall grass; scattered Trees & Impenetrable \\
\hline 1005RC43 & 1 & $0-30$ & 0 & Negative & 10YR 2/1 & Clay & Cow pasture; tall grass; Inundation in patches & Impenetrable \\
\hline
\end{tabular}




\begin{tabular}{|c|c|c|c|c|c|c|c|c|}
\hline Shovel Test \# & $\begin{array}{l}\begin{array}{l}\text { Level } \\
\text { (Strat) }\end{array} \\
\end{array}$ & $\begin{array}{l}\text { Depth } \\
\text { (cmbs) }\end{array}$ & GSV \% & $\begin{array}{c}\begin{array}{c}\text { Positive or } \\
\text { Negative }\end{array} \\
\end{array}$ & $\begin{array}{c}\text { Munsell } \\
\text { Color }\end{array}$ & Soil Texture Description & Description (Area; Vegetation) & Reason for Termination \\
\hline 1005WE02 & 1 & $0-10$ & 0 & Negative & 10YR $3 / 1$ & Silty loam & Wetland; high grass; roots & Soil change \\
\hline 1005WE02 & 2 & $10-15$ & 0 & Negative & $10 \mathrm{YYR} 3 / 1$ & Silty loam & Wetland; high grass; roots & Subsoil \\
\hline 1005WE06 & 1 & $0-10$ & 0 & Negative & 10YR $3 / 1$ & Clay loam & Forest; many roots; wetland & Soil change \\
\hline 1005WE06 & 2 & $10-20$ & 0 & Negative & 10YR $3 / 1$ & Clay loam & Forest; many roots; wetland & Subsoil \\
\hline 1005WE07 & 1 & $0-10$ & 0 & Negative & $10 Y R 3 / 1$ & Loam & Wetland; tall grass; trees on edge of corridor & Soil change \\
\hline 1005WE07 & 2 & $10-15$ & 0 & Negative & $10 Y R 3 / 1$ & Loam & Wetland; tall grass; trees on edge of corridor & Subsoil \\
\hline 1005WE09 & 1 & $0-10$ & 0 & Negative & $10 Y R 3 / 1$ & Clay loam & Wetland; tall grass; trees on edge of corridor & Soil change \\
\hline 1005WE09 & 2 & $10-15$ & 0 & Negative & $10 Y R 3 / 1$ & Clay loam & Wetland; tall grass; trees on edge of corridor & Subsoil \\
\hline 1005WE11 & 1 & $0-25$ & 0 & Negative & $10 \mathrm{Yr} 3 / 1$ & Loam & Wetland; cow pasture & Subsoil \\
\hline 1005WE14 & 1 & $0-20$ & 5 & Negative & 10YR $3 / 1$ & Loam & Wetland; cow pasture; tall grass; near a shed & Soil change \\
\hline 1005WE14 & 2 & $20-25$ & 5 & Negative & 10YR $3 / 1$ & Loam & Wetland; cow pasture; tall grass; near a shed & Subsoil \\
\hline 1005WE16 & 1 & $0-15$ & 0 & Negative & 10YR 3/1 & Loam & Wetland; cow pasture & Soil change \\
\hline 1005WE16 & 2 & $15-20$ & 0 & Negative & 10YR 3/1 & Loam & Wetland; cow pasture & Subsoil \\
\hline 1005WE18 & 1 & $0-10$ & 0 & Negative & 10YR 3/1 & Loam & Cow pasture; tall grass & Soil change \\
\hline 1005WE18 & 2 & $10-15$ & 0 & Negative & 10YR $3 / 1$ & Loam & Cow pasture; tall grass & Subsoil \\
\hline 1005WE22 & 1 & $0-10$ & 0 & Negative & 10YR $3 / 1$ & Clay loam & Cow pasture; tall grass & Soil change \\
\hline 1005WE22 & 2 & $10-15$ & 0 & Negative & 10YR $3 / 1$ & Clay loam & Cow pasture; tall grass & Subsoil \\
\hline 1005WE25 & 1 & $0-10$ & 0 & Negative & 10YR $3 / 1$ & Clay loam & Cow pasture; tall grass & Inundation \\
\hline 1005WE27 & 1 & $0-10$ & 0 & Negative & 10YR $3 / 1$ & Clay & Cow pasture; close to dirt road & Impenetrable \\
\hline 1005WE27 & 2 & $10-15$ & 0 & Negative & 10YR 3/1 & Clay & Cow pasture; close to dirt road & Impenetrable \\
\hline 1005WE29 & 1 & $0-5$ & 0 & Negative & 10YR $3 / 1$ & Clay loam & Cow pasture; tall grass & Inundation \\
\hline 1005WE31 & 1 & $0-10$ & 0 & Negative & $10 Y R 3 / 1$ & Silty loam & Cow pasture; tall grass & Subsoil \\
\hline 1005WE31 & 2 & $10-15$ & 0 & Negative & 10YR 3/1 & Silty loam & Cow pasture; tall grass & Subsoil \\
\hline 1005WE33 & 1 & $0-15$ & 0 & Negative & 10YR $2 / 1$ & Clay & Cow pasture; tall grass; iron inclusions & Impenetrable \\
\hline 1005WE35 & 1 & $0-10$ & 0 & Negative & 10YR $2 / 1$ & Clay & Cow pasture; tall grass & Impenetrable \\
\hline 1005WE35 & 2 & $10-15$ & 0 & Negative & $10 Y R 2 / 1$ & Clay & Cow pasture; tall grass & Impenetrable \\
\hline 1005WE38 & 1 & $0-5$ & 1 & Negative & 10YR 3/1 & Clay & Cow pasture; tall Grass; Open field & Impenetrable \\
\hline 1005WE38 & 2 & $5-10$ & 1 & Negative & 10YR $3 / 1$ & Silty clay & Cow pasture; tall Grass; Open field & Impenetrable \\
\hline 1005WE41 & 1 & $0-5$ & 0 & Negative & 10YR $3 / 1$ & Clay & Cow pasture; tall Grass; Open field & Impenetrable \\
\hline 1005WE41 & 2 & $5-10$ & 0 & Negative & 10YR 3/1 & Clay & Cow pasture; tall Grass; Open field & Impenetrable \\
\hline 1005WE44 & 1 & $0-10$ & 0 & Negative & 10YR 3/1 & Clay loam & Cow pasture; tall Grass; Open field & Soil change \\
\hline 1005WE44 & 2 & $10-15$ & 0 & Negative & 10YR $3 / 1$ & Clay loam & Cow pasture; tall Grass; Open field & Subsoil \\
\hline 1005JL01 & 1 & $0-30$ & 5 & Negative & 10YR $4 / 2$ & Silty sand & Disturbed by road sand & Disturbed \\
\hline 1005JL02 & 1 & $0-30$ & 5 & Negative & $\begin{array}{c}10 Y R \\
4 / 2 ; 6 / 2 \\
\end{array}$ & Silty clay & Disturbed by road sand; mottled with pea gravel & Disturbed \\
\hline 1005JL03 & 1 & $0-30$ & 5 & Negative & $\begin{array}{c}10 Y R \\
4 / 2 ; 6 / 2 \\
\end{array}$ & Silty clay & Disturbed next to dug out berm & Disturbed \\
\hline $1005 \mathrm{JL} 04$ & 1 & $0-30$ & 5 & Negative & $10 \mathrm{YR} 4 / 2$ & Sandy clay & Imported sand mixed with berm clay & Disturbed \\
\hline 1005RK05 & 1 & $0-30$ & 0 & Negative & $10 Y R 4 / 2$ & Clay & $A$ and $B$ horizon is mixed; near two-track & Disturbed \\
\hline 1005RK06 & 1 & $0-30$ & 0 & Negative & 1 OYR $4 / 2$ & Clay & Disturbed; next to artificial berm & Disturbed \\
\hline 1005JL07 & 1 & $0-30$ & 15 & Negative & $10 Y R 3 / 2$ & Silty clay & In tree line; hit water at $35 \mathrm{cmbs}$ & Inundation \\
\hline 1005JL07 & 2 & $0-30$ & 15 & Negative & 10YR 4/1 & Clay & In tree line; hit water at $35 \mathrm{cmbs}$ & Inundation \\
\hline 1005JL08 & 1 & $0-30$ & 5 & Negative & $\begin{array}{c}10 Y R \\
4 / 2 ; 3 / 2 \\
\end{array}$ & Sandy clay & Imported sand mixed with clay & Disturbed \\
\hline 1005JL09 & 1 & $0-60$ & 5 & Negative & $10 Y R 4 / 2$ & Silty sand & Imported sand from dirt road & Disturbed \\
\hline 1005RK10 & 1 & $0-30$ & 10 & Negative & $10 Y R 4 / 2$ & Silty loam & Inside treeline; near artificial berm & Soil change \\
\hline 1005RK10 & 2 & $30-40$ & 10 & Negative & $10 Y R 5 / 2$ & Silty clay & Inside treeline; near artificial berm & Disturbed \\
\hline 1005RK11 & 1 & $0-60$ & 5 & Negative & $10 Y R 5 / 2$ & Silty clay & Imported sand from road & Disturbed \\
\hline 1005RK12 & 1 & $0-60$ & 5 & Negative & 10YR 5/2 & Silty clay & Surface clay & Disturbed \\
\hline 1005JL13 & 1 & $0-15$ & 5 & Negative & 10YR $4 / 2$ & Sand & Top is imported sand & Impenetrable \\
\hline 1005JL13 & 2 & $15-40$ & 5 & Negative & 1OYR $4 / 1$ & Silty clay & Top is imported sand & Impenetrable \\
\hline 1005JL14 & 1 & $0-30$ & 5 & Negative & 10YR $3 / 2$ & Silty loam & Near wetland & Impenetrable \\
\hline 1005JL14 & 2 & $30-40$ & 5 & Negative & 1OYR $4 / 1$ & Silty clay & Near wetland & Disturbed \\
\hline 1005JL15 & 1 & $0-40$ & 0 & Negative & 10YR $4 / 2$ & Silty sand & Old road bed & Disturbed \\
\hline 1005RK16 & 1 & $0-40$ & 10 & Negative & $\begin{array}{c}10 Y R \\
3 / 1 ; 4 / 1\end{array}$ & Silty clay & Disturbed; on berm near treeline & Disturbed \\
\hline
\end{tabular}




\begin{tabular}{|c|c|c|c|c|c|c|c|c|}
\hline Shovel Test \# & $\begin{array}{l}\begin{array}{l}\text { Level } \\
\text { (Strat) }\end{array} \\
\end{array}$ & $\begin{array}{l}\text { Depth } \\
\text { (cmbs) }\end{array}$ & GSV \% & $\begin{array}{c}\begin{array}{c}\text { Positive or } \\
\text { Negative }\end{array} \\
\end{array}$ & $\begin{array}{c}\text { Munsell } \\
\text { Color }\end{array}$ & Soil Texture Description & Description (Area; Vegetation) & Reason for Termination \\
\hline 1005RK17 & 1 & $0-40$ & 0 & Negative & $\begin{array}{c}10 Y R \\
3 / 1 ; 4 / 1\end{array}$ & Silty clay & Disturbed; cow pasture (land owner bulldozes the area) & Disturbed \\
\hline 1005JL18 & 1 & $0-30$ & 0 & Negative & $\begin{array}{c}10 Y R \\
3 / 1 ; 4 / 1\end{array}$ & Silty clay & Disturbed in cow pasture & Disturbed \\
\hline 1005JL19 & 1 & $0-30$ & 0 & Negative & $\begin{array}{c}10 Y R \\
3 / 1 ; 4 / 1\end{array}$ & Silty clay & In cow pasture & Impenetrable \\
\hline $1005 J L 20$ & 1 & $0-30$ & 0 & Negative & $\begin{array}{c}10 Y R \\
3 / 1 ; 4 / 1 \\
\end{array}$ & Silty clay & In cow pasture & Impenetrable \\
\hline $1005 J L 20$ & 2 & $0-30$ & 0 & Negative & $\begin{array}{c}10 Y R \\
3 / 1 ; 4 / 1 \\
\end{array}$ & Silty clay & In cow pasture & Impenetrable \\
\hline 1005JL21 & 1 & $0-30$ & 0 & Negative & $\begin{array}{c}10 Y R \\
3 / 1 ; 4 / 1 \\
\end{array}$ & Silty clay & In cow pasture & Impenetrable \\
\hline 1005JL21 & 2 & $0-30$ & 0 & Negative & $\begin{array}{c}10 Y R \\
3 / 1 ; 4 / 1\end{array}$ & Silty clay & In cow pasture & Impenetrable \\
\hline 1005JL22 & 1 & $0-30$ & 5 & Negative & $\begin{array}{c}10 Y R \\
3 / 2 ; 6 / 4\end{array}$ & Silty clay & In bulldozed cow pasture & Disturbed \\
\hline $1005 \mathrm{JL} 23$ & 1 & $0-15$ & 5 & Negative & 10YR $4 / 1$ & Silty loam & On $10 \%$ slope & Soil change \\
\hline $1005 \mathrm{JL} 23$ & 2 & $0-15$ & 5 & Negative & 10YR $6 / 4$ & Silty clay & On 10\% slope & Subsoil \\
\hline $1005 J L 24$ & 1 & $0-10$ & 5 & Negative & 10YR $2 / 1$ & Silty clay & $5 \mathrm{~m}$ from swamp & Inundation \\
\hline $1005 J L 24$ & 2 & $0-10$ & 5 & Negative & 10YR $2 / 1$ & Silty clay & $5 \mathrm{~m}$ from swamp & Inundation \\
\hline 1005JL25 & 1 & $0-40$ & 5 & Negative & $\begin{array}{c}10 Y R \\
3 / 1 ; 4 / 1\end{array}$ & Silty clay & Disturbed from bulldozer & Disturbed \\
\hline $1005 J \mathrm{JL} 6$ & 1 & $0-30$ & 5 & Negative & $\begin{array}{c}10 Y R \\
3 / 1 ; 4 / 1 \\
\end{array}$ & Silty clay & Disturbed from bulldozer & Disturbed \\
\hline 1005JL26 & 2 & $0-30$ & 5 & Negative & $\begin{array}{c}10 Y R \\
3 / 1 ; 4 / 1\end{array}$ & Silty clay & Disturbed from bulldozer & Disturbed \\
\hline $1005 J L 27$ & 1 & $0-10$ & 5 & Negative & 10YR $4 / 2$ & Sand & Disturbed with sand on top & Disturbed \\
\hline 1005JL27 & 2 & $10-40$ & 5 & Negative & $\begin{array}{c}10 Y R \\
3 / 1 ; 4 / 1 \\
\end{array}$ & Silty clay & Disturbed with sand on top & Disturbed \\
\hline 1005RK29 & 1 & $0-30$ & 0 & Negative & 10YR $3 / 2$ & Silty loam & In cow pasture & Soil change \\
\hline 1005RK29 & 2 & $30-40$ & 0 & Negative & 10YR 3/1 & Silty clay & In cow pasture & Subsoil \\
\hline 1005RK31 & 1 & $0-15$ & 0 & Negative & 10YR $3 / 1$ & Silty clay & Inundation at $10 \mathrm{cmbs}$ & Inundation \\
\hline 1005RK31 & 2 & $0-15$ & 0 & Negative & 10YR 3/1 & Silty clay & Inundation at $10 \mathrm{cmbs}$ & Inundation \\
\hline 1005RK34 & 1 & $0-15$ & 0 & Negative & 10YR $3 / 1$ & Silty clay & Water at $15 \mathrm{cmbs}$ & Inundation \\
\hline 1005RK34 & 2 & $0-15$ & 0 & Negative & 10YR $3 / 1$ & Silty Cay & Water at $15 \mathrm{cmbs}$ & Inundation \\
\hline 1005RK36 & 1 & $0-25$ & 0 & Negative & 10YR $2 / 1$ & Silty clay & Very compact and wet soils & Inundation \\
\hline 1005RK36 & 2 & $0-25$ & 0 & Negative & 10YR 3/1 & Silty clay & Very compact and wet soils & Inundation \\
\hline 1005RK38 & 1 & $0-25$ & 0 & Negative & $\begin{array}{c}\text { 10YR } \\
3 / 2+7.5 \mathrm{yr} 5 \\
13\end{array}$ & Silty loam & In cow pasture; has a few iron concretions & Soil change \\
\hline 1005RK38 & 2 & $25-35$ & 0 & Negative & 10YR $2 / 1$ & Silty clay & In cow pasture; has a few iron concretions & Subsoil \\
\hline 1005RK40 & 1 & $0-25$ & 0 & Negative & \begin{tabular}{|c|}
10 YR \\
$3 / 2+7.5 y r 5$ \\
13 \\
\end{tabular} & Silty loam & In cow pasture; has a few iron concretions & Soil change \\
\hline 1005RK40 & 2 & $25-35$ & 0 & Negative & 10YR 2/1 & Silty clay & In cow pasture; has a few iron concretions & Subsoil \\
\hline 1005RK42 & 1 & $0-30$ & 0 & Negative & 10YR $2 / 1$ & Silty clay & Inundated & Inundation \\
\hline 1005RK44 & 1 & $0-30$ & 0 & Negative & \begin{tabular}{|c|}
$10 Y R$ \\
$3 / 2 ; 6 / 4 ; 3 /$ \\
1 \\
\end{tabular} & Silty clay & Disturbed & Inundation \\
\hline 1005RK46 & 1 & $0-30$ & 0 & Negative & \begin{tabular}{|c|}
$10 \mathrm{YR}$ \\
$3 / 2 ; 6 / 4 ; 3 /$ \\
1 \\
\end{tabular} & Clay & Disturbed by line & Disturbed \\
\hline 1005RK48 & 1 & $0-30$ & 0 & Negative & 10YR $3 / 1$ & Silty clay & In cow pasture & Soil change \\
\hline 1005RK48 & 2 & $30-45$ & 0 & Negative & $\begin{array}{c}10 Y R \\
4 / 1 ; 6 / 4\end{array}$ & Silty clay & In cow pasture & Subsoil \\
\hline 1005 RK50 & 1 & $0-30$ & 0 & Negative & 10YR $3 / 1$ & Silty clay & In cow pasture & Soil change \\
\hline
\end{tabular}




\begin{tabular}{|c|c|c|c|c|c|c|c|c|}
\hline Shovel Test \# & $\begin{array}{l}\begin{array}{l}\text { Level } \\
\text { (Strat) }\end{array} \\
\end{array}$ & $\begin{array}{l}\text { Depth } \\
\text { (cmbs) }\end{array}$ & GSV \% & $\begin{array}{c}\begin{array}{c}\text { Positive or } \\
\text { Negative }\end{array} \\
\end{array}$ & $\begin{array}{c}\text { Munsell } \\
\text { Color }\end{array}$ & Soil Texture Description & Description (Area; Vegetation) & Reason for Termination \\
\hline 1005RK50 & 2 & $30-45$ & 0 & Negative & $\begin{array}{c}10 Y R \\
4 / 1 ; 6 / 4 \\
\end{array}$ & Silty clay & In cow pasture & Subsoil \\
\hline 1005 JL28 & 1 & $0-28$ & 5 & Negative & $10 Y R 3 / 1$ & Clay & Cow pasture; ankle high grass; near highway & Impenetrable \\
\hline $1005 J L 30$ & 1 & $0-28$ & 5 & Negative & 10YR 3/1 & Clay & Cow pasture; ankle high grass & Impenetrable \\
\hline $1005 J L 33$ & 1 & $0-20$ & 5 & Negative & 10YR 4/1 & Clay & Cow pasture; ankle high grass; wet & Inundation \\
\hline $1005 J L 35$ & 1 & $0-30$ & 5 & Negative & 10YR 2/1 & Clay & Cow pasture; ankle high grass & Impenetrable \\
\hline $1005 J L 37$ & 1 & $0-30$ & 5 & Negative & 10YR $2 / 1$ & Clay & Cow pasture; ankle high grass & Impenetrable \\
\hline $1005 J L 39$ & 1 & $0-30$ & 5 & Negative & $10 \mathrm{Yr} 2 / 1$ & Clay & Cow pasture; ankle high grass & Impenetrable \\
\hline 1005JL41 & 1 & $0-30$ & 5 & Negative & 10YR $4 / 1$ & Clay & Cow pasture; ankle high grass; iron inclusions & Impenetrable \\
\hline $1005 \mathrm{JL} 43$ & 1 & $0-30$ & 5 & Negative & 10YR 4/1 & Clay & Cow pasture; ankle high grass & Impenetrable \\
\hline $1005 J \mathrm{~J} 45$ & 1 & $0-30$ & 5 & Negative & 10YR $2 / 1$ & Clay & Cow pasture; iron inclusions & Impenetrable \\
\hline $1005 J L 47$ & 1 & $0-30$ & 5 & Negative & 10YR $2 / 1$ & Clay & Cow pasture; iron inclusions & Impenetrable \\
\hline $1005 J L 49$ & 1 & $0-15$ & 5 & Negative & 10YR 4/1 & Clay & cow pasture; hit water table & Inundation \\
\hline 1005JL51 & 1 & $0-28$ & 5 & Negative & 10YR $3 / 1$ & Clay & Cow pasture; ankle high grass & Impenetrable \\
\hline 1006WE01 & 1 & $0-5$ & 0 & Negative & 10YR $3 / 1$ & Clay & Pasture; tall grass; near wetland & Inundation \\
\hline 1006WE03 & 1 & $0-5$ & 0 & Negative & 10YR $3 / 1$ & Clay & Pasture; tall grass; near wetland & Inundation \\
\hline 1006WE05 & 1 & $0-5$ & 0 & Negative & 10YR $3 / 1$ & Clay & Pasture; tall grass; near wetland & Inundation \\
\hline 1006WE07 & 1 & $0-10$ & 0 & Negative & 10YR $3 / 1$ & Clay & Pasture; tall grass & Soil change \\
\hline 1006WE07 & 2 & $10-15$ & 0 & Negative & 10YR $3 / 1$ & Clay loam & Pasture; tall grass & Subsoil \\
\hline 1006WE09 & 1 & $0-20$ & 0 & Negative & $10 Y R 6 / 4$ & Clay & Red and grey color; cow pasture; tall grass & Impenetrable \\
\hline 1006WE11 & 1 & $0-15$ & 0 & Negative & 10YR $3 / 1$ & Clay & Wetland area & Inundation \\
\hline 1006WE13 & 1 & $0-15$ & 0 & Negative & $10 \mathrm{YYR} 3 / 1$ & Clay & Wetland area; close to stream; near trees along fence line; sticky soil & Impenetrable \\
\hline 1006WE15 & 1 & $0-15$ & 0 & Negative & 10YR $3 / 1$ & Clay & Pasture; Tall grass; close to fence line; sticky soil & Impenetrable \\
\hline 1006WE18 & 1 & $0-10$ & 0 & Negative & $10 \mathrm{Yr} 4 / 1$ & Silty loam & Pasture; open field; barn nearby & Soil change \\
\hline 1006WE18 & 2 & $10-25$ & 0 & Negative & 10YR 4/1 & Silty loam & Pasture; open field; barn nearby & Subsoil \\
\hline 1006WE20 & 1 & $0-15$ & 0 & Negative & 10YR $4 / 1$ & Silty loam & Pasture & Soil change \\
\hline 1006WE20 & 2 & $15-20$ & 0 & Negative & 10YR 4/1 & Silty loam & Pasture & Subsoil \\
\hline 1006WE23 & 1 & $0-5$ & 0 & Negative & $10 Y R 3 / 1$ & Clay & Wetland & Inundation \\
\hline 1006WE25 & 1 & $0-15$ & 0 & Negative & 10YR 3/1 & Clay & Pasture between wetland and stream & Impenetrable \\
\hline 1006WE29 & 1 & $0-15$ & 0 & Negative & 10YR 3/1 & Clay & Pasture; near stream & Impenetrable \\
\hline 1006WE35 & 1 & $0-20$ & 0 & Negative & $10 Y R 6 / 4$ & Clay & Pasture; tall grass; red-grey clay & Impenetrable \\
\hline 1006RK02 & 1 & $0-30$ & 0 & Negative & \begin{tabular}{|c|}
$10 Y R$ \\
$3 / 1 ; 5 / 1 ; 6 /$ \\
4
\end{tabular} & Silty clay & Disturbed & Disturbed \\
\hline 1006RK04 & 1 & $0-40$ & 0 & Negative & \begin{tabular}{|c|}
$10 Y R$ \\
$3 / 1 ; 4 / 1$ \\
\end{tabular} & Silty clay & In cow pasture; very sticky soil & Impenetrable \\
\hline 1006RK06 & 1 & $0-40$ & 0 & Negative & $\begin{array}{c}10 Y R \\
3 / 1 ; 4 / 1 \\
\end{array}$ & Silty clay & In cow pasture; very sticky soil & Impenetrable \\
\hline 1006RK08 & 1 & $0-40$ & 0 & Negative & $\begin{array}{c}10 Y R \\
3 / 1 ; 4 / 1 \\
\end{array}$ & Silty clay & In cow pasture; very sticky soil & Impenetrable \\
\hline 1006RK10 & 1 & $0-40$ & 0 & Negative & $\begin{array}{c}10 Y R \\
3 / 1 ; 4 / 1\end{array}$ & Silty clay & In cow pasture; very sticky soil & Impenetrable \\
\hline 1006RK12 & 1 & $0-40$ & 0 & Negative & $\begin{array}{c}10 Y R \\
3 / 1 ; 4 / 1\end{array}$ & Silty clay & In cow pasture; very sticky soil & Impenetrable \\
\hline 1006RK14 & 1 & $0-30$ & 10 & Negative & $\begin{array}{c}10 Y R \\
3 / 1 ; 2 / 1\end{array}$ & Silty clay & In cow pasture; very sticky soil; $2 \mathrm{~m}$ west of creek & Impenetrable \\
\hline 1006RK16 & 1 & $0-40$ & 0 & Negative & $\begin{array}{c}10 Y R \\
4 / 1 ; 3 / 1 \\
\end{array}$ & Silty clay & In cow pasture with tall grasses & Impenetrable \\
\hline 1006RK18 & 1 & $0-30$ & 0 & Negative & $\begin{array}{c}10 Y R \\
2 / 1 ; 3 / 1\end{array}$ & Clay & In tall grass; hit water at $25 \mathrm{cmbs}$ & Inundation \\
\hline 1006RK20 & 1 & $0-40$ & 0 & Negative & $\begin{array}{c}10 \mathrm{YR} \\
2 / 1 ; 3 / 1 \\
\end{array}$ & Clay & In tall grass; $\mathrm{A}$ and $\mathrm{A} 2$ mixed with iron inclusions & Impenetrable \\
\hline 1006RK26 & 1 & $0-40$ & 0 & Negative & $\begin{array}{c}10 Y R \\
2 / 1 ; 3 / 1 \\
\end{array}$ & Clay & In tall grass; A and A2 mixed with iron inclusions & Impenetrable \\
\hline 1006RK30 & 1 & $0-10$ & 0 & Negative & $10 \mathrm{YR} 2 / 1$ & Clay & Water at $5 \mathrm{cmbs}$ & Inundation \\
\hline
\end{tabular}




\begin{tabular}{|c|c|c|c|c|c|c|c|c|}
\hline Shovel Test \# & $\begin{array}{l}\text { Level } \\
\text { (Strat) }\end{array}$ & $\begin{array}{l}\text { Depth } \\
\text { (cmbs) }\end{array}$ & GSV \% & $\begin{array}{l}\text { Positive or } \\
\text { Negative }\end{array}$ & $\begin{array}{l}\text { Munsell } \\
\text { Color }\end{array}$ & Soil Texture Description & Description (Area; Vegetation) & Reason for Termination \\
\hline 1006RK32 & 1 & $0-30$ & 0 & Negative & \begin{tabular}{|c|}
$10 Y R$ \\
$3 / 1 ; 4 / 1 ; 6 /$ \\
4 \\
\end{tabular} & Clay & Disturbed & Disturbed \\
\hline 1006RC02 & 1 & $0-30$ & 0 & Negative & 10YR $3 / 1$ & Clay & Open cow pasture; adjacent to wetland; tall grass; trees along fence line & Impenetrable \\
\hline 1006RC04 & 1 & $0-15$ & 0 & Negative & 1OYR $3 / 1$ & Clay & Open cow pasture; adjacent to wetland; tall grass; trees along fence line & Inundation \\
\hline $1006 \mathrm{RC} 06$ & 1 & $0-15$ & 0 & Negative & $10 Y R 3 / 1$ & Clay & Open cow pasture; adjacent to wetland; tall grass; trees along fence line & Inundation \\
\hline 1006RC08 & 1 & $0-20$ & 0 & Negative & $10 Y R 3 / 1$ & Clay & Open cow pasture; adjacent to wetland; tall grass; trees along fence line & Inundation \\
\hline $1006 \mathrm{RC10}$ & 1 & $0-30$ & 0 & Negative & $10 \mathrm{YR} 3 / 1$ & Clay & Open cow pasture; adjacent to wetland; tall grass; trees along fence line & Inundation \\
\hline $1006 \mathrm{RC} 12$ & 1 & $0-30$ & 0 & Negative & $10 \mathrm{YR} 3 / 1$ & Clay & Open cow pasture; adjacent to wetland; tall grass; trees along fence line & Inundation \\
\hline 1006RC14 & 1 & $0-30$ & 0 & Negative & 10YR $3 / 1$ & Clay & Open cow pasture; adjacent to canal; trees along fence line & Impenetrable \\
\hline $1006 \mathrm{RC16}$ & 1 & $0-30$ & 0 & Negative & 10YR $3 / 1$ & Clay & Open cow pasture; adjacent to canal; trees along fence line & Impenetrable \\
\hline 1006RC17 & 1 & $0-50$ & 0 & Negative & 10YR $3 / 1$ & Clay loam & Open field; tall grass; trees along fence line; scattered brush & Subsoil \\
\hline 1006RC19 & 1 & $0-50$ & 0 & Negative & $10 \mathrm{YR} 3 / 1$ & Clay loam & Open field; tall grass; trees along fence line; scattered brush & Subsoil \\
\hline $1006 \mathrm{RC22}$ & 1 & $0-15$ & 0 & Negative & $10 Y R 3 / 1$ & Clay loam & Open field; tall grass; trees along fence line; scattered brush & Inundation \\
\hline 1006RC24 & 1 & $0-30$ & 0 & Negative & 10YR $3 / 1$ & Clay loam & Open field; tall grass; trees along fence line; scattered brush & Impenetrable \\
\hline 1006 RC28 & 1 & $0-30$ & 0 & Negative & $10 \mathrm{YR} 3 / 1$ & Clay loam & Open field; tall grass; trees along fence line; scattered brush & Impenetrable \\
\hline 1006RC34 & 1 & $0-30$ & 0 & Negative & 10YR $3 / 1$ & Clay loam & Open field; tall grass; trees along fence line; scattered brush & Impenetrable \\
\hline 1006」L01 & 1 & $0-30$ & 5 & Negative & $\begin{array}{c}10 Y R \\
3 / 1 ; 2 / 1\end{array}$ & Clay & Cow pasture; ankle high grass; next to dug out ditch & Impenetrable \\
\hline 1006」L03 & 1 & $0-30$ & 5 & Negative & $\begin{array}{c}10 Y R \\
3 / 1 ; 2 / 1\end{array}$ & Clay & Cow pasture; ankle high grass; next to dug out ditch & Impenetrable \\
\hline 1006JL05 & 1 & $0-10$ & 5 & Negative & $\begin{array}{c}10 Y R \\
2 / 1 ; 4 / 1\end{array}$ & Clay & Cow pasture; ankle high grass; next to dug out ditch; Disturbed & Disturbed \\
\hline 1006」L07 & 1 & $0-30$ & 5 & Negative & $\begin{array}{c}10 Y R \\
3 / 1 ; 6 / 4\end{array}$ & Clay & Cow pasture; ankle high grass & Impenetrable \\
\hline 1006」L09 & 1 & $0-12$ & 5 & Negative & $\begin{array}{c}10 Y R \\
3 / 1 ; 4 / 1\end{array}$ & Clay & Cow pasture; disturbed & Disturbed \\
\hline 1006JL11 & 1 & $0-12$ & 5 & Negative & $\begin{array}{c}10 Y R \\
3 / 1 ; 4 / 1\end{array}$ & Clay & Cow pasture; disturbed & Disturbed \\
\hline 1006JL13 & 1 & $0-15$ & 5 & Negative & $10 Y R 2 / 1$ & Clay & Cow pasture; disturbed; charcoal & Disturbed \\
\hline 1006JL15 & 1 & $0-30$ & 5 & Negative & 10YR $4 / 1$ & Clay & Cow pasture near stream & Impenetrable \\
\hline 1006JL17 & 1 & $0-8$ & 5 & Negative & 10YR $4 / 1$ & Clay & Cow pasture; hit water table & Inundation \\
\hline 1006JL19 & 1 & $0-10$ & 5 & Negative & 10YR $4 / 1$ & Clay & Cow pasture; hit water table & inundation \\
\hline 1006JL21 & 1 & $0-35$ & 5 & Negative & $\begin{array}{c}10 Y R \\
3 / 1 ; 4 / 1\end{array}$ & Clay & Cow pasture; slight slope; waist high grass & Impenetrable \\
\hline 1006JL22 & 1 & $0-12$ & 5 & Negative & $\begin{array}{c}10 Y R \\
2 / 1 ; 4 / 1\end{array}$ & Clay & near pipeline; disturbed & Disturbed \\
\hline 1006JL27 & 1 & $0-15$ & 5 & Negative & $\begin{array}{c}10 Y R \\
2 / 1 ; 4 / 1 \\
\end{array}$ & Clay & near pipeline; disturbed & Disturbed \\
\hline 1006JL31 & 1 & $0-8$ & 5 & Negative & 10YR $3 / 1$ & Clay & Inundation & Inundation \\
\hline 1006」L33 & 1 & $0-10$ & 5 & Negative & $\begin{array}{c}10 Y R \\
3 / 1 ; 6 / 4\end{array}$ & Clay & Disturbed; imported gravel & Disturbed \\
\hline 1008RC01 & 1 & $0-40$ & 0 & Negative & 10YR $3 / 3$ & Clay loam & $\begin{array}{l}\begin{array}{l}\text { Open field; wetland and upland areas; trees along boundary; tall grass; scattered brush; large } \\
\text { inundation spots. }\end{array}\end{array}$ & Soil change \\
\hline 1008RC01 & 2 & $40-50$ & 0 & Negative & 10YR $5 / 6$ & Silty clay & $\begin{array}{c}\begin{array}{c}\text { Open field; wetland and upland areas; trees along boundary; tall grass; scattered brush; large } \\
\text { inundation spots. }\end{array}\end{array}$ & Subsoil \\
\hline 1008RC03 & 1 & $0-30$ & 0 & Negative & 10YR $3 / 3$ & Clay loam & $\begin{array}{l}\text { Open field; wetland and upland areas; trees along boundary; tall grass; scattered brush; large } \\
\text { inundation spots. }\end{array}$ & Soil change \\
\hline $1008 \mathrm{RC} 03$ & 2 & $30-40$ & 0 & Negative & 10YR $5 / 6$ & Clay loam & $\begin{array}{l}\begin{array}{l}\text { Open field; wetland and upland areas; trees along boundary; tall grass; scattered brush; large } \\
\text { inundation spots. }\end{array}\end{array}$ & Subsoil \\
\hline 1008RC04 & 1 & $0-30$ & 0 & Negative & 10YR $3 / 3$ & Clay loam & $\begin{array}{l}\text { Open field; wetland and upland areas; trees along boundary; tall grass; scattered brush; large } \\
\text { inundation spots. }\end{array}$ & Soil change \\
\hline 1008RC04 & 2 & $30-40$ & 0 & Negative & 10YR $5 / 6$ & Clay loam & $\begin{array}{l}\text { Open field; wetland and upland areas; trees along boundary; tall grass; scattered brush; large } \\
\text { inundation spots. }\end{array}$ & Subsoil \\
\hline 1008WE02 & 1 & $0-10$ & 0 & Negative & 1OYR $3 / 10$ & Clay loam & Wetland; tall grass; thick brush & Soil change \\
\hline 1008WE02 & 2 & $10-15$ & 0 & Negative & 10 YYR 5/6 & Clay loam & Wetland; tall grass; thick brush & Subsoil \\
\hline
\end{tabular}




\begin{tabular}{|c|c|c|c|c|c|c|c|c|}
\hline Shovel Test \# & $\begin{array}{l}\begin{array}{l}\text { Level } \\
\text { (Strat) }\end{array} \\
\end{array}$ & $\begin{array}{l}\text { Depth } \\
\text { (cmbs) }\end{array}$ & GSV \% & $\begin{array}{l}\begin{array}{c}\text { Positive or } \\
\text { Negative }\end{array} \\
\end{array}$ & $\begin{array}{c}\text { Munsell } \\
\text { Color }\end{array}$ & Soil Texture Description & Description (Area; Vegetation) & Reason for Termination \\
\hline 1008WE05 & 1 & $0-5$ & 0 & Negative & 10YR $3 / 10$ & Clay loam & Wetland; tall grass; thick brush; close to forest & Inundation \\
\hline 1008WE06 & 1 & $0-10$ & 0 & Negative & $10 Y R 5 / 6$ & Clay & Open field; tall grass & Impenetrable \\
\hline 1009WE02 & 1 & $0-10$ & 0 & Negative & 10YR $4 / 1$ & Clay loam & Cow pasture & Soil change \\
\hline 1009WE02 & 2 & $10-15$ & 0 & Negative & 10YR 4/1 & Clay loam & Cow pasture & Subsoil \\
\hline 1009WE03 & 1 & $0-10$ & 0 & Negative & $10 \mathrm{YYR} 3 / 2$ & Silty loam & Forest; tall trees; short grass; little brush; adjacent to pasture & Soil change \\
\hline 1009WE03 & 2 & $10-15$ & 0 & Negative & $10 \mathrm{YrR} 3 / 2$ & Silty loam & Forest; tall trees; short grass; little brush; adjacent to pasture & Subsoil \\
\hline 1009WE06 & 1 & $0-5$ & 0 & Negative & $10 Y R 3 / 2$ & Loam & Near a trail; adjacent to ditch and field; gravel inclusions & Disturbed \\
\hline 1009WE10 & 1 & $0-15$ & 0 & Negative & $10 \mathrm{YR} 3 / 2$ & Silty loam & Next to treeline; tall grass; open field & Soil change \\
\hline 1009WE10 & 2 & $15-20$ & 0 & Negative & 10YR $3 / 2$ & Silty loam & Next to treeline; tall grass; open field & Subsoil \\
\hline 1009WE13 & 1 & $0-25$ & 0 & Negative & $10 \mathrm{YR} 3 / 2$ & Silty loam & Next to creak; tall grass; open field & Inundation \\
\hline 1009WE17 & 1 & $0-35$ & 0 & Negative & $10 Y R 3 / 2$ & Silty clay & Open field & Inundation \\
\hline 1009WE20 & 1 & $0-30$ & 0 & Negative & 10YR $3 / 2$ & Silty clay & Open field; tall grass & Soil change \\
\hline 1009WE20 & 2 & $30-35$ & 0 & Negative & $10 \mathrm{YYR} 3 / 2$ & Silty clay & Open field; tall grass & Subsoil \\
\hline 1009WE24 & 1 & $0-30$ & 0 & Negative & 10YR $3 / 2$ & Silty loam & Open field; tall grass & Inundation \\
\hline 1009WE27 & 1 & $0-35$ & 0 & Negative & $10 \mathrm{YYR} 3 / 2$ & Silty loam & Open field; tall grass & Inundation \\
\hline 1009TR04 & 1 & $0-30$ & 0 & Negative & 10YR $4 / 1$ & Clay & Swampy oak woodlands; dry on surface & Subsoil \\
\hline 1009TR07 & 1 & $0-20$ & 0 & Negative & $10 \mathrm{Yr} 3 / 2$ & Loam & Grassy pasture; heavy-moderate inundation & Inundation \\
\hline 1009TR09 & 1 & $0-35$ & 0 & Negative & 10YR $3 / 2$ & Loam & Grassy pasture 12-24 inch grass; dry on surface & Subsoil \\
\hline 1009TR11 & 1 & $0-35$ & 0 & Negative & $10 \mathrm{YR} 3 / 2$ & Loam & Grassy pasture 12-24 inch grass; dry on surface & Subsoil \\
\hline 1009TR15 & 1 & $0-35$ & 0 & Negative & 10YR $3 / 2$ & Loam & Grassy pasture 6-12 inch grass; dry on surface & Subsoil \\
\hline 1009TR19 & 1 & $0-40$ & 0 & Negative & $10 Y R 3 / 2$ & Loam & Grassy pasture 12-18 inch grass; dry on surface & Subsoil \\
\hline 1009TR23 & 1 & $0-35$ & 0 & Negative & $10 \mathrm{YR} 3 / 2$ & Loam & Grassy pasture 12-18 inch grass; dry on surface & Subsoil \\
\hline 1009TR26 & 1 & $0-35$ & 0 & Negative & 10YR $3 / 2$ & Loam & Grassy pasture 24-36 inch grass; moderately Inundation; some riparian grasses & Subsoil \\
\hline 1009TR29 & 1 & $0-35$ & 0 & Negative & $10 \mathrm{YYR} 3 / 2$ & Loam & Grassy pasture 36-72 inch grass; dry on surface & Subsoil \\
\hline 1009RC01 & 1 & $0-30$ & 0 & Negative & 10YR 4/1 & Clay & Cow pasture; tract LI-47; short grass; scattered brush & Subsoil \\
\hline 1009RC05 & 1 & $0-50$ & 0 & Negative & $10 \mathrm{YR} 3 / 2$ & Loam & Forest cover; tall pine trees; thick rose briar; heavy vegetation & Subsoil \\
\hline 1009RC05 & 2 & $50-60$ & 0 & Negative & $10 \mathrm{YR} 3 / 2$ & Loam & Forest cover; tall pine trees; thick rose briar; heavy vegetation & Soil change \\
\hline 1009RC08 & 1 & $0-40$ & 0 & Negative & $10 \mathrm{YR} 3 / 2$ & Loam & Open pasture; tall grass; trees along property line; some inundation & Inundation \\
\hline 1009RC12 & 1 & $0-50$ & 0 & Negative & 10YR $3 / 2$ & Loam & Open pasture; tall grass; trees along property line; some inundation & Soil change \\
\hline 1009RC12 & 2 & $50-60$ & 0 & Negative & $10 \mathrm{YYR} 3 / 2$ & Loam & Open pasture; tall grass; trees along property line; some inundation & Inundation \\
\hline 1009RC14 & 1 & $0-30$ & 0 & Negative & $10 \mathrm{YR} 3 / 2$ & Loam & Open pasture; tall grass; trees along property line; some inundation & Inundation \\
\hline $1009 \mathrm{RC} 16$ & 1 & $0-30$ & 0 & Negative & $10 \mathrm{YR} 3 / 2$ & Loam & Open pasture; tall grass; trees along property line; some inundation & Inundation \\
\hline $1009 \mathrm{RC} 18$ & 1 & $0-40$ & 0 & Negative & $10 \mathrm{YR} 3 / 3$ & Loam & Open pasture; tall grass; trees along property line; some inundation & Inundation \\
\hline 1009RC21 & 1 & $0-30$ & 0 & Negative & $10 \mathrm{YR} 3 / 2$ & Loam & Open pasture; tall grass; trees along property line; some inundation & Inundation \\
\hline 1009RC22 & 1 & $0-40$ & 0 & Negative & $10 \mathrm{YR} 3 / 3$ & Loam & Open grass pasture; some inundation & Subsoil \\
\hline 1009RC25 & 1 & $0-30$ & 0 & Negative & $10 \mathrm{YR} 3 / 3$ & Loam & Open grass pasture; some inundation & Subsoil \\
\hline 1009RC28 & 1 & $0-60$ & 0 & Negative & $10 \mathrm{YR} 3 / 3$ & Loam & Open grass pasture; some inundation & Subsoil \\
\hline 1010TR03 & 1 & $0-4$ & 100 & Negative & 10YR $4 / 1$ & Silty clay & Swampy woodland; thin briar understory & Soil change \\
\hline 1010TR03 & 2 & 4-10 & 100 & Negative & 10YR $3 / 1$ & Silty clay & Swampy woodland; thin briar understory & Subsoil \\
\hline 101018WE12 & 1 & $0-10$ & 0 & Negative & 10YR $3 / 1$ & Silty clay & Swampy woodland; thin briar understory & Subsoil \\
\hline 1010TR13 & 1 & $0-8$ & 0 & Negative & $10 Y R 5 / 2$ & Loam & Grassy field surrounded by pines; utility poles and berm through field; grass $36-48$ inches tall & Soil change \\
\hline 1010TR13 & 2 & $8-18$ & 0 & Negative & $7.5 Y R 6 / 8$ & Loam & Grassy field surrounded by pines; utility poles and berm through field; grass $36-48$ inches tall & Subsoil \\
\hline 1010TR16 & 1 & $0-8$ & 5 & Negative & 10YR 5/2 & Loam & Grassy field adjacent to yard and home; grass $12-48$ inches tall & Soil change \\
\hline 1010TR16 & 2 & $8-18$ & 5 & Negative & $7.5 Y R 6 / 8$ & Loam & Grassy field adjacent to yard and home; grass $12-48$ inches tall & Subsoil \\
\hline 1010TR19 & 1 & $0-4$ & 10 & Negative & $10 \mathrm{YR} 5 / 2$ & Loam & Moderately dense woodland with moderately dense understory & Subsoil \\
\hline 1010TR21 & 1 & $0-5$ & 0 & Negative & 10YR 5/2 & Loam & Moderately dense woodland with moderately dense understory & Soil change \\
\hline 1010TR21 & 2 & $5-18$ & 0 & Negative & $7.5 Y R 6 / 8$ & Loam & Moderately dense woodland with moderately dense understory & Subsoil \\
\hline 1010TR24 & 1 & $0-10$ & 0 & Negative & $10 \mathrm{YR} 5 / 2$ & Loam & Grassy field adjacent to yard and home; grass $12-36$ inches tall & Soil change \\
\hline 1010TR24 & 2 & $10-20$ & 0 & Negative & $7.5 Y R 6 / 8$ & Loam & Grassy field adjacent to yard and home; grass $12-36$ inches tall & Subsoil \\
\hline 1010TR28 & 1 & $0-5$ & 0 & Negative & $10 \mathrm{YR} 5 / 2$ & Loam & Grassy field with utility line and berm. $12-60$ inch grass & Soil change \\
\hline 1010TR28 & 2 & $5-20$ & 0 & Negative & 7.5YR $6 / 8$ & Loam & Grassy field with utility line and berm. 12-60 inch grass & Subsoil \\
\hline 1010TR32 & 1 & $0-20$ & 0 & Negative & $10 Y R 5 / 2$ & Loam & Grassy field with utility line and berm. $12-60$ inch grass; mixed A and B horizons & Inundation \\
\hline 1010TR32 & 2 & $20-22$ & 0 & Negative & 7.5 YR $6 / 8$ & Clay & Grassy field with utility line and berm. $12-60$ inch grass; mixed $A$ and $B$ horizons & Inundation \\
\hline
\end{tabular}




\begin{tabular}{|c|c|c|c|c|c|c|c|c|}
\hline Shovel Test \# & $\begin{array}{l}\text { Level } \\
\text { (Strat) }\end{array}$ & $\begin{array}{l}\text { Depth } \\
\text { (cmbs) }\end{array}$ & GSV \% & $\begin{array}{l}\text { Positive or } \\
\text { Negative }\end{array}$ & $\begin{array}{c}\text { Munsell } \\
\text { Color }\end{array}$ & Soil Texture Description & Description (Area; Vegetation) & Reason for Termination \\
\hline 1010TR35 & 1 & $0-10$ & 0 & Negative & 10YR $5 / 2$ & Loam & Grassy field with utility line and berm. 12-60 inch grass; mixed A and B horizons & Subsoil \\
\hline 1010TR35 & 2 & $10-22$ & 0 & Negative & $7.5 Y R 6 / 8$ & Clay & Grassy field with utility line and berm. $12-60$ inch grass; mixed $A$ and $B$ horizons & Subsoil \\
\hline 1010WE02 & 1 & $0-15$ & 0 & Negative & 10YR $4 / 1$ & Clay & Adjacent to stream; tall grass; red-gray color & Impenetrable \\
\hline 1010WE02 & 2 & $15-20$ & 0 & Negative & 10YR $3 / 1$ & Clay & Adjacent to stream; tall grass; red-gray color & Impenetrable \\
\hline 1010WE11 & 1 & $0-20$ & 0 & Negative & $10 Y R 5 / 2$ & Clay & Open field; adjacent to berm & Impenetrable \\
\hline 1010WE15 & 1 & $0-20$ & 0 & Negative & $10 Y R 5 / 2$ & Clay & Open field; adjacent to road & Impenetrable \\
\hline 1010WE17 & 1 & $0-15$ & 0 & Negative & $10 Y R 5 / 2$ & Clay & Pine forest & Impenetrable \\
\hline 1010WE22 & 1 & $0-15$ & 0 & Negative & 10YR $5 / 2$ & Clay & Pine forest; wide O horizon & Impenetrable \\
\hline 1010WE25 & 1 & $0-20$ & 0 & Negative & $10 \mathrm{YR} 5 / 2$ & Clay & Open field & Impenetrable \\
\hline 1010WE29 & 1 & $0-20$ & 0 & Negative & 10YR $5 / 2$ & Clay & Open field; tall grass & Impenetrable \\
\hline 1010WE31 & 1 & $0-5$ & 0 & Negative & 10YR $5 / 2$ & Loamy clay & Open field; tall grass & Inundation \\
\hline 1010WE31 & 2 & $5-15$ & 0 & Negative & 10YR $5 / 2$ & Loamy clay & Open field; tall grass & Inundation \\
\hline 1010WE34 & 1 & $0-20$ & 0 & Negative & 10 YR $5 / 2$ & Clay & Open field; tall grass & Impenetrable \\
\hline 1010RC01 & 1 & $0-30$ & 0 & Negative & 10YR 5/1 & Clay & TX-LI-055.000; short grass; cow pasture; heavy rooted soil; large Inundation areas. & Soil change \\
\hline 1010RC01 & 2 & $30-40$ & 0 & Negative & 10YR $3 / 1$ & Clay & TX-LI-055.000; short grass; cow pasture; heavy rooted soil; large Inundation areas. & Subsoil \\
\hline 1010RC04 & 1 & $0-30$ & 0 & Negative & $10 Y R 5 / 1$ & Clay & TX-LI-055.000; short grass; cow pasture; heavy rooted soil; large Inundation areas. & Soil change \\
\hline 1010RC04 & 2 & $30-40$ & 0 & Negative & 10YR $3 / 1$ & Clay & TX-LI-055.000; short grass; cow pasture; heavy rooted soil; large Inundation areas. & Subsoil \\
\hline 1010RC08 & 1 & $0-20$ & 0 & Negative & $10 \mathrm{YR} 5 / 1$ & Clay & TX-LI-055.000; short grass; cow pasture; heavy rooted soil; large Inundation areas. & Inundation \\
\hline 1010RC10 & 1 & $0-15$ & 0 & Negative & $10 \mathrm{YR} 5 / 2$ & Clay & TX-LI-062/063; medium-tall grass; centerline has scattered brush; some forest cover & Soil change \\
\hline 1010RC10 & 2 & $15-20$ & 0 & Negative & $7.5 Y R 6 / 8$ & Clay & TX-LI-062/063; medium-tall grass; centerline has scattered brush; some forest cover & Subsoil \\
\hline 1010RC14 & 1 & $0-20$ & 0 & Negative & $10 \mathrm{YR} 5 / 2$ & Clay & TX-LI-062/063; medium-tall grass; centerline has scattered brush; some forest cover & Soil change \\
\hline 1010RC14 & 2 & $20-25$ & 0 & Negative & 7.5YR $6 / 8$ & Clay & TX-LI-062/063; medium-tall grass; centerline has scattered brush; some forest cover & Subsoil \\
\hline 1010RC17 & 1 & $0-15$ & 0 & Negative & $10 \mathrm{YR} 5 / 2$ & Clay & TX-LI-062/063; medium-tall grass; centerline has scattered brush; some forest cover & Soil change \\
\hline 1010RC17 & 2 & $15-20$ & 0 & Negative & 7.5YR $6 / 8$ & Clay & TX-LI-062/063; medium-tall grass; centerline has scattered brush; some forest cover & Subsoil \\
\hline 1010RC20 & 1 & $0-15$ & 0 & Negative & $10 \mathrm{Yr} 5 / 2$ & Clay & TX-LI-062/063; medium-tall grass; centerline has scattered brush; some forest cover & Soil change \\
\hline 1010RC20 & 2 & $15-20$ & 0 & Negative & 7.5 YR $6 / 8$ & Clay & TX-LI-062/063; medium-tall grass; centerline has scattered brush; some forest cover & Subsoil \\
\hline 1010RC23 & 1 & $0-15$ & 0 & Negative & 10YR $5 / 2$ & Clay & TX-LI-062/063; medium-tall grass; centerline has scattered brush; some forest cover & Soil change \\
\hline 1010RC23 & 2 & $15-20$ & 0 & Negative & $7.5 Y R 6 / 8$ & Clay & TX-LI-062/063; medium-tall grass; centerline has scattered brush; some forest cover & Subsoil \\
\hline 1010RC26 & 1 & $0-15$ & 0 & Negative & 10YR 5/2 & Clay & Very sticky clay; tall grass; some forest cover; some thick vegetation & Soil change \\
\hline 1010RC26 & 2 & $15-20$ & 0 & Negative & 7.5YR $6 / 8$ & Clay & Very sticky clay; tall grass; some forest cover; some thick vegetation & Subsoil \\
\hline 1010RC27 & 1 & $0-15$ & 0 & Negative & $10 \mathrm{YR} 5 / 2$ & Clay & Very sticky clay; tall grass; some forest cover; some thick vegetation & Soil change \\
\hline 1010RC27 & 2 & $15-20$ & 0 & Negative & 7.5YR 6/8 & Clay & Very sticky clay; tall grass; some forest cover; some thick vegetation & Subsoil \\
\hline 1010RC30 & 1 & $0-15$ & 0 & Negative & $10 \mathrm{YR} 5 / 2$ & Clay & Very sticky clay; tall grass; some forest cover; some thick vegetation & Soil change \\
\hline 1010RC30 & 2 & $15-20$ & 0 & Negative & $7.5 Y R 6 / 8$ & Clay & Very sticky clay; tall grass; some forest cover; some thick vegetation & Subsoil \\
\hline 1010RC33 & 1 & $0-15$ & 0 & Negative & $10 \mathrm{YR} 5 / 2$ & Clay & Very sticky clay; tall grass; some forest cover; some thick vegetation & Soil change \\
\hline 1010RC33 & 2 & $15-20$ & 0 & Negative & 7.5YR $6 / 8$ & Clay & Very sticky clay; tall grass; some forest cover; some thick vegetation & Subsoil \\
\hline 1011WE18 & 1 & $0-10$ & 0 & Negative & 10YR $4 / 1$ & Clay & Open field; tall grass; cow pasture & Soil change \\
\hline 1011WE18 & 2 & $10-15$ & 0 & Negative & 10YR $3 / 1$ & Clay & Open field; tall grass; cow pasture & Subsoil \\
\hline 1011RC16 & 1 & $0-15$ & 0 & Negative & 10YR 4/1 & Clay & Open cow pasture; rutted soil; tall unmanicured grass; scattered brush & Subsoil \\
\hline 1011TR17 & 1 & $0-12$ & 0 & Negative & 10YR 4/1 & Clay & $\begin{array}{l}\text { Grassy field; 4-8 inch grass; } 12-24 \text { inch shrubs; near a small artificial drain; fence; berm and } 2 \\
\text { exposed pipelines }\end{array}$ & Soil change \\
\hline 1011TR17 & 2 & $12-22$ & 0 & Negative & 10YR $3 / 1$ & Clay & $\begin{array}{l}\text { Grassy field; 4-8 inch grass; } 12-24 \text { inch shrubs; near a small artificial drain; fence; berm and } 2 \\
\text { exposed pipelines }\end{array}$ & Subsoil \\
\hline 1012RC03 & 1 & $0-8$ & 10 & Negative & 10YR 4/2 & Clay & Tract TX-LI-064.000; row cleared; shot grass; scattered tree limbs & Subsoil \\
\hline 1012RC06 & 1 & $0-10$ & 10 & Negative & 10YR 4/2 & Clay & Heavily forested; thick vines; palmettos; south of row & Subsoil \\
\hline 1012RC09 & 1 & $0-10$ & 10 & Negative & $10 Y R 4 / 2$ & Clay & Row cleared; short grass; trees along south edge & Subsoil \\
\hline 1012RC12 & 1 & $0-15$ & 0 & Negative & $10 \mathrm{YYR} 4 / 2$ & Clay & Heavy forest; mature tree cover; palmettos; thick vines & Subsoil \\
\hline 1012RC15 & 1 & $0-10$ & 10 & Negative & 1OYR $4 / 2$ & Clay & Heavy forest; mature tree cover; palmettos; thick vines & Subsoil \\
\hline 1012RC17 & 1 & $0-15$ & 10 & Negative & $10 \mathrm{YYR} 4 / 2$ & Clay & Heavy forest; mature tree cover; palmettos; thick vines & Subsoil \\
\hline 1012RC20 & 1 & $0-20$ & 10 & Negative & 10YR $4 / 2$ & Clay & Heavy forest; mature tree cover; palmettos; thick vines & Subsoil \\
\hline 1012RC22 & 1 & $0-15$ & 10 & Negative & 10YR $4 / 2$ & Clay & Heavy forest; mature tree cover; palmettos; thick vines & Subsoil \\
\hline 1012 RC25 & 1 & $0-10$ & 10 & Negative & $10 \mathrm{Yr} 4 / 2$ & Clay & Heavy forest; mature tree cover; palmettos; thick vines & Subsoil \\
\hline 1012RC27 & 1 & $0-15$ & 10 & Negative & 10YR 3/1 & Clay & Heavy forest; mature tree cover; palmettos; thick vines & Subsoil \\
\hline 1012RC30 & 1 & $0-15$ & 10 & Negative & 10YR $4 / 2$ & Clay & Heavy forest; mature tree cover; palmettos; thick vines & Subsoil \\
\hline 1012RC33 & 1 & $0-10$ & 10 & Negative & $10 \mathrm{YYR} 4 / 2$ & Clay & Heavy forest; mature tree cover; palmettos; thick vines & Subsoil \\
\hline $1012 \mathrm{RC} 36$ & 1 & $0-20$ & 10 & Negative & $10 \mathrm{YR} 7 / 4$ & Clay & Heavy forest; mature tree cover; palmettos; thick vines & Subsoil \\
\hline 1012RC38 & 1 & $0-15$ & 10 & Negative & $10 \mathrm{YR} 7 / 4$ & Clay & Heavy forest; mature tree cover; palmettos; thick vines & Subsoil \\
\hline
\end{tabular}




\begin{tabular}{|c|c|c|c|c|c|c|c|c|}
\hline Shovel Test \# & $\begin{array}{l}\text { Level } \\
\text { (Strat) }\end{array}$ & $\begin{array}{l}\text { Depth } \\
\text { (cmbs) }\end{array}$ & GSV \% & $\begin{array}{c}\text { Positive or } \\
\text { Negative }\end{array}$ & $\begin{array}{l}\text { Munsell } \\
\text { Color }\end{array}$ & Soil Texture Description & Description (Area; Vegetation) & Reason for Termination \\
\hline 1012RC41 & 1 & $0-20$ & 10 & Negative & $10 Y R 7 / 4$ & Clay & Heavy forest; mature tree cover; palmettos; thick vines & Subsoil \\
\hline 1012RC44 & 1 & $0-30$ & 10 & Negative & $10 \mathrm{YR} 7 / 1$ & Clay & Heavy forest; mature tree cover; palmettos; thick vines & Subsoil \\
\hline 1012RC47 & 1 & $0-30$ & 10 & Negative & $10 Y R 7 / 4$ & Clay & Forest cover; mature trees; palmettos; leaf ground cover & Subsoil \\
\hline 1012RC50 & 1 & $0-40$ & 10 & Negative & $10 Y R 7 / 4$ & Clay & Forest cover; mature trees; palmettos; leaf ground cover & Subsoil \\
\hline 1012RC52 & 1 & $0-30$ & 10 & Negative & $10 Y R 7 / 4$ & Clay & Row cleared; short grass; scattered brush & Subsoil \\
\hline 1012RC55 & 1 & $0-15$ & 10 & Negative & $10 Y R 7 / 4$ & Clay & Row cleared; short grass; scattered brush & Subsoil \\
\hline 1012RC58 & 1 & $0-30$ & 10 & Negative & $10 Y R 7 / 4$ & Silt & Row cleared; short grass; scattered brush & Soil change \\
\hline 1012RC58 & 2 & $30-40$ & 10 & Negative & $10 Y R 7 / 4$ & Silt & Row cleared; short grass; scattered brush & Subsoil \\
\hline $1012 \mathrm{RC} 60$ & 1 & $0-40$ & 10 & Negative & $10 \mathrm{YR} 7 / 4$ & Silt & Row cleared; short grass; scattered brush & Subsoil \\
\hline 1012RC63 & 1 & $0-20$ & 10 & Negative & $10 Y R 7 / 4$ & Clay & Row cleared; short grass; scattered brush & Subsoil \\
\hline 1012RC66 & 1 & $0-10$ & 10 & Negative & $10 Y R 7 / 4$ & Silt & Row cleared; short grass; scattered brush & Disturbed \\
\hline 1012RC68 & 1 & $0-10$ & 0 & Negative & $10 \mathrm{YR} 4 / 4$ & Clay loam & Row cleared; cut short grass & Subsoil \\
\hline 1012RC71 & 1 & $0-10$ & 0 & Negative & $10 Y R 7 / 4$ & Clay loam & Row cleared; cut short grass & Soil change \\
\hline 1012RC71 & 2 & $10-15$ & 0 & Negative & $10 Y R 7 / 4$ & Clay loam & Row cleared; cut short grass & Subsoil \\
\hline 1012RC73 & 1 & $0-10$ & 10 & Negative & 10YR $4 / 1$ & Clay loam & Forest cover & Subsoil \\
\hline 1012RC76 & 1 & $0-10$ & 10 & Negative & $10 Y R 4 / 1$ & Clay loam & Forest cover & Soil change \\
\hline 1012RC76 & 2 & $10-20$ & 10 & Negative & 10YR 5/1 & Clay loam & Forest cover & Subsoil \\
\hline 1012RC77 & 1 & $0-10$ & 10 & Negative & $10 \mathrm{YR} 4 / 1$ & Clay loam & Forest cover & Soil change \\
\hline 1012RC77 & 2 & $10-30$ & 10 & Negative & 10YR $5 / 1$ & Clay loam & Forest cover & Subsoil \\
\hline 1012TR01 & 1 & $0-15$ & 10 & Negative & 10YR $4 / 2$ & Loamy clay & Grassy road corridor between two wooded areas & Subsoil \\
\hline 1012TR01 & 2 & $15-25$ & 10 & Negative & 10YR $5 / 2$ & Loamy clay & Grassy road corridor between two wooded areas & Subsoil \\
\hline 1012TR04 & 1 & $0-7$ & 5 & Negative & $10 Y R 4 / 2$ & Loamy clay & Mixed oak woodland; thin understory; thick leaf duff & Soil change \\
\hline 1012TR04 & 2 & $7-24$ & 5 & Negative & $10 Y R 5 / 2$ & Loamy clay & Mixed oak woodland; thin understory; thick leaf duff & Subsoil \\
\hline 1012TR08 & 1 & $0-10$ & 5 & Negative & 10YR $4 / 2$ & Loamy clay & Mixed oak woodland; thin understory; thick leaf duff; friable soil & Soil change \\
\hline 1012TR08 & 2 & $10-20$ & 5 & Negative & $10 Y R 5 / 2$ & Loamy clay & Mixed oak woodland; thin understory; thick leaf duff; friable soil & Subsoil \\
\hline 1012TR11 & 1 & $0-15$ & 0 & Negative & 10YR $4 / 2$ & Loam & Mixed oak woodland; thin understory; thick leaf duff; root disturbance & Soil change \\
\hline 1012TR11 & 2 & $15-25$ & 0 & Negative & 10YR $5 / 2$ & Loam & Mixed oak woodland; thin understory; thick leaf duff; root disturbance & Subsoil \\
\hline 1012TR14 & 1 & $0-35$ & 0 & Negative & 10YR 4/1 & Loam & Mixed pine forest; thick pine needle and leaf duff & Roots \\
\hline 1012TR19 & 1 & $0-60$ & 0 & Negative & 10YR 4/1 & Clay loam & Mixed pine forest; thick pine needle and leaf duff & Impenetrable \\
\hline 1012TR32 & 1 & $0-20$ & 5 & Negative & 10YR 4/1 & Loam & Mixed oak and pine woodland; moderate understory; some palms & Roots \\
\hline 1012TR34 & 1 & $0-20$ & 70 & Negative & $10 \mathrm{YYR} 4 / 2$ & Sandy clay & Mixed oak and pine woodland; moderate understory; some palms & Soil change \\
\hline 1012TR34 & 2 & $20-40$ & 70 & Negative & $10 Y R 5 / 2$ & Clay & Mixed oak and pine woodland; moderate understory; some palms & Subsoil \\
\hline 1012TR43 & 1 & $0-15$ & 10 & Negative & $10 \mathrm{YR} 4 / 2$ & Sandy loam & Mixed oak and pine woodland; moderate understory; moderate growth of palms & Soil change \\
\hline 1012TR43 & 2 & $15-30$ & 10 & Negative & $10 Y R 5 / 2$ & Sandy clay & Mixed oak and pine woodland; moderate understory; moderate growth of palms & Subsoil \\
\hline 1012TR51 & 1 & $0-15$ & 0 & Negative & $10 Y R 4 / 2$ & Sandy loam & Mixed oak woodland; thin understory; thick leaf duff & Soil change \\
\hline 1012TR51 & 2 & $15-30$ & 0 & Negative & 10YR $5 / 2$ & Sandy loam & Mixed oak woodland; thin understory; thick leaf duff & Subsoil \\
\hline 1012TR57 & 1 & $0-50$ & 90 & Negative & $10 Y R 7 / 4$ & Sandy loam & Within row; soil is very homogenous; likely imported fill & Disturbed \\
\hline 1012TR61 & 1 & $0-30$ & 0 & Negative & $10 Y R 7 / 4$ & Sandy loam & Within row; soil is very homogenous; likely imported fill & Disturbed \\
\hline 101218WE42 & 1 & $0-10$ & 50 & Negative & 10YR $4 / 2$ & Silty loam & Grassy row between two forested areas & Soil change \\
\hline 101218WE42 & 2 & $10-25$ & 50 & Negative & 10YR $5 / 2$ & Silty clay & Grassy row between two forested areas & Subsoil \\
\hline 1012TR71 & 1 & $0-25$ & 100 & Negative & 10YR $4 / 2$ & Silty loam & Grassy row between two forested areas & Soil change \\
\hline 1012TR71 & 2 & $25-35$ & 100 & Negative & $10 Y R 5 / 2$ & Silty loam & Grassy row between two forested areas & Subsoil \\
\hline 101218RC64 & 1 & $0-10$ & 10 & Negative & 10YR $4 / 2$ & Silty loam & Mixed oak and pine woodland; thin understory; some palms & Soil change \\
\hline 101218RC64 & 2 & $10-30$ & 10 & Negative & 10YR $5 / 2$ & Silty clay & Mixed oak and pine woodland; thin understory; some palms & Subsoil \\
\hline 1012WE05 & 1 & $0-10$ & 2 & Negative & 10YR 4/1 & Clay & Utility corridor; edge of forest; short grass & Soil change \\
\hline 1012WE05 & 2 & $10-15$ & 2 & Negative & 10YR 3/1 & Clay & Utility corridor; edge of forest; short grass & Subsoil \\
\hline 1012WE07 & 1 & $0-10$ & 1 & Negative & 10YR $4 / 1$ & Clay loam & Utility corridor; edge of forest; short grass & Soil change \\
\hline 1012WE07 & 2 & $10-15$ & 1 & Negative & $10 \mathrm{Yr} 3 / 1$ & Clay loam & Utility corridor; edge of forest; short grass & Subsoil \\
\hline 1012WE10 & 1 & $0-10$ & 10 & Negative & 10YR $4 / 1$ & Clay loam & Forest; roots & Soil change \\
\hline 1012WE10 & 2 & $10-15$ & 10 & Negative & 10YR 3/1 & Clay loam & Forest; roots & Subsoil \\
\hline 1012WE13 & 1 & $0-10$ & 10 & Negative & 10YR $4 / 1$ & Sandy clay & Forest; roots & Soil change \\
\hline 1012WE13 & 2 & $10-15$ & 10 & Negative & 10YR $3 / 1$ & Sandy clay & Forest; roots & Subsoil \\
\hline 1012WE17 & 1 & $0-10$ & 5 & Negative & 10YR 4/1 & Clay loam & Pine forest & Soil change \\
\hline
\end{tabular}




\begin{tabular}{|c|c|c|c|c|c|c|c|c|}
\hline Shovel Test \# & $\begin{array}{l}\text { Level } \\
\text { (Strat) }\end{array}$ & $\begin{array}{l}\text { Depth } \\
\text { (cmbs) }\end{array}$ & GSV \% & $\begin{array}{c}\text { Positive or } \\
\text { Negative }\end{array}$ & $\begin{array}{l}\text { Munsell } \\
\text { Color }\end{array}$ & Soil Texture Description & Description (Area; Vegetation) & Reason for Termination \\
\hline 1012WE17 & 2 & $10-15$ & 5 & Negative & 10YR $3 / 1$ & Clay loam & Pine forest & Subsoil \\
\hline 1012WE18 & 1 & $0-10$ & 2 & Negative & 10YR $4 / 1$ & Clay loam & Forest & Soil change \\
\hline 1012WE18 & 2 & $10-20$ & 2 & Negative & 10YR $3 / 1$ & Clay loam & Forest & Subsoil \\
\hline 1012WE21 & 1 & $0-8$ & 2 & Negative & 10YR $4 / 1$ & Clay loam & Pine forest & Soil change \\
\hline 1012WE21 & 2 & $8-15$ & 2 & Negative & $10 Y R 3 / 1$ & Clay loam & Pine forest & Subsoil \\
\hline 1012WE24 & 1 & $0-10$ & 5 & Negative & 10YR $4 / 1$ & Loamy clay & Forest & Soil change \\
\hline 1012WE24 & 2 & $10-15$ & 5 & Negative & 10YR $3 / 1$ & Loamy clay & Forest & Subsoil \\
\hline 1012WE26 & 1 & $0-10$ & 2 & Negative & 10YR 4/1 & Sandy clay & Forest; roots & Soil change \\
\hline 1012WE26 & 2 & $10-15$ & 2 & Negative & $10 Y R 3 / 1$ & Sandy clay & Forest; roots & Subsoil \\
\hline 1012WE28 & 1 & $0-20$ & 1 & Negative & 10YR $4 / 1$ & Sand & Forest; roots; sandy hill & Soil change \\
\hline 1012WE28 & 2 & $20-25$ & 1 & Negative & 10YR $3 / 1$ & Sand & Forest; roots; sandy hill & Subsoil \\
\hline 1012WE31 & 1 & $0-10$ & 0 & Negative & 10YR $4 / 1$ & Clay & Cleared path in forest area & Soil change \\
\hline 1012WE31 & 2 & $10-15$ & 0 & Negative & 10YR $3 / 1$ & Clay & Cleared path in forest area & Subsoil \\
\hline 1012WE35 & 1 & $0-15$ & 5 & Negative & 10YR $4 / 1$ & Sand & Forest; thick brush and thorns; roots & Soil change \\
\hline 1012WE35 & 2 & $15-25$ & 5 & Negative & 10YR $3 / 1$ & Sand & Forest; thick brush and thorns; roots & Subsoil \\
\hline 1012WE38 & 1 & $0-40$ & 2 & Negative & $10 Y R 7 / 4$ & Loam & Forest; thick brush and thorns; roots; likely imported fill & Soil change \\
\hline 1012WE41 & 1 & $0-10$ & 3 & Negative & 10YR $4 / 1$ & Clay loam & Forest; thick brush and thorns; roots & Subsoil \\
\hline 1012WE41 & 2 & $10-15$ & 3 & Negative & $10 \mathrm{YY} 3 / 1$ & Clay loam & Forest; thick brush and thorns; roots & Soil change \\
\hline 1012WE45 & 1 & $0-10$ & 5 & Negative & 10YR $4 / 1$ & Clay loam & Forest; thick brush; roots & Subsoil \\
\hline 1012WE45 & 2 & $10-15$ & 5 & Negative & 10YR $3 / 1$ & Clay loam & Forest; thick brush; roots & Soil change \\
\hline 1012WE48 & 1 & $0-10$ & 2 & Negative & 10YR 4/1 & Clay loam & Forest; thick brush; roots & Subsoil \\
\hline 1012WE48 & 2 & $10-15$ & 2 & Negative & $10 Y R 3 / 1$ & Clay loam & Forest; thick brush; roots & Soil change \\
\hline 1012WE50 & 1 & $0-10$ & 1 & Negative & 10YR $4 / 1$ & Clay loam & Forest; thick brush; roots & Subsoil \\
\hline 1012WE50 & 2 & $10-15$ & 1 & Negative & 10YR $3 / 1$ & Clay loam & Forest; thick brush; roots & Soil change \\
\hline 1012WE53 & 1 & $0-10$ & 0 & Negative & $10 Y R 4 / 1$ & Clay & Utility corridor; edge of forest; short grass & Subsoil \\
\hline 1012WE53 & 2 & $10-15$ & 0 & Negative & 10YR $3 / 1$ & Clay & Utility corridor; edge of forest; short grass & Soil change \\
\hline 1012WE54 & 1 & $0-8$ & 1 & Negative & 10YR $4 / 1$ & Clay & Utility corridor; edge of forest; short grass & Subsoil \\
\hline 1012WE54 & 2 & $8-15$ & 1 & Negative & 10YR $3 / 1$ & Clay & Utility corridor; edge of forest; short grass & Soil change \\
\hline 1012WE56 & 1 & $0-5$ & 1 & Negative & 10YR $4 / 1$ & Silty clay & Utility corridor; short grass & Subsoil \\
\hline 1012WE56 & 2 & $5-10$ & 1 & Negative & 10YR $3 / 1$ & Silty clay & Utility corridor; short grass & Soil change \\
\hline 1012WE58 & 1 & $0-50$ & 1 & Negative & $10 Y R 7 / 4$ & Sandy loam & Utility corridor; homogenous soil; likely imported fill & Subsoil \\
\hline 1012WE63 & 1 & $0-10$ & 1 & Negative & 10YR $4 / 1$ & Clay loam & Utility corridor; short grass & Soil change \\
\hline 1012WE63 & 2 & $10-15$ & 1 & Negative & $10 \mathrm{Yr} 3 / 1$ & Clay loam & Utility corridor; short grass & Subsoil \\
\hline 1012WE65 & 1 & $0-30$ & 1 & Negative & $10 Y R 7 / 4$ & Sandy loam & Utility corridor; homogenous soil; likely imported fill & Disturbed \\
\hline 1012WE69 & 1 & $0-10$ & 0 & Negative & 10YR $4 / 1$ & Clay loam & Utility corridor; short grass & Subsoil \\
\hline 1012WE69 & 2 & $10-20$ & 0 & Negative & 10YR $3 / 1$ & Clay & Utility corridor; short grass & Subsoil \\
\hline 101218TR74 & 1 & $0-10$ & 0 & Negative & 10YR $4 / 1$ & Clay loam & Utility corridor; short grass & Soil change \\
\hline 101218TR74 & 2 & $10-20$ & 0 & Negative & 10YR $3 / 1$ & Clay & Utility corridor; short grass & Subsoil \\
\hline 101218TR67 & 1 & $0-10$ & 0 & Negative & 10YR $4 / 1$ & Clay loam & Utility corridor; short grass & Soil change \\
\hline 101218TR67 & 2 & $10-15$ & 0 & Negative & 10YR $3 / 1$ & Clay & Utility corridor; short grass & Subsoil \\
\hline 101218TR39 & 1 & $0-10$ & 0 & Negative & 10YR $4 / 1$ & Clay loam & Utility corridor; short grass & Soil change \\
\hline 101218TR39 & 2 & $10-20$ & 0 & Negative & 10YR $3 / 1$ & Clay & Utility corridor; short grass & Subsoil \\
\hline 101218TR35 & 1 & $0-10$ & 0 & Negative & 10YR $4 / 1$ & Clay loam & Utility corridor; short grass & Soil change \\
\hline 101218TR35 & 2 & $10-15$ & 0 & Negative & 10YR $3 / 1$ & Clay & Utility corridor; short grass & Subsoil \\
\hline 101218TR23 & 1 & $0-10$ & 0 & Negative & 10YR $4 / 1$ & Clay loam & Utility corridor; short grass & Soil change \\
\hline 101218TR23 & 2 & $10-20$ & 0 & Negative & 10YR $3 / 1$ & Clay & Utility corridor; short grass & Subsoil \\
\hline 1012WE73 & 1 & $0-10$ & 0 & Negative & 10YR $4 / 1$ & Clay & Utility corridor; short grass & Soil change \\
\hline
\end{tabular}




\begin{tabular}{|c|c|c|c|c|c|c|c|c|}
\hline Shovel Test \# & $\begin{array}{l}\text { Level } \\
\text { (Strat) }\end{array}$ & $\begin{array}{l}\text { Depth } \\
\text { (cmbs) }\end{array}$ & GSV \% & $\begin{array}{c}\text { Positive or } \\
\text { Negative }\end{array}$ & $\begin{array}{l}\text { Munsell } \\
\text { Color }\end{array}$ & Soil Texture Description & Description (Area; Vegetation) & Reason for Termination \\
\hline 1012WE73 & 2 & $10-15$ & 0 & Negative & 10YR $3 / 1$ & Clay & Utility corridor; short grass & Subsoil \\
\hline 1012 WE76 & 1 & $0-10$ & 2 & Negative & 10YR $4 / 1$ & Clay loam & Forest & Soil change \\
\hline 1012WE76 & 2 & $10-15$ & 2 & Negative & 10YR $3 / 1$ & Clay loam & Forest & Subsoil \\
\hline 1013WE02 & 1 & $0-15$ & 0 & Negative & $10 Y R 7 / 2$ & Clay & Cow pasture; grassy field & Soil change \\
\hline 1013WE02 & 2 & $15-20$ & 0 & Negative & $10 Y R 7 / 2$ & Clay & Cow pasture; grassy field & Subsoil \\
\hline 1013WE05 & 1 & $0-10$ & 0 & Negative & $10 Y R 7 / 2$ & Clay & Cow pasture; grassy field & Inundation \\
\hline 1013WE05 & 2 & $10-15$ & 0 & Negative & $10 Y R 7 / 2$ & Clay & Cow pasture; grassy field & Inundation \\
\hline 1013WE07 & 1 & $0-5$ & 0 & Negative & $10 Y R 7 / 2$ & Clay & Cow pasture; grassy field & Soil change \\
\hline 1013WE07 & 2 & $5-10$ & 0 & Negative & $10 \mathrm{YR} 7 / 2$ & Clay & Cow pasture; grassy field & Subsoil \\
\hline 1013WE10 & 1 & $0-10$ & 0 & Negative & $10 Y R 7 / 2$ & Clay & Cow pasture; tall grass; between fence and forest & Soil change \\
\hline 1013WE10 & 2 & $10-15$ & 0 & Negative & $10 Y R 7 / 2$ & Clay & Cow pasture; tall grass; between fence and forest & Subsoil \\
\hline 1013WE12 & 1 & $0-10$ & 1 & Negative & $10 \mathrm{YR} 7 / 2$ & Clay & Forest; Short trees & Soil change \\
\hline 1013WE12 & 2 & $10-15$ & 1 & Negative & $10 \mathrm{YR} 7 / 2$ & Clay & Forest; Short trees & Subsoil \\
\hline 1013WE15 & 1 & $0-10$ & 0 & Negative & $10 Y R 7 / 4$ & Clay loam & Utility corridor between trees; short grass & Soil change \\
\hline 1013WE15 & 2 & $10-15$ & 0 & Negative & $10 Y R 7 / 4$ & Clay loam & Utility corridor between trees; short grass & Subsoil \\
\hline 1013WE19 & 1 & $0-10$ & 0 & Negative & $10 \mathrm{YR} 7 / 4$ & Silty clay & Field between forest and fence & Soil change \\
\hline 1013WE19 & 2 & $10-15$ & 0 & Negative & $10 Y R 7 / 4$ & Silty clay & Field between forest and fence & Subsoil \\
\hline 1013WE21 & 1 & $0-15$ & 0 & Negative & $10 Y R 7 / 4$ & Sandy loam & Cow pasture; short grass & Soil change \\
\hline 1013WE21 & 2 & $15-20$ & 0 & Negative & $10 \mathrm{YR} 7 / 4$ & Sandy loam & Cow pasture; short grass & Subsoil \\
\hline 1013WE24 & 1 & $0-15$ & 0 & Negative & $10 Y R 7 / 4$ & Silty clay & Cow pasture adjacent to wetland & Soil change \\
\hline 1013WE24 & 2 & $15-20$ & 0 & Negative & 10YR $7 / 4$ & Silty clay & Cow pasture adjacent to wetland & Subsoil \\
\hline 1013WE26 & 1 & $0-20$ & 0 & Negative & 10YR $7 / 4$ & Sandy loam & Open cow pasture & Soil change \\
\hline 1013WE26 & 2 & $20-30$ & 0 & Negative & $10 Y R 7 / 4$ & Sandy loam & Open cow pasture & Subsoil \\
\hline 1013WE28 & 1 & $0-50$ & 0 & Negative & $10 Y R 7 / 4$ & Sandy loam & Cow pasture; deep homogenous soil & Subsoil \\
\hline 1013WE31 & 1 & $0-30$ & 0 & Negative & $10 Y R 7 / 4$ & Sandy clay & Open cow pasture & Soil change \\
\hline 1013WE31 & 2 & $30-40$ & 0 & Negative & $10 Y R 7 / 4$ & Sandy clay & Open cow pasture & Subsoil \\
\hline 1013WE35 & 1 & $0-10$ & 0 & Negative & $10 Y R 7 / 2$ & Clay & Forest; cleared area & Soil change \\
\hline 1013WE35 & 2 & $10-20$ & 0 & Negative & $10 \mathrm{YR} 7 / 2$ & Clay & Forest; cleared area & Subsoil \\
\hline 1013WE37 & 1 & $0-15$ & 0 & Negative & $10 \mathrm{YR} 7 / 2$ & Sandy clay & Open field between fence and forest & Soil change \\
\hline 1013WE37 & 2 & $15-25$ & 0 & Negative & $10 Y R 7 / 2$ & Sandy clay & Open field between fence and forest & Subsoil \\
\hline 1013WE40 & 1 & $0-15$ & 0 & Negative & $10 \mathrm{YR} 7 / 2$ & Clay & Open cow pasture & Soil change \\
\hline 1013WE40 & 2 & $15-25$ & 0 & Negative & $10 \mathrm{YR} 7 / 2$ & Clay & Open cow pasture & Subsoil \\
\hline 1013TR01 & 1 & $0-10$ & 50 & Negative & $10 \mathrm{YR} 4 / 2$ & Loamy Clay & Grassy field boarded by woods grass 6 inches tall, lots of tracks & Soil change \\
\hline 1013TR01 & 2 & $10-25$ & 50 & Negative & $10 Y R 4 / 3$ & Loamy Clay & Grassy field boarded by woods grass 6 inches tall, lots of tracks & Subsoil \\
\hline 1013TR04 & 1 & $0-25$ & 30 & Negative & $10 Y R 4 / 2$ & Loamy Clay & Grassy field boarded by woods grass 3 inches tall, lots of tracks & Soil change \\
\hline 1013TR04 & 2 & $25-35$ & 30 & Negative & 10YR $4 / 3$ & Clay & Grassy field boarded by woods grass 3 inches tall, lots of tracks & Subsoil \\
\hline 1013TR09 & 1 & $0-15$ & 30 & Negative & 10YR $4 / 2$ & Loamy Clay & Grassy field boarded by woods grass 3 inches tall, lots of tracks & Soil change \\
\hline 1013TR09 & 2 & $15-25$ & 30 & Negative & $10 Y R 4 / 3$ & Clay & Grassy field boarded by woods grass 3 inches tall, lots of tracks & Subsoil \\
\hline 1013TR14 & 1 & $0-15$ & 0 & Negative & $10 Y R 4 / 2$ & Loamy Clay & Grassy open canopy surrounded by woods, grass 6-12 inches & Soil change \\
\hline 1013TR14 & 2 & $15-25$ & 0 & Negative & $10 Y R 4 / 3$ & Clay & Grassy field boarded by woods grass 3 inches tall, lots of tracks & Subsoil \\
\hline 1013TR18 & 1 & $0-15$ & 0 & Negative & 10YR $4 / 2$ & Loamy Clay & Grassy open canopy surrounded by woods, grass 6-12 inches & Soil change \\
\hline 1013TR18 & 2 & $15-25$ & 0 & Negative & $10 \mathrm{YR} 4 / 3$ & Clay & Grassy field boarded by woods grass 3 inches tall, lots of tracks & Subsoil \\
\hline 1013TR22 & 1 & $0-55$ & 0 & Negative & $10 \mathrm{YR} 7 / 4$ & Sandy loam & Grassy field, surrounded by woods grass 6-12 inches tall, thin shrubbery & Subsoil \\
\hline 1013TR27 & 1 & $0-55$ & 0 & Negative & $10 Y R 7 / 4$ & Sandy loam & Grassy field, surrounded by woods grass 6-12 inches tall, thin shrubbery & Subsoil \\
\hline 1013TR30 & 1 & $0-40$ & 0 & Negative & $10 \mathrm{YR} 7 / 5$ & Sandy loam & Grassy field, surrounded by woods grass 6-12 inches tall, thin shrubbery & Subsoil \\
\hline 1013TR33 & 1 & $0-15$ & 50 & Negative & 1OYR $7 / 4$ & Clay & Grassy field, surrounded by woods grass $6-12$ inches tall, thin shrubbery & Soil change \\
\hline 1013TR33 & 2 & $15-25$ & 50 & Negative & $10 \mathrm{YR} 7 / 2$ & Clay & Grassy field, surrounded by woods grass 6-12 inches tall, thin shrubbery & Subsoil \\
\hline 1013TR38 & 1 & $0-10$ & 0 & Negative & 10YR $7 / 4$ & Clay & Grassy field, surrounded by woods grass 6-12 inches tall, thin shrubbery & Soil change \\
\hline 1013TR38 & 2 & $10-30$ & 0 & Negative & $10 \mathrm{YR} 7 / 2$ & Clay loam & Grassy field, surrounded by woods grass 6-12 inches tall, thin shrubbery & Subsoil \\
\hline 1013TR41 & 1 & $0-25$ & 0 & Negative & $10 \mathrm{YR} 7 / 4$ & Clay loam & Grassy field, surrounded by woods grass $6-12$ inches tall, thin shrubbery & Subsoil \\
\hline 1013RC03 & 1 & $0-20$ & 0 & Negative & $10 Y R 7 / 4$ & Clay loam & Open cow pasture; short-medium grass; trees along property line & Subsoil \\
\hline $1013 \mathrm{RC} 06$ & 1 & $0-30$ & 0 & Negative & $10 \mathrm{YR} 7 / 4$ & Clay loam & Open cow pasture; short-medium grass; trees along property line & Subsoil \\
\hline 1013RC08 & 1 & $0-20$ & 0 & Negative & $10 \mathrm{YR} 7 / 4$ & Clay loam & Open cow pasture; short-medium grass; trees along property line & Subsoil \\
\hline 1013RC11 & 1 & $0-20$ & 0 & Negative & 10YR $7 / 4$ & Clay loam & Open cow pasture; short-medium grass; trees along property line & Subsoil \\
\hline 1013RC12 & 1 & $0-15$ & 0 & Negative & $10 \mathrm{YR} 7 / 4$ & Clay loam & Open cow pasture; short-medium grass; trees along property line & Subsoil \\
\hline 1013RC15 & 1 & $0-20$ & 0 & Negative & $10 Y R 7 / 4$ & Clay loam & Open cow pasture; short-medium grass; trees along property line & Subsoil \\
\hline 1013RC17 & 1 & $0-15$ & 0 & Negative & $10 Y R 7 / 4$ & Clay loam & Open cow pasture; short-medium grass; trees along property line & Subsoil \\
\hline
\end{tabular}




\begin{tabular}{|c|c|c|c|c|c|c|c|c|}
\hline Shovel Test \# & $\begin{array}{l}\text { Level } \\
\text { (Strat) }\end{array}$ & $\begin{array}{l}\text { Depth } \\
\text { (cmbs) }\end{array}$ & GSV \% & $\begin{array}{l}\text { Positive or } \\
\text { Negative }\end{array}$ & $\begin{array}{l}\text { Munsell } \\
\text { Color }\end{array}$ & Soil Texture Description & Description (Area; Vegetation) & Reason for Termination \\
\hline 1013RC20 & 1 & $0-15$ & 0 & Negative & 10YR $7 / 4$ & Clay loam & Open cow pasture; short-medium grass; trees along property line & Subsoil \\
\hline 1013RC23 & 1 & $0-20$ & 0 & Negative & 10YR $7 / 4$ & Clay loam & Open grass cow pasture; short grass & Subsoil \\
\hline 1013RC25 & 1 & $0-20$ & 0 & Negative & 10YR $7 / 4$ & Clay loam & Open grass cow pasture; short grass & Subsoil \\
\hline 1013RC28 & 1 & $0-60$ & 0 & Negative & 10YR $7 / 4$ & Silty loam & Open grass cow pasture; short grass & Subsoil \\
\hline 1013RC31 & 1 & $0-50$ & 0 & Negative & 10YR $7 / 4$ & Silty loam & Open grass cow pasture; short grass & Subsoil \\
\hline 1013RC34 & 1 & $0-30$ & 0 & Negative & 10YR $7 / 4$ & Clay loam & Forest cover; thick brush & Subsoil \\
\hline 1013RC36 & 1 & $0-30$ & 0 & Negative & 10YR $7 / 4$ & Clay loam & Forest cover; thick brush & Subsoil \\
\hline 1013RC39 & 1 & $0-30$ & 0 & Negative & 10YR $7 / 4$ & Clay loam & Open grass pasture; short grass & Subsoil \\
\hline 1018RC03 & 1 & $0-30$ & 10 & Negative & 10YR $4 / 1$ & Silty loam & Cow pasture very wet ground, trees along property live, snorf grass & Soil change \\
\hline 1018RC03 & 2 & $30-50$ & 10 & Negative & 10YR $4 / 2$ & Silty loam & Cow pasture very wet ground, trees along property live, snorf grass & Subsoil \\
\hline $1018 \mathrm{RCO6}$ & 1 & $0-15$ & 10 & Negative & 10YR $4 / 1$ & Silty loam & Cow pasture very wet ground, trees along property live, snorf grass & Soil change \\
\hline 1018RC06 & 2 & $15-20$ & 10 & Negative & 10YR $4 / 2$ & Silty loam & Cow pasture very wet ground, trees along property live, snorf grass & Subsoil \\
\hline $1018 \mathrm{RC10}$ & 1 & $0-15$ & 10 & Negative & 10YR $4 / 1$ & Silty loam & Cow pasture very wet ground, trees along property live, snorf grass & Soil change \\
\hline 1018RC10 & 2 & $15-20$ & 10 & Negative & 10YR $4 / 2$ & Silty loam & Cow pasture very wet ground, trees along property live, snorf grass & Subsoil \\
\hline $1018 \mathrm{RC13}$ & 1 & $0-15$ & 10 & Negative & 10YR $4 / 1$ & Silty loam & Forest cover, tall grass brush vines & Subsoil \\
\hline 1018RC17 & 1 & $0-10$ & 10 & Negative & 10YR $4 / 1$ & Silty loam & Forest cover, tall grass brush vines & Subsoil \\
\hline 1018RC19 & 1 & $0-15$ & 10 & Negative & 10YR $4 / 2$ & Silty loam & Cow pasture, very rutty, trees along, property & Soil change \\
\hline 1018RC19 & 2 & $15-20$ & 10 & Negative & 10YR $5 / 1$ & Silty loam & Cow pasture, very rutty, trees along, property & Subsoil \\
\hline 1018RC22 & 1 & $0-10$ & 10 & Negative & 10YR 4/1 & Silty loam & Open cow pasture; short-medium grass; trees along property line & Soil change \\
\hline $1018 \mathrm{RC} 22$ & 2 & $10-15$ & 10 & Negative & 10YR $5 / 1$ & Silty loam & Open cow pasture; short-medium grass; trees along property line & Subsoil \\
\hline 1018RC24 & 1 & $0-10$ & 10 & Negative & 10YR $4 / 1$ & Silty loam & Cow pasture short-med, grass. Some inundation & Soil change \\
\hline 1018RC24 & 2 & $10-15$ & 10 & Negative & 10YR $4 / 2$ & Silty loam & Cow pasture short-med, grass. Some inundation & Subsoil \\
\hline 1018RC32 & 1 & $0-15$ & 10 & Negative & 10YR $4 / 1$ & Clay loam & Cow pasture short-med, grass. Some inundation & Soil change \\
\hline 1018RC32 & 2 & $15-20$ & 10 & Negative & 10YR $4 / 1$ & Clay loam & Cow pasture short-med, grass. Some inundation & Subsoil \\
\hline 1018RC47 & 1 & $0-10$ & 0 & Negative & 10YR $4 / 1$ & Silty loam & Cow pasture short-med, grass. Some inundation & Soil change \\
\hline 1018RC47 & 2 & $10-20$ & 0 & Negative & 10YR $4 / 1$ & Silty loam & Cow pasture short-med, grass. Some inundation & Subsoil \\
\hline 1018WE01 & 1 & $0-30$ & 0 & Negative & 10YR $4 / 2$ & Sandy loam & Cow Pasture & Soil change \\
\hline 1018WE01 & 2 & $30-60$ & 0 & Negative & 10YR $4 / 2$ & Sandy loam & Cow Pasture & Subsoil \\
\hline 1018WE04 & 1 & $0-10$ & 0 & Negative & 10YR $4 / 2$ & Sandy loam & Cow Pasture & Soil change \\
\hline 1018WE04 & 2 & $10-40$ & 0 & Negative & 10YR $4 / 2$ & Sandy loam & Cow Pasture & Subsoil \\
\hline 1018WE07 & 1 & $0-15$ & 0 & Negative & 10YR $4 / 2$ & Sandy loam & Cow Pasture & Soil change \\
\hline 1018WE07 & 2 & $15-30$ & 0 & Negative & 10YR $4 / 2$ & Sandy loam & Cow Pasture & Subsoil \\
\hline 1018WE09 & 1 & $0-15$ & 0 & Negative & 10YR $4 / 2$ & Silty loam & Clear cow pasture & Soil change \\
\hline 1018WE09 & 2 & $15-30$ & 0 & Negative & 10YR $4 / 2$ & Silty loam & Clear cow pasture & Subsoil \\
\hline 1018WE12 & 1 & $0-10$ & 0 & Negative & 10YR $4 / 2$ & Silty clay & Edge of forest utility corridor & Soil change \\
\hline 1018WE12 & 2 & $10-20$ & 0 & Negative & 10YR $4 / 2$ & Silty clay & Edge of forest utility corridor & Subsoil \\
\hline 1018WE14 & 1 & $0-10$ & 0 & Negative & 10YR $4 / 2$ & Silty clay & Edge of forest utility corridor & Soil change \\
\hline 1018WE14 & 2 & $10-20$ & 0 & Negative & 10YR $4 / 2$ & Silty clay & Edge of forest utility corridor & Subsoil \\
\hline 1018WE16 & 1 & $0-10$ & 0 & Negative & 10YR $4 / 2$ & Silty clay & Edge of forest utility corridor & Soil change \\
\hline 1018WE16 & 2 & $10-25$ & 0 & Negative & 10YR $4 / 2$ & Silty clay & Edge of forest utility corridor & Subsoil \\
\hline 1018WE18 & 1 & $0-10$ & 0 & Negative & 10YR $4 / 2$ & Silty loam & Open cow pasture & Soil change \\
\hline 1018WE18 & 2 & $10-15$ & 0 & Negative & 10YR $4 / 2$ & Silty loam & Open cow pasture & Subsoil \\
\hline 1018WE20 & 1 & $0-15$ & 0 & Negative & 10YR $4 / 2$ & Silty clay & Open cow pasture & Soil change \\
\hline 1018WE20 & 2 & $15-25$ & 0 & Negative & 10YR $4 / 2$ & Silty clay & Open cow pasture & Subsoil \\
\hline 1018WE23 & 1 & $0-10$ & 0 & Negative & 10YR $4 / 2$ & Silty clay & Disturbed, next to pump station & Soil change \\
\hline 1018WE23 & 2 & $10-15$ & 0 & Negative & 10YR $4 / 2$ & Silty clay & Disturbed, next to pump station & Subsoil \\
\hline 1018WE25 & 1 & $0-15$ & 0 & Negative & 10YR $4 / 2$ & Silty clay & Open cow pasture & Soil change \\
\hline 1018WE25 & 2 & $15-25$ & 0 & Negative & 10YR $4 / 2$ & Silty clay & Open cow pasture & Subsoil \\
\hline 1018WE26 & 1 & $0-20$ & 0 & Negative & 10YR $4 / 2$ & Silty clay & Open cow pasture & Soil change \\
\hline 1018WE26 & 2 & $20-40$ & 0 & Negative & 10YR $4 / 2$ & Silty clay & Open cow pasture & Subsoil \\
\hline 1018WE27 & 1 & $0-15$ & 0 & Negative & 10YR $4 / 2$ & Silty clay & Open cow pasture & Soil change \\
\hline 1018WE27 & 2 & $15-35$ & 0 & Negative & 10YR $4 / 2$ & Silty clay & Open cow pasture & Subsoil \\
\hline 1018WE28 & 1 & $0-15$ & 0 & Negative & 10YR $4 / 2$ & Silty clay & Open cow pasture & Soil change \\
\hline 1018WE28 & 2 & $15-30$ & 0 & Negative & 10YR $4 / 2$ & Silty clay & Open cow pasture & Subsoil \\
\hline 1018WE30 & 1 & $0-10$ & 0 & Negative & 10YR $4 / 2$ & Clay & Open cow pasture, near canal & Soil change \\
\hline 1018WE30 & 2 & $10-15$ & 0 & Negative & 10YR $4 / 2$ & Clay & Open cow pasture, near canal & Subsoil \\
\hline 1018WE34 & 1 & $0-15$ & 0 & Negative & 10YR $4 / 2$ & Sandy clay & Open cow pasture & Soil change \\
\hline
\end{tabular}




\begin{tabular}{|c|c|c|c|c|c|c|c|c|}
\hline Shovel Test \# & $\begin{array}{l}\text { Level } \\
\text { (Strat) }\end{array}$ & $\begin{array}{l}\text { Depth } \\
\text { (cmbs) }\end{array}$ & GSV \% & $\begin{array}{c}\text { Positive or } \\
\text { Negative }\end{array}$ & $\begin{array}{l}\text { Munsell } \\
\text { Color }\end{array}$ & Soil Texture Description & Description (Area; Vegetation) & Reason for Termination \\
\hline 1018WE34 & 2 & $15-25$ & 0 & Negative & 10YR $4 / 2$ & Sandy clay & Open cow pasture & Subsoil \\
\hline 1018WE36 & 1 & $0-10$ & 0 & Negative & 1OYR 4/1 & Clay & Open cow pasture & Soil change \\
\hline 1018WE36 & 2 & $10-15$ & 0 & Negative & 10YR 4/1 & Clay & Open cow pasture & Subsoil \\
\hline 1018WE42 & 1 & $0-15$ & 0 & Negative & 10YR $4 / 2$ & Silty clay & Open cow pasture & Soil change \\
\hline 1018WE42 & 2 & $15-25$ & 0 & Negative & 1OYR $4 / 2$ & Silty clay & Open cow pasture & Subsoil \\
\hline 101818TR8 & 1 & $0-15$ & 0 & Negative & 10YR 4/1 & Silty clay & Open cow pasture & Soil change \\
\hline 101818TR8 & 2 & $15-25$ & 0 & Negative & 1OYR $4 / 2$ & Clay & Open cow pasture & Subsoil \\
\hline 101818TR5 & 1 & $0-10$ & 0 & Negative & 1OYR 4/1 & Silty clay & Open cow pasture & Soil change \\
\hline 101818TR5 & 2 & $10-15$ & 0 & Negative & 10YR $4 / 2$ & Clay & Open cow pasture & Subsoil \\
\hline 101818TR11 & 1 & $0-15$ & 0 & Negative & 10YR $4 / 1$ & Silty clay & Open cow pasture & Soil change \\
\hline 101818TR11 & 2 & $15-25$ & 0 & Negative & 10YR $4 / 2$ & Clay & Open cow pasture & Subsoil \\
\hline 101818TR2 & 1 & $0-15$ & 0 & Negative & 10YR 4/1 & Silty clay & Open cow pasture & Soil change \\
\hline 101818TR2 & 2 & $15-25$ & 0 & Negative & 1OYR $4 / 2$ & Clay & Open cow pasture & Subsoil \\
\hline 101818RC43 & 1 & $0-10$ & 0 & Negative & 10YR $4 / 1$ & Silty clay & Open cow pasture & Soil change \\
\hline 101818RC43 & 2 & $10-15$ & 0 & Negative & 10YR $4 / 2$ & Clay & Open cow pasture & Subsoil \\
\hline 101818RC41 & 1 & $0-15$ & 0 & Negative & 10YR 4/1 & Silty clay & Open cow pasture & Soil change \\
\hline 101818RC41 & 2 & $15-25$ & 0 & Negative & 1OYR $4 / 2$ & Clay & Open cow pasture & Subsoil \\
\hline 101818RC39 & 1 & $0-15$ & 0 & Negative & 10YR $4 / 1$ & Silty clay & Open cow pasture & Soil change \\
\hline 101818RC39 & 2 & $15-25$ & 0 & Negative & 10YR $4 / 2$ & Clay & Open cow pasture & Subsoil \\
\hline 101818RC37 & 1 & $0-10$ & 0 & Negative & 10YR $4 / 1$ & Silty clay & Open cow pasture & Soil change \\
\hline 101818RC37 & 2 & $10-15$ & 0 & Negative & 1OYR $4 / 2$ & Clay & Open cow pasture & Subsoil \\
\hline 101818RC21 & 1 & $0-15$ & 0 & Negative & 10YR $4 / 1$ & Silty clay & Open cow pasture & Soil change \\
\hline 101818RC21 & 2 & $15-25$ & 0 & Negative & 1OYR $4 / 2$ & Clay & Open cow pasture & Subsoil \\
\hline 1018WE48 & 1 & $0-15$ & 0 & Negative & 10YR $4 / 2$ & Silty clay & Wet, marshlands, wild grasses cattle turbation & Soil change \\
\hline 1018WE48 & 2 & $15-25$ & 0 & Negative & $10 \mathrm{YR} 4 / 2$ & Silty clay & Wet, marshlands, wild grasses cattle turbation & Subsoil \\
\hline 1018WK31 & 1 & $0-20$ & 0 & Negative & $10 \mathrm{YrR} 4 / 2$ & Silty clay loam & Wet, marshlands, wild grasses cattle turbation & Subsoil \\
\hline 1018WK35 & 1 & $0-25$ & 0 & Negative & 10YR $4 / 2$ & Silty clay loam & Wet, marshlands, wild grasses cattle turbation & Soil change \\
\hline 1018WK35 & 2 & $25-35$ & 0 & Negative & $10 \mathrm{YR} 6 / 2$ & Silty clay loam & Wet, marshlands, wild grasses cattle turbation & Subsoil \\
\hline 1018WK45 & 1 & $0-40$ & 5 & Negative & 7.5 YR $5 / 4$ & Silty loam & Stp location 100m west of forest margin. B horizon looked "washed out of hydric" & Subsoil \\
\hline 1018 WK45 & 2 & $40-50$ & 5 & Negative & 10YR 4/1 & Silty loam & Stp location 100m west of forest margin. B horizon looked "washed out of hydric" & Subsoil \\
\hline 1018WK49 & 1 & $0-15$ & 5 & Negative & $10 \mathrm{YR} 4 / 2$ & Silty loam & Wet marshland, wild grasses cattle turbation & Soil change \\
\hline 1018WK49 & 2 & $15-35$ & 5 & Negative & $10 \mathrm{YR} 6 / 2$ & Silty clay loam & Wet marshland, wild grasses cattle turbation & Subsoil \\
\hline 1018JL29 & 1 & $0-10$ & 100 & Negative & 10YR 4/1 & Clay & Inundation, cow pasture & Inundation \\
\hline 1018JL33 & 1 & $0-12$ & 100 & Negative & $10 \mathrm{YR} 4 / 2$ & Clay & Grass two- track, near canal & Soil change \\
\hline 1018JL33 & 2 & $12-35$ & 100 & Negative & $10 Y R 4 / 2$ & Clay & Grass two- track, near canal & Subsoil \\
\hline 1018JL38 & 1 & $0-10$ & 5 & Negative & $10 Y R 4 / 2$ & Silty clay loam & Cow pasture semi-inundation & Subsoil \\
\hline 1018JL40 & 1 & $0-12$ & 10 & Negative & 10YR $4 / 2$ & Silty clay loam & Cow pasture semi-inundation & Soil change \\
\hline $1018 J \mathrm{JL} 40$ & 2 & $12-35$ & 10 & Negative & 10YR $6 / 2$ & Silty clay loam & Cow pasture semi-inundation & Subsoil \\
\hline $1018 J \mathrm{~J} 44$ & 1 & $0-12$ & 10 & Negative & $10 \mathrm{YR} 4 / 2$ & Silty clay loam & Cow pasture semi-inundation & Soil change \\
\hline $1018 \mathrm{JL} 44$ & 2 & $12-35$ & 10 & Negative & 10YR $6 / 2$ & Silty clay loam & Cow pasture semi-inundation & Subsoil \\
\hline 1018JL46 & 1 & $0-12$ & 10 & Negative & 10YR $4 / 2$ & Silty clay loam & Cow pasture semi-inundation & Soil change \\
\hline 1018JL46 & 2 & $12-35$ & 10 & Negative & $10 \mathrm{YYR} 6 / 2$ & Silty clay loam & Cow pasture semi-inundation & Subsoil \\
\hline $1018 \mathrm{JL} 50$ & 1 & $0-20$ & 10 & Negative & $10 Y R 4 / 2$ & Silty clay loam & Cow pasture semi-inundation & Soil change \\
\hline $1018 \mathrm{JL} 50$ & 2 & $20-30$ & 10 & Negative & $10 Y R 6 / 2$ & Silty clay loam & Cow pasture semi-inundation & Subsoil \\
\hline
\end{tabular}




\begin{tabular}{|c|c|c|c|c|c|c|c|c|}
\hline Shovel Test \# & $\begin{array}{l}\text { Level } \\
\text { (Strat) }\end{array}$ & $\begin{array}{l}\text { Depth } \\
\text { (cmbs) }\end{array}$ & GSV \% & $\begin{array}{l}\text { Positive or } \\
\text { Negative }\end{array}$ & $\begin{array}{l}\text { Munsell } \\
\text { Color }\end{array}$ & Soil Texture Description & Description (Area; Vegetation) & Reason for Termination \\
\hline 1019RC04 & 1 & $0-15$ & 0 & Negative & 10YR $4 / 1$ & Clay loam & Cow pasture, short-med. Grass, rutted soil, trees along property line & Subsoil \\
\hline 1019RC09 & 1 & $0-15$ & 0 & Negative & 10YR 4/1 & Clay loam & Cow pasture, short-med. Grass, rutted soil, trees along property line & Inundation \\
\hline 1019RC10 & 1 & $0-15$ & 0 & Negative & 10YR $4 / 1$ & Clay loam & Cow pasture, short-med. Grass, rutted soil, trees along property line & Soil change \\
\hline 1019RC10 & 2 & $15-20$ & 0 & Negative & 10YR $4 / 2$ & Clay loam & Cow pasture, short-med. Grass, rutted soil, trees along property line & Subsoil \\
\hline 1019RC14 & 1 & $0-30$ & 0 & Negative & 10YR $4 / 1$ & Clay loam & Cow pasture, short-med. Grass, rutted soil, trees along property line & Soil change \\
\hline 1019RC14 & 2 & $30-40$ & 0 & Negative & 10YR $4 / 2$ & Clay loam & Cow pasture, short-med. Grass, rutted soil, trees along property line & Subsoil \\
\hline 1019RC22 & 1 & $0-10$ & 0 & Negative & 10YR $4 / 1$ & Clay loam & Cow pasture, short-med. Grass, rutted soil, trees along property line & Soil change \\
\hline 1019RC22 & 2 & $10-20$ & 0 & Negative & 10YR $4 / 2$ & Clay loam & Cow pasture, short-med. Grass, rutted soil, trees along property line & Subsoil \\
\hline $1019 \mathrm{RC} 26$ & 1 & $0-10$ & 0 & Negative & 10YR $4 / 1$ & Clay loam & Cow pasture, short-med. Grass, rutted soil, trees along property line & Soil change \\
\hline $1019 \mathrm{RC26}$ & 2 & $10-20$ & 0 & Negative & 10YR $4 / 2$ & Clay loam & Cow pasture, short-med. Grass, rutted soil, trees along property line & Subsoil \\
\hline 101918RC35 & 1 & $0-30$ & 0 & Negative & 10YR $4 / 1$ & Clay loam & Cow pasture, short-med. Grass, rutted soil, trees along property line & Soil change \\
\hline 101918RC30 & 1 & $0-30$ & 0 & Negative & 10YR 4/1 & Clay loam & Cow pasture, short-med. Grass, rutted soil, trees along property line & Subsoil \\
\hline 1019RC40 & 1 & $0-30$ & 0 & Negative & 10YR $4 / 1$ & Clay loam & Cow pasture, short-med. Grass, rutted soil, trees along property line & Soil change \\
\hline 1019RC44 & 1 & $0-50$ & 0 & Negative & 10YR $4 / 1$ & Clay loam & Cow pasture & Subsoil \\
\hline 1019RC52 & 1 & $0-10$ & 0 & Negative & 10YR $4 / 1$ & Clay loam & Cow pasture & Soil change \\
\hline 1019RC52 & 2 & $10-30$ & 0 & Negative & 10YR $4 / 2$ & Clay loam & Cow pasture & Subsoil \\
\hline 1019RC56 & 1 & $0-15$ & 0 & Negative & 10YR 4/1 & Clay loam & Cow pasture & Inundation \\
\hline $1019 \mathrm{RC} 60$ & 1 & $0-20$ & 0 & Negative & 10YR 4/1 & Clay loam & Cow pasture & Inundation \\
\hline 1019RC63 & 1 & $0-10$ & 0 & Negative & 10YR 4/1 & Clay & Cow pasture & Subsoil \\
\hline 1019WK02 & 1 & $0-25$ & $0-5$ & Negative & $\begin{array}{c}10 Y R 4 / 2 \\
5 / 2\end{array}$ & Silty clay loam & Wet marsh/pasture. Forest median $\sim 150 \mathrm{~m}$ Stp & Inundation \\
\hline 1019WK06 & 1 & $0-10$ & $0-5$ & Negative & $\begin{array}{c}10 Y R ~ 4 / 2 \\
5 / 2\end{array}$ & Silty clay loam & Wet marsh/ pasture. Standing water $<4 \mathrm{~m}$ west of stp & Inundation \\
\hline 1019WK08 & 1 & $0-25$ & $0-5$ & Negative & 10YR $4 / 2$ & Silty clay loam & Wet marsh/ pasture & Soil change \\
\hline 1019WK08 & 2 & $25-30$ & $0-5$ & Negative & 10YR $5 / 6$ & Silty clay loam & Wet marsh/pasture & Subsoil \\
\hline 1019WK14 & 1 & $0-10$ & 0 & Negative & $\begin{array}{c}10 Y R 4 / 2 \\
5 / 2\end{array}$ & Silty clay loam & $\begin{array}{c}\text { Wet marsh/ pasture. Raised two-track } \sim 7 \mathrm{~m} \text { south of stp. Fence and canal } ~ 10-20 \mathrm{~m} \text { north. High } \\
\text { grass }\end{array}$ & Soil change \\
\hline 1019WK14 & 2 & $10-30$ & 0 & Negative & 10YR $4 / 1$ & Silty clay loam & $\begin{array}{c}\text { Wet marsh/ pasture. Raised two-track } 7 \mathrm{~m} \text { south of stp. Fence and canal } ~ 10-20 \mathrm{~m} \text { north. High } \\
\text { grass }\end{array}$ & Subsoil \\
\hline 1019WK18 & 1 & $0-15$ & $70-75$ & Negative & $\begin{array}{c}10 Y R 4 / 2 \\
5 / 2\end{array}$ & Silty clay loam & $\begin{array}{c}\text { Area is a small storage area for hay and tractors/ farming equipment. Area washed out/ subject to } \\
\text { periods of inundation }\end{array}$ & Soil change \\
\hline 1019WK18 & 2 & $15-30$ & $70-75$ & Negative & 10YR $4 / 1$ & Silty clay loam & $\begin{array}{c}\text { Area is a small storage area for hay and tractors/ farming equipment. Area washed out/ subject to } \\
\text { periods of inundation }\end{array}$ & Subsoil \\
\hline 1019WK20 & 1 & $0-25$ & $10-15$ & Negative & $\begin{array}{c}\text { 10YR } 4 / 2 \\
5 / 2 \\
\end{array}$ & Silty clay loam & Wet marshy pasture & Soil change \\
\hline 1019WK20 & 2 & 25-35 & $10-15$ & Negative & 10YR $4 / 1$ & Silty clay loam & Wet marshy pasture & Subsoil \\
\hline 1019WK24 & 1 & $0-25$ & $0-5$ & Negative & $\begin{array}{c}10 Y R 4 / 2 \\
5 / 2\end{array}$ & Silty clay loam & Wet marshy pasture & Soil change \\
\hline 1019WK24 & 2 & $25-45$ & $0-5$ & Negative & 10YR 4/1 & Silty clay loam & Wet marshy pasture & Subsoil \\
\hline 1019WK28 & 1 & $0-5$ & $0-5$ & Negative & $\begin{array}{c}10 Y R 4 / 2 \\
5 / 2\end{array}$ & Silty clay loam & Wet marshy pasture. Stp was in a deflated area - grass was different/ poor growth & Soil change \\
\hline 1019WK28 & 2 & $5-30$ & $0-5$ & Negative & 10YR $4 / 1$ & Silty clay loam & Wet marshy pasture. Stp was in a deflated area - grass was different/ poor growth & Subsoil \\
\hline 1019WK32 & 1 & $0-30$ & 45 & Negative & $\begin{array}{l}10 Y R 4 / 2 \\
5 / 2\end{array}$ & Silty clay loam & Wet marshy pasture/ deflated area & Soil change \\
\hline 1019WK32 & 2 & $30-40$ & 45 & Negative & 10YR 4/1 & Silty clay loam & Wet marshy pasture/deflated area & Subsoil \\
\hline 1019WK36 & 1 & $0-5$ & 20 & Negative & $\begin{array}{c}10 Y R 4 / 2 \\
5 / 2\end{array}$ & Silty clay loam & Wet marshy pasture/ deflated area sandstone $\sim 12 \mathrm{cmbs}$ & Soil change \\
\hline 1019WK36 & 2 & $5-30$ & 20 & Negative & 10YR $4 / 1$ & Silty loam & Wet marshy pasture/ deflated area sandstone $\sim 12 \mathrm{cmbs}$ & Subsoil \\
\hline 1019Wк39 & 1 & $0-30$ & 0 & Negative & $\begin{array}{c}10 Y R 4 / 2 \\
5 / 2\end{array}$ & Clay loam & Wet marshy pasture - very high grass & Inundation \\
\hline 1019WK43 & 1 & $0-35$ & 0 & Negative & $\begin{array}{c}10 Y R 4 / 2 \\
5 / 2\end{array}$ & Silty clay loam & Wet marshy pasture - very high grass & Inundation \\
\hline 1019WK47 & 1 & $0-20$ & 0 & Negative & $\begin{array}{c}10 Y R 4 / 2 \\
5 / 2\end{array}$ & Silty clay loam & Wet marshy pasture - very high grass & Inundation \\
\hline
\end{tabular}




\begin{tabular}{|c|c|c|c|c|c|c|c|c|}
\hline Shovel Test \# & $\begin{array}{l}\begin{array}{l}\text { Level } \\
\text { (Strat) }\end{array} \\
\end{array}$ & $\begin{array}{l}\text { Depth } \\
\text { (cmbs) }\end{array}$ & GSV \% & $\begin{array}{l}\begin{array}{c}\text { Positive or } \\
\text { Negative }\end{array} \\
\end{array}$ & $\begin{array}{c}\text { Munsell } \\
\text { Color }\end{array}$ & Soil Texture Description & Description (Area; Vegetation) & Reason for Termination \\
\hline $1019 W K 50$ & 1 & $0-10$ & 0 & Negative & $\begin{array}{c}10 Y R \quad 4 / 2 \\
5 / 2\end{array}$ & Silty clay loam & Wet marshy pasture - very high grass & Subsoil \\
\hline 1019WK54 & 1 & $0-10$ & $0-5$ & Negative & $\begin{array}{c}10 Y R 4 / 2 \\
5 / 2\end{array}$ & Silty clay loam & Wet marshy pasture - very high grass & Soil change \\
\hline 1019WK54 & 2 & $10-30$ & $0-5$ & Negative & 10YR 4/1 & Silty clay loam & Wet marshy pasture - very high grass & Subsoil \\
\hline 1019WK58 & 1 & $0-5$ & $70-75$ & Negative & \begin{tabular}{|c|}
1 OYR $4 / 2$ \\
$5 / 2$ \\
\end{tabular} & Silty clay loam & Wet marshy pasture - very high grass & Soil change \\
\hline 1019WK58 & 2 & $5-30$ & $70-75$ & Negative & 10YR $4 / 1$ & Silty clay loam & Wet marshy pasture - very high grass & Subsoil \\
\hline 1019WK62 & 1 & $0-10$ & $0-5$ & Negative & 10YR 4/2 & Silty clay loam & Wet marshy pasture, stp is $5 \mathrm{~m}$ south of two-track & Soil change \\
\hline 1019WK62 & 2 & $10-30$ & $0-5$ & Negative & 10YR 4/1 & Silty clay loam & Wet marshy pasture, stp is $5 \mathrm{~m}$ south of two-track & Subsoil \\
\hline 1019WE01 & 1 & $0-10$ & 0 & Negative & 10YR $4 / 1$ & Clay & Open cow pasture & Inundation \\
\hline 1019WE05 & 1 & $0-15$ & 0 & Negative & 10YR $4 / 1$ & Clay & Open cow pasture & Soil change \\
\hline 1019WE05 & 2 & $15-30$ & 0 & Negative & 10YR 4/1 & Clay loam & Open cow pasture & Subsoil \\
\hline 1019WE13 & 1 & $0-10$ & 0 & Negative & 10YR $4 / 1$ & Clay & Open cow pasture, near fence line \& stream & Soil change \\
\hline 1019WE13 & 2 & $10-20$ & $0-5$ & Negative & 10YR $4 / 1$ & Clay & Open cow pasture, near fence line \& stream & Subsoil \\
\hline 1019WE17 & 1 & $0-20$ & 0 & Negative & 10YR $4 / 2$ & Silty clay & Hay yard, viney plants & Soil change \\
\hline 1019WE17 & 2 & $20-30$ & 0 & Negative & $10 \mathrm{Yr} 4 / 2$ & Silty clay & Hay yard, viney plants & Subsoil \\
\hline 1019WE21 & 1 & $0-15$ & 0 & Negative & 10YR 4/1 & Clay & Open field & Soil change \\
\hline 1019WE21 & 2 & $15-25$ & 0 & Negative & 10YR 4/1 & Clay & Open field & Subsoil \\
\hline 1019WE25 & 1 & $0-15$ & $0-5$ & Negative & 10YR 4/1 & Clay & Open cow pasture & Soil change \\
\hline 1019WE25 & 2 & $15-20$ & 0 & Negative & 10YR 4/1 & Clay & Open cow pasture & Subsoil \\
\hline 1019WE31 & 1 & $0-10$ & 0 & Negative & 10YR 4/1 & Clay & Open cow pasture & Soil change \\
\hline 1019WE31 & 2 & $10-15$ & 0 & Negative & 1OYR $4 / 1$ & Clay & Open cow pasture & Subsoil \\
\hline 1019WE34 & 1 & $0-15$ & 0 & Negative & 1 OYR $4 / 2$ & Clay & Open cow pasture & Soil change \\
\hline 1019WE34 & 2 & $15-25$ & $0-5$ & Negative & 10YR 4/2 & Clay loam & Open cow pasture & Subsoil \\
\hline 1019WE38 & 1 & $0-15$ & 0 & Negative & 10YR $4 / 1$ & Clay & Open cow pasture & Soil change \\
\hline 1019WE38 & 2 & $15-20$ & 0 & Negative & 1OYR $4 / 1$ & Clay & Open cow pasture & Subsoil \\
\hline 1019WE42 & 1 & $0-15$ & 0 & Negative & 1OYR 4/1 & Clay & Open cow pasture & Soil change \\
\hline 1019WE42 & 2 & $15-20$ & 0 & Negative & 10YR $4 / 1$ & Clay & Open cow pasture & Subsoil \\
\hline 1019WE46 & 1 & $10-20$ & 0 & Negative & 10YR 4/1 & Clay & Open cow pasture & Soil change \\
\hline 1019WE46 & 2 & $0-20$ & 0 & Negative & 10YR $4 / 1$ & Clay & Open cow pasture & Subsoil \\
\hline 1019WE49 & 1 & $0-10$ & 0 & Negative & 10YR $4 / 1$ & Clay & Open cow pasture & Soil change \\
\hline 1019WE49 & 2 & $10-15$ & 0 & Negative & 10YR $4 / 1$ & Clay & Open cow pasture & Subsoil \\
\hline 1019WE53 & 1 & $0-20$ & 0 & Negative & $10 Y R 4 / 2$ & Clay loam & Open cow pasture & Soil change \\
\hline 1019WE53 & 2 & $20-25$ & 0 & Negative & 10YR $4 / 2$ & Clay loam & Open cow pasture & Subsoil \\
\hline 1019WE59 & 1 & $0-5$ & 0 & Negative & $10 Y R 4 / 2$ & Silty clay & Open cow pasture, near pump station & Soil change \\
\hline 1019WE59 & 2 & $5-20$ & 0 & Negative & $10 \mathrm{YR} 4 / 2$ & Silty clay & Open cow pasture, near pump station & Subsoil \\
\hline 1019WE61 & 1 & $0-15$ & 0 & Negative & 10YR $4 / 2$ & Silty clay & Open cow pasture, wetland area & Soil change \\
\hline 1019WE61 & 2 & $15-25$ & 0 & Negative & $10 Y R 4 / 2$ & Silty clay & Open cow pasture, wetland area & Subsoil \\
\hline 1019JL03 & 1 & $0-20$ & 10 & Negative & 10YR $4 / 1$ & Silty clay & Cow pasture, semi-inundated area & Inundation \\
\hline 1019JL07 & 1 & $0-20$ & 10 & Negative & 10YR $4 / 1$ & Silty clay & Cow pasture, near dirt road & Soil change \\
\hline 1019JL07 & 2 & $20-30$ & 10 & Negative & 10YR $4 / 2$ & Clay & Cow pasture, near dirt road & Subsoil \\
\hline 1019JL16 & 1 & $0-20$ & 10 & Negative & 10YR $4 / 1$ & Silty clay & In between road and canal & Soil change \\
\hline 1019JL16 & 2 & $20-30$ & 10 & Negative & $10 \mathrm{YR} 4 / 2$ & Clay & In between road and canal & Subsoil \\
\hline 1019JL19 & 1 & $0-20$ & 10 & Negative & 10YR $4 / 1$ & Silty clay & off of gravel road, $60 \mathrm{~m}$ from pump station & Soil change \\
\hline 1019JL19 & 2 & $20-30$ & 10 & Negative & $10 Y R 4 / 2$ & Clay & off of gravel road, $60 \mathrm{~m}$ from pump station & Subsoil \\
\hline 1019JL23 & 1 & $0-20$ & 10 & Negative & 10YR 4/1 & Silty clay & Cow pasture, semi-inundated area & Soil change \\
\hline 1019JL23 & 2 & $20-30$ & 10 & Negative & $10 \mathrm{YYR} 4 / 2$ & Clay & Cow pasture, semi-inundated area & Subsoil \\
\hline 1019JL27 & 1 & $0-30$ & 10 & Negative & 10YR 4/1 & Silty clay & Cow pasture, semi-inundated area & Soil change \\
\hline 1019JL27 & 2 & $30-40$ & 10 & Negative & 10YR $4 / 2$ & Clay & Cow pasture, semi-inundated area & Subsoil \\
\hline $1019 \mathrm{JL} 29$ & 1 & $0-20$ & 10 & Negative & 10YR $4 / 1$ & Silty clay & Cow pasture, semi-inundated area & Soil change \\
\hline 1019JL29 & 2 & $20-30$ & 10 & Negative & 10YR $4 / 2$ & Clay & Cow pasture, semi-inundated area & Subsoil \\
\hline 1019JL33 & 1 & $0-20$ & 10 & Negative & 10YR 4/1 & Silty clay & Cow pasture, semi-inundated area & Soil change \\
\hline $1019 \mathrm{JL} 33$ & 2 & $20-30$ & 10 & Negative & 10YR 4/1 & Clay & Cow pasture, semi-inundated area & Subsoil \\
\hline 1019JL37 & 1 & $0-10$ & 10 & Negative & 10YR $4 / 1$ & Clay & In wetland, cow pasture & Inundation \\
\hline 1019JL41 & 1 & $0-10$ & 10 & Negative & 10YR 4/1 & Clay & In wetland, cow pasture & Inundation \\
\hline
\end{tabular}




\begin{tabular}{|c|c|c|c|c|c|c|c|c|}
\hline Shovel Test \# & $\begin{array}{l}\text { Level } \\
\text { (Strat) }\end{array}$ & $\begin{array}{l}\text { Depth } \\
\text { (cmbs) }\end{array}$ & GSV \% & $\begin{array}{c}\text { Positive or } \\
\text { Negative }\end{array}$ & $\begin{array}{l}\text { Munsell } \\
\text { Color }\end{array}$ & Soil Texture Description & Description (Area; Vegetation) & Reason for Termination \\
\hline $1019 J L 45$ & 1 & $0-10$ & 10 & Negative & 10YR $4 / 1$ & Clay & In wetland, cow pasture & Inundation \\
\hline $1019 J L 48$ & 1 & $0-10$ & 10 & Negative & 10YR 4/1 & Clay & In wetland, cow pasture & Inundation \\
\hline 1019JL51 & 1 & $0-10$ & 10 & Negative & 10YR $4 / 1$ & Clay & In wetland, cow pasture & Inundation \\
\hline 1019JL55 & 1 & $0-10$ & 10 & Negative & 10YR $4 / 1$ & Clay & In wetland, cow pasture & Inundation \\
\hline 1019JL59 & 1 & $0-15$ & 10 & Negative & 10YR $4 / 1$ & Clay & Near gravel road, semi-inundated area, disturbed & Disturbed \\
\hline 1020JL03 & 1 & $0-20$ & 10 & Negative & 10YR $4 / 1$ & Silty clay & Cow pasture & Soil change \\
\hline $1020 \mathrm{JL} 03$ & 2 & $20-30$ & 10 & Negative & $10 Y R 4 / 2$ & Clay & Cow pasture & Subsoil \\
\hline 1020JL14 & 1 & $0-20$ & 10 & Negative & 10YR $4 / 1$ & Silty clay & Cow pasture & Soil change \\
\hline 1020JL14 & 2 & $20-30$ & 10 & Negative & $10 \mathrm{YR} 4 / 2$ & Clay & Cow pasture & Subsoil \\
\hline 1020JL22 & 1 & $0-20$ & 10 & Negative & 10YR $4 / 1$ & Silty clay & Cow pasture & Soil change \\
\hline $1020 \mathrm{JL} 22$ & 2 & $20-30$ & 10 & Negative & 10YR $4 / 2$ & Clay & Cow pasture & Subsoil \\
\hline $1020 J L 26$ & 1 & $0-10$ & 10 & Negative & 10YR $4 / 1$ & Silty clay, wet & Cow pasture, inundated & Inundation \\
\hline 1020JL29 & 1 & $0-10$ & 10 & Negative & 10YR $4 / 1$ & Silty clay, wet & Open field, knee-high grass, wetland & Inundation \\
\hline 1020JL32 & 1 & $0-10$ & 10 & Negative & 10YR $4 / 1$ & Clay, wet & Open field, wetland & Disturbed \\
\hline 1020JL36 & 1 & $0-20$ & 10 & Negative & 10YR $4 / 1$ & Silty clay & Open field, wetland & Soil change \\
\hline $1020 \mathrm{JL} 36$ & 2 & $20-30$ & 10 & Negative & $10 \mathrm{YR} 4 / 2$ & Clay & Open field, wetland & Subsoil \\
\hline 1020JL40 & 1 & $0-20$ & 10 & Negative & 10YR $4 / 1$ & Silty clay & Open field, wetland & Soil change \\
\hline $1020 \mathrm{JL40}$ & 2 & $20-30$ & 10 & Negative & $10 \mathrm{YY} 4 / 2$ & Clay & Open field, wetland & Subsoil \\
\hline 1020JL44 & 1 & $0-20$ & 10 & Negative & 10YR $4 / 1$ & Silty clay & Open field, wetland & Soil change \\
\hline $1020 J L 44$ & 2 & $20-30$ & 10 & Negative & 10YR $4 / 2$ & Clay & Open field, wetland & Subsoil \\
\hline $1020 \mathrm{JL} 48$ & 1 & $0-20$ & 10 & Negative & 10YR $4 / 1$ & Silty clay & Open field, knee-high grass, & Soil change \\
\hline $1020 J L 48$ & 2 & $20-30$ & 10 & Negative & $10 Y R 4 / 2$ & Clay & Open field, knee-high grass, & Subsoil \\
\hline $1020 \mathrm{JL} 52$ & 1 & $0-20$ & 10 & Negative & 10YR 4/1 & Silty clay & Open field, knee-high grass, inundated & Soil change \\
\hline 1020JL52 & 2 & $20-30$ & 10 & Negative & 10YR $4 / 2$ & Clay & Open field, knee-high grass, inundated & Subsoil \\
\hline 1020WK01 & 1 & $0-15$ & $0-5$ & Negative & $\begin{array}{c}10 Y R 4 / 2 \\
5 / 2\end{array}$ & Silty clay loam & Wet marshy pasture & Soil change \\
\hline 1020WK01 & 2 & $15-30$ & $0-5$ & Negative & 10YR $4 / 1$ & Silty clay loam & Wet marshy pasture & Subsoil \\
\hline 1020WK15 & 1 & $0-20$ & 0 & Negative & $\begin{array}{c}10 Y R 4 / 2 \\
5 / 2\end{array}$ & Silty clay loam & Gravel inclusions/sandstone small $\sim 4 \mathrm{~m}$ east of canal berm, not clustered, disturbed & Soil change \\
\hline 1020WK15 & 2 & $20-30$ & 0 & Negative & 10YR $4 / 1$ & Silty clay loam & Gravel inclusions/sandstone small $4 \mathrm{~m}$ east of canal berm, not clustered, disturbed & Subsoil \\
\hline 1020WK21 & 1 & $0-25$ & 0 & Negative & $\begin{array}{c}10 Y R 4 / 2 \\
5 / 2\end{array}$ & Silty clay loam & Wet marsh, thigh-high grasses & Soil change \\
\hline 1020WK21 & 2 & $25-35$ & 0 & Negative & 10YR 4/1 & Loamy clay & Wet marsh, thigh-high grasses & Subsoil \\
\hline 1020WK25 & 1 & $0-5$ & 0 & Negative & $\begin{array}{c}10 Y R 4 / 2 \\
5 / 2\end{array}$ & Silty clay loam & Wet marsh & Inundation \\
\hline 1020WK28 & 1 & $0-25$ & 0 & Negative & $\begin{array}{c}10 Y R 4 / 2 \\
5 / 2\end{array}$ & Silty clay loam & Wet marsh, thigh-high grasses & Soil change \\
\hline 1020 WK28 & 2 & $25-35$ & 0 & Negative & 10YR 4/1 & Loamy clay & Wet marsh, thigh-high grasses & Subsoil \\
\hline 1020WK33 & 1 & $0-25$ & 0 & Negative & $\begin{array}{c}10 Y R 4 / 2 \\
5 / 2\end{array}$ & Silty clay loam & Wet marsh, thigh-high grasses & Inundation \\
\hline 1020WK37 & 1 & $0-13$ & 0 & Negative & 10YR $4 / 2$ & Silty clay loam & Wet marshy area & Soil change \\
\hline 1020WK37 & 2 & $13-20$ & 0 & Negative & 10YR $4 / 1$ & Loamy clay & Wet marshy area & Soil change \\
\hline 1020 WK37 & 3 & $20-30$ & 0 & Negative & $10 Y R 5 / 6$ & Silty clay & Wet marshy area & Subsoil \\
\hline 1020WK41 & 1 & $0-20$ & 5-10 & Negative & $\begin{array}{c}10 Y R 4 / 2 \\
5 / 2\end{array}$ & Silty clay loam & Wet marshy area. Found gravel bits in both horizons - disturbed area & Soil change \\
\hline 1020WK41 & 2 & $20-30$ & $5-10$ & Negative & 10YR $4 / 1$ & Silty clay loam & Wet marshy area. Found gravel bits in both horizons - disturbed area & Subsoil \\
\hline 1020WK45 & 1 & $0-13$ & $0-5$ & Negative & $\begin{array}{c}10 Y R 4 / 2 \\
5 / 2\end{array}$ & Silty clay loam & Wet marshy area. Sandstone bits found in A horizons & Soil change \\
\hline 1020WK45 & 2 & $13-30$ & $0-5$ & Negative & 10YR $4 / 1$ & Silty clay loam & Wet marshy area. Sandstone bits found in A horizons & Subsoil \\
\hline 1020WK49 & 1 & $0-20$ & $10-15$ & Negative & $\begin{array}{c}10 Y R 4 / 2 \\
5 / 2 \\
\end{array}$ & Silty clay loam & Wet marshy area & Soil change \\
\hline 1020WK49 & 2 & $20-25$ & $10-15$ & Negative & 10YR $4 / 1$ & Silty clay loam & Wet marshy area & Inundation \\
\hline 1020WK53 & 1 & $0-14$ & $0-5$ & Negative & $\begin{array}{c}10 Y R 4 / 2 \\
5 / 2\end{array}$ & Silty clay loam & Wet marshy area & Soil change \\
\hline 1020 WK53 & 2 & $14-30$ & $0-5$ & Negative & 10YR 4/1 & Silty clay loam & Wet marshy area & Subsoil \\
\hline 1020WE01 & 1 & $0-10$ & 0 & Negative & 10YR $4 / 1$ & Clay & Open cow pasture & Soil change \\
\hline
\end{tabular}




\begin{tabular}{|c|c|c|c|c|c|c|c|c|}
\hline Shovel Test \# & $\begin{array}{l}\text { Level } \\
\text { (Strat) }\end{array}$ & $\begin{array}{l}\text { Depth } \\
\text { (cmbs) }\end{array}$ & GSV \% & $\begin{array}{c}\text { Positive or } \\
\text { Negative }\end{array}$ & $\begin{array}{l}\text { Munsell } \\
\text { Color }\end{array}$ & Soil Texture Description & Description (Area; Vegetation) & Reason for Termination \\
\hline 1020WE01 & 2 & $10-15$ & 0 & Negative & 10YR $4 / 1$ & Clay & Open cow pasture & Subsoil \\
\hline 1020WE13 & 1 & $0-10$ & 0 & Negative & 10YR $4 / 1$ & Clay & Cow pasture, near canal & Soil change \\
\hline 1020WE13 & 2 & $10-15$ & 0 & Negative & 10YR $4 / 1$ & Clay & Cow pasture, near canal & Subsoil \\
\hline 1020WE20 & 1 & $0-10$ & 0 & Negative & 10YR $4 / 1$ & Clay & Open field, wetland area & Soil change \\
\hline 1020WE20 & 2 & $10-15$ & 0 & Negative & 10YR $4 / 1$ & Clay & Open field, wetland area & Subsoil \\
\hline 1020WE24 & 1 & $0-5$ & 0 & Negative & 10YR $4 / 1$ & Clay & Open field, wetland area & Inundation \\
\hline 1020WE27 & 1 & $0-10$ & 0 & Negative & 10YR 4/1 & Clay & Open field, wetland area & Soil change \\
\hline 1020WE27 & 2 & $10-15$ & 0 & Negative & 10YR 4/1 & Clay & Open field, wetland area & Subsoil \\
\hline 1020WE31 & 1 & $0-10$ & 0 & Negative & 10YR $4 / 1$ & Clay & Wetland, open field, tall grass & Soil change \\
\hline 1020WE31 & 2 & $10-15$ & 0 & Negative & 10YR $4 / 1$ & Clay & Wetland, open field, tall grass & Subsoil \\
\hline 1020WE35 & 1 & $0-15$ & 0 & Negative & 10YR 4/1 & Clay & Wetland, open field, tall grass & Soil change \\
\hline 1020WE35 & 2 & $15-25$ & 0 & Negative & 10YR $4 / 1$ & Clay & Wetland, open field, tall grass & Subsoil \\
\hline 1020WE39 & 1 & $0-10$ & 0 & Negative & 10YR 4/1 & Clay & Wetland, open field, tall grass & Soil change \\
\hline 1020WE39 & 2 & $10-20$ & 0 & Negative & 10YR 4/1 & Clay & Wetland, open field, tall grass & Subsoil \\
\hline 1020WE42 & 1 & $0-20$ & 0 & Negative & 10YR $4 / 1$ & Clay & Wetland area, cow pasture & Soil change \\
\hline 1020WE42 & 2 & $20-25$ & 0 & Negative & $10 \mathrm{YR} 4 / 2$ & Sandy clay & Wetland area, cow pasture & Subsoil \\
\hline 1020WE46 & 1 & $0-15$ & 0 & Negative & 10YR $4 / 1$ & Clay & Cow pasture, open field & Soil change \\
\hline 1020WE46 & 2 & $15-20$ & 0 & Negative & 10YR $4 / 1$ & Clay & Cow pasture, open field & Subsoil \\
\hline 1020WE50 & 1 & $0-15$ & 0 & Negative & 10YR 4/1 & Clay & Open field, tall grass & Soil change \\
\hline 1020WE50 & 2 & $15-20$ & 0 & Negative & 10YR $4 / 1$ & Clay & Open field, tall grass & Subsoil \\
\hline 1020WE55 & 1 & $0-10$ & 0 & Negative & 10YR 4/1 & Clay & Open field, wetland & Soil change \\
\hline 1020WE55 & 2 & $10-15$ & 0 & Negative & 10YR $4 / 1$ & Clay & Open field, wetland & Subsoil \\
\hline 1020RC12 & 1 & $0-30$ & 0 & Negative & 10YR 4/1 & Clay loam & Cow pasture, open field, tall grass. Scattered brush, occasional tree & Subsoil \\
\hline 1020RC19 & 1 & $0-30$ & 0 & Negative & 10YR $4 / 1$ & Clay loam & Cow pasture, open field, tall grass. Scattered brush, occasional tree & Subsoil \\
\hline 1020RC23 & 1 & $0-15$ & 0 & Negative & 10YR 4/1 & Clay loam & Cow pasture, open field, tall grass. Scattered brush, occasional tree & Subsoil \\
\hline 1020RC30 & 1 & $0-10$ & 0 & Negative & 10YR 4/1 & Clay loam & Cow pasture, open field, tall grass. Scattered brush, occasional tree & Subsoil \\
\hline 1020RC34 & 1 & $0-15$ & 0 & Negative & 10YR $4 / 1$ & Clay loam & Cow pasture, open field, tall grass. Scattered brush, occasional tree & Subsoil \\
\hline 1020RC38 & 1 & $0-15$ & 0 & Negative & 10YR 4/1 & Clay loam & Cow pasture. Med-tall grass. Scattered brush, trees along tree line & Subsoil \\
\hline 1020RC38 & 2 & $15-25$ & 0 & Negative & $10 Y R 4 / 2$ & Clay loam & Cow pasture. Med-tall grass. Scattered brush, trees along tree line & Subsoil \\
\hline 1020RC43 & 1 & $0-40$ & 0 & Negative & 10YR $4 / 1$ & Clay loam & Cow pasture. Med-tall grass. Scattered brush, trees along tree line & Subsoil \\
\hline 1020RC47 & 1 & $0-30$ & 0 & Negative & $10 \mathrm{YYR} 4 / 1$ & Clay loam & Cow pasture. Med-tall grass. Scattered brush, trees along tree line & Subsoil \\
\hline 1020RC51 & 1 & $0-30$ & 0 & Negative & 10YR 4/1 & Clay loam & Cow pasture. Med-tall grass. Scattered brush, trees along tree line & Subsoil \\
\hline 1020RC54 & 1 & $0-30$ & 0 & Negative & $10 \mathrm{Yr} 4 / 1$ & Clay loam & Cow pasture. Med-tall grass. Scattered brush, trees along tree line & Subsoil \\
\hline 1022WE01 & 1 & $0-10$ & 0 & Negative & $10 Y R 4 / 2$ & Clay loam & Cow pasture, near fence & Soil change \\
\hline 1022WE01 & 2 & $10-20$ & 0 & Negative & $10 Y R 4 / 2$ & Clay loam & Cow pasture, near fence & Subsoil \\
\hline 1022WE05 & 1 & $0-10$ & 0 & Negative & 10YR $4 / 2$ & Clay loam & Cow pasture & Soil change \\
\hline 1022WE05 & 2 & $10-20$ & 0 & Negative & 10YR $4 / 2$ & Clay loam & Cow pasture & Subsoil \\
\hline 1022WE08 & 1 & $0-25$ & 0 & Negative & $10 Y R 4 / 2$ & Loamy clay & Cow pasture & Soil change \\
\hline 1022WE08 & 2 & $25-30$ & 0 & Negative & 10YR $4 / 2$ & Clay & Cow pasture & Subsoil \\
\hline 1022WE12 & 1 & $0-15$ & 0 & Negative & 10YR 4/1 & Clay & Cow pasture & Soil change \\
\hline 1022WE12 & 2 & $15-25$ & 0 & Negative & 10YR $4 / 1$ & Clay & Cow pasture & Subsoil \\
\hline 1022WE16 & 1 & $0-15$ & 0 & Negative & 10YR $4 / 1$ & Clay & Cow pasture & Soil change \\
\hline 1022WE16 & 2 & $15-25$ & 0 & Negative & 10YR $4 / 1$ & Clay & Cow pasture & Subsoil \\
\hline 1022WE20 & 1 & $0-15$ & 0 & Negative & 10YR $4 / 1$ & Clay & Cow pasture & Soil change \\
\hline 1022WE20 & 2 & $15-25$ & 0 & Negative & 10YR 4/1 & Clay & Cow pasture & Subsoil \\
\hline 1022WE29 & 1 & $0-20$ & 0 & Negative & 1OYR $4 / 2$ & Sandy clay & Cow pasture, wetland area & Soil change \\
\hline 1022WE29 & 2 & $20-25$ & 0 & Negative & $10 Y R 4 / 2$ & Sandy clay & Cow pasture, wetland area & Subsoil \\
\hline 1022WE33 & 1 & $0-10$ & 0 & Negative & 10YR $4 / 2$ & Silty clay & Cow pasture, wetland area & Soil change \\
\hline 1022WE33 & 2 & $10-20$ & 0 & Negative & $10 Y R 4 / 2$ & Silty clay & Cow pasture, wetland area & Subsoil \\
\hline 1022WE36 & 1 & $0-10$ & 0 & Negative & 10YR 4/1 & Clay & Open field, close to reeds & Soil change \\
\hline 1022WE36 & 2 & $10-20$ & 0 & Negative & 10YR $4 / 1$ & Clay & Open field, close to reeds & Subsoil \\
\hline 1022WE40 & 1 & $0-5$ & 0 & Negative & $10 \mathrm{YR} 4 / 2$ & Clay & Open field, wetland & Inundation \\
\hline 1022WE44 & 1 & $0-5$ & 0 & Negative & 10YR $4 / 2$ & Clay & Open field, wetland & Inundation \\
\hline 1022WE50 & 1 & $0-10$ & 0 & Negative & 10YR $4 / 2$ & Silty clay & Open field, wetland & Soil change \\
\hline 1022WE50 & 2 & $10-20$ & 0 & Negative & $10 Y R 4 / 2$ & Silty clay & Open field, wetland & Subsoil \\
\hline 1022WE54 & 1 & $0-10$ & 0 & Negative & $10 Y R 4 / 2$ & Clay loam & Forest, many roots & Soil change \\
\hline 1022WE54 & 2 & $10-15$ & 0 & Negative & $10 Y R 4 / 2$ & Clay loam & Forest, many roots & Subsoil \\
\hline
\end{tabular}




\begin{tabular}{|c|c|c|c|c|c|c|c|c|}
\hline Shovel Test \# & $\begin{array}{l}\text { Level } \\
\text { (Strat) }\end{array}$ & $\begin{array}{l}\text { Depth } \\
\text { (cmbs) }\end{array}$ & GSV \% & $\begin{array}{c}\text { Positive or } \\
\text { Negative }\end{array}$ & $\begin{array}{l}\text { Munsell } \\
\text { Color }\end{array}$ & Soil Texture Description & Description (Area; Vegetation) & Reason for Termination \\
\hline 1022WE58 & 1 & $0-20$ & 0 & Negative & 10YR $4 / 1$ & Clay & Open field & Soil change \\
\hline 1022WE58 & 2 & $20-25$ & 0 & Negative & 10YR 4/1 & Clay & Open field & Subsoil \\
\hline 1022WE65 & 1 & $0-5$ & 0 & Negative & 10YR $4 / 2$ & Clay & Wetland, reeds \& tall brush & Inundation \\
\hline 1022WE69 & 1 & $0-20$ & 0 & Negative & $10 Y R 4 / 2$ & Sandy clay & Cow pasture & Soil change \\
\hline 1022WE69 & 2 & $20-25$ & 0 & Negative & $10 Y R 4 / 2$ & Sandy clay & Cow pasture & Subsoil \\
\hline $1022 \mathrm{RC} 04$ & 1 & $0-20$ & 10 & Negative & 10YR $4 / 1$ & Silt loam & Cow pasture, fenced. Scattered trees, tall-med grass & Soil change \\
\hline 1022RC04 & 2 & $20-30$ & 0 & Negative & $10 Y R 4 / 2$ & Silt loam & Cow pasture, fenced. Scattered trees, tall-med grass & Subsoil \\
\hline $1022 \mathrm{RC} 10$ & 1 & $0-10$ & 0 & Negative & 10YR $4 / 1$ & Silt loam & Cow pasture, fenced. Scattered trees, tall-med grass & Soil change \\
\hline $1022 \mathrm{RC} 10$ & 2 & $10-30$ & 0 & Negative & $10 \mathrm{YR} 4 / 2$ & Silt loam & Cow pasture, fenced. Scattered trees, tall-med grass & Subsoil \\
\hline 1022RC15 & 1 & $0-10$ & 0 & Negative & 10YR $4 / 1$ & Clay loam & Cow pasture, fenced. Scattered trees, tall-med grass & Inundationlogged @ 10cm \\
\hline 1022RC19 & 1 & $0-15$ & 0 & Negative & 10YR 4/1 & Clay loam & Cow pasture, fenced. Scattered trees, tall-med grass & Subsoil \\
\hline $1022 \mathrm{RC21}$ & 1 & $0-15$ & 0 & Negative & 10YR 4/1 & Clay loam & Cow pasture. Open field, trees along fence line short-med grass & Soil change \\
\hline 1022RC21 & 2 & $15-20$ & 0 & Negative & 10YR $4 / 2$ & Clay loam & Cow pasture. Open field, trees along fence line short-med grass & Subsoil \\
\hline $1022 \mathrm{RC26}$ & 1 & $0-10$ & 0 & Negative & $10 \mathrm{YYR} 4 / 1$ & Clay loam & Cow pasture. Open field, trees along fence line short-med grass & Soil change \\
\hline $1022 \mathrm{RC} 26$ & 2 & $10-20$ & 0 & Negative & 10YR $4 / 2$ & Clay loam & Cow pasture. Open field, trees along fence line short-med grass & Subsoil \\
\hline 1022RC32 & 1 & $0-10$ & 0 & Negative & $10 Y R 4 / 1$ & Silt loam & Cow pasture. Open field, trees along fence line short-med grass & Soil change \\
\hline 1022RC32 & 2 & $10-25$ & 0 & Negative & 10YR $4 / 2$ & Silt loam & Cow pasture. Open field, trees along fence line short-med grass & Subsoil \\
\hline $1022 \mathrm{RC} 38$ & 1 & $0-10$ & 0 & Negative & 10YR $4 / 1$ & Clay loam & Cow pasture. Open field, trees along fence line short-med grass & Inundationlogged \\
\hline 1022RC43 & 1 & $0-15$ & 0 & Negative & 10YR 4/1 & Clay loam & Open pasture, cow pasture. Short-medium grass & Inundationlogged \\
\hline $1022 \mathrm{RC} 45$ & 1 & $0-10$ & 0 & Negative & 10YR 4/1 & Silt loam & Open pasture, cow pasture. Short-medium grass & Soil change \\
\hline 1022RC45 & 2 & $10-20$ & 0 & Negative & 10YR 4/2 & Silt loam & Open pasture, cow pasture. Short-medium grass & Subsoil \\
\hline $1022 \mathrm{RC48}$ & 1 & $0-40$ & 0 & Negative & 10YR 4/1 & Silt loam & Open pasture, cow pasture. Short-medium grass & Soil change \\
\hline $1022 \mathrm{RC48}$ & 2 & $40-50$ & 0 & Negative & $10 Y R 4 / 2$ & Silt loam & Open pasture, cow pasture. Short-medium grass & Subsoil \\
\hline 1022RC52 & 1 & $0-30$ & 0 & Negative & 10YR $4 / 1$ & Silt loam & Open pasture, cow pasture. Short-medium grass & Soil change \\
\hline 1022RC52 & 2 & $30-60$ & 0 & Negative & $10 Y R 4 / 2$ & Silt loam & Open pasture, cow pasture. Short-medium grass & Subsoil \\
\hline 1022RC55 & 1 & $0-30$ & 0 & Negative & 10YR 4/1 & Silt loam & Open pasture, cow pasture. Short-medium grass & Soil change \\
\hline 1022RC55 & 2 & $30-60$ & 0 & Negative & 10YR $4 / 2$ & Silt loam & Open pasture, cow pasture. Short-medium grass & Subsoil \\
\hline 1022RC59 & 1 & $0-15$ & 0 & Negative & $10 \mathrm{YR} 4 / 1$ & Clay loam & Open pasture, cow pasture. Short-medium grass & Inundationlogged \\
\hline 1022RC63 & 1 & $0-15$ & 0 & Negative & 10YR $4 / 1$ & Clay loam & Open pasture, cow pasture. Short-medium grass & Inundationlogged \\
\hline 1022RC67 & 1 & $0-30$ & 0 & Negative & 10YR 4/1 & Silt loam & Open pasture, cow pasture. Short-medium grass & Soil change \\
\hline 1022RC67 & 2 & $30-60$ & 0 & Negative & 10YR 4/1 & Silt loam & Open pasture, cow pasture. Short-medium grass & Subsoil \\
\hline 1022$\lrcorner\llcorner 03$ & 1 & $0-20$ & 10 & Negative & 10YR 4/1 & Silt loam & Open cow pasture & Soil change \\
\hline $1022 J L 03$ & 2 & $20-30$ & 10 & Negative & $10 \mathrm{YR} 4 / 2$ & Silty clay & Open cow pasture & Subsoil \\
\hline $1022 J L 07$ & 1 & $0-20$ & 10 & Negative & 1OYR $4 / 1$ & Silt loam & Open cow pasture & Soil change \\
\hline $1022 J L 07$ & 2 & $20-30$ & 10 & Negative & $10 \mathrm{YR} 4 / 2$ & Silty clay & Open cow pasture & Subsoil \\
\hline 1022JL10 & 1 & $0-20$ & 10 & Negative & 10YR $4 / 1$ & Silt loam & Open cow pasture & Soil change \\
\hline $1022 J L 10$ & 2 & $20-30$ & 10 & Negative & 10YR $4 / 2$ & Silty clay & Open cow pasture & Subsoil \\
\hline 1022JL13 & 1 & $0-15$ & 10 & Negative & 10YR $4 / 1$ & Silt clay & Open cow pasture & Soil change \\
\hline 1022JL13 & 2 & $15-30$ & 10 & Negative & $10 Y R 4 / 2$ & Clay & Open cow pasture & Subsoil \\
\hline 1022JL18 & 1 & $0-10$ & 10 & Negative & 10YR 4/1 & Silt clay & Open cow pasture, semi-inundated & Soil change \\
\hline 1022JL18 & 2 & $10-25$ & 10 & Negative & 10YR $4 / 3$ & Clay & Open cow pasture, semi-inundated & Subsoil \\
\hline $1022 J L 27$ & 1 & $0-20$ & 10 & Negative & 10YR $4 / 1$ & Silt loam & Open cow pasture & Soil change \\
\hline $1022 J L 27$ & 2 & $20-30$ & 10 & Negative & $10 \mathrm{Yr} 4 / 2$ & Silty clay & Open cow pasture & Subsoil \\
\hline $1022 J L 31$ & 1 & $0-20$ & 10 & Negative & 10YR $4 / 1$ & Silt loam & Open cow pasture & Soil change \\
\hline $1022 \mathrm{~J} L 31$ & 2 & $20-30$ & 10 & Negative & 10YR $4 / 2$ & Silty clay & Open cow pasture & Subsoil \\
\hline 1022JL35 & 1 & $0-20$ & 10 & Negative & 10YR 4/1 & Silt loam & Open cow pasture, near wetland & Soil change \\
\hline $1022 J L 35$ & 2 & $20-30$ & 10 & Negative & $10 Y R 4 / 3$ & Silty clay & Open cow pasture, near wetland & Subsoil \\
\hline 1022JL39 & 1 & $0-10$ & 10 & Negative & 10YR $4 / 1$ & Silty clay & Open cow pasture, near wetland & Inundation \\
\hline $1022 J L 42$ & 1 & $0-10$ & 10 & Negative & 10YR 4/1 & Silty clay & Open cow pasture, near wetland & Inundation \\
\hline $1022 J\llcorner 47$ & 1 & $0-15$ & 10 & Negative & 10YR 4/1 & Silt loam & Open cow pasture, near wetland & Soil change \\
\hline $1022 J\llcorner 47$ & 2 & $15-25$ & 10 & Negative & 10YR $4 / 3$ & Silty clay & Open cow pasture, near wetland & Subsoil \\
\hline $1022 J L 51$ & 1 & $0-30$ & 10 & Negative & 10YR 4/1 & Silt loam & Open cow pasture, near wetland & Soil change \\
\hline 1022JL51 & 2 & $30-40$ & 10 & Negative & 10YR $4 / 3$ & Silty clay & Open cow pasture, near wetland & Subsoil \\
\hline $1022 J L 56$ & 1 & $0-20$ & 10 & Negative & 10YR 4/1 & Silt loam & Open field, semi-inundated & Soil change \\
\hline $1022 J L 56$ & 2 & $20-30$ & 10 & Negative & $10 Y R 4 / 3$ & Silty clay & Open field, semi-inundated & Subsoil \\
\hline $1022 J L 60$ & 1 & $0-10$ & 10 & Negative & 10YR $4 / 1$ & Silty clay & Open field, semi-inundated & Inundation \\
\hline 1022JL61 & 1 & $0-10$ & 10 & Negative & 10YR 4/1 & Silty clay & Open field, semi-inundated & Inundation \\
\hline
\end{tabular}




\begin{tabular}{|c|c|c|c|c|c|c|c|c|}
\hline Shovel Test \# & $\begin{array}{l}\text { Level } \\
\text { (Strat) }\end{array}$ & $\begin{array}{l}\text { Depth } \\
\text { (cmbs) }\end{array}$ & GSV \% & $\begin{array}{l}\text { Positive or } \\
\text { Negative }\end{array}$ & $\begin{array}{c}\text { Munsell } \\
\text { Color }\end{array}$ & Soil Texture Description & Description (Area; Vegetation) & Reason for Termination \\
\hline $1022 J L 62$ & 1 & $0-10$ & 10 & Negative & 10YR $4 / 1$ & Silty clay & $\begin{array}{l}\text { Open field, semi-inundated } \\
\end{array}$ & Inundation \\
\hline 1022JL66 & 1 & $0-15$ & 10 & Negative & 10YR 4/1 & Silt loam & Open field, semi-inundated. At edge of wetland & Soil change \\
\hline $1022 J L 66$ & 2 & $15-30$ & 10 & Negative & 10YR $4 / 2$ & Silty clay & Open field, semi-inundated. At edge of wetland & Subsoil \\
\hline 1022JL71 & 1 & $0-30$ & 10 & Negative & 10YR 4/1 & Silt loam & Open cow pasture & Soil change \\
\hline 1022JL71 & 2 & $30-40$ & 10 & Negative & 10YR $4 / 2$ & Silty clay & Open cow pasture & Subsoil \\
\hline 102218RC71 & 1 & $0-30$ & 10 & Negative & 10YR $4 / 1$ & Silt loam & Open cow pasture & Subsoil \\
\hline 1022WK68 & 1 & $0-20$ & 0 & Negative & 10YR $4 / 2$ & Silt loam & Marshy pasture $\mathrm{w} / \mathrm{grass}$ and rose briars & Soil change \\
\hline 1022 WK68 & 2 & $20-35$ & 0 & Negative & 10YR 4/1 & Silt loam & Marshy pasture w/grass and rose briars & Soil change \\
\hline 1022 WK68 & 3 & $35-45$ & 0 & Negative & 10YR $5 / 6$ & Sand & Marshy pasture w/grass and rose briars & Subsoil \\
\hline 1022WK02 & 1 & $0-35$ & $25-30$ & Negative & 10YR $4 / 2$ & Silty clay loam & Pasture w/ wild grass. Iron 2 nd strat had orange deposits & Soil change \\
\hline 1022WK02 & 2 & $35-45$ & $25-30$ & Negative & 10YR $5 / 6$ & Silty clay loam & Pasture w/ wild grass. Iron 2 nd strat had orange deposits & Subsoil \\
\hline 1022WK06 & 1 & $0-17$ & $5-10$ & Negative & 10YR $4 / 2$ & Silty loam & Pasture $\mathrm{w} /$ wild grass - areas of inundation & Soil change \\
\hline 1022WK06 & 2 & $17-40$ & 5-10 & Negative & $\begin{array}{c}\text { 10YR } 4 / 1 \\
5 / 2 \\
\end{array}$ & Silty clay & Pasture $\mathrm{w} /$ wild grass - areas of inundation & Subsoil \\
\hline 1022WK09 & 1 & $0-40$ & $0-5$ & Negative & 10YR $4 / 2$ & Silty loam & Pasture $\mathrm{w} /$ wild grass - areas of inundation & Soil change \\
\hline 1022WK09 & 2 & $40-50$ & $0-5$ & Negative & 10YR 4/1 & Silty loam & Pasture $\mathrm{w} /$ wild grass - areas of inundation & Subsoil \\
\hline 1022WK14 & 1 & $0-25$ & $0-5$ & Negative & 10YR $4 / 2$ & Silty clay loam & Pasture $\mathrm{w} /$ wild grass - areas of inundation & Inundation \\
\hline 1022WK17 & 1 & $0-15$ & $0-5$ & Negative & 10YR $4 / 2$ & Silty clay loam & $\begin{array}{l}\text { Pasture } w / \text { wild grass - areas of inundation } \\
\end{array}$ & Soil change \\
\hline 1022WK17 & 2 & $15-25$ & $0-5$ & Negative & 10YR $4 / 1$ & Silty clay loam & Pasture w/ wild grass - areas of inundation. Mottling occurred just before inundation & Inundation \\
\hline 1022WK26 & 1 & $0-15$ & $0-5$ & Negative & $\begin{array}{c}10 Y R 4 / 2 \\
5 / 2\end{array}$ & Silty clay loam & Marshy pasture w/ wild grasses & Soil change \\
\hline 1022WK26 & 2 & $15-30$ & $0-5$ & Negative & 10YR 4/1 & Silty clay loam & Marshy pasture w/ wild grasses & Subsoil \\
\hline 1022WK30 & 1 & $0-28$ & $20-25$ & Negative & $\begin{array}{c}10 Y R 4 / 2 \\
5 / 2 \\
\end{array}$ & Silty clay loam & Marshy pasture $\mathrm{w} /$ wild grasses & Soil change \\
\hline 1022 WK30 & 2 & $28-40$ & $20-25$ & Negative & 10YR $5 / 6$ & Silt & Marshy pasture $\mathrm{w} /$ wild grasses & Subsoil \\
\hline 1022 WK34 & 1 & $0-30$ & $0-5$ & Negative & 10YR $4 / 2$ & Silty loam & Marshy pasture w/wild grasses. STP 3m east of inundated area & Soil change \\
\hline 1022 WK34 & 2 & $30-40$ & $0-5$ & Negative & 10YR 4/1 & Clay & Marshy pasture w/wild grasses. STP $3 \mathrm{~m}$ east of inundated area & Subsoil \\
\hline 1022 WK37 & 1 & $0-20$ & $0-5$ & Negative & 10YR $4 / 2$ & Silty clay loam & Marshy pasture - STP located in large patch $(20 \times 20 \mathrm{~m})$ of rose briar & Soil change \\
\hline 1022WK37 & 2 & $20-30$ & $0-5$ & Negative & 10YR 4/1 & Clay & Marshy pasture - STP located in large patch $(20 \times 20 \mathrm{~m})$ of rose briar & Subsoil \\
\hline 1022WK41 & 1 & $0-15$ & $0-5$ & Negative & 10YR $4 / 2$ & Silty clay loam & Marshy pasture w/wild grasses and rose briar & Inundation \\
\hline 1022 WK46 & 1 & $0-15$ & $0-5$ & Negative & 10YR $4 / 2$ & Silty loam & Marshy pasture $\mathrm{w} /$ wild grasses and rose briar & Soil change \\
\hline 1022WK46 & 2 & $15-35$ & $0-5$ & Negative & $\begin{array}{c}10 Y R 5 / 6 \\
4 / 1\end{array}$ & Silty clay & Marshy pasture w/wild grasses and rose briar & Subsoil \\
\hline 1022WK49 & 1 & $0-10$ & $0-5$ & Negative & 10YR $4 / 2$ & Silty loam & Marshy pasture $w /$ wild grasses and rose briar & Soil change \\
\hline 1022 WK49 & 2 & $10-30$ & $0-5$ & Negative & 10YR 4/1 & Silty clay & Marshy pasture w/wild grasses and rose briar & Subsoil \\
\hline 1022 WK53 & 1 & $0-30$ & $0-5$ & Negative & 10YR $4 / 2$ & Silty loam & Marshy pasture $w /$ wild grasses and rose briar & Soil change \\
\hline 1022WK53 & 2 & $30-40$ & $0-5$ & Negative & $\begin{array}{c}\text { 10YR } 5 / 6 \\
4 / 1 \\
\end{array}$ & Silty clay & Marshy pasture w/wild grasses and rose briar & Subsoil \\
\hline 1022WK57 & 1 & $0-25$ & 0 & Negative & 10YR $4 / 2$ & Silty clay loam & Marshy pasture $w /$ wild grasses & Inundation \\
\hline 1022WK64 & 1 & $0-10$ & 0 & Negative & 10YR $4 / 2$ & Silty clay loam & Marshy pasture w/wild grasses & Soil change \\
\hline 1022WK64 & 2 & $10-30$ & 0 & Negative & 10YR 4/1 & Clay & Marshy pasture w/wild grasses & Subsoil \\
\hline 1023WK02 & 1 & $0-20$ & $0-5$ & Negative & 10YR $4 / 2$ & Silty clay loam & Wetland w/wild grasses and briars & Soil change \\
\hline 1023WK02 & 2 & $20-35$ & $0-5$ & Negative & 10YR 4/1 & Silty clay loam & Wetland w/wild grasses and briars & Subsoil \\
\hline 1023WK06 & 1 & $0-15$ & $0-5$ & Negative & 10YR $4 / 2$ & Silty clay loam & Wetland w/wild grasses and briars & Soil change \\
\hline 1023WK06 & 2 & $15-30$ & $0-5$ & Negative & 10YR 4/1 & Silty clay loam & Wetland $\mathrm{w} /$ wild grasses and briars & Subsoil \\
\hline 1023WK13 & 1 & $0-20$ & $0-5$ & Negative & 10YR $4 / 2$ & Silty clay loam & Wetland w/wild grasses and briars & Soil change \\
\hline 1023WK13 & 2 & $20-30$ & $0-5$ & Negative & 10YR 4/1 & Silty clay loam & Wetland w/wild grasses and briars & Subsoil \\
\hline 1023RC04 & 1 & $0-30$ & 0 & Negative & 10YR 4/1 & Clay loan & Open cow pasture. Tall grass & Subsoil \\
\hline 1023RC08 & 1 & $0-20$ & 0 & Negative & 10YR 4/1 & Clay loan & Open cow pasture. Tall grass & Subsoil \\
\hline 1023RC12 & 1 & $0-30$ & 0 & Negative & 10YR 4/1 & Clay loan & Open cow pasture. Tall grass & Subsoil \\
\hline 1023JL03 & 1 & $0-40$ & 10 & Negative & 10YR 4/1 & Clay & Open field, semi-inundated & Subsoil \\
\hline 1023JL07 & 1 & $0-10$ & 10 & Negative & 10YR $4 / 1$ & Clay & Open field, semi-inundated & Inundation \\
\hline 1023JL10 & 1 & $0-10$ & 10 & Negative & 10YR $4 / 1$ & Clay & Open field, semi-inundated & Inundation \\
\hline
\end{tabular}




\begin{tabular}{|c|c|c|c|c|c|c|c|c|}
\hline Shovel Test \# & $\begin{array}{l}\begin{array}{l}\text { Level } \\
\text { (Strat) }\end{array} \\
\end{array}$ & $\begin{array}{l}\text { Depth } \\
\text { (cmbs) }\end{array}$ & GSV \% & $\begin{array}{c}\begin{array}{c}\text { Positive or } \\
\text { Negative }\end{array} \\
\end{array}$ & $\begin{array}{c}\text { Munsell } \\
\text { Color }\end{array}$ & Soil Texture Description & Description (Area; Vegetation) & Reason for Termination \\
\hline 1023WE01 & 1 & $0-20$ & 0 & Negative & 10YR $4 / 2$ & Silty clay & Cow pasture & Soil change \\
\hline 1023WE01 & 2 & $20-25$ & 0 & Negative & 10YR $4 / 2$ & Silty clay & Cow pasture & Subsoil \\
\hline 1023WE05 & 1 & $0-15$ & 0 & Negative & 10YR $4 / 1$ & Clay & Cow pasture & Soil change \\
\hline 1023WE05 & 2 & $15-20$ & 0 & Negative & 10YR $4 / 1$ & Clay & Cow pasture & Subsoil \\
\hline 1023WE09 & 1 & $0-10$ & 0 & Negative & 10YR $4 / 1$ & Clay & Tall reeds, wetland & Inundation \\
\hline 1023WE11 & 1 & $0-20$ & 0 & Negative & 10YR 4/1 & Clay & Cow pasture & Soil change \\
\hline 1023WE11 & 2 & $20-25$ & 0 & Negative & 10YR $4 / 1$ & Clay & Cow pasture & Subsoil \\
\hline 1024JL10 & 1 & $0-18$ & 0 & Negative & 10YR $3 / 2$ & Sandy loam & Inside wooded area. Dense vegetation & Soil change \\
\hline 1024JL10 & 2 & $18-35$ & 0 & Negative & 10YR $3 / 3$ & Loam & Inside wooded area. Dense vegetation & Subsoil \\
\hline 1024WK02 & 1 & $0-20$ & 0 & Negative & 10YR4/1 & Silty clay loam & Wetland with intermediate amounts of grass and briars. Area subject to periods of inundation. & Inundation \\
\hline 1024WK09 & 1 & $0-40$ & 0 & Negative & $10 Y R 3 / 3$ & Sandy clay & Stp 15M off road (north). Wetland area overgrown with grasses, vines and hardwoods & Soil change \\
\hline 1024WK09 & 2 & $40-50$ & 0 & Negative & $10 \mathrm{YR} 5 / 6$ & Sandy loam & Stp 15M off road (north). Wetland area overgrown with grasses, vines and hardwoods & Subsoil \\
\hline 1024WE01 & 1 & $0-10$ & 0 & Negative & 10YR $4 / 2$ & Clay & Tall vegetation, open field, wetland area & Soil change \\
\hline 1024WE01 & 2 & $10-15$ & 0 & Negative & 10YR $4 / 2$ & Clay & Tall vegetation, open field, wetland area & Subsoil \\
\hline 1024WE08 & 1 & $0-10$ & 0 & Negative & 10YR $4 / 2$ & Silty clay & Forest, thick with brush & Soil change \\
\hline 1024WE08 & 2 & $10-15$ & 0 & Negative & 10YR $4 / 1$ & Clay & Forest, thick with brush & Subsoil \\
\hline 1024RC11 & 1 & $0-30$ & 0 & Negative & $10 \mathrm{YR} 5 / 2$ & Silt loam & Forest corner. Thick vegetation adjacent to wetland. Wet \& inundated & Subsoil \\
\hline 1025WK01 & 1 & $0-25$ & 0 & Negative & 10YR $3 / 1$ & Silty clay loam & Wetland area with wild grasses. Acting as pasture. STP 5M east of road and fencing & Soil change \\
\hline 1025WK01 & 2 & $25-40$ & 0 & Negative & $\begin{array}{c}10 Y R 3 / 1 \\
\& 5 / 6\end{array}$ & Clay loam & Wetland area with wild grasses. Acting as pasture. STP $5 \mathrm{M}$ east of road and fencing & Subsoil \\
\hline 1025WK04 & 1 & $0-15$ & 0 & Negative & $10 Y R 3 / 1$ & Silty clay loam & Wetland area with wild grasses. Acting as pasture. About $5 \mathrm{M}$ from berm & Soil change \\
\hline 1025WK04 & 2 & $15-30$ & 0 & Negative & $\begin{array}{c}10 Y R 3 / 1 \\
\& 5 / 6\end{array}$ & Clay & Wetland area with wild grasses. Acting as pasture. About $5 \mathrm{M}$ from berm & Subsoil \\
\hline 1025WK05 & 1 & $0-10$ & 0 & Negative & $10 \mathrm{YR} 4 / 1$ & Clay loam & STP is on wetland on corridor with swamp on either side & Soil change \\
\hline 1025WK05 & 2 & $10-30$ & 0 & Negative & 10YR $3 / 1$ & Clay & STP is on wetland on corridor with swamp on either side & Subsoil \\
\hline 1025WK07 & 1 & $0-10$ & 0 & Negative & 10YR $4 / 1$ & Clay loam & STP is on wetland on corridor with swamp on either side & Soil change \\
\hline 1025WK07 & 2 & $10-30$ & 0 & Negative & 10YR $3 / 1$ & Clay & STP is on wetland on corridor with swamp on either side & Subsoil \\
\hline 1025WK09 & 1 & $0-15$ & 0 & Negative & 10YR $4 / 1$ & Clay loam & STP is on wetland on corridor with swamp on either side & Soil change \\
\hline 1025WK09 & 2 & $15-30$ & 0 & Negative & 10YR $3 / 1$ & Clay & STP is on wetland on corridor with swamp on either side & Subsoil \\
\hline 1025WK11 & 1 & $0-35$ & 0 & Negative & 10YR $3 / 1$ & Silty clay loam & STP is on wetland on corridor with swamp on either side. Inundated at $35 \mathrm{cmbs}$ & Inundation \\
\hline 1026 WK10 & 1 & $0-25$ & 0 & Negative & 10YR $4 / 1$ & Silty clay loam & Wetland/ pasture with inundation everywhere but in high areas & Soil change \\
\hline 1026 WK10 & 2 & $25-40$ & 0 & Negative & 10YR $5 / 1$ & Clay & Wetland/ pasture with inundation everywhere but in high areas & Subsoil \\
\hline 1026JL02 & 1 & $0-10$ & 10 & Negative & 1OYR $4 / 1$ & Clay & Inundated cow pasture. Slight upland area & Inundation \\
\hline 1026JL04 & 1 & $0-10$ & 10 & Negative & $10 Y R 4 / 1$ & Clay & Inundated cow pasture. Slight upland area & Inundation \\
\hline 102618RC4 & & $0-10$ & 10 & Negative & 10YR 4/1 & Clay & Inundated cow pasture. Slight upland area & Inundation \\
\hline 1026JL06 & 1 & $0-15$ & 10 & Negative & 10YR $4 / 1$ & Clay & Inundated cow pasture. Slight upland area & Soil change \\
\hline $1026 \mathrm{JL} 06$ & 2 & $15-30$ & 10 & Negative & $10 \mathrm{YR} 3 / 1$ & Clay & Inundated cow pasture. Slight upland area & Subsoil \\
\hline $1026 J L 08$ & 1 & $0-15$ & 10 & Negative & 10YR $4 / 1$ & Clay & Inundated cow pasture. Slight upland area & Soil change \\
\hline $1026 J \mathrm{~L} 08$ & 2 & $15-30$ & 10 & Negative & 10YR $3 / 1$ & Clay & Inundated cow pasture. Slight upland area & Subsoil \\
\hline 1026JL11 & 1 & $0-15$ & 10 & Negative & 10YR $4 / 1$ & Clay & Inundated cow pasture. Slight upland area & Soil change \\
\hline 1026JL11 & 2 & $15-30$ & 10 & Negative & $10 \mathrm{YY} 3 / 1$ & Clay & Inundated cow pasture. Slight upland area & Subsoil \\
\hline 1026WE01 & 1 & $0-15$ & 0 & Negative & 10YR $4 / 2$ & Silty clay & Wetland area, cow pasture, grasses & Soil change \\
\hline 1026WE01 & 2 & $15-30$ & 0 & Negative & 10YR $4 / 1$ & Clay & Wetland area, cow pasture, grasses & Subsoil \\
\hline 1026WE03 & 1 & $0-15$ & 0 & Negative & 10YR $4 / 2$ & Silty clay & Wetland area, cow pasture, grasses & Soil change \\
\hline 1026WE03 & 2 & $15-30$ & 0 & Negative & 10YR $4 / 1$ & Clay & Wetland area, cow pasture, grasses & Subsoil \\
\hline 1026WE05 & 1 & $0-10$ & 0 & Negative & 10YR $4 / 2$ & Silty clay & Wetland area, cow pasture, grasses & Soil change \\
\hline 1026WE05 & 2 & $10-25$ & 0 & Negative & 10YR $4 / 1$ & Clay & Wetland area, cow pasture, grasses & Subsoil \\
\hline 1026WE09 & 1 & $0-20$ & 0 & Negative & $10 \mathrm{YR} 4 / 2$ & Silty clay & Wetland area, cow pasture, grasses & Soil change \\
\hline 1026WE09 & 2 & $20-25$ & 0 & Negative & 10YR 4/1 & Clay & Wetland area, cow pasture, grasses & Subsoil \\
\hline 1027RC04 & 1 & $0-10$ & 0 & Negative & 10YR $4 / 1$ & Clay loam & Cow pasture. Short Grasses. Water at $10 \mathrm{cmbs}$ & Inundation \\
\hline 1027JL01 & 1 & $0-10$ & 0 & Negative & $10 \mathrm{Yr} 4 / 1$ & Clay loam & Semi-wooded area, inundated area, high ground & Inundation \\
\hline $1027 J L 05$ & 1 & $0-10$ & 0 & Negative & $10 \mathrm{YR} 4 / 1$ & Clay loam & Open cow pasture, mostly inundated & Inundation \\
\hline $1027 \mathrm{JL} 05$ & 2 & $10-20$ & 0 & Negative & $10 \mathrm{YR} 4 / 6$ & Clay & Open cow pasture, mostly inundated & Inundation \\
\hline 1027 WK06 & 1 & $0-25$ & 0 & Negative & 10 YR $4 / 1$ & Clay loam & Wetland being utilized as a pasture inundated throughout most of area & Soil change \\
\hline 1027 WK06 & 2 & $25-35$ & 0 & Negative & $10 \mathrm{YR} 4 / 6$ & Clay & Wetland being utilized as a pasture inundated throughout most of area & Subsoil \\
\hline $102718 \mathrm{JL} 8$ & 1 & $0-10$ & 0 & Negative & 10 YR $4 / 1$ & Clay loam & Open cow pasture, mostly inundated & Inundation \\
\hline
\end{tabular}




\begin{tabular}{|c|c|c|c|c|c|c|c|c|}
\hline Shovel Test \# & $\begin{array}{l}\text { Level } \\
\text { (Strat) }\end{array}$ & $\begin{array}{l}\text { Depth } \\
\text { (cmbs) }\end{array}$ & GSV \% & $\begin{array}{l}\text { Positive or } \\
\text { Negative }\end{array}$ & $\begin{array}{c}\text { Munsell } \\
\text { Color }\end{array}$ & Soil Texture Description & Description (Area; Vegetation) & Reason for Termination \\
\hline 1027WK09 & 1 & $0-25$ & 0 & Negative & $10 \mathrm{YR} 4 / 1$ & Clay loam & Wetland being utilized as a pasture inundated throughout most of area & Soil change \\
\hline 1027WK09 & 2 & $25-35$ & 0 & Negative & $10 \mathrm{YR} 4 / 6$ & Clay & Wetland being utilized as a pasture inundated throughout most of area & Subsoil \\
\hline 1027WE02 & 1 & $0-15$ & 0 & Negative & 10YR $4 / 1$ & Clay & Tall grass & Inundation \\
\hline 1027WE07 & 1 & $0-15$ & 0 & Negative & 10 YR $4 / 1$ & Clay loam & Wetland and cow pasture. & Inundation \\
\hline 1027WE07 & 2 & $15-25$ & 0 & Negative & 1OYR $4 / 6$ & Clay & Wetland and cow pasture. & Inundation \\
\hline 1027WE10 & 1 & $0-10$ & 0 & Negative & $10 \mathrm{YR} 4 / 1$ & Clay loam & Wetland and cow pasture. & Inundation \\
\hline 1027WE10 & 2 & $10-20$ & 0 & Negative & $10 \mathrm{YYR} 4 / 6$ & Clay & Wetland and cow pasture. & Inundation \\
\hline 1027TR03 & 1 & $0-10$ & 0 & Negative & 10YR 5/1 & Clay & Clear-cut zone containing tall grasses and shrubs, lightly inundated & Soil change \\
\hline 1027TR03 & 2 & $10-20$ & 0 & Negative & 10YR $4 / 1$ & Clay & Clear-cut zone containing tall grasses and shrubs, lightly inundated & Subsoil \\
\hline 1029TR02 & 1 & $0-15$ & 0 & Negative & 10YR $4 / 1$ & Clay & $\begin{array}{c}\text { Grassy field surrounded by mature trees (mesquite, Japanese maple, American Elm, Chinese } \\
\text { Tallow }\end{array}$ & Soil change \\
\hline 1029TR02 & 2 & $15-25$ & 0 & Negative & 10YR 5/1 & Clay & $\begin{array}{c}\text { Grassy field surrounded by mature trees (mesquite, Japanese maple, American Elm, Chinese } \\
\text { Tallow }\end{array}$ & Subsoil \\
\hline 1029WK03 & 1 & $0-15$ & 0 & Negative & 10YR 5/1 & Clay & Area was wetland/ pasture; areas of inundation around STP & Soil change \\
\hline 1029WK03 & 2 & $15-30$ & 0 & Negative & 10YR $4 / 1$ & Clay & Area was wetland/ pasture; areas of inundation around STP & Subsoil \\
\hline 1029JL04 & 1 & $0-10$ & 0 & Negative & 10YR $4 / 1$ & Clay & In open field; inundated at $10 \mathrm{cmbs}$ & Inundation \\
\hline 1029WE01 & 1 & $0-10$ & 0 & Negative & 10YR $4 / 2$ & Clay & Cow pasture. Open field; tall grasses & Soil change \\
\hline 1029WE01 & 2 & $10-15$ & 0 & Negative & $10 \mathrm{YR} 4 / 2$ & Clay & Cow pasture. Open field; tall grasses & Subsoil \\
\hline 1030TR01 & 1 & $0-15$ & 0 & Negative & $10 \mathrm{YR} 4 / 2$ & Clay & Rice field; near dirt road & Soil change \\
\hline 1030TR01 & 2 & $15-25$ & 0 & Negative & 10YR $5 / 2$ & Clay & Rice field; near dirt road & Subsoil \\
\hline 1030TR03 & 1 & $0-15$ & 0 & Negative & $10 Y R 4 / 2$ & Clay & Rice field; lightly inundated, surrounded by berms & Soil change \\
\hline 1030TR03 & 2 & $15-25$ & 0 & Negative & $10 Y R 5 / 2$ & Clay & Rice field; lightly inundated, surrounded by berms & Subsoil \\
\hline 1030TR06 & 1 & $0-15$ & 0 & Negative & $10 Y R 4 / 2$ & Clay & Rice field; lightly inundated, surrounded by berms & Soil change \\
\hline 1030TR06 & 2 & $15-25$ & 0 & Negative & $10 Y R 5 / 2$ & Clay & Rice field; lightly inundated, surrounded by berms & Subsoil \\
\hline 1030TR08 & 1 & $0-12$ & 0 & Negative & $10 Y R 4 / 2$ & Clay & Rice field; lightly inundated, surrounded by berms & Soil change \\
\hline 1030TR08 & 2 & $12-25$ & 0 & Negative & 10YR $5 / 2$ & Clay & Rice field; lightly inundated, surrounded by berms & Subsoil \\
\hline 1030TR10 & 1 & $0-8$ & 100 & Negative & 10YR $4 / 2$ & Clay & Sparsely vegetated field, heavily disturbed by machinery & Soil change \\
\hline 1030TR10 & 2 & $8-18$ & 100 & Negative & $10 \mathrm{YR} 5 / 2$ & Clay & Sparsely vegetated field, heavily disturbed by machinery & Subsoil \\
\hline 1030TR12 & 1 & $0-5$ & 100 & Negative & 1OYR $4 / 2$ & Clay & Sparsely vegetated field, heavily disturbed by machinery & Soil change \\
\hline 1030TR12 & 2 & $5-15$ & 100 & Negative & 10YR 5/2 & Clay & Sparsely vegetated field, heavily disturbed by machinery & Subsoil \\
\hline 1030TR14 & 1 & $0-5$ & 100 & Negative & $10 \mathrm{YYR} 4 / 2$ & Clay & Sparsely vegetated field, heavily disturbed by machinery & Soil change \\
\hline 1030TR14 & 2 & $5-15$ & 100 & Negative & $10 Y R 5 / 2$ & Clay & Sparsely vegetated field, heavily disturbed by machinery & Subsoil \\
\hline 1030TR16 & 1 & $0-5$ & 100 & Negative & $10 Y R 4 / 2$ & Clay & Sparsely vegetated field, heavily disturbed by machinery & Inundation \\
\hline 1030TR18 & 1 & $0-5$ & 100 & Negative & $10 Y R 4 / 2$ & Clay & Sparsely vegetated field, heavily disturbed by machinery & Soil change \\
\hline 1030TR18 & 2 & $5-15$ & 100 & Negative & $10 Y R 5 / 2$ & Clay & Sparsely vegetated field, heavily disturbed by machinery & Subsoil \\
\hline 1030TR20 & 1 & $0-10$ & 100 & Negative & 10YR $4 / 2$ & Clay & Sparsely vegetated field, heavily disturbed by machinery & Soil change \\
\hline 1030TR20 & 2 & $10-20$ & 100 & Negative & $10 Y R 5 / 2$ & Clay & Sparsely vegetated field, heavily disturbed by machinery & Subsoil \\
\hline 1030TR22 & 1 & $0-15$ & 50 & Negative & $10 \mathrm{YYR} 4 / 2$ & Clay & Sparsely vegetated field, heavily disturbed by machinery & Soil change \\
\hline 1030TR22 & 2 & $15-25$ & 50 & Negative & $10 Y R 5 / 2$ & Clay & Sparsely vegetated field, heavily disturbed by machinery & Subsoil \\
\hline 1030TR24 & 1 & $0-3$ & 100 & Negative & $10 Y R 4 / 2$ & Clay & Sparsely vegetated field, heavily disturbed by machinery & Soil change \\
\hline 1030TR24 & 2 & 3-15 & 100 & Negative & 10YR $5 / 2$ & Clay & Sparsely vegetated field, heavily disturbed by machinery & Subsoil \\
\hline 1030TR26 & 1 & $0-10$ & 100 & Negative & $10 \mathrm{YR} 4 / 2$ & Clay & Sparsely vegetated field, heavily disturbed by machinery & Soil change \\
\hline 1030TR26 & 2 & $10-20$ & 100 & Negative & $10 \mathrm{YR} 5 / 2$ & Clay & Sparsely vegetated field, heavily disturbed by machinery & Subsoil \\
\hline 1030TR28 & 1 & $0-5$ & 100 & Negative & $10 Y R 4 / 2$ & Clay & Sparsely vegetated field, heavily disturbed by machinery & Soil change \\
\hline 1030TR28 & 2 & 5-15 & 100 & Negative & $10 \mathrm{YR} 5 / 2$ & Clay & Sparsely vegetated field, heavily disturbed by machinery & Subsoil \\
\hline 1030TR10 & 1 & $0-10$ & 50 & Negative & 10YR $4 / 2$ & Clay & Elm woodland with some pine and oak & Soil change \\
\hline 1030TR10 & 2 & $10-20$ & 50 & Negative & $10 \mathrm{YR} 5 / 2$ & Clay & Elm woodland with some pine and oak & Subsoil \\
\hline 1030TR14 & 1 & $0-10$ & 0 & Negative & 1OYR $4 / 2$ & Clay & Marsh vegetated land between woods and modified grassy area & Soil change \\
\hline 1030TR14 & 2 & $10-25$ & 0 & Negative & $10 \mathrm{YR} 5 / 2$ & Clay & Marsh vegetated land between woods and modified grassy area & Subsoil \\
\hline 1030TR17 & 1 & $0-10$ & 0 & Negative & 10YR $4 / 2$ & Clay & $\begin{array}{l}\text { Marsh vegetated land between woods and modified grassy area. Lightly inundated with American } \\
\text { elm, pine, black oak }\end{array}$ & Soil change \\
\hline 1030TR17 & 2 & $10-20$ & 0 & Negative & 10YR $5 / 2$ & Clay & $\begin{array}{l}\text { Marsh vegetated land between woods and modified grassy area. Lightly inundated with American } \\
\text { elm, pine, black oak }\end{array}$ & Subsoil \\
\hline 1030WE01 & 1 & $0-20$ & 0 & Negative & 10YR 3/1 & Clay & Cow pasture, short grass & Soil change \\
\hline 1030WE01 & 2 & $20-30$ & 0 & Negative & $10 \mathrm{YR} 3 / 1$ & Clay & Cow pasture, short grass & Subsoil \\
\hline 1030WE04 & 1 & $0-20$ & 0 & Negative & 10YR $4 / 1$ & Clay & Cow pasture, short grass & Soil change \\
\hline 1030WE04 & 2 & $20-30$ & 0 & Negative & $10 \mathrm{YR} 4 / 2$ & Clay & Cow pasture, short grass & Subsoil \\
\hline
\end{tabular}




\begin{tabular}{|c|c|c|c|c|c|c|c|c|}
\hline Shovel Test \# & $\begin{array}{l}\text { Level } \\
\text { (Strat) }\end{array}$ & $\begin{array}{l}\text { Depth } \\
\text { (cmbs) }\end{array}$ & GSV \% & $\begin{array}{l}\text { Positive or } \\
\text { Negative }\end{array}$ & $\begin{array}{l}\text { Munsell } \\
\text { Color }\end{array}$ & Soil Texture Description & Description (Area; Vegetation) & Reason for Termination \\
\hline 1030WE07 & 1 & $0-20$ & 0 & Negative & 10YR $4 / 1$ & Clay & Cow pasture, short grass & Subsoil \\
\hline 1030WE09 & 1 & $0-20$ & 0 & Negative & 10YR $4 / 1$ & Clay & Cow pasture, short grass & Soil change \\
\hline 1030WE09 & 2 & $20-30$ & 0 & Negative & 10YR $4 / 1$ & Clay & Cow pasture, short grass & Subsoil \\
\hline 1030WE11 & 1 & $0-20$ & 0 & Negative & 10YR $5 / 2$ & Clay & Disturbed area by river & Soil change \\
\hline 1030WE11 & 2 & $20-30$ & 0 & Negative & 10YR $4 / 2$ & Clay & Disturbed area by river & Subsoil \\
\hline 1030JL02 & 1 & $0-10$ & 20 & Negative & 10YR $4 / 1$ & Clay & $\begin{array}{l}\text { Rice field } \\
\end{array}$ & Inundation \\
\hline 1030JL04 & 1 & $0-10$ & 20 & Negative & 10YR $4 / 1$ & Clay & Rice field & Inundation \\
\hline 1030JL05 & 1 & $0-10$ & 20 & Negative & 10YR $4 / 1$ & Clay & Rice field & Inundation \\
\hline 1030JL07 & 1 & $0-15$ & 20 & Negative & 10YR $4 / 1$ & Clay & Rice field & Inundation \\
\hline 1030JL07 & 2 & $15-25$ & 20 & Negative & 10YR $3 / 1$ & Clay & Rice field & Inundation \\
\hline 1030JLO9 & 1 & $0-15$ & 90 & Negative & 10YR $4 / 1$ & Clay & Open field, cut field & Soil change \\
\hline 1030JL09 & 2 & $15-25$ & 90 & Negative & 10YR $3 / 1$ & Clay & Open field, cut field & Subsoil \\
\hline 1030JL11 & 1 & $0-15$ & 90 & Negative & 10YR $4 / 1$ & Clay & Open field, cut field & Soil change \\
\hline 1030JL11 & 2 & $15-25$ & 90 & Negative & 10YR $3 / 1$ & Clay & Open field, cut field & Subsoil \\
\hline 1030JL13 & 1 & $0-15$ & 90 & Negative & 10YR $4 / 1$ & Clay & Open field, cut field & Soil change \\
\hline 1030JL13 & 2 & $15-25$ & 90 & Negative & 10YR $3 / 1$ & Clay & Open field, cut field & Subsoil \\
\hline 1030JL15 & 1 & $0-15$ & 90 & Negative & 10YR $4 / 1$ & Clay & Open field, cut field & Soil change \\
\hline 1030JL15 & 2 & $15-25$ & 90 & Negative & 10YR $3 / 1$ & Clay & Open field, cut field & Subsoil \\
\hline 1030JL17 & 1 & $0-10$ & 90 & Negative & 10YR 4/1 & Clay & Open field, cut field & Subsoil \\
\hline 1030JL19 & 1 & $0-10$ & 90 & Negative & 10YR $4 / 1$ & Clay & Open field, cut field & Soil change \\
\hline 1030JL19 & 2 & $10-20$ & 90 & Negative & 10YR $3 / 1$ & Clay & Open field, cut field & Subsoil \\
\hline 1030JL21 & 1 & $0-10$ & 90 & Negative & 10YR $4 / 1$ & Clay & Open field, cut field & Soil change \\
\hline 1030JL21 & 2 & $10-20$ & 90 & Negative & 10YR $3 / 1$ & Clay & Open field, cut field & Subsoil \\
\hline 1030JL23 & 1 & $0-10$ & 90 & Negative & 10YR $4 / 1$ & Clay & Open field, cut field & Subsoil \\
\hline 1030JL27 & 1 & $0-15$ & 90 & Negative & 10YR $4 / 1$ & Clay & Open field, cut field & Soil change \\
\hline 1030JL27 & 2 & $15-25$ & 90 & Negative & 10YR $3 / 1$ & Clay & Open field, cut field & Subsoil \\
\hline 1030JL29 & 1 & $0-15$ & 90 & Negative & 10YR $4 / 1$ & Clay & Open field, cut field & Soil change \\
\hline 1030JL29 & 2 & $15-25$ & 90 & Negative & 10YR $3 / 1$ & Clay & Open field, cut field & Subsoil \\
\hline 1030JL12 & 1 & $0-10$ & 0 & Negative & 10YR $4 / 1$ & Clay & In semi-inundated wooded area, charcoal present & Soil change \\
\hline $1030 \mathrm{JL} 12$ & 2 & $10-20$ & 0 & Negative & 10YR $3 / 1$ & Clay & In semi-inundated wooded area, charcoal present & Subsoil \\
\hline 1030JL16 & 1 & $0-10$ & 0 & Negative & 10YR $4 / 1$ & Clay & Tall grass; waist high & Subsoil \\
\hline 1030WK02 & 1 & $0-20$ & 10 & Negative & 10YR $3 / 1$ & Silty clay loam & Wetland with several areas of inundation; utilized as pasture & Soil change \\
\hline 1030WK02 & 2 & $20-30$ & 10 & Negative & 10YR $4 / 1$ & Silty clay & Wetland with several areas of inundation; utilized as pasture & Subsoil \\
\hline 1030WK05 & 1 & $0-20$ & 10 & Negative & 10YR $4 / 1$ & Silty clay & Wetland with several areas of inundation; utilized as pasture & Soil change \\
\hline 1030WK08 & 1 & $0-25$ & 10 & Negative & 10YR $4 / 1$ & Loam & Wetland with several areas of inundation; utilized as pasture & Soil change \\
\hline 1030WK08 & 2 & $25-35$ & 10 & Negative & 10YR 5/1 & Clay loam & Wetland with several areas of inundation; utilized as pasture & Subsoil \\
\hline 1030WK13 & 1 & $0-25$ & 10 & Negative & 10YR $4 / 2$ & Loam & Wetland with several areas of inundation; very disturbed from construction & Soil change \\
\hline 1030WK13 & 2 & $25-35$ & 10 & Negative & 10YR $5 / 2$ & Clay loam & Wetland with several areas of inundation; very disturbed from construction & Subsoil \\
\hline 1030WK18 & 1 & $0-15$ & 10 & Negative & 10YR $4 / 1$ & Loam & Wetland; however it is forested with hardwoods, lots of leaf litter & Soil change \\
\hline 1030WK18 & 2 & $15-30$ & 10 & Negative & 10YR $4 / 6$ & Clay loam & Wetland; however it is forested with hardwoods, lots of leaf litter & Subsoil \\
\hline 1030RC03 & 1 & $0-15$ & 0 & Negative & 10YR $4 / 1$ & Clay loam & TX-Je -030 open cow pasture; short grass; scattered trees & Inundation \\
\hline $1030 \mathrm{RC} 06$ & 1 & $0-15$ & 0 & Negative & 10YR $4 / 1$ & Clay & TX-Je -030 open cow pasture; short grass; scattered trees & Inundation \\
\hline $1030 \mathrm{RC15}$ & 1 & $0-20$ & 0 & Negative & 10YR $4 / 1$ & Clay & Open grass pasture. Tx Li-025 short grass. Wetlands and rolling terrain & Inundation \\
\hline $1030 \mathrm{RC} 18$ & 1 & $0-20$ & 0 & Negative & 10YR $4 / 1$ & Clay & Open grass pasture. Tx Li-025 short grass. Wetlands and rolling terrain & Inundation \\
\hline $1031 \mathrm{RCO} 2$ & 1 & $0-20$ & 0 & Negative & 10YR $4 / 1$ & Clay loam & Tall brush, difficult to walk through & Inundation \\
\hline 1031RC05 & 1 & $0-10$ & 0 & Negative & 10YR $4 / 1$ & Clay loam & Tall brush, difficult to walk through & Inundation \\
\hline 1031RC08 & 1 & $0-15$ & 0 & Negative & 10YR $4 / 1$ & Clay loam & Tall brush, difficult to walk through & Inundation \\
\hline 1031RC13 & 1 & $0-20$ & 0 & Negative & 10YR $4 / 1$ & Clay loam & Tall brush, difficult to walk through & Inundation \\
\hline 1031RC15 & 1 & $0-10$ & 0 & Negative & 10YR $4 / 1$ & Clay loam & Tall brush, difficult to walk through & Inundation \\
\hline 1031WK04 & 1 & $0-25$ & 0 & Negative & 10YR $3 / 1$ & Loam & STP near existing pipeline; area is disturbed and inundated & Soil change \\
\hline 1031WK04 & 2 & $25-35$ & 0 & Negative & 10YR $4 / 1$ & Clay loam & STP near existing pipeline; area is disturbed and inundated & Subsoil \\
\hline 1031WK09 & 1 & $0-25$ & 0 & Negative & 10YR $3 / 1$ & Loam & STP near existing pipeline; area is disturbed and inundated & Soil change \\
\hline 1031WK12 & 1 & $0-30$ & 0 & Negative & 10YR $3 / 1$ & Loam & STP near existing pipeline; area is disturbed and inundated & Soil change \\
\hline 1031WK12 & 2 & $30-40$ & 0 & Negative & 10YR $4 / 1$ & Clay loam & STP near existing pipeline; area is disturbed and inundated & Subsoil \\
\hline 1031 WK16 & 1 & $0-15$ & 0 & Negative & 10YR $3 / 1$ & Loam & STP near existing pipeline; area is disturbed and inundated & Soil change \\
\hline 1031TR03 & 1 & $0-20$ & 0 & Negative & 10YR $2 / 1$ & Clay & Marshland surrounded by mature trees & Soil change \\
\hline 1031TR03 & 2 & $20-30$ & 0 & Negative & 10YR $3 / 1$ & Clay & Marshland surrounded by mature trees & Subsoil \\
\hline
\end{tabular}




\begin{tabular}{|c|c|c|c|c|c|c|c|c|}
\hline Shovel Test \# & $\begin{array}{l}\text { Level } \\
\text { (Strat) }\end{array}$ & $\begin{array}{l}\text { Depth } \\
\text { (cmbs) }\end{array}$ & GSV \% & $\begin{array}{c}\text { Positive or } \\
\text { Negative }\end{array}$ & $\begin{array}{c}\text { Munsell } \\
\text { Color }\end{array}$ & Soil Texture Description & Description (Area; Vegetation) & Reason for Termination \\
\hline 1031TR07 & 1 & $0-20$ & 0 & Negative & $10 Y R 2 / 1$ & Clay & Marshland surrounded by mature trees & Soil change \\
\hline 1031TR07 & 2 & $20-30$ & 0 & Negative & 10YR $3 / 1$ & Clay & Marshland surrounded by mature trees & Subsoil \\
\hline 1031TR13 & 1 & $0-20$ & 0 & Negative & 10YR $2 / 1$ & Clay & Marshland surrounded by mature trees & Soil change \\
\hline 1031TR13 & 2 & $20-30$ & 0 & Negative & 10YR $3 / 1$ & Clay & Marshland surrounded by mature trees & Subsoil \\
\hline 1031TR17 & 1 & $0-15$ & 0 & Negative & $10 Y R 2 / 1$ & Clay & Marshland surrounded by mature trees & Soil change \\
\hline 1031TR17 & 2 & $15-25$ & 0 & Negative & 10YR $3 / 1$ & Clay & Marshland surrounded by mature trees & Subsoil \\
\hline 1031WE01 & 1 & $0-10$ & 0 & Negative & 10YR $4 / 1$ & Clay & Wetland area with tall grass & Soil change \\
\hline 1031WE01 & 2 & $10-20$ & 0 & Negative & 10YR $3 / 1$ & Clay & Wetland area with tall grass & Subsoil \\
\hline 1031WE06 & 1 & $0-15$ & 0 & Negative & $10 Y R 4 / 1$ & Clay & Heavy rush with wild flowers. Wetland area & Soil change \\
\hline 1031WE06 & 2 & $15-25$ & 0 & Negative & 10YR $3 / 1$ & Clay & Heavy rush with wild flowers. Wetland area & Subsoil \\
\hline 1031WE10 & 1 & $0-10$ & 0 & Negative & 10YR 4/1 & Clay & Short grass with open field & Soil change \\
\hline 1031WE10 & 2 & $10-20$ & 0 & Negative & 10YR $3 / 1$ & Clay & Short grass with open field & Subsoil \\
\hline 1031WE14 & 1 & $0-15$ & 0 & Negative & 10YR $4 / 1$ & Clay & Short grass with open field & Soil change \\
\hline 1031WE14 & 2 & $15-25$ & 0 & Negative & 10YR $3 / 1$ & Clay & Short grass with open field & Subsoil \\
\hline 1031СK02 & 1 & $0-30$ & 0 & Negative & $\begin{array}{c}10 Y R 4 / 1 \\
\& 3 / 1\end{array}$ & Clay & Tall grass. Evidence of inundation. Clay inclusions denote subsoil & Soil change \\
\hline 1031CK02 & 2 & $30-40$ & 0 & Negative & 10YR 5/1 & Clay & Tall grass. Evidence of inundation. Clay inclusions denote subsoil & Subsoil \\
\hline 1031 CK04 & 1 & $0-30$ & 0 & Negative & $10 Y R 3 / 1$ & Clay & Tall grass. Evidence of inundation. Clay inclusions denote subsoil & Soil change \\
\hline 1031 СK04 & 2 & $30-40$ & 0 & Negative & 10YR $5 / 1$ & Clay & Tall grass. Evidence of inundation. Clay inclusions denote subsoil & Subsoil \\
\hline 1031Ск06 & 1 & $0-20$ & 0 & Negative & $\begin{array}{c}\text { 10YR 4/1 } \\
\& 3 / 1 \& \\
7 / 1 \\
\end{array}$ & Clay & Hydric soil. H2O@ $20 \mathrm{cmbs}$ & Inundation \\
\hline 1031СК08 & 1 & $0-20$ & 0 & Negative & $\begin{array}{c}\text { 10YR 4/1 } \\
\& 3 / 1 \& \\
7 / 1\end{array}$ & Clay & Hydric soil. H2O@ 20cmbs & Inundation \\
\hline 1031СК11 & 1 & $0-30$ & 0 & Negative & $\begin{array}{c}\text { 10YR 4/1 } \\
\& 3 / 1 \& \\
7 / 1\end{array}$ & Clay & In hydric soils. Clay inclusions denote sub & Soil change \\
\hline 1031CK11 & 2 & $30-40$ & 0 & Negative & 10YR $4 / 3$ & Clay & In hydric soils. Clay inclusions denote sub & Subsoil \\
\hline $1031 \mathrm{CK} 12$ & 1 & $0-30$ & 0 & Negative & $\begin{array}{c}10 Y R \text { 4/1 } \\
\& 3 / 1 \& \\
7 / 1\end{array}$ & Clay & In hydric soils. Clay inclusions denote sub. Heavy mottling & Soil change \\
\hline 1031 CK12 & 2 & $30-40$ & 0 & Negative & $10 \mathrm{YR} 4 / 3$ & Clay & In hydric soils. Clay inclusions denote sub. Heavy mottling & Subsoil \\
\hline $1031 \mathrm{CK} 13$ & 1 & $0-50$ & 0 & Negative & $\begin{array}{c}\text { 10YR 4/1 } \\
\& 3 / 1 \& \\
7 / 1\end{array}$ & Clay & In hydric soils. Clay inclusions denote sub. Heavy mottling & Soil change \\
\hline 1031 CK13 & 2 & $50-70$ & 0 & Negative & $10 \mathrm{YR} 4 / 3$ & Clay & In hydric soils. Clay inclusions denote sub. Heavy mottling & Subsoil \\
\hline 1031CK14 & 1 & $0-50$ & 0 & Negative & $\begin{array}{c}\text { 10YR 4/1 } \\
\& 3 / 1 \& \\
7 / 1\end{array}$ & Clay & In hydric soils. Clay inclusions denote sub. Heavy mottling & Soil change \\
\hline $1031 \mathrm{CK} 14$ & 2 & $50-70$ & 0 & Negative & 10YR $4 / 3$ & Clay & In hydric soils. Clay inclusions denote sub. Heavy mottling & Subsoil \\
\hline 1031CK15 & 1 & $0-70$ & 0 & Negative & $10 Y R 4 / 3$ & Sand & $120 \mathrm{M}$ south of shoreline. Very loose and medium grain sand. Clay inclusions denote subsoil & Soil change \\
\hline $1031 \mathrm{CK} 15$ & 2 & $70-80$ & 0 & Negative & 10YR $6 / 5$ & Clay & $120 \mathrm{M}$ south of shoreline. Very loose and medium grain sand. Clay inclusions denote subsoil & Subsoil \\
\hline 1031СК17 & 1 & $0-70$ & 0 & Negative & 10YR $4 / 3$ & Sand & $120 \mathrm{M}$ south of shoreline. Very loose and medium grain sand. Clay inclusions denote subsoil & Soil change \\
\hline 1031СK17 & 2 & $70-80$ & 0 & Negative & 10YR $6 / 5$ & Clay & $120 \mathrm{M}$ south of shoreline. Very loose and medium grain sand. Clay inclusions denote subsoil & Subsoil \\
\hline 1031CK18 & 1 & $0-70$ & 0 & Negative & 10YR $4 / 3$ & Sand & $120 \mathrm{M}$ south of shoreline. Very loose and medium grain sand. Clay inclusions denote subsoil & Soil change \\
\hline 1031СК18 & 2 & $70-80$ & 0 & Negative & 10YR $6 / 5$ & Clay & $120 \mathrm{M}$ south of shoreline. Very loose and medium grain sand. Clay inclusions denote subsoil & Subsoil \\
\hline 1031 CK20 & 1 & $0-70$ & 0 & Negative & 10YR $4 / 3$ & Sand & $120 \mathrm{M}$ south of shoreline. Very loose and medium grain sand. Clay inclusions denote subsoil & Soil change \\
\hline 1031 СK20 & 2 & $70-80$ & 0 & Negative & 10YR $6 / 5$ & Clay & $120 \mathrm{M}$ south of shoreline. Very loose and medium grain sand. Clay inclusions denote subsoil & Subsoil \\
\hline
\end{tabular}




\begin{tabular}{|c|c|c|c|c|c|c|c|c|}
\hline Shovel Test \# & $\begin{array}{l}\text { Level } \\
\text { (Strat) }\end{array}$ & $\begin{array}{l}\text { Depth } \\
\text { (cmbs) }\end{array}$ & GSV \% & $\begin{array}{l}\begin{array}{c}\text { Positive or } \\
\text { Negative }\end{array} \\
\end{array}$ & $\begin{array}{c}\text { Munsell } \\
\text { Color }\end{array}$ & Soil Texture Description & Description (Area; Vegetation) & Reason for Termination \\
\hline 1031 CK22 & 1 & $0-70$ & 0 & Negative & $10 Y R 4 / 3$ & Sand & $120 \mathrm{M}$ south of shoreline. Very loose and medium grain sand. Clay inclusions denote subsoil & Soil change \\
\hline 1031СК22 & 2 & $70-80$ & 0 & Negative & 10YR $6 / 5$ & Clay & $120 \mathrm{M}$ south of shoreline. Very loose and medium grain sand. Clay inclusions denote subsoil & Subsoil \\
\hline 1031 Ск23 & 1 & $0-70$ & 0 & Negative & $10 \mathrm{YR} 4 / 3$ & Sand & $120 \mathrm{M}$ south of shoreline. Very loose and medium grain sand. Clay inclusions denote subsoil & Soil change \\
\hline 1031 CK23 & 2 & $70-80$ & 0 & Negative & 10YR $6 / 5$ & Clay & $120 \mathrm{M}$ south of shoreline. Very loose and medium grain sand. Clay inclusions denote subsoil & Subsoil \\
\hline 1031 СK26 & 1 & $0-70$ & 0 & Negative & 1OYR $4 / 3$ & Sand & $120 \mathrm{M}$ south of shoreline. Very loose and medium grain sand. Clay inclusions denote subsoil & Soil change \\
\hline 1031 СK26 & 2 & $70-80$ & 0 & Negative & $10 Y R 6 / 5$ & Clay & $120 \mathrm{M}$ south of shoreline. Very loose and medium grain sand. Clay inclusions denote subsoil & Subsoil \\
\hline 1031 СK27 & 1 & $0-70$ & 0 & Negative & 1OYR $4 / 3$ & Sand & $120 \mathrm{M}$ south of shoreline. Very loose and medium grain sand. Clay inclusions denote subsoil & Soil change \\
\hline 1031 СK27 & 2 & $70-80$ & 0 & Negative & 10YR $6 / 5$ & Clay & $120 \mathrm{M}$ south of shoreline. Very loose and medium grain sand. Clay inclusions denote subsoil & Subsoil \\
\hline $1031 \mathrm{HS} 1$ & 1 & $0-10$ & 0 & Negative & 10YR $4 / 3$ & Sand & Open, grassy area btw heavy veg. brush + standing family & Soil change \\
\hline $1031 \mathrm{HS1}$ & 2 & $10-30$ & 0 & Negative & $10 \mathrm{YR} 6 / 5$ & Clay & Open, grassy area btw heavy veg. brush + standing family & Subsoil \\
\hline $1031 \mathrm{HS} 3$ & 1 & $0-35$ & 30 & Negative & 10YR $4 / 3$ & Sand & Forested area with scarce leaf ground cover & Soil change \\
\hline $1031 \mathrm{HS} 3$ & 2 & $35-45$ & 30 & Negative & $10 \mathrm{YR} 6 / 5$ & Clay & Forested area with scarce leaf ground cover & Subsoil \\
\hline $1031 \mathrm{HS} 5$ & 1 & $0-35$ & 0 & Negative & 10YR 4/3 & Sand & Open grassy area, next to heavily forested area & Soil change \\
\hline $1031 \mathrm{HS} 5$ & 2 & $35-45$ & 0 & Negative & $10 Y R 6 / 5$ & Clay & Open grassy area, next to heavily forested area & Subsoil \\
\hline $1031 \mathrm{HS7}$ & 1 & $0-20$ & 0 & Negative & $10 \mathrm{YR} 4 / 3$ & Sand & Open grassy area, next to heavily forested area & Subsoil \\
\hline $1031 \mathrm{HS9}$ & 1 & $0-20$ & 0 & Negative & $10 Y R 6 / 5$ & Sand & Open grassy area, next to heavily forested area & Soil change \\
\hline $1031 \mathrm{HSg}$ & 2 & $20-30$ & 0 & Negative & $10 \mathrm{Yr} 4 / 3$ & Clay & Open grassy area, next to heavily forested area & Subsoil \\
\hline $1031 \mathrm{HS} 16$ & 1 & $0-100$ & 0 & Negative & $10 Y R 4 / 4$ & Sand & Open grassy area, next to heavily forested area & Subsoil \\
\hline 1031CK29 & 1 & $0-30$ & 0 & Negative & 10 YR $4 / 3$ & Clay loam & Thick vegetation, surrounded by inundation & Soil change \\
\hline 1031CK29 & 2 & $30-40$ & 0 & Negative & 10 YR $3 / 2$ & Clay & Thick vegetation, surrounded by inundation & Subsoil \\
\hline $103118 \mathrm{HS} 30$ & 1 & $0-25$ & 0 & Negative & 10 YR $3 / 2$ & Clay & Located in heavily wooded & Subsoil \\
\hline 103118 HS28 & 1 & $0-20$ & 0 & Negative & 10 YR $3 / 2$ & Clay & Located in heavily wooded & Subsoil \\
\hline 103118 HS25 & 1 & $0-20$ & 0 & Negative & 10 YR $3 / 2$ & Clay & Located in heavily wooded & Subsoil \\
\hline $103118 \mathrm{HS} 24$ & 1 & $0-30$ & 0 & Negative & 10 YR $3 / 2$ & Clay & Located in heavily wooded & Subsoil \\
\hline 103118 HS21 & 1 & $0-25$ & 0 & Negative & 10 YR $3 / 2$ & Clay & Located in heavily wooded & Subsoil \\
\hline 103118HS19 & 1 & $0-20$ & 0 & Negative & 10 YR $3 / 2$ & Clay & Located in heavily wooded & Subsoil \\
\hline 1031CK31 & 1 & $0-20$ & 0 & Negative & 10 YR $3 / 2$ & Clay & Located in heavily wooded area surrounded by inundation & Subsoil \\
\hline 1217 CK03 & 1 & $0-5$ & 0 & Negative & $10 Y R 5 / 1$ & Clay loam & Located in heavily wooded area surrounded by inundation & Inundation \\
\hline 1217WK02 & 1 & $0-15$ & 0 & Negative & 1OYR $4 / 1$ & Clay & $\begin{array}{c}\begin{array}{c}\text { Area is a creek/ drainage with dense vegetation, older timber, brush, and grasses. Herbaceous } \\
\text { ground cover obscure GSV }\end{array}\end{array}$ & Inundation \\
\hline $1217 \mathrm{~J} 01$ & 1 & $0-10$ & 0 & Negative & $10 \mathrm{YR} 4 / 1$ & Clay & Within powerline corridor. Possible disturbed & Inundation \\
\hline 1219WK38 & 1 & $0-20$ & 30-35 & Negative & N 5/0 & Clay & $\begin{array}{l}\text { Area is a forested wetland; lots of older timbers, understory primarily palmettos, briar, grasses. } \\
\text { Tree litter is primary GSV obfuscation- inundated }\end{array}$ & Soil change \\
\hline 1219WK38 & 2 & 20-35 & $30-35$ & Negative & 10YR $4 / 1$ & Clay & $\begin{array}{l}\text { Area is a forested wetland; lots of older timbers, understory primarily palmettos, briar, grasses. } \\
\text { Tree litter is primary GSV obfuscation- inundated }\end{array}$ & Subsoil \\
\hline 1219WK41 & 1 & 0 & $30-35$ & Negative & Inundated & Inundated & $\begin{array}{l}\text { Area is a forested wetland; lots of older timbers, understory primarily palmettos, briar, grasses. } \\
\text { Tree litter is primary GSV obfuscation- inundated }\end{array}$ & Inundation \\
\hline 1219WK43 & 1 & $0-25$ & $0-5$ & Negative & $N 5 / 0$ & Clay & $\begin{array}{l}\text { Area is a right-of-way with tall grasses and briars. Signs of A.H. disturbances and primarily } \\
\text { inundated }\end{array}$ & Soil change \\
\hline 1219WK43 & 2 & $25-35$ & $0-5$ & Negative & 10YR $4 / 1$ & Clay & $\begin{array}{l}\text { Area is a right-of-way with tall grasses and briars. Signs of A.H. disturbances and primarily } \\
\text { inundated }\end{array}$ & Subsoil \\
\hline 1219WK45 & 1 & $0-20$ & $5-10$ & Negative & N $5 / 0$ & Clay & $\begin{array}{l}\begin{array}{c}\text { Area is a right of way with tall grasses and briars. Signs of A.H. disturbances and primarily } \\
\text { inundated }\end{array} \\
\end{array}$ & Inundation \\
\hline
\end{tabular}




\begin{tabular}{|c|c|c|c|c|c|c|c|c|}
\hline Shovel Test \# & $\begin{array}{l}\text { Level } \\
\text { (Strat) }\end{array}$ & $\begin{array}{l}\text { Depth } \\
\text { (cmbs) }\end{array}$ & GSV \% & $\begin{array}{l}\text { Positive or } \\
\text { Negative }\end{array}$ & $\begin{array}{c}\text { Munsell } \\
\text { Color }\end{array}$ & Soil Texture Description & Description (Area; Vegetation) & Reason for Termination \\
\hline 1219WK51 & 1 & $0-15$ & $0-5$ & Negative & $\mathrm{N} 5 / 0$ & Clay & Area is a right of way near an ag ditch. Clear of timber, but with short grasses. & Soil change \\
\hline 1219WK51 & 2 & $15-35$ & $0-5$ & Negative & 10YR 4/1 & Clay & Area is a right of way near an ag ditch. Clear of timber, but with short grasses. & Subsoil \\
\hline 1219WK54 & 1 & $0-20$ & $10-15$ & Negative & N 5/0 & Clay & $\begin{array}{l}\text { Area is forested/wooded with juvenile to old timber and dense with saw palmettos. Area most } \\
\text { likely subject to periods of inundation - soil oxygenation }\end{array}$ & Soil change \\
\hline 1219WK54 & 2 & $20-35$ & $10-15$ & Negative & 10YR $4 / 1$ & Clay & $\begin{array}{l}\text { Area is forested/wooded with juvenile to old timber and dense with saw palmettos. Area most } \\
\text { likely subject to periods of inundation - soil oxygenation }\end{array}$ & Subsoil \\
\hline 1219WK59 & 1 & $0-15$ & $0-5$ & Negative & $\mathrm{N} 5 / 0$ & Clay & $\begin{array}{l}\text { Area is forested/wooded with juvenile to old timber and dense with saw palmettos. Area most } \\
\text { likely subject to periods of inundation - soil oxygenation }\end{array}$ & Soil change \\
\hline 1219WK59 & 2 & $15-30$ & $0-5$ & Negative & 10YR 4/1 & Clay & $\begin{array}{l}\text { Area is forested/wooded with juvenile to old timber and dense with saw palmettos. Area most } \\
\text { likely subject to periods of inundation - soil oxygenation }\end{array}$ & Subsoil \\
\hline 1219WK62 & 1 & $0-15$ & $0-5$ & Negative & N $5 / 0$ & Clay & $\begin{array}{l}\text { Area is forested/wooded with juvenile to old timber and dense with saw palmettos. Area most } \\
\text { likely subject to periods of inundation - soil oxygenation }\end{array}$ & Soil change \\
\hline 1219WK62 & 2 & $15-30$ & $0-5$ & Negative & 10YR 4/1 & Clay & $\begin{array}{l}\text { Area is forested/wooded with juvenile to old timber and dense with saw palmettos. Area most } \\
\text { likely subject to periods of inundation - soil oxygenation }\end{array}$ & Subsoil \\
\hline 1219WK64 & 1 & $0-25$ & $0-5$ & Negative & $N 5 / 0$ & Clay & $\begin{array}{l}\text { Area is forested/wooded with juvenile to old timber and dense with saw palmettos. Area most } \\
\text { likely subject to periods of inundation - soil oxygenation }\end{array}$ & Inundation \\
\hline 1219WK69 & 1 & $0-15$ & $0-5$ & Negative & N $5 / 0$ & Clay & $\begin{array}{l}\text { Area is forested/wooded with juvenile to old timber and dense with saw palmettos. Area most } \\
\text { likely subject to periods of inundation - soil oxygenation }\end{array}$ & Inundation \\
\hline 1219WK71 & 1 & 0 & 0 & Negative & Inundated & Inundated & Area is a powerline right of way & Inundation \\
\hline 1219WK74 & 1 & $0-30$ & $5-10$ & Negative & Disturbed & Disturbed & $\begin{array}{l}\text { Area is a powerline right of way with lots of dense grasses (chest height), pockets of standing } \\
\text { water, and signs of pig turbation }\end{array}$ & Dusturbed \\
\hline 1219 CK 40 & 1 & $0-20$ & 0 & Negative & N $5 / 0$ & Clay loam & Survey area located in woods. Heavily inundated. Subsoil by compact clay. Bevil clay series & Soil change \\
\hline 1219СK40 & 2 & $20-35$ & 0 & Negative & 10YR $4 / 1$ & Clay & Survey area located in woods. Heavily inundated. Subsoil by compact clay. Bevil clay series & Subsoil \\
\hline 1219СK42 & 1 & $0-20$ & 0 & Negative & $\mathrm{N} 5 / 0$ & Clay loam & Survey area located in woods. Heavily inundated. Subsoil by compact clay. Bevil clay series & Soil change \\
\hline 1219CK42 & 2 & 20-35 & 0 & Negative & 10YR $4 / 1$ & Clay & Survey area located in woods. Heavily inundated. Subsoil by compact clay. Bevil clay series & Subsoil \\
\hline 1219 СК 46 & 1 & $0-20$ & 0 & Negative & N 5/0 & Clay loam & Survey area located in woods. Heavily inundated. Subsoil by compact clay. Bevil clay series & Soil change \\
\hline 1219CK46 & 2 & 20-35 & 0 & Negative & 10YR $4 / 1$ & Clay & Survey area located in woods. Heavily inundated. Subsoil by compact clay. Bevil clay series & Subsoil \\
\hline 1219 CK50 & 1 & $0-20$ & 0 & Negative & $\mathrm{N} 5 / 0$ & Clay loam & Survey area located in woods. Heavily inundated. Subsoil by compact clay. Bevil clay series & Soil change \\
\hline 1219 CK50 & 2 & $20-35$ & 0 & Negative & 10YR $4 / 1$ & Clay & Survey area located in woods. Heavily inundated. Subsoil by compact clay. Bevil clay series & Subsoil \\
\hline 1219 CK52 & 1 & $0-20$ & 0 & Negative & $\mathrm{N} 5 / 0$ & Loamy clay & Heavily wooded \& inundation. Bevil clay. H20 @ 22 25 cmbs & Soil change \\
\hline 1219 CK52 & 2 & $20-25$ & 0 & Negative & 10YR 4/1 & Clay & Heavily wooded \& inundation. Bevil clay. $\mathrm{H} 20 @ 22^{\sim} 25 \mathrm{cmbs}$ & Subsoil \\
\hline 1219 CK55 & 1 & $0-20$ & 0 & Negative & $\mathrm{N} 5 / 0$ & Loamy clay & Heavily wooded \& inundation. Bevil clay. $\mathrm{H} 20 @ 22^{\sim} 25 \mathrm{cmbs}$ & Soil change \\
\hline 1219 CK55 & 2 & $20-25$ & 0 & Negative & 10YR 4/1 & Clay & Heavily wooded \& inundation. Bevil clay. $\mathrm{H} 20 @ 22 \sim 25 \mathrm{cmbs}$ & Subsoil \\
\hline 1219 CK57 & 1 & $0-20$ & 0 & Negative & $\mathrm{N} 5 / 0$ & Loamy clay & Heavily wooded \& inundation. Bevil clay. $\mathrm{H} 20 @ 22^{\sim} 25 \mathrm{cmbs}$ & Soil change \\
\hline 1219 CK57 & 2 & $20-25$ & 0 & Negative & 10YR 4/1 & Clay & Heavily wooded \& inundation. Bevil clay. $\mathrm{H} 20 @ 22^{\sim} 25 \mathrm{cmbs}$ & Subsoil \\
\hline 1219 СK60 & 1 & $0-20$ & 0 & Negative & $\mathrm{N} 5 / 0$ & Loamy clay & Heavily wooded \& inundation. Bevil clay. $\mathrm{H} 20 @ 22^{2} 25 \mathrm{cmbs}$ & Soil change \\
\hline 1219 CK60 & 2 & $20-25$ & 0 & Negative & 1OYR $4 / 1$ & Clay & Heavily wooded \& inundation. Bevil clay. H20 @ 22 25 cmbs & Subsoil \\
\hline 1219 CK63 & 1 & $0-20$ & 0 & Negative & $\mathrm{N} 5 / 0$ & Loamy clay & Heavily wooded \& inundation. Bevil clay. H20@22 25 cmbs & Soil change \\
\hline 1219 CK63 & 2 & $20-25$ & 0 & Negative & 10YR 4/1 & Clay & Heavily wooded \& inundation. Bevil clay. H20@22 25 cmbs & Subsoil \\
\hline 1219 CK66 & 1 & $0-20$ & 0 & Negative & $\mathrm{N} 5 / 0$ & Loamy clay & Heavily wooded \& inundation. Bevil clay. H20@22 25 cmbs & Soil change \\
\hline 1219СK66 & 2 & $20-25$ & 0 & Negative & 10YR 4/1 & Clay & Heavily wooded \& inundation. Bevil clay. H20 @ 22 25 cmbs & Subsoil \\
\hline 1219 CK70 & 1 & $0-40$ & 0 & Negative & $\begin{array}{c}10 Y R 4 / 1 \\
5 / 14 / 3 \\
\text { and gravel }\end{array}$ & Clay & Within open powerline corridor. Heavily disturbed \& undulating surface. & Disturbed \\
\hline 1219 CK73 & 1 & $0-40$ & 0 & Negative & $\begin{array}{c}10 Y R 4 / 1 \\
5 / 14 / 3\end{array}$ & Clay w/gravel & Within open powerline corridor. Heavily disturbed \& undulating surface. & Disturbed \\
\hline
\end{tabular}




\begin{tabular}{|c|c|c|c|c|c|c|c|c|}
\hline Shovel Test \# & $\begin{array}{l}\text { Level } \\
\text { (Strat) }\end{array}$ & $\begin{array}{l}\text { Depth } \\
\text { (cmbs) }\end{array}$ & GSV \% & $\begin{array}{l}\text { Positive or } \\
\text { Negative }\end{array}$ & $\begin{array}{l}\text { Munsell } \\
\text { Color }\end{array}$ & Soil Texture Description & Description (Area; Vegetation) & Reason for Termination \\
\hline $1219 \mathrm{CK} 76$ & 1 & $0-40$ & 0 & Negative & $\begin{array}{c}\text { 10YR } 4 / 1 \\
5 / 14 / 3\end{array}$ & Clay w/gravel & Within open powerline corridor. Heavily disturbed \& undulating surface. & Disturbed \\
\hline 1219JL39 & 1 & $0-10$ & 20 & Negative & 10YR $4 / 6$ & Clay & Forested area. Inundated & Inundation \\
\hline $1219 \mathrm{JL} 46$ & 1 & $0-10$ & 10 & Negative & 10YR $4 / 6$ & Clay & Inundated powerline corridor & Inundation \\
\hline 1219JL48 & 2 & $0-10$ & 10 & Negative & 10YR $4 / 6$ & Clay & Inundated powerline corridor & Inundation \\
\hline 1219JL49 & 1 & $0-23$ & 10 & Negative & 10YR $4 / 6$ & Clay & On corridor between wooded area \& powerline corridor & Soil change \\
\hline 1219JL49 & 2 & $23-58$ & 10 & Negative & 10YR $4 / 1$ & Clay & On corridor between wooded area \& powerline corridor & Subsoil \\
\hline 1219JL53 & 1 & $0-15$ & 20 & Negative & 10YR $4 / 6$ & Clay & Inundated forest & Inundation \\
\hline $1219 \mathrm{JL} 56$ & 1 & $0-15$ & 20 & Negative & 10YR $4 / 6$ & Clay & Inundated forest & Inundation \\
\hline 1219JL58 & 1 & $0-15$ & 20 & Negative & 10YR $4 / 6$ & Clay & Inundated forest & Inundation \\
\hline 1219JL61 & 1 & $0-23$ & 20 & Negative & 10YR $4 / 6$ & Clay & Forest, near small waterbody & Soil change \\
\hline 1219JL61 & 2 & $23-58$ & 20 & Negative & 10YR $4 / 1$ & Clay & Forest, near small waterbody & Subsoil \\
\hline 1219JL65 & 1 & $0-20$ & 30 & Negative & 10YR $4 / 6$ & Clay & Inundated forest & Inundation \\
\hline 1219JL68 & 1 & $0-20$ & 10 & Negative & 10YR $4 / 6$ & Clay & Inundated powerline corridor & Inundation \\
\hline 1219JL72 & 1 & $0-20$ & 20 & Negative & 10YR $4 / 6$ & Clay & Inundated powerline corridor & Inundation \\
\hline $1219 \mathrm{JL} 75$ & 1 & $0-20$ & 40 & Negative & 10YR $4 / 6$ & Clay & Inundated powerline corridor & Inundation \\
\hline 1219JL77 & 1 & $0-20$ & 10 & Negative & 10YR $4 / 6$ & Clay & Inundated powerline corridor & Inundation \\
\hline $1220 \mathrm{CKO6}$ & 1 & $0-15$ & 0 & Negative & 10YR $4 / 1$ & Clay & South of $41 \mathrm{jf} 66$. Surrounded by inundation. Open field/ not heavily modified. & Soil change \\
\hline $1220 \mathrm{CKO6}$ & 2 & $15-30$ & 0 & Negative & 10YR 5/1 & Clay & South of $41 \mathrm{jf66}$. Surrounded by inundation. Open field/ not heavily modified. & Subsoil \\
\hline 1220 CKO9 & 1 & $0-10$ & 0 & Negative & 10YR $4 / 1$ & Clay & South of $41 \mathrm{jf} 66$. Surrounded by inundation. Open field/ not heavily modified. & Inundation \\
\hline 1220 CK13 & 1 & $0-10$ & 0 & Negative & 10YR $3 / 1$ & Sandy clay & Located in marshland. Surrounded by water. H20 @ $10 \mathrm{cmbs}$. & Inundation \\
\hline 1220 CK14 & 1 & $0-10$ & 0 & Negative & 10YR $3 / 1$ & Sandy clay & Located in marshland. Surrounded by water. $\mathrm{H} 20 @ 10 \mathrm{cmbs}$. & Inundation \\
\hline $1220 \mathrm{CK} 16$ & 1 & $0-10$ & 0 & Negative & 10YR $3 / 1$ & Sandy clay & Located in marshland. Surrounded by water. $\mathrm{H} 20 @ 10 \mathrm{cmbs}$. & Inundation \\
\hline $1220 \mathrm{CK} 18$ & 1 & $0-10$ & 0 & Negative & 10YR $3 / 1$ & Sandy clay & Located in marshland. Surrounded by water. H20 @ 10 cmbs. & Inundation \\
\hline $122018 \mathrm{JL} 21$ & & $0-10$ & 0 & Negative & 10YR $3 / 1$ & Sandy clay & Located in marshland. Surrounded by water. $\mathrm{H} 20 @ 10 \mathrm{cmbs}$. & Inundation \\
\hline $1220 \mathrm{CK} 23$ & 1 & $0-10$ & 0 & Negative & 10YR $3 / 1$ & Sandy clay & Located in marshland. Surrounded by water. H20 @ 10 cmbs. & Inundation \\
\hline $1220 \mathrm{CK} 27$ & 1 & $0-10$ & 0 & Negative & 10YR $3 / 1$ & Sandy clay & Located in marshland. Surrounded by water. H20 @ $10 \mathrm{cmbs}$. & Inundation \\
\hline $1220 \mathrm{CK} 29$ & 1 & $0-40$ & 0 & Negative & 10YR $3 / 1$ & Sandy clay loam & Open cow pasture/ knee-high grass. Surrounded by inundation. West of ag ditch & Soil change \\
\hline 1220 CK29 & 2 & $40-50$ & 0 & Negative & 10YR 5/1 & Sandy clay loam & Open cow pasture/ knee-high grass. Surrounded by inundation. West of ag ditch & Subsoil \\
\hline 1220 CK32 & 1 & $0-40$ & 0 & Negative & $10 \mathrm{YR} 3 / 1$ & Sandy clay loam & Open cow pasture/ knee-high grass. Surrounded by inundation. West of ag ditch & Soil change \\
\hline 1220 CK32 & 2 & $40-50$ & 0 & Negative & 10YR 5/1 & Sandy clay loam & Open cow pasture/ knee-high grass. Surrounded by inundation. West of ag ditch & Subsoil \\
\hline $1220 \mathrm{CK} 35$ & 1 & $0-15$ & 0 & Negative & $10 Y R 3 / 1$ & Clay & Open field, knee-high grass. Undisturbed. H20 @ 15cmbs & Inundation \\
\hline $1220 \mathrm{CK} 38$ & 1 & $0-17$ & 0 & Negative & $10 \mathrm{YR} 3 / 1$ & Clay & Open field, knee-high grass. Undisturbed. H20 @ 15cmbs & Inundation \\
\hline 1220JL01 & 1 & $0-23$ & 10 & Negative & $\begin{array}{c}10 Y R 3 / 1 \\
4 / 1\end{array}$ & Clay & On cut grass, near sports complex \& paved road & Soil change \\
\hline 1220」L01 & 2 & $23-50$ & 10 & Negative & $\begin{array}{c}10 Y R 3 / 1 \\
4 / 1\end{array}$ & Clay & On cut grass, near sports complex \& paved road & Subsoil \\
\hline 1220JL04 & 1 & $0-20$ & 10 & Negative & Disturbed & Clay & Inundated grass, near sports complex parking lot & Disturbed \\
\hline 1220JL07 & 1 & $0-23$ & 10 & Negative & 10YR 4/1 & Clay & Inundated pasture. Near historic farmstead & Soil change \\
\hline 1220JL07 & 2 & $23-48$ & 10 & Negative & 10YR 5/1 & Clay & Inundated pasture. Near historic farmstead & Subsoil \\
\hline 1220JL10 & 1 & $0-23$ & 10 & Negative & 10YR $4 / 1$ & Clay & Inundated pasture. Near historic farmstead & Soil change \\
\hline 1220JL10 & 2 & $23-48$ & 10 & Negative & $10 Y R 5 / 1$ & Clay & Inundated pasture. Near historic farmstead & Subsoil \\
\hline 1220JL11 & 1 & $0-15$ & 10 & Negative & Disturbed & Clay & Near gravel road \& gated station & Disturbed \\
\hline 1220JL17 & 1 & $0-13$ & 10 & Negative & 10YR 4/1 & Clay & Pipeline corridor, inundated & Inundation \\
\hline 1220JL19 & 1 & $0-13$ & 10 & Negative & 10YR $4 / 1$ & Clay & Pipeline corridor, inundated & Inundation \\
\hline $1220 \mathrm{JL} 24$ & 1 & $0-13$ & 10 & Negative & 10YR $4 / 1$ & Clay & Pipeline corridor, inundated & Inundation \\
\hline $1220 \mathrm{JL} 25$ & 1 & $0-15$ & 10 & Negative & 10YR $4 / 1$ & Clay & Waist-high grass, inundated & Inundation \\
\hline $1220 \mathrm{JL} 30$ & 1 & $0-23$ & 10 & Negative & 10YR $4 / 1$ & Clay & Open cow pasture & Soil change \\
\hline $1220 \mathrm{JL} 30$ & 2 & $23-48$ & 10 & Negative & 10YR 5/1 & Clay & Open cow pasture & Subsoil \\
\hline 1220JL33 & 1 & $0-23$ & 10 & Negative & 10YR 4/1 & Clay & Open cow pasture & Soil change \\
\hline 1220JL33 & 2 & $23-48$ & 10 & Negative & 10YR 5/1 & Clay & Open cow pasture & Subsoil \\
\hline $1220 \mathrm{JL} 40$ & 1 & $0-15$ & 10 & Negative & 10YR $4 / 1$ & Clay & Wooded area, near powerline corridor & Soil change \\
\hline $1220 \mathrm{JL} 40$ & 2 & $15-28$ & 10 & Negative & 10YR $3 / 1$ & Clay & Wooded area, near powerline corridor & Subsoil \\
\hline 1220 WK02 & 1 & $0-15$ & 0 & Negative & 10YR $3 / 1$ & Clay & Area is manicured lawn adjacent to a very large parking lot and sports compound. & Soil change \\
\hline 1220 WK02 & 2 & $15-35$ & 0 & Negative & 10YR 4/1 & Clay & Area is manicured lawn adjacent to a very large parking lot and sports compound. & Subsoil \\
\hline 1220WK03 & 1 & $0-20$ & 0 & Negative & 10YR $3 / 1$ & Clay & Area is manicured lawn adjacent to a very large parking lot and sports compound. & Soil change \\
\hline 1220WK03 & 2 & $20-35$ & 0 & Negative & 10YR $4 / 1$ & Clay & Area is manicured lawn adjacent to a very large parking lot and sports compound. & Subsoil \\
\hline
\end{tabular}




\begin{tabular}{|c|c|c|c|c|c|c|c|c|}
\hline Shovel Test \# & $\begin{array}{l}\text { Level } \\
\text { (Strat) }\end{array}$ & $\begin{array}{l}\text { Depth } \\
\text { (cmbs) }\end{array}$ & GSV \% & $\begin{array}{c}\text { Positive or } \\
\text { Negative }\end{array}$ & $\begin{array}{c}\text { Munsell } \\
\text { Color }\end{array}$ & Soil Texture Description & Description (Area; Vegetation) & Reason for Termination \\
\hline 1220WK05 & 1 & $0-25$ & 0 & Negative & 10YR $4 / 1$ & Clay & Area in an upland/wetland with close-cut grass. Areas of inundated and gravel road bisecting ESA & Soil change \\
\hline 1220WK05 & 2 & $25-40$ & 0 & Negative & $\begin{array}{c}10 Y R 5 / 1 \\
\& 4 / 6\end{array}$ & Clay & Area in an upland/wetland with close-cut grass. Areas of inundated and gravel road bisecting ESA & Subsoil \\
\hline 1220WK08 & 1 & $0-15$ & 0 & Negative & 10YR $4 / 1$ & Clay & Area in an upland/wetland with close-cut grass. Areas of inundated and gravel road bisecting ESA & Disturbed \\
\hline 1220WK08 & 2 & $15-40$ & 0 & Negative & $\begin{array}{c}10 Y R 5 / 1 \\
\& 4 / 6\end{array}$ & Clay & Area in an upland/wetland with close-cut grass. Areas of inundated and gravel road bisecting ESA & Disturbed \\
\hline 1220WK12 & 1 & $0-30$ & 30 & Negative & 10YR 4/1 & Clay & Area is thoroughly inundated wetland. STP spot is on a dry patch with young pine. & Soil change \\
\hline 1220WK12 & 2 & $30-40$ & 30 & Negative & 10YR $5 / 1$ & Clay & Area is thoroughly inundated wetland. STP spot is on a dry patch with young pine. & Subsoil \\
\hline 1220WK15 & 1 & $0-20$ & 30 & Negative & 10YR $3 / 1$ & Clay & Area is thoroughly inundated wetland. STP spot is on a dry patch with young pine. & Soil change \\
\hline 1220WK15 & 2 & $20-30$ & 30 & Negative & 10YR $4 / 1$ & Clay & Area is thoroughly inundated wetland. STP spot is on a dry patch with young pine. & Subsoil \\
\hline 1220 WK20 & 1 & $0-25$ & 15 & Negative & 10YR $5 / 1$ & Clay & Area subject to frequent periods of inundation & Inundation \\
\hline 1220WK22 & 1 & $0-15$ & 15 & Negative & 10YR $4 / 1$ & Clay & Area subject to frequent periods of inundation & Inundation \\
\hline 1220WK26 & 1 & $0-5$ & 0 & Negative & 10YR $4 / 1$ & Clay & $\begin{array}{c}\text { Area is a tall-grass pasture with standing water throughout; tread going through area has } \\
\text { disturbed soil }\end{array}$ & Inundation \\
\hline 1220WK28 & 1 & $0-20$ & 0 & Negative & $\begin{array}{c}10 Y R 4 / 1 \\
\& 5 / 1\end{array}$ & Clay & $\begin{array}{l}\text { Area is a tall-grass pasture with standing water throughout; tread going through area has } \\
\text { disturbed soil }\end{array}$ & Disturbed \\
\hline 1220WK31 & 1 & $0-25$ & 20 & Negative & $\begin{array}{c}10 Y R 3 / 1 \\
\& 5 / 6\end{array}$ & Silty clay loam & Area is mostly open field with dense green grasses & Soil change \\
\hline 1220WK31 & 2 & $25-35$ & 20 & Negative & 10YR $3 / 1$ & Silty clay & Area is mostly open field with dense green grasses & Subsoil \\
\hline 1220WK34 & 1 & $0-20$ & 0 & Negative & $10 Y R 3 / 1$ & Silty clay loam & Area is mostly open field with dense green grasses & Inundation \\
\hline 1220WK39 & 1 & $0-15$ & 0 & Negative & 10YR $3 / 1$ & Clay & Area is a forested/ wooded area wetland. & Soil change \\
\hline 1220WK39 & 2 & $15-30$ & 0 & Negative & 10YR $4 / 1$ & Clay & Area is a forested/ wooded area wetland. & Subsoil \\
\hline 1221CK02 & 1 & $0-10$ & 0 & Negative & $10 Y R 5 / 1$ & Clay & Open field with knee high grass. $\mathrm{H} 2 \mathrm{O}$ at $10 \mathrm{cmbs}$ & Inundation \\
\hline 1221CK03 & 1 & $0-10$ & 0 & Negative & 10YR 5/1 & Clay & Open field with knee high grass. $\mathrm{H} 2 \mathrm{O}$ at $10 \mathrm{cmbs}$ & Inundation \\
\hline 1221CK06 & 1 & $0-10$ & 0 & Negative & 10YR 5/1 & Clay & Open field with knee high grass. $\mathrm{H} 2 \mathrm{O}$ at $10 \mathrm{cmbs}$ & Inundation \\
\hline 1221CK08 & 1 & $0-10$ & 0 & Negative & 10YR 5/1 & Clay & Open field with knee high grass. $\mathrm{H} 2 \mathrm{O}$ at $10 \mathrm{cmbs}$ & Inundation \\
\hline 1221CK11 & 1 & $0-10$ & 0 & Negative & 10YR 5/1 & Clay & Open field with knee high grass. $\mathrm{H} 2 \mathrm{O}$ at $10 \mathrm{cmbs}$ & Inundation \\
\hline 1221CK14 & 1 & $0-15$ & 0 & Negative & 10YR 5/1 & Clay & Open field with knee high grass. & Soil change \\
\hline 1221CK14 & 2 & $15-30$ & 0 & Negative & $10 Y R 3 / 1$ & Clay & Open field with knee high grass. & Subsoil \\
\hline 1221CK15 & 1 & $0-15$ & 0 & Negative & 10YR 5/1 & Clay & Open field with knee high grass. & Soil change \\
\hline 1221CK15 & 2 & $15-30$ & 0 & Negative & $10 \mathrm{YR} 3 / 1$ & Clay & Open field with knee high grass. & Subsoil \\
\hline 1221CK18 & 1 & $0-15$ & 0 & Negative & 10YR $5 / 1$ & Clay & Open field with knee high grass. & Soil change \\
\hline 1221CK18 & 2 & $15-30$ & 0 & Negative & $10 \mathrm{YR} 3 / 1$ & Clay & Open field with knee high grass. & Subsoil \\
\hline 1221CK22 & 1 & $0-5$ & 0 & Negative & 10YR 5/1 & Clay & Open field- fallow. Heavily inundated with tall grass. Water at $5 \mathrm{cmbs}$. & Inundation \\
\hline $1221 \mathrm{CK} 25$ & 1 & $0-5$ & 0 & Negative & 10YR 5/1 & Clay & Open field- fallow. Heavily inundated with tall grass. Water at $5 \mathrm{cmbs}$. & Inundation \\
\hline $1221 \mathrm{CK} 28$ & 1 & $0-5$ & 0 & Negative & 10YR 5/1 & Clay & Open field- fallow. Heavily inundated with tall grass. Water at $5 \mathrm{cmbs}$. & Inundation \\
\hline 1221CK31 & 1 & $0-5$ & 0 & Negative & 10YR 5/1 & Clay & Open field- fallow. Heavily inundated with tall grass. Water at $5 \mathrm{cmbs}$. & Inundation \\
\hline 1221 CK33 & 1 & $0-5$ & 0 & Negative & 10YR 5/1 & Clay & Open field- fallow. Heavily inundated with tall grass. Water at $5 \mathrm{cmbs}$. & Inundation \\
\hline 1221CK34 & 1 & $0-5$ & 0 & Negative & 10YR 5/1 & Clay & Open field- fallow. Heavily inundated with tall grass. Water at $5 \mathrm{cmbs}$. & Inundation \\
\hline 1221JL09 & 1 & $0-15$ & 0 & Negative & $\begin{array}{c}10 Y R 4 / 1 \\
\& 5 / 1\end{array}$ & Clay & Waist-high grasses and brush & Inundation \\
\hline 1221JL10 & 1 & $0-15$ & 0 & Negative & $\begin{array}{c}10 Y R ~ 4 / 1 \\
\& 5 / 1\end{array}$ & Clay & Waist-high grasses and brush & Inundation \\
\hline 1221JL13 & 1 & $0-15$ & 0 & Negative & $\begin{array}{c}10 Y R 4 / 1 \\
\& 5 / 1\end{array}$ & Clay & Waist-high grasses and brush & Inundation \\
\hline 1221JL16 & 1 & $0-15$ & 0 & Negative & $\begin{array}{c}10 Y R 4 / 1 \\
\& 5 / 1\end{array}$ & Clay & Waist-high grasses and brush & Inundation \\
\hline 1221JL19 & 1 & $0-15$ & 0 & Negative & $\begin{array}{c}10 Y R 4 / 1 \\
\& 5 / 1\end{array}$ & Clay & Waist-high grasses and brush & Inundation \\
\hline $1221 J\llcorner 20$ & 1 & $0-15$ & 0 & Negative & $\begin{array}{c}10 Y R 4 / 1 \\
\& 5 / 1\end{array}$ & Clay & Open field & Soil change \\
\hline $1221 \mathrm{JL} 20$ & 2 & $15-25$ & 0 & Negative & 10YR $3 / 1$ & Clay & Open field & Subsoil \\
\hline 1221JL24 & 1 & $0-15$ & 100 & Negative & Disturbed & Clay & Open field & Disturbed \\
\hline 122127 & 1 & $0-10$ & 100 & Negative & $\begin{array}{c}10 Y R 4 / 1 \\
\& 5 / 1\end{array}$ & Clay & Open field. Inundated & Inundation \\
\hline
\end{tabular}




\begin{tabular}{|c|c|c|c|c|c|c|c|c|}
\hline Shovel Test \# & $\begin{array}{l}\text { Level } \\
\text { (Strat) }\end{array}$ & $\begin{array}{l}\text { Depth } \\
\text { (cmbs) }\end{array}$ & GSV \% & $\begin{array}{l}\begin{array}{l}\text { Positive or } \\
\text { Negative }\end{array} \\
\end{array}$ & $\begin{array}{c}\text { Munsell } \\
\text { Color }\end{array}$ & Soil Texture Description & Description (Area; Vegetation) & Reason for Termination \\
\hline $1221 / 30$ & 1 & $0-15$ & 10 & Negative & $\begin{array}{c}\text { 10YR } 4 / 1 \\
\& 5 / 1\end{array}$ & Clay & Open field. Inundated & Inundation \\
\hline 1221JL32 & 1 & $0-15$ & 10 & Negative & $\begin{array}{c}\text { 10YR 4/1 } \\
\& 5 / 1\end{array}$ & Clay & Open field. & Inundation \\
\hline 1221JL32 & 1 & $15-28$ & 10 & Negative & $\begin{array}{c}\text { 10YR } 4 / 1 \\
\& 5 / 1 \\
\end{array}$ & Clay & Open field. & Inundation \\
\hline 1221JL36 & 1 & $0-10$ & 10 & Negative & $\begin{array}{c}\text { 10YR } 4 / 1 \\
\& 5 / 1 \\
\end{array}$ & Clay & Open field. & Inundation \\
\hline 1221WK01 & 1 & $0-25$ & 30 & Negative & 1OYR $4 / 1$ & Granular clay & Area is a two track adjacent to wetland; very disturbed. & Disturbed \\
\hline 1221WK04 & 1 & $0-20$ & 0 & Negative & 10YR $4 / 1$ & Granular clay & Wetland with standing water throughout. Briar and grass obscure GSV & Soil change \\
\hline 1221WK04 & 2 & $20-30$ & 0 & Negative & 10YR $3 / 1$ & Angular clay & Wetland with standing water throughout. Briar and grass obscure GSV & Subsoil \\
\hline 1221WK05 & 1 & $0-25$ & 0 & Negative & 10YR $4 / 1$ & Granular clay & Wetland with standing water throughout. Briar and grass obscure GSV & Soil change \\
\hline 1221WK05 & 2 & $25-35$ & 0 & Negative & 10YR $5 / 1$ & Angular clay & Wetland with standing water throughout. Briar and grass obscure GSV & Subsoil \\
\hline 1221WK07 & 1 & $0-15$ & 0 & Negative & 10YR $4 / 1$ & Granular clay & Wetland with standing water throughout. Briar and grass obscure GSV & Soil change \\
\hline 1221WK07 & 2 & $15-30$ & 0 & Negative & $\begin{array}{c}7.5 Y R 5 / 1 \\
\& 5 / 6\end{array}$ & Angular clay & Wetland with standing water throughout. Briar and grass obscure GSV & Subsoil \\
\hline 1221WK12 & 1 & $0-20$ & 0 & Negative & 10YR $4 / 1$ & Granular clay & Wetland with standing water throughout. Briar and grass obscure GSV & Soil change \\
\hline 1221WK12 & 2 & 20-25 & 0 & Negative & $\begin{array}{c}7.5 \text { YR } 5 / 1 \\
\& 5 / 6\end{array}$ & Angular clay & Wetland with standing water throughout. Briar and grass obscure GSV & Subsoil \\
\hline 1221WK17 & 1 & $0-15$ & 0 & Negative & 1OYR $4 / 1$ & Granular clay & Wetland with standing water throughout. Briar and grass obscure GSV & Soil change \\
\hline 1221WK17 & 2 & $15-30$ & 0 & Negative & $\begin{array}{c}7.5 Y R 5 / 1 \\
\& 5 / 6\end{array}$ & Angular clay & Wetland with standing water throughout. Briar and grass obscure GSV & Subsoil \\
\hline 1221WK21 & 1 & $0-25$ & 0 & Negative & 10YR $4 / 1$ & Granular clay & $\begin{array}{l}\text { Area is an Ag. Field (rice) and inundated } \\
\end{array}$ & Inundation \\
\hline 1221WK23 & 1 & $0-15$ & 10 & Negative & 10YR $4 / 1$ & Granular clay & Area is adjacent to gravel road and inundated with overgrowth. Area is very disturbed & Inundation \\
\hline 1221WK26 & 1 & $0-15$ & 0 & Negative & 1OYR $4 / 1$ & Granular clay & Area is adjacent to gravel road and inundated with overgrowth. Area is very disturbed & Inundation \\
\hline 1221WK29 & 1 & $0-15$ & 0 & Negative & 10YR $4 / 1$ & Granular clay & Area is adjacent to gravel road and inundated with overgrowth. Area is very disturbed & Inundation \\
\hline 1221WK35 & 1 & $0-15$ & 0 & Negative & 10YR $4 / 1$ & Granular clay & Area is adjacent to gravel road and inundated with overgrowth. Area is very disturbed & Inundation \\
\hline 0125WE01 & 1 & $0-30$ & 5 & Negative & 10 YR $5 / 4$ & Clay loam & Mixed horizon disturbed by pigs and temporary log road, open field, tall grass & Disturbed \\
\hline 0125RK01 & 1 & $0-23$ & 0 & Negative & 10 YR $4 / 1$ & Sandy clay loam & In cow pasture with light inundation surrounding,. Soil is very wet, short grasses & Soil change \\
\hline 0125RK01 & 2 & $23-33$ & 0 & Negative & 10 YR $5 / 2$ & Clay loam & In cow pasture with light inundation surrounding,. Soil is very wet, short grasses & Subsoil \\
\hline $0125 \mathrm{RK} 03$ & 1 & $0-23$ & 0 & Negative & 10 YR $4 / 1$ & Sandy clay loam & In cow pasture with light inundation surrounding,. Soil is very wet, short grasses & Soil change \\
\hline $0125 \mathrm{RK} 03$ & 2 & $23-33$ & 0 & Negative & 10 YR $5 / 2$ & Clay loam & In cow pasture with light inundation surrounding,. Soil is very wet, short grasses & Subsoil \\
\hline 0125 RK05 & 1 & $0-2$ & 0 & Negative & $10 \mathrm{YR} 3 / 1$ & Sandy Clay & Water just under grass at $2 \mathrm{cmbs}$ & Inundation \\
\hline $0125 \mathrm{RK} 08$ & 1 & $0-20$ & 0 & Negative & 10 YR $4 / 1$ & Sandy clay loam & in dense briar with mature pine, complete inundation to the south & Soil change \\
\hline 0125RK08 & 2 & $20-30$ & 0 & Negative & 10 YR $5 / 2$ & Clay loam & In cow pasture with light inundation surrounding,. Soil is very wet, short grasses & Subsoil \\
\hline 0125RK10 & 1 & $0-20$ & 0 & Negative & 10 YR $4 / 1$ & Sandy clay loam & In cow pasture with light inundation surrounding,. Soil is very wet, short grasses & Soil change \\
\hline 0125RK10 & 2 & $20-30$ & 0 & Negative & 10 YR $5 / 2$ & Clay loam & In cow pasture with light inundation surrounding,. Soil is very wet, short grasses & Subsoil \\
\hline 0125RK12 & 1 & $0-20$ & 0 & Negative & 10 YR $4 / 1$ & Sandy clay loam & In cow pasture with light inundation surrounding,. Soil is very wet, short grasses & Soil change \\
\hline 0125RK12 & 2 & $20-30$ & 0 & Negative & 10 YR $5 / 2$ & Clay loam & In cow pasture with light inundation surrounding,. Soil is very wet, short grasses & Subsoil \\
\hline 0125RK15 & 1 & $0-20$ & 0 & Negative & 10 YR $4 / 1$ & Sandy clay loam & In cow pasture with light inundation surrounding,. Soil is very wet, short grasses & Soil change \\
\hline 0125RK15 & 2 & $20-30$ & 0 & Negative & 10 YR $5 / 2$ & Clay loam & In cow pasture with light inundation surrounding,. Soil is very wet, short grasses & Subsoil \\
\hline $0125 \mathrm{RC} 02$ & 1 & $0-15$ & 0 & Negative & 10 YR $5 / 2$ & Clay loam & Tall grass, rase, briar & Subsoil \\
\hline $0125 \mathrm{RC} 04$ & 1 & $0-15$ & 0 & Negative & 10 YR $5 / 2$ & Clay loam & Tall grass, rase, briar & Subsoil \\
\hline $0125 \mathrm{RC} 06$ & 1 & $0-10$ & 0 & Negative & 10 YR $5 / 2$ & Clay loam & Tall grass, rase, briar & Subsoil \\
\hline 0125RC07 & 1 & $0-20$ & 0 & Negative & 10 YR $5 / 2$ & Clay loam & Tall grass, rase, briar & Subsoil \\
\hline $0125 \mathrm{RC09}$ & 1 & $0-20$ & 0 & Negative & 10 YR $5 / 2$ & Clay loam & Tall grass, rase, briar & Subsoil \\
\hline 0125RC11 & 1 & $0-20$ & 0 & Negative & 10 YR $5 / 2$ & Clay loam & Tall grass, rase, briar & Subsoil \\
\hline $0125 \mathrm{RC13}$ & 1 & $0-20$ & 0 & Negative & 10 YR $5 / 2$ & Clay loam & Tall grass, rase, briar & Subsoil \\
\hline 0125RC14 & 1 & $0-10$ & 0 & Negative & 10 YR $5 / 2$ & Clay loam & Tall grass, rase, briar & Subsoil \\
\hline 0125RC15 & 1 & $0-10$ & 0 & Negative & 10 YR $5 / 2$ & Clay loam & Tall grass, rase, briar & Subsoil \\
\hline 092619WE02 & 1 & $0-15$ & 10 & Negative & 10 YR $4 / 1$ & Clay & Forest with trash piles, mixed soil horizons & Disturbed \\
\hline 092619WE02 & 2 & $15-35$ & 10 & Negative & 10YR $5 / 4$ & Clay & Forest with trash piles, mixed soil horizons & Disturbed \\
\hline 092619WE04 & 1 & $0-30$ & 10 & Negative & 1OYR $4 / 3$ & Clay & Tall grasses near wetlands, motteled clay & Disturbed \\
\hline
\end{tabular}




\begin{tabular}{|c|c|c|c|c|c|c|c|c|}
\hline Shovel Test \# & $\begin{array}{l}\begin{array}{l}\text { Level } \\
\text { (Strat) }\end{array} \\
\end{array}$ & $\begin{array}{l}\text { Depth } \\
\text { (cmbs) }\end{array}$ & GSV \% & $\begin{array}{c}\begin{array}{c}\text { Positive or } \\
\text { Negative }\end{array} \\
\end{array}$ & $\begin{array}{c}\text { Munsell } \\
\text { Color }\end{array}$ & Soil Texture Description & Description (Area; Vegetation) & Reason for Termination \\
\hline 092619WE06 & 1 & $0-20$ & 10 & Negative & 10YR $4 / 1$ & Clay loam & Lawn in front of prison, mottled subsoil & Disturbed \\
\hline 092619WE06 & 2 & $20-50$ & 10 & Negative & $10 Y R 4 / 3$ & Clay & Lawn in front of prison, mottled subsoil & Disturbed \\
\hline 092619WE08 & 1 & $0-20$ & 10 & Negative & $10 \mathrm{YR} 4 / 3$ & Clay & Open field, medium sized grasses, scattered trees, mottled soil & Innundation \\
\hline 092619AM01 & 1 & $0-15$ & 0 & Negative & 10YR $4 / 1$ & Clay & Wooded area with a laye of leafs on ground & Impenetrable \\
\hline 092619AM01 & 2 & $15-28$ & 0 & Negative & 10YR $3 / 1$ & Clay & Wooded area with a laye of leafs on ground & Impenetrable \\
\hline 092619AM03 & 1 & $0-15$ & 0 & Negative & 10YR $4 / 1$ & Clay & Heavily vegetated & Soil change \\
\hline 092619AM03 & 2 & $15-28$ & 0 & Negative & 10YR 3/1 & Clay & Heavily vegetated & Soil change \\
\hline 092619SB5 & 1 & $0-15$ & 10 & Negative & 1OYR 4/1 & Clay loam & Lawn in front of prison, mottled subsoil & Disturbed \\
\hline 092619SB5 & 2 & $15-35$ & 10 & Negative & $10 \mathrm{YR} 4 / 3$ & Clay & Lawn in front of prison, mottled subsoil & Disturbed \\
\hline 092619SB7 & 1 & $0-15$ & 10 & Negative & 10YR 4/1 & Clay loam & Lawn in front of prison, mottled subsoil & Disturbed \\
\hline 092619SB7 & 2 & $15-35$ & 10 & Negative & $10 \mathrm{YR} 4 / 3$ & Clay & Lawn in front of prison, mottled subsoil & Disturbed \\
\hline 092619SB9 & 1 & $0-15$ & 10 & Negative & 10YR 4/1 & Clay loam & Lawn in front of prison, mottled subsoil & Disturbed \\
\hline 092619SB9 & 2 & $15-35$ & 10 & Negative & 10YR $4 / 3$ & Clay & Lawn in front of prison, mottled subsoil & Disturbed \\
\hline 092719AM04 & 1 & $0-15$ & 15 & Negative & 10YR $4 / 1$ & Clay & Heavily vegetated, Beaumont soil & Disturbed \\
\hline \begin{tabular}{|l|} 
092719WE1 \\
\end{tabular} & 1 & $0-15$ & 15 & Negative & 10YR 4/1 & Clay & Forest, damp soil, dense briar & Inundation \\
\hline 092719WE2 & 1 & $0-15$ & 0 & Negative & 10YR $3 / 1$ & Clay & Open Field, med/ tall grasses, mixed horizons & Disturbed \\
\hline \begin{tabular}{|l|} 
092719WE2 \\
\end{tabular} & 2 & $15-30$ & 0 & Negative & $10 Y R 6 / 2$ & Clay & Open Field, med/ tall grasses, mixed horizons & Disturbed \\
\hline 092719WE4 & 1 & $0-10$ & 0 & Negative & 10 YR $5 / 4$ & Clay & Open Field, med/tall grasses, & Disturbed \\
\hline 092719WE4 & 2 & $10-20$ & 0 & Negative & 10YR $6 / 2$ & Clay & Open Field, med/tall grasses, & Disturbed \\
\hline \begin{tabular}{|l} 
092719WE5 \\
\end{tabular} & 1 & $0-25$ & 0 & Negative & $10 Y R 4 / 4$ & Clay & Short grass by fence adjacent to wetland & Soil change \\
\hline 092719WE5 & 2 & $25-50$ & 0 & Negative & 10YR 3/1 & Clay & Short grass by fence adjacent to wetland & Subsoil \\
\hline \begin{tabular}{|l} 
092719WE8 \\
\end{tabular} & 1 & $0-30$ & 0 & Negative & 10YR 4/1 & Clay & Short grass by fence adjacent to wetland & Soil change \\
\hline 092719WE8 & 2 & $30-50$ & 0 & Negative & 10YR $3 / 1$ & Clay & Short grass by fence adjacent to wetland & Subsoil \\
\hline 092719WE10 & 1 & $0-30$ & 0 & Negative & 10YR $4 / 1$ & Clay & Short grass by fence adjacent to wetland & Soil change \\
\hline 092719WE10 & 2 & $30-45$ & 0 & Negative & 10YR $3 / 1$ & Clay & Short grass by fence adjacent to wetland & Subsoil \\
\hline 092719WE11 & 1 & $0-30$ & 0 & Negative & 10YR $4 / 1$ & Clay & Short grass by fence adjacent to wetland & Soil change \\
\hline 092719WE11 & 2 & $30-45$ & 0 & Negative & 10YR $3 / 1$ & Clay & Short grass by fence adjacent to wetland & Subsoil \\
\hline 092719WE13 & 1 & $0-15$ & 0 & Negative & 10YR $4 / 1$ & Clay & Short grass by fence adjacent to wetland & Soil change \\
\hline 092719WE13 & 2 & $15-40$ & 0 & Negative & 10YR $3 / 1$ & Clay & Short grass by fence adjacent to wetland & Subsoil \\
\hline 092719WE15 & 1 & $0-25$ & 0 & Negative & 10YR $4 / 1$ & Clay & Short grass by fence adjacent to wetland & Soil change \\
\hline 092719WE15 & 2 & $25-35$ & 0 & Negative & 10YR $3 / 1$ & Clay & Short grass by fence adjacent to wetland & Subsoil \\
\hline 092719SB9 & 1 & $0-15$ & 0 & Negative & 10YR 4/1 & Clay & Short grass by fence adjacent to wetland & Soil change \\
\hline 092719SB9 & 2 & $15-40$ & 0 & Negative & 10YR 3/1 & Clay & Short grass by fence adjacent to wetland & Subsoil \\
\hline $092719 S B 7$ & 1 & $0-15$ & 0 & Negative & 10YR 4/1 & Clay & Short grass by fence adjacent to wetland & Soil change \\
\hline 092719SB7 & 2 & $15-40$ & 0 & Negative & 10YR $3 / 1$ & Clay & Short grass by fence adjacent to wetland & Subsoil \\
\hline 092719SB6 & 1 & $0-15$ & 0 & Negative & 10YR 4/1 & Clay & Short grass by fence adjacent to wetland & Soil change \\
\hline 092719SB6 & 2 & $15-40$ & 0 & Negative & 10YR $3 / 1$ & Clay & Short grass by fence adjacent to wetland & Subsoil \\
\hline 092719SB22 & 1 & $0-15$ & 0 & Negative & 10YR $4 / 1$ & Clay & Short grass by fence adjacent to wetland & Soil change \\
\hline 092719SB22 & 2 & $15-40$ & 0 & Negative & 10YR 3/1 & Clay & Short grass by fence adjacent to wetland & Subsoil \\
\hline
\end{tabular}




\begin{tabular}{|c|c|c|c|c|c|c|c|c|}
\hline Shovel Test \# & $\begin{array}{l}\text { Level } \\
\text { (Strat) }\end{array}$ & $\begin{array}{l}\text { Depth } \\
\text { (cmbs) }\end{array}$ & GSV \% & $\begin{array}{l}\text { Positive or } \\
\text { Negative }\end{array}$ & $\begin{array}{c}\text { Munsell } \\
\text { Color }\end{array}$ & Soil Texture Description & Description (Area; Vegetation) & Reason for Termination \\
\hline $092719 S B 21$ & 1 & $0-15$ & 0 & Negative & 10YR $4 / 1$ & Clay & Short grass by fence adjacent to wetland & Soil change \\
\hline 092719SB21 & 2 & $15-40$ & 0 & Negative & 10YR $3 / 1$ & Clay & Short grass by fence adjacent to wetland & Subsoil \\
\hline 092719SB20 & 1 & $0-15$ & 0 & Negative & 10YR $4 / 1$ & Clay & Short grass by fence adjacent to wetland & Soil change \\
\hline 092719SB20 & 2 & $15-40$ & 0 & Negative & 10YR $3 / 1$ & Clay & Short grass by fence adjacent to wetland & Subsoil \\
\hline 092719SB19 & 1 & $0-15$ & 0 & Negative & 10YR $4 / 1$ & Clay & Short grass by fence adjacent to wetland & Soil change \\
\hline 092719SB19 & 2 & $15-40$ & 0 & Negative & 10YR $3 / 1$ & Clay & Short grass by fence adjacent to wetland & Subsoil \\
\hline 092719SB18 & 1 & $0-15$ & 0 & Negative & 10YR $4 / 1$ & Clay & Short grass by fence adjacent to wetland & Soil change \\
\hline 092719SB18 & 2 & $15-40$ & 0 & Negative & 10YR $3 / 1$ & Clay & Short grass by fence adjacent to wetland & Subsoil \\
\hline 092719SB17 & 1 & $0-15$ & 0 & Negative & 10YR $4 / 1$ & Clay & Short grass by fence adjacent to wetland & Soil change \\
\hline 092719SB17 & 2 & $15-40$ & 0 & Negative & 10YR $3 / 1$ & Clay & Short grass by fence adjacent to wetland & Subsoil \\
\hline 092719SB16 & 1 & $0-15$ & 0 & Negative & 10YR $4 / 1$ & Clay & Short grass by fence adjacent to wetland & Soil change \\
\hline 092719SB16 & 2 & $15-40$ & 0 & Negative & 10YR $3 / 1$ & Clay & Short grass by fence adjacent to wetland & Subsoil \\
\hline 092719SB14 & 1 & $0-15$ & 0 & Negative & 10YR $4 / 1$ & Clay & Short grass by fence adjacent to wetland & Soil change \\
\hline 092719SB14 & 2 & $15-40$ & 0 & Negative & 10YR $3 / 1$ & Clay & Short grass by fence adjacent to wetland & Subsoil \\
\hline 092719SB12 & 1 & $0-15$ & 0 & Negative & 10YR $4 / 1$ & Clay & Short grass by fence adjacent to wetland & Soil change \\
\hline 092719SB12 & 2 & $15-40$ & 0 & Negative & 10YR $3 / 1$ & Clay & Short grass by fence adjacent to wetland & Subsoil \\
\hline 081518SB9 & 1 & $0-30$ & 10 & Negative & 10YR $3 / 1$ & Clay & In grassy cow pasture; smaller grasses & Subsoil \\
\hline $081518 S B 72$ & 1 & $0-35$ & 10 & Negative & 10YR $3 / 1$ & Clay & In grassy cow pasture; smaller grasses & Subsoil \\
\hline 081518SB70 & 1 & $0-30$ & 10 & Negative & 10YR $3 / 1$ & Clay & In grassy cow pasture; smaller grasses & Subsoil \\
\hline $081518 \mathrm{SB} 7$ & 1 & $0-30$ & 10 & Negative & 10YR $3 / 1$ & Clay & In grassy cow pasture; smaller grasses & Subsoil \\
\hline 081518SB68 & 1 & $0-35$ & 10 & Negative & 10YR $3 / 1$ & Clay & In grassy cow pasture; smaller grasses & Subsoil \\
\hline 081518SB65 & 1 & $0-30$ & 10 & Negative & 10YR $3 / 1$ & Clay & In grassy cow pasture; smaller grasses & Subsoil \\
\hline 081518 SB64 & 1 & $0-30$ & 10 & Negative & 10YR $3 / 1$ & Clay & In grassy cow pasture; smaller grasses & Subsoil \\
\hline 081518SB62 & 1 & $0-35$ & 10 & Negative & 10YR $3 / 1$ & Clay & In grassy cow pasture; smaller grasses & Subsoil \\
\hline 081518SB60 & 1 & $0-35$ & 10 & Negative & 10YR $3 / 1$ & Clay & In grassy cow pasture; smaller grasses & Subsoil \\
\hline 081518SB59 & 1 & $0-30$ & 10 & Negative & 10YR $3 / 1$ & Clay & In grassy cow pasture; smaller grasses & Subsoil \\
\hline 081518SB57 & 1 & $0-30$ & 10 & Negative & 10YR $3 / 1$ & Clay & In grassy cow pasture; smaller grasses & Subsoil \\
\hline $0815185 B 56$ & 1 & $0-35$ & 10 & Negative & 10YR $3 / 1$ & Clay & In grassy cow pasture; smaller grasses & Subsoil \\
\hline $0815185 B 54$ & 1 & $0-30$ & 10 & Negative & 10YR $3 / 1$ & Clay & In grassy cow pasture; smaller grasses & Subsoil \\
\hline
\end{tabular}




\begin{tabular}{|c|c|c|c|c|c|c|c|c|}
\hline Shovel Test \# & $\begin{array}{l}\begin{array}{l}\text { Level } \\
\text { (Strat) }\end{array} \\
\end{array}$ & $\begin{array}{l}\text { Depth } \\
\text { (cmbs) }\end{array}$ & GSV \% & $\begin{array}{l}\begin{array}{c}\text { Positive or } \\
\text { Negative }\end{array} \\
\end{array}$ & $\begin{array}{c}\text { Munsell } \\
\text { Color }\end{array}$ & Soil Texture Description & Description (Area; Vegetation) & Reason for Termination \\
\hline $081518 \mathrm{SB} 52$ & 1 & $0-35$ & 10 & Negative & 10YR $3 / 1$ & Clay & In grassy cow pasture; smaller grasses & Subsoil \\
\hline 081518SB50 & 1 & $0-30$ & 10 & Negative & 10YR $3 / 1$ & Clay & In grassy cow pasture; smaller grasses & Subsoil \\
\hline 081518SB5 & 1 & $0-30$ & 10 & Negative & 10YR $3 / 1$ & Clay & In grassy cow pasture; smaller grasses & Subsoil \\
\hline 081518SB47 & 1 & $0-35$ & 10 & Negative & 10YR $3 / 1$ & Clay & In grassy cow pasture; smaller grasses & Subsoil \\
\hline 081518SB45 & 1 & $0-35$ & 10 & Negative & 10YR $3 / 1$ & Clay & In grassy cow pasture; smaller grasses & Subsoil \\
\hline $081518 \mathrm{SB} 43$ & 1 & $0-30$ & 10 & Negative & 10YR $3 / 1$ & Clay & In grassy cow pasture; smaller grasses & Subsoil \\
\hline $081518 S B 42$ & 1 & $0-30$ & 10 & Negative & 10YR $3 / 1$ & Clay & In grassy cow pasture; smaller grasses & Subsoil \\
\hline 081518SB4 & 1 & $0-35$ & 10 & Negative & 10YR $3 / 1$ & Clay & In grassy cow pasture; smaller grasses & Subsoil \\
\hline 081518SB38 & 1 & $0-30$ & 10 & Negative & 10YR $3 / 1$ & Clay & In grassy cow pasture; smaller grasses & Subsoil \\
\hline 081518 SB37 & 1 & $0-35$ & 10 & Negative & 10YR $3 / 1$ & Clay & In grassy cow pasture; smaller grasses & Subsoil \\
\hline 081518SB35 & 1 & $0-30$ & 10 & Negative & 10YR $3 / 1$ & Clay & In grassy cow pasture; smaller grasses & Subsoil \\
\hline $081518 S B 33$ & 1 & $0-30$ & 10 & Negative & 10YR $3 / 1$ & Clay & In grassy cow pasture; smaller grasses & Subsoil \\
\hline $081518 S B 31$ & 1 & $0-35$ & 10 & Negative & 10YR $3 / 1$ & Clay & In grassy cow pasture; smaller grasses & Subsoil \\
\hline $081518 S B 28$ & 1 & $0-35$ & 10 & Negative & 10YR $3 / 1$ & Clay & In grassy cow pasture; smaller grasses & Subsoil \\
\hline $081518 S B 25$ & 1 & $0-30$ & 10 & Negative & 10YR $3 / 1$ & Clay & In grassy cow pasture; smaller grasses & Subsoil \\
\hline 081518SB2 & 1 & $0-30$ & 10 & Negative & 10YR $3 / 1$ & Clay & In grassy cow pasture; smaller grasses & Subsoil \\
\hline 081518SB17 & 1 & $0-35$ & 10 & Negative & 10YR $3 / 1$ & Clay & In grassy cow pasture; smaller grasses & Subsoil \\
\hline 081518SB16 & 1 & $0-30$ & 10 & Negative & 10YR $3 / 1$ & Clay & In grassy cow pasture; smaller grasses & Subsoil \\
\hline 081518SB12 & 1 & $0-30$ & 10 & Negative & 10YR $3 / 1$ & Clay & In grassy cow pasture; smaller grasses & Subsoil \\
\hline 101019SB01 & 1 & $0-20$ & 0 & Negative & 10YR $5 / 2$ & Clay & Disturbed; within pipeline corridor & Disturbed \\
\hline 101019SB02 & 1 & $0-25$ & 0 & Negative & 10YR $5 / 3$ & Clay & Disturbed; within pipeline corridor & Disturbed \\
\hline 101019SB03 & 1 & $0-20$ & 0 & Negative & 10YR $5 / 4$ & Clay & Disturbed; within pipeline corridor & Disturbed \\
\hline 101019SB04 & 1 & $0-20$ & 0 & Negative & 10YR $5 / 5$ & Clay & Disturbed; within pipeline corridor & Disturbed \\
\hline 101019SB05 & 1 & $0-20$ & 0 & Negative & 10YR $5 / 6$ & Clay & Disturbed; within pipeline corridor & Disturbed \\
\hline 101019SB06 & 1 & $0-10$ & 1 & Negative & 10YR $5 / 7$ & Clay & Soil wet; inundation at $10 \mathrm{cmbs}$ & Inundation \\
\hline $101019 S B 07$ & 1 & $0-10$ & 2 & Negative & 10YR $5 / 8$ & Clay & Soil wet; inundation at $10 \mathrm{cmbs}$ & Inundation \\
\hline 101019SB08 & 1 & $0-10$ & 3 & Negative & 10YR $5 / 9$ & Clay & Soil wet; inundation at $10 \mathrm{cmbs}$ & Inundation \\
\hline 101019SB09 & 1 & $0-10$ & 4 & Negative & 10YR 5/10 & Clay & Soil wet; inundation at $10 \mathrm{cmbs}$ & Inundation \\
\hline $101019 S B 10$ & 1 & $0-10$ & 5 & Negative & 10YR 5/11 & Clay & Soil wet; inundation at $10 \mathrm{cmbs}$ & Inundation \\
\hline 1106WE1 & 1 & $0-25$ & 3 & Negative & 10YR $6 / 1$ & Sandy Clay Loam & Thin brush forest, short grass by trinity River & Subsoil \\
\hline 1106WE1 & 2 & $25-30$ & & Negative & 10YR 5/1 & Sandy Clay Loam & Thin brush forest, short grass by trinity River & Subsoil \\
\hline
\end{tabular}




\begin{tabular}{|c|c|c|c|c|c|c|c|c|}
\hline Shovel Test \# & $\begin{array}{l}\text { Level } \\
\text { (Strat) }\end{array}$ & $\begin{array}{l}\text { Depth } \\
\text { (cmbs) }\end{array}$ & GSV \% & $\begin{array}{c}\text { Positive or } \\
\text { Negative }\end{array}$ & $\begin{array}{l}\text { Munsell } \\
\text { Color }\end{array}$ & Soil Texture Description & Description (Area; Vegetation) & Reason for Termination \\
\hline 1106WE1 & 3 & $30-50$ & & Negative & 10YR $3 / 1$ & Sandy Loam & Thin brush forest, short grass by trinity River & Subsoil \\
\hline 1106MT2 & 1 & $0-50$ & 0 & Negative & $10 \mathrm{YR} 5 / 4$ & Sandy Clay Loam & Low grasses and weeds; mixed hardwoods & Subsoil \\
\hline 1106WE3 & 1 & $0-25$ & 3 & Negative & 10YR $6 / 1$ & Sandy Clay Loam & Thin brush forest, short grass by trinity River & Subsoil \\
\hline 1106WE3 & 2 & $25-35$ & & Negative & $10 \mathrm{YR} 2 / 1$ & Sandy Clay Loam & Thin brush forest, short grass by trinity River & Subsoil \\
\hline 1106WE3 & 3 & $35-50$ & & Negative & 1OYR $4 / 2$ & Sandy Loam & Thin brush forest, short grass by trinity River & Subsoil \\
\hline 1106JT4 & 1 & $0-25$ & 0 & Negative & 10YR $5 / 1$ & Sandy Clay Loam & River valley mixed hardwood, thin understory & Subsoil \\
\hline 1106JT4 & 2 & $25-50$ & & Negative & $10 Y R 3 / 1$ & Clay & River valley mixed hardwood, thin understory & Subsoil \\
\hline 1106MT6 & 1 & $0-20$ & 0 & Negative & 10YR $5 / 4$ & Sandy Clay Loam & Low grasses and weeds; mixed hardwoods & Subsoil \\
\hline 1106MT6 & 2 & $20-45$ & & Negative & $10 \mathrm{YR} 5 / 4$ & Sand & Low grasses and weeds; mixed hardwoods & Subsoil \\
\hline 1106MT6 & 3 & $45-65$ & & Negative & 10YR $5 / 4$ & Sandy Clay Loam & Low grasses and weeds; mixed hardwoods & Subsoil \\
\hline 1106WE7 & 1 & $0-30$ & 0 & Negative & 10YR $8 / 1$ & Sand & Tall grass, thin brush and forest by Trinity River & Subsoil \\
\hline 1106WE7 & 2 & $30-50$ & & Negative & 10YR 5/1 & Sandy Loam & Tall grass, thin brush and forest by Trinity River & Subsoil \\
\hline $1106 \mathrm{JT} 8$ & 1 & $0-40$ & 0 & Negative & 10YR 6/1 & Sandy Clay Loam & River valley mixed hardwood, thin understory & Subsoil \\
\hline 1106JT8 & 2 & $40-50$ & & Negative & 10YR 4/1 & Clay & River valley mixed hardwood, thin understory & Subsoil \\
\hline 1106MT9 & 1 & $0-20$ & 0 & Negative & 10YR $5 / 4$ & Sandy Clay Loam & Low grasses and weeds; mixed hardwoods & Subsoil \\
\hline 1106MT9 & 2 & $20-60$ & & Negative & $10 Y R 5 / 4$ & Sandy Clay Loam & Low grasses and weeds; mixed hardwoods & Subsoil \\
\hline 1106WE10 & 1 & $0-25$ & 10 & Negative & 10YR $6 / 1$ & Sand & Short grass, forest, thin brush by Trinity River & Subsoil \\
\hline 1106WE10 & 2 & $25-35$ & & Negative & $10 \mathrm{YR} 7 / 1$ & Sand & Short grass, forest, thin brush by Trinity River & Subsoil \\
\hline 1106WE10 & 3 & $35-50$ & & Negative & 10YR 5/1 & Sandy Loam & Short grass, forest, thin brush by Trinity River & Subsoil \\
\hline 1106JT11 & 1 & $0-40$ & 0 & Negative & $10 \mathrm{YR} 6 / 3$ & Sandy Clay Loam & River valley mixed hardwood, thin understory & Subsoil \\
\hline 1106JT11 & 2 & $40-50$ & & Negative & $10 \mathrm{YR} 5 / 1$ & Sandy Clay Loam & River valley mixed hardwood, thin understory & Subsoil \\
\hline 1106WE12 & 1 & $0-25$ & 5 & Negative & 10YR $6 / 1$ & Sand & Short grass, forest, thin brush by Trinity River & Subsoil \\
\hline 1106WE12 & 2 & $25-50$ & & Negative & 10YR 4/1 & Sandy Loam & Short grass, forest, thin brush by Trinity River & Subsoil \\
\hline 1106JT13 & 1 & $0-10$ & 0 & Negative & 10YR $6 / 1$ & Sandy Clay Loam & River valley mixed hardwood, thin understory & Subsoil \\
\hline 1106JT13 & 2 & $10-30$ & & Negative & $10 \mathrm{YYR} 5 / 1$ & Clay loam & River valley mixed hardwood, thin understory & Subsoil \\
\hline 1106WE14 & 1 & $0-25$ & 5 & Negative & 10YR $6 / 1$ & Sand & Short grass, forest, thin brush by Trinity River & Subsoil \\
\hline 1106WE14 & 2 & $25-50$ & & Negative & 10YR $4 / 1$ & Sandy Loam & Short grass, forest, thin brush by Trinity River & Subsoil \\
\hline 1106MT15 & 1 & $0-30$ & 0 & Negative & $10 \mathrm{YR} 5 / 4$ & Sandy Clay Loam & Low grasses and weeds shrubby hardwoods & Subsoil \\
\hline 1106MT15 & 2 & $30-70$ & & Negative & $10 Y R 5 / 4$ & Sandy Clay Loam & Low grasses and weeds shrubby hardwoods & Subsoil \\
\hline 1106MVR16 & 1 & $0-100$ & 0 & Negative & $10 \mathrm{YR} 5 / 4$ & Sandy Clay Loam & Low grasses and weeds mixed hardwoods & Subsoil \\
\hline 1106WE17 & 1 & $0-25$ & 5 & Negative & $10 \mathrm{YYR} 7 / 1$ & Sand & Tall grass, thin chrush, young forest & Subsoil \\
\hline 1106WE17 & 2 & $25-50$ & & Negative & 10YR 5/1 & Sandy Clay Loam & Tall grass, thin chrush, young forest & Subsoil \\
\hline 1106WE18 & 1 & $0-20$ & 0 & Negative & $10 \mathrm{YR} 7 / 1$ & Sand & Tall vegetation near swamp & Subsoil \\
\hline 1106WE18 & 2 & $20-50$ & & Negative & 1OYR $4 / 1$ & Sandy Loam & Tall vegetation near swamp & Subsoil \\
\hline 1106MVR19 & 1 & $0-60$ & 0 & Negative & $10 \mathrm{YR} 5 / 4$ & Sand & Low grasses and weeds mixed hardwoods & Subsoil \\
\hline 1106MVR19 & 2 & $60-80$ & & Negative & 10YR $5 / 4$ & Sandy Clay Loam & Low grasses and weeds mixed hardwoods & Subsoil \\
\hline $1106 \mathrm{MT} 20$ & 1 & $0-60$ & 0 & Negative & 10YR 5/4 & Sandy Clay Loam & Low grasses and weeds, mid story willows with cottonwoods & Subsoil \\
\hline 1106WE21 & 1 & $0-30$ & 0 & Negative & 10YR 6/1 & Sand & Tall grass, saplings between older forest & Subsoil \\
\hline 1106WE21 & 2 & $30-50$ & & Negative & 10YR 4/1 & Sandy Clay Loam & Tall grass, saplings between older forest & Subsoil \\
\hline 1106JT22 & 1 & $0-20$ & 0 & Negative & 10YR $6 / 1$ & Sandy Clay Loam & River valley mixed hardwood, thin understory & Subsoil \\
\hline 1106JT22 & 2 & $20-50$ & & Negative & $10 \mathrm{YR} 4 / 2$ & Sandy Clay Loam & River valley mixed hardwood, thin understory & Subsoil \\
\hline 1106WE23 & 1 & $0-20$ & 0 & Negative & 10YR $6 / 1$ & Sand & Tall grass, saplings between older forest, frequent flooding & Subsoil \\
\hline 1106WE23 & 2 & $20-50$ & & Negative & $10 \mathrm{YR} 5 / 1$ & Sandy Clay Loam & Tall grass, saplings between older forest, frequent flooding & Subsoil \\
\hline 1106MVR24 & 1 & $0-60$ & 0 & Negative & $10 \mathrm{YR} 5 / 4$ & Sand & Low grasses and weeds mixed hardwoods & Subsoil \\
\hline 1106MVR24 & 2 & $60-80$ & & Negative & 10YR $5 / 4$ & Sandy Clay Loam & Low grasses and weeds mixed hardwoods & Subsoil \\
\hline 1106MT25 & 1 & $0-20$ & 0 & Negative & 10YR $5 / 4$ & Sandy Clay Loam & Low grasses, hardwoods & Subsoil \\
\hline 1106WE26 & 1 & $0-30$ & 0 & Negative & $10 \mathrm{YYR} 6 / 1$ & Sand & Tall grass, saplings between older forest, frequent flooding & Subsoil \\
\hline 1106WE26 & 2 & $30-50$ & & Negative & 10YR 5/2 & Sandy Clay Loam & Tall grass, saplings between older forest, frequent flooding & Subsoil \\
\hline $1106 \mathrm{MT} 27$ & 1 & $0-60$ & 0 & Negative & $10 Y R 5 / 4$ & Sandy Clay Loam & Some mixed vines mixed hardwoods & Subsoil \\
\hline 1106JT28 & 1 & $0-10$ & 0 & Negative & $10 \mathrm{Yr} 4 / 2$ & Sandy Clay Loam & River valley mixed hardwood, thin understory & Subsoil \\
\hline 1106JT28 & 2 & $10-60$ & & Negative & 10YR $6 / 2$ & Sandy Loam & River valley mixed hardwood, thin understory & Subsoil \\
\hline 1106MVR29 & 1 & $0-100$ & 0 & Negative & $10 \mathrm{YR} 5 / 4$ & Sandy Clay Loam & Low grasses and weeds mixed hardwoods & Subsoil \\
\hline 1106WE30 & 1 & $0-25$ & 0 & Negative & $10 \mathrm{YR} 2 / 1$ & Sand & Lilly pads/marsh plants between forest and Bayou & Subsoil \\
\hline 1106WE30 & 2 & $25-50$ & & Negative & 10YR $4 / 1$ & Sandy Clay Loam & Lilly pads/marsh plants between forest and Bayou & Subsoil \\
\hline $1106 \mathrm{MY} 31$ & 1 & $0-65$ & 0 & Negative & $10 Y R 5 / 4$ & Sandy Clay Loam & Ground vines and shrubs, grasses & Subsoil \\
\hline
\end{tabular}




\begin{tabular}{|c|c|c|c|c|c|c|c|c|}
\hline Shovel Test \# & $\begin{array}{l}\text { Level } \\
\text { (Strat) }\end{array}$ & $\begin{array}{l}\text { Depth } \\
\text { (cmbs) }\end{array}$ & GSV \% & $\begin{array}{c}\text { Positive or } \\
\text { Negative }\end{array}$ & $\begin{array}{c}\text { Munsell } \\
\text { Color }\end{array}$ & Soil Texture Description & Description (Area; Vegetation) & Reason for Termination \\
\hline 1106WE32 & 1 & $0-30$ & 10 & Negative & 10YR $6 / 1$ & Sand & Tall grass, saplings & Subsoil \\
\hline 1106WE32 & 2 & $30-50$ & & Negative & 10YR $4 / 1$ & Sandy Clay Loam & Tall grass, saplings & Subsoil \\
\hline $1106 \mathrm{JT} 33$ & 1 & $0-40$ & 0 & Negative & 10YR $6 / 2$ & Sandy Clay Loam & River valley mixed hardwood, thin understory & Subsoil \\
\hline $1106 \mathrm{JT} 33$ & 2 & $40-60$ & & Negative & 10YR $4 / 1$ & Sandy Clay Loam & River valley mixed hardwood, thin understory & Subsoil \\
\hline 1106WE34 & 1 & $0-30$ & 0 & Negative & 10YR $6 / 1$ & Sand & Forest by River frequent flooding / Sand deposits & Subsoil \\
\hline 1106WE34 & 2 & $30-55$ & & Negative & 10YR $4 / 1$ & Sandy Clay Loam & Forest by River frequent flooding / Sand deposits & Subsoil \\
\hline 1106MVR35 & 1 & $0-100$ & 0 & Negative & $10 Y R 4 / 3$ & Sandy Clay Loam & Low grasses and weeds mixed hardwoods & Subsoil \\
\hline 1106WE36 & 1 & $0-30$ & 0 & Negative & 10YR $6 / 1$ & Sand & Tall grass, edge of ROW & Subsoil \\
\hline 1106WE36 & 2 & $30-50$ & & Negative & 10YR $4 / 1$ & Sandy Clay Loam & Tall grass, edge of ROW & Subsoil \\
\hline $1106 \mathrm{MT} 37$ & 1 & $0-60$ & 0 & Negative & 10YR $5 / 4$ & Sandy Clay Loam & Ground vines and shrubs, grasses & Subsoil \\
\hline 1106WE38 & 1 & $0-30$ & 0 & Negative & 10YR $6 / 1$ & Sand & Tall grass, saplings & Subsoil \\
\hline 1106WE38 & 2 & $30-55$ & & Negative & 10YR $4 / 1$ & Sandy Clay Loam & Tall grass, saplings & Subsoil \\
\hline 1106MVR39 & 1 & $0-100$ & 0 & Negative & $10 \mathrm{YR} 5 / 3$ & Sandy Loam & Low grasses and weeds mixed hardwoods & Subsoil \\
\hline $1106 \mathrm{JT} 40$ & 1 & $0-20$ & 0 & Negative & $10 Y R 6 / 2$ & Sandy Clay Loam & River valley mixed hardwood, thin understory & Subsoil \\
\hline $1106 \mathrm{JT} 40$ & 2 & $20-60$ & & Negative & 10YR $5 / 2$ & Sandy Clay Loam & River valley mixed hardwood, thin understory & Subsoil \\
\hline 1106WE41 & 1 & $0-25$ & 0 & Negative & $10 Y R 6 / 1$ & Sand & Thin brush in forest between River and creek, frequent flooding & Subsoil \\
\hline 1106WE41 & 2 & $25-50$ & & Negative & $10 Y R 4 / 2$ & Sandy Clay Loam & Thin brush in forest between River and creek, frequent flooding & Subsoil \\
\hline 1106MVR42 & 1 & $0-80$ & 0 & Negative & 10YR $4 / 3$ & Sandy Clay Loam & Low grasses and weeds mixed hardwoods & Subsoil \\
\hline 1106MVR42 & 2 & $80-100$ & & Negative & 1OYR $3 / 3$ & Sandy Clay Loam & Low grasses and weeds mixed hardwoods & Subsoil \\
\hline 1106WE43 & 1 & $0-15$ & 0 & Negative & $10 \mathrm{YR} 3 / 3$ & Sandy Clay Loam & Tall grass and rose vines in ROW & Subsoil \\
\hline 1106WE43 & 2 & $15-50$ & & Negative & $10 Y R 5 / 3$ & Sandy Clay Loam & Tall grass and rose vines in ROW & Subsoil \\
\hline $1106 \mathrm{JT} 44$ & 1 & 0 & 0 & Negative & $10 \mathrm{YR} 5 / 2$ & Sandy Clay Loam & River valley mixed hardwood, thin understory & Subsoil \\
\hline 1106MVR45 & 1 & $0-70$ & 0 & Negative & $10 Y R 6 / 3$ & Sandy Clay Loam & Low grasses and weeds mixed hardwoods & Subsoil \\
\hline 1106WE46 & 1 & $0-30$ & 0 & Negative & $10 \mathrm{YYR} 6 / 1$ & Sandy Clay Loam & Forest, thin brush, frequent flooding, mattled soil & Subsoil \\
\hline 1106WE46 & 2 & $30-45$ & & Negative & 10YR 5/1 & Sandy Clay Loam & Forest, thin brush, frequent flooding, mattled soil & Subsoil \\
\hline 1106WE46 & 3 & $45-55$ & & Negative & 10YR $3 / 1$ & Sandy Clay Loam & Forest, thin brush, frequent flooding, mattled soil & Subsoil \\
\hline 1106WE47 & 1 & $0-30$ & 0 & Negative & 10YR $6 / 1$ & Sandy Clay Loam & Tall grass, saplings, edge of ROW & Subsoil \\
\hline 1106WE47 & 2 & $30-50$ & & Negative & $10 Y R 4 / 2$ & Sandy Clay Loam & Tall grass, saplings, edge of ROW & Subsoil \\
\hline 1107WE1 & 1 & $0-30$ & 0 & Negative & $10 Y R 6 / 1$ & Sandy Clay Loam & Forest, thin brush, frequent flooding & Subsoil \\
\hline 1107WE1 & 2 & $30-50$ & & Negative & 10YR 4/1 & Sandy Clay Loam & Forest, thin brush, frequent flooding & Subsoil \\
\hline 1107JT2 & 1 & $0-10$ & 0 & Negative & $10 Y R 5 / 2$ & Sandy Clay Loam & River valley mixed hardwood, thin understory & Subsoil \\
\hline $1107 \mathrm{JT2}$ & 2 & $10-50$ & & Negative & $10 Y R 5 / 1$ & Sandy Clay Loam & River valley mixed hardwood, thin understory & Subsoil \\
\hline 1107MVR3 & 1 & $0-100$ & 0 & Negative & $10 Y R 6 / 3$ & Sandy Clay Loam & Low grasses and weeds, mixed hardwoods, beside drainage & Subsoil \\
\hline 1107MT4 & 1 & $0-30$ & 0 & Negative & $10 Y R 5 / 2$ & Sandy Clay Loam & Low grasses and shrubs mixed hardwoods & Subsoil \\
\hline 1107MT4 & 2 & $30-70$ & & Negative & 10YR $5 / 2$ & Sandy Clay Loam & Low grasses and shrubs mixed hardwoods & Subsoil \\
\hline 1107WE5 & 1 & $0-30$ & 0 & Negative & $10 Y R 7 / 1$ & Sandy Clay Loam & Tall grass, line of trees in ROW & Subsoil \\
\hline 1107WE5 & 2 & $30-50$ & & Negative & 10YR 5/1 & Sandy Clay Loam & Tall grass, line of trees in ROW & Subsoil \\
\hline 1107WE6 & 1 & $0-25$ & 0 & Negative & $10 Y R 7 / 1$ & Sandy Clay Loam & Thin brush, forest & Subsoil \\
\hline 1107WE6 & 2 & $25-50$ & & Negative & 10YR 4/1 & Sandy Clay Loam & Thin brush, forest & Subsoil \\
\hline $1107 \mathrm{JT7}$ & 1 & $0-15$ & 0 & Negative & 10YR $4 / 2$ & Sandy Clay Loam & River valley mixed hardwood, thin understory & Subsoil \\
\hline 1107MT8 & 1 & $0-55$ & 0 & Negative & $10 \mathrm{YR} 5 / 4$ & Sandy Clay Loam & Low ground vines and shrubs; mixed hardwoods & Subsoil \\
\hline 1107WE09 & 1 & $0-25$ & 0 & Negative & $10 \mathrm{YR} 5 / 1$ & Sandy Clay Loam & Tall grass, trees in RIOW & Subsoil \\
\hline 1107WE09 & 2 & $25-50$ & & Negative & 10YR $3 / 1$ & Sandy Clay Loam & Tall grass, trees in RIOW & Subsoil \\
\hline 1107MVR10 & 1 & $0-25$ & 0 & Negative & 10 Y $5 / 3$ & Sandy Clay Loam & Beside drainage, no vegetation & Subsoil \\
\hline 1107MVR10 & 2 & $25-100$ & & Negative & 10YR $6 / 3$ & Sandy Clay Loam & Beside drainage, no vegetation & Subsoil \\
\hline 1107JT11 & 1 & $0-50$ & 0 & Negative & 1OYR 5/1 & Sandy Clay Loam & River valley mixed hardwood, thin understory & Subsoil \\
\hline 1107JT11 & 2 & $50-60$ & & Negative & 10 YY $5 / 2$ & Sandy Clay Loam & River valley mixed hardwood, thin understory & Subsoil \\
\hline 1107WE12 & 1 & $0-30$ & 0 & Negative & $10 Y R 7 / 1$ & Sandy Clay Loam & Forest by creek bank, thin brush & Subsoil \\
\hline 1107WE12 & 2 & $30-50$ & & Negative & 10YR $4 / 2$ & Sandy Clay Loam & Forest by creek bank, thin brush & Subsoil \\
\hline 1107MT13 & 1 & $0-20$ & 0 & Negative & $10 \mathrm{YR} 5 / 4$ & Sandy Clay Loam & Grasses and Shrubs & Subsoil \\
\hline 1107MT13 & 2 & $20-50$ & & Negative & 1OYR $5 / 2$ & Sandy Clay Loam & Grasses and Shrubs & Subsoil \\
\hline 1107MVR14 & 1 & $0-20$ & 0 & Negative & 10 YR $5 / 3$ & Sandy Clay Loam & Wooded area, heavy leaf litter, low grasses/weeds & Subsoil \\
\hline 1107MVR14 & 2 & $20-80$ & & Negative & 10YR6/3 & Sandy Clay Loam & Wooded area, heavy leaf litter, low grasses/weeds & Subsoil \\
\hline 1107WE15 & 1 & $0-40$ & 0 & Negative & $10 Y R 7 / 1$ & Sandy Clay Loam & Forest by creek bank, thin brush & Subsoil \\
\hline
\end{tabular}




\begin{tabular}{|c|c|c|c|c|c|c|c|c|}
\hline Shovel Test \# & $\begin{array}{l}\text { Level } \\
\text { (Strat) }\end{array}$ & $\begin{array}{l}\text { Depth } \\
\text { (cmbs) }\end{array}$ & GSV \% & $\begin{array}{l}\text { Positive or } \\
\text { Negative }\end{array}$ & $\begin{array}{l}\text { Munsell } \\
\text { Color }\end{array}$ & Soil Texture Description & Description (Area; Vegetation) & Reason for Termination \\
\hline 1107WE15 & 2 & $40-55$ & & Negative & 10YR $4 / 2$ & Sandy Clay Loam & Forest by creek bank, thin brush & Subsoil \\
\hline $1107 \mathrm{JT} 16$ & 1 & $0-10$ & 0 & Negative & 10YR $4 / 2$ & Sandy Clay Loam & River valley mixed hardwood, thin understory & Subsoil \\
\hline 1107MT17 & 1 & $0-20$ & 0 & Negative & 10YR $5 / 4$ & Sandy Clay Loam & Grasses and ground vines & Subsoil \\
\hline 1107MT17 & 2 & $20-30$ & & Negative & 10YR 5/4 & Sandy Clay Loam & Grasses and ground vines & Subsoil \\
\hline 1107WE18 & 1 & $0-30$ & 0 & Negative & 10YR $7 / 1$ & Sandy Clay Loam & Forest, thin brush, frequent flooding & Subsoil \\
\hline 1107WE18 & 2 & $30-50$ & 0 & Negative & 10YR $5 / 1$ & Sandy Clay Loam & Forest, thin brush, frequent flooding & Subsoil \\
\hline 1112MVR1 & 1 & $0-20$ & 40 & Negative & 10YR $5 / 2$ & Sandy Loam & Low grasses and weeds, mixed hardwoods & Soil Change \\
\hline 1112MVR1 & 2 & $20-40$ & & Negative & 10YR $5 / 3$ & Sandy Clay Loam & Low grasses and weeds, mixed hardwoods & Root Impasse \\
\hline $1112 \mathrm{JJ} 2$ & 1 & $0-22$ & 100 & Negative & 10YR $5 / 1$ & Silty Clay Loam & Grass coverage & Soil Change \\
\hline $1112 \mathrm{JJ} 2$ & 2 & $22-60$ & & Negative & 10YR $5 / 1$ & Silty Clay & Grass coverage & Subsoil \\
\hline $1112 \mathrm{TI3}$ & 1 & $0-16$ & 70 & Negative & 10YR 5/1 & Silty Clay Loam & Blue mist flower, Periscaria, Iva Annua & Soil Change \\
\hline $1112 T 13$ & 2 & $16-40$ & & Negative & 10YR $4 / 1$ & Silty Clay Loam & Blue mist flower, Periscaria, Iva Annua & Root Impasse \\
\hline 1112WE4 & 1 & $0-30$ & 0 & Negative & 10YR 6/1 & Sandy Loam & Tall grass and saplings, edge of forest & Soil Change \\
\hline 1112WE4 & 2 & $30-55$ & & Negative & 10YR 4/1 & Sandy Clay Loam & Tall grass and saplings, edge of forest & Subsoil \\
\hline 1112MVR5 & 1 & $0-50$ & 30 & Negative & 10YR $6 / 2$ & Sandy Loam & Low grass and weeds, mixed hardwoods, beside stream & Soil Change \\
\hline 1112MVR5 & 2 & $50-65$ & & Negative & 10YR $5 / 3$ & Sandy Clay Loam & Low grass and weeds, mixed hardwoods, beside stream & Root Impasse \\
\hline $1112 \mathrm{JJ} 6$ & 1 & 1 & 95 & Negative & 1 & 1 & Stream & Inundation \\
\hline 1112WE7 & 1 & $0-30$ & 0 & Negative & 10YR 5/1 & Sandy Clay Loam & Tall grass in R.O.W. & Soil Change \\
\hline 1112WE7 & 2 & $30-50$ & & Negative & 10YR $6 / 1$ & Sandy Clay Loam & Tall grass in R.O.W. & Subsoil \\
\hline $1112 \mathrm{JJ} 8$ & 1 & $0-45$ & 0 & Negative & 10YR 5/1 & Silty Clay & Shrub layer & Root Impasse \\
\hline 1112MVR9 & 1 & $0-40$ & 0 & Negative & 10YR $4 / 1$ & Clay & High shrubs and low grasses & Subsoil \\
\hline 1112 TI10 & 1 & $0-12$ & 60 & Negative & 10YR 5/1 & Silty Clay Loam & Iva Annua, Bunge's Hackberry, Dwarf Palmetto & Soil Change \\
\hline 1112 TI10 & 2 & $12-17$ & & Negative & 10YR $4 / 1$ & Silty Clay Loam & Iva Annua, Bunge's Hackberry, Dwarf Palmetto & Inundation \\
\hline 1112WE11 & 1 & $0-35$ & 20 & Negative & 10YR $6 / 1$ & Sand & Forest by creek, thin brush & Soil Change \\
\hline 1112WE11 & 2 & $35-60$ & & Negative & 10YR $4 / 3$ & Sandy Loam & Forest by creek, thin brush & Subsoil \\
\hline $1112 \mathrm{JJ} 12$ & 1 & $0-50$ & 20 & Negative & 10YR $5 / 1$ & Silty Clay & Trees and shrubs, forested wetland & Inundation \\
\hline 1112WE13 & 1 & $0-25$ & 0 & Negative & 10YR 5/1 & Clay Loam & Tall grass in R.O.W. & Inundation \\
\hline 1112MVR14 & 1 & $0-40$ & 0 & Negative & 10YR $3 / 1$ & Clay & High shrubs and low grasses, compact soil & Subsoil \\
\hline $1112 \mathrm{TI} 15$ & 1 & $0-10$ & 80 & Negative & 10YR $5 / 1$ & Silty Clay Loam & Cocklebur, Spring Chloracantha, Sumpweed, Swamp Smartweed, R.O.W. & Soil Change \\
\hline 1112 TI15 & 2 & $10-16$ & & Negative & 10YR $4 / 1$ & Silty Clay Loam & Cocklebur, Spiny Chloracantha, Sumpweed, Swamp Smartweed, R.O.W. & Inundation \\
\hline 1112WE16 & 1 & $0-15$ & 20 & Negative & 10YR 5/1 & Clay Loam & Forest, thin brush, damp soil & Inundation \\
\hline $1112 \mathrm{JJ} 17$ & 1 & $0-20$ & 100 & Negative & 10YR 5/1 & Silty Clay & Trees and grasses, forested wetland & Inundation \\
\hline 1112WE18 & 1 & $0-15$ & 0 & Negative & 10YR $5 / 1$ & Clay Loam & Tall grass in R.O.W. & Inundation \\
\hline $1112 \mathrm{JJ} 19$ & 1 & $0-5$ & 95 & Negative & 10YR $5 / 3$ & Silty Clay & Trees and grasses, forested wetland & Inundation \\
\hline $1112 \mathrm{JJ} 20$ & 1 & $0-10$ & 95 & Negative & 10YR $5 / 1$ & Silty Clay & $30 \%$ tree coverage, $65 \%$ herb coverage & Inundation \\
\hline 1112WE21 & 1 & $0-20$ & 15 & Negative & 10YR 5/1 & Clay & Forest, thin brush & Soil Change \\
\hline 1112WE21 & 2 & $20-45$ & & Negative & 10YR $3 / 1$ & Clay & Forest, thin brush & Subsoil \\
\hline $1112 \mathrm{~T} 122$ & 1 & $0-10$ & 10 & Negative & 10YR 5/1 & Silty Clay Loam & American Elm, Green Ash & Soil Change \\
\hline 1112 TI22 & 2 & $10-16$ & & Negative & 10YR $4 / 1$ & Silty Clay Loam & American Elm, Green Ash & Inundation \\
\hline 1112WE23 & 1 & 1 & 0 & Negative & 1 & 1 & R.O.W. & Inundation \\
\hline 1112WE24 & 1 & $0-5$ & 15 & Negative & 10YR 5/1 & Clay & Forest, thin brush & Inundation \\
\hline $1112 \mathrm{JJ} 25$ & 1 & $0-40$ & 100 & Negative & 10YR $5 / 6$ & Silty Clay & Herbaceous layer dominated by Cockleburs & Subsoil \\
\hline $1112 \mathrm{~T} / 26$ & 1 & $0-9$ & 60 & Negative & 10YR $5 / 1$ & Silty Clay Loam & Spiny Chlorocantha, Tallow, Green Ash, woodland & Soil Change \\
\hline 1112 TI26 & 2 & $9-15$ & & Negative & 10YR $4 / 1$ & Silty Clay Loam & Spiny Chlorocantha, Tallow, Green Ash, woodland & Inundation \\
\hline 1112MVR27 & 1 & $0-20$ & 25 & Negative & 10YR $4 / 1$ & Clay & Low grasses and weeds, mixed hardwoods, saturated soil & Subsoil \\
\hline 1112WE28 & 1 & $0-20$ & 25 & Negative & 10YR $4 / 1$ & Clay & Forest, thin brush & Soil Change \\
\hline 1112WE28 & 2 & $20-40$ & & Negative & 10YR $3 / 1$ & Clay & $\begin{array}{l}\text { Forest, thin brush } \\
\end{array}$ & Subsoil \\
\hline 1112MVR29 & 1 & $0-20$ & 25 & Negative & 10YR $4 / 1$ & Clay & Low grasses, weeds, mixed hardwoods, saturated soil & Subsoil \\
\hline 1112WE30 & 1 & $0-20$ & 2 & Negative & 10YR $4 / 1$ & Clay & Forest, thin brush & Soil Change \\
\hline 1112WE30 & 2 & $20-40$ & & Negative & 10YR $3 / 1$ & Clay & Forest, thin brush & Subsoil \\
\hline $1112 \mathrm{JJ} 31$ & 1 & $0-16$ & 30 & Negative & 10YR $5 / 4$ & Clay & Forested wetland, herbaceous layer & Inundation \\
\hline $1112 \mathrm{~T} / 32$ & 1 & $0-9$ & 40 & Negative & 10YR 5/1 & Silty Clay Loam & Button bush, Green Ash & Inundation \\
\hline 1112WE33 & 1 & $0-15$ & 5 & Negative & 10YR $4 / 1$ & Clay & Forest, thin brush & Soil Change \\
\hline 1112 WE33 & 2 & $15-40$ & & Negative & 10YR $3 / 1$ & Clay & Forest, thin brush & Subsoil \\
\hline $1112 \mathrm{~T} \mid 34$ & 1 & $0-10$ & 40 & Negative & 10YR 4/1 & Silty Clay Loam & Cocklebur $100 \%$ & Inundation \\
\hline 1112WE35 & 1 & $0-20$ & 5 & Negative & 10YR $4 / 1$ & Clay & Edge of forest near R.O.W. & Soil Change \\
\hline 1112WE35 & 2 & $20-40$ & & Negative & 10YR $3 / 1$ & Clay & Edge of forest near R.O.W. & Subsoil \\
\hline
\end{tabular}


Appendix D - Shovel Test Data

\begin{tabular}{|c|c|c|c|c|c|c|c|c|}
\hline Shovel Test \# & $\begin{array}{l}\text { Level } \\
\text { (Strat) }\end{array}$ & $\begin{array}{l}\text { Depth } \\
\text { (cmbs) }\end{array}$ & GSV \% & $\begin{array}{c}\text { Positive or } \\
\text { Negative }\end{array}$ & $\begin{array}{l}\text { Munsell } \\
\text { Color }\end{array}$ & Soil Texture Description & Description (Area; Vegetation) & Reason for Termination \\
\hline $1112 \mathrm{JJ} 36$ & 1 & $0-45$ & 35 & Negative & 10YR $5 / 4$ & Clay & Forested wetland & Subsoil \\
\hline $1112 \mathrm{~T} 137$ & 1 & $0-3$ & 90 & Negative & 10YR 4/1 & Silty Clay Loam & Button bush, Green Ash, forest & Inundation \\
\hline $1112 \mathrm{JJ} 38$ & 1 & $0-24$ & 85 & Negative & 10YR 5/1 & Silty Clay & Dominated by grasses & Inundation \\
\hline 1112WE39 & 1 & $0-20$ & 5 & Negative & $10 Y R 4 / 1$ & Clay & Edge of forest near R.O.W. & Soil Change \\
\hline 1112WE39 & 2 & $20-35$ & & Negative & 10YR $3 / 1$ & Clay & Edge of forest near R.O.W. & Inundation \\
\hline 1112 TI40 & 1 & $0-10$ & 90 & Negative & 10YR $4 / 1$ & Silty Clay Loam & Green Ash, Black Willow, Balloon Vine & Inundation \\
\hline 1112MVR41 & 1 & $0-25$ & 70 & Negative & 10YR $4 / 2$ & Clay & Low grasses and weeds, mixed hardwoods, highly saturated Clay & Subsoil \\
\hline $1112 \mathrm{~T} 142$ & 1 & $0-10$ & 50 & Negative & 10YR 4/1 & Silty Clay Loam & Green Ash, Black Willow, Balloon Vine & Inundation \\
\hline
\end{tabular}


APPENDIX E: Shovel TeSt DATA For Code-Permitted Tracts 
Appendix E - Shovel Test Data for Code-Permitted Tracts

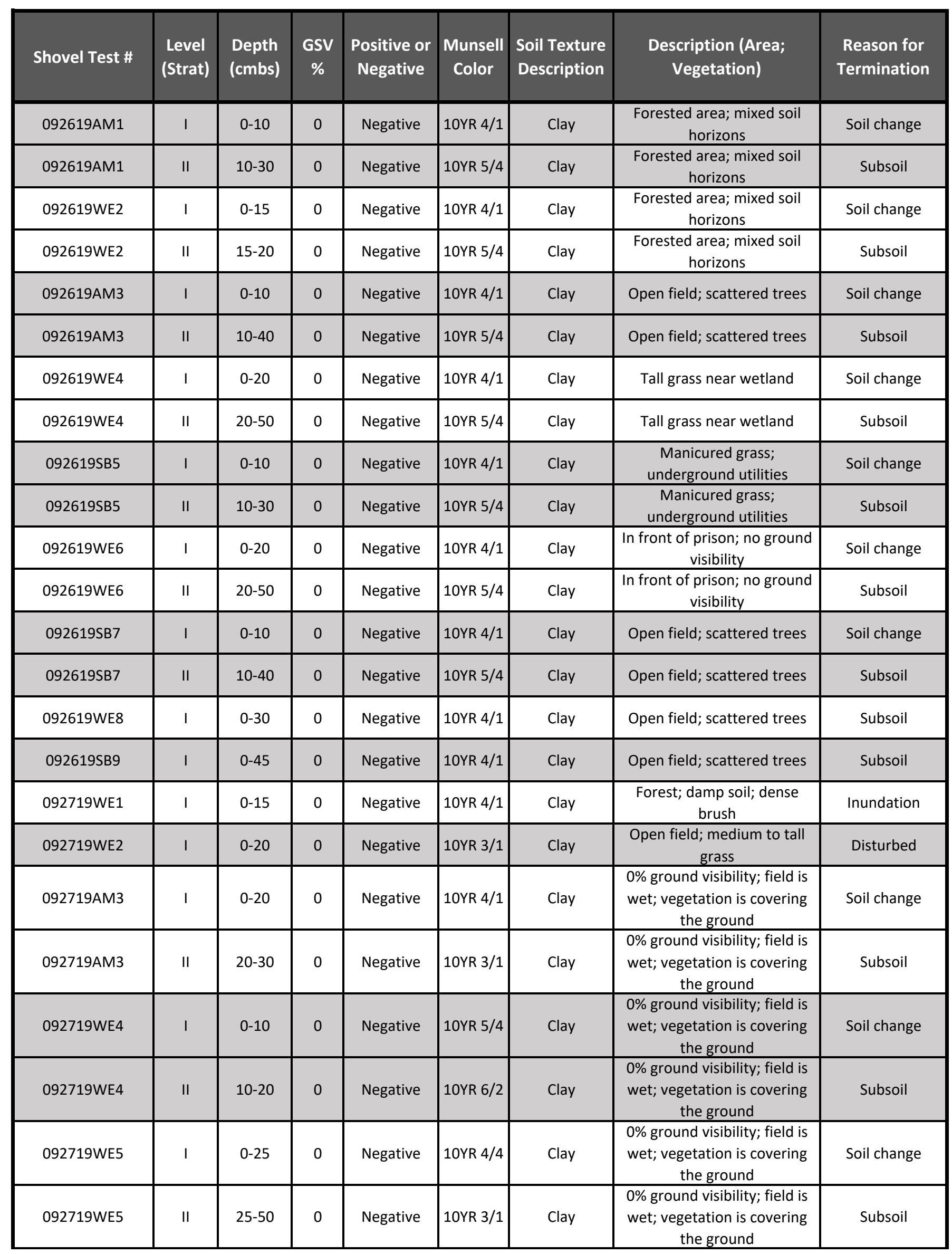


Appendix E - Shovel Test Data for Code-Permitted Tracts

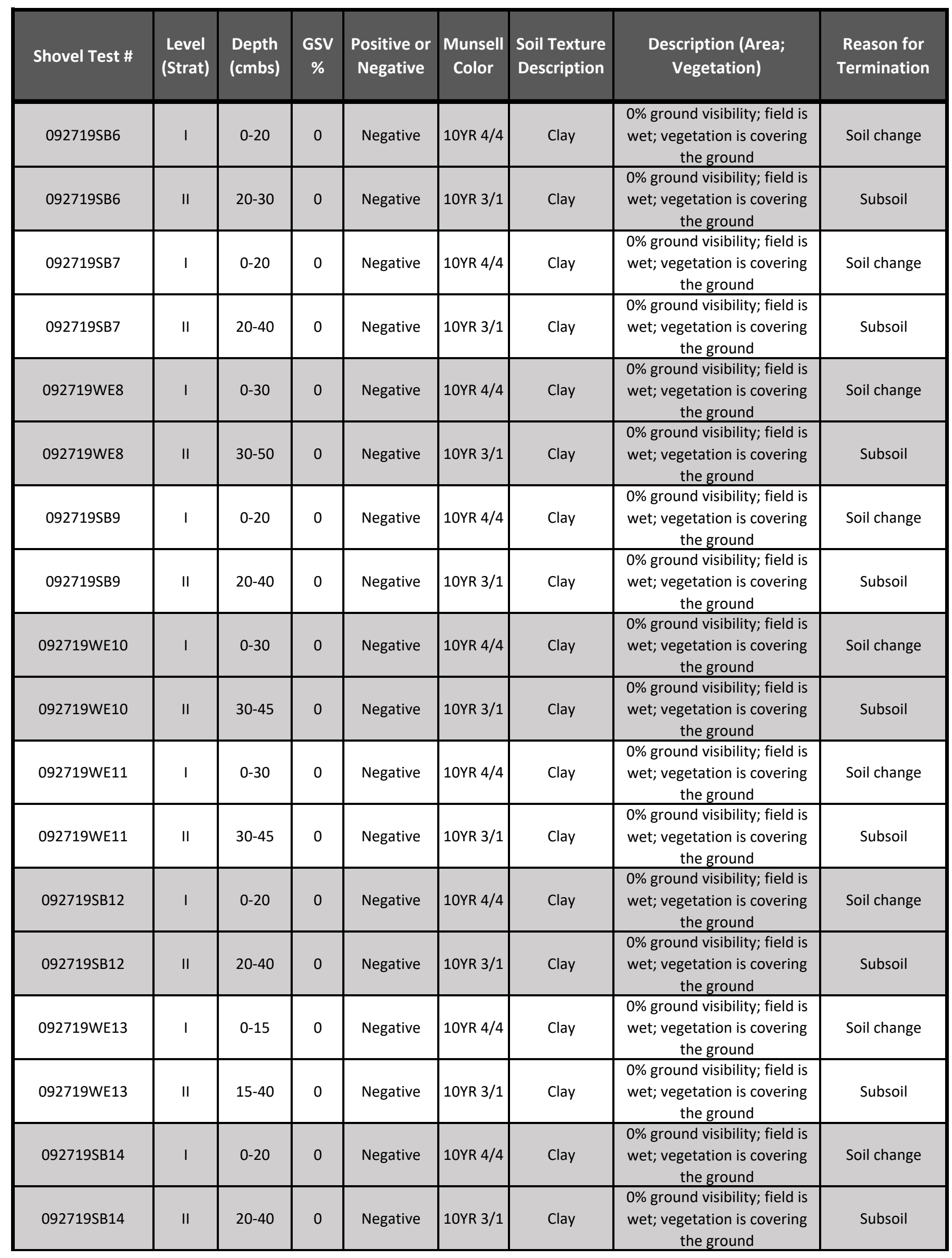


Appendix E - Shovel Test Data for Code-Permitted Tracts

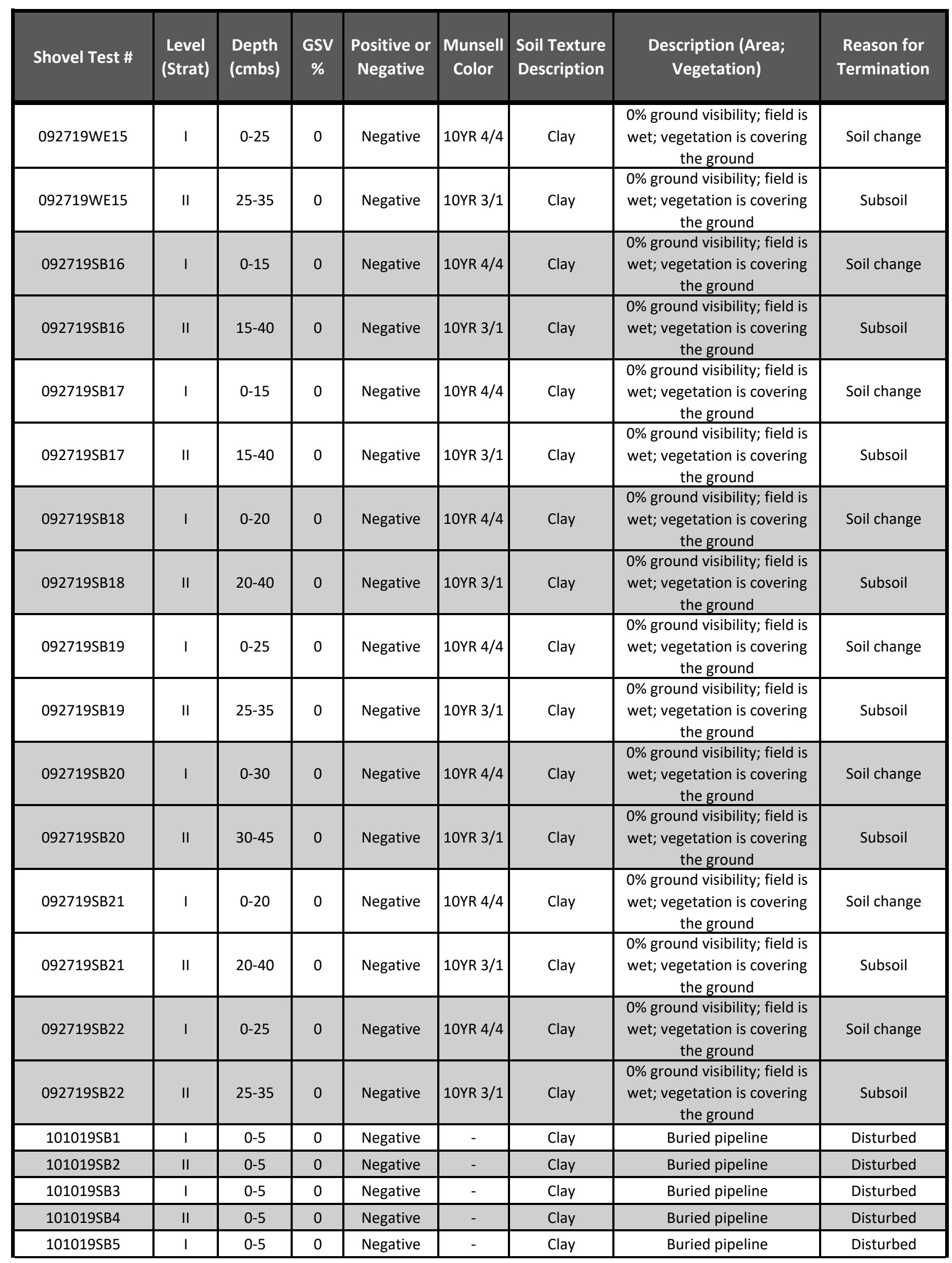


Appendix E - Shovel Test Data for Code-Permitted Tracts

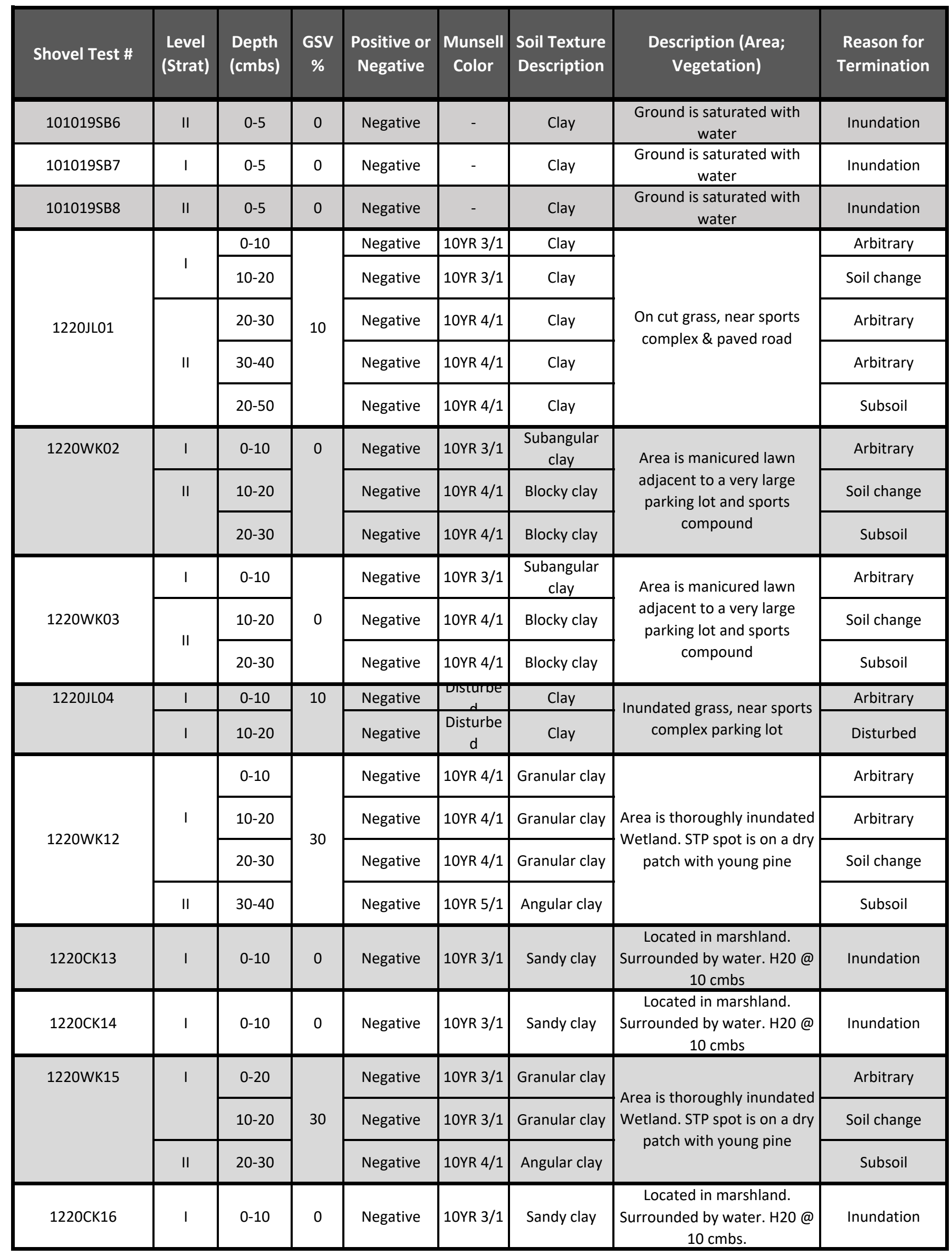




\section{Appendix E - Shovel Test Data for Code-Permitted Tracts}

\begin{tabular}{|c|c|c|c|c|c|c|c|c|}
\hline Shovel Test \# & $\begin{array}{l}\text { Level } \\
\text { (Strat) }\end{array}$ & $\begin{array}{l}\text { Depth } \\
\text { (cmbs) }\end{array}$ & $\begin{array}{c}\text { GSV } \\
\%\end{array}$ & $\begin{array}{l}\text { Positive or } \\
\text { Negative }\end{array}$ & $\begin{array}{c}\text { Munsell } \\
\text { Color }\end{array}$ & $\begin{array}{l}\text { Soil Texture } \\
\text { Description }\end{array}$ & $\begin{array}{c}\text { Description (Area; } \\
\text { Vegetation) }\end{array}$ & $\begin{array}{l}\text { Reason for } \\
\text { Termination }\end{array}$ \\
\hline 1220JL17 & । & $0-10$ & 10 & Negative & 10YR 4/1 & Clay & Pipeline corridor, inundated & Inundation \\
\hline $1220 \mathrm{CK} 18$ & 1 & $0-10$ & 0 & Negative & 10YR 3/1 & Sandy clay & $\begin{array}{c}\text { Located in marshland. } \\
\text { Surrounded by water. H20 @ } \\
10 \text { cmbs. }\end{array}$ & Inundation \\
\hline 1220JL19 & 1 & $0-10$ & 10 & Negative & 10YR 4/1 & Clay & Pipeline corridor, inundated & Inundation \\
\hline \multirow{2}{*}{$1220 W K 20$} & \multirow{2}{*}{ I } & $0-10$ & \multirow{2}{*}{15} & Negative & 10YR 5/1 & Granular clay & \multirow{2}{*}{$\begin{array}{l}\text { Area subject to frequent } \\
\text { periods of inundation }\end{array}$} & Arbitrary \\
\hline & & $10-20$ & & Negative & 10YR 5/1 & Granular clay & & Inundation \\
\hline 1220JL21 & I & $0-25$ & 15 & Negative & 10YR 5/1 & Granular clay & $\begin{array}{l}\text { Area subject to frequent } \\
\text { periods of inundation }\end{array}$ & Inundation \\
\hline 1220WK22 & I & $0-10$ & 15 & Negative & 10YR 4/1 & Granular clay & $\begin{array}{l}\text { Area subject to frequent } \\
\text { periods of inundation }\end{array}$ & Inundation \\
\hline 1220 CK23 & I & $0-10$ & 0 & Negative & 10YR 3/1 & Sandy clay & $\begin{array}{c}\text { Located in marshland. } \\
\text { Surrounded by water. H20 @ } \\
10 \text { cmbs. }\end{array}$ & Inundation \\
\hline 1220JL24 & I & $0-10$ & 10 & Negative & 10YR 4/1 & Clay & Pipeline corridor, inundated & Inundation \\
\hline \multirow[t]{2}{*}{ 1221WK01 } & \multirow{2}{*}{ I } & $0-10$ & \multirow{2}{*}{30} & Negative & 10YR 4/1 & Granular clay & \multirow{2}{*}{$\begin{array}{l}\text { Area is a two track adjacent } \\
\text { to Wetland; very disturbed. }\end{array}$} & Arbitrary \\
\hline & & $10-20$ & & Negative & 10YR 4/1 & Granular clay & & Disturbed \\
\hline 1221CK02 & I & $0-10$ & 0 & Negative & 10YR 5/1 & Clay & $\begin{array}{l}\text { Open field with knee high } \\
\text { grass. } \mathrm{H} 2 \mathrm{O} \text { at } 10 \mathrm{cmbs}\end{array}$ & Inundation \\
\hline 1221СК03 & I & $0-10$ & 0 & Negative & 10YR 5/1 & Clay & $\begin{array}{l}\text { Open field with knee high } \\
\text { grass. } \mathrm{H} 2 \mathrm{O} \text { at } 10 \mathrm{cmbs}\end{array}$ & Inundation \\
\hline \multirow[t]{3}{*}{ 1221WK04 } & \multirow{2}{*}{ I } & $0-10$ & \multirow{3}{*}{0} & Negative & 10YR 4/1 & Granular clay & \multirow{3}{*}{$\begin{array}{c}\text { Wetland with standing water } \\
\text { throughout. Briar and grass } \\
\text { obscure ground surface } \\
\text { visibility }\end{array}$} & Arbitrary \\
\hline & & $10-20$ & & Negative & 10YR 4/1 & Granular clay & & Soil change \\
\hline & ॥ & $20-30$ & & Negative & 10YR 3/1 & Angular clay & & Subsoil \\
\hline \multirow[t]{4}{*}{$1221 W K 05$} & \multirow[t]{2}{*}{1} & $0-10$ & \multirow{4}{*}{0} & Negative & 10YR 4/1 & Granular clay & \multirow{4}{*}{$\begin{array}{c}\text { Wetland with standing water } \\
\text { throughout. Briar and grass } \\
\text { obscure ground surface } \\
\text { visibility }\end{array}$} & Arbitrary \\
\hline & & $10-20$ & & Negative & 10YR 4/1 & Granular clay & & Soil change \\
\hline & \multirow[t]{2}{*}{ II } & $20-30$ & & Negative & 10YR 5/1 & Angular clay & & Arbitrary \\
\hline & & $30-40$ & & Negative & 10YR 5/1 & Angular clay & & Subsoil \\
\hline 1221СK06 & I & $0-10$ & 0 & Negative & 10YR 5/1 & Clay & $\begin{array}{l}\text { Open field with knee high } \\
\text { grass. } \mathrm{H} 2 \mathrm{O} \text { at } 10 \mathrm{cmbs}\end{array}$ & Inundation \\
\hline \multirow[t]{3}{*}{ 1221WK07 } & I & $0-10$ & \multirow[t]{3}{*}{0} & Negative & 10YR 4/1 & Granular clay & \multirow[t]{3}{*}{$\begin{array}{l}\text { Wetland with standing water } \\
\text { throughout. Briar and grass }\end{array}$} & Arbitrary \\
\hline & \multirow[t]{2}{*}{ ॥ } & $10-20$ & & Negative & 7.5YR 5/1 & Angular clay & & Soil change \\
\hline & & $20-30$ & & Negative & 7.5YR 5/1 & Angular clay & & Subsoil \\
\hline
\end{tabular}


Appendix E - Shovel Test Data for Code-Permitted Tracts

\begin{tabular}{|c|c|c|c|c|c|c|c|c|}
\hline Shovel Test \# & $\begin{array}{l}\text { Level } \\
\text { (Strat) }\end{array}$ & $\begin{array}{l}\text { Depth } \\
\text { (cmbs) }\end{array}$ & $\begin{array}{c}\text { GSV } \\
\%\end{array}$ & $\begin{array}{c}\text { Positive or } \\
\text { Negative }\end{array}$ & $\begin{array}{c}\text { Munsell } \\
\text { Color }\end{array}$ & $\begin{array}{l}\text { Soil Texture } \\
\text { Description }\end{array}$ & $\begin{array}{c}\text { Description (Area; } \\
\text { Vegetation) }\end{array}$ & $\begin{array}{l}\text { Reason for } \\
\text { Termination }\end{array}$ \\
\hline 1221CK08 & I & $0-10$ & 0 & Negative & 10YR 5/1 & Clay & $\begin{array}{l}\text { Open field with knee high } \\
\text { grass. } \mathrm{H} 2 \mathrm{O} \text { at } 10 \mathrm{cmbs}\end{array}$ & Inundation \\
\hline 1221JL09 & 1 & $0-10$ & 0 & Negative & $\begin{array}{c}\text { 10YR 4/1 } \\
\& 10 Y R \\
5 / 1\end{array}$ & Clay & Waist-high grasses and brush & Inundation \\
\hline 1221JL10 & I & $0-10$ & 0 & Negative & $\begin{array}{c}\text { 10YR 4/1 } \\
\& 10 Y R \\
5 / 1\end{array}$ & Clay & Waist-high grasses and brush & Inundation \\
\hline 1221CK11 & I & $0-10$ & 0 & Negative & 10YR 5/1 & Clay & $\begin{array}{l}\text { Open field with knee high } \\
\text { grass. } \mathrm{H} 2 \mathrm{O} \text { at } 10 \mathrm{cmbs}\end{array}$ & Inundation \\
\hline \multirow{3}{*}{$1221 W K 12$} & \multirow{2}{*}{ I } & $0-10$ & \multirow{3}{*}{0} & Negative & 10YR 4/1 & Granular clay & \multirow{3}{*}{$\begin{array}{c}\text { Wetland with standing water } \\
\text { throughout. Briar and grass } \\
\text { obscure ground surface } \\
\text { visibility }\end{array}$} & Arbitrary \\
\hline & & $10-20$ & & Negative & 10YR 4/1 & Granular clay & & Soil change \\
\hline & II & $20-25$ & & Negative & 7.5YR 5/1 & Angular clay & & Subsoil \\
\hline 1221JL13 & I & $0-10$ & 0 & Negative & 10YR 4/1 & Clay & Waist-high grasses and brush & Inundation \\
\hline \multirow{3}{*}{ 1221CK14 } & I & $0-10$ & \multirow{3}{*}{0} & Negative & 10YR 5/1 & Clay & \multirow{3}{*}{$\begin{array}{l}\text { Open field with knee high } \\
\text { grass. }\end{array}$} & Arbitrary \\
\hline & \multirow{2}{*}{ II } & $10-20$ & & Negative & 10YR 3/1 & Clay & & Soil change \\
\hline & & $20-30$ & & Negative & 10YR 3/1 & Clay & & Subsoil \\
\hline \multirow[t]{3}{*}{ 1221CK15 } & 1 & $0-10$ & \multirow[t]{3}{*}{0} & Negative & 10YR 5/1 & Clay & \multirow[t]{3}{*}{$\begin{array}{l}\text { Open field with knee high } \\
\text { grass. }\end{array}$} & Arbitrary \\
\hline & \multirow[t]{2}{*}{ II } & $10-20$ & & Negative & 10YR 3/1 & Clay & & Soil change \\
\hline & & $20-30$ & & Negative & 10YR 3/1 & Clay & & Subsoil \\
\hline 1221JL16 & I & $0-10$ & 0 & Negative & 10YR 4/1 & Clay & Waist-high grasses and brush & Inundation \\
\hline \multirow[t]{3}{*}{ 1221WK17 } & I & $0-10$ & \multirow[t]{3}{*}{0} & Negative & 10YR 4/1 & Granular clay & \multirow{3}{*}{$\begin{array}{c}\text { Wetland with standing water } \\
\text { throughout. Briar and grass } \\
\text { obscure ground surface } \\
\text { visibility }\end{array}$} & Arbitrary \\
\hline & \multirow[t]{2}{*}{ II } & $10-20$ & & Negative & 7.5YR 5/1 & Angular clay & & Soil change \\
\hline & & $20-30$ & & Negative & 7.5YR 5/1 & Angular clay & & Subsoil \\
\hline \multirow{3}{*}{ 1221CK18 } & I & $0-10$ & \multirow{3}{*}{0} & Negative & 10YR 5/1 & Clay & \multirow{3}{*}{$\begin{array}{l}\text { Open field with knee high } \\
\text { grass. }\end{array}$} & Arbitrary \\
\hline & \multirow{2}{*}{ II } & $10-20$ & & Negative & 10YR 3/1 & Clay & & Soil change \\
\hline & & $20-30$ & & Negative & 10YR 3/1 & Clay & & Subsoil \\
\hline 1221JL19 & 1 & $0-10$ & 0 & Negative & 10YR 4/1 & Clay & Waist-high grasses and brush & Inundation \\
\hline
\end{tabular}




\section{APPENDIX F: Archeological Monitoring Plan}




\section{ENERGY}

TRANSFER

Energy Transfer Company

Archeological Monitoring Plan

Orbit Pipeline Project

January 2020 


\section{Archeological Monitoring Plan for the Orbit Pipeline Project}

\section{A. INTRODUCTION}

Energy Transfer Company (ETC) is proposing to construct the Orbit Pipeline Project (Project) located in Jefferson, Liberty, and Chambers counties, Texas. The Project consists of approximately 68.7 miles (mi) (110.6 kilometer [km]) of new 20.0-inch (in) (50.8 centimeter [cm]) diameter pipeline that will be used to transmit ethane and propane. This document describes the procedures for archeological monitoring during the construction phase of the Project at select areas (herein defined as Permit Areas) as defined by the US Army Corps of Engineers (USACE), and outlined in Table 1. It is intended to:

- Maintain compliance with applicable Federal and State laws and regulations during construction of the Project; and,

- Describe the procedure the archeological monitor and construction personnel will follow in the event that archeological resources are identified during the construction phase of the Project.

The Archeological Monitor will be a Secretary of the Interior (SOI) qualified archeologist, and would work collaboratively with the Environmental Inspection team to ensure compliance with Section 106 of the National Historic Preservation Act (NHPA), and USACE permitting provisions.

The Environmental Project Manager, Construction Supervisor, or Lead Environmental Inspector (EI) will be responsible for providing ample notice (i.e. 48 to 72 hours) to the archeological monitor when ground disturbing activities are anticipated to occur within the Permit Areas. For purposes of this Plan, ground disturbing activities are defined as any activities that have the potential to impact cultural resources. These include vegetation clearing, grading, and trenching activities. Certain activities during construction would not require monitoring such as the lowering in of the pipe, clean up or back-filling, transportation of equipment, or installation of temporary fencing.

The Archeological Monitor would actively observe ground disturbing activities to look for evidence of cultural materials exposed by construction equipment, and would be required to follow all safety procedures and protocols while on site. For safety purposes, the monitor would not be allowed to enter any trenches at any point unless approval is provided by the Construction Supervisor and proper shoring is in place. Instead, the monitor would inspect excavated soils behind construction equipment at a safe distance in accordance with safety protocols.

At Horizontal Direction Drill (HDD) workspaces, monitoring will take place at the entry and exit drill points. In the event that an inadvertent return occurs, the monitor would be present to inspect clean-up activities.

The Archeological Monitor would be responsible for submitting daily reports to the El team and the Archeological Principal Investigator that details the areas monitored, results of monitoring activities, and representative photographs. Upon completion of the monitoring responsibilities, a monitoring report would be produced for review by the USACE and the Texas Historical Commission (THC) to conclude Section 106 consultations for the Project. 


\section{B. PROCEDURES FOR THE DISCOVERY OF CULTURAL RESOURCES}

In the event that a cultural resource is discovered within a Permit Area, the following plan will be implemented:

1. All work within 100 feet of both sides of the discovery will immediately stop and the El will be notified. The area of work stoppage will be adequate to provide for the security, protection, and integrity of the materials.

2. The El and Archeological Monitor will take appropriate steps to protect the discovery site. This will include flagging the immediate area of discovery and stop work or exclusion zone as well as notifying the Environmental Project Manager. All cultural materials will be fully delineated in accordance with THC guidelines within the project area by the archaeologist, and possibly additional personnel, in order to determine if further work and/or avoidance is recommended. If the monitor determines that the find constitutes an archeological site, then additional evaluation and assessment may be necessary.

3. Work in the immediate area will not resume until treatment of the discovery has been completed.

4. If the discovery is determined to have the potential for eligibility, the archaeologist will consult with the USACE and SHPO on how best to avoid, minimize, or otherwise mitigate further impacts. Treatment measures may include, but are not limited to, mapping, photography, sample collection, or excavation activity.

\section{PROCEDURES FOR THE DISCOVERY OF HUMAN REMAINS}

In the event that human remains, possible human remains, or grave goods are encountered during either construction or maintenance activities, the following plan outlines the specific procedures to be followed. These procedures meet or exceed the Policy Statement Regarding Treatment of Burial Sites, Human Remains, and Funerary Objects adopted by the Advisory Council on Historic Preservation, "Protection of Historic and Cultural Properties" (36 CFR Part 800); Procedures for the Protection of Historic Properties (33 CFR 325 Appendix C); the Archaeological and Historic Preservation Act, and Texas Health and Safety Code (Title 8, Chapters 711-714).

All activity that might disturb the remains shall cease and may not resume until authorized by appropriate law enforcement officials, the USACE Staff Archeologist, or the State Archaeologist. Any human remains, burial sites, or burial related materials that are discovered during construction will at all times be treated with dignity and respect.

1. The Lead El or archaeologist, will notify the Environmental Project Manager, the Texas Historical Commission Regional Archaeologist, the law enforcement agency and the coroner of the jurisdiction where the site or remains are located within 24 hours of the discovery. The USACE Staff Archeologist also be contacted to assist with identifying the remains, as required.

2. Any activity that may disturb the unmarked burial site, human skeletal remains, or burial artifacts associated with the site will immediately cease on discovery. The site will be carefully covered and secured for protection from degradation by weather or unauthorized 
individuals.

3. The El will be responsible for taking appropriate steps to protect the discovery. This will include fencing off the immediate area of discovery and flagging the area as an exclusion zone. No activity may resume until authorized by the agency authority governing the disposition of the human remains.

4. If the unmarked burial site, human skeletal remains, or funerary objects can be shown to have ethnic affinity with a living Native American tribe, the USACE would take necessary steps to assist in determining the tribe(s), if any, who may have historic ties to the region and represent descendants of any Native American remains.

5. If the District Coroner finds that the unmarked burial site is over 50 years old and that there is no need for a legal inquiry by their office or for a criminal investigation, and if no direct relations to any Native American tribe are found, then the USACE will have jurisdiction of the site, human skeletal remains, and the burial artifacts.

Should the find be determined to constitute a cemetery, a notice of existence of a cemetery will be filed within 10 days of the discovery per requirement under Ch 711 of the Texas Health \& Safety Code.

\section{PROJECT CONTACTS}

\section{Environmental Inspector}

Attn: TBD

Phone:

\section{ETC Environmental Project Manager}

Attn: Jonathan Minton

Phone: (o) 713-989-2127

Email: jonathan.minton@energytransfer.com

\section{USACE - Galveston}

Attn: Jerry Androy, Staff Archeologist

2000 Fort Point Road

Galveston, Texas 77550

Phone: 409-766-3821

Email: jerry.l.androy@usace.army.mil

\section{Texas Historical Commission}

Bill Martin

Team Lead, Review and Compliance

P.O. Box 12276, Austin, TX 78711

Phone: (512) 463-5867

Texas Health and Safety Code (Title 8, Chapters 711-714)

Maggie Moore

Regional Archeologist/ Project Reviewer

P.O. Box 12276, Austin, TX 78711

Phone: (512) 463-6508 


\begin{tabular}{|c|c|c|c|c|c|c|}
\hline \multicolumn{7}{|c|}{ Orbit Permit Areas } \\
\hline Feature ID & Milepost & $\begin{array}{l}\text { Tract } \\
\text { ID }\end{array}$ & $\begin{array}{c}\text { Land } \\
\text { Ownership }\end{array}$ & $\begin{array}{c}\text { Reason } \\
\text { Surveys } \\
\text { Not } \\
\text { conducted }\end{array}$ & $\begin{array}{l}\text { Archeological } \\
\text { Monitor } \\
\text { Needed }\end{array}$ & $\begin{array}{c}\text { Appendix } \\
\text { B Aerial } \\
\text { Map Pg. } \\
\text { No. }\end{array}$ \\
\hline WP9CH004_DT & 2.7 & $\begin{array}{l}\text { TX-CH- } \\
019.200\end{array}$ & Private & $\begin{array}{c}\text { Survey } \\
\text { permission } \\
\text { denied }\end{array}$ & $\begin{array}{l}\text { No-Workspace } \\
\text { change; feature } \\
\text { will not be } \\
\text { impacted }\end{array}$ & 5 \\
\hline $\begin{array}{l}\text { WP4LI002_PFO (First } \\
\text { Crossing) }\end{array}$ & 16.9 & $\begin{array}{l}\text { TX-LI- } \\
029.000\end{array}$ & Private & $\begin{array}{c}\text { Flooded } \\
\text { tract }\end{array}$ & Yes & 23 \\
\hline $\begin{array}{l}\text { WP4LI002_PFO_C } \\
\text { (First Crossing) }\end{array}$ & 17 & $\begin{array}{l}\text { TX-LI- } \\
029.000\end{array}$ & Private & $\begin{array}{l}\text { Flooded } \\
\text { tract }\end{array}$ & Yes & 23 \\
\hline $\begin{array}{l}\text { WP4LI002_PFO } \\
\text { (Second Crossing) }\end{array}$ & 17 & $\begin{array}{l}\text { TX-LI- } \\
029.000\end{array}$ & Private & $\begin{array}{c}\text { Flooded } \\
\text { tract }\end{array}$ & Yes & 23 \\
\hline $\begin{array}{l}\text { WP4LI002_PFO_C } \\
\text { (Second Crossing) }\end{array}$ & 17.2 & $\begin{array}{c}\text { TX-LI- } \\
029.000\end{array}$ & Private & $\begin{array}{c}\text { Flooded } \\
\text { tract }\end{array}$ & Yes & 23 \\
\hline $\begin{array}{c}\text { WP3LI007_PFO (First } \\
\text { Crossing) }\end{array}$ & 17.8 & $\begin{array}{c}\text { TX-LI- } \\
029.000 \\
\end{array}$ & Private & $\begin{array}{l}\text { Originally } \\
\text { flooded tract }\end{array}$ & $\begin{array}{l}\text { No - survey has } \\
\text { been completed }\end{array}$ & 24 \\
\hline $\begin{array}{l}\text { WP3LI007_PFO } \\
\text { (Second Crossing) }\end{array}$ & 17.8 & $\begin{array}{l}\text { TX-LI- } \\
029.000\end{array}$ & Private & $\begin{array}{l}\text { Originally } \\
\text { flooded tract }\end{array}$ & $\begin{array}{l}\text { No - survey has } \\
\text { been completed }\end{array}$ & 24 \\
\hline $\begin{array}{l}\text { WP3LI007_PFO } \\
\text { (Third Crossing) }\end{array}$ & 17.8 & $\begin{array}{c}\text { TX-LI- } \\
029.000 \\
\end{array}$ & Private & $\begin{array}{l}\text { Originally } \\
\text { flooded tract }\end{array}$ & $\begin{array}{l}\text { No - survey has } \\
\text { been completed }\end{array}$ & 24 \\
\hline $\begin{array}{l}\text { WP4LI002_PEM } \\
\text { (Second Crossing) }\end{array}$ & 17.8 & $\begin{array}{l}\text { TX-LI- } \\
029.000\end{array}$ & Private & $\begin{array}{l}\text { Originally } \\
\text { flooded tract }\end{array}$ & $\begin{array}{l}\text { No - survey has } \\
\text { been completed }\end{array}$ & 24 \\
\hline WP3LI007_PEM & 17.9 & $\begin{array}{l}\text { TX-LI- } \\
029.000\end{array}$ & Private & $\begin{array}{l}\text { Originally } \\
\text { flooded tract }\end{array}$ & $\begin{array}{l}\text { No - survey has } \\
\text { been completed }\end{array}$ & 24 \\
\hline $\begin{array}{l}\text { WP3LI007_PFO } \\
\text { (Fourth Crossing) }\end{array}$ & 17.9 & $\begin{array}{c}\text { TX-LI- } \\
029.000 \\
\end{array}$ & Private & $\begin{array}{c}\text { Originally } \\
\text { flooded tract }\end{array}$ & $\begin{array}{l}\text { No - survey has } \\
\text { been completed }\end{array}$ & 24 \\
\hline $\begin{array}{l}\text { WP3LI007_PFO } \\
\text { (Fifth Crossing) }\end{array}$ & 17.9 & $\begin{array}{c}\text { TX-LI- } \\
029.000 \\
\end{array}$ & Private & $\begin{array}{l}\text { Originally } \\
\text { flooded tract }\end{array}$ & $\begin{array}{l}\text { No - survey has } \\
\text { been completed }\end{array}$ & 24 \\
\hline $\begin{array}{l}\text { WP3LI007_PFO } \\
\text { (Sixth Crossing) }\end{array}$ & 18 & $\begin{array}{l}\text { TX-LI- } \\
029.000\end{array}$ & Private & $\begin{array}{l}\text { Originally } \\
\text { flooded tract }\end{array}$ & $\begin{array}{l}\text { No - survey has } \\
\text { been completed }\end{array}$ & 24 \\
\hline $\begin{array}{c}\text { WP3LI007_PFO } \\
\text { (Seventh Crossing) }\end{array}$ & 18 & $\begin{array}{c}\text { TX-LI- } \\
029.000 \\
\end{array}$ & Private & $\begin{array}{l}\text { Originally } \\
\text { flooded tract }\end{array}$ & $\begin{array}{l}\text { No - survey has } \\
\text { been completed }\end{array}$ & 24 \\
\hline $\begin{array}{l}\text { WP3LI007_PFO } \\
\text { (Eighth Crossing) }\end{array}$ & 18 & $\begin{array}{l}\text { TX-LI- } \\
029.000\end{array}$ & Private & $\begin{array}{l}\text { Originally } \\
\text { flooded tract }\end{array}$ & $\begin{array}{l}\text { No - survey has } \\
\text { been completed }\end{array}$ & 24 \\
\hline $\begin{array}{l}\text { WP3LI007_PFO } \\
\text { (Ninth Crossing) }\end{array}$ & 18.1 & $\begin{array}{c}\text { TX-LI- } \\
029.000 \\
\end{array}$ & Private & $\begin{array}{l}\text { Originally } \\
\text { flooded tract }\end{array}$ & $\begin{array}{l}\text { No - survey has } \\
\text { been completed }\end{array}$ & 24 \\
\hline $\begin{array}{l}\text { WP3LI007_PFO } \\
\text { (Tenth Crossing) }\end{array}$ & 18.2 & $\begin{array}{c}\text { TX-LI- } \\
029.000\end{array}$ & Private & $\begin{array}{l}\text { Originally } \\
\text { flooded tract }\end{array}$ & $\begin{array}{l}\text { No - survey has } \\
\text { been completed }\end{array}$ & 24 \\
\hline $\begin{array}{c}\text { WP3LI007_PFO } \\
\text { (Eleventh Crossing) }\end{array}$ & 18.3 & $\begin{array}{c}\text { TX-LI- } \\
029.000 \\
\end{array}$ & Private & $\begin{array}{l}\text { Originally } \\
\text { flooded tract }\end{array}$ & $\begin{array}{l}\text { No - survey has } \\
\text { been completed }\end{array}$ & 25 \\
\hline WP3LI006_PSS & 18.3 & $\begin{array}{c}\text { TX-LI- } \\
029.000\end{array}$ & Private & $\begin{array}{l}\text { Originally } \\
\text { flooded tract }\end{array}$ & $\begin{array}{l}\text { No - survey has } \\
\text { been completed }\end{array}$ & 25 \\
\hline $\begin{array}{l}\text { WP3LI006_PFO (First } \\
\text { Crossing) }\end{array}$ & 18.3 & $\begin{array}{l}\text { TX-LI- } \\
029.000\end{array}$ & Private & $\begin{array}{l}\text { Originally } \\
\text { flooded tract }\end{array}$ & $\begin{array}{l}\text { No - survey has } \\
\text { been completed }\end{array}$ & 25 \\
\hline $\begin{array}{c}\text { WP3LI006_PFO } \\
\text { (Second Crossing) }\end{array}$ & 18.4 & $\begin{array}{l}\text { TX-LI- } \\
029.000\end{array}$ & Private & $\begin{array}{l}\text { Originally } \\
\text { flooded tract }\end{array}$ & $\begin{array}{l}\text { No - survey has } \\
\text { been completed }\end{array}$ & 25 \\
\hline WP3LI006_PEM & 18.4 & $\begin{array}{c}\text { TX-LI- } \\
029.000 \\
\end{array}$ & Private & $\begin{array}{l}\text { Originally } \\
\text { flooded tract }\end{array}$ & $\begin{array}{l}\text { No - survey has } \\
\text { been completed }\end{array}$ & 25 \\
\hline
\end{tabular}




\begin{tabular}{|c|c|c|c|c|c|c|}
\hline \multicolumn{7}{|c|}{ Orbit Permit Areas } \\
\hline Feature ID & Milepost & $\begin{array}{c}\text { Tract } \\
\text { ID }\end{array}$ & $\begin{array}{c}\text { Land } \\
\text { Ownership }\end{array}$ & $\begin{array}{c}\text { Reason } \\
\text { Surveys } \\
\text { Not } \\
\text { conducted }\end{array}$ & $\begin{array}{l}\text { Archeological } \\
\text { Monitor } \\
\text { Needed }\end{array}$ & $\begin{array}{c}\text { Appendix } \\
\text { B Aerial } \\
\text { Map Pg. } \\
\text { No. }\end{array}$ \\
\hline $\begin{array}{l}\text { WP3LI006_PFO } \\
\text { (Third Crossing) }\end{array}$ & 18.5 & $\begin{array}{l}\text { TX-LI- } \\
029.000\end{array}$ & Private & $\begin{array}{l}\text { Originally } \\
\text { flooded tract }\end{array}$ & $\begin{array}{l}\text { No - survey has } \\
\text { been completed }\end{array}$ & 25 \\
\hline $\begin{array}{l}\text { WP3LI006_PFO } \\
\text { (Fourth Crossing) }\end{array}$ & 18.6 & $\begin{array}{c}\text { TX-LI- } \\
029.000\end{array}$ & Private & $\begin{array}{c}\text { Originally } \\
\text { flooded tract }\end{array}$ & $\begin{array}{l}\text { No - survey has } \\
\text { been completed }\end{array}$ & 25 \\
\hline $\begin{array}{l}\text { WP3LI006_PFO } \\
\text { (Fifth Crossing) }\end{array}$ & 18.6 & $\begin{array}{l}\text { TX-LI- } \\
029.000\end{array}$ & Private & $\begin{array}{l}\text { Originally } \\
\text { flooded tract }\end{array}$ & $\begin{array}{l}\text { No - survey has } \\
\text { been completed }\end{array}$ & 25 \\
\hline $\begin{array}{l}\text { WP3LI006_PFO } \\
\text { (Sixth Crossing) }\end{array}$ & 18.8 & $\begin{array}{c}\text { TX-LI- } \\
029.000\end{array}$ & Private & $\begin{array}{c}\text { Originally } \\
\text { flooded tract }\end{array}$ & $\begin{array}{l}\text { No - survey has } \\
\text { been completed }\end{array}$ & 25 \\
\hline $\begin{array}{l}\text { WP3LI006_PFO } \\
\text { (Seventh Crossing) }\end{array}$ & 18.9 & $\begin{array}{l}\text { TX-LI- } \\
029.000 \\
\end{array}$ & Private & $\begin{array}{l}\text { Originally } \\
\text { flooded tract }\end{array}$ & $\begin{array}{l}\text { No - survey has } \\
\text { been completed }\end{array}$ & 25 \\
\hline $\begin{array}{l}\text { WP3LI002_PEM } \\
\text { (First Crossing) }\end{array}$ & 19 & $\begin{array}{l}\text { TX-LI- } \\
031.000\end{array}$ & Private & $\begin{array}{l}\text { Originally } \\
\text { flooded tract }\end{array}$ & $\begin{array}{l}\text { No - Avoided } \\
\text { via HDD/Bore }\end{array}$ & 26 \\
\hline WP3LI002_PSS_B & 19.1 & $\begin{array}{c}\text { TX-LI- } \\
031.000\end{array}$ & Private & $\begin{array}{l}\text { Originally } \\
\text { flooded tract }\end{array}$ & $\begin{array}{l}\text { No - Avoided } \\
\text { via HDD/Bore }\end{array}$ & 26 \\
\hline $\begin{array}{l}\text { WP3LI002_PEM } \\
\text { (Second Crossing) }\end{array}$ & 19.1 & $\begin{array}{c}\text { TX-LI- } \\
031.000\end{array}$ & Private & $\begin{array}{l}\text { Originally } \\
\text { flooded tract }\end{array}$ & $\begin{array}{l}\text { No - Avoided } \\
\text { via HDD/Bore }\end{array}$ & 26 \\
\hline $\begin{array}{l}\text { WP3LI002_PEM } \\
\text { (Third Crossing) }\end{array}$ & 19.1 & $\begin{array}{c}\text { TX-LI- } \\
031.000\end{array}$ & Private & $\begin{array}{l}\text { Originally } \\
\text { flooded tract }\end{array}$ & $\begin{array}{l}\text { No - Avoided } \\
\text { via } \mathrm{HDD} / \text { Bore }\end{array}$ & 26 \\
\hline WP3LI004 & 19.1 & $\begin{array}{l}\text { TX-LI- } \\
031.000\end{array}$ & Private & $\begin{array}{l}\text { Originally } \\
\text { flooded tract }\end{array}$ & $\begin{array}{l}\text { No - Avoided } \\
\text { via HDD/Bore }\end{array}$ & 26 \\
\hline $\begin{array}{l}\text { WP3LI005_PEM } \\
\text { (First Crossing) }\end{array}$ & 19.1 & $\begin{array}{c}\text { TX-LI- } \\
031.000\end{array}$ & Private & $\begin{array}{l}\text { Originally } \\
\text { flooded tract }\end{array}$ & $\begin{array}{l}\text { No - Avoided } \\
\text { via HDD/Bore }\end{array}$ & 26 \\
\hline WP3LI005_PFO_CYP & 19.2 & $\begin{array}{l}\text { TX-LI- } \\
031.000\end{array}$ & Private & $\begin{array}{l}\text { Originally } \\
\text { flooded tract }\end{array}$ & $\begin{array}{l}\text { No - Avoided } \\
\text { via } \mathrm{HDD} / \text { Bore }\end{array}$ & 26 \\
\hline WP4LI020_PSS_B & 26.5 & $\begin{array}{c}\text { TX-LI- } \\
054.910 \\
\end{array}$ & Private & $\begin{array}{l}\text { Originally } \\
\text { flooded tract }\end{array}$ & $\begin{array}{l}\text { No - Avoided } \\
\text { via HDD/Bore }\end{array}$ & 33 \\
\hline $\begin{array}{c}\text { WP4LI020_PSS (First } \\
\text { Crossing) }\end{array}$ & 26.5 & $\begin{array}{l}\text { TX-LI- } \\
054.910\end{array}$ & Private & $\begin{array}{l}\text { Originally } \\
\text { flooded tract }\end{array}$ & $\begin{array}{l}\text { No - Avoided } \\
\text { via HDD/Bore }\end{array}$ & 33 \\
\hline $\begin{array}{c}\text { WP4LI020_PEM } \\
\text { (First Crossing) }\end{array}$ & 26.5 & $\begin{array}{c}\text { TX-LI- } \\
054.910 \\
\end{array}$ & Private & $\begin{array}{l}\text { Originally } \\
\text { flooded tract }\end{array}$ & $\begin{array}{l}\text { No - Avoided } \\
\text { via HDD/Bore }\end{array}$ & 33 \\
\hline $\begin{array}{c}\text { WP4LI020_PSS } \\
\text { (Second Crossing) }\end{array}$ & 26.6 & $\begin{array}{c}\text { TX-LI- } \\
054.910\end{array}$ & Private & $\begin{array}{l}\text { Originally } \\
\text { flooded tract }\end{array}$ & $\begin{array}{l}\text { No - Avoided } \\
\text { via } \mathrm{HDD} / \text { Bore }\end{array}$ & 33 \\
\hline $\begin{array}{l}\text { WP4LI020_PSS } \\
\text { (Third Crossing) }\end{array}$ & 26.6 & $\begin{array}{l}\text { TX-LI- } \\
054.910\end{array}$ & Private & $\begin{array}{l}\text { Originally } \\
\text { flooded tract }\end{array}$ & $\begin{array}{l}\text { No - Avoided } \\
\text { via } \mathrm{HDD} / \text { Bore }\end{array}$ & 33 \\
\hline $\begin{array}{l}\text { WP4LI020_PEM } \\
\text { (Second Crossing) }\end{array}$ & 26.7 & $\begin{array}{c}\text { TX-LI- } \\
054.910\end{array}$ & Private & $\begin{array}{l}\text { Originally } \\
\text { flooded tract }\end{array}$ & $\begin{array}{l}\text { No - Avoided } \\
\text { via HDD/Bore }\end{array}$ & 33 \\
\hline WP4LI021_PSS & 29.5 & $\begin{array}{c}\text { TX-LI- } \\
063.000\end{array}$ & Private & None & $\begin{array}{l}\text { No - Avoided } \\
\text { via } \mathrm{HDD} / \text { Bore }\end{array}$ & 36 \\
\hline WP4LI021_PEM & 29.5 & $\begin{array}{l}\text { TX-LI- } \\
063.000\end{array}$ & Private & None & $\begin{array}{l}\text { No - Avoided } \\
\text { via HDD/Bore }\end{array}$ & 36 \\
\hline $\begin{array}{l}\text { WP9JE010_PFO_DT } \\
\text { (First Crossing) }\end{array}$ & 47.5 & $\begin{array}{c}\text { TX-JE- } \\
018.000\end{array}$ & Private & $\begin{array}{l}\text { Originally } \\
\text { flooded tract }\end{array}$ & $\begin{array}{l}\text { No - Avoided } \\
\text { via HDD/Bore }\end{array}$ & 55 \\
\hline WP9JE010_PEM_DT & 47.6 & $\begin{array}{c}\text { TX-JE- } \\
018.000 \\
\end{array}$ & Private & $\begin{array}{l}\text { Originally } \\
\text { flooded tract }\end{array}$ & $\begin{array}{l}\text { No - Avoided } \\
\text { via HDD/Bore }\end{array}$ & 56 \\
\hline $\begin{array}{c}\text { WP9JE010_PFO_DT } \\
\text { (Second Crossing) }\end{array}$ & 47.6 & $\begin{array}{l}\text { TX-JE- } \\
018.000\end{array}$ & Private & $\begin{array}{l}\text { Originally } \\
\text { flooded tract }\end{array}$ & $\begin{array}{l}\text { No - Avoided } \\
\text { via HDD/Bore }\end{array}$ & 56 \\
\hline
\end{tabular}




\begin{tabular}{|c|c|c|c|c|c|c|}
\hline \multicolumn{7}{|c|}{ Orbit Permit Areas } \\
\hline Feature ID & Milepost & $\begin{array}{c}\text { Tract } \\
\text { ID }\end{array}$ & $\begin{array}{c}\text { Land } \\
\text { Ownership }\end{array}$ & $\begin{array}{c}\text { Reason } \\
\text { Surveys } \\
\text { Not } \\
\text { conducted }\end{array}$ & $\begin{array}{l}\text { Archeological } \\
\text { Monitor } \\
\text { Needed }\end{array}$ & $\begin{array}{c}\text { Appendix } \\
\text { B Aerial } \\
\text { Map Pg. } \\
\text { No. }\end{array}$ \\
\hline WP4JE060 & 47.7 & $\begin{array}{l}\text { TX-JE- } \\
018.000 \\
\end{array}$ & Private & $\begin{array}{l}\text { Originally } \\
\text { flooded tract }\end{array}$ & $\begin{array}{l}\text { No - Avoided } \\
\text { via HDD/Bore }\end{array}$ & 56 \\
\hline SP9CH002_DT & 2.5 & $\begin{array}{l}\text { TX-CH- } \\
017.000 \\
\end{array}$ & Private & $\begin{array}{l}\text { Originally } \\
\text { flooded tract }\end{array}$ & $\begin{array}{l}\text { No - Avoided } \\
\text { via HDD/Bore }\end{array}$ & 5 \\
\hline SP9CH013_DT & 2.9 & $\begin{array}{l}\text { TX-CH- } \\
019.200\end{array}$ & Private & $\begin{array}{c}\text { Survey } \\
\text { permission } \\
\text { denied }\end{array}$ & $\begin{array}{l}\text { No - Avoided } \\
\text { via HDD/Bore }\end{array}$ & 5 \\
\hline $\begin{array}{l}\text { SP4LI004 (First } \\
\text { Crossing) }\end{array}$ & 17 & $\begin{array}{l}\text { TX-LI- } \\
029.000\end{array}$ & Private & $\begin{array}{l}\text { Flooded } \\
\text { tract }\end{array}$ & Yes & 23 \\
\hline SP3LI004 & 17.8 & $\begin{array}{c}\text { TX-LI- } \\
029.000 \\
\end{array}$ & Private & $\begin{array}{l}\text { Originally } \\
\text { flooded tract }\end{array}$ & $\begin{array}{l}\text { No - survey has } \\
\text { been completed }\end{array}$ & 24 \\
\hline SP3LI003 & 18.3 & $\begin{array}{l}\text { TX-LI- } \\
029.000\end{array}$ & Private & $\begin{array}{l}\text { Originally } \\
\text { flooded tract }\end{array}$ & $\begin{array}{l}\text { No - survey has } \\
\text { been completed }\end{array}$ & 25 \\
\hline $\begin{array}{l}\text { SP3LI001(First } \\
\text { Crossing) }\end{array}$ & 19 & $\begin{array}{c}\text { TX-LI- } \\
031.000 \\
\end{array}$ & Private & $\begin{array}{l}\text { Originally } \\
\text { flooded tract }\end{array}$ & $\begin{array}{l}\text { No - Avoided } \\
\text { via HDD/Bore }\end{array}$ & 26 \\
\hline $\begin{array}{l}\text { SP3LI001 (Second } \\
\text { Crossing) }\end{array}$ & 19 & $\begin{array}{c}\text { TX-LI- } \\
031.000\end{array}$ & Private & $\begin{array}{l}\text { Originally } \\
\text { flooded tract }\end{array}$ & $\begin{array}{l}\text { No - Avoided } \\
\text { via HDD/Bore }\end{array}$ & 26 \\
\hline $\begin{array}{l}\text { SP3LI002 (First } \\
\text { Crossing) }\end{array}$ & 19.1 & $\begin{array}{c}\text { TX-LI- } \\
031.000\end{array}$ & Private & $\begin{array}{l}\text { Originally } \\
\text { flooded tract }\end{array}$ & $\begin{array}{l}\text { No - Avoided } \\
\text { via HDD/Bore }\end{array}$ & 26 \\
\hline $\begin{array}{l}\text { SP3LI002 (Second } \\
\text { Crossing) }\end{array}$ & 19.1 & $\begin{array}{r}\text { TX-LI- } \\
031.000 \\
\end{array}$ & Private & $\begin{array}{l}\text { Originally } \\
\text { flooded tract }\end{array}$ & $\begin{array}{c}\text { No - Avoided } \\
\text { via HDD/Bore }\end{array}$ & 26 \\
\hline SP9LI018_DT & 22.7 & $\begin{array}{c}\text { TX-LI- } \\
041.000\end{array}$ & Private & $\begin{array}{c}\text { Survey } \\
\text { permission } \\
\text { denied }\end{array}$ & $\begin{array}{l}\text { No - Avoided } \\
\text { via HDD/Bore }\end{array}$ & 29 \\
\hline SP9LI016_DT & 23 & $\begin{array}{l}\text { TX-LI- } \\
041.000\end{array}$ & Private & $\begin{array}{c}\text { Survey } \\
\text { permission } \\
\text { denied }\end{array}$ & $\begin{array}{l}\text { No - Avoided } \\
\text { via HDD/Bore }\end{array}$ & 30 \\
\hline SP9LI017_DT & 23.4 & $\begin{array}{l}\text { TX-LI- } \\
041.000\end{array}$ & Private & $\begin{array}{c}\text { Survey } \\
\text { permission } \\
\text { denied }\end{array}$ & $\begin{array}{l}\text { No - Avoided } \\
\text { via HDD/Bore }\end{array}$ & 30 \\
\hline SP4LI009 & 26.5 & $\begin{array}{c}\text { TX-LI- } \\
054.910\end{array}$ & Private & $\begin{array}{l}\text { Flooded } \\
\text { tract }\end{array}$ & $\begin{array}{l}\text { No - Avoided } \\
\text { via HDD/Bore }\end{array}$ & 33 \\
\hline SP9JE012_DT & 46.3 & $\begin{array}{c}\text { TX-JE- } \\
014.110\end{array}$ & Private & $\begin{array}{c}\text { Flooded } \\
\text { tract }\end{array}$ & $\begin{array}{l}\text { No - Avoided } \\
\text { via } \mathrm{HDD} / \text { Bore }\end{array}$ & 54 \\
\hline SP9JE011_DT & 46.5 & $\begin{array}{l}\text { TX-JE- } \\
016.900\end{array}$ & $\begin{array}{c}\text { Public - } \\
\text { Jefferson } \\
\text { County } \\
\text { Drainage } \\
\text { District } 6 \\
\end{array}$ & $\begin{array}{l}\text { Survey } \\
\text { permission } \\
\text { denied }\end{array}$ & $\begin{array}{l}\text { No - Avoided } \\
\text { via HDD/Bore }\end{array}$ & 54 \\
\hline SP9JE010_DT & 46.9 & $\begin{array}{l}\text { TX-JE- } \\
017.000\end{array}$ & Private & $\begin{array}{c}\text { Survey } \\
\text { permission } \\
\text { denied }\end{array}$ & $\begin{array}{l}\text { No - Avoided } \\
\text { via HDD/Bore }\end{array}$ & 55 \\
\hline SP9JE009_DT & 47 & $\begin{array}{l}\text { TX-JE- } \\
017.000\end{array}$ & Private & $\begin{array}{c}\text { Survey } \\
\text { permission } \\
\text { denied }\end{array}$ & $\begin{array}{l}\text { No - Avoided } \\
\text { via HDD/Bore }\end{array}$ & 55 \\
\hline
\end{tabular}




\begin{tabular}{|c|c|c|c|c|c|c|}
\hline \multicolumn{7}{|c|}{ Orbit Permit Areas } \\
\hline Feature ID & Milepost & $\begin{array}{c}\text { Tract } \\
\text { ID }\end{array}$ & $\begin{array}{c}\text { Land } \\
\text { Ownership }\end{array}$ & $\begin{array}{c}\text { Reason } \\
\text { Surveys } \\
\text { Not } \\
\text { conducted }\end{array}$ & $\begin{array}{c}\text { Archeological } \\
\text { Monitor } \\
\text { Needed }\end{array}$ & $\begin{array}{c}\text { Appendix } \\
\text { B Aerial } \\
\text { Map Pg. } \\
\text { No. }\end{array}$ \\
\hline SP9JE008_DT & 47.2 & $\begin{array}{l}\text { TX-JE- } \\
017.902\end{array}$ & $\begin{array}{c}\text { Public - } \\
\text { Jefferson } \\
\text { County } \\
\text { Drainage } \\
\text { District } 6 \\
\end{array}$ & $\begin{array}{c}\text { Survey } \\
\text { Permission } \\
\text { denied }\end{array}$ & $\begin{array}{l}\text { No - Avoided } \\
\text { via HDD/Bore }\end{array}$ & 55 \\
\hline SP9JE007_DT & 47.4 & $\begin{array}{l}\text { TX-JE- } \\
018.000\end{array}$ & Private & $\begin{array}{c}\text { Survey } \\
\text { permission } \\
\text { denied }\end{array}$ & $\begin{array}{l}\text { No - Avoided } \\
\text { via HDD/Bore }\end{array}$ & 55 \\
\hline SP9JE019_DT & 47.5 & $\begin{array}{l}\text { TX-JE- } \\
018.000\end{array}$ & Private & $\begin{array}{c}\text { Survey } \\
\text { permission } \\
\text { denied }\end{array}$ & $\begin{array}{l}\text { No - Avoided } \\
\text { via HDD/Bore }\end{array}$ & 55 \\
\hline SP4JE049 & 47.6 & $\begin{array}{l}\text { TX-JE- } \\
018.000\end{array}$ & Private & $\begin{array}{c}\text { Survey } \\
\text { permission } \\
\text { denied }\end{array}$ & $\begin{array}{l}\text { No - Avoided } \\
\text { via HDD/Bore }\end{array}$ & 56 \\
\hline SP9JE050_DT & 47.8 & $\begin{array}{l}\text { TX-JE- } \\
018.000\end{array}$ & Private & $\begin{array}{c}\text { Survey } \\
\text { permission } \\
\text { denied }\end{array}$ & $\begin{array}{l}\text { No - Avoided } \\
\text { via HDD/Bore }\end{array}$ & 56 \\
\hline $\begin{array}{l}\text { SP9JE001_DT (First } \\
\text { Crossing) }\end{array}$ & 48.1 & $\begin{array}{l}\text { TX-JE- } \\
018.000\end{array}$ & Private & $\begin{array}{c}\text { Survey } \\
\text { permission } \\
\text { denied }\end{array}$ & $\begin{array}{l}\text { No - Avoided } \\
\text { via HDD/Bore }\end{array}$ & 56 \\
\hline $\begin{array}{c}\text { SP9JE001_DT } \\
\text { (Second Crossing) }\end{array}$ & 48.1 & $\begin{array}{l}\text { TX-JE- } \\
018.000\end{array}$ & Private & $\begin{array}{c}\text { Survey } \\
\text { permission } \\
\text { denied }\end{array}$ & $\begin{array}{l}\text { No - Avoided } \\
\text { via HDD/Bore }\end{array}$ & 56 \\
\hline SP9JE003_DT & 48.4 & $\begin{array}{l}\text { TX-JE- } \\
021.000\end{array}$ & Private & $\begin{array}{c}\text { Survey } \\
\text { permission } \\
\text { denied }\end{array}$ & $\begin{array}{l}\text { No - Avoided } \\
\text { via HDD/Bore }\end{array}$ & 56 \\
\hline SP9JE004_DT & 48.8 & $\begin{array}{l}\text { TX-JE- } \\
021.000\end{array}$ & Private & $\begin{array}{c}\text { Survey } \\
\text { permission } \\
\text { denied }\end{array}$ & $\begin{array}{l}\text { No - Avoided } \\
\text { via HDD/Bore }\end{array}$ & 57 \\
\hline SP9JE005_DT & 49 & $\begin{array}{l}\text { TX-JE- } \\
021.000\end{array}$ & Private & $\begin{array}{c}\text { Survey } \\
\text { permission } \\
\text { denied }\end{array}$ & $\begin{array}{l}\text { No - Avoided } \\
\text { via HDD/Bore }\end{array}$ & 57 \\
\hline SP9JE006_DT & 49.2 & $\begin{array}{l}\text { TX-JE- } \\
022.000\end{array}$ & Private & $\begin{array}{c}\text { Survey } \\
\text { permission } \\
\text { denied }\end{array}$ & $\begin{array}{l}\text { No - Avoided } \\
\text { via HDD/Bore }\end{array}$ & 57 \\
\hline
\end{tabular}


APPENDIX G: UnANTICIPATEd Discovery Plan 


\title{
Energy Transfer Company
}

Plan for the Unanticipated Discovery of Cultural Resources and Human Remains

\author{
Orbit Pipeline Project
}

October 2019 


\section{PLAN FOR THE UNANTICIPATED DISCOVERY OF CULTURAL RESOURCES AND HUMAN REMAINS}

\section{A. INTRODUCTION}

Energy Transfer Company (ETC) proposes to construct the Orbit Pipeline Project (Project) located in Jefferson, Liberty, and Chambers counties, Texas. The Project consists of approximately 68.7 miles (mi) (110.6 kilometer [km]) of new 20.0 inch (in) (50.8 centimeter [cm]) diameter pipeline that will be used to transmit ethane and propane. This document describes the procedures for dealing with unanticipated discoveries during the course of project construction. It is intended to:

- Maintain compliance with applicable Federal and State laws and regulations during construction of the Project;

- Describe to regulatory and review agencies the procedure the Project or its representative will follow to prepare for and deal with unanticipated discoveries; and

- Provide direction and guidance to project personnel as to the proper procedure to be followed should an unanticipated discovery occur.

\section{B. PROCEDURES FOR THE DISCOVERY OF CULTURAL RESOURCES}

In the event that any member of the construction work force believes that a cultural resource discovery is encountered the following plan will be implemented:

1. All work within 100 feet both sides of the discovery will immediately stop and the Environmental Inspector will be notified. The area of work stoppage will be adequate to provide for the security, protection, and integrity of the materials. A cultural resource can be prehistoric or historic and could consist of, but not be limited to:

- An accumulation of shell, burned rocks, ceramics or other subsistence related materials

- An area of charcoal or very dark soil with artifacts

- Stone tools, arrowheads, or dense concentrations of stone artifacts

- A cluster of bones in association with shell, charcoal, burned rocks, stone artifacts, ceramics, or other culturally-modified items. A historic structure or assemblage of historic materials older than 50 years

2. If the Environmental Inspector believes that the discovery is a cultural resource, the Environmental Inspector will take appropriate steps to protect the discovery site. This will include flagging the immediate area of discovery and stop work or exclusion zone, as well as notifying the Environmental Project Manager and/or Company Representative. Work in the immediate area will not resume until treatment of the discovery has been completed.

3. ETC or its representative will arrange for the discovery to be evaluated by a qualified archaeologist who meets the Secretary of Interior Standards for archeology. The archaeologist will evaluate the remains and provide recommendations for how to manage the resource under the State's Historic Preservation Plan. 
4. The archaeologist will seek consultation with the SHPO and US Army Corps of Engineer (USACE) staff archaeologist regarding the National Register eligibility status of the discovery. If the discovery is determined to have the potential for eligibility, the archaeologist will consult with the SHPO on how best to avoid, minimize, or otherwise mitigate further impacts. Treatment measures may include mapping, photography, sample collection, or excavation activity.

5. The archaeologist will implement the appropriate treatment measure(s) and provide a report on its methods and results. The investigation and technical report will be performed in compliance with the Secretary of the Interior's Standards and Guidelines for Archaeological Documentation (48 CFR 44734-44737); the Advisory Council on Historic Preservation (ACHP) publication "Treatment of Archaeological Properties" (ACHP 1980); and follow the guidelines set forth by the Texas State Historic Preservation Office.

\section{PROCEDURES FOR THE DISCOVERY OF HUMAN REMAINS}

In the event that human remains are encountered during either construction or maintenance activities, the following plan outlines the specific procedures to be followed. These procedures meet or exceed the Policy Statement Regarding Treatment of Burial Sites, Human Remains, and Funerary Objects adopted by the ACHP, "Protection of Historic and Cultural Properties" (36 CFR Part 800); Procedures for the Protection of Historic Properties (33 CFR 325 Appendix C); the Archaeological and Historic Preservation Act, and Texas Health and Safety Code (Title 8, Chapters 711-714).

All activity that might disturb the remains shall cease and may not resume until authorized by appropriate law enforcement officials or the State Archaeologist. Any human remains, burial sites, or burial related materials that are discovered during construction will at all times be treated with dignity and respect.

1. The Site Manager- or archaeologist, if present, will notify ETC's Environmental Project Manager, the law enforcement agency and the coroner of the jurisdiction where the site or remains are located within two days of the discovery. The State Archaeologist will also be contacted to assist with identifying the remains, as required. The USACE archeologist (Galveston District) will also be notified.

2. Any activity that may disturb the unmarked burial site, human skeletal remains, or burial artifacts associated with the site will immediately cease on discovery. The site will be carefully covered and secured for protection from degradation by weather or unauthorized individuals.

3. The Environmental Inspector will be responsible for taking appropriate steps to protect the discovery. This will include fencing off the immediate area of discovery and flagging the area as an exclusion zone. No activity may resume until authorized by the agency authority governing the disposition of the human remains.

4. If the unmarked burial site, human skeletal remains, or funerary objects can be shown to have ethnic affinity with a living Native American tribe, the Project's Environmental Manager will notify the SHPO and USACE to assist in determining the tribe(s), if any, 
who may have historic ties to the region and represent descendants of any Native American remains. If direct relations to a Native American tribe are verified, the tribe will have control of the disposition of the human skeletal remains.

5. If the District Coroner finds that the unmarked burial site is over 50 years old and that there is no need for a legal inquiry by their office or for a criminal investigation, and if no direct relations to any Native American tribe are found, then the SHPO will have jurisdiction of the site, human skeletal remains, and the burial artifacts.

\section{PROJECT CONTACTS}

\section{Environmental Inspector}

Attn: TBD

Phone:

\section{ETC Environmental Project Manager}

Attn: Jonathan Minton

Phone: (0) 713-989-2127

c 281-433-9428

\section{Texas Historical Commission}

Bill Martin

Team Lead, Review and Compliance

1511 Colorado, Austin, TX 78701

Phone: (512) 463-5867

Texas Health and Safety Code (Title 8, Chapters 711-714)

Maggie Moore

Regional Archeologist/ Project Reviewer

1511 Colorado, Austin, TX 78701

Phone: (512) 463-6508USACE Archaeologist

Attn: Jerry Androy

Phone: (409) 766-3821

\begin{tabular}{|c|c|c|}
\hline \multicolumn{3}{|c|}{ Sheriff Contact Information } \\
\hline Jefferson County & Address & Phone \\
\hline Sheriff & $\begin{array}{r}1001 \text { Pearl St. \#103 } \\
\text { Beaumont, TX 77701 }\end{array}$ & $409-835-8411$ \\
\hline Zena Stephens & Address & Phone \\
\hline Liberty County & $\begin{array}{c}\text { 2400 Beaumont Ave. } \\
\text { Liberty, TX 77575 }\end{array}$ & $936-336-4505$ \\
\hline Sheriff & \multicolumn{1}{|c|}{ Address } & Phone \\
\hline Bobby Rader & $\begin{array}{r}\text { 201 North Court } \\
\text { Anahuac, TX 77514 }\end{array}$ & 409-267-2500 \\
\hline Brian C. Hawthorne & \multicolumn{2}{|c|}{}
\end{tabular}

